\title{
Les hauts revenus face aux modifications des taux marginaux supérieurs de l'impôt sur le revenu en France, 1970-1996 *
}

Thomas Piketty (CNRS, URA928)

$n^{\circ} 9812$

* Etude réalisée dans le cadre d'un contrat RCB avec la Direction de la Prévision portant sur "L'impact du système fiscalo-social français sur l'offre de travail". Je remercie Jean-Philippe Cotis (directeur de la DP), Jean-Luc Tavernier (sousdirecteur de la sous-direction Finances Publiques (sous-direction D)), Jean-Paul Depecker (chef du Bureau des Etudes Fiscales (bureau D2)), ainsi que François Ecalle et Didier Eyssartier (respectivement sous-directeur de la sous-direction D et chef du bureau D2 lors du lancement de cette étude), pour leur soutien. Je remercie également Florence Toquet et Eric Morel (inspecteurs des impôts au BEF) pour leur aide précieuse, notamment lors de l'utilisation des fichiers de déclarations de revenus, ainsi que Olivier Gutman et Bertrand Saint-Aubin (administrateurs au BEF). Enfin, je remercie Bruno Rousselet et Pierre Cruchet (respectivement chef de bureau et inspecteur des impôts au Bureau C1 du Service de la Législation Fiscale), Pascal Ardilly et Christian Chataing (respectivement chef de bureau et inspecteur des impôts au bureau IIC de la Direction Générale des Impôts), et Stéphane Lollivier, JeanMichel Hourriez et Jean-Paul Lorgnet (Division "Revenus et patrimoine des ménages " de l'INSEE) pour l'aide qu'ils m'ont apportée à différentes étapes de cette étude. Les résultats et conclusions de cette étude sont uniquement ceux de leur auteur, et ils ne sauraient en aucun cas engager la Direction de la Prévision ou le Ministère des Finances. Tous les commentaires sont les bienvenus (thomas.piketty@cepremap.cnrs.fr). 
Les hauts revenus face aux modifications des taux marginaux supérieurs de l'impôt sur le revenu en France, 1970-1996

Résumé : Cette étude propose une estimation de l'élasticité du revenu imposable vis-à-vis des taux marginaux supérieurs de l'impôt sur le revenu en France. Pour cela, nous utilisons les deux plus fortes variations de taux marginaux des 25 dernières années (création de la tranche à $65 \%$ et plafonnement du quotient familial en 1981-1982, suppression de la tranche à $65 \%$ et abaissement des taux marginaux supérieurs en 1986-1987) comme des "expériences naturelles": nous comparons l'évolution du niveau et de la composition des revenus des contribuables concernés par ces variations de taux marginaux et des contribuables qui n'étaient pas concernés, et nous estimons l'ampleur des variations de revenu imposable qui peuvent être attribuées aux variations de taux marginaux. Afin de distinguer les éventuels effets incitatifs dus aux variations de taux marginaux des effets "mécaniques" du cycle économique sur la répartition des revenus, nous étudions les fluctuations de court-terme enregistrés par les hauts et les très hauts revenus sur l'ensemble de la période 1970-1996.

Les résultats obtenus suggèrent que les épisodes de 1981-1982 et 1986-1987 n'ont conduit à aucune variation importante des revenus concernés : la répartition des hauts revenus est globalement très stable sur la période 1970-1996, et les fluctuations observées s'expliquent principalement par le cycle économique, et non pas par la fiscalité. Les hauts revenus baissent en 1982-1983, mais ils retrouvent dès 1985 leur niveau de 1980-1981. De même, les hauts revenus progressent très rapidement durant le boom économique de 1986-1989, mais cette hausse est entièrement annulée par la récession de 1991-1993. De plus, en utilisant le fait que le mécanisme de plafonnement du quotient familial mis en place en 1981 conduit à des variations de taux marginaux à des niveaux de revenu imposable très différents suivant le nombre de parts de quotient familial, il apparaît que les baisses de 19821983 (comme les hausses de 1986-1989) n'ont pas été causées directement par les variations des taux marginaux effectifs d'imposition. Nous estimons des élasticités maximales de l'ordre de 0,1-0,2, c'est-à-dire des élasticités sensiblement inférieures aux niveaux requis pour que des baisses des taux marginaux supérieurs de l'IR puissent être auto-financées. 
High-Income Taxpayers' Reactions to Marginal Income Tax Rates Changes in France, 1970-1996

Abstract : This paper uses the main income tax law changes that occured in France over the 1970-1996 period as "natural experiments" in order to estimate the elasticity of high-income taxpayers' taxable income with respect to marginal tax rates. Given the large pro-cyclicity of the very-high-income taxpayers' income share over the entire period, we use annual tax returns data about the level and composition of income within the top decile and the top centile of the taxpayers distribution of taxable income. The general conclusion is that the relatively large changes in top marginal income tax rates (both in 1981-1982 and 1986-1987) did not induce any important structural change in the distribution. The distribution of taxable income among high-income taxpayers is extremly stable in France over the entire 1970-1996 period, and short-term fluctuations are better explained by the business cycle than by tax-induced behavioral changes.

Keywords : Income Taxation, Labor Supply, Income Distribution JEL Classification Numbers : H21, H22, J22 


\section{Introduction}

\subsection{Objectif et méthodologie de cette étude}

L'objectif de cette étude est d'estimer l'impact des taux marginaux supérieurs de l'impôt sur le revenu sur le comportement des contribuables en France. Les taux marginaux du barème de I'IR sont-ils actuellement à un niveau tel que tout relèvement significatif se traduirait par une diminution suffisamment forte du revenu imposable pour que les recettes fiscales diminuent? Autrement dit, le sommet de la "courbe de Laffer" a-t-il déjà été dépassé en France, et faut-il abaisser les taux marginaux supérieurs pour stimuler l'activité économique et permettre une progression des recettes fiscales? Ou bien doit-on considérer que les effets desincitatifs des taux marginaux de I'IR sont suffisamment faibles pour qu'un relèvement des taux permette d'accroître les recettes, et inversement que toute diminution des taux se traduise inévitablement par une perte de recettes ?

Dans le cadre de cette étude, nous nous intéresserons donc à l'ensemble des facteurs qui font que le revenu imposable des contribuables peut varier en réponse à des modifications des taux marginaux. De façon générale, les effets desincitatifs de taux marginaux élevés sur les comportements individuels peuvent en effet prendre différentes formes. Tout d'abord, des taux marginaux élevés peuvent potentiellement conduire les contribuables à choisir de travailler moins, c'est-à-dire à réduire leur "offre de travail". Mais cette éventuelle réduction de l'offre de travail doit être envisagée dans un sens large, et non dans le sens étroit d'une réduction de la durée du travail, qui est la seule variable d'ajustement prise en compte par la littérature économétrique traditionnelle sur "l'élasticité de l'offre de travail". Par exemple, on peut imaginer que des taux marginaux élevés conduisent des cadres à consacrer moins d'effort à leur stratégie de promotion interne et d'ascension professionnelle, des entrepreneurs ou des professions libérales à consacrer moins d'énergie à la progression de leurs bénéfices, ou même des possesseurs de patrimoines à consacrer moins d'attention aux rendements de leurs placements. Enfin, des taux marginaux élevés peuvent conduire certains contribuables à développer différentes stratégies d' "optimisation fiscale " (voire de fraude fiscale ou de délocalisation) de façon à réduire le montant de leurs revenus effectivement soumis à l'impôt. Toutes ces variables d'ajustement concourent à la formation du revenu imposable, et elles sont potentiellement beaucoup plus importantes que les variations de la durée du travail offerte par les salariés, notamment si l'on s'intéresse aux revenus élevés. Outre que la frontière exacte entre ces différents types de comportements peut en pratique être assez ambigue, tous ces effets desincitatifs ont la même valeur du point de vue des recettes fiscales: seul compte l'effet global des taux marginaux sur le montant du revenu imposable effectivement déclaré, indépendamment des comportements individuels spécifiques qui sont à l'origine de cet effet global. Dans cette étude, nous nous intéresserons donc non pas à "l'élasticité de l'offre de travail " (entendue dans un sens étroit), mais à "l'élasticité du revenu imposable ", c'est-à- 
dire à l'ampleur des variations de revenu imposable induites par des variations des taux marginaux d'imposition.

Comment mesurer une telle élasticité ? Par exemple, comment prédire quels seraient les conséquences d'un abaissement ou d'un relèvement du taux marginal supérieur de l'impôt sur le revenu (actuellement de 54\%) sur le niveau des revenus déclarés dans cette tranche d'imposition? La méthode la plus naturelle pour répondre à cette question consiste à étudier minutieusement quel a été l'impact des modifications de taux marginaux qui sont intervenues dans le passé sur le revenu imposable des contribuables concernés. Mais pour qu'une telle méthodologie puisse conduire à des estimations fiables, deux conditions doivent être remplies.

\subsubsection{Les " expériences naturelles" utilisées}

Tout d'abord, il est nécessaire que les modifications de taux marginaux étudiées soient d'une ampleur suffisamment importante, et qu'elles ne concernent qu'une fraction bien délimitée de la population, de façon à pouvoir comparer l'évolution du revenu imposable des contribuables touchés par la réforme et de ceux qui n'étaient pas concernés. Par exemple, il serait illusoire de vouloir estimer l'élasticité du revenu imposable à partir de la baisse des taux marginaux intervenue en 1996: le taux marginal supérieur est certes passé de $56,8 \%$ à $54 \%$, mais outre qu'il s'agit d'une baisse d'une très faible ampleur, tous les taux marginaux du barème ont été réduit dans les mêmes proportions, si bien qu'il est totalement impossible de distinguer l'effet éventuel de la fiscalité sur le niveau des revenus imposables des multiples autres effets dus au cycle économique et à des facteurs macroéconomiques.

Dans le cadre de cette étude, nous avons donc choisi de nous concentrer sur les deux seuls épisodes de la période contemporaine remplissant ces critères, à savoir la création en 1981-1982 d'une tranche d'imposition à 65\% et d'un mécanisme de plafonnement des effets du quotient familial, et la suppression en 1986-1987 de la tranche à $65 \%$, accompagnée de la baisse du taux de $60 \%$. Certes, ces variations de taux marginaux de 1981-1982 et 1986-1987 n'ont pas l'ampleur des réformes appliquées dans un certain nombre de pays étrangers, notamment aux Etats-Unis, où le taux marginal supérieur de l'impôt fédéral sur le revenu est passé de $70 \%$ en 1979 à $28 \%$ en 1986, avant de remonter à $39 \%$ en 1993. Mais il s'agit tout de même de variations importantes. Par exemple, les contribuables qui étaient concernés par la tranche à $65 \%$ de 1982 à 1985 ont vu leur taux marginal d'imposition passer de $65 \%$ pour leurs revenus de 1985 à $58 \%$ pour leurs revenus de 1986 puis $56,8 \%$ pour leurs revenus de 1987 et des années suivantes. Autrement dit, pour 1 franc supplémentaire de revenu imposable, ces contribuables conservaient 35 centimes jusqu'en 1985 et 43,2 centimes à partir de 1987, soit une progression de près de $25 \%$ de leur " taux de rétention ". ${ }^{1}$ Dans le même temps, les autres contribuables, et ${ }^{1}$ Le " taux de rétention" est défini comme l'inverse du taux marginal d'imposition : il mesure combien
de centimes restent à la disposition du contribuable quand son revenu imposable augmente de 1 
notamment ceux qui disposaient d'un revenu imposable par nombre de parts de quotient familial légèrement inférieur au seuil de l'ex-tranche à $60 \%$, voyaient leur taux marginal d'imposition inchangé (ou réduit dans des proportions insignifiantes). Si le niveau des taux marginaux avait des effets significatifs sur les comportements individuels, on devrait donc s'attendre à ce qu'ils se manifestent en une telle occasion. En prenant en compte à la fois la création de tranche à $65 \%$ et le plafonnement du quotient familial, l'alourdissement de l'imposition des hauts revenus intervenue en 1981-1982 a conduit à des variations du taux de rétention d'une ampleur comparable à celles de 1986-1987, et ces variations concernaient là encore un groupe spécifique de contribuables et non pas l'ensemble de la population. La mise en place en 1981 du mécanisme de plafonnement des effets du quotient familial constitue une "expérience naturelle" particulièrement significative, car ce mécanisme ne concernait ni les foyers de célibataires ayant 1 seule part de quotient familial, ni les foyers de couples mariés ayant seulement 2 parts de quotient familial. Par exemple, ce plafonnement a conduit un certain nombre de contribuables célibataires ayant 1,5 ou 2 parts à passer directement de la tranche à $40 \%$ en 1980 à la tranche à $55 \%$ en 1981 (soit une baisse de $25 \%$ de leur taux de rétention), alors que les contribuables célibataires ayant 1 part de quotient familial et le même niveau de revenu voyaient leur taux marginal inchangé. En comparant les évolutions observées à différents niveaux de revenu pour ces différents groupes de contribuables, on peut ainsi espérer identifier les effets des taux marginaux effectifs d'imposition sur les comportements individuels.

Précisons d'emblée que le fait que nous choisissions de nous concentrer sur les variations observées dans la tranche supérieure de l'IR ne signifie aucunement que cette tranche constitue le principal enjeu de l'impôt sur le revenu. Il est bien connu en effet que cette tranche a une importance budgétaire limitée (bien que non totalement négligeable dans un contexte budgétaire restrictif), du fait du faible nombre de contribuables concernés. Actuellement, environ 210000 foyers fiscaux (environ 0,7\% du nombre total de foyers) se trouvent dans la tranche supérieure du barème de l'IR, c'est-à-dire que 210000 foyers sont soumis à un taux marginal de $54 \%$ sur la fraction de leur revenu imposable par part de quotient familial supérieure à 291270 francs (pour les revenus de 1997). L'assiette totale de la tranche supérieure est de l'ordre de 75 milliards de francs, soit environ 2,9\% du revenu imposable total de l'ensemble des foyers fiscaux. ${ }^{2}$ Autrement dit, les recettes supplémentaires procurées par le relèvement de 1 point du taux marginal supérieur (de $54 \%$ à $55 \%$ ) de la tranche

\footnotetext{
franc. Le passage d'un taux marginal de $65 \%$ à un taux marginal de $56,8 \%$ équivaut donc à une augmentation d'environ $23,4 \%$ du taux de rétention, qui passe de $35 \%$ à $43,2 \%(8,2 / 35=0,234)$.

${ }^{2}$ Les 210000 foyers de la tranche supérieure détiennent environ $6,7 \%$ du revenu imposable total, mais seule la fraction de leurs revenus supérieure à 291270 francs par part est soumise au taux marginal de 54\%, ce qui conduit à diviser par 2,3 environ l'assiette considérée. Ces estimations du rendement de la tranche supérieure ont été obtenues à partir de l'échantillon léger de déclarations de revenus 1995 (cf. Annexe F), après prise en compte des effets du plafonnement du quotient familial. Sans prise en compte du plafonnement du quotient familial, seuls 120000 foyers environ seraient concernés par la tranche supérieure (environ $0,4 \%$ des foyers), pour une assiette totale d'environ 55 milliards de francs.
} 
supérieure seraient de l'ordre de 750 millions de FF, de même que les recettes perdues en cas d'abaissement de 1 point (en supposant que les revenus déclarés demeurent inchangés dans les deux cas). Si l'on pouvait taxer à un taux marginal de $100 \%$ les revenus de la tranche supérieure (ce qui reviendrait à imposer un revenu légal maximal de 291270 francs par part de quotient familial) sans induire la moindre perte de revenu déclaré, alors les recettes supplémentaires seraient de l'ordre de 35 milliards de francs, soit à peine plus de $10 \%$ des recettes totales de l'IR (environ 300 milliards). La raison pour laquelle nous nous concentrons sur la tranche supérieure découle simplement du fait qu'aucune autre tranche du barème de I'IR n'a connu des variations de taux marginaux aussi importantes que la tranche supérieure, si bien que la tranche supérieure nous fournit la seule " expérience naturelle " d'une ampleur suffisante que nous puissions utiliser (avec le plafonnement des effets du quotient familial). En outre, l'avantage d'une estimation fondée sur les hauts revenus de la tranche supérieure est que les élasticités obtenues peuvent être considérées comme des bornes supérieures pour les élasticités caractérisant les autres groupes de revenus. En effet, les hauts revenus, en particulier du fait de la multiplicité de leurs sources de revenus et de la prépondérance de leurs revenus non-salariaux, disposent potentiellement de beaucoup plus d'opportunités que les revenus moyens pour faire varier leur revenu imposable en fonction de leurs incitations financières. Les hauts revenus sont donc vraisemblablement plus "élastiques " que les revenus moins élevés. Par exemple, s'il apparaissait que des variations importantes du taux marginal supérieur induisent des variations faibles des revenus de la tranche supérieure, alors on pourrait raisonnablement en déduire qu'il en irait de même pour les tranches inférieures. Bien que la tranche supérieure de l'IR ait en elle-même une importance budgétaire relativement limitée, l'étude des fluctuations de la tranche supérieure permet donc d'évaluer les effets incitatifs d'éventuelles réformes aux conséquences budgétaires beaucoup plus importantes, telles que les réformes de 1993 ou $1996 .^{3}$

\subsubsection{La nécessité d'utiliser des données annuelles sur longue période}

La deuxième condition indispensable pour qu'une telle méthodologie d'estimation de l'élasticité du revenu imposable soit viable est de disposer de données adéquates. Pour espérer pouvoir isoler les éventuels effets de variations des taux marginaux d'imposition, il est indispensable de disposer de données annuelles sur la distribution du revenu imposable portant sur une période suffisamment longue. En effet, outre d'éventuels trends de longue période, les hauts revenus connaissent des variations liées au cycle économique d'une ampleur extrêmement importante (comparés aux autres revenus). Si l'on se contentait de comparer les distributions observées pendant quelques années consécutives, ou bien les distributions observées sur longue période mais à des dates espacées dans le temps, on risquerait donc d'attribuer à des variations de taux marginaux des évolutions qui n'ont en fait rien à

\footnotetext{
${ }^{3}$ Pour un rappel du coût budgétaire de ces réformes, cf. section 2.2, tableau 2-4 infra.
} 
voir avec la fiscalité (et inversement). C'est pourquoi les études de Lindsey (1987), qui évalue l'effet de la réforme fiscale américaine de 1981 à partir des distributions des années 1980-1984, et de Feldstein (1995), qui évalue l'effet de la réforme de 1986 à partir des distributions des années 1985 et $1988,{ }^{4}$ ne font guère l'unanimité aux Etats-Unis. De plus, l'expérience américaine a montré qu'une forte variation des taux marginaux concernant les hauts revenus pouvait parfois induire des variations très importantes des revenus déclarés durant l'année suivant immédiatement la réforme, bien que ces variations puissent disparaître ensuite au bout de quelques années. Par exemple, Goolsbee (1997) a montré de façon convaincante que le relèvement à 39\% du taux marginal supérieur de l'IR américain intervenu en 1993 avait causé en 1993 une baisse importante du revenu déclaré par les contribuables concernés, mais que dès 1994 les hauts revenus avaient repris leur trend des années précédentes. La baisse purement temporaire observée en 1993 était due en grande partie à la baisse des plus-values, qui aux Etats-Unis sont soumises au barème progressif, et que les contribuables concernés ont massivement réalisé en 1992 (dès l'annonce de la réforme applicable en 1993), et plus généralement aux multiples formes de rémunérations dont les hauts revenus peuvent décider d'avancer ou de retarder de quelques années la date officielle d'encaissement (primes de cadres supérieurs, bénéfices de non-salariés, etc..). Goolsbee en conclut que la véritable élasticité à long-terme du revenu imposable est en fait extrêmement faible, et en particulier très inférieure au niveau requis pour qu'un relèvement de taux tel que celui de 1993 conduise à une baisse de recettes fiscales. ${ }^{5}$ Afin de distinguer rigoureusement les effets dus à des fluctuations de court-terme et/ou à des trends de long-terme des éventuels effets dus aux variations de taux marginaux intervenues en France en 1981-1982 et 1986-1987, et d'espérer ainsi pouvoir mesurer de véritables élasticités de long-terme, nous avons donc utilisé dans le cadre de cette étude des données annuelles portant sur la distribution du revenu imposable sur la période 1970-1996. L'avantage de faire débuter notre période d'étude à l'année 1970, outre que ce choix permet de mieux identifier les éventuels trends précédent l'épisode de 1981-1982, est que cela nous offre l'opportunité d'étudier l'impact de la crise de 1974-1975 sur les hauts revenus, ce qui apporte des informations utiles sur les effets habituellement observés du cycle économique sur les hauts revenus.

\subsection{Sources et données utilisées}

\footnotetext{
${ }^{4}$ Cf. également Navratil (1995), qui applique la même méthode que Feldstein pour estimer l'effet de la réforme de 1981 (Navratil utilise un panel de déclarations pour les années 1980 et 1983, alors que Feldstein(1995) utilise un panel de déclarations pour les années 1985 et 1988 ; Lindsay(1987) utilise des échantillons de déclarations pour les années 1980, 1981, 1982, 1983 et 1984, mais sans identifiant individuel permettant de suivre les mêmes contribuables, contrairement à Feldstein et Nivratil).

${ }^{5}$ Cf. également Slemrod(1995a, 1995b) et Gordon et Slemrod(1997), qui montrent que l'essentiel de la progression des hauts revenus observée entre 1986 et 1988 est en fait due à un transfert entre l'impôt sur les sociétés et l'impôt sur le revenu individuel, sans que l'assiette totale augmente sensiblement (l'élasticité calculée par Feldstein(1995), qui oublie de prendre en compte ce phénomène, est donc fortement surévaluée, indépendamment du problème des fluctuations cycliques).
} 
Idéalement, une telle étude devrait être menée à partir d'une série d'échantillons représentatifs de déclarations de revenus permettant d'étudier de façon fine l'évolution de la répartition des hauts et des très hauts revenus soumis à I'IR sur la période 1970-1996. Malheureusement, les échantillons de déclarations constitués chaque année par la DGI ne sont disponibles sous un format informatique exploitable que pour la période 1988-1996. L'INSEE effectue des enquêtes sur les "Revenus fiscaux" des ménages à partir d'échantillons de déclarations de revenus depuis 1956, mais ces enquêtes ne sont menées que tous les 5-6 ans, si bien qu'elles ne permettent pas de prendre en compte correctement la cyclicité des hauts revenus. En outre, contrairement aux échantillons constitués par la DGI, qui au-delà d'un certain niveau de revenu contiennent toutes les déclarations de revenus déposées en France métropolitaine, le taux de sondage des enquêtes "Revenus fiscaux" de I'INSEE ne permet pas une étude suffisamment fine des très hauts revenus.

Il est donc apparu que la seule source statistique permettant d'étudier l'évolution de la distribution du revenu imposable de façon continue sur longue période était constituée par les états statistiques portant sur l'ensemble des déclarations déposées par les contribuables (les "Etats 1921 "), et que l'administration fiscale établit chaque année depuis que l'IR existe. Ces statistiques fiscales ne peuvent cependant pas être utilisées à l'état brut. En effet, les Etats 1921 contiennent des tableaux statistiques sur le nombre de contribuables et le montant des revenus déclarés en fonction d'un certain nombre de tranches de revenu imposable exprimées en francs courants (les seuils nominaux des tranches utilisées dans ces tableaux étant ajustées relativement peu fréquemment), si bien que l'inflation et la croissance générale des revenus réels rendent les chiffres bruts difficilement comparables d'une année sur l'autre. Nous avons donc utilisé les chiffres de ces tableaux pour estimer chaque année les paramètres structurels de la distribution du revenu imposable des hauts et des très hauts revenus, paramètres qui nous ont ensuite permis d'estimer le niveau et la composition des revenus des $10 \%$ des foyers fiscaux ayant le revenu imposable le plus élevé, des $5 \%$ ayant le revenu imposable le plus élevé, des $1 \%$, des 0,5\%, etc..., pour chaque année de la période 1970-1996. Tous les résultats et analyses présentés dans cette étude sont issus de cette procédure d'estimation, les échantillons de déclarations de la DGI (1988-1995) et les enquêtes "Revenus fiscaux" de l'INSEE (1970, 1975, 1979, 1984 et 1990) ayant uniquement été utilisés pour tester la fiabilité de nos estimations. De fait, cette procédure permet d'obtenir des estimations extrêmement précises de l'évolution des hauts revenus, avec des marges d'erreur totalement négligeables comparées à l'ampleur des fluctuations observées d'une année sur l'autre, car la distribution des hauts revenus est remarquablement bien approximée par une loi de Pareto. La propriété caractéristique de la loi de Pareto est que le revenu moyen des contribuables dont le revenu est supérieur à un certain seuil de revenu est toujours égal au produit d'une constante $b$ et de ce seuil de revenu, cette constante $b$ ne dépendant pas (ou peu) du seuil de revenu considéré (en France, b est de l'ordre de 
1,7-1,8 sur l'ensemble de la période 1970-1996). II suffit donc d'estimer ces paramètres structurels pour une année donnée pour connaître l'ensemble de la distribution des hauts revenus de cette année. Une méthodologie similaire avait déjà été utilisée par Feenberg et Poterba (1993) afin d'estimer l'évolution des centiles supérieurs de la distribution américaine du revenu imposable sur la période 19501989. En comparant les résultats de leurs estimations aux échantillons de déclarations disponibles pour la période récente, Feenberg et Poterba aboutissent également à la conclusion que cette procédure d'estimation conduit à des résultats extrêmement fiables. ${ }^{6}$ Le choix des sources statistiques mobilisables pour cette étude, l'ensemble des tableaux statistiques et des procédures d'estimation utilisées, ainsi que les résultats des principaux tests de fiabilité effectués, sont décrits de façon détaillée dans une série d'annexes techniques placées à la fin de ce texte (cf. Annexes $\mathrm{A}$ à $\mathrm{H}$ ).

Apparemment, les statistiques fiscales des Etats 1921 n'ont jamais été utilisé de cette façon. En particulier, les rapports du Conseil des Impôts portant sur l'impôt sur le revenu se contentent de reproduire les chiffres bruts issus des Etats 1921, sans aucune tentative pour homogénéiser les chiffres ou établir des séries continues sur la répartition des revenus imposables. ${ }^{7}$ II en va de même des études sur l'impôt sur le revenu effectuées par la DGl et publiées chaque année dans "Statistiques et Etudes Financières " jusqu'à la disparition de cette publication du Ministère des Finances dans les années 1980, qui se contentent généralement de décrire les principales variations enregistrés dans les Etats 1921 d'une année sur l'autre. ${ }^{8}$ L'INSEE a consacré des publications importantes à chacune des enquêtes "Revenus fiscaux" effectuées depuis 1956, mais, outre que ces études raisonnent en termes de

\footnotetext{
${ }^{6}$ Feenberg et Poterba disposent d'échantillons de déclarations depuis les revenus de 1979 (le US Treasury diffuse de tels échantillons depuis 1968, mais le NBER ne les utilise que depuis 1979), ce qui leur permet de tester la fiabilité de l'approximation par une loi de Pareto dans une période de transformation extrêmement rapide de la distribution américaine des revenus : la coefficient $b$ (ratio entre le revenu moyen et le seuil de revenu considéré) passe progressivement d'environ 1,8 en 1979 à environ 2,7 en 1988-1989, mais leurs estimations restent toujours aussi précises (leur estimation de la part du revenu imposable détenue par les $0,5 \%$ les plus riches passent de $6,04 \%$ en $1979(6,06 \%$ d'après l'échantillon de déclarations de revenus 1979) à $12,02 \%$ en 1988 (12,05\% d'après l'échantillon de déclarations de revenus 1988)).

${ }^{7}$ Le Conseil des Impôts a consacré ses rapports annuels à l'impôt sur le revenu en 1972, 1974, 1979, 1984 et 1990 (les trois premiers rapports ont été publiés dans "Statistiques et Etudes Financières", série bleue, $n^{\circ} 287$ (1972), $n^{\circ} 311$ (1974) et $n^{\circ} 361-362$ (1979)). Ces rapports constituent des sources précieuses, notamment pour tout ce qui concerne l'évolution de la législation et les comparaisons internationales. Outre les Etats 1921, qui constituent toujours la source statistique essentielle, ces rapports utilisent également parfois les enquêtes "Revenus fiscaux" (cf. annexe G). Le fait que ces rapports ne se posent jamais directement la question des éventuels effets desincitatifs de l'impôt sur le revenu s'explique sans doute en partie par des considérations politiques: par exemple, on imagine mal que le Conseil des Impôts en 1984 se saisisse de la question brûlante des éventuels fuites de hauts revenus consécutives à l'alternance de $1981 \ldots$

${ }^{8} \mathrm{Cf}$. Annexe B.3. II en va de même des grands rapports administratifs sur les prélèvements. Cf. par exemple le rapport de la commission Ducamin consacré à l'étude des prélèvements pesant sur les ménages, dont l'annexe 23, intitulée "Evolution de la structure des revenus élevés déclarés à l'impôt sur le revenu", se contente en fait de reproduire les chiffres bruts des Etats 1921 concernant la composition des revenus imposables supérieurs à 400000 ou 500000 francs courants de 1981 à 1992, sans chercher à corriger le fait que la proportion de contribuables concernés a été multiplié par plus de 2 durant cette période (cf. tableau D-1, annexe D.1).
} 
ménages et non de foyers fiscaux et ne qu'elles ne s'intéressent pas spécifiquement aux hauts revenus, I'INSEE n'a apparemment jamais cherché à exploiter les statistiques fiscales annuelles issues des Etats $1921 .^{9}$

De façon générale, les travaux portant sur l'impôt sur le revenu en France sont relativement peu nombreux. Les travaux d'origine non administrative sont principalement le fait des juristes, dont les ouvrages portent exclusivement sur la législation en cours et ne contiennent généralement aucune analyse statistique des revenus déclarés. ${ }^{10}$ Les quelques travaux sur I'IR effectués par des économistes sont généralement consacrés à l'analyse descriptive et prospective de la progressivité de l'impôt et de son évolution en tant que telles, ${ }^{11}$ et non pas à l'estimation empirique des éventuels effets des taux marginaux d'imposition sur les comportements individuels. Les rares estimations sur données françaises de l'élasticité de l'offre de travail confirment les résultats habituels de la littérature économétrique traditionnelle, à savoir que les seules populations pour lesquelles l'élasticité n'est pas voisine de 0-0,1 (valeurs habituellement obtenues pour les populations ayant déjà un emploi) sont les populations se trouvant à la frontière entre l'emploi et le non-emploi et dont les comportements de participation au marché du travail sont relativement élastiques, notamment pour ce qui est de l'offre de travail des femmes. ${ }^{12}$ Mais il s'agit là d'estimations de l'élasticité de l' "offre de travail" entendue au sens strict de la durée du travail offerte, et non d'estimations de l'élasticité du revenu imposable, si bien que ces estimations peuvent difficilement être utilisées pour estimer le niveau de la pression fiscale limite pour les contribuables aisés, dont les comportements susceptibles d'être affectés par les taux marginaux d'imposition sont d'une nature différente (cf. section 1.1. supra). En dehors de ces estimations de l'élasticité de l'offre de travail, les seules études sur données françaises des phénomènes de type "courbe de Laffer " se résument à des comparaisons au niveau macroéconomique entre l'évolution des recettes totales de l'Etat et des taux globaux d'imposition, ${ }^{13}$ méthodologie très fruste et qui a déjà été abondamment critiquée (à juste titre) ${ }^{14}$ En particulier, aucune étude ne semble avoir

\footnotetext{
${ }^{9} \mathrm{Cf}$. Annexe G pour la liste des publications de l'INSEE consacrées aux enquêtes "Revenus fiscaux" depuis 1970.

${ }^{10}$ L'excellent livre de droit de David (1987) est une exception à cette règle, puisqu'il inclut également un grand nombre de tableaux statistiques issus des rapports du Conseil des Impôts et des études publiées par l'INSEE et dans "Statistiques et Etudes Financières ", sans toutefois proposer d'analyse statistique originale.

${ }^{11}$ Cf. par exemple Maillard (1989), Trannoy, Jehanne, Lebreton et Moyes (1991), Roquet, Rafaliarison et Trannoy (1993), et Bourguignon et Chiappori (1997). Maillard propose plusieurs mesures de la progressivité de l'IR en France. Trannoy et al. étudient dans quelle mesure les différents barèmes de I'IR et des cotisations sociales depuis 1959 peuvent être ordonnées en termes de dominance au sens de Lorenz (la réponse étant largement négative). Bourguignon et Chiappori étudient dans quelle mesure la progressivité actuelle du couple IR/prestations sociales pourrait être égalée ou dépassée par un système d'allocation universelle et de taux marginal unique.

${ }_{12}$ Pour un survey relativement récent des études internationales sur l'élasticité de l'offre de travail, cf. Blundell (1995). Pour des études sur données françaises, cf. par exemple Bourguignon et Magnac (1985) et Piketty (1998) et les références citées dans ces articles.

${ }_{13}$ Cf. par exemple Fourçans (1985).

${ }^{14}$ Cf. par exemple Fleurbaey, Ould Biya, Ralle et Rousse (1987) et Théret et Uri (1987).
} 
tenté de comparer l'évolution des revenus déclarés par différents groupes de revenus en fonction des variations de la pression fiscale auxquels ils sont soumis. ${ }^{15}$ Ce vide relatif contraste singulièrement avec la vigueur des propos souvent tenus au sujet des effets desincitatifs de l'impôt. ${ }^{16}$

\subsection{Organisation de ce texte}

\section{Cette étude est organisée de la façon suivante.}

La section 2 commence par rappeler les grandes lignes de l'évolution générale de l'impôt sur le revenu de 1970 à 1996 , afin de situer le contexte général dans lequel s'insèrent les épisodes étudiés. La section 2.1 présente les grandes évolutions des règles de calculs de l'IR de 1970 à 1996, et la section 2.2 décrit la croissance générale du nombre de foyers fiscaux, des revenus imposables et des recettes de I'IR de 1970 à 1996.

La section 3 décrit ensuite l'évolution générale de la distribution du revenu imposable de l'ensemble des foyers fiscaux (tous nombres de parts de quotient familial confondus) de 1970 à 1996. Nous commençons par fixer les ordres de grandeur atteints par les hauts, les très hauts et les très hauts revenus au milieu des

\footnotetext{
${ }^{15}$ Le " travail " dont la méthodologie se rapproche le plus de celle que nous mettons en oeuvre ici est celui de Lacoude (1995). Mais cette "étude empirique de l'effet Laffer en France " se résume en fait à un unique tableau, sans aucune précision sur les sources et la méthodologie utilisées (cf. tableau 8 , p.141; "Source: Ministère des Finances ” ...), qui prétend donner des chiffres portant sur la part du revenu imposable total détenue par le centile supérieur et les centiles immédiatement inférieurs de la distribution du revenu imposable en 1979, 1984, 1988, 1990 et 1991, et selon lesquels la part des $1 \%$ les plus riches serait passer de 1,83\% du revenu total en 1984 à environ $8 \%$ en 1988-1991, preuve, selon l'auteur, que les baisses d'impôts de 1986-1987 ont puissamment stimulé l'offre de travail des contribuables concernées! Bien évidemment, ces chiffres sont totalement farfelus : jamais personne n'a observé un quadruplement du revenu relatif des $1 \%$ les plus riches en 4 ans, et le simple bon sens devrait conduire à rejeter l'idée que la part des $1 \%$ les plus riches puisse être en 1984 de moins de $2 \%$ du revenu total (ce qui impliquerait que les $1 \%$ les plus riches ont un revenu moyen qui est moins de 2 fois plus élevé que le revenu moyen de l'ensemble de la distribution, ce qui là encore ne s'est tout simplement jamais vu...). Nos résultats montrent que les variations de la part des $1 \%$ les plus riches, des $0,5 \%$ etc..., qu'elles soient causées par le cycle économique ou par la fiscalité, sont toujours d'une ampleur beaucoup plus faible (cf. par exemple les tableaux 3-3 à 3-5, section 3.2). Après contact avec l'auteur, il s'est avéré impossible de déterminer avec certitude si les chiffres totalement farfelus de 1979 et 1984 (les chiffres de 1988-1991 sont vaguement réalistes) étaient dus à une erreur grossière (mais sincère) de calcul ou à une tentative délibérée de manipulation des chiffres. Le fait qu'un tel article puisse être publiée dans la "Revue Française d'Economie " mérite d'être retenu (le reste de l'article se contente de réciter les anecdotes de la littérature de gare américaine sur la courbe de Laffer).

${ }^{16}$ Par exemple, le Projet de Loi de Finances pour 1987 annonce que "dans le cadre de la politique d'abaissement de la pression fiscale poursuivie par les pouvoirs publics, et afin d'éviter les effets décourageants des prélèvements actuels, la tranche à $65 \%$ serait supprimée dès l'imposition des revenus de 1986 ; le taux le plus élevé serait ramené à 58\% " (exposé des motifs de l'article 2, PLF87, pp.16-17). Cette formulation laisse donc clairement entendre que les revenus déclarés par les contribuables concernés allaient progresser grâce à cette mesure. Pourtant, le chiffrage du PLF87, tout comme le chiffrage du PLF83 lors de la création de la tranche à $65 \%$, ne prend en compte aucun effet incitatif éventuel et se contente de donner le coût mécanique de la mesure à assiette fiscale inchangée (le coût de la suppression du taux d'imposition de 65\% est chiffré à 1,520 milliard, et celui de la limitation du taux supérieur à $58 \%$ à 700 millions; cf. PLF87, "Evaluation des voies et moyens", pp.11-12). Aucune étude émanant du gouvernement ou des partis politiques ne semble être revenue ultérieurement sur le chiffrage des "effets décourageants des prélèvements actuels".
} 
années 1990, ordres de grandeur qu'il est utile d'avoir présent à l'esprit pour la suite de cette étude (section 3.1). Puis la section 3-2 décrit l'évolution de la part du revenu imposable total détenue par les $10 \%$ des foyers fiscaux (tous nombres de parts confondus) ayant le revenu imposable le plus élevé, des $5 \%$ ayant le revenu imposable le plus élevé, des $1 \%$, des $0,5 \%$, etc.., sur la période 1970-1996. La section 3-3 présente l'évolution de la composition du revenu imposable de ces différents groupes de contribuables de 1970 à 1996.

La section 4 contient l'ensemble des estimations de l'élasticité du revenu imposable. La section 4-1 présente des estimations obtenues à partir de l'évolution de la distribution du revenu imposable des foyers ayant 1 part de quotient familial. Puis les sections 4-2 et 4-3 présentent des estimations obtenues à partir de l'évolution de la distribution du revenu imposable des foyers de célibataires ( $Q F=1,1,5$ ou 2B) et des foyers de couples mariés ( $Q F=2 A, 2,5$ ou 3 ). Les estimations des sections 4.2 et 4.3 confirment globalement les estimations obtenues en section 4.1 à partir des foyers fiscaux ayant 1 part de quotient familial et permettent de les affiner sur certains points, notamment en utilisant le fait que le mécanisme du quotient familial et son plafonnement à partir de 1981 impliquent que les variations de taux marginaux s'appliquent à des niveaux de revenus qui dépendent de façon fine du nombre de parts de quotient familial. Toutes les estimations de l'élasticité du revenu imposable présentés dans cette section sont des estimations obtenues de façon "simple", c'est-à-dire en comparant " à la main " les évolutions des revenus concernés par des modifications de taux marginaux et des revenus qui n'étaient pas concernés et en rapportant ces évolutions à l'ampleur des modifications de taux marginaux susceptibles de les avoir causées. ${ }^{17}$

Enfin, la section 5 conclut.

Une série d'annexes techniques a été placée à la fin du texte. Outre les annexes $A$ à $H$, qui décrivent de façon détaillée l'ensemble des données et des procédures d'estimation utilisées, la liste des annexes inclut l'annexe I, qui décrit l'évolution des seuils de plafonnement du quotient familial depuis sa mise en place en 1981, seuils que nous utilisons dans la section 4 pour calculer les taux marginaux effectifs en fonction du niveau de revenu et du nombre de parts de quotient familial, ainsi que l'annexe J, qui contient l'ensemble des références citées dans le texte.

\footnotetext{
17 L'autre possibilité, en apparence plus sophistiquée, consisterait à régresser la part du revenu imposable des différents fractiles (P90-95, P95-99, P99-99,5, etc..) sur une liste de variables explicatives incluant non seulement toutes les modifications de taux marginaux applicables à ces différents fractiles, mais également une série de variables dummies permettant de prendre en compte les éventuels effets du cycle économique, et ce sur l'ensemble de la période 1970-1996. Cette technique aurait l'avantage d'exploiter toute l'information disponible en une seule fois et de conduire à une estimation globale de l'élasticité du revenu imposable (cf. par exemple Saez (1998a)). Cependant, outre qu'une telle régression conduirait à des résultats similaires à ceux présentés ici, le prix à payer est que cette technique aboutit à obscurcir considérablement la source exacte des estimations obtenues : on ne sait plus très bien quelles années et quelle combinaison de variables explicatives ont produit tel ou tel résultat. Nous avons donc choisi de présenter des estimations intelligibles par tous, et de laisser le lecteur intéressé effectuer ses propres estimations à partir des données brutes présentées dans les tableaux de résultats.
} 


\section{L'impôt sur le revenu de 1970 à 1996 : quelques données de cadrage}

\subsection{Les règles de calcul de l'impôt sur le revenu de 1970 à $1996{ }^{18}$}

\subsubsection{L'évolution du barème de l'impôt sur le revenu}

Le tableau 2-1, qui décrit l'évolution des barèmes de l'impôt sur le revenu applicables aux revenus des années 1970 (déclarés en 1971) à 1997 (déclarés en 1998), montre que la structure générale du barème n'a guère évolué sur la période 1970-1997. Les barèmes décrits sur le tableau 2-1 sont les barèmes "bruts ", i.e. avant prise en compte des éventuels décotes, majorations/minorations, réductions d'impôt, plafonnement des effets du quotient familial, etc... (cf. infra).

Insérer Tableau 2-1 : Le barème de l'impôt sur le revenu de 1970 à 1997

Le nombre de tranches était sensiblement plus élevé entre 1974 et 1992 qu'en 1970-1973 et 1993-1997, mais il s'agit là de changements relativement mineurs, l'effet principal d'un nombre de tranches plus élevé étant simplement de lisser la courbe des taux marginaux (la courbe des taux moyens étant toujours continue, par construction), sans que cela implique nécessairement la moindre redistribution substantielle entre groupes de revenus. De fait, les réformes de 1974 et 1993 n'ont entraîné aucun changement substantiel de taux marginal d'imposition pour quelque groupe de contribuable que ce soit, et ces réformes ne peuvent donc pas être exploitées pour estimer l'élasticité du revenu imposable.

Pendant les années 1970, seules les tranches inférieures du barème étaient entièrement indexées sur l'inflation, alors que les tranches supérieures bénéficiaient d'une indexation partielle (voire nulle pour certaines années), si bien par exemple que le nombre de contribuables concernés par la tranche à $60 \%$ a fortement progressé durant cette période. ${ }^{19}$ Cependant, outre qu'elles ont concerné de très larges couches de revenus moyens et de revenus élevés, ces modifications des taux marginaux ont pris place très graduellement (au rythme de l'inflation), et elles sont donc difficilement exploitables. La Loi de Finances pour 1982 (dispositions applicables aux revenus de 1981), institue le principe de l'indexation intégrale de toutes les tranches du barèmes sur l'inflation, principe qui sera appliqué depuis lors.

\footnotetext{
${ }^{18}$ II existe de multiples sources permettant d'étudier l'évolution de la législation de l'impôt sur le revenu. Outre les Lois de Finances pour l'année $n+1$, publiées chaque année au JO "Lois et Décrets " du 31/12/n, et qui par définition contiennent l'ensemble des mesures nouvelles applicables aux revenus de l'année $n$, nous avons consulté les "Brochure Pratique $n+1$ (Déclaration des revenus n)" publiées chaque année depuis 1979 par la DGI et les "Guide Pratique du contribuable n+1 (revenus n)" publiées chaque année depuis 1932 par le SNUI. Les rapports du Conseil des Impôts consacrés à l'impôt sur le revenu contiennent également des descriptions synthétiques de l'évolution de la législation, ainsi que les $n^{\circ}$ de "Statistiques et Etudes Financières " consacrées à "L'impôt sur le revenu en 19.. " (cf. Annexe B.3). Le livre de Nizet (1991) fournit également une bonne description des orientations générales de la législation fiscale de 1945 à 1990.

${ }^{19}$ Cf. graphiques 4-1 et 4-2 (section 4)
} 
Tableau 2-1: Les barêmes de l'impôt sur le revenu de 1970 à 1997 (en francs courants)

\begin{tabular}{|c|c|c|c|c|c|c|c|c|c|c|c|c|c|}
\hline 1970 & & 1971 & & 1972 & & 1973 & & 1974 & & 1975 & & 1976 & \\
\hline 2900 & 10 & 3100 & 10 & 3300 & 10 & 4950 & 5 & 5500 & 5 & 6125 & 5 & 6725 & \\
\hline 5100 & 15 & 5400 & 15 & 5750 & 15 & 5200 & 10 & 5825 & 10 & 6425 & 10 & 7050 & 10 \\
\hline 8500 & 20 & 8950 & 20 & 9500 & 20 & 6250 & 15 & 7000 & 15 & 7700 & 15 & 8450 & 15 \\
\hline 12600 & 30 & 13250 & 30 & 14050 & 30 & 9900 & 20 & 11100 & 20 & 12225 & 20 & 13400 & \\
\hline 20050 & 40 & 21050 & 40 & 22000 & 40 & 14900 & 30 & 15050 & 25 & 16575 & 25 & 17575 & \\
\hline 40100 & 50 & 42100 & 50 & 43500 & 50 & 22000 & 40 & 19000 & 30 & 20900 & 30 & 22150 & \\
\hline \multirow[t]{6}{*}{80200} & 60 & 84200 & 60 & 86500 & 60 & 46325 & 50 & 24450 & 35 & 25250 & 35 & 26775 & \\
\hline & & & & & & 92125 & 60 & 26475 & 40 & 29125 & 40 & 30875 & \\
\hline & & & & & & & & 45825 & 45 & 50400 & 45 & 53425 & \\
\hline & & & & & & & & 64900 & 50 & 71375 & 50 & 73525 & \\
\hline & & & & & & & & 84000 & 55 & 92400 & 55 & 95175 & \\
\hline & & & & & & & & 103150 & 60 & 113450 & 60 & 113450 & \\
\hline 1977 & & 1978 & & 1979 & & 1980 & & 1981 & & 1982 & & 1983 & \\
\hline 7250 & 5 & 7925 & 5 & 8725 & 5 & 9890 & 5 & 11230 & 5 & 12620 & 5 & 13770 & \\
\hline 7600 & 10 & 8300 & 10 & 9125 & 10 & 10340 & 10 & 11740 & 10 & 13190 & 10 & 14390 & 10 \\
\hline 9100 & 15 & 9925 & 15 & 10825 & 15 & 12270 & 15 & 13930 & 15 & 15640 & 15 & 17070 & 5 \\
\hline 14400 & 20 & 15700 & 20 & 17125 & 20 & 19410 & 20 & 22030 & 20 & 24740 & 20 & 26990 & \\
\hline 18900 & 25 & 20625 & 25 & 22275 & 25 & 24950 & 25 & 28320 & 25 & 31810 & 25 & 34700 & \\
\hline 23800 & 30 & 25925 & 30 & 28000 & 30 & 31360 & 30 & 35590 & 30 & 39970 & 30 & 43610 & \\
\hline 28775 & 35 & 31350 & 35 & 33875 & 35 & 37970 & 35 & 43060 & 35 & 48370 & 35 & 52760 & \\
\hline 33200 & 40 & 36175 & 40 & 39075 & 40 & 43770 & 40 & 49680 & 40 & 55790 & 40 & 60870 & 40 \\
\hline 57425 & 45 & 62600 & 45 & 65125 & 45 & 72940 & 45 & 82790 & 45 & 92970 & 45 & 101430 & \\
\hline 79025 & 50 & 86125 & 50 & 89575 & 50 & 100320 & 50 & 113860 & 50 & 127860 & 50 & 139500 & \\
\hline 100900 & 55 & 105950 & 55 & 105950 & 55 & 118660 & 55 & 134680 & 55 & 151250 & 55 & 165010 & \\
\hline \multirow[t]{2}{*}{119100} & 60 & 125050 & 60 & 125050 & 60 & 135000 & 60 & 153200 & 60 & 172040 & 60 & 187700 & \\
\hline & & & & & & & & & & 195000 & 65 & 212750 & \\
\hline 1984 & & 1985 & & 1986 & & 1987 & & 1988 & & 1989 & & 1990 & \\
\hline 14820 & 5 & 15650 & 5 & 16030 & 5 & 16560 & 5 & 17000 & 5 & 17570 & 5 & 18140 & \\
\hline 15490 & 10 & 16360 & 10 & 16760 & 10 & 17320 & 9,6 & 17780 & 9,6 & 18370 & 9,6 & 18960 & \\
\hline 18370 & 15 & 19400 & 15 & 19870 & 15 & 20530 & 14,4 & 21070 & 14,4 & 21770 & 14,4 & 22470 & \\
\hline 29050 & 20 & 30680 & 20 & 31420 & 20 & 32460 & 19,2 & 33310 & 19,2 & 34410 & 19,2 & 35520 & 19,2 \\
\hline 37340 & 25 & 39440 & 25 & 40390 & 25 & 41730 & 24 & 42820 & 24 & 44240 & 24 & 45660 & \\
\hline 46920 & 30 & 49550 & 30 & 50740 & 30 & 52410 & 28,8 & 53770 & 28,8 & 55540 & 28,8 & 57320 & 28,8 \\
\hline 56770 & 35 & 59950 & 35 & 61390 & 35 & 63420 & 33,6 & 65070 & 33,6 & 67220 & 33,6 & 69370 & 33,6 \\
\hline 65500 & 40 & 69170 & 40 & 70830 & 40 & 73170 & 38,4 & 75070 & 38,4 & 77550 & 38,4 & 80030 & 38,4 \\
\hline 109140 & 45 & 115250 & 45 & 118020 & 45 & 121910 & 43,2 & 125080 & 43,2 & 129210 & 43,2 & 133340 & 43,2 \\
\hline 150100 & 50 & 158510 & 50 & 162310 & 50 & 167670 & 49 & 172030 & 49 & 177710 & 49 & 183400 & 49 \\
\hline 177550 & 55 & 187490 & 55 & 191990 & 55 & 198330 & 53,9 & 203490 & 53,9 & 210210 & 53,9 & 216940 & 53,9 \\
\hline 201970 & 60 & 213280 & 60 & 218400 & 58 & 225610 & 56,8 & 231480 & 56,8 & 239120 & 56,8 & 246770 & 56,8 \\
\hline 228920 & 65 & 241740 & 65 & & & & & & & & & & \\
\hline
\end{tabular}

$\begin{array}{rrrrrrrrrrrrrr}1991 & & 1992 & & 1993 & & 1994 & & 1995 & & 1996 & & 1997 & \\ 18690 & 5 & 19220 & 5 & 21900 & 12 & 22210 & 12 & 22610 & 12 & 25610 & 10,5 & 25890 & 10,5 \\ 19530 & 9,6 & 20080 & 9,6 & 47900 & 25 & 48570 & 25 & 49440 & 25 & 50380 & 24 & 50930 & 24 \\ 23150 & 14,4 & 23800 & 14,4 & 84300 & 35 & 85480 & 35 & 87020 & 35 & 88670 & 33 & 89650 & 33 \\ 36590 & 19,2 & 37620 & 19,2 & 136500 & 45 & 138410 & 45 & 140900 & 45 & 143580 & 43 & 145160 & 43 \\ 47030 & 24 & 48350 & 24 & 222100 & 50 & 225210 & 50 & 229260 & 50 & 233620 & 48 & 236190 & 48 \\ 59040 & 28,8 & 60690 & 28,8 & 273900 & 56,8 & 277730 & 56,8 & 282730 & 56,8 & 288100 & 54 & 291270 & 54 \\ 71450 & 33,6 & 73450 & 33,6 & & & & & & & & & & \\ 82430 & 38,4 & 84740 & 38,4 & & & & & & & & & & \\ 137340 & 43,2 & 141190 & 43,2 & & & & & & & & & & \\ 188900 & 49 & 194190 & 49 & & & & & & & & & & \end{array}$

Lecture : Au titre de l'imposition des revenus de 1970 (déclarés en 1971), la fraction du revenu imposable par part de quotient familial comprise entre 0 franc et 2900 francs est soumise à un taux marginal de $0 \%$, la fraction comprise entre 2900 francs et 5100 francs est soumise à un taux marginal de $10 \%$, etc..., et la fraction supérieure à 80200 francs est soumise à un taux marginal de $60 \%$. 
Les seules modifications importantes du barème sur la période 1970-1996 concernent donc la tranche supérieure. Des revenus de 1970 aux revenus de 1981, le taux marginal de la tranche supérieure est de 60\%. L'alternance politique de 1981 conduit à la création d'une $13^{\text {ème }}$ tranche : des revenus de 1982 (LF83) aux revenus de 1985 (LF86), le taux supérieur est de 65\%. L'augmentation de la pression fiscale applicable aux hauts revenus est encore plus importante si l'on prend en compte les majorations d'impôts et le plafonnement du quotient familial introduits en 1981-1982. Le retour de la droite au gouvernement en 1986 conduit à inverser les orientations de 1981. Dans un premier temps, la tranche à $65 \%$ est supprimée, et le taux de la tranche à $60 \%$ est ramené à $58 \%$ (LF 87 , applicable aux revenus de 1986). Dans un second temps, tous les taux marginaux du barème sont abaissés de 2 à $4 \%$ de leur niveau initial, si bien que le taux supérieur passe à $56,8 \%$ (LF88, applicable aux revenus de 1987). Ce taux supérieur de $56,8 \%$ s'est appliqué aux revenus des années 1987 à 1995. En particulier, contrairement à ce qui s'était produit lors de la première cohabitation, la réforme entreprise en 1993 (LF94) lors de la seconde cohabitation s'est contentée de réduire le nombre de tranches du barème, sans toucher ni au seuil ni au taux de la tranche supérieure (bien que la "simplification" du barème de 1993 se soit accompagnée d'une baisse générale de la pression fiscale, notamment pour les revenus élevés; cf. section 2.2 infra). En 1996 (LF97), tous les taux du barème sont à nouveau abaissés dans des proportions variables, et le taux supérieur passe à $54 \%$. Ce plan de baisse des taux de l'impôt sur le revenu devait se poursuivre pendant 5 ans, pour conduire à un taux marginal supérieur de $45 \%$, mais le retour de la gauche au pouvoir en 1997 y a mis fin, même si le nouveau gouvernement socialiste, tout comme son prédécesseur en 1988, a choisi de ne pas revenir sur la baisse de taux décidée par le gouvernement précédent. Seuls les épisodes de 1981-1982 et 1986-1987 peuvent donc être utilisées pour estimer l'élasticité du revenu imposable.

\subsubsection{Décote, majorations et minorations d'impôts}

Le tableau 2-1 décrit uniquement le barème de l'IR stricto sensu, sans aucune prise en compte des autres règles qui viennent modifier le calcul de l'impôt issu du barème (décote, majorations, minorations, plafonnement des effets du quotient familial, etc...), et dont nous résumons ici les grandes évolutions. La décote, qui existe depuis l'imposition des revenus de 1981, était initialement réservée aux contribuables célibataires dont l'impôt était inférieur à un certain seuil, l'objectif étant d'éviter l'imposabilité des célibataires rémunérés au niveau du SMIC (les couples disposant d'un seul SMIC étant déjà exonérés, grâce au système du quotient familial). ${ }^{20}$ Ce mécanisme a été étendu aux familles à compter de l'imposition des

\footnotetext{
${ }^{20}$ Un mécanisme de décote similaire à celui qui a été introduit en 1981 avait déjà été appliqué lors de l'imposition des revenus des années 1970 à 1972, puis supprimé en 1973 avec l'élargissement de la tranche à taux 0 . Ce mécanisme avait ensuite été remplacé par un mécanisme ad hoc de réduction d'impôt destiné aux célibataires smicards, supprimé en 1981 avec le retour de la décote.
} 
revenus de 1986, ce qui a entraîné une forte augmentation du nombre de foyers nonimposables (cf. section 2.2 infra). Depuis 1986, la décote s'applique donc à tous les foyers fiscaux dont l'impôt est inférieur à un certain seuil (3260 francs pour l'imposition des revenus de 1996). L'élargissement de la tranche à taux 0 qui a accompagné les réformes du barème de 1993 et 1996 (cf. tableau 2.1) a permis d'abaisser sensiblement ce seuil (il était de 5110 francs en 1992), et l'objectif initial de la réforme sur 5 ans mise en place en 1996 était d'aboutir à un élargissement suffisamment important de la tranche à taux 0 pour pouvoir supprimer complètement la décote (cet objectif a été abandonné en même temps que la baisse programmée des taux du barème). Le système de la décote aboutit à des modifications importantes des taux marginaux effectifs applicables à des zones spécifiques de revenus moyens et faibles, ${ }^{21}$ mais les données disponibles permettent difficilement d'en mesurer les effets, ${ }^{22}$ et nous n'avons donc pas cherché à exploiter ces variations.

L'alternance de 1981 marque également le retour des mécanismes de majorations et de minorations d'impôt. ${ }^{23}$ Un système de minorations pour les impôts faibles et de majorations pour les impôts élevés existait déjà en 1970-1972, mais il était d'une ampleur relativement modeste, puisque la majoration maximum applicable aux impôts les plus élevés ne dépassait pas le taux de $3 \%{ }^{24}$ La Loi de Finances Rectificative pour 1974 du 16/7/74 (LFR74) introduit une " majoration exceptionnelle de l'impôt sur le revenu de 1973, instituée dans le cadre du plan de rétablissement des équilibres économiques et financiers", qui se traduit par des augmentations d'impôt pour tous les contribuables dont l'impôt due au titre des revenus de 1973 est supérieur à 2500 francs, à un taux qui atteint $20 \%$ pour les impôts les plus élevés. II

\footnotetext{
${ }^{21}$ La décote fonctionne de la façon suivante : les contribuables dont l'impôt I est inférieur au seuil s de la décote voient leur impôt réduit de (s-I). Les contribuables dont l'impôt I est inférieur à s/2 deviennent donc non imposables du fait de la décote, et les contribuables dont l'impôt est compris entre s/2 et s voient leur impôt passer de I à 2l-s. Autrement dit, le taux marginal du premier groupe est ramené à $0 \%$, alors que celui du second est multiplié par 2 .

${ }^{22}$ La difficulté vient du fait que les contribuables concernés par la décote ont par définition des revenus imposables situés à la marge des seuils d'imposabilité. Les Etats 1921 ne conservant des informations statistiques sur les foyers non-imposables que depuis 1985, il est donc difficile d'étudier de façon fiable les modifications fines de la distribution des revenus concernés par l'introduction de la décote en 1981 (en outre, la distribution des revenus faibles et moyens ne peut pas être correctement approximée par une loi de Pareto, et il faudrait donc mettre au point une autre technique d'estimation pour obtenir des résultats en fractiles à partir des tableaux en tranche des Etats 1921).

${ }_{23}$ Pour une intéressante rétrospective des majorations d'impôt appliquées de 1974 à 1983, cf. "Statistiques et Etudes Financières", série rouge, n³94 (1984), pp.28-32.

${ }^{24}$ Les contribuables dont l'impôt dû au titre des revenus de 1970 était inférieur à 5000 francs bénéficiaient de minorations d'impôt progressives à des taux allant de $15 \%$ à $3 \%$ (un système similaire de minorations a été appliqué aux revenus de 1971 et 1972) ; les contribuables dont l'impôt dû au titre des revenus de 1970 était supérieur à 20000 francs devaient acquitter une majoration de $3 \%$ (ce taux a été ramené à $2 \%$ pour les revenus de 1971 , puis supprimé à compter des revenus de 1972). Outre ce système de majorations et de minorations, le barème de l'IR applicable au revenus de 1970 et 1971 comportait un mécanisme permettant de taxer d'avantage les revenus non-salariaux : le barème " officiel" avait de taux allant de $3 \%, 13 \%, \ldots$, à $63 \%$ (et non de $0 \%, 10 \%, \ldots$, à $60 \%$ ), mais tous les contribuables bénéficiaient d'une réduction d'impôt égale à $3 \%$ du montant net de leurs salaires et pensions. Ce mécanisme a été supprimé à compter des revenus de 1972, si bien que le taux marginal effectif applicable aux revenus non-salariaux les plus élevés est passé de $64,9 \%$ $(63 \% \times 1,03)$ en 1970 à $64,3 \%(63 \% \times 1,02)$ en 1971 et $60 \%$ en 1972.
} 
est cependant impossible d'exploiter cette majoration exceptionnelle pour mesurer l'élasticité du revenu imposable, dans la mesure où elle s'est appliquée uniquement aux revenus de 1973, qui avaient déjà été déclarés par les contribuables au printemps 1974 avant que la majoration soit annoncé en juillet 1974 : en principe, les comportements n'ont donc pas eu le temps de s'ajuster aux nouvelles incitations. II en va de même de la " majoration exceptionnelle de l'impôt sur le revenu de 1975, instituée dans le cadre de la lutte contre l'inflation et du financement de l'aide aux agriculteurs victimes de la sécheresse ", introduite par la LFR75 du 29/10/76, et dont les taux atteignaient $8 \%$ pour les impôts les plus élevés, et de la "majoration exceptionnelle de l'impôt sur les revenus élevés de l'année 1980, instituée pour financer diverses mesures en faveur de l'emploi et dans le cadre d'un effort de solidarité nationale ", introduite par la LFR80 du 3/8/81, et qui impose une majoration d'impôt de 25\% pour les contribuables dont l'IR dû au titre des revenus de 1980 est supérieur à 100000 francs.

La différence importante entre les majorations exceptionnelles de 1974 et 1976 et celle de 1981 et que cette dernière s'inscrit dans une orientation générale de hausse de la pression fiscale sur les hauts revenus, alors que les premières ont été d'une application purement ponctuelle et "exceptionnelle". On peut donc penser que, contrairement aux majorations de 1974 et 1976, la majoration de 1981 a agit sur les anticipations des contribuables concernés quand aux taux marginaux auxquels ils seront soumis dans le futur, et donc potentiellement sur leurs comportements. De fait, outre la création de la tranche à 65\% applicable à compter des revenus de 1982 (LF83), qui concernait à peu près le même groupe de contribuables que la majoration exceptionnelle de $1981,{ }^{25}$ le système des "majorations exceptionnelles" devient quasi-permanent après 1981. La Loi de Finances pour 1982 (LF82) remplace la majoration exceptionnelle applicable aux revenus de 1980 par une "majoration exceptionnelle de l'impôt sur le revenu de 1981, instituée pour financer une partie de l'indemnisation des travailleurs sans emploi ", qui impose une majoration d'impôt de $10 \%$ aux contribuables dont l'impôt dû au titre des revenus de 1981 est supérieur à 25000 francs. $^{26}$ La Loi de Finances pour 1983 (LF83) introduit une " majoration exceptionnelle de l'impôt sur le revenu de 1982, ayant le même objet que la majoration prévue par la loi de finances du 30 décembre 1981 ", dont le taux de $7 \%$ s'applique aux contribuables dont l'impôt dû au titre des revenus de 1982 est supérieur à 28000 francs. La Loi de Finances pour 1984 (LF84) la remplace par une “ majoration progressive de l'impôt sur le revenu de 1983, instituée en raison des

\footnotetext{
${ }^{25}$ Pour les contribuables ayant 1 part de quotient familial, un impôt de 100000 francs correspondait à un revenu imposable de 220000 francs en 1980, c'est-à-dire à un niveau légèrement supérieur au seuil de la tranche à 65\% applicable à compter des revenus de 1982 (195000 francs en 1982). Pour les contribuables ayant un nombre plus élevé de parts, le seuil d'application de la majoration exceptionnelle de 1981 était légèrement inférieur au seuil de la tranche à $65 \%$.

${ }^{26}$ Tous les contribuables dont l'impôt dû au titre des revenus de 1981 est supérieur à 5000 francs devront en outre s'acquitter d'un "emprunt obligatoire ayant pour objet l'accroissement des ressources d'épargne et le financement des actions engagées par l'Etat en faveur du développement industriel et du soutien de l'emploi ", institué par l'ordonnance du 30/4/83, égal à $10 \%$ de l'impôt dû au titre des revenus de 1981 (l'emprunt était remboursable après 3 ans, avec un intérêt de 11\% l'an).
} 
exigences de la situation conjoncturelle et dans un souci de solidarité ", qui impose une majoration d'impôt de 5\% aux contribuables dont l'impôt dû au titre des revenus de 1983 est supérieur à 20000 francs et de $8 \%$ pour les contribuables dont l'impôt dû est supérieur à 30000 francs. Un système de minorations d'impôt est introduit à compter des revenus de 1984 (LF85) : les contribuables dont l'impôt dû au titre des revenus de 1984 est inférieur à 21520 francs voient leur impôt minoré de $5 \%$, alors que les contribuables dont l'impôt dû est supérieur à 32080 francs voient leur impôt majoré de $3 \%$. Les majorations d'impôt sont définitivement abolis à compter des revenus de 1985 (LF86), et tous les contribuables dont l'impôt dû au titre des revenus de 1985 est inférieur à 34090 francs bénéficient de minorations d'impôt à des taux allant de $8 \%$ (pour les contribuables dont l'impôt est inférieur à 22730 francs) à $3 \%$ (pour les contribuables dont l'impôt est compris entre 28410 francs et 34090 francs). Le nouveau gouvernement issu des législatives de 1986 augmentera à nouveau les taux des minorations d'impôt applicables aux revenus de 1986 (LF87), ${ }^{27}$ et ce système de minorations restera inchangé jusqu'à l'imposition des revenus de 1992, avant que la réforme de 1993 n'intègre les minorations dans le barème. Compte tenu des modifications incessantes du système de majorations d'impôt intervenues entre 1981 et 1985, il est cependant difficile d'estimer avec certitude les taux marginaux effectifs perçus par les différents groupes de revenu durant ces années. De plus, et surtout, les majorations applicables aux revenus des années 1981-1984 concernent un nombre beaucoup plus important de contribuables que les modifications des taux marginaux supérieurs du barème sur lesquelles nous nous concentrons dans cette étude. En particulier, tous les contribuables concernés par les tranche à $55 \%$ et à $60 \%$ (56,8\% depuis 1987), et a fortiori, par la tranche à $65 \%$, ont toujours été concernés par ces majorations, généralement au même taux, et c'est pourquoi nous ne les avons pas prises en compte dans les estimations de l'élasticité du revenu imposable présentées dans la section 4, qui reposent principalement sur la comparaison entre les contribuables concernés par la tranche à $65 \%$ et ceux qui étaient concernés uniquement par celles à $60 \%$ (ou à $55 \%$ ). ${ }^{28}$ Quoi qu'il en soit, la prise en compte des majorations d'impôt de 1981-1984 ne pourrait

\footnotetext{
${ }^{27}$ Tous les contribuables dont le revenu imposable par part est inférieur à 295000 francs en 1986 bénéficient de minorations d'impôt, à des taux allant de $11 \%$ pour les contribuables dont l'impôt dû au titre des revenus de 1986 est inférieur à 22730 francs à $6 \%$ pour les contribuables dont l'impôt dû est compris entre 29090 et 34910 francs et $3 \%$ pour les contribuables dont l'impôt dû est supérieur à 41060 francs (mais dont le revenu imposable par part est inférieur à 295000 francs). Ces taux seront intégralement reconduits de 1986 à 1992, les seuils étant chaque année indexés sur l'inflation.

${ }^{28}$ Pour les contribuables ayant 1 part de quotient familial, les majorations d'impôts applicables aux revenus des années 1981-1984 correspondaient à un seuil de revenu imposable de l'ordre de 100000 francs (89000 francs en 1981, 100000 francs en 1982, 83000 en 1983, 115000 francs en 1984), et elles concernaient donc entre $1,5 \%$ et $2 \%$ des contribuables (cf. tableau D-4, annexe D.2). Cette proportion était légèrement plus élevée pour les contribuables ayant 1,5 ou $2 \mathrm{~B}$ parts de quotient familial (environ $2 \%$ des contribuables), et sensiblement plus élevé pour les contribuables ayant $2 \mathrm{~A}$, 2,5 ou 3 parts de quotient familial (entre 5 et $10 \%$ des contribuables). L'emprunt obligatoire de 1983 concernait une proportion de contribuables encore plus élevée (plus de 10\% en moyenne). Dans tous les cas, tous les foyers du centile supérieur étaient concernés, si bien que les différentiels de taux marginaux entre foyers du centile supérieur estimés dans la section 4 ne sont pas affectés par ces majorations.
} 
que conduire à réviser à la baisse les élasticités obtenues sans les prendre en compte, puisque cela aboutirait à une estimation plus élevée de la hausse du taux marginal effectif applicable aux hauts revenus, pour une même baisse observée des hauts revenus.

\subsubsection{Modifications apportées au mécanisme du quotient familial}

Le mécanisme du quotient familial a également connu des modifications importantes dans les années 1980-1990, après plusieurs décennies sans aucune modification importante. Les règles de base servant au calcul du nombre de parts de quotient familial n'ont cependant jamais été modifiées. Les foyers fiscaux de célibataires (célibataires, veufs ou divorcés) ont droit à 1 part et les foyers de couples mariés à 2 parts, auxquelles s'ajoutent une demi-part par enfant à charge. Outre l'attribution de demi-parts supplémentaires aux personnes invalides, ${ }^{29} \mathrm{ce}$ système des demi-parts a toujours comporté des exceptions, notamment pour les contribuables célibataires. D'une part, les contribuables célibataires ayant eu au moins un enfant majeur (ou faisant l'objet d'une imposition distincte) ont toujours bénéficié d'une demi-part supplémentaire. La conséquence est qu'une partie importante des contribuables célibataires sans enfant à charge, et en particulier l'immense majorité des personnes âgées vivant seules (toutes celles qui ont eu au moins un enfant) ont droit à 1,5 parts de quotient familial et non à 1 part. De fait, la distribution du revenu imposable des contribuables ayant 1,5 parts de quotient familial est sensiblement plus inégalitaire que celle de tous les autres groupes de quotient familial, même si cet écart a eu tendance à se réduire. ${ }^{30}$ D'autre part, les contribuables célibataires ayant des enfants à charges ont toujours eu droit à une part entière (et non à un demi-part) pour leur premier enfant à charge. Un contribuable célibataire ayant 1 enfant à charge à donc droit à 2 parts de quotient familial et non à 1,5 parts. ${ }^{31}$ Parmi les foyers fiscaux ayant 2 parts de quotient familial, les statistiques fiscales des Etats 1921 distinguent ainsi les contribuables de célibataires (dits " $2 \mathrm{~B}$ ") et les contribuables de couples mariés sans enfant à charge (dits " $2 A$ "), et nous utiliserons également cette distinction.

A compter des revenus de 1979 (LF80), une demi-part supplémentaire est accordée aux foyers ayant au moins 5 enfants à charge : le nombre de parts d'un couple marié ayant 5 enfants à charge passe de 4,5 à 5 , le nombre de parts d'un couple marié ayant 6 enfants à charge passe de 5 à 5,5 , etc... A compter des

\footnotetext{
${ }^{29}$ A compter des revenus de 1981, ces demi-parts sont attribués pour toutes les personnes à charge invalides du foyer, et non plus seulement pour les ascendants et enfants à charge invalides.

${ }^{30}$ Cf. les tableaux D-5 et D-6, annexe D.2. La forte concentration des revenus observée pour ce groupe de contribuables s'explique par le fait qu'il regroupe en grande partie des personnes âgées, tranche d'âge où se mêlent à la fois un grand nombre de personnes seules aux revenus très modestes et un petit nombre de contribuables ayant un patrimoine et des revenus très élevés.

${ }^{31}$ Les veufs dont les enfants sont issus du mariage avec le conjoint décédé ont également droit à une autre demi-part supplémentaire, si bien qu'un contribuable veuf ayant 1 enfant à charge (issu du mariage avec le conjoint décédé) a droit à 2,5 parts (comme un couple marié ayant 1 enfant à charge).
} 
revenus de 1980, cette demi-part supplémentaire s'appliquera aux foyers ayant au moins 3 enfants à charge (le nombre de parts d'un couple marié ayant 3 enfants à charge passe donc de 3,5 à 4 , et celui d'un couple ayant 4 enfants passe de 4 à 4,5). Le gouvernement issu des législatives de 1986 généralisera ce système de la "prime aux familles nombreuses " : depuis l'imposition des revenus de 1986 (LF87), tous les enfants à charge de rang supérieur ou égal à 3 donnent droit à une part complète (un couple marié avec 3 enfants à charge à droit à 4 parts, un couple marié avec 4 enfants à charge à droit à 5 parts, etc...). Ces réformes ont conduit mécaniquement à une augmentation importante du nombre de contribuables ayant un nombre élevé de parts de quotient familial, ${ }^{32}$ et à une baisse sensible de la pression fiscale imposée aux familles nombreuses. Cependant, ces demi-parts supplémentaires n'ont pas pour autant conduit à des baisses spectaculaires des taux marginaux d'imposition des foyers concernés: pour la plupart des contribuables, l'attribution d'une demi-part supplémentaire n'est pas suffisante pour changer de tranche d'imposition, et, lorsqu'elle le permet, la baisse de taux marginal n'est jamais supérieure à $5 \%$. De plus, la mise en place en 1981 d'un système de plafonnement des effets du quotient familial a conduit à obscurcir un peu plus le tableau. Au final, les contribuables ayant 3 enfants (ou plus) à charge ont connu entre 1979 et 1986 des variations multiples et contradictoires de leur taux marginal de variation, si bien qu'il est difficile d'exploiter ces variations pour estimer l'élasticité du revenu imposable.

Par contre, la mise en place du système de plafonnement du quotient familial a conduit à des effets clairs et massifs sur les taux marginaux d'imposition des contribuables de célibataires ayant 1,5 ou 2B parts et des contribuables de couples mariés, ainsi que sur les taux marginaux d'impositions des contribuables de couples mariés ayant 1 ou 2 enfants à charge (c'est-à-dire 2,5 ou 3 parts de quotient familial). Nous nous concentrerons donc sur ces 4 groupes de contribuables, ainsi que sur les contribuables célibataires ayant 1 part de quotient familial et les contribuables de couples mariés sans enfant à charge (c'est-à-dire ayant 2A parts de quotient familial), pour effectuer les estimations de l'élasticité du revenu imposable présentées dans la section $4 .{ }^{33}$ Le système de plafonnement des effets du quotient

\footnotetext{
${ }^{32}$ Cf. les tableaux C-6 et C-7 (annexe C.3).

${ }^{33}$ Les Etats 1921 ne font pas la distinction entre les contribuables de couples mariés ayant plus de 2 parts de quotient familial et les contribuables de célibataires ayant plus de 2 parts de quotient familial ; pour estimer les effets du plafonnement du quotient familial sur les taux marginaux effectifs d'imposition, nous ferons l'hypothèse que tous les contribuables ayant 2,5 ou 3 parts sont des couples mariés (d'après les échantillons légers de déclarations de revenus des années 1988-1995 (variable " mat "), tel est le cas de plus de $80 \%$ des foyers ayant 2,5 parts et de plus de $98 \%$ des foyers ayant 3 parts). Cette difficulté implique également qu'il serait difficile de suivre correctement l'évolution de la distribution du revenu imposable des foyers ayant plus de 3 parts (outre que les familles nombreuses ont connu des variations multiples et contradictoires, ce qui diminue leur intérêt du point de vue de l'estimation de l'élasticité du revenu imposable) : les multiples changements des règles d'attribution de demi-parts supplémentaires font que la distribution des nombres élevés de parts de quotient familial a beaucoup changé pendant les années 1980, si bien les couples mariés ayant un nombre élevé de parts pour des raisons "normales" sont difficiles à distinguer des autres foyers ayant pendant quelques années le même nombre élevé de parts pour d'autres raisons (demi-parts supplémentaires des célibataires, des personnes invalides) ; cf. tableaux C-6 et C-7 (annexe C.3).
} 
familial mis en place à compter des revenus de 1981 (LF82) fonctionne de la façon suivante : si la réduction d'impôt procurée par chaque demi-part de quotient familial est supérieure à un certain seuil (7500 francs en 1981), alors cette réduction est réduite au niveau de ce seuil, et l'impôt dû est calculé dans ces demi-parts. Ce plafonnement s'applique à chaque demi-part de quotient familial au-delà de 1 part pour les contribuables de célibataires (célibataires, veufs ou divorcés) et au-delà de 2 parts pour les contribuables de couples mariés. La conséquence est que les contribuables ayant 1,5, 2B , 2,5 ou 3 parts de quotient familial ont connu une forte hausse de leur taux marginal d'imposition en 1981, et ce à des niveaux de revenus sensiblement inférieurs au seuil de la tranche à $65 \%$, et donc à des niveaux de revenus auxquels les contribuables ayant 1 ou 2A parts de quotient familial voyaient leur taux marginal inchangé. Depuis l'imposition des revenus de 1986 (LF87), le seuil de plafonnement du quotient familial est sensiblement plus faible pour les deux demiparts accordées au premier enfant à charge des célibataires que pour toutes les autres demi-parts, si bien que les contribuables ayant 2B parts de quotient familial ont connu en 1986 une hausse importante et extrêmement ciblée de leur taux marginal d'imposition, hausse que nous exploiterons également dans la section $4 .^{34}$ Tous les seuils de plafonnement du quotient familial appliqués depuis 1981, ainsi que les barèmes d'imposition après prise en compte des effets du plafonnement, sont décrits dans l'annexe I.

Plus récemment, de nouvelles modifications du mécanisme du quotient familial ont été mises en place. A compter de l'imposition des revenus de 1995 (LF96), les contribuables célibataires doivent faire la preuve qu'ils élèvent seuls leur enfant pour bénéficier d'une part entière au titre de leur premier enfant à charge. Cette innovation, destinée à faire la chasse aux concubins notoires supposés bénéficier de ce système, a conduit à une forte diminution du nombre de foyers ayant 2B parts de quotient familial et à une hausse correspondante du nombre de foyers ayant 1,5 parts de quotient familial. ${ }^{35}$ A compter de l'imposition des revenus de 1997 (LF98), un nouveau plafond de réduction d'impôt a été institué pour les contribuables célibataires ayant 1,5 parts grâce au fait qu'ils ont eu au moins un enfant: dorénavant, la réduction d'impôt procurée par cette demi-part ne peut excéder 6100 francs, au lieu des 16380 francs (en 1997) du plafond général. II est actuellement envisagé d'abaisser le plafond général lui-même et de le porter à environ 11000 francs à compter de l'imposition des revenus de 1998. Toutes ces modifications

\footnotetext{
${ }^{34}$ Cependant, le nouveau plafond mis en place en 1986 ne s'applique pas aux veufs dont l'enfant à charge est issu du mariage avec le conjoint décédé (qui continuent de bénéficier du plafond général). Les Etats 1921 ne nous permettant pas de distinguer ces contribuables parmi les foyers fiscaux ayant 2B parts de quotient familial, et pour estimer les taux marginaux effectifs d'imposition nous avons fait I'hypothèse que ce nouveau seuil s'appliquait à l'ensemble des foyers fiscaux ayant 2B parts de quotient familial (pour 1994, c'est-à-dire pour la dernière année avant le durcissement des conditions d'attribution de la part entière au $1^{\text {er }}$ enfant à charge des célibataires, l'échantillon léger de déclarations de revenus (variable "mat") indique qu'environ 9,5\% des contribuables ayant $2 \mathrm{~B}$ parts de quotient familial étaient des veufs, contre environ $45 \%$ de célibataires et $45 \%$ de divorcés ; la proportion de contribuables continuant de bénéficier de l'ancien seuil est encore inférieure, puisque tous les enfants à charge des veufs ne sont pas issus du mariage avec le conjoint décédé).

${ }^{35}$ Cf. tableaux C-5 et C-6 (annexe C.3).
} 
conduisent elles aussi à des modifications importantes et ciblées de taux marginaux d'imposition, mais elles sont trop récentes pour que l'on puisse les utiliser pour estimer l'élasticité du revenu imposable.

\subsection{Le nombre de foyers, le revenu moyen et les recettes de l'IR de 1970 à 1996}

Le tableau 2-2 rappelle quelle a été la très forte croissance du nombre absolu de foyers fiscaux de 1970 à 1996.

Insérer Tableau 2-2 : La croissance du nombre de foyers fiscaux de 1970 à 1996

Le nombre de foyers fiscaux a ainsi progressé de plus de $45 \%$ durant les 25 dernières années, passant d'environ 21 millions de foyers en 1970 à plus de 30,5 millions en 1995. Sur 25 ans, cette progression a été sensiblement la même que celle du nombre de ménages, qui est passé de 16,3 millions en 1970 à près de 23,2 millions en 1996 (soit une progression de 42\%). En 1970 comme en 1995, on compte ainsi environ 1,3 foyers fiscaux par ménage (en moyenne). Cette stabilité sur 25 ans masque cependant des évolutions structurelles profondes, puisque le nombre moyen de foyers par ménage a diminué régulièrement pendant la première moitié de la période, pour atteindre 1,22 en 1983, avant de progresser très rapidement à partir de 1983 et de retrouver son niveau de 1970 dès la fin des années 80 . Autrement dit, les forces structurelles poussant à la diminution du nombre moyen de foyers par ménage (la baisse séculaire du nombre de ménages complexes où cohabitent plusieurs familles et/ou plusieurs générations) l'ont emporté pendant les années 70 , alors que les forces poussant dans la direction opposée (la progression du nombre de couples non-mariés, ainsi que la croissance récente du nombre de grands enfants habitant chez leurs parents dans l'attente d'un emploi) l'ont emporté dans les années 80 et 90. Au final, c'est l'équilibre entre ces différentes forces qui a fait que le nombre moyen de foyers par ménage est sensiblement le même en 1970 et en 1995.

L'évolution de la taille moyenne des foyers fiscaux est plus transparente : le tableau 2-2 indique que celle-ci a diminué tous les ans depuis 1970, passant de 2,4 en 1970 à 1,9 en 1995. Cette évolution n'est pas seulement due à la diminution du nombre moyen d'enfants par foyers : le nombre moyen de personnes âgées de plus de 15 ans par foyer fiscal a également fortement diminué (le même constat s'appliquerait si I'on se limitait aux personnes âgées de plus de 18 ans ou de plus de 25 ans). Cette évolution lourde est la conséquence à la fois du vieillissement de la population (et donc de l'augmentation du nombre de personnes âgées vivant seules) et de la croissance du nombre de jeunes célibataires (concubins ou non) et de parents isolés. Le pourcentage de foyers fiscaux ayant une seule part de quotient familial est ainsi passé progressivement d'environ $28 \%$ en 1970 à plus de $34 \%$ en $1995 .{ }^{36}$

\footnotetext{
${ }^{36}$ Les tableaux C-5 et C-6 (annexe C.3) décrivent l'évolution du nombre et du revenu moyen des contribuables en fonction de leur nombre de parts de quotient familial de 1970 à 1996 .
} 
Tableau 2-2 : La croissance du nombre de foyers fiscaux de 1970 à 1996

\begin{tabular}{ccccccccc} 
& N.foyers & \%f.imp. & Pop.tot. &. N.foy. & Pop. $15+$ & .N.foy. & \multicolumn{2}{c}{ N.ménages N.f./N.m. } \\
1970 & 21033 & 50,0 & 50528 & 2,40 & 37946 & 1,80 & 16310 & 1,29 \\
1971 & 21355 & 51,6 & 51016 & 2,39 & 38389 & 1,80 & 16611 & 1,29 \\
1972 & 21653 & 53,1 & 51486 & 2,38 & 38794 & 1,79 & 16911 & 1,28 \\
1973 & 21921 & 55,2 & 51916 & 2,37 & 39186 & 1,79 & 17204 & 1,27 \\
1974 & 22161 & 57,6 & 52321 & 2,36 & 39581 & 1,79 & 17493 & 1,27 \\
1975 & 22364 & 60,3 & 52600 & 2,35 & 39944 & 1,79 & 17745 & 1,26 \\
1976 & 22497 & 63,3 & 52798 & 2,35 & 40230 & 1,79 & 17977 & 1,25 \\
1977 & 22709 & 61,7 & 53019 & 2,33 & 40579 & 1,79 & 18221 & 1,25 \\
1978 & 22939 & 63,5 & 53271 & 2,32 & 40925 & 1,78 & 18480 & 1,24 \\
1979 & 23186 & 64,7 & 53481 & 2,31 & 41272 & 1,78 & 18730 & 1,24 \\
1980 & 23457 & 65,2 & 53731 & 2,29 & 41643 & 1,78 & 18998 & 1,23 \\
1981 & 23750 & 63,4 & 54029 & 2,27 & 42005 & 1,77 & 19289 & 1,23 \\
1982 & 24043 & 63,7 & 54335 & 2,26 & 42369 & 1,76 & 19589 & 1,23 \\
1983 & 24283 & 62,8 & 54650 & 2,25 & 42712 & 1,76 & 19834 & 1,22 \\
1984 & 24572 & 61,9 & 54895 & 2,23 & 43033 & 1,75 & 20057 & 1,23 \\
1985 & 25144 & 60,7 & 55157 & 2,19 & 43374 & 1,73 & 20290 & 1,24 \\
1986 & 25534 & 52,1 & 55411 & 2,17 & 43714 & 1,71 & 20522 & 1,24 \\
1987 & 26341 & 50,8 & 55682 & 2,11 & 44087 & 1,67 & 20764 & 1,27 \\
1988 & 26791 & 50,3 & 55966 & 2,09 & 44477 & 1,66 & 21015 & 1,27 \\
1989 & 27360 & 50,7 & 56270 & 2,06 & 44856 & 1,64 & 21276 & 1,29 \\
1990 & 28029 & 51,0 & 56577 & 2,02 & 45188 & 1,61 & 21542 & 1,30 \\
1991 & 28607 & 51,2 & 56893 & 1,99 & 45481 & 1,59 & 21984 & 1,30 \\
1992 & 29052 & 50,8 & 57217 & 1,97 & 45760 & 1,58 & 22297 & 1,30 \\
1993 & 29558 & 50,4 & 57530 & 1,95 & 46072 & 1,56 & 22532 & 1,31 \\
1994 & 30038 & 49,9 & 57779 & 1,92 & 46347 & 1,54 & 22840 & 1,32 \\
1995 & 30585 & 50,6 & 58020 & 1,90 & 46634 & 1,52 & 23156 & 1,32 \\
$1996\left(^{*}\right)$ & 30725 & 48,8 & 58256 & 1,90 & 46945 & 1,53 & 23451 & 1,31 \\
$1970-1995$ & $+45,4 \%$ & & & & & & & \\
& $+1,5 \%$ & & $+14,8 \%$ & $-21,0 \%$ & $+22,9 \%$ & $-15,5 \%$ & $+42,0 \%$ & $+2,4 \%$ \\
& & $+0,6 \%$ & $-0,9 \%$ & $+0,8 \%$ & $-0,7 \%$ & $+1,4 \%$ & $+0,1 \%$
\end{tabular}

Sources: Nombre de foyers et \% de foyers imposables: Etats 1921 (1985-1995) et estimations de l'auteur à partir des Etats 1921 et d'estimations DGI (1970-1984) (cf. Annexe C)

Population métropolitaine totale et population métropolitaine âgée de 15 ans ou plus (au 1/1 de chaque année): Comptabilité Nationale (cf. INSEE-Résultats n547-548-549 (série "Economie générale" n¹45-146-147), "Comptes et indicateurs économiques - Rapport sur les Comptes de la Nation 1996" (juin 1997), p.39)

Nombres de ménages ordinaires de France métropolitaine: résultats des recensements pour 1975, 1982 et 1990 (cf. INSEE-Résultats n³36 (série "Démographie-société" n³5), "Structure des ménages par région et par département" (septembre 1994), p.22); pour les années intercensitaires (1970-1974,1976-1981,1983-1989), estimations obtenues par interpolation linéaire à partir des chiffres des recensements de 1968, 1975, 1982 et 1990; pour les années 1991-1996, estimations issues des Enquêtes Emploi (calées sur le recensement de 1990)

Lecture: En 1970, la France compte 21,033 millions de foyers fiscaux, dont 50,0\% de foyers imposables, 50,528 millions d'habitants (soit 2,40 habitants par foyer fiscal), 37,946 millions d'habitants âgés de plus de 15 ans (soit 1,80 habitants de plus de 15 ans par foyer fiscal), et 16,310 millions de ménages (soit 1,29 foyers fiscaux par ménage).

Notes: (i) 1996(*): chiffres provisoires pour les foyers fiscaux (rôles émis au 31/12/97)

(ii) Tous les chiffres sont exprimés en milliers d'unités

(iii) La ligne "1970-1975" indique le pourcentage d'augmentation entre 1970 et 1995, et la ligne suivante indique le taux de croissance annuel moyen. 
Le tableau 2-2 indique également l'évolution du pourcentage de foyers imposables, c'est-à-dire le pourcentage de foyers fiscaux dont l'impôt dû au fisc est strictement positif. ${ }^{37}$ On remarque que le pourcentage de foyers imposables a suivi une "courbe en $\cap$ " au cours de la période 1970-1996: ce pourcentage est passé progressivement d'environ $50 \%$ en 1970 à plus de de $65 \%$ en 1980, avant de diminuer légèrement de 1981 à 1985, puis de chuter brutalement en 1986 et de se stabiliser autour de 50\% depuis 1986-1987 (la baisse enregistrée en 1996 étant due à l'élargissement de la tranche à taux 0 qui a accompagné la réforme du barème de 1996). La forte progression des années 70 s'explique par la très forte croissance des revenus imposables pendant cette période: dans un contexte de croissance des revenus réels, l'indexation des tranches inférieures du barème sur l'inflation ne suffit pas à éviter qu'un nombre croissant de contribuables franchisse les seuils d'imposabilité. Cette évolution "naturelle" d'un impôt progressif sur le revenu dans un contexte de croissance des revenus réels s'était déjà observée pendant les années 60. Le retournement observé depuis 1980 s'explique à la fois par des changements de législation et par le très fort ralentissement du rythme de progression du revenu imposable. D'une part, la création de la décote en 1981, et surtout son extension à l'ensemble des foyers fiscaux en 1986, ont conduit à exonérer de l'impôt sur le revenu près de 4 millions de foyers fiscaux. D'autre part, le revenu imposable moyen ne progresse plus en termes réels depuis la fin des années 70 , ce qui explique que l'évolution " naturelle " de l'IR soit désormais caractérisée par la relative fixité (légèrement pro-cyclique) du pourcentage du revenu imposable (à législation inchangée et à tranches du barème parfaitement indexées).

Insérer Tableau 2-3 : La croissance du revenu imposable de 1970 à 1996

Le tableau 2-3 indique en effet l'existence d'une rupture profonde dans l'évolution des revenus réels entre les années 1970 et les années 1980-1990. Sur l'ensemble de la période 1970-1995, le revenu imposable total (exprimé en FF 1996) a progressé de plus de $90 \%$, soit un taux de croissance annuel moyen de $2,6 \%$. Mais compte tenu de la très forte progression du nombre de foyers fiscaux, dont le taux de croissance annuel moyen a été de 1,5\% sur la période 1970-1995 (cf. tableau 2-2), le revenu imposable moyen (exprimé en FF 1996) a en fait progressé d'à peine plus de $30 \%$, soit un taux de croissance annuel moyen de $1,1 \%$. Surtout, l'intégralité de cette hausse de $30 \%$ s'est faite entre 1970 et 1978 : depuis la fin des années 1970 , le revenu imposable moyen des foyers fiscaux stagne aux alentours de 90000 francs (en FF 1996). Compte tenu du fait que la taille moyenne des foyers a fortement diminué pendant cette même période, cela ne signifie évidemment pas que le niveau de vie moyen n'a pas progressé pendant les années 1980-1990 : par

\footnotetext{
${ }^{37}$ Par convention, les statistiques fiscales incluent toujours les foyers bénéficiant d'une restitution partielle d'avoir fiscal (c'est-à-dire les foyers dont l'impôt avant imputation de l'avoir fiscal est strictement positif mais inférieur au montant de l'avoir fiscal) dans les foyers imposables, et les foyers bénéficiant d'une restitution totale d'avoir fiscal (c'est-à-dire les foyers dont l'impôt avant imputation de l'avoir fiscal est nul) dans les foyers non-imposables.
} 
Tableau 2-3 : La croissance du revenu imposable de 1970 à 1996

\begin{tabular}{|c|c|c|c|c|c|c|c|c|c|}
\hline & \multicolumn{2}{|c|}{$\begin{array}{l}\text { Rimp tot. Rimp moy. } \\
\text { (en FF courants) }\end{array}$} & \multirow[t]{2}{*}{$\begin{array}{c}\text { Taux } \\
\text { d'inflat. }\end{array}$} & \multicolumn{3}{|c|}{$\begin{array}{l}\text { Rimp tot. ./Pop. } \\
\text { (en FF 1996) }\end{array}$} & \multirow{2}{*}{$\begin{array}{c}\text { PIB (FF } \\
\text { courants) } \\
793519\end{array}$} & $\begin{array}{c}\text { PIB } \\
\text { (FF } 1996\end{array}$ & \multirow[t]{2}{*}{$\begin{array}{l}\text { Tx de } \\
\text { crois. }\end{array}$} \\
\hline 1970 & 266545 & 12673 & & 1402801 & 27763 & 66695 & & 4176216 & \\
\hline 1971 & 296472 & 13883 & 5,5 & 1478963 & 28990 & 69257 & 884186 & 4410795 & 5,6 \\
\hline 1972 & 331905 & 15328 & 6,2 & 1559060 & 30281 & 72002 & 987947 & 4640688 & \\
\hline 1973 & 375957 & 17150 & 7,2 & 1647373 & 31732 & 75150 & 1129835 & 4950726 & 6,7 \\
\hline 1974 & 440525 & 19879 & 13,8 & 1696222 & 32420 & 76542 & 1302978 & 5017053 & 1,3 \\
\hline 1975 & 510468 & 22826 & 11,8 & 1758080 & 33424 & 78613 & 1467884 & 5055470 & 0,8 \\
\hline 1976 & 589300 & 26195 & 9,7 & 1850119 & 35041 & 82238 & 1700553 & 5338920 & 5,6 \\
\hline 1977 & 674519 & 29702 & 9,3 & 1937479 & 36543 & 85317 & 1917803 & 5508674 & 3,2 \\
\hline 1978 & 772638 & 33682 & 9,1 & 2034203 & 38186 & 88679 & 2182588 & 5746323 & 4,3 \\
\hline 1979 & 882419 & 38058 & 10,8 & 2096783 & 39206 & 90432 & 2481097 & 5895522 & 2,6 \\
\hline 1980 & 1012484 & 43163 & 13,5 & 2119682 & 39450 & 903 & 2808295 & 5879297 & $-0,3$ \\
\hline 1981 & 1163064 & 48972 & 13,4 & 2147203 & 39742 & 90 & 3164804 & 5842738 & $-0,6$ \\
\hline 1982 & 1329965 & 55317 & 11,8 & 2196180 & 40419 & & 3626021 & 5987674 & 2,5 \\
\hline 1983 & 1468961 & 60493 & & 221 & 40498 & & 4006498 & 6036458 & 0,8 \\
\hline 1984 & 1579767 & 64291 & 7 & 221 & 40371 & & 913 & 6119134 & \\
\hline 1985 & 1692625 & 673 & 5,8 & & 40690 & 89260 & 143 & 6232157 & 1,8 \\
\hline 1986 & 1789 & 7008 & 2,7 & 23 & 41698 & 904 & 5069296 & 6544923 & 5,0 \\
\hline 1987 & 1888205 & 71682 & 3,1 & 236 & 42465 & 89766 & 5336652 & 6682933 & 2,1 \\
\hline 1988 & 1985182 & 74098 & 2,7 & 2420628 & 43252 & 90351 & 5735092 & 6993075 & 4,6 \\
\hline 1989 & 2111464 & 77173 & 3,6 & 2485145 & 44165 & 90831 & 6159680 & 7249802 & 3,7 \\
\hline 1990 & 2250842 & 80303 & 3,4 & 2562079 & 45285 & 91407 & 6509488 & 7409592 & 2,2 \\
\hline 1991 & 2358505 & 82446 & 3,2 & 2601385 & 45724 & 90936 & 6776231 & 7474050 & 0,9 \\
\hline 1992 & 2434864 & 83810 & 2,4 & 2622663 & 45837 & 90274 & 6999546 & 7539416 & 0,9 \\
\hline 1993 & 2488985 & 84206 & 2,1 & 2625816 & 45643 & 88836 & 7077087 & 7466148 & $-1,0$ \\
\hline 1994 & 2544299 & 84702 & 1,7 & 2639303 & 45679 & 87865 & 7389654 & 7665584 & 2,7 \\
\hline 1995 & 2627503 & 85908 & 1,7 & 2680053 & 46192 & 87626 & 7662391 & 7815639 & 2,0 \\
\hline $1996\left(^{*}\right)$ & 2686789 & 87446 & 2,0 & 2686789 & 46120 & 87446 & 7860517 & 7860517 & 0,6 \\
\hline $1510-1$ & & & & $\begin{array}{c}+91,1 \% \\
+2,6 \%\end{array}$ & $\begin{array}{c}+66,4 \% \\
+2,1 \%\end{array}$ & $\begin{array}{c}+31,4 \% \\
+1,1 \%\end{array}$ & & $\begin{array}{l}+87,1 \% \\
+2,5 \%\end{array}$ & \\
\hline
\end{tabular}

Sources: Revenu imposable total: Etats 1921 (1985-1995) et estimations de l'auteur à partir des Etats 1921 et des enquêtes "Revenus fiscaux" de l'INSEE (1970-1984) (cf. Annexe C)

Taux d'inflation (taux d'évolution en moyenne annuelle de l'indice des prix à la consommation): Comptabilité Nationale (cf. le "Rapport sur les Comptes de la Nation 1996", op.cit., p.131)

PIB en FF courants: Comptabilité Nationale (p.25) (PIB en FF 1996 calculé à partir du taux d'inflation issu de l'indice des prix à la consommation; taux de croissance en moyenne annuelle).

Lecture: En 1970, le revenu imposable total des foyers fiscaux (imposables et non-imposables) était de 266,545 milliards de francs courants, et le revenu imposable moyen par foyer fiscal était de 12673 francs courants; exprimés en francs de 1996, le revenu imposable total était de 1402,801 milliards de francs, le revenu imposable moyen par habitant de 27763 francs, et le revenu imposable moyen par foyer fiscal de 66695 francs; le PIB était de 793,519 milliards de francs courants, soit 4176,216 milliards de francs de 1996; entre 1970 et 1971, l'indice des prix à la consommation a progressé de $5,5 \%$, et le PIB réel de $5,6 \%$.

Notes: (i) 1996(*): chiffres provisoires pour le revenu imposable (rôles émis au 31/12/97)

(ii) Les chiffres agrégés sont exprimés en millions de FF, et les chiffres moyens en FF.

(iii) La ligne "1970-1975" indique le pourcentage d'augmentation entre 1970 et 1995, et la ligne suivante indique le taux de croissance annuel moyen. 
exemple, le revenu imposable moyen par habitant a progressé de près de $38 \%$ entre 1970 et 1978 , puis à nouveau d'environ $21 \%$ entre 1978 et 1995 . II reste que la rupture entre les années 1970 et les années 1980-1990 est bien réelle : le taux de croissance annuel moyen du revenu imposable moyen par habitant est passé de 4,1\% sur la période 1970-1978 à 1,1\% par an sur la période 1978-1995.

Pour l'essentiel, cette rupture est due non pas à du décrochage du revenu imposable des ménages vis-à-vis du PIB, mais bel et bien à un abaissement structurel du taux de croissance de l'économie française. Sur longue période, le revenu imposable des ménages évolue en effet au même rythme que le PIB : le PIB réel de l'économie française a progressé de près de $90 \%$ entre 1970 et 1995, c'est-àdire exactement dans les mêmes proportions que le revenu imposable. ${ }^{38}$ Sur l'ensemble de la période 1970-1995, le revenu imposable des ménages représente de l'ordre de $34-35 \%$ du PIB. Le fait qu'à peine plus d'un tiers du PIB se retrouve dans l'assiette imposable de l'impôt sur le revenu s'explique de la façon suivante. Tout d'abord, le revenu primaire brut (RPB) des ménages, au sens de la Comptabilité Nationale, représente généralement environ $74-75 \%$ du PIB. ${ }^{39}$ Mais le revenu primaire brut des ménages inclut de nombreuses catégories de revenus qui ne sont pas soumises à l'impôt sur le revenu: prestations familiales, remboursements d'assurance-maladie, loyers fictifs, revenus du capital exonérés d'impôt (intérêts du Livret $A$, etc..) ou soumis au prélèvement libératoire (intérêts d'obligations, etc..), revenus bruts des non-salariés correspondant à la dépréciation de leur capital, etc... Au final, le montant des revenus que les contribuables sont tenus de déclarer sur leur déclaration de revenus représentent à peine $70 \%$ du revenu primaire brut des ménages, soit environ $50 \%$ du PIB. ${ }^{40}$ Enfin, les revenus imposables représentent environ $70 \%$ des revenus déclarés, compte tenu des abattements et déductions auxquels donnent droit les différents types de revenus (et notamment les abattements de $10 \%$ et $20 \%$ des salaires et des retraites), si bien que le revenu imposable représente entre $45 \%$ et $50 \%$ du revenu primaire brut des ménages, et à peine $35 \%$ du PIB.

Sur longue période, les recettes de l'impôt sur le revenu ont également progressé dans les mêmes proportions que le revenu imposable : l'impôt émis au titre des

\footnotetext{
${ }^{38}$ Le tableau 2-3 utilise le même déflateur pour le revenu imposable des ménages que pour le PIB, à savoir l'indice des prix à la consommation (calculé en moyenne annuelle). Mais sur longue période, le choix du déflateur n'a guère d'importance : l'indice des prix à la consommation a été multiplié par 5,26 entre 1970 et 1996, alors que le déflateur du PIB habituellement utilisé en Comptabilité Nationale (calculé à partir des séries de PIB en prix courants et en prix de 1980 données dans le Rapport sur les Comptes de la Nation 1996, pp.25-26) a été multiplié par 5,29 entre 1970 et 1996.

${ }^{39}$ Le reste du PIB (environ un quart) est composé de la consommation des administrations et des revenus des entreprises non distribués au ménages qui les possèdent. Le ratio RPB/PIB a progressivement passé de $74-75 \%$ au début des années 1970 à plus de $78 \%$ en 1981, avant de retrouver son niveau de $74-75 \%$ dans les années 1980-1990. Cette évolution, qui explique une partie (minoritaire) de la rupture enregistrée par les taux de croissance du revenu imposable moyen, doit être mise en parallèle avec l'évolution bien connue de la part des salaires dans la valeur ajoutée des entreprises, qui a connu un sommet historique à la fin des années 1970 et au début des années 1980 (cf. par exemple Piketty (1997a, p.40, tableau VIII ; p.47, tableau IX)).

${ }_{40}$ Le ratio (revenu imposable)/RPB a probablement légèrement augmenté pendant les années 1970, du fait de la diminution de la part des revenus des entreprises individuelles (cf. Annexe C.2).
} 
Tableau 2-4 : Les recettes de l'impôt sur le revenu de 1970 à 1996

\begin{tabular}{cccccccccc}
\multicolumn{2}{c}{$\begin{array}{c}\text { Rev. imp. Imp.émis } \\
\text { (en FF courants) }\end{array}$} & $\begin{array}{c}\text { Impôt/Rimp } \\
(\%)\end{array}$ & $\begin{array}{c}\text { Impôt/Rfisc } \\
(\%)\end{array}$ & $\begin{array}{c}\text { Impôt/PIB } \\
(\%)\end{array}$ & $\begin{array}{c}\text { Recettes } \\
\text { (FF cour.) }\end{array}$ & $\begin{array}{c}\text { Rec./ } \\
\text { Rimp(\%) }\end{array}$ & $\begin{array}{c}\text { Rec./ } \\
\text { Rfisc(\%) }\end{array}$ & $\begin{array}{c}\text { Rec./PIB } \\
(\%)\end{array}$ \\
1970 & 266545 & 26807 & 10,1 & 7,3 & 3,4 & 29721 & 11,2 & 8,1 & 3,7 \\
1971 & 296472 & 31217 & 10,5 & 7,6 & 3,5 & 32547 & 11,0 & 7,9 & 3,7 \\
1972 & 331905 & 34566 & 10,4 & 7,5 & 3,5 & 34447 & 10,4 & 7,4 & 3,5 \\
1973 & 375957 & 41657 & 11,1 & 7,9 & 3,7 & 45087 & 12,0 & 8,5 & 4,0 \\
1974 & 440525 & 49832 & 11,3 & 8,0 & 3,8 & 48412 & 11,0 & 7,8 & 3,7 \\
1975 & 510468 & 61316 & 12,0 & 8,4 & 4,2 & 63356 & 12,4 & 8,7 & 4,3 \\
1976 & 589300 & 74723 & 12,7 & 8,8 & 4,4 & 77260 & 13,1 & 9,1 & 4,5 \\
1977 & 674519 & 82937 & 12,3 & 8,5 & 4,3 & 89900 & 13,3 & 9,2 & 4,7 \\
1978 & 772638 & 97392 & 12,6 & 8,7 & 4,5 & 100150 & 13,0 & 8,9 & 4,6 \\
1979 & 882419 & 113497 & 12,9 & 8,8 & 4,6 & 118510 & 13,4 & 9,2 & 4,8 \\
1980 & 1012484 & 132393 & 13,1 & 9,0 & 4,7 & 137885 & 13,6 & 9,4 & 4,9 \\
1981 & 1163064 & 155609 & 13,4 & 9,3 & 4,9 & 162160 & 13,9 & 9,7 & 5,1 \\
1982 & 1329965 & 172679 & 13,0 & 9,1 & 4,8 & 182880 & 13,8 & 9,6 & 5,0 \\
1983 & 1468961 & 194182 & 13,2 & 9,3 & 4,8 & 197100 & 13,4 & 9,5 & 4,9 \\
1984 & 1579767 & 197011 & 12,5 & 8,9 & 4,5 & 207300 & 13,1 & 9,3 & 4,8 \\
1985 & 1692625 & 206942 & 12,2 & 8,7 & 4,4 & 213120 & 12,6 & 8,9 & 4,5 \\
1986 & 1789572 & 213605 & 11,9 & 8,5 & 4,2 & 222600 & 12,4 & 8,8 & 4,4 \\
1987 & 1888205 & 224510 & 11,9 & 8,4 & 4,2 & 233000 & 12,3 & 8,7 & 4,4 \\
1988 & 1985182 & 227851 & 11,5 & 8,1 & 4,0 & 243830 & 12,3 & 8,7 & 4,3 \\
1989 & 2111464 & 254693 & 12,1 & 8,5 & 4,1 & 263100 & 12,5 & 8,8 & 4,3 \\
1990 & 2250842 & 277332 & 12,3 & 8,6 & 4,3 & 301100 & 13,4 & 9,4 & 4,6 \\
1991 & 2358505 & 286049 & 12,1 & 8,5 & 4,2 & 314000 & 13,3 & 9,3 & 4,6 \\
1992 & 2434864 & 285651 & 11,7 & 8,2 & 4,1 & 306400 & 12,6 & 8,7 & 4,4 \\
1993 & 2488985 & 270532 & 10,9 & 7,5 & 3,8 & 296700 & 11,9 & 8,2 & 4,2 \\
1994 & 2544299 & 272536 & 10,7 & 7,4 & 3,7 & 296000 & 11,6 & 8,0 & 4,0 \\
1995 & 2627503 & 280785 & 10,7 & 7,3 & 3,7 & 312300 & 11,9 & 8,2 & 4,1 \\
$1996\left(^{*}\right)$ & 2686789 & 256480 & 9,5 & 6,6 & 3,3 & 290000 & 10,8 & 7,4 & 3,7
\end{tabular}

Sources: Impôt net émis au titre des revenus de l'année n: Etats 1921, tableaux III (foyers imposables) (cf. Annexe B)

Recettes: recettes de l'impôt sur le revenu perçues pendant l'année $\mathrm{n}+1$ (estimation publiée en septembre $\mathrm{n}+1$ dans le PLF pour l'année $\mathrm{n}+2$ ).

Lecture: En 1970, le revenu imposable total était de 266,545 milliards de francs courants; le total des impositions établies en 1971 et 1972 au titre des revenus de 1970 était de 26,807 milliards de francs courants, soit $10,1 \%$ du revenu imposalbe total, environ $7,3 \%$ du revenu fiscal déclaré (avant abattements, déductions etc..) et 3,4\% du PIB; les recettes recouvrées en 1971 au titre de l'impôt sur le revenu (portant principalement sur les revenus de 1970) étaient de 29,721 milliards de francs, soit $11,2 \%$ du revenu imposable de $1970,8,1 \%$ du revenu fiscal de 1970 , et $3,7 \%$ du PIB de 1970 .

Notes: (i) 1996(*): chiffres provisoires pour le revenu imposable et l'impôt émis (rôles émis au $31 / 12 / 97)$

(ii) Tous les chiffres sont exprimés en millions de FF. 
revenus de 1970 représentait $10,1 \%$ du revenu imposable de 1970, et l'impôt émis au titre des revenus de 1995 représente 10,7\% du revenu imposable de 1995 (cf. tableau 2-4).

Insérer Tableau 2-4 : La croissance des recettes de l'impôt sur le revenu de 1970 à 1996

Le tableau 2-4 distingue deux types de statistiques pour les recettes fiscales : d'une part l'impôt net émis au titre des revenus de l'année n, et d'autre part les recettes effectivement recouvrées durant l'année $n+1$. L'impôt net émis au titre des revenus de l'année $\mathrm{n}$ est défini comme l'ensemble des émissions d'impôt effectuées au $31 / 12 / n+2$ à partir des déclarations de revenus de l'année $n$, telles qu'elles sont comptabilisées dans les Etats 1921. Par exemple, s'agissant de l'année 1995, 280,785 milliards d'émissions d'impôts au titre des revenus de 1995 ont été effectuées en 1996 et en $1997 .{ }^{41}$ Par opposition, les 312,300 milliards de recettes recouvrées durant l'année 1996 incluent non seulement les émissions effectuées et recouvrées en 1996 au titre des revenus de 1995, mais également des reliquats d'émissions effectués au titre des revenus de 1994 et des années antérieures et recouvrés en 1996, ainsi que le produit du contrôle fiscal et des éventuels redressements d'impôt. Cette dernière composante explique que les recettes effectivement recouvrés soient toujours structurellement supérieures à l'impôt émis, avec un écart de l'ordre de 5 à $10 \%$ suivant les années. Dans le tableau 2-4, nous avons retenu les évaluations de recettes recouvrées pendant l'année $n+1$ publiées à l'automne $n+1$ dans les Projet de Loi de Finances pour l'année $n+2$, évaluations qui diffèrent généralement assez peu des évaluations initiales de la Loi de Finances pour $\mathrm{n}+1 .{ }^{42}$ Exprimées en pourcentage du revenu imposable, les recettes de l'IR passent ainsi de $11,2 \%$ en 1970 à $11,9 \%$ en 1995 . Le tableau $2-4$ indique également l'évolution de l'impôt émis et des recettes en pourcentage du revenu fiscal (avant déductions et abattements): le revenu imposable étant égal à environ $70 \%$ du revenu déclaré, les recettes de l'IR représentent en 1970 comme en 1995 de l'ordre de $8 \%$ du revenu fiscal, soit entre 3,5 et $4 \%$ du PIB. ${ }^{43}$

\footnotetext{
41 Jusqu'aux revenus de 1986 (inclus), l'Etat 1921 définitif était établi au $31 / 3 / n+2$ et non au $31 / 12 / n+2$. Cela n'introduit aucun biais important, car en pratique l'immense majorité des émissions se fait pendant l'année $n+1$ et au début de l'année $n+2$.

${ }^{42}$ Par exemple, la Loi de Finances pour 1996 prévoyait des recettes d'IR de 310,130 milliards (cf. JO "Lois et décrets" du 31/12/95, p.19047), évaluation révisée à 312,300 milliards dans le PLF97 (cf. PLF 97, "Evaluation des voies et moyens ", tome 1 ("Les prévisions de recettes "), p.10). La LF97 escomptait 291,845 milliards(cf. JO "Lois et décrets" du 31/12/96, p.19511), évaluation révisée à 290,000 milliards dans le PLF98 ((cf. PLF98, "Evaluation des voies et moyens", tome 1 ("Les prévisions de recettes "), p.12)). Les prévisions de recettes pour l'IR ne sont distinguées de celles des autres impôts directs perçus par voie d'émissions de rôles uniquement depuis PLF78; les estimations de recettes pour 1970-1975 indiquées dans le tableau 2-4 sont égales aux prévisions de recettes du total des impôts directs perçus par voie d'émissions de rôles pour les années 1971-1976, telles qu'elles ont été publiées dans les PLF72-77, multipliées par un coefficient de 0,95 (ce qui correspond à peu près à la part de l'IR dans le total des émissions de rôles d'impôts directs durant cette période).

${ }^{43}$ Les colonnes (impôt émis)/(revenu fiscal) et recettes/(revenu fiscal) du tableau 2-4 ont été calculées en supposant que le taux d'imposabilité moyen avait évolué de façon linéaire entre les enquêtes
} 
De la même façon que pour le pourcentage de foyers imposables, cette stabilité de long-terme observée entre 1970 et 1995 masque une évolution "en $\cap$ " observée au cours des 25 dernières années: les recettes de l'IR ont atteint près de $10 \%$ du revenu fiscal (et plus de $5 \%$ du PIB) en 1981, avant de tomber à $7,4 \%$ du revenu fiscal $(3,7 \%$ du PIB) en 1996. Sur la période récente, la baisse du rendement de l'IR n'est cependant pas la conséquence de la diminution du pourcentage de foyers imposables, ce dernier étant extrêmement stable depuis 1987. La baisse du rendement de I'IR observée pendant les années 1990 est pour l'essentiel la conséquence du gonflement progressif du poids des mécanismes de réductions d'impôt (emplois à domicile, etc...), et surtout des réductions générales des taux du barème mises en place en 1993 et en 1996. Cette baisse tendancielle est extrêmement significative : exprimées en pourcentage du PIB, les recettes de l'IR ont perdu près d'un point de PIB entre 1991 et 1996, et plus de 2,5 points de revenu imposable. Si les recettes perçues par l'Etat en 1997 (ou en 1998) au titre de l'impôt sur le revenu représentaient le même pourcentage du revenu des ménages qu'en 1992, alors l'Etat disposerait de recettes supplémentaires de l'ordre de 70 milliards de francs. ${ }^{44}$

\footnotetext{
"Revenus fiscaux" de 1970, 1975, 1979 et 1984 et les échantillons légers de déclarations de revenus des années 1988 à 1995 (cf. les tableaux des annexes $F$ et G). Les éventuelles erreurs induites sont très faibles, puisque le taux d'imposabilité moyen est toujours compris entre $69 \%$ et $72 \%$ environ.

${ }^{44}$ Le fait que la baisse des taux marginaux du barème de 1993-1996 ait mécaniquement conduit à une baisse des recettes fiscales suggère assez clairement que le sommet de la courbe de Laffer n'a pas été atteint en France. Mais en toute rigueur, cette " expérience naturelle " n'est pas satisfaisante, car rien ne permet d'exclure que sans ces baisses de taux marginaux l'ensemble des revenus déclarés par les contribuables (et éventuellement les recettes fiscales) seraient en 1996-1997 plus faibles qu'ils ne le sont : pour estimer les effets de variations de taux marginal sur les comportements, il faut que ces variations concernent uniquement un groupe spécifique de contribuables, ce qui n'est pas le cas des baisses de 1993-1996.
} 


\section{L'évolution de la part et de la composition des hauts revenus de 1970 à 1996}

\subsection{Hauts revenus, très hauts revenus et très très hauts revenus : premiers repères}

Le coeur de notre étude consistant à comparer l'évolution du revenu des $10 \%$ les plue riches, des $1 \%$ les plus riches, des $0,5 \%$ les plus riches, etc.., il est utile d'avoir présent à l'esprit les ordres de grandeur atteints par les hauts, les très hauts et les très hauts revenus au milieu des années 1990. Le tableau 3-1 décrit la distribution du revenu fiscal déclaré et du revenu imposable en 1995, telle qu'elle nous est fournie par l'échantillon léger de déclarations de revenus 1995.

Insérer Tableau 3-1: La distribution du revenu fiscal déclaré et du revenu imposable en 1995

Le revenu fiscal moyen des foyers fiscaux était en 1995 de 126057 francs, soit environ 10500 francs par mois. Compte tenu du fait que chaque ménage comprend en moyenne 1,3 foyers fiscaux (cf. section 2.2 supra), cela signifie que le revenu fiscal moyen des ménages était en 1995 de l'ordre de 164000 francs, soit environ 13700 francs par mois. Le revenu fiscal médian des foyers fiscaux était de 94888 francs, soit environ 7900 francs par mois, ce qui correspond à un revenu médian des ménages de l'ordre de 123000 francs, ou 10300 francs par mois. Ces ordres de grandeurs (entre 10000 et 11000 francs par mois de revenu médian, et environ 14000 francs par mois de revenu moyen) correspondent assez bien aux ordres de grandeurs mesurés par des enquêtes sur les revenus telles que l'enquête "Budget

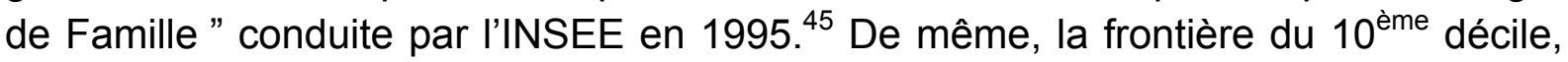
qui se situe à 256189 francs d'après le tableau 3-1, soit environ 21300 francs par mois, correspond bien aux ordres de grandeurs habituellement retenus, comme par exemple lors du récent débat sur le plafonnement des allocations familiales : environ $10 \%$ des ménages disposent de plus de 25000 francs par mois.

Dans le tableau 3-1, comme dans l'ensemble de cette étude, " P90-100 " désigne le groupe des $10 \%$ des foyers fiscaux ayant le revenu le plus élevé (c'est-à-dire le $10^{\text {ème }}$ décile de la distribution des revenus), "P90-95" désigne la moitié la plus pauvre de ce $10^{\text {ème }}$ décile, "P95-100 " désigne le groupe des $5 \%$ des foyers fiscaux ayant le revenu le plus élevé, et ainsi de suite. "P99,999-100" regroupe donc les $0,001 \%$ des foyers fiscaux ayant le revenu le plus élevé, c'est-à-dire les 300 foyers fiscaux les plus fortunés de France. Pour faire partie de ce groupe, il faut disposer de plus de 11,2 millions de francs de revenu fiscal, et les membres de ce groupe

\footnotetext{
${ }^{45}$ Cette enquête évalue à environ 11700 francs le revenu mensuel médian des ménages en 1994, et à environ 14190 francs le revenu mensuel moyen (cf. par exemple Piketty (1997a, p.13, tableau IV)). Les revenus estimés par les enquêtes "Budget de Famille " sont légèrement supérieurs aux revenus fiscaux déclarés, car ces derniers n'incluent pas les revenus non-imposables (ils excluent notamment les prestations sociales non-imposables (allocations familiales, minimas sociaux, ...), si bien que la sous-estimation est particulièrement forte pour les bas revenus).
} 
Tableau 3-1:

\section{La distribution du revenu fiscal déclaré et du revenu imposable en 1995}

Revenu fiscal déclaré

$\begin{array}{lrc}\begin{array}{l}\text { N.foyers fisc. } \\ \text { rfisc.moyen }\end{array} & \begin{array}{r}29958336 \\ 126057\end{array} \\ \text { rfisc.médian } & 94888 & \\ & \begin{array}{r}\text { rfisc.moy. } \\ \text { lim.inf. }\end{array} \\ \text { P90-100 } & 403411 & 256189 \\ \text { P90-95 } & 287273 & \\ \text { P95-100 } & 520147 & 329144 \\ \text { P95-99 } & 408812 & \\ \text { P99-100 } & 963259 & 589531 \\ \text { P99-99,5 } & 661192 & \\ \text { P99,5-100 } & 1265717 & 754057 \\ \text { P99,5-99,9 } & 967034 & \\ \text { P99,9-100 } & 2459152 & 1426985 \\ \text { P99,9-99,99 } & 1974986 & \\ \text { P99,99-100 } & 6816325 & 3774938 \\ \text { P99,99-99,999 } & 5524843 & \\ \text { P99,999-100 } & 18461260 & 11217410\end{array}$

Revenu imposable

$\begin{array}{lrrr}\begin{array}{l}\text { N.foyers fisc. } \\ \text { rimp.moyen }\end{array} & \begin{array}{r}29958336 \\ 86476\end{array} & 68,6 \\ \text { rimp.médian } & 63706 & & \\ & \text { rimp.moy. } & \text { lim.inf. } & \text { \%rimp/rfisc } \\ \text { P90-100 } & 290810 & 177755 & \\ \text { P90-95 } & 199685 & & 69,5 \\ \text { P95-100 } & 381732 & 230183 & \\ \text { P95-99 } & 291750 & & 71,4 \\ \text { P99-100 } & 741825 & 431162 & \\ \text { P99-99,5 } & 484953 & & 73,3 \\ \text { P99,5-100 } & 988474 & 558820 & \\ \text { P99,5-99,9 } & 729328 & & 75,4 \\ \text { P99,9-100 } & 2075102 & 1109499 & \\ \text { P99,9-99,99 } & 1628548 & & 82,5 \\ \text { P99,99-100 } & 6094976 & 3307816 & \\ \text { P99,99-99,999 } & 4889172 & & 88,5 \\ \text { P99,999-100 } & 16967380 & 10192690 & 91,9\end{array}$

Source: Calculs de l'auteur à partir de l'échantillon léger de déclarations de revenus 1995 (cf. Annexe F). Le taux de sondage moyen de l'échantillon est de 1/741 (40442 observations pour 29958336 foyers), mais il passe de $1 / 1338$ sur P0-90 (20147 observations pour 26962502 foyers) à $1 / 741$ sur P90-95 (2022 observations pour 1497917 foyers), 1/238 sur P95-99 (5036 observations pour 1199413 foyers), 1/99 sur P99-99,5 (1511 observations pour 149792 foyers), 1/41 sur P99,5-99,9 (2901 observations pour 119941 foyers), 1/5 sur P99,9-99,99 (5830 observations pour 26963 foyers), et 1/1 sur P99,99-100 (2995 observations pour 2995 foyers).

Lecture: "P90-100" regroupe les $10 \%$ des foyers fiscaux ayant le revenu fiscal (1er tableau) ou le revenu imposable (2nd tableau) le plus élevé; leur revenu fiscal moyen est de 403411FF en 1995, et ils disposent tous de plus de 256189FF de revenu fiscal. "P95-100" regroupe les $5 \%$ des foyers fiscaux ayant le revenu le plus élevé, "P99-100" regroupe les $1 \%$ des foyers fiscaux ayant le revenu le plus élevé, etc.. 
disposent en moyenne de près de 18,5 millions de revenu fiscal. Pour faire partie des $1 \%$ des foyers fiscaux ayant le revenu fiscal le plus élevé, c'est-à-dire des 300000 foyers fiscaux les plus fortunés, il faut disposer de plus de 589531 francs de revenu annuel (soit environ 49100 francs par mois), et les membres de ce groupe dispose en moyenne de 963259 francs de revenu fiscal (soit environ 80300 francs par mois). La frontière à dépasser pour faire partie de P99,9-100, c'est-à-dire des $0,1 \%$ des foyers fiscaux ayant le revenu fiscal le plus élevé, soit environ 30000 foyers, était en 1995 de 1426985 francs (environ 118900 francs par mois).

Le tableau 3-1 décrit également la distribution du revenu imposable. Le revenu imposable moyen était en 1995 de 86476 francs, soit environ $68,6 \%$ du revenu fiscal déclaré moyen. La dernière colonne du tableau 3-1 indique que ce "taux d'imposabilité " est une fonction croissante du niveau de revenu, notamment à l'intérieur du centile supérieur. Le revenu imposable moyen des $0,001 \%$ des foyers fiscaux ayant le revenu imposable le plus élevé était ainsi de 16,967 millions de francs en 1995, soit $91,9 \%$ des 18,461 millions de francs de revenu fiscal moyen des $0,001 \%$ des foyers fiscaux ayant le revenu fiscal le plus élevé. Le fait que les taux d'imposabilité atteignent des niveaux aussi élevés pour les très hauts revenus s'explique par plusieurs facteurs: tout d'abord, à ces niveaux de revenus, les plafonnements des déductions et abattements de $10 \%$ et $20 \%$ sur les pensions et retraites sont généralement largement dépassés, si bien que les salaires et retraites imposable tend à se rapprocher du salaires et retraites déclaré ; ensuite et surtout, les revenus de capitaux mobiliers occupent une place très importante pour les très hauts revenus, et ces revenus catégoriels sont imposables à 100\% (au-delà d'un modeste abattement forfaitaire). Ce même phénomène de croissance du taux d'imposabilité s'observe dans des proportions identiques sur l'ensemble de la période 1970-1995. ${ }^{46}$

De fait, le tableau 3-2 indique que la composition moyenne du revenu fiscal évolue de façon extrêmement rapide à l'intérieur du centile supérieur de la distribution.

\section{Insérer Tableau 3-2 : La composition du revenu fiscal déclaré en 1995}

Tous foyers fiscaux confondus, les salaires et les pensions de retraites représentaient en 1995 plus de $86 \%$ du revenu fiscal déclaré $(63,1 \%$ pour les salaires, et $23,4 \%$ pour les retraites). Ce pourcentage atteint $92 \%$ pour P0-90 (les 9 premiers déciles de la distributions): $63,9 \%$ pour les salaires et $28,1 \%$ pour les retraites. La part des salaires et pensions pour P90-95 continue d'être supérieure à la moyenne de la distribution : $87,8 \%$, dont $69,6 \%$ pour les salaires. La part des salaires continue d'être supérieure à la moyenne sur P95-99 : ces foyers disposent de $65,1 \%$ de leur revenu total sous forme de salaires. Mais à partir de P99, la part des salaires et des pensions baisse rapidement, et les revenus du patrimoine et des professions non-salariés deviennent progressivement majoritaires.

\footnotetext{
${ }^{46} \mathrm{Cf}$. les tableaux des annexes $\mathrm{F}$ et $\mathrm{G}$.
} 
Tableau 3-2 : La composition du revenu fiscal déclaré en 1995

$\begin{array}{lccccccccc}\text { En \%: } & \text { BA } & \text { BIC } & \text { BNC } & \text { RGA } & \text { RF } & \text { RCM } & \text { SAL } & \text { PEN } & \\ \text { P0-100 } & 1,2 & 3,4 & 3,3 & 0,3 & 2,5 & 2,8 & 63,1 & 23,4 & 100,0 \\ \text { P90-100 } & 1,4 & 5,4 & 8,8 & 0,5 & 4,0 & 5,0 & 61,4 & 13,5 & 100,0 \\ \text { P95-100 } & 1,6 & 6,4 & 12,1 & 0,7 & 4,6 & 6,8 & 57,2 & 10,6 & 100,0 \\ \text { P99-100 } & 1,3 & 8,0 & 20,9 & 1,1 & 6,1 & 13,5 & 43,7 & 5,4 & 100,0 \\ \text { P99,5-100 } & 1,3 & 8,5 & 22,8 & 1,1 & 6,3 & 17,8 & 38,4 & 3,8 & 100,0 \\ \text { P99,9-100 } & 1,2 & 7,3 & 19,5 & 1,1 & 6,4 & 30,7 & 31,5 & 2,4 & 100,0 \\ \text { P99,99-100 } & 0,8 & 6,8 & 11,2 & 0,6 & 5,4 & 53,3 & 20,7 & 1,4 & 100,0 \\ \text { P99,999-100 } & 0,1 & 10,4 & 8,4 & 0,3 & 3,1 & 62,4 & 14,2 & 1,1 & 100,0 \\ & & & & & & & & & \\ \text { En \%: } & \text { BA } & \text { BIC } & \text { BNC } & \text { RGA } & \text { RF } & \text { RCM } & \text { SAL } & \text { PEN } & \\ \text { P0-90 } & 1,1 & 2,4 & 0,7 & 0,1 & 1,8 & 1,8 & 63,9 & 28,1 & 100,0 \\ \text { P90-95 } & 1,1 & 3,7 & 2,7 & 0,2 & 2,8 & 1,8 & 69,2 & 18,6 & 100,0 \\ \text { P95-99 } & 1,7 & 5,4 & 7,0 & 0,5 & 3,7 & 2,9 & 65,1 & 13,7 & 100,0 \\ \text { P99-99,5 } & 1,5 & 7,0 & 17,3 & 1,1 & 5,6 & 5,4 & 53,8 & 8,3 & 100,0 \\ \text { P99,5-99,9 } & 1,3 & 9,3 & 24,9 & 1,2 & 6,2 & 9,6 & 42,8 & 4,7 & 100,0 \\ \text { P99,9-99,99 } & 1,3 & 7,5 & 22,6 & 1,3 & 6,8 & 22,0 & 35,6 & 2,8 & 100,0 \\ \text { P99,99-99,999 } & 1,0 & 5,5 & 12,3 & 0,6 & 6,2 & 49,9 & 23,1 & 1,5 & 100,0 \\ \text { P99,999-100 } & 0,1 & 10,4 & 8,4 & 0,3 & 3,1 & 62,4 & 14,2 & 1,1 & 100,0\end{array}$

Source: Cf. tableau 3-1.

Lecture: Le revenu fiscal des $0,001 \%$ des foyers fiscaux ayant le revenu fiscal le plus élevé est composé pour $0,1 \%$ de bénéfices agricoles (BA), pour 10,4\% de bénéfices industriels et commerciaux (BIC), pour $8,4 \%$ de bénéfices noncommerciaux (BNC), pour $0,3 \%$ de rémunération de gérants et associés (RGA), pour $3,1 \%$ de revenus fonciers (RF), pour $62,4 \%$ de revenus de capitaux mobiliers (RCM), pour $14,2 \%$ de salaires (SAL), et pour $1,1 \%$ de pensions de retraite (PEN). 
Au delà de cette caractéristique bien connue, deux faits importants révélés par le tableau 3-2 méritent d'être retenus. Tout d'abord, il faut monter très haut dans la distribution du revenu pour que les salaires et pensions cessent d'être majoritaires. Les foyers de P99-99,5, c'est-à-dire de l'avant-dernier demi-centile, disposent en moyenne de 10 fois plus de revenus sous forme de salaires (53,8\% de leur revenu total) que de revenus de capitaux mobiliers ( $5,4 \%$ de leur revenu total). La part des revenus de capitaux mobiliers ne dépasse celle des salaires qu'à partir de P99,99100 , c'est-à-dire pour les 3000 foyers fiscaux les plus riches, et ce bien que l'année 1995 soit une année particulièrement haute pour les revenus de capitaux mobiliers (cf. les tableaux de l'Annexe F, ainsi que le tableau 3-6 infra). Ce résultat n'est pas véritablement étonnant : il exprime simplement le fait qu'il est beaucoup plus courant de disposer de 80000 francs de revenu mensuel (ce qui représente le revenu mensuel moyen de P99-100) grâce à son salaire de cadre supérieur dans une grande entreprise que de disposer du même revenu grâce à ses loyers et à ses dividendes.

Le second enseignement important est que si la part des salaires et pensions diminue régulièrement de $\mathrm{P} 90-95$ à $\mathrm{P} 99,999-100$ et que celle des revenus de capitaux mobiliers augmente régulièrement de P90-95 à P99,999-100, il n'en va pas de même de la part des BIC, des BNC et des revenus fonciers, qui connaissent des évolutions beaucoup plus complexes. En effet, l'importance de ces trois catégories de revenus augmente régulièrement jusqu'à $P 99,5-99,9$, puis se met à décroître à l'intérieur de P99,9-100, pour céder la place aux revenus de capitaux mobiliers, qui pour les 300 foyers les plus riches deviennent trois fois plus importants que l'ensemble des BIC, BNC et revenus fonciers. Autrement dit, les très très hauts revenus reposent d'avantage sur leurs placements financiers que sur leurs loyers ou leurs activités non-salariées, contrairement aux très hauts revenus, pour qui ces sources de revenus sont prépondérantes. Remarquons également que la part des BIC augmente à nouveau au niveau de P99,999. Cela traduit le fait que si de façon générale il existe beaucoup plus de médecins et d'avocats que de commerçants parmi les foyers aisés (la part des BNC est de 20,9\% dans P99-100, contre 8,0\% pour les BIC, bien que les BIC comme les BNC représentent en moyenne 3,3-3,4\% de l'ensemble des revenus des foyers), il existe cependant un petit nombre de gros commerçants et d'entrepreneurs très, très aisés (la part des BIC augmente à 10,4\% dans $\mathrm{P} 99,999-100$, contre $8,4 \%$ pour les BNC). ${ }^{47}$

\subsection{L'évolution de la part des hauts revenus de 1970 à 1996}

Quelles ont été les grandes lignes de l'évolution de l'inégalité du revenu imposable pendant la période 1970-1996 ? Les tableaux 3-3 et 3-4 décrivent l'évolution de la part des $10 \%$ des foyers fiscaux ayant le revenu imposable le plus élevé (P90-100), des 5\% (P95-100), des 1\% (P99-100), des 0,5\% (P99,5-100), des 0,1\% (P99,9-100)

\footnotetext{
${ }^{47}$ Les échantillons légers de déclarations de revenus permettent d'observer ce même phénomène sur l'ensemble de la période 1988-1995 (cf. les tableaux de l'annexe F).
} 
et des $0,01 \%$ (P99,99-100). Tout comme celles des tableaux 3-1 et 3-2, ces estimations concernent l'ensemble des foyers fiscaux, tous nombres de parts de quotient familial confondus.

Insérer Tableaux 3-3 et 3-4 : L'évolution de la part des hauts revenus dans le revenu imposable de 1970 à 1996

Le premier constat est celui d'une relative stabilité de la part des hauts revenus sur la période 1970-1996. La part de P90-100 varie entre un minimum de $31,15 \%$ en 1982 et un maximum de $35,41 \%$ en 1973 , et se situe la plupart du temps autour de $33-34 \%$. Une part de $33,71 \%$ en 1995 signifie que les foyers fiscaux du $10^{\text {ème }}$ décile ont en 1995 un revenu moyen qui est 3,37 fois plus élevé que le revenu moyen de l'ensemble des foyers fiscaux, ce qui correspond à peu près aux chiffres de 290810 francs de revenu moyen pour P90-100 et de 86476 francs de revenu moyen pour l'ensemble des foyers fiscaux mentionnés sur le tableau 3-1 $(290810 / 86476=$ $3,36) .{ }^{48}$ Autrement dit, sur l'ensemble de la période 1970-1996, le revenu moyen du $10^{\text {ème }}$ décile se situe toujours entre 3,1 et 3,5 fois le revenu moyen de l'ensemble des foyers fiscaux, et généralement autour de 3,3-3,4 fois le revenu moyen. De même, la part de P99-100 varie entre 7,84\% du revenu total en 1983 à 10,00\% en 1973 , ce qui signifie que le revenu moyen des $1 \%$ les plus riches se situe toujours autour de 8-10 fois le revenu moyen de l'ensemble des foyers fiscaux. En 1995, ce ratio est de 8,62, ce qui correspond à peu près au chiffre de 741825 francs de revenu moyen pour P99-100 mentionnés sur le tableau 3-1 $(741825 / 86476=8,58)$. A titre de comparaison, la part du revenu imposable détenue par les $0,5 \%$ des foyers fiscaux américains les plus aisés est passé d'environ $6 \%$ à la fin des années 1970 à près de $12 \%$ à la fin des années 1980 (auparavant, elle était passée progressivement d'environ $9 \%$ en 1950 à moins de $6 \%$ dans les années 1970). ${ }^{49}$ En France, cette part de P99,5-100 est de l'ordre de 5-6\% sur l'ensemble de la période 1970-1996. Au Royaume-Uni, la part du revenu imposable des $1 \%$ des foyers les plus riches a diminué régulièrement de plus de $11 \%$ en 1950 à à peine plus de $5 \%$ à la fin des années $1970,{ }^{50}$ alors que cette part est restée relativement stable autour de $8-9 \%$ en France sur la période 1970-1996. La principale caractéristique de la distribution française des revenus pendant les 25 dernières années est donc sa très grande inertie, si on la compare à ce qui s'observe parfois à l'étranger en l'espace d'une ou deux décennies. Le revenu imposable nominal a été multiplié par 10, et le revenu imposable réel par près de deux, mais sa répartition a globalement très peu changé.

Au-delà de ce constat général, les tableaux 3-3 et 3-4 montrent que les parts des hauts revenus ont connu deux grands types de fluctuations sur la période 1970-1996. D'une part, le tableau 3-3 atteste l'existence d'une certaine "courbe en U " de

\footnotetext{
${ }^{48} \mathrm{Cf}$. Annexe E.1 pour une comparaison systématique entre les estimations établies à partir des Etats 1921 et des estimations issues des échantillons légers.

${ }^{49}$ Cf. Feenberg et Poterba (1993).

${ }^{50}$ Cf. Dolan (1989, p.202).
} 
Tableau 3-3 :

L'évolution de la part des hauts revenus dans le revenu imposable de 1970 à 1996 (ensemble des foyers fiscaux)

$\begin{array}{rrrrrrr} & \mathrm{P} 90-100 & \mathrm{P} 95-100 & \mathrm{P} 99-100 & \mathrm{P} 99,5-100 & \mathrm{P} 99,9-100 & \mathrm{P} 99,99-100 \\ 1970 & 34,49 & 23,31 & 9,32 & 6,26 & 2,45 & 0,68 \\ 1971 & 34,81 & 23,56 & 9,54 & 6,43 & 2,53 & 0,68 \\ 1972 & 34,49 & 23,43 & 9,59 & 6,49 & 2,58 & 0,71 \\ 1973 & 35,41 & 24,13 & 10,00 & 6,81 & 2,75 & 0,80 \\ 1974 & 34,78 & 23,55 & 9,57 & 6,46 & 2,54 & 0,68 \\ 1975 & 34,86 & 23,51 & 9,54 & 6,41 & 2,53 & 0,68 \\ 1976 & 34,63 & 23,35 & 9,49 & 6,38 & 2,53 & 0,68 \\ 1977 & 33,02 & 22,05 & 8,76 & 5,89 & 2,35 & 0,64 \\ 1978 & 32,71 & 21,90 & 8,77 & 5,89 & 2,34 & 0,63 \\ 1979 & 32,37 & 21,75 & 8,80 & 5,94 & 2,39 & 0,65 \\ 1980 & 32,00 & 21,41 & 8,58 & 5,78 & 2,31 & 0,63 \\ 1981 & 32,03 & 21,33 & 8,49 & 5,70 & 2,29 & 0,62 \\ 1982 & 31,15 & 20,59 & 7,94 & 5,31 & 2,08 & 0,55 \\ 1983 & 31,64 & 20,73 & 7,84 & 5,18 & 1,97 & 0,50 \\ 1984 & 31,73 & 20,78 & 7,88 & 5,19 & 1,99 & 0,51 \\ 1985 & 32,29 & 21,20 & 8,07 & 5,35 & 2,06 & 0,53 \\ 1986 & 32,67 & 21,57 & 8,35 & 5,57 & 2,18 & 0,57 \\ 1987 & 33,05 & 21,99 & 8,71 & 5,90 & 2,39 & 0,65 \\ 1988 & 33,44 & 22,25 & 8,90 & 6,07 & 2,49 & 0,70 \\ 1989 & 33,82 & 22,71 & 9,23 & 6,34 & 2,65 & 0,76 \\ 1990 & 34,04 & 22,84 & 9,25 & 6,35 & 2,65 & 0,76 \\ 1991 & 33,79 & 22,53 & 8,95 & 6,09 & 2,49 & 0,69 \\ 1992 & 33,55 & 22,21 & 8,69 & 5,87 & 2,37 & 0,64 \\ 1993 & 33,52 & 22,11 & 8,58 & 5,79 & 2,32 & 0,63 \\ 1994 & 33,67 & 22,21 & 8,64 & 5,85 & 2,37 & 0,65 \\ 1995 & 33,71 & 22,23 & 8,62 & 5,82 & 2,34 & 0,63 \\ 1996\left(^{*}\right) & 33,51 & 22,07 & 8,50 & 5,73 & 2,29 & 0,62 \\ & & & & & & \end{array}$

Source: Estimations de l'auteur à partir des Etats 1921 (cf. Annexe D.1)

Lecture: En 1970, les 10\% des foyers fiscaux ayant le revenu imposable le plus élevé détiennent $34,49 \%$ du revenu imposable total, les $5 \%$ ayant le revenu imposable le plus élevé détiennent $25,41 \%$ du revenu imposable total, etc...

Note: $1996\left(^{*}\right)$ : chiffres provisoires (rôles émis au 31/12/97) 
Tableau 3-4 :

L'évolution de la part des hauts revenus dans le revenu imposable de 1970 à 1996 (ensemble des foyers fiscaux)

$\begin{array}{rrrrrr} & \text { P90-95 } & \text { P95-99 } & \text { P99-99,5 } & \text { P99,5-99,9 } & \text { P99,9-100 } \\ 1970 & 11,18 & 13,98 & 3,07 & 3,81 & 2,45 \\ 1971 & 11,25 & 14,02 & 3,11 & 3,90 & 2,53 \\ 1972 & 11,06 & 13,84 & 3,10 & 3,91 & 2,58 \\ 1973 & 11,29 & 14,13 & 3,19 & 4,05 & 2,75 \\ 1974 & 11,23 & 13,98 & 3,11 & 3,91 & 2,54 \\ 1975 & 11,35 & 13,97 & 3,13 & 3,88 & 2,53 \\ 1976 & 11,28 & 13,86 & 3,12 & 3,84 & 2,53 \\ 1977 & 10,97 & 13,28 & 2,87 & 3,54 & 2,35 \\ 1978 & 10,82 & 13,13 & 2,88 & 3,55 & 2,34 \\ 1979 & 10,62 & 12,95 & 2,86 & 3,55 & 2,39 \\ 1980 & 10,59 & 12,83 & 2,81 & 3,46 & 2,31 \\ 1981 & 10,69 & 12,84 & 2,79 & 3,41 & 2,29 \\ 1982 & 10,56 & 12,65 & 2,63 & 3,22 & 2,08 \\ 1983 & 10,91 & 12,89 & 2,67 & 3,20 & 1,97 \\ 1984 & 10,95 & 12,90 & 2,69 & 3,19 & 1,99 \\ 1985 & 11,09 & 13,12 & 2,72 & 3,29 & 2,06 \\ 1986 & 11,10 & 13,23 & 2,77 & 3,39 & 2,18 \\ 1987 & 11,07 & 13,28 & 2,81 & 3,51 & 2,39 \\ 1988 & 11,19 & 13,35 & 2,83 & 3,58 & 2,49 \\ 1989 & 11,11 & 13,48 & 2,89 & 3,69 & 2,65 \\ 1990 & 11,19 & 13,59 & 2,90 & 3,70 & 2,65 \\ 1991 & 11,26 & 13,58 & 2,86 & 3,60 & 2,49 \\ 1992 & 11,33 & 13,52 & 2,82 & 3,51 & 2,37 \\ 1993 & 11,40 & 13,54 & 2,79 & 3,47 & 2,32 \\ 1994 & 11,47 & 13,57 & 2,79 & 3,48 & 2,37 \\ 1995 & 11,48 & 13,61 & 2,80 & 3,48 & 2,34 \\ 1996\left(^{*}\right) & 11,44 & 13,57 & 2,77 & 3,44 & 2,29\end{array}$

Source: Calculs effectués à partir des résultats du tableau 3-3 (P90-95 = P90-100 - P95-100; P95-99 = P95-100 - P99-100; etc..)

Note: $1996\left({ }^{*}\right)$ : chiffres provisoires (rôles émis au 31/12/97) 
l'inégalité des revenus en France pendant cette période. La part de P90-100 passe progressivement de $34,5-35 \%$ au début des années 1970 à à peine plus de $31 \%$ en 1982, avant de remonter dans les années 1980 et de stabiliser autour de 33,5-34\% dans les années 1990. II s'agit là de variations d'une ampleur limitée, mais qui traduisent cependant l'existence de deux trends bien réels allant en sens contraire dans les années 1970 et dans les années 1980-1990. Dans les années 1970, la compression des inégalités de salaires, entraînée notamment par les très fortes revalorisations du SMIC, a conduit à une certaine diminution des inégalités de revenus. ${ }^{51}$ Ce phénomène de compression des inégalités salariales a pris fin au début des années 1980, et la force dominante pendant les années 1980-1990 est devenue la croissance du chômage et du sous-emploi (temps partiel, travail intermittent, ...), que la montée en charge des allocations chômage et des transferts sociaux n'a pu entièrement compenser, si bien qu'elle s'est traduite par une certaine augmentation des inégalités de revenus (là encore à un rythme modéré). ${ }^{22}$ Ces phénomènes de long-terme ont déjà été étudiés par ailleurs, ${ }^{53}$ et leur analyse détaillée dépasse de très loin le cadre de cette étude. ${ }^{54}$

\footnotetext{
${ }^{51}$ Le fait que la "grande compression" des inégalités salariales françaises entre 1968 et 1983 n'ait conduit qu'à une diminution modérée de la part de P90-100 peut s'expliquer de plusieurs façons : tout d'abord, la diminution modérée de la part de P90-100 peut masquer une diminution plus importante de l'inégalité dans les parties inférieures de la distribution, par exemple telle qu'elle peut être mesurée par les ratios interdéciles P90/P10 ou P50/P10 ; ensuite, la compression des inégalités salariales françaises ne s'est finalement traduite que par une baisse d'environ $15 \%$ du ratio P90/P10 de la distribution des salaires, qui est passé d'environ 3,7 à la fin des années 1960 à environ 3,2 au début des années 1980 : la France n'a jamais connu ni les ratios extrêmement élevés de l'ordre de 4,5-5 observés aux Etats-Unis dans les années 1990, ni les ratios extrêmement faibles de l'ordre de 2-2,5 observés traditionnellement dans les pays scandinaves (cf. Piketty (1997a, p.12, tableau III ; p.19, tableau VII)).

${ }^{52}$ En utilisant les enquêtes "Budget de Famille " conduite par l'INSEE en 1979, 1984-1985, 1989 et 1994-1995, Bourguignon et Martinez (1997) ont montré que la croissance des inégalités de revenu disponible, qui en pratique semble avoir été extrêmement limitée (voire insignifiante), aurait été beaucoup plus élevée si la montée du sous-emploi n'avait pas été compensée par les transferts sociaux, et notamment par les allocations chômage (qui, dans les déclarations de revenus, entrent dans la catégorie des " traitements et salaires "). La baisse continue de l'inégalité parmi les retraités a également contribué à ce que l'inégalité totale augmente très peu, malgré une augmentation sensible des inégalités de revenus parmi les ménages d'âge actif entre 1984-1985 et 1994-1995 (cf. InseeSynthèses $n^{\circ} 5$, "Revenus et patrimoines des ménages, édition 1996 ", août 1996, p.37). La baisse de l'inégalité parmi les retraités a également contribué à la baisse globale des inégalités pendant les années 1970, mais aucune étude ne semble avoir tenté de quantifier l'importance relative de ce trend comparé au trend concernant les ménages d'âge actif.

${ }^{53}$ En fait, s'il existe d'excellents travaux sur l'évolution des inégalités salariales dans le long-terme (cf. par exemple Insee-Résultats $n^{\circ} 457$, "Séries longues sur les salaires ", 1996), il existe relativement peu d'études de long-terme sur les inégalités de revenus. Le $\mathrm{n}^{\circ} 1$ de Insee-Synthèses ("Revenus et patrimoines des ménages, édition 1995 ", juin 1995) indique que le rapport interdécile P90/P10 en revenu disponible par unité de consommation (après imputation des prestations sociales non imposables et des impôts) est passé de 4,04 dans l'enquête "Revenus fiscaux" de 1975 à 3,65 dans l'enquête de 1979, 3,67 dans l'enquête de 1984 et 3,42 dans l'enquête de 1990 (cf. pp.31-52); aucune diminution significative n'est observable si l'on omet le passage en UC (cf. Synthèses $n^{\circ} 1$, annexe 6). Le $n^{\circ} 5$ de Insee-Synthèses ("Revenus et patrimoines des ménages, édition 1996 ", août 1996) indique que le rapport interdécile P90/P10 en revenu déclaré par UC est passé de 4,20 dans l'enquête "Budget de Famille" de 1979 à 3,81 dans l'enquête de 1984-1985, 3,89 dans l'enquête de 1989 et 4,01 dans l'enquête de 1994-1995; en calant les revenus du capital déclarés par les ménages sur les revenus du capital de la Comptabilité Nationale, le ratio P90/P10 passe de 4,07 en 1984-1985 à 4,19 en 1989 et 4,48 en 1994-1995 (I'Insee précise toutefois que la méthodologie des
} 
D'autre part, et surtout, les tableaux 3-3 et 3-4 montrent l'importance des fluctuations de court-terme que connaissent les hauts et les très hauts revenus. La part de P90-95 ne connaît pas de fluctuations importantes de court-terme: relativement stable de 1970 à 1976, elle baisse légèrement (mais régulièrement) de 1976 à 1980, puis elle progresse régulièrement, mais à un rythme extrêmement lent, depuis 1982. Cela traduit le fait que les revenus des foyers de P90-95 sont composés dans leur immense majorité de salaires et de pensions de retraite qui ne sont pas (ou peu) affectés par les fluctuations de court-terme dues au cycle économique. Inversement, à mesure que l'on monte dans la hiérarchie des hauts revenus, les fluctuations de court-terme deviennent de plus en plus importantes. Le tableau 3-5 montre clairement la relation entre ces fluctuations de court-terme et le cycle économique.

Insérer Tableau 3-5: Les taux de croissance annuels de P95-99/P90-95, P9999,5/P90-95, etc..., de 1971 à 1996

En moyenne sur la période 1970-1995, les parts de P95-99, P99-99,5, P99,5-99,9 et P99,9-100 ne connaissent pas de trend significatif relativement à la part de P90-95, si ce n'est un (très) léger trend décroissant: le ratio P95-99/P90-95 diminue en moyenne de 0,2\% par an, les ratios P99-99,5/P90-95 et P99,5-99,9/P90-95 diminuent en moyenne de $0,5 \%$ par an, et le ratio P99,9-100/P90-95 diminue en moyenne de 0,3\% par an. Les taux de croissance annuels de P95-99/P90-95 fluctuent relativement peu autour de cette moyenne : en un an, les revenus de P9599 ne perdent jamais plus de 1,5\% sur les revenus de P90-95 (en 1977), et ne gagnent jamais plus de 1,7\% (en 1989). Autrement dit, les foyers de P95-99 sont encore des foyers qui sont relativement épargnées par les fluctuations dues au cycle économique. Les fluctuations des revenus du centile supérieur vis-à-vis des revenus de P90-95 sont beaucoup plus importantes: en un an, les revenus de P99,9-100 peuvent perdre jusqu'à $8,2 \%$ sur les revenus de P90-95 (en 1983), et peuvent gagner jusqu'à $9,8 \%$ (en 1987). Ces fluctuations sont d'autant plus fortes que l'on monte dans les strates supérieures du centile supérieur (elles seraient encore plus fortes pour P99,99-100 que pour P99,9-100). La comparaison avec les taux de croissance du PIB montrent clairement que ces fluctuations sont très étroitement liées au cycle économique :

(i) De 1970 à 1973, période de très forte croissance de l'économie française, les

enquêtes a trop changé entre 1979 et 1984-1985 pour que ces deux enquêtes soient véritablement comparables, et que la légère augmentation de l'inégalité observée depuis l'enquête de 1984-1985 "avoisine les précisions de la mesure", car certains indicateurs de l'inégalité donnent une évolution allant dans la direction opposée).

${ }^{54}$ En outre, les données utilisées dans cette étude ne sont pas adaptées à l'étude de l'ensemble de la distribution des revenus: les revenus faibles disposent notamment de prestations sociales nonimposables et qui ne figurent donc pas dans les statistiques fiscales, et surtout les Etats 1921 ne conservent la trace des revenus déclarés par les foyers non-imposables que depuis 1985. 
Tableau 3-5 :

Les taux de croissance annuels de P95-99/P90-95, P99-99,5/P90-95, etc.., de 1971 à 1996 (ensemble des foyers fiscaux)

$\begin{array}{rrrrrr} & P 95-99 & P 99-99,5 & P 99,5-99,9 & P 99,9-100 & g(P I B) \\ 1971 & -0,3 & 0,9 & 1,7 & 2,7 & 5,6 \\ 1972 & 0,3 & 1,2 & 2,1 & 3,8 & 5,2 \\ 1973 & 0,1 & 0,9 & 1,5 & 4,6 & 6,7 \\ 1974 & -0,6 & -2,0 & -3,0 & -7,2 & 1,3 \\ 1975 & -1,0 & -0,5 & -1,9 & -1,6 & 0,8 \\ 1976 & -0,2 & 0,3 & -0,3 & 0,8 & 5,6 \\ 1977 & -1,5 & -5,2 & -5,5 & -4,5 & 3,2 \\ 1978 & 0,3 & 1,6 & 1,8 & 0,9 & 4,3 \\ 1979 & 0,5 & 1,4 & 1,9 & 4,1 & 2,6 \\ 1980 & -0,7 & -1,7 & -2,1 & -3,0 & -0,3 \\ 1981 & -0,9 & -1,5 & -2,6 & -2,0 & -0,6 \\ 1982 & -0,3 & -4,6 & -4,2 & -8,0 & 2,5 \\ 1983 & -1,3 & -1,9 & -3,8 & -8,2 & 0,8 \\ 1984 & -0,4 & 0,6 & -0,7 & 0,6 & 1,4 \\ 1985 & 0,5 & -0,2 & 1,7 & 2,0 & 5,0 \\ 1986 & 0,7 & 1,9 & 3,0 & 5,8 & 2,1 \\ 1987 & 0,7 & 1,6 & 3,8 & 9,8 & 4,6 \\ 1988 & -0,5 & -0,3 & 0,9 & 3,2 & 3,7 \\ 1989 & 1,7 & 2,9 & 3,8 & 7,1 & 2,2 \\ 1990 & 0,1 & -0,4 & -0,5 & -0,8 & 0,9 \\ 1991 & -0,7 & -2,0 & -3,3 & -6,4 & 0,9 \\ 1992 & -1,1 & -2,2 & -3,2 & -5,6 & -1,0 \\ 1993 & -0,5 & -1,6 & -1,8 & -2,4 & 2,7 \\ 1994 & -0,3 & -0,5 & 0,0 & 1,3 & 2,0 \\ 1995 & 0,2 & 0,3 & -0,1 & -1,2 & 0,6 \\ 1996\left(^{*}\right) & 0,0 & -0,8 & -1,0 & -1,6 & 2,5 \\ & & & & & \end{array}$

Source: Calculs effectués à partir des résultats du tableau 3-4 (pour l'année $n$, le taux de croissance de P95-99/P90-95 (-0,3\%) est défini par [(P95-99/P90-95)(n) - (P95-99/ P90-95)(n-1)]/(P95-99/P90-95)(n-1). (Taux de croissance du PIB: cf. tableau 2.3)

Lecture: Entre 1970 et 1971, le revenu imposable des foyers fiscaux de P95-99 a diminué de $0,3 \%$ relativement au revenu imposable des foyers de P90-95; celui des foyers de P99,9-100 a progresssé de 2,7\% relativement au revenu imposable des foyers de P90-95. La ligne "1970-1995" indique les taux de croissance annuels moyens de P95-99/P90-95, P99-99,5/P90-95, P99,5-99,9/P90-95 et P99,9-100/P90-95.

Note: $1996\left(^{*}\right)$ : chiffres provisoires (rôles émis au 31/12/97) 
revenus de P99,9-100 gagnent plus de $11 \%$ sur les revenus de P90-95 et de P9599 , plus de $8 \%$ sur les revenus de P99-99,5, et près de $6 \%$ sur ceux de P99,599,9.

(ii) Pendant la récession (ou plutôt la très faible croissance) de 1974-1975, les revenus de P99,9-100 perdent près de $9 \%$ sur ceux de $\mathrm{P} 90-95$, plus de $7 \%$ sur ceux de P95-99, plus de $6 \%$ sur ceux de P99-99,5 et près de $4 \%$ sur ceux de $P 99,5-99,9$. Le centile supérieur perd à nouveau de l'ordre de $5 \%$ de revenus sur P90-95 quand la croissance s'essouffle en 1977, après la forte reprise de 1976.

(iii) Pendant les années de récession (ou de très faible croissance) de 1980-1983, les revenus de P99,9-100 perdent au total plus de $22 \%$ sur ceux de P90-95, plus de $19 \%$ sur ceux de P95-99, plus de $12 \%$ sur ceux de P99-99,5, et près de $10 \%$ sur ceux de P99,5-99,9.

(iv) La reprise s'amorce dès 1985-1986, et les années de croissance élevée de la fin des années 1980 s'accompagneront d'un gonflement extrêmement important des hauts revenus : au total, entre 1985 et 1989, les revenus de P99,9-100 gagneront ainsi plus de $28 \%$ sur ceux de P90-95, près de $26 \%$ sur ceux de P9599, plus de $22 \%$ sur ceux de P99-99,5, et plus de $16 \%$ sur ceux de P99,5-99,9.

(v) Les années de récession de 1991-1993 conduisent à annuler une partie importante du chemin parcouru en 1986-1989: entre 1989 et 1993, les revenus de P99,9-100 perdent environ $16 \%$ sur ceux de P90-95, plus de $13 \%$ sur ceux de P9599 , près de $10 \%$ sur ceux de $\mathrm{P} 99-99,5$, et plus de $7 \%$ sur ceux de $\mathrm{P} 99,5-99,9$.

On voit immédiatement la difficulté liée à l'utilisation des épisodes de 1981-1982 et 1986-1987 pour estimer l'élasticité du revenu imposable: la hausse des taux marginaux imposée aux hauts revenus en 1981-1982 prend place dans un contexte de très faible croissance économique, tandis que la baisse des taux marginaux de1986-1987 prend place dans un contexte de très forte croissance. Compte tenu de l'importance des fluctuations cycliques des hauts revenus observées à plusieurs reprises en l'absence de toute modification importante des taux marginaux imposés aux hauts revenus (en 1970-1973, en 1974-1975, et surtout en 1991-1993), il serait extrêmement hasardeux d'attribuer aux modifications fiscales l'intégralité des fluctuations observées en 1981-1983 et en 1986-1989. L'ampleur des fluctuations observés fait que l'enjeu est de taille : si l'on attribuait intégralement à la fiscalité les quelques $20 \%$ de baisse des hauts revenus de $1980-1983$ et les quelques $20 \%$ de hausse des hauts revenus observés en 1985-1989, on aboutirait à la conclusion que la hausse des taux marginaux de 1981-1982 a fait perdre des recettes fiscales à l'Etat et que la baisse de 1986-1987 lui en a fait gagner (cf. section 4). L'objectif du reste de cette étude est donc de parvenir à distinguer de façon aussi convaincante que possible la partie des fluctuations enregistrées durant ces épisodes qui peut être considérée comme l'effet "normal" du cycle économique de celle que l'on peut éventuellement attribuer à la fiscalité. Les tableaux 3-1 à 3-3 ne permettent pas d'effectuer une telle décomposition, car ils regroupent l'ensemble des foyers fiscaux, sans aucune prise en compte du nombre de parts de quotient familial, si bien qu'il est 
impossible d'identifier précisément les tranches d'imposition et les éventuelles modifications de taux marginaux concernant les différents fractiles de foyers fiscaux. Afin de comparer de façon fine les foyers concernés par de fortes variations de taux marginaux d'imposition et les autres foyers, il faut donc passer à l'étude de la distribution du revenu imposable en se restreignant à la sous-population des foyers fiscaux ayant un même nombre de parts de quotient familial (cf. section 4 infra).

Notons tout d'abord que cette très forte cyclicité des hauts revenus semble être un phénomène universel. Dans tous les pays pour lesquels des études sont disponibles, la part des très hauts revenus a tendance à croître pendant les périodes de forte croissance, notamment du fait du gonflement des revenus de capitaux mobiliers et des bénéfices des professions non salariés durant ces périodes et de la surreprésentation de ces catégories de revenus parmi les très hauts revenus, et inversement la part des très hauts revenus tend à baisser pendant les récessions et les périodes de faible croissance, notamment du fait du dégonflement de ces catégories de revenus. ${ }^{55}$ Ce phénomène de pro-cyclicité de l'inégalité (l'inégalité des revenus augmente dans les phases hautes du cycle économique, et diminue dans les phases basses) ne concerne cependant que les très hauts revenus, et il est dominé pour le reste de la distribution par un phénomène allant en sens inverse, ce qui explique sans doute pourquoi il n'a pas retenu toute l'attention qu'il mérite. La littérature économétrique sur la cyclicité de la distribution des revenus s'est en effet concentrée sur le phénomène de contra-cyclicité de l'inégalité due aux fluctuations du chômage : la diminution du chômage généralement liée aux périodes de forte croissance conduit à un gonflement de la part des revenus des déciles inférieurs de la distribution, et inversement la progression du chômage des périodes de récessions ou de faible croissance conduit généralement à une diminution de la part des déciles inférieurs. ${ }^{56}$ De fait, si l'on mesure l'inégalité des revenus uniquement par les parts des revenus des 5 quintiles de la distribution des revenus, comme le fait généralement cette littérature, alors seul ce second phénomène de contra-cyclicité de l'inégalité est perceptible (la part du quintile inférieur augmente et celle du quintile supérieur diminue dans les phases hautes du cycle, et inversement dans les phases basses), car le premier phénomène de pro-cyclicité de l'inégalité se produit uniquement à l'intérieur du quintile supérieur (et principalement à l'intérieur du décile supérieur ou même du centile supérieur). ${ }^{57}$ Dans le cadre de cette étude, seul le

\footnotetext{
${ }^{55}$ Cf. par exemple Nolan (1987a, 1987b) pour une étude détaillée portant sur le Royaume-Uni. Cette même cyclicité est perceptible dans les séries annuelles de Feenberg et Poterba (1993), même s'ils omettent de l'étudier en tant que telle. Cf. également Saez (1998a), qui constate le même phénomène aux Etats-Unis pendant les années 1920 et 1930 (à partir des statistiques fiscales américaines de l'époque), ce qui suggère qu'il s'agit là d'un phénomène à l'oeuvre depuis toujours dans les économies de marché.

${ }^{56}$ Cf. par exemple Nolan $(1987 a, 1989)$ et Jântti $(1994)$ et les références données dans ces études. Cette littérature a également montré que cette contra-cyclicité de l'inégalité découlait pour l'essentiel de l'effet du taux de chômage (en particulier, le taux d'inflation a généralement un impact beaucoup plus ambigu sur la distribution des revenus).

${ }^{57}$ Bien qu'aucune étude détaillée sur données françaises ne semble exister, ce phénomène peut également être observé en France. A partir des échantillons légers de déclarations de revenus (cf. annexe F), nous avons calculé que la part de P0-20 était passée de $4,1 \%$ à $3,7 \%$ entre 1989 et 1993 ,
} 
phénomène de pro-cyclicité de l'inégalité concernant les hauts revenus est pertinent, et notre objectif est de savoir dans quelle mesure les "cycles fiscaux" de 1981-1982 et 1986-1987 ont pu en amplifier son ampleur. La première étape naturelle de cette analyse consiste à étudier dans quelle mesure les fluctuations de la composition des hauts revenus observés durant ces épisodes se distinguent des fluctuations observés lors des autres épisodes cycliques de la période 1970-1996.

\section{3.. L'évolution de la composition des hauts revenus de 1970 à 1996}

Le tableau 3-6 décrit l'évolution de la composition du revenu imposable des hauts revenus de 1970 à 1996. Compte tenu des données disponibles et de l'évolution très rapide de la composition des revenus à l'intérieur du centile supérieur (cf. tableaux 31 et $3-2),{ }^{58}$ nous n'avons pas cherché à estimer l'évolution de la composition au-delà de P99,5-100 (cf. Annexe D.3).

Insérer Tableau 3-6 : L'évolution de la composition du revenu imposable des hauts revenus de 1970 à 1996

Les grandes tendances de la composition des hauts revenus sur la période 19701996 sont les mêmes que celles observées pour l'ensemble des foyers fiscaux à partir des enquêtes "Revenus fiscaux" sur la période 1970-1990 (cf. Annexe G) : forte progression de la part des retraites, légère diminution de la part des salaires, forte baisse de la part des BIC, relative stabilité de la part des BNC et des revenus de capitaux mobiliers, légère progression de la part des revenus fonciers. La part des BIC dans le revenu imposable de P90-95 passe ainsi de $15,0 \%$ en 1970 à $5,1 \%$ en 1995, alors que celle des retraites passe de moins de $10 \%$ au début des années 1970 à plus de $17 \%$ en 1995.

Les résultats du tableau 3-6 montrent également le rôle joué par la cyclicité de la composition du revenu imposable pour expliquer la cyclicité des hauts revenus. Par exemple, la part des BNC connaît des fluctuations qui sont globalement procycliques : elle baisse lors de la récession de 1974, elle baisse à nouveau lors de la récession du début des années 1980, elle progresse très fortement lors de la reprise de la fin des années 1980, puis elle diminue à nouveau lors de la récession de 19901993, avant de stabiliser à un niveau plus élevé depuis 1994-1995. De même, la part des BIC connaît des fluctuations pro-cycliques autour de son trend de décroissance :

alors que celle de $\mathrm{P} 20-40$ passait de $10,0 \%$ à $9,8 \%$, celle de $\mathrm{P} 40-60$ de $15,0 \%$ à $15,0 \%$, celle de $\mathrm{P} 60$ 80 de $22,6 \%$ à $22,8 \%$, et celle de P $80-100$ de $48,4 \%$ à $48,7 \%$ (et ce bien que la part de P99-100 ait fortement baissée pendant cette même période).

${ }_{58}$ Les estimations du tableau 3-6 ne sont pas directement comparables à celles du tableau 3-2, car les premières concernent la composition du revenu imposable, alors que les secondes traitent de la composition du revenu déclaré. Compte tenu des fortes variations du taux d'imposabilité des différents types de revenus catégoriels (cf. section 3.1 supra), les différences peuvent être importantes: par exemple, la part des salaires et retraites dans le revenu imposable est toujours sensiblement plus faible que dans le revenu déclaré, car leur taux d'imposabilité moyen est inférieur à celui des autres revenus catégoriels. En appliquant les principales règles de la législation concernant les déductions et abattements catégoriels, on aboutit cependant à une excellente correspondance entre les estimations de la composition du revenu imposable issues des échantillons légers et celles issues des Etats 1921. 


\begin{tabular}{|c|c|c|c|c|c|c|c|c|c|c|c|c|c|c|c|c|}
\hline 1970 & BA & $\mathrm{BIC}$ & $\mathrm{BNC}$ & RGA & RF & RCM & SAL PEN & 1978 & BA & $\mathrm{BIC}$ & $\mathrm{BNC}$ & RGA & RF & $\mathrm{RCM}$ & SAL & PEN \\
\hline P90-95 & 1,7 & 15,0 & 2,7 & 0,3 & 2,4 & 1,9 & 76,0 & P90-95 & 1,5 & 11,2 & 2,3 & 0,2 & 2,2 & 1,1 & 71,7 & \\
\hline $95-99$ & 2,2 & 19,4 & 6,9 & 0,6 & 3,4 & 2,7 & 64,9 & D95-99 & 1,6 & 17,7 & 7,9 & 0,4 & 3,6 & 2,0 & 58,8 & 8,0 \\
\hline $99-99,5$ & 2,7 & 21,9 & 16,9 & 0,9 & 4,6 & 4,8 & 48,3 & $99-99,5$ & 1,7 & 21,4 & 16,6 & 0,6 & 4,6 & 4,0 & 45,2 & 5,9 \\
\hline $99,5-100$ & 2,4 & 5,0 & 19,2 & 1,0 & 4,9 & 13,7 & 33,8 & P99,5-100 & 1,9 & 22,6 & 24,7 & 0,9 & 5,5 & 12,6 & 28,5 & 3,4 \\
\hline 971 & BA & BIC & BNC & RGA & RF & $\mathrm{RCM}$ & SAL PEN & 1979 & BA & $\mathrm{BIC}$ & BNC & RGA & RF & RCM & SAL & \\
\hline $90-95$ & 1,7 & 14,1 & 2,0 & 0,3 & 2,4 & 1,5 & 78,1 & P90-95 & 1,4 & 11,5 & 2,7 & 0,2 & 2,4 & 1,4 & 70,5 & \\
\hline 95-99 & 2,1 & 18,5 & 7,0 & 0,5 & 3,5 & 2,7 & 65,6 & P95-99 & 1,6 & 17,5 & 7,4 & 0,4 & 3,6 & 2,0 & 58,9 & 8,0 \\
\hline $99-99,5$ & 2,5 & 21,8 & 16,6 & 0,8 & 4,8 & 4,5 & 49,0 & د99-99,5 & 2,0 & 22,1 & 20,4 & 0,7 & 5,0 & 4,5 & 40,0 & \\
\hline P99,5-100 & 2,2 & 25,5 & 20,2 & 1,0 & 5,2 & 13,5 & 32,4 & P99,5-100 & 2,3 & 21,8 & 24,0 & 0,8 & 5,6 & 12,9 & 29,2 & \\
\hline 1972 & BA & $\mathrm{BIC}$ & $\mathrm{BNC}$ & RGA & RF & $\mathrm{RCM}$ & SAL PEN & 1980 & BA & $\mathrm{BIC}$ & $\mathrm{BNC}$ & RGA & RF & $\mathrm{RCM}$ & SAL & \\
\hline P90-95 & 0,6 & 13,3 & 2,0 & 0,2 & 2,3 & 1,5 & 80,1 & P90-95 & 1,4 & 10,2 & 2,2 & 0,2 & 2,1 & 1,4 & 72,0 & \\
\hline $95-99$ & 0,7 & 18,2 & 6,8 & 0,6 & 3,5 & 2,4 & 67,7 & D95-99 & 1,7 & 17,3 & 7,9 & 0,4 & 3,7 & 2,5 & 57,9 & \\
\hline $99-99,5$ & 0,9 & 23,5 & 16,1 & 0,9 & 4,8 & 5,1 & 48,8 & P99-99,5 & 2,1 & 22,2 & 21,5 & 0,7 & 5,2 & 4,7 & 38,6 &,+ 9 \\
\hline P99,5-100 & 1,2 & 26,4 & 20,1 & 1,1 & 5,1 & 12,9 & 33,3 & P99,5-100 & 2,4 & 21,5 & 23,3 & 0,8 & 5,6 & 13,5 & 29,5 & \\
\hline 1973 & BA & $\mathrm{BIC}$ & BNC & RGA & RF & $\mathrm{RCM}$ & SAL PEN & 1981 & BA & $\mathrm{BIC}$ & $\mathrm{BNC}$ & RGA & RF & RCM & SAL & \\
\hline P90-95 & 2,0 & 12,6 & 2,3 & 0,3 & 2,3 & 1,5 & 78,9 & P90-95 & 1,3 & 11,3 & 3,0 & 0,2 & 2,8 & 1,5 & 69,2 & \\
\hline P95-99 & 2,7 & 17,8 & 6,7 & 0,6 & 3,4 & 2,4 & 66,4 & P95-99 & 1,5 & 14,7 & 7,6 & 0,3 & 3,7 & 2,6 & 60,6 & 9,0 \\
\hline P99-99,5 & 3,1 & 24,1 & 17,1 & 0,9 & 4,7 & 4,5 & 45,7 & P99-99,5 & 2,0 & 20,4 & 19,3 & 0,6 & 5,4 & 4,9 & 41,8 & \\
\hline P99,5-100 & 2,6 & 24,4 & 19,9 & 1,2 & 4,8 & 12,3 & 34,7 & P99,5-100 & 2,2 & 20,1 & 22,8 & 0,8 & 6,2 & 14,4 & 30,0 & 3, \\
\hline 1974 & BA & $\mathrm{BIC}$ & $\mathrm{BNC}$ & RGA & RF & $\mathrm{RCM}$ & SAL PEN & 1982 & BA & $\mathrm{BIC}$ & $\mathrm{BNC}$ & RGA & RF & RCM & SAL & \\
\hline P90-95 & 1,5 & 11,7 & 2,1 & 0,2 & 2,1 & 1,4 & 80,9 & P90-95 & 1,4 & 9,8 & 2,6 & 0,2 & 2,6 & 1,6 & 70,1 & \\
\hline P95-99 & 2,1 & 17,5 & 6,8 & 0,6 & 3,3 & 2,4 & 67,2 & & 1,8 & 14,8 & 8,4 & 0,4 & 4,0 & 2,4 & 58,8 & \\
\hline P99-99,5 & 2,7 & 25,2 & 18,8 & 1,0 & 4,7 & 4,1 & 43,5 & P99-99,5 & 2,3 & 19,1 & 16,3 & 0,6 & 5,3 & 3,5 & 46,4 & 6,7 \\
\hline P99,5-100 & 2,7 & 25,5 & 19,2 & 1,3 & 4,8 & 11,9 & 34,7 & P99,5-100 & 2,5 & 19,8 & 22,9 & 0,8 & 6,5 & 13,2 & 30,8 & \\
\hline 1975 & BA & $\mathrm{BIC}$ & $\mathrm{BNC}$ & RGA & RF & $\mathrm{RCM}$ & SAL PEN & 1983 & BA & $\mathrm{BIC}$ & BNC & RGA & RF & RCM & SAL & \\
\hline P90-95 & 1,3 & 11,9 & 2,7 & 0,3 & 2,2 & 1,6 & $70,0 \quad 10,0$ & P90-95 & 1,3 & 8,9 & 2,5 & 0,1 & 2,6 & 1,6 & 1,0 & \\
\hline P95-99 & 1,5 & 17,1 & 7,4 & 0,5 & 3,3 & 2,4 & $59,5 \quad 8,4$ & P95-99 & 1,9 & 14,3 & 8,9 & 0,3 & 4,2 & 2,3 & 59,1 & 8,9 \\
\hline P99-99,5 & 1,5 & 22,6 & 15,9 & 0,9 & 4,3 & 3,4 & 45,5 & P99-9 & 2,4 & 17,0 & 15,0 & 0,5 & 5,3 & 3,5 & 49,6 & \\
\hline P99,5-100 & 2,0 & 24,3 & 22,2 & 1,1 & 4,9 & 11,8 & 30,5 & P99,5-100 & 3,1 & 18,0 & 24,7 & 0,7 & 6,9 & 11,9 & 31,5 & \\
\hline 1976 & BA & $\mathrm{BIC}$ & BNC & RGA & RF & $\mathrm{RCM}$ & SAL PEN & 1984 & BA & $\mathrm{BIC}$ & $\mathrm{BNC}$ & RGA & RF & $\mathrm{RCM}$ & SAL & \\
\hline P90-95 & 1,1 & 11,3 & 2,3 & 0,2 & 2,1 & 1,3 & $70,7 \quad 11,0$ & P90-95 & 1,5 & 8,4 & 2,3 & 0,1 & 2,7 & 1,6 & 70,5 & \\
\hline P95-99 & 1,2 & 18,4 & 7,6 & 0,5 & 3,3 & 2,0 & $58,2 \quad 8,9$ & P95-99 & 2,2 & 12,6 & 8,6 & 0,3 & 4,2 & 2,4 & 60,0 & 9 \\
\hline P99-99,5 & 1,2 & 23,2 & 14,5 & 0,7 & 4,2 & 4,1 & 45,4 & P99-99,5 & 3,3 & 16,4 & 18,8 & 0,5 & 5,8 & 4,3 & 44,8 & 6,1 \\
\hline P99,5-100 & 1,4 & 24,7 & 22,9 & 1,0 & 4,8 & 11,7 & 30,4 & P99,5-100 & 3,9 & 16,3 & 23,5 & 0,7 & 6,8 & 12,1 & 33,1 & 3,6 \\
\hline 1977 & BA & $\mathrm{BIC}$ & BNC & RGA & RF & $\mathrm{RCM}$ & SAL PEN & 1985 & BA & $\mathrm{BIC}$ & $\mathrm{BNC}$ & RGA & RF & $\mathrm{RCM}$ & SAL & \\
\hline P90-95 & 1,0 & 11,2 & 2,3 & 0,2 & 2,1 & 1,0 & 72,5 & P90-95 & 1,4 & 7,9 & 2,2 & 0,1 & 2,8 & 1,8 & 70,5 & \\
\hline P95-99 & 1,2 & 17,6 & 7,9 & 0,5 & 3,3 & 2,0 & 59,7 & P95-99 & 2,3 & 12,0 & 9,2 & 0,3 & 4,4 & 2,7 & 59,2 & 9,8 \\
\hline P99-99,5 & 1,3 & 21,0 & 12,6 & 0,7 & 4,1 & 3,4 & 50,3 & P99-99,5 & 3,3 & 15,2 & 17,6 & 0,5 & 5,7 & 3,9 & 47,1 & 6,8 \\
\hline P99,5-100 & 1,4 & 23,8 & 22,3 & 1,0 & 5,3 & 13,2 & 29,5 & P99,5-100 & 4,0 & 16,1 & 24,6 & 0,6 & 6,9 & 12,2 & 32,0 & \\
\hline
\end{tabular}




\begin{tabular}{|c|c|c|c|c|c|c|c|c|c|c|c|c|c|c|c|c|c|}
\hline 1986 & BA & $\mathrm{BIC}$ & BNC & RGA & RF & RCM & SAL & PEN & 1992 & BA & $\mathrm{BIC}$ & BNC & RGA & RF & RCM & SAL & PEN \\
\hline P90-95 & 1,4 & 8,2 & 3,1 & 0,1 & 3,0 & 2,1 & 68,5 & 13,6 & P90-95 & 1,3 & 5,9 & 2,9 & 0,2 & 3,7 & 1,4 & 68,8 & 15,8 \\
\hline $95-99$ & 2,1 & 12,1 & 9,4 & 0,3 & 4,5 & 2,7 & 58,8 & 10,2 & P95-99 & 1,7 & 8,8 & 9,9 & 0,5 & 6,0 & 2,4 & 59,6 & 1,0 \\
\hline $99-99,5$ & 2,8 & 15,1 & 16,9 & 0,4 & 5,7 & 3,7 & 48,1 & 7,3 & د99-99,5 & 1,8 & 9,5 & 12,0 & 0,6 & 6,6 & 2,6 & 6,8 & 0,0 \\
\hline P99,5-100 & 3,6 & 16,3 & 26,4 & 0,6 & 6,9 & 11,9 & 31,1 & 3,2 & P99,5-100 & 2,3 & 11,7 & 28,7 & 0,8 & 10,0 & 15,8 & 28,0 & 2,6 \\
\hline 1987 & BA & $\mathrm{BIC}$ & BNC & RGA & RF & $\mathrm{RCM}$ & SAL & PEN & 1993 & BA & $\mathrm{BIC}$ & BNC & RGA & $\mathrm{RF}$ & RCM & SAL & $\mathrm{PE}$ \\
\hline P90-95 & 1,7 & 8,3 & 3,2 & 0,1 & 3,1 & 2,1 & 67,6 & 13,9 & P90-95 & 1,2 & 5,4 & 2,6 & 0,3 & 3,7 & 1,2 & 68,6 & 17,0 \\
\hline $95-99$ & 2,2 & 12,2 & 9,7 & 0,3 & 4,7 & 2,8 & 57,8 & 10,1 & $95-99$ & 1,4 & 8,0 & 9,9 & 0,5 & 6,0 & 2,5 & 9,9 & 11,8 \\
\hline P99-99,5 & 2,6 & 14,6 & 15,7 & 0,4 & 5,8 & 3,5 & 49,7 & 7,7 & P99-99,5 & 1,5 & 8,5 & 11,6 & 0,6 & 6,5 & 2,6 & 7,9 & 1,0 \\
\hline P99,5-100 & 3,2 & 16,1 & 26,2 & 0,6 & 7,1 & 13,7 & 30,3 & 2,9 & $99,5-100$ & 1,4 & 10,8 & 28,7 & 0,9 & 9,8 & 17,3 & 8,1 & 2,9 \\
\hline 1988 & BA & $\mathrm{BIC}$ & $\mathrm{BNC}$ & RGA & RF & RCM & SAL & PEN & 1994 & BA & $\mathrm{BIC}$ & BNC & RGA & $\mathrm{RF}$ & RCM & SAL & PEN \\
\hline P90-95 & 1,84 & 7,9 & 3,1 & 0,15 & 3,3 & 2,1 & 67,6 & 14,1 & P90-95 & 1,4 & 5,1 & 2,6 & 0,3 & 3,7 & 1,0 & 8,4 & 17,5 \\
\hline $95-99$ & 2,37 & 11,9 & 10,2 & 0,33 & 5,16 & 3,0 & 57,1 & 9,9 & P95-99 & 1,5 & 7,6 & 9,6 & 0,6 & 6,0 & 2,7 & 59,8 & 12,2 \\
\hline P99-99,5 & 2,63 & 13,7 & 14,9 & 0,42 & 6,07 & 3,4 & 50,9 & 8,0 & P99-99,5 & 1,5 & 8,0 & 10,8 & 0,7 & 6,4 & 2,3 & 58,7 & 11,5 \\
\hline P99,5-100 & 2,94 & 15,9 & 26,8 & 0,58 & 7,6 & 14,5 & 29,1 & 2,6 & P99,5-100 & 1,2 & 10,1 & 27,9 & 1,0 & 9,7 & 19,5 & 27,6 & 2,9 \\
\hline 1989 & BA & $\mathrm{BIC}$ & BNC & RGA & RF & $\mathrm{RCM}$ & SAL & PEN & & BA & $\mathrm{BIC}$ & BNC & RGA & RF & $\mathrm{RCM}$ & AL & PE \\
\hline P90-95 & 2,0 & 7,5 & 3,0 & 0,2 & 3,5 & 2,0 & 67,6 & 14,3 & P90-95 & 1,5 & 5,1 & 3,4 & 0,4 & 3,8 & 1,5 & 67,0 & 17,2 \\
\hline P95-99 & 2,5 & 11,5 & 10,8 & 0,3 & 5,6 & 3,2 & 56,3 & 9,7 & P95-99 & 1,7 & 7,3 & 9,5 & 0,7 & 5,7 & 3,0 & 59,8 & 12,3 \\
\hline P99-99,5 & 2,7 & 12,8 & 14,1 & 0,4 & 6,3 & 3,3 & 52,1 & 8,4 & P99-99,5 & 1,8 & 7,6 & 10,6 & 0,8 & 6,1 & 2,9 & 58,6 & 11,6 \\
\hline P99,5-100 & 2,7 & 15,8 & 27,5 & 0,6 & 8,1 & 15,3 & 27,9 & 2,2 & P99,5-100 & 1,4 & 9,7 & 27,5 & 1,2 & 8,7 & 21,8 & 26,9 & 2,8 \\
\hline 1990 & BA & $\mathrm{BIC}$ & BNC & RGA & RF & $\mathrm{RCM}$ & SAL & PEN & $1996\left(^{*}\right)$ & BA & $\mathrm{BIC}$ & BNC & RGA & $\mathrm{RF}$ & $\mathrm{RCM}$ & SAL & PEN \\
\hline P90-95 & 1,7 & 7,0 & 2,8 & 0,2 & 3,5 & 1,8 & 68,4 & 14,7 & P90-95 & 1,5 & 4,7 & 3,2 & 0,0 & 3,8 & 1,7 & 67,1 & 17,9 \\
\hline P95-99 & 2,3 & 10,7 & 10,6 & 0,4 & 5,7 & 3,0 & 57,4 & 9,9 & P95-99 & 1,8 & 6,7 & 9,4 & 0,0 & 5,8 & 3,3 & 60,2 & 12,7 \\
\hline P99-99,5 & 2,4 & 11,7 & 13,3 & 0,5 & 6,4 & 3,0 & 54,0 & 8,8 & P99-99,5 & 1,9 & 7,0 & 10,1 & 0,0 & 6,1 & 3,1 & 59,6 & 12,3 \\
\hline P99,5-100 & 2,9 & 14,4 & 27,6 & 0,6 & 8,7 & 15,7 & 27,9 & 2,2 & P99,5-100 & 1,7 & 8,9 & 26,5 & 0,0 & 9,0 & 22,7 & 28,3 & 2,9 \\
\hline
\end{tabular}

1991 BA BIC BNC RGA RF RCM SAL PEN

$\begin{array}{lllllllll}\mathrm{P} 90-95 & 1,4 & 6,4 & 2,6 & 0,2 & 3,6 & 1,5 & 69,1 & 15,2\end{array}$

$\begin{array}{lllllllll}\text { P95-99 } & 2,1 & 9,9 & 10,5 & 0,5 & 5,9 & 2,8 & 58,4 & 10,1\end{array}$

$\begin{array}{lllllllll}\mathrm{P} 99-99,5 & 2,2 & 10,6 & 12,5 & 0,5 & 6,4 & 2,7 & 55,9 & 9,2\end{array}$

$\begin{array}{lllllllll}\mathrm{P} 99,5-100 & 3,1 & 13,0 & 27,7 & 0,7 & 9,3 & 16,1 & 27,9 & 2,2\end{array}$

Source: Estimations de l'auteur à partir des Etats 1921 (cf. Annexe D.3).

Lecture: En 1996, les 0,5\% des foyers fiscaux ayant le revenu imposable le plus élevé (P99,5-100) disposent en moyenne de $1,7 \%$ de leurs revenus sous forme de bénéfices agricoles (BA), de $8,9 \%$ de leurs revenus sous forme de bénéfices industriels et commerciaux (BIC), de $26,5 \%$ de leurs revenus sous forme de bénéfices non commerciaux (BNC), de $0,0 \%$ sous forme de rémunérations de gérants et associés (RGA), de $9,0 \%$ sous forme de revenus fonciers (RF), de $22,7 \%$ sous forme de revenus de capitaux mobiliers (RCM), de $28,3 \%$ sous forme de salaires (SAL), et de $2,9 \%$ sous formes de pensions de retraite (PEN).

Notes: (i) $1996\left(^{*}\right)$ : chiffres provisoires (rôles émis au 31/12/97)

(ii) La décomposition des salaires et pensions de retraites n'est fournie dans les Etats 1921 qu'à partir des revenus de 1975 . 
cette décroissance est particulièrement rapide en 1974, 1980, 1982-1983, puis elle est inversée en 1985-1989, années où la part des BIC progresse ou baisse très légèrement, avant de baisser à nouveau très rapidement en 1990-1993 et de se stabiliser en 1994-1995. La part des revenus fonciers ne connaît pas de véritable cyclicité, mais leur très forte progression entre 1984 et 1992, dans un contexte de boom immobilier, contribue à gonfler les très hauts revenus. De la même façon que pour les BIC/BNC, les revenus fonciers sont en effet sur-représentés parmi les hauts revenus, si bien que même si leur poids augmente dans les mêmes proportions pour P90-95 que pour P99,5-100, par exemple du fait d'un phénomène macroéoconomique général de progression de ce type de revenus, alors cela se traduit mécaniquement par une hausse du ratio P99,5-100/P90-95, indépendamment de tout changement de comportement dû à des modifications de taux marginaux. Cela est particulièrement frappant pour les revenus fonciers, dont le poids augmente dans les mêmes proportions pour P90-95 que pour P99,5-100 entre 1984-1985 et 1992, ce qui montre que cette évolution n'a rien à voir avec la baisse des taux marginaux des très hauts revenus intervenue en 1986-1987. II en va de même pour la croissance de la part des BIC entre 1985 et 1987 : cette croissance touche d'avantage les revenus de P90-95 que ceux de P99,5-100, ce qui suggère là encore que cette évolution n'est pas due aux réformes fiscales de 1986-1987.

La part des revenus de capitaux mobiliers connaît des fluctuations plus complexes : elle est pro-cyclique en 1974, 1982-1983 et 1986-1989, ce qui contribue de la même façon que pour les BIC/BNC à la pro-cyclicité de l'inégalité, mais elle semble contracyclique pour les très hauts revenus de P99,5-100 lors de la récession de 19901993, bien que nettement pro-cyclique pour les hauts revenus (jusqu'à P99-99,5). En fait, le niveau absolu des revenus de capitaux mobiliers détenus par le centile supérieur n'a pas progressé en 1990-1993 : il est resté à peu près stable, dans un contexte de chute du niveau absolu du revenu imposable de ces contribuables, si bien que le poids relatif des revenus de capitaux mobiliers a progressé pour les très hauts revenus entre 1990 et 1993. Les échantillons légers de déclarations de revenus pour 1988-1995 permettent d'étudier dans le détail cet épisode (cf. les tableaux de l'annexe F). D'une part, le principal "effet compositionel " expliquant la chute de la part des très hauts revenus entre 1990 et 1993 est la baisse très importante des BIC et des BNC. D'autre part, en dépit de la chute importante des revenus de capitaux mobiliers pour les foyers inférieurs au centile P99,5, les très hauts revenus (au delà de $\mathrm{P} 99,5$ ) semblent avoir réussi à préserver le niveau absolu de leurs revenus de capitaux mobiliers, peut-être du fait de placements mieux informés (sans toutefois éviter une stagnation de ces revenus, comparée à la très forte hausse enregistrée en 1986-1989). Les résultats du tableau 3-6 suggèrent également que, sur l'ensemble de la période étudiée, la cyclicité des BIC/BNC joue un rôle plus important que la cyclicité des revenus de capitaux mobiliers pour expliquer la cyclicité des hauts revenus. Nolan (1987b) est conduit à la même conclusion dans son étude sur données britanniques, et il l'attribue au fait que la cyclicité des revenus de capitaux mobiliers est atténuée par le comportement des 
entreprises, qui cherchent généralement à "lisser" leurs distributions de dividendes au cours du cycle économique, alors que les professions non-salariées supportent intégralement et individuellement les variations de leurs bénéfices.

Cet épisode de 1990-1993, qui est le seul de la période 1970-1996 à exhiber une part contra-cyclique des revenus de capitaux mobiliers des très hauts revenus, et qui est également le seul que les données disponibles nous permettent d'étudier de façon détaillée, incite cependant à la prudence. II est probable que chaque cycle économique ait ses caractéristiques propres du point de l'importance relative des mouvements de BIC, BNC, revenus fonciers et revenus de capitaux mobiliers, et il est donc difficile d'identifier une éventuelle particularité "fiscale" des "cycles compositionels" de 1981-1983 et 1986-1989 des autres épisodes de la période 1970-1996.

De plus, et surtout, les estimations du tableau 3-6 montrent que la cyclicité de la composition du revenu imposable n'explique qu'une faible part de la cyclicité des hauts revenus. Par exemple, la baisse de la part des revenus de capitaux mobiliers de P99,5-100 enregistrée entre 1981 et 1983 (de 14,4\% à 11,9\%), qui est pourtant l'une des variations les plus rapides observées sur l'ensemble de la période, ne peut expliquer au maximum qu'une baisse de l'ordre de 2,8\% de P99,5-100/P95-100, soit une baisse relativement insignifiante comparée à la baisse totale de P99,5-100/P95100 enregistrée sur cette période (cf. tableau 2-3 supra). ${ }^{59}$ Cela montre que la cyclicité des hauts revenus est due principalement à la cyclicité des distributions intra-catégorielles, et non pas à des " effets compositionels ". Cela est confirmé par l'analyse détaillée de la récession de 1990-1993 que l'on peut faire à partir des échantillons légers de déclarations de revenus: si l'on multiplie tous les revenus catégoriels individuels déclarés en 1990 par le taux de variation de chaque type de revenu catégoriel observé au niveau agrégé entre 1990 et 1993, alors on n'explique ainsi qu'environ $30-40 \%$ de la baisse de la part des très hauts revenus effectivement observée entre 1990 et 1993. Autrement dit, les 2/3 de la baisse des très hauts revenus observée entre 1990 et 1993 s'explique par des évolutions intracatégorielles. En particulier, les BIC et BNC des très hauts revenus ont beaucoup plus baissé que le reste des BIC/BNC. Ces évolutions intra-catégorielles s'observent également pour les salaires: le montant des salaires perçus par les foyers de P99,99-100 a baissé sensiblement relativement au montant des salaires perçus par les foyers de P99,9-99,99, le montant des salaires perçus par les foyers de P99,999,99 a baissé relativement à celui perçu par les foyers de P99-99,9, etc.., et ce dans des proportions très comparables à celles observées pour la moyenne des autres revenus. Cela montre que la cyclicité des hauts revenus n'est pas seulement

\footnotetext{
${ }^{59} \mathrm{Si}$ seuls les revenus de capitaux mobiliers avaient baissé entre 1981 et 1983 , et si tous les autres revenus catégoriels de P99,5-100 avaient conservé leur niveau de 1983, alors la baisse de $\mathrm{x} \%$ de la part de P99,5-100 compatible avec une baisse de $14,4 \%$ à $11,9 \%$ de la part des RCM est donnée par : $0,119=(0,144-x) /(1-x)$, soit $x=0,028$, ou $2,8 \%$. En outre, cette estimation haute ne vaut que pour P99,5-100/P90-95 : compte tenu du fait que la part des RCM a baissé de près de 1,5 points pour P99-99,5 (de 4,9\% en 1981 à 3,5\% en 1983), la baisse des RCM peut expliquer au maximum une baisse de l'ordre de $1,5 \%$ du ratio P99,5-100/P99-99,5.
} 
due au fait que les très hauts revenus supportent les risques liés aux revenus nonsalariaux : ce même partage du risque entre très hauts revenus et hauts revenus, qui est à l'origine du phénomène de pro-cyclicité de l'inégalité parmi les hauts revenus, se retrouvent également pour les revenus salariaux. Ce résultat n'est pas vraiment surprenant, si l'on considère que les très hauts salaires comportent une part importante de primes liées à l'activité économique, de la même façon que les revenus non-salariaux. L'importance relative de ces différents mécanismes de partage des risques au cours du cycle économique est fortement susceptible de varier au cours du temps, et il est donc illusoire de vouloir caractériser tel ou tel épisode particulier du cycle des hauts revenus uniquement par l'éventuelle spécificité de l'évolution observée de la composition.

En conclusion, les données disponibles sur l'évolution de la composition des hauts revenus ne permettent pas de distinguer clairement les cycles de 1981-1983 et 19861989 des autres cycles. Rien dans l'évolution de la composition ne permet de dire dans quel mesure les épisodes de 1981-1983 et de 1986-1989 sont le produit "naturel" du cycle économique ou ont été amplifiés par l'évolution des taux marginaux (au moins en partie). Afin d'estimer l'élasticité du revenu imposable à partir de ces épisodes, la seule possibilité est donc de comparer de façon aussi fine que possible les fluctuations observées pour les niveaux de revenus soumis à des variations importantes de taux marginaux à celles observées pour les niveaux de revenu qui n'étaient pas concernés. Pour cela, il nous faut passer à l'étude de la distribution du revenu imposable en fonction du nombre de parts de quotient familial. 


\section{Estimations de l'élasticité du revenu imposable}

\subsection{Estimations effectuées à partir des foyers ayant 1 part de quotient familial}

Les tableaux 4-1, 4-2 et 4-3 décrivent l'évolution de la distribution du revenu imposable des foyers fiscaux ayant 1 part de quotient familial.

Insérer : Tableaux 4-1 et 4-2: L'évolution de la part des hauts revenus dans le revenu imposable de 1970 à 1996 (foyers fiscaux ayant 1 part de quotient familial)

Insérer: Tableaux 4-3: Les taux de croissance annuels de P98-99/P95-98, P9999,5/P95-98, etc..., de 1971 à 1996 (foyers fiscaux ayant 1 part de quotient familial)

Le premier constat est que les hauts revenus ayant 1 part de quotient familial (relativement aux autres foyers fiscaux ayant 1 part de QF) connaissent le même type de cyclicité que l'ensemble des hauts revenus : augmentation en 1970-1973, baisse en 1974-1975 et en 1982-1983, augmentation en 1986-1989 et baisse en 1990-1993. Nous retrouvons cette même cyclicité pour les foyers fiscaux ayant 1,5 parts, 2 parts, 2,5 parts et 3 parts de quotient familial. ${ }^{60}$ Le fait que ces fluctuations de court-terme existent séparément pour chaque groupe de contribuables ayant le même nombre de part de quotient familial, et non seulement pour l'ensemble des contribuables, montre la robustesse et l' " universalité " de ce phénomène de cyclicité des hauts revenus.

Le graphique 4-1 décrit l'évolution sur la période 1970-1996 des seuils des tranches supérieures du barème de I'IR et des seuils P99, P99,5, P99,7 et P99,9 de la distribution du revenu imposable des foyers ayant 1 part du quotient familial, tous ces montant étant exprimés en francs de $1996 .{ }^{61}$ Ce graphique montre notamment l'importance de la non-indexation des tranches supérieures du barème pendant les années 1970 : alors qu'en 1970 la tranche à $60 \%$ concernait moins de $0,1 \%$ des foyers fiscaux ayant 1 part de quotient familial, cette même tranche en concerne plus de $0,3 \%$ en 1980. Depuis 1981, les seuils des tranches sont parfaitement indexés sur l'inflation (cf. section 2.1.1), et ils dessinent donc des lignes horizontales sur le graphique 4-1.

\footnotetext{
${ }^{60}$ Cf. sections 4.2 et 4.3 infra, ainsi que les tableaux de l'annexe D.2 pour les résultats complets de l'estimation de la distribution du revenu imposable pour ces 5 groupes de quotient familial (tableaux D-5 à D-14). Ces tableaux indiquent également que pour chacune de ces distributions, les parts de P90-95, P95-99, P99-99,5, etc.., sont globalement stables sur la période 1970-1996 (à l'exception de la distribution des contribuables ayant 1,5 parts de quotient familial, qui a connu un trend significatif de décroissance du ratio P99,9-100/P95-98 au cours de cette période, au rythme moyen de 1,1\% par an ; cf. tableau D-6).

${ }^{61}$ Les montants en francs courants ont été convertis en francs de 1996 en utilisant la série de taux d'inflation (taux de variation des indices des prix à la consommation en moyenne annuelle) reproduite sur le tableau 2-3. Les seuils P99, P99,5, etc..., en francs courants sont issus des estimations effectués à partir des Etats 1921 (cf. Annexe D.2, tableau D-4). Les seuils des tranches supérieures en francs courants sont ceux du tableau 2-1.
} 
Tableau 4-1 :

L'évolution de la part des hauts revenus dans le revenu imposable de 1970 à 1996 (foyers fiscaux ayant 1 part de quotient familial)

\begin{tabular}{|c|c|c|c|c|c|c|c|}
\hline & P95-100 & P98-100 & P99-100 & P99,5-100 & P99,7-100 & P99,9-100 & P99,99-100 \\
\hline 1970 & 18,33 & 10,40 & 6,85 & 4,55 & 3,38 & 1,80 & 0,49 \\
\hline 1971 & 18,45 & 10,53 & 6,96 & 4,65 & 3,47 & 1,86 & 0,51 \\
\hline 1972 & 18,46 & 10,56 & 7,01 & 4,70 & 3,52 & 1,90 & 0,52 \\
\hline 1973 & 19,13 & 11,14 & 7,51 & 5,13 & 3,90 & 2,20 & 0,72 \\
\hline 1974 & 18,25 & 10,40 & 6,92 & 4,65 & 3,49 & 1,90 & 0,57 \\
\hline 1975 & 18,25 & 10,40 & 6,90 & 4,64 & 3,48 & 1,90 & 0,62 \\
\hline 1976 & 17,92 & 10,16 & 6,73 & 4,53 & 3,40 & 1,86 & 0,56 \\
\hline 1977 & 17,13 & 9,68 & 6,38 & 4,26 & 3,21 & 1,76 & 0,55 \\
\hline 1978 & 16,89 & 9,55 & 6,33 & 4,25 & 3,19 & 1,75 & 0,52 \\
\hline 1979 & 16,69 & 9,53 & 6,35 & 4,29 & 3,23 & 1,77 & 0,51 \\
\hline 1980 & 16,51 & 9,36 & 6,19 & 4,17 & 3,13 & 1,72 & 0,50 \\
\hline 1981 & 16,91 & 9,58 & 6,35 & 4,23 & 3,19 & 1,73 & 0,50 \\
\hline 1982 & 16,35 & 9,12 & 5,95 & 3,93 & 2,91 & 1,53 & 0,41 \\
\hline 1983 & 16,90 & 9,39 & 6,10 & 4,01 & 2,96 & 1,54 & 0,41 \\
\hline 1984 & 17,15 & 9,50 & 6,19 & 4,07 & 3,01 & 1,58 & 0,43 \\
\hline 1985 & 17,72 & 9,91 & 6,49 & 4,30 & 3,20 & 1,71 & 0,48 \\
\hline 1986 & 17,98 & 10,13 & 6,67 & 4,45 & 3,32 & 1,80 & 0,51 \\
\hline 1987 & 18,31 & 10,39 & 6,88 & 4,62 & 3,47 & 1,90 & 0,55 \\
\hline 1988 & 18,63 & 10,65 & 7,10 & 4,81 & 3,64 & 2,04 & 0,61 \\
\hline 1989 & 18,95 & 10,91 & 7,32 & 5,00 & 3,80 & 2,17 & 0,68 \\
\hline 1990 & 18,94 & 10,83 & 7,21 & 4,89 & 3,69 & 2,08 & 0,64 \\
\hline 1991 & 18,70 & 10,59 & 7,00 & 4,70 & 3,53 & 1,98 & 0,60 \\
\hline 1992 & 18,54 & 10,41 & 6,84 & 4,57 & 3,42 & 1,90 & 0,57 \\
\hline 1993 & 18,60 & 10,39 & 6,80 & 4,53 & 3,38 & 1,87 & 0,56 \\
\hline 1994 & 18,78 & 10,48 & 6,86 & 4,58 & 3,42 & 1,90 & 0,57 \\
\hline 1995 & 18,76 & 10,43 & 6,81 & 4,53 & 3,37 & 1,87 & 0,56 \\
\hline $1996\left(^{*}\right)$ & 18,64 & 10,35 & 6,75 & 4,48 & 3,28 & 1,85 & 0,56 \\
\hline
\end{tabular}

Source: Estimations de l'auteur à partir des Etats 1921 (cf. annexes D.1 et D.2)

Lecture: En 1970, les 5\% des foyers fiscaux ayant 1 part de quotient familial dont le revenu imposable est la plus élevé détiennent $18,33 \%$ du revenu imposable total des foyers fiscaux ayant 1 part de quotient familial; les $2 \%$ ayant le revenu imposable le plus élevé en détiennent $10,40 \%$; etc... Note: $1996\left(^{*}\right)$ : chiffres provisoires (rôles émis au 31/12/97) 
Tableau 4-2:

L'évolution de la part des hauts revenus dans le revenu imposable de 1970 à 1996 (foyers fiscaux ayant 1 part de quotient familial)

$\begin{array}{ccccccc} & \text { P95-98 } & \text { P98-99 } & \text { P99-99,5 } & \text { P99,5-99,7 } & \text { P99,7-100 } & \text { P99,9-100 } \\ 1970 & 7,93 & 3,55 & 2,30 & 1,17 & 3,38 & 1,80 \\ 1971 & 7,92 & 3,57 & 2,31 & 1,18 & 3,47 & 1,86 \\ 1972 & 7,90 & 3,55 & 2,30 & 1,19 & 3,52 & 1,90 \\ 1973 & 7,99 & 3,63 & 2,38 & 1,23 & 3,90 & 2,20 \\ 1974 & 7,85 & 3,48 & 2,28 & 1,16 & 3,49 & 1,90 \\ 1975 & 7,85 & 3,51 & 2,26 & 1,16 & 3,48 & 1,90 \\ 1976 & 7,76 & 3,44 & 2,19 & 1,13 & 3,40 & 1,86 \\ 1977 & 7,45 & 3,30 & 2,12 & 1,05 & 3,21 & 1,76 \\ 1978 & 7,35 & 3,22 & 2,08 & 1,06 & 3,19 & 1,75 \\ 1979 & 7,15 & 3,18 & 2,06 & 1,06 & 3,23 & 1,77 \\ 1980 & 7,16 & 3,16 & 2,03 & 1,04 & 3,13 & 1,72 \\ 1981 & 7,33 & 3,23 & 2,11 & 1,04 & 3,19 & 1,73 \\ 1982 & 7,23 & 3,18 & 2,02 & 1,02 & 2,91 & 1,53 \\ 1983 & 7,51 & 3,29 & 2,10 & 1,05 & 2,96 & 1,54 \\ 1984 & 7,64 & 3,31 & 2,12 & 1,06 & 3,01 & 1,58 \\ 1985 & 7,81 & 3,42 & 2,19 & 1,10 & 3,20 & 1,71 \\ 1986 & 7,85 & 3,47 & 2,22 & 1,12 & 3,32 & 1,80 \\ 1987 & 7,92 & 3,51 & 2,26 & 1,15 & 3,47 & 1,90 \\ 1988 & 7,98 & 3,55 & 2,29 & 1,17 & 3,64 & 2,04 \\ 1989 & 8,04 & 3,59 & 2,32 & 1,20 & 3,80 & 2,17 \\ 1990 & 8,11 & 3,61 & 2,33 & 1,20 & 3,69 & 2,08 \\ 1991 & 8,10 & 3,59 & 2,30 & 1,17 & 3,53 & 1,98 \\ 1992 & 8,13 & 3,57 & 2,27 & 1,15 & 3,42 & 1,90 \\ 1993 & 8,22 & 3,59 & 2,27 & 1,15 & 3,38 & 1,87 \\ 1994 & 8,30 & 3,61 & 2,28 & 1,16 & 3,42 & 1,90 \\ 1995 & 8,33 & 3,62 & 2,29 & 1,16 & 3,37 & 1,87 \\ 1996\left(^{*}\right) & 8,29 & 3,60 & 2,27 & 1,20 & 3,28 & 1,85\end{array}$

Source: Calculs effectués à partir des résultats du tableau 4-1 (P95-98 = P95-100 - P98-100; P98-99 = P98-100 - P99-100; etc..)

Note: $1996\left(^{*}\right)$ : chiffres provisoires (rôles émis au 31/12/97) 
Tableau 4-3 :

Les taux de croissance annuels de P98-99/P95-98, P99-99,5/P95-98, etc.., de 1971 à 1996 (foyers fiscaux ayant 1 part de quotient familial)

\begin{tabular}{|c|c|c|c|c|c|c|}
\hline & P98-99 & P99-99,5 & P99,5-99,7 & P99,7-100 & P99,9-100 & $g(P I B)$ \\
\hline 1971 & 0,7 & 0,8 & 1,2 & 2,7 & 3,4 & 5,6 \\
\hline 1972 & $-0,1$ & $-0,1$ & 0,8 & 1,6 & 2,3 & 5,2 \\
\hline 1973 & 1,0 & 2,2 & 2,6 & 9,7 & 14,4 & 6,7 \\
\hline 1974 & $-2,4$ & $-2,7$ & $-4,2$ & $-8,9$ & $-11,8$ & 1,3 \\
\hline 1975 & 0,7 & $-0,9$ & 0,0 & $-0,1$ & 0,0 & 0,8 \\
\hline 1976 & $-0,9$ & $-1,7$ & $-1,3$ & $-1,3$ & $-1,2$ & 5,6 \\
\hline 1977 & 0,1 & 0,5 & $-2,7$ & $-1,8$ & $-1,2$ & 3,2 \\
\hline 1978 & $-1,0$ & $-0,4$ & 1,7 & 0,8 & 0,4 & 4,3 \\
\hline 1979 & 1,4 & 1,8 & 2,9 & 4,1 & 4,2 & 2,6 \\
\hline 1980 & $-0,6$ & $-1,7$ & $-1,6$ & $-3,3$ & $-3,0$ & $-0,3$ \\
\hline 1981 & $-0,3$ & 1,8 & $-2,3$ & $-0,4$ & $-1,6$ & $-0,6$ \\
\hline 1982 & $-0,2$ & $-3,2$ & $-0,6$ & $-7,6$ & $-10,3$ & 2,5 \\
\hline 1983 & $-0,5$ & 0,0 & $-1,2$ & $-2,1$ & $-3,0$ & 0,8 \\
\hline 1984 & $-0,9$ & $-0,6$ & $-0,6$ & 0,0 & 0,4 & 1,4 \\
\hline 1985 & 1,1 & 1,1 & 1,8 & 4,2 & 6,2 & 1,8 \\
\hline 1986 & 0,8 & 0,9 & 1,2 & 3,3 & 4,8 & 5,0 \\
\hline 1987 & 0,5 & 0,7 & 1,1 & 3,6 & 4,8 & 2,1 \\
\hline 1988 & 0,3 & 0,6 & 1,3 & 3,9 & 6,1 & 4,6 \\
\hline 1989 & 0,3 & 0,6 & 1,3 & 3,7 & 5,6 & 3,7 \\
\hline 1990 & $-0,3$ & $-0,6$ & $-0,7$ & $-3,7$ & $-4,8$ & 2,2 \\
\hline 1991 & $-0,6$ & $-1,2$ & $-1,9$ & $-4,2$ & $-5,0$ & 0,9 \\
\hline 1992 & $-0,8$ & $-1,4$ & $-2,0$ & $-3,5$ & $-4,1$ & 0,9 \\
\hline 1993 & $-0,6$ & $-1,1$ & $-1,3$ & $-2,1$ & $-2,6$ & $-1,0$ \\
\hline 1994 & $-0,3$ & $-0,4$ & $-0,4$ & 0,1 & 0,7 & 2,7 \\
\hline 1995 & $-0,3$ & $-0,3$ & $-0,3$ & $-1,9$ & $-2,2$ & 2,0 \\
\hline $1996\left(^{*}\right)$ & $-0,1$ & 0,0 & 3,8 & $-2,0$ & $-0,5$ & 0,6 \\
\hline 1970-1995 & $-0,1$ & $-0,2$ & $-0,2$ & $-0,2$ & $-0,1$ & 2,5 \\
\hline
\end{tabular}

Source: Calculs effectués à partir des résultats du tableau 4-2 (pour l'année $\mathrm{n}$, le taux de croissance de P98-99/P95-98 est défini par [(P98-99/P95-98)(n) - (P98-99/

P95-98)(n-1)]/(P98-99/P95-98)(n-1). (Taux de croissance du PIB: cf. tableau 2.3)

Lecture: Entre 1970 et 1971, le revenu imposable des foyers fiscaux de P98-99 a progressé de $0,7 \%$ relativement au revenu imposable des foyers de P95-98; celui des foyers de P99,9-100 a progresssé de 3,4\% relativement au revenu imposable des foyers de P95-98. La ligne "1970-1995" indique les taux de croissance annuels moyens de P95-99/P90-95, P99-99,5/P90-95, P99,5-99,9/P90-95 et P99,9-100/P90-95.

Note: $1996\left(^{*}\right)$ : chiffres provisoires (rôles émis au 31/12/97) 
Insérer : Graphique 4-1 : Les seuils des fractiles et des tranches supérieures de 1970 à 1996 (foyers fiscaux ayant 1 part de quotient familial)

Seuls les $0,3 \%$ des foyers ayant le revenu imposable le plus élevé (parmi les foyers ayant 1 part de QF) étaient concernés par la création de la tranche à $65 \%$ en 1982 : le seuil de cette tranche (environ 320000 francs de 1996) se situait en 1982-1985 entre les seuils P99,7 et P99,8. Les foyers fiscaux ayant 1 part de QF n'étant pas concernés par le plafonnement du QF mis en place en 1981, seuls les foyers de P99,7-100 ont donc connu une augmentation importante de leur taux marginal (de $60 \%$ à $65 \%$ ): tous les autres foyers, et en particulier les foyers de P99-99,5, ont vu leur taux marginal inchangé. De la même façon, seuls les foyers de P99,7-100 étaient concernés par la suppression de la tranche à $65 \%$ en 1986 . Ces foyers ont donc connu une baisse substantielle de leur taux marginal (de 65\% en 1982-1985 à $56,8 \%$ depuis 1987), alors que les foyers de P99,5-99,7 connaissaient une baisse beaucoup plus légère (de $60 \%$ à $56,8 \%$ ), et que tous les autres foyers (et en particulier ceux de P99-99,5) connaissaient des baisses insignifiantes (de $55 \%$ à $53,9 \%$, de $50 \%$ à $49 \%$, etc..). Le tableau $4-4$ utilise l'évolution des revenus de P9999,5 et P99,7-100 durant ces deux épisodes pour obtenir une première estimation de l'élasticité du revenu imposable.

Insérer Tableau 4-4: Estimation de l'élasticité du revenu imposable à partir des foyers fiscaux ayant 1 part de quotient familial.

\subsubsection{La création de la tranche à $65 \%(1981-1982)$}

Le tableau 4-4 indique que la part de P99-99,5 est restée pratiquement stable entre 1981 et 1983, alors que celle de P99,7-100 diminuait de plus de 7\%, si bien que le ratio P99,7-100/P99-99,5 diminuait de 6,6\%. En 1981 comme en 1983, les foyers de P99-99,5 étaient pour environ $60 \%$ d'entre eux dans la tranche à $50 \%$ et pour environ $40 \%$ dans la tranche à $55 \%$, soit un taux marginal moyen de $52 \%{ }^{62}$ Les foyers de P99,7-100 étaient tous dans la tranche à $60 \%$ en 1981, et ils sont (presque) tous passés dans la tranche à $65 \%$ en 1983 , soit un diminution de $12,5 \%$ de leur taux de rétention (qui est passé de $40 \%$ à $35 \%$, soit une baisse de 5 points sur une base de 40). Le ratio entre les taux de rétention de P99,7-100 et de P99-99,5 a donc diminué de $12,5 \%$. Si l'on attribuait l'intégralité de la baisse des revenus de P99,7-100 à celle de leur taux de rétention, on aboutirait ainsi à une élasticité du revenu imposable e $=0,53(0,53=6,6 / 12,5)$.

Afin de fixer les ordres de grandeurs, le tableau 4-4 (de même que les tableaux suivants) indique également les taux marginaux $t^{*}$ et $t^{* *}$ maximisant les recettes fiscales impliqués par ces élasticités. Ces taux $t^{*}$ et $t^{\prime *}$ mesurent donc le sommet de la " courbe de Laffer". Ils correspondent à "taux socialement optimaux" associés à

\footnotetext{
${ }^{62}$ Tous les taux marginaux moyens mentionnée dans les tableaux 4-4 à 4-10 ont été estimés à partir de nos estimations des seuils P99, P99,1,..., P99, 9 effectués à partir des Etats 1921 (cf. Annexe D.2).
} 
Graphique 4-1: Les seuils des fractiles et des tranches supérieures de 1970 à 1996

(foyers fiscaux ayant 1 part de quotient familial)

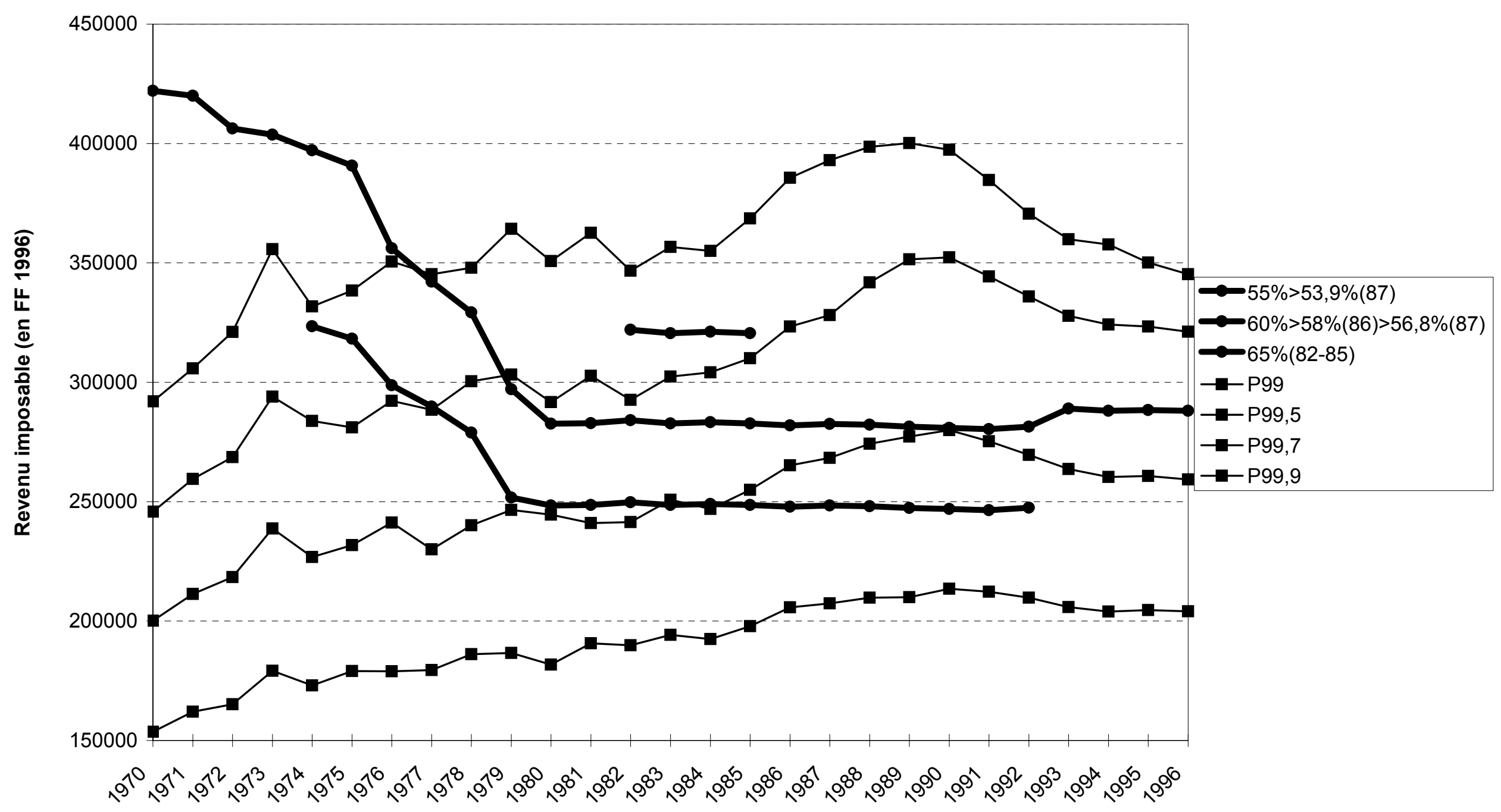


Tableau 4-4: Estimation de l'élasticité du revenu imposable à partir des foyers fiscaux ayant 1 part de quotient familial

\section{(a) 1981-1985}

\begin{tabular}{|c|c|c|c|c|c|c|c|}
\hline & P99-99,5 & P99,7-100 & ratio & & P99-99,5 & $P 99,7-100$ & ratio \\
\hline y (1981) & 2,11 & 3,19 & 1,51 & y (1981) & 2,11 & 3,19 & 1,51 \\
\hline y (1983) & 2,10 & 2,96 & 1,41 & y (1985) & 2,19 & 3,20 & 1,4 \\
\hline dy/y (\%) & $-0,8$ & $-7,3$ & $-6,6$ & dy/y (\%) & 3,6 & 0,3 & $-3,2$ \\
\hline$t^{\prime}(1981)$ & 52 & 60 & 0,83 & $t^{\prime}(1981)$ & 52 & 60 & \\
\hline$t^{\prime}(1983)$ & 52 & 65 & 0,73 & $t^{\prime}(1985)$ & 52 & 65 & \\
\hline $\mathrm{dt}^{\prime} /\left(1-\mathrm{t}^{\prime}\right)(\%)$ & 0,0 & $-12,5$ & $-12,5$ & $\mathrm{dt}^{\prime} /\left(1-\mathrm{t}^{\prime}\right)(\%)$ & 0,0 & $-12,5$ & \\
\hline \multicolumn{3}{|l|}{ élasticité e } & 0,53 & \multicolumn{3}{|l|}{ élasticité e } & \\
\hline \multicolumn{3}{|c|}{ taux marginal $t^{*}=1 /(1+e)(\%)$} & 65,4 & \multicolumn{3}{|c|}{ taux marginal $t^{*}=1 /(1+e)(\%)$} & \\
\hline \multicolumn{3}{|c|}{ taux marginal $\mathrm{t}^{\prime * *}=1 /(1+a e)(\%)$} & 45,1 & \multicolumn{3}{|c|}{ taux marginal $t^{\prime *}=1 /(1+a e)(\%)$} & \\
\hline
\end{tabular}

\section{(b) 1985-1995}

\begin{tabular}{|c|c|c|c|c|c|c|c|}
\hline & P99-99,5 & P99,7-100 & ratio & & P99-99,5 & P99,7-100 & ratio \\
\hline y (1985) & 2,19 & 3,20 & 1,46 & y (1985) & 2,19 & 3,20 & 1,46 \\
\hline y (1989) & 2,32 & 3,80 & 1,64 & y (1995) & 2,29 & 3,37 & 1,4 \\
\hline dy/y (\%) & 6,0 & 18,8 & 12,0 & dy/y (\%) & 4,5 & 5,2 & 0,7 \\
\hline$t^{\prime}(1985)$ & 52 & 65 & 0,73 & $t^{\prime}(1985)$ & 52 & 65 & 0,7 \\
\hline$t^{\prime}(1989)$ & 51 & 56,8 & 0,88 & $t^{\prime}(1995)$ & 51 & 56,8 & 0, \\
\hline $\mathrm{dt}^{\prime} /\left(1-\mathrm{t}^{\prime}\right)(\%)$ & 2,2 & 23,4 & 20,8 & $\mathrm{dt}^{\prime} /\left(1-\mathrm{t}^{\prime}\right)(\%)$ & 2,2 & 23,4 & 20,8 \\
\hline \multicolumn{3}{|l|}{ élasticité e } & 0,58 & \multicolumn{3}{|l|}{ élasticité e } & 0,0 \\
\hline \multicolumn{3}{|c|}{ taux marginal $t^{*}=1 /(1+e)(\%)$} & 63,3 & \multicolumn{3}{|c|}{ taux marginal $t^{*}=1 /(1+e)(\%)$} & \\
\hline \multicolumn{3}{|c|}{ taux marginal $\mathrm{t}^{\prime * *}=1 /(1+a e)(\%)$} & 42,9 & \multicolumn{3}{|c|}{ taux marginal $t^{\prime \prime *}=1 /(1+a e)(\%)$} & \\
\hline
\end{tabular}

Source: Calculs effectués à partir des résultats du tableau 4-2.

Lecture: Entre 1981 et 1983, la part du revenu imposable détenue par les foyers de P99-99,5 est passée de $2,11 \%$ à $2,10 \%$, soit une baisse de $0,8 \%$; la part de $\mathrm{P} 99,7-100$ est passée de $3,19 \%$ à $2,96 \%$, soit une baisse de 7,3\%. Le ratio entre la part de $\mathrm{P} 99,7-100$ et la part de P99-99,5 est donc passée de 1,51 à 1,41 , soit une baisse de 6,6\%. Le taux marginal moyen des foyers de P99-99,5 est passé de $52 \%$ en 1981 à $52 \%$ en 1983 , soit une hausse de $0,0 \%$ de leur taux de rétention; celui des foyers de P99,7-100 est passé de $60 \%$ à $65 \%$, soit une baisse de $12,5 \%$ de leur taux de rétention. Le ratio entre le taux de rétention de P99,7-100 et celui de P99-99,5 est donc passé de 0,83 à 0,73 , soit une baisse de $12,5 \%$. L'élasticité du revenu imposable correspondant à ces variations est égale à e $=0,53(=6,6 / 12,5)$, et les taux marginaux $t^{*}=1 /(1+e)$ et $t^{\prime *}=1 /(1+a e)(a=2,3)$ maximisant les recettes fiscales impliqués par ces élasticités sont égaux à $65,4 \%$ et $45,1 \%$. 
un objectif social de type rawlsien, consistant à maximiser les ressources disponibles pour venir en aide aux personnes les plus défavorisées. En outre, ces taux constituent des bornes supérieures des taux socialement optimaux correspondant à des objectifs sociaux plus nuancés accordant un poids positif au bien-être des personnes les plus fortunées (comme l'objectif utilitariste), et ils ont le mérite d'être aisément calculables à partir de l'élasticité du revenu imposable. Si un même taux marginal était appliqué à tous les revenus, ce qui correspondrait à une impôt linéaire, alors le taux maximisant les recettes fiscales (noté $t^{*}$ ) serait donné par la formule bien connue $t^{*}=1 /(1+e) .{ }^{63}$ Dans le cas présent $(e=0,53)$, on obtient $t^{*}=65,4 \%$ : si tous les revenus répondaient toujours dans les mêmes proportions aux variations de taux marginaux que les revenus examinés ici, alors le taux marginal linéaire maximisant les recettes fiscales serait de $65,4 \%$. En pratique, les taux marginaux supérieurs ne sont cependant appliqués qu'à une fraction des revenus des contribuables les plus fortunés : pour une élasticité donnée du revenu imposable, le taux marginal supérieur maximisant les recettes fiscales est donc toujours inférieur lorsqu'il ne s'applique qu'au delà d'un certain seuil que lorsqu'il s'applique à tous les revenus. On peut montrer que si la distribution des hauts revenus suit une loi de Pareto de coefficient $a>1$, alors le taux marginal appliqué uniquement aux hauts revenus et maximisant les recettes fiscales (noté $t^{* *}$ ) est donnée par la formule $t^{\prime *}=1 /(1+a e) .{ }^{64}$ En pratique, c'est

\footnotetext{
${ }^{63}$ On peut retrouver cette formule de la façon suivante: supposons que le taux marginal (unique) $t$ passe de $t$ à $t+d t$; le taux de rétention 1-t (qui est équivalent au taux de salaire net (1-t)w dans le cas où l'on considère uniquement un revenu du travail classique $\mathrm{y}=\mathrm{wl}$ ) diminue $\mathrm{de} \mathrm{dt} /(1-\mathrm{t}) \%$, et le revenu imposable de edt/(1-t)\% ; les recettes fiscales passent de $R$ à $R+d R$, avec $d R=y d t$ - tyedt/(1-t) ; la maximisation des recettes fiscales est atteinte pour un taux $t^{*}$ tel que $d R=0$, c'est-à-dire $t^{*}=1 /(1+e)$. Les valeurs de $t^{*}$ données dans les tableaux 4-4 à 4-10 sont en réalité des sous-estimations du taux linéaire maximisant les recettes fiscales, car la formule $t^{*}=1 /(1+e)$ exige en toute rigueur que e soit l'élasticité non-compensée $e_{N C}$, c'est-à-dire la somme de l'élasticité compensée $e_{C}$ (positive) et de l'élasticité revenu $\mathrm{e}_{\mathrm{y}}$ (négative) (cf. Piketty (1997b, p.195, note 8)), alors que l'élasticité empirique e estimée dans les tableaux 4-4 à 4-10 est en fait une moyenne pondérée de l'élasticité compensée et de l'élasticité non-compensée (cf. note suivante), et elle est donc toujours supérieure (ou égale, si l'élasticité revenu est nulle) à l'élasticité compensée.

${ }^{64}$ Supposons que le taux marginal supérieur $\mathrm{t}^{\prime}$, applicable à la fraction des revenus supérieure à $\mathrm{y}_{0}$, passe de t' à t'+dt', soit une baisse de $\mathrm{dt}^{\prime} /\left(1-\mathrm{t}^{\prime}\right) \%$ du taux marginal de rétention 1-t'. Si l'élasticité est égale à e, alors tous les contribuables dont le revenu y est supérieur à yo voient leur revenu baisser de edt'/(1-t')\% ; l'assiette de la tranche supérieure, c'est-à-dire $y-y_{0}$, baisse donc de eydt'/[(1-t')(y$\left.\left.\mathrm{y}_{0}\right)\right] \%$. Les recettes rapportées par la tranche supérieure passent de $\mathrm{R}$ à $\mathrm{R}+\mathrm{dR}$, avec $\mathrm{dR}=\mathrm{dt}^{\prime}\left(\mathrm{y}^{*}\left(\mathrm{y}_{0}\right)\right.$ $\left.\mathrm{y}_{0}\right)-\mathrm{t}^{\prime}\left(\mathrm{y}^{*}\left(\mathrm{y}_{0}\right)-\mathrm{y}_{0}\right)$ ey $\mathrm{y}^{*}\left(\mathrm{y}_{0}\right) \mathrm{dt} /\left[\left(1-\mathrm{t}^{\prime}\right)\left(\mathrm{y}^{*}\left(\mathrm{y}_{0}\right)-\mathrm{y}_{0}\right)\right]$, où $\mathrm{y}^{*}\left(\mathrm{y}_{0}\right)$ est le revenu moyen des contribuables dont le revenu est supérieur à $y_{0}$. Le taux $t^{\prime *}$ vérifie $d R=0$, soit $t^{\prime *}=1 /\left[1+e y^{*}\left(y_{0}\right) /\left(y^{*}\left(y_{0}\right)-y_{0}\right)\right]$. Si les revenus supérieurs à $\mathrm{y}_{0}$ suivent une loi de Pareto de paramètre $a>1$, alors $\mathrm{y}^{*}\left(\mathrm{y}_{0}\right) /\left(\mathrm{y}^{*}\left(\mathrm{y}_{0}\right)-\mathrm{y}_{0}\right)=a$ (cf. Annexe $\mathrm{D}$ $1)$, d'où la formule $t^{\prime *}=1 /(1+a e)$. L'élasticité " empirique " e utilisée dans cette formule est en fait une moyenne pondérée de l'élasticité non-compensée $e_{N C}$ et de l'élasticité compensée $e_{C}$ : pour un contribuable dont le revenu est égal à $y>y_{0}$, on peut montrer que e $=e_{N C}\left(y-y_{0}\right) / y+e_{C} y_{0} / y\left(s i ~ y=y_{0}\right.$, alors la hausse de t' à t'+dt' du taux marginal supérieur s'apparente à une baisse compensée du taux de rétention (aucun effet revenu) ; inversement, pour y très élevé, l'effet revenu t'( $\left(y-y_{0}\right)$ tend vers t'y, si bien que la hausse de t' s'apparente à une baisse non-compensée du taux de rétention); en moyenne sur la tranche supérieure, on a $e=e_{N C} / a+e_{C}(a-1) / a$, si bien que la formule $t^{\prime *}=1 /(1+a e)$ est bien équivalente à la formule $t^{\prime *}=1 /\left(1+e_{N C}+(a-1) e_{C}\right)$ développée par Saez (1998b).Toutes les études empiriques estimant des élasticités avec une méthode du type "le taux de rétention de la tranche supérieure a varié de $x \%$, le revenu moyen de la tranche supérieure a varié de $y \%$, donc e $=y / x \%$ ", telles que celles de Lindsay (1987) et Feldstein (1995), estiment en fait cette élasticité pondérée, même s'ils omettent généralement de le signaler (en l'absence d'effet revenu $e=e_{N C}=e_{C}$, et ces distinctions n'ont aucune importance).
} 
ce taux marginal t'* qui est pertinent pour évaluer si les effets d'une modification du taux marginal supérieur sur les finances publiques (pour le tableau 4-4 comme pour les tableaux suivants, nous avons retenu une valeur moyenne de 2,3 pour le paramètre a caractérisant les hauts revenus (cf. annexes D.1 et D. 2 pour des estimations empiriques de ce paramètre) $).{ }^{65}$ Dans le cas présent $(e=0,53)$, on obtient donc $t^{\prime *}=45,1 \%$. Si l'élasticité du revenu imposable était de 0,53 , alors le taux marginal supérieur maximisant les recettes fiscales serait seulement de $45,1 \%$. Autrement dit, si la baisse des revenus de P99,7-100 enregistrée entre 1981 et 1983 pouvait être attribuée à la création de la tranche à $65 \%$, alors il faudrait conclure que la création de la tranche à $65 \%$ a en fait conduit à une baisse des recettes fiscales.

Cependant, une telle conclusion serait pour le moins hâtive, puisque le tableau 4-1 indique que la part de P99,7-100 s'est mise à progresser dès 1984, et a retrouvé (et même dépassé) dès 1985 son niveau de 1981. La part de P99-99,5 a également progressé entre 1983 et 1985, mais moins rapidement que celle de P99,7-100, si bien que la ratio P99,7-100/P99-99,5 a diminué de seulement 3,2\% entre 1981 et 1985. En comparant les années 1981 et 1985, on aboutit ainsi à une élasticité $\mathrm{e}=0,26(0,26=3,2 / 12,5)$, soit un taux marginal $\mathrm{t}^{* *}=62,9 \%$ (cf. tableau 4-4).

Plusieurs facteurs peuvent expliquer pourquoi la part de P99,7-100 a diminué entre 1981 et 1983, avant de remonter dès 1984. Tout d'abord, il est possible que la chute de 1981 n'ait rien à voir avec la création de la tranche à $65 \%$, et soit simplement la conséquence mécanique de la mauvaise conjoncture économique: la reprise économique qui s'amorce dès 1984-1985, notamment pour les profits des entreprises, aurait alors conduit à un retour mécanique des hauts revenus à leur niveau relatif de 1980-1981, et ce indépendamment du taux marginal supporté par les contribuables concernés (la tranche à $65 \%$ ne sera supprimée qu'en 1986). L'autre possibilité est que la création de la tranche à $65 \%$ a conduit dans un premier temps à des comportements de reports de rémunérations, ce qui expliquerait (en partie) la chute observée en 1982-1983, et que ces reports ont rapidement pris fin. Un tel phénomène de baisse immédiate des revenus concernés lors d'une hausse du taux marginal supérieur, suivie d'un retour aux niveaux antérieures en l'espace de quelques années, est similaire au phénomène observé en 1993 aux Etats-Unis et analysé par Goolsbee (cf. section 1.1.2). Compte tenu de l'importance de la chute enregistrée en 1982, du caractère purement temporaire de cette baisse et de son léger décalage avec le cycle économique, cette seconde interprétation nous semble la plus plausible. On peut cependant noter que les très hauts revenus des foyers de P99,99-100 expliquent en 1981-1983 comme en 1973-1974 et en 1990-1993 environ la moitié de la baisse des revenus de P99,9-100 (cf. tableau 4-1). Cette "homothéticité" entre les fluctuations observées pour les hauts et les très hauts revenus suggère que les fluctuations de 1981-1983 sont pour l'essentiel de la même

\footnotetext{
${ }^{65}$ C'est ce même coefficient de 2,3 qui explique pourquoi l'assiette de la tranche supérieure de l'impôt représente actuellement environ $2,9 \%$ du revenu imposable total, bien que le revenu imposable des contribuables concernés par la tranche supérieure représente environ $6,7 \%$ du revenu imposable total $(6,7 / 2,9=2,3)$ (cf. section 1.1.1, p.3).
} 
nature que celles observés lors des récessions de 1973-1974 et 1990-1993, et que le phénomène de "report" ne peut expliquer qu'une partie minoritaire des fluctuations de 1981-1983. Si la hausse du taux marginal d'imposition (et non pas le cycle économique) était responsable de la baisse des hauts revenus de 1981-1983, on devrait en effet s'attendre à ce que tous les revenus de P99,7-100 aient été touché dans les mêmes proportions, ou tout du moins dans des proportions moins "inégalitaires " que celles qui caractérisent un cycle économique normal. ${ }^{66}$

Quoi qu'il en soit, le fait est que les revenus de P99,7-100 ont retrouvé dès 1985 leur niveau relatif de 1981, malgré la hausse de leur taux marginal d'imposition, ce qui suggère que l'élasticité de long-terme du revenu imposable vis-à-vis du taux marginal d'imposition est relativement faible (au maximum 0,26 ). Cette élasticité maximale de 0,26 correspond à un taux marginal supérieur $\mathrm{t}^{\text {*** }}=62,9 \%$, soit un niveau intermédiaire entre 60 et $65 \%$ : autrement dit, la légère baisse des revenus de la tranche supérieure (comparée aux revenus de P99-99,5) observée entre 1981 et 1985 équilibre la hausse du taux marginal supérieur, si bien que l'effet net sur les recettes de la création de la tranche à $65 \%$ est pratiquement nul (légèrement positif). En outre, il semble relativement plausible de supposer que le rattrapage des hauts revenus observée en 1984-1985 se serait poursuivi dans les années suivantes, y compris si la tranche à $65 \%$ n'avait pas été supprimée en 1986, ce qui conduirait à réviser à la baisse l'élasticité de 0,26 estimée en comparant les années 1981 et 1985 , et donc à conclure que l'effet net sur les recettes est nettement positif. La tranche à $65 \%$ ayant été supprimée en 1986 , personne ne pourra jamais dire ce qui se serait passée en l'absence de cette décision. Par contre, les réformes mises en place en 1986-1987 n'ayant pas été remises en cause par les gouvernements suivants, l'épisode de 1986-1987 permet des estimations plus fiables de la véritable élasticité de long-terme, et ces estimations suggèrent des élasticités voisines de 0 0,1 (cf. section 4.1.2 infra). De plus, on peut affiner les estimations de l'élasticité du revenu imposable sur la période 1981-1985 en comparant les foyers ayant 1 part de quotient familial aux autres foyers de célibataires, et on aboutit ainsi à la conclusion robuste que cette élasticité de 0,26 est très fortement surévaluée (cf. section 4.2.1 infra).

Cette première estimation de l'élasticité du revenu imposable, tout comme les estimations suivantes, fait l'hypothèse que les taux marginaux perçus par les contribuables fortunés au début des années 1980 coïncidaient avec les taux marginaux effectifs. On pourrait cependant imaginer que tous les contribuables du centile supérieur se soient sentis concernés par la création de la tranche à $65 \%$, même si cette dernière ne concernait en fait que le tiers supérieur du centile supérieur. Dans ce cas, il faudrait comparer l'évolution des revenus de P99-100 à

\footnotetext{
${ }^{66}$ II est cependant difficile d'utiliser directement l'homothéticité des fluctuations observés (i.e. le fait que les revenus de P99,9-100 fluctuent "normalement" $x$ fois plus que ceux de P99,5-99,9, etc..) pour obtenir des estimations de l'élasticité du revenu imposable, car on peut imaginer que les élasticités soient plus élevées pour les très hauts revenus que pour les hauts revenus, ce qui permettrait de rendre compte de l'homothéticité observée y compris si les fluctuations étaient dues aux variations de taux marginaux.
} 
ceux des centiles inférieurs, plutôt que de comparer l'évolution de P99,7-100 et P9999,5. L'hypothèse d'une connaissance approximative de la législation par les contribuables aisés semble relativement douteuse, si l'on en juge par les ressources (conseillers fiscaux, presse spécialisée,...) que ces contribuables dépensent pour en connaître les moindres recoins, ${ }^{67}$ mais elle n'est pas totalement absurde, compte tenu du climat d'effervescence politique et symbolique entourant l'impôt sur le revenu en 1981-1983. Quoi qu'il en soit, une telle hypothèse ne conduirait pas à des estimations plus élevées de l'élasticité du revenu imposable. La part de P95-98 a progressé de $9,1 \%$ entre 1980 et 1985 , celle de P98-99 a progressé de $8,2 \%$, alors que celles de P99-99,5 progressait de $8,0 \%$ et celle de P99,5-100 progressait de $3,2 \%$ (cf. tableaux $4-2$ et $4-3$ ). Autrement dit, les revenus des foyers concernés par la création de la tranche à $65 \%$ n'ont beaucoup baissé ni vis-à-vis des foyers immédiatement inférieurs de P99-99,5, ni vis-à-vis des foyers plus distants de P9598 ou P98-99. ${ }^{68}$ En comparant l'évolution des revenus de la tranche supérieure (ou du centile supérieure) et de ceux de P95-98 ou P98-99, on aboutirait donc à des ordres de grandeur similaires à ceux obtenus en comparant P99,7-100 et P99-99,5, c'est-à-dire à des élasticités maximales de l'ordre de $0,2-0,3{ }^{69}$

\subsubsection{La suppression de la tranche à $65 \%$ (1986-1987)}

L'avantage de l'épisode de 1986-1987 est que, contrairement à l'épisode de 19811982, l'expérience a été conduite jusqu'à son terme : le taux supérieur de $56,8 \%$ s'est appliqué sans discontinuer des revenus de 1987 aux revenus de 1995, si bien que nous pouvons comparer au cours d'un cycle économique complet l'évolution des revenus des foyers concernés par la suppression de la tranche à $65 \%$ et de ceux des foyers qui n'étaient pas concernés. Cette comparaison nous permet de constater que la hausse des revenus des foyers de P99,7-100 observée entre 1986 et 1989 a été entièrement annulée lors de la phase basse du cycle économique entre 1990 et 1993. Entre 1985 et 1989, la part de P99,7-100 augmentait ainsi de $18,8 \%$, alors que celle de P99-99,5 augmentait de "seulement" $6 \%$, si bien que le ratio P99,7$100 /$ P99-99,5 progressait de $12,0 \%$. Le taux marginal des premiers passait de $65 \%$ à $56,8 \%$, soit une hausse de $23,4 \%$ de leur taux de rétention, alors que les seconds bénéficiaient uniquement des très légères baisses accordés aux autres taux

\footnotetext{
${ }^{67}$ En 1982, parmi les foyers ayant 1 part de QF, les foyers de P99-99,5 avaient un revenu imposable compris entre 115000 et 145000 francs, alors que la tranche à $65 \%$ s'appliquait uniquement à la fraction des revenus supérieure à 195000 francs (cf. Annexe D.2, tableau D-4). Ces foyers n'avaient donc pas besoin d'un conseiller fiscal à plein temps pour comprendre qu'ils n'étaient pas concernés...

${ }^{68}$ En 1982, les foyers de P95-98 avaient des revenus imposables compris entre 65000 et 90000 francs (cf. Annexe D-2, tableau D-4), et on imagine donc mal comment ils auraient pu se sentir concernés par la création de la tranche à 65\% (qui s'appliquait aux revenus supérieurs à 195000 francs).

${ }^{69}$ En outre, si l'on estimait l'élasticité du revenu imposable en comparant P99,7-100 (ou P99-100) et P95-98, alors il faudrait prendre en compte les majorations d'impôts, qui concernaient les foyers de P99-100 mais pas ceux de P95-98 (cf. section 2.1.2 supra), ce qui conduirait à réviser à la baisse l'élasticité estimée.
} 
marginaux du barème. ${ }^{70} \mathrm{Si}$ l'on attribuait la hausse du revenu relatif de P99,7-100 observée entre 1985 et 1989 à la hausse de leur taux relatif de rétention, alors on aboutirait à une élasticité $e=0,58$ et à un taux marginal $\mathrm{t}^{\prime *}=42,9 \%$ (cf. tableau $4-4$ ). Mais le fait est que cette hausse a été entièrement annulée lors des 4 années suivantes : entre 1985 et 1995 , les parts de P99-99,5 et P99,7-100 ont progressé exactement dans les mêmes proportions $(4,5 \%$ et $5,2 \%)$, ce qui correspond à une élasticité extrêmement faible $(e=0,04)$ et à des taux marginaux maximisant les recettes fiscales supérieures à $90 \%$ (cf. tableau 4-4).

Autrement dit, sur la période 1985-1995, les revenus imposables des foyers de P99,7-100 et de P99-99,5 ont progressé au même rythme, bien que les premiers aient vu leur taux de rétention relevé soudainement de près de $25 \%$, alors que les seconds bénéficiaient d'une hausse insignifiante de leur taux de rétention. Les années 1985 et 1995 se situant à des phases similaires du cycle économique, cette estimation des effets incitatifs de la tranche à $65 \%$ est probablement la moins mauvaise que l'on puisse obtenir. Certes, on ne pourra jamais déterminer avec certitude ce qui se serait passé en l'absence de l'abaissement à $56,8 \%$ du taux supérieur de l'IR. Par exemple, rien dans les données disponibles ne permet d'exclure avec certitude la possibilité que sans cette baisse de taux marginal, les revenus de P99,7-100 se seraient subitement mis à baisser relativement à ceux de P99-99,5, du fait d'un trend structurel nouveau, et que seuls cette baisse de taux marginal leur a permis (grâce au renforcement de leurs incitations au travail, au sens large) de préserver le niveau de leur revenu relatif. Mais il y a tout lieu d'être sceptique quand à une telle possibilité : rien dans l'évolution générale de l'inégalité et de la composition des revenus entre 1970 et 1996 ne laisse présager un tel trend subit, et tout laisse supposer que les fluctuations de P99,7-100/P99-99,5 enregistrées en 1986-1989 et 1990-1993 correspondent aux deux phases symétriques d'un cycle économique classique, qui n'a rien à voir avec les variations de taux marginaux d'imposition. La régularité de la "courbe en $\cap$ " dessinée en 1985-1993 par le seuil P99,9 (graphique 4-1) suggère bel et bien l'existence d'un cycle économique normal. De même, la stabilisation de la part de P99,7-100 enregistrée depuis 1993 et la fin de la récession contredit l'hypothèse d'un trend structurel lourd (cf. tableau 4-2). Notons également que de même qu'en 1970-1973, 1973-1974 et 1981-1983, les très hauts revenus de P99,99-100 expliquent environ la moitié des fluctuations de la part de P99,9-100 en 1986-1989 puis 1990-1993 (cf. tableau 4-1). Cette très grande similarité entre ces différents épisodes suggère là encore que les fluctuations de 1986-1989 et 1990-1993 relèvent d'un phénomène cyclique de type classique, et non d'une perturbation due à la fiscalité. La seule perturbation que les données permettent de déceler est de la même nature que lors de l'épisode de 1982-1983: de la même façon qu'il est possible qu'une partie de la chute des hauts revenus observée en 1982 soit due à des reports purement temporaires de la date de paiement de certaines rémunérations, il est possible qu'un

\footnotetext{
${ }^{70}$ En 1985 , environ $60 \%$ des foyers de P99-99,5 sont dans la tranche à $50 \%$, environ $40 \%$ dans la tranche à $55 \%$. Les proportions sont similaires en 1989 , mais les taux sont passés à $49 \%$ et $53,9 \%$.
} 
même phénomène de report explique une partie de la hausse des hauts revenus de 1987-1988. Mais de la même façon qu'aux Etats-Unis (cf. section 1.1.2), de tels phénomènes sont par nature purement temporaires, et n'ont aucun impact sur l'élasticité à long-terme du revenu imposable.

Enfin, de même que lors de l'épisode de 1981-1983, l'hypothèse selon laquelle l'ensemble du centile supérieure se serait senti concerné par la suppression de la tranche à $65 \%$ en 1986-1987 ne modifierait guère les élasticités obtenues. Entre 1985 et 1995, la part de P95-98 a progressé de 6,7\%, celle de P98-99 a progressé de $5,8 \%$, alors que celles de P99-99,5 progressait de $4,5 \%$ et celle de P99,5-100 progressait de 5,3\% (cf. tableaux 4-1 et 4-2). Autrement dit, tous les revenus des foyers de P95-100 ont progressé dans des proportions analogues entre 1985 et 1995, et pas seulement les revenus du centile supérieur. En comparant l'évolution des revenus de la tranche supérieure (ou du centile supérieure) et de ceux de P9598 ou P98-99, on aboutirait donc au même type d'élasticités, à savoir des élasticités inférieures à 0,1 (ou négatives).

\subsection{Estimations effectuées à partir des foyers de célibataires ( $Q F=1,1,5$ et $2 B)$}

\subsubsection{La création de la tranche à $65 \%$ et la mise en place du plafonnement des effets du quotient familial (1981-1982)}

Par définition, les foyers ayant une seule part de quotient familial n'étaient pas concernés par le mécanisme de plafonnement des effets du quotient familial mis en place en 1981. Par contre, les autres foyers de célibataires, et notamment les foyers ayant 1,5 ou 2B parts de quotient familial étaient concernés (cf. section 2.1.3 supra). Le graphique 4-2 décrit l'évolution des seuils des tranches supérieures du barème applicables aux foyers ayant 1,5 part de quotient familial (après prise en compte des effets du plafonnement des effets du quotient familial à partir de 1981) et des seuils P98, P99, P99,5 et P99,9 de la distribution du revenu imposable des foyers ayant 1,5 part du quotient familial, tous ces montant étant exprimés en francs de $1996 .{ }^{71}$

Insérer : Graphique 4-2 : Les seuils des fractiles et des tranches supérieures de 1970 à 1996 (foyers fiscaux ayant 1,5 parts de quotient familial)

Environ $0,5 \%$ des foyers fiscaux ayant 1,5 parts de QF étaient concernés par la création de la tranche à $65 \%$ (contre environ $0,3 \%$ des foyers fiscaux ayant 1 part de quotient familial). Mais une proportion beaucoup plus élevée de foyers fiscaux ayant 1,5 parts de QF ont vu leur taux marginal augmenter dans des proportions importantes, du fait du plafonnement des effets du quotient familial. Le graphique 4-

\footnotetext{
${ }^{71}$ De même que pour le graphique 4-1, les montants en francs courants ont été convertis en francs de 1996 en utilisant la série de taux d'inflation du tableau 2-3. Les seuils P99, P99,5, etc..., en francs courants sont issus des estimations effectués à partir des Etats 1921 (cf. Annexe D.2, tableau D-6). Les seuils de plafonnement des effets du quotient familial, ainsi que le barème après prise en compte de ce plafonnement, sont données dans l'annexe I.
} 
Graphique 4-2: Les seuils des fractiles et des tranches supérieures de 1970 à 1996

(foyers fiscaux ayant 1,5 parts de quotient familial)

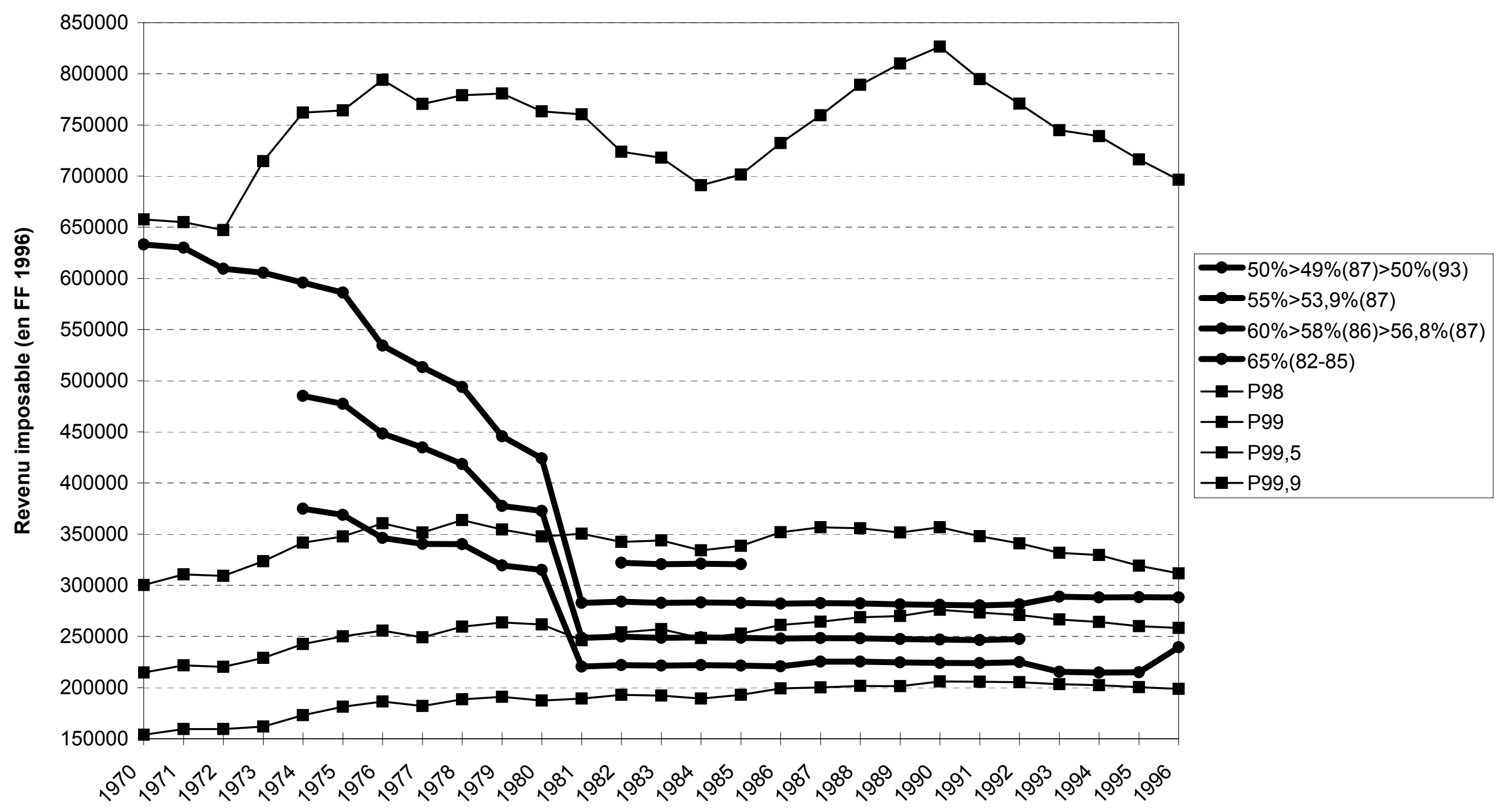


2 montre que ce plafonnement a conduit à un baisse massive en 1981 du seuil d'application des tranches à $50 \%, 55 \%$ et $60 \%$. Le seuil d'application du plafonnement correspondait à un revenu imposable légèrement inférieur au seuil P99 de la distribution du revenu imposable des foyers ayant 1,5 parts de QF, si bien que l'ensemble du centile supérieur de cette distribution (et non seulement P99,5-100) a connu une hausse substantielle de taux marginal. De la même façon, environ $0,5 \%$ des foyers fiscaux ayant $2 \mathrm{~B}$ parts de quotient familial étaient concernés par la création de la tranche à $65 \%$, mais l'ensemble du centile supérieure de cette distribution (et non seulement P99,5-100) a connu une hausse substantielle de taux marginal. ${ }^{72}$ Dans les deux cas, la hausse de taux marginal entraînée pour les contribuables de P99-99,5 par le plafonnement des effets du quotient familial a même été supérieure à la hausse entraînée pour les contribuables de P99,5-100 par la création de la tranche à $65 \%$. Si les taux marginaux d'imposition avaient un impact sensible sur les comportements des contribuables, on devrait donc s'attendre à que la part de P99-99,5 ait baissée au moins autant que celle de P99,5-100 pour les foyers ayant 1,5 ou 2B parts de quotient familial, ou au minimum que le ratio P99,5100/P99-99,5 ait moins baissé pour ces foyers que pour les foyers ayant 1 part de quotient familial. Or, les tableaux 4-5 et 4-6 montrent que tel n'a pas été le cas : les fluctuations des revenus à l'intérieur du centile supérieur ont été sensiblement les mêmes pour tous les foyers de célibataires.

Insérer Tableau 4-5: Estimation de l'élasticité du revenu imposable à partir des foyers fiscaux ayant 1,5 parts de quotient familial.

Insérer Tableau 4-6: Estimation de l'élasticité du revenu imposable à partir des foyers fiscaux ayant 2B parts de quotient familial.

Le tableau 4-5 indique que le taux marginal moyen des foyers de P99-99,5 ait passé de $46 \%$ en 1980 (environ $80 \%$ étaient dans la tranche à $45 \%$ et $20 \%$ dans la tranche à $50 \%$ ) à $58 \%$ à partir de $1981-1982$ (environ $60 \%$ se retrouvent dans la tranche à $55 \%, 20 \%$ dans la tranche à $60 \%$ et $20 \%$ dans la tranche à $65 \%$ ), soit une baisse de plus de $22 \%$ de leur taux de rétention. Dans le même temps, le taux marginal moyen des ménages de P99,5-100 est passé de $58 \%$ en 1980 (40\% dans la tranche à $55 \%$ et $60 \%$ dans la tranche à $60 \%$ ) à $65 \%$ à partir de 1982 , soit une baisse de $16,7 \%$ de leur taux de rétention. Pourtant, entre 1980 et 1983, le ratio P99,5-100/P99-99,5 a baissé d'environ $6 \%$ pour les foyers ayant 1,5 de quotient familial, c'est-à-dire exactement dans les mêmes proportions que pour les foyers ayant 1 part de quotient familial. ${ }^{73}$ La seule différence est que cette baisse a été

\footnotetext{
${ }^{72}$ Cf. annexe D.2 pour les résultats détaillés de l'estimation de la distribution du revenu imposable des foyers ayant 1,5 ou 2B parts de quotient familial, et annexe I pour les seuils de plafonnement du QF et les barèmes après plafonnement applicables aux contribuables ayant 1,5 parts et 2B parts de quotient familial.

${ }^{73}$ Le tableau 4-4 compare les années 1981 à 1983 (puisque contrairement au plafonnement des effets du quotient familial, applicable dès les revenus de 1981, la tranche à $65 \%$ ne s'est appliquée qu'à compter des revenus de 1982), mais les résultats seraient similaires si l'on comparait les années 1980 et 1983 (cf. tableaux 4-1 et 4-2).
} 
Tableau 4-5: Estimation de l'élasticité du revenu imposable à partir des foyers fiscaux ayant 1,5 parts de quotient familial

\section{(a) 1980-1985}

\begin{tabular}{|c|c|c|c|c|c|c|c|}
\hline & P99-99,5 & P99,5-100 & ratio & & P99-99,5 & P99,5-100 & ratio \\
\hline y (1980) & 2,79 & 6,68 & 2,40 & y (1980) & 2,79 & 6,68 & 2,40 \\
\hline y (1983) & 2,60 & 5,86 & 2,25 & y (1985) & 2,59 & 5,84 & 2,25 \\
\hline dy/y (\%) & $-6,5$ & $-12,4$ & $-6,3$ & dy/y (\%) & $-7,0$ & $-12,7$ & $-6,1$ \\
\hline$t^{\prime}(1980)$ & 46 & 58 & 0,78 & t' (1980) & 46 & 58 & 0,78 \\
\hline$t^{\prime}(1983)$ & 58 & 65 & 0,83 & $t^{\prime}(1985)$ & 58 & 65 & 0,83 \\
\hline $\mathrm{dt}^{\prime} /\left(1-\mathrm{t}^{\prime}\right)(\%)$ & $-22,2$ & $-16,7$ & 7,1 & $\mathrm{dt}^{\prime} /\left(1-\mathrm{t}^{\prime}\right)(\%)$ & $-22,2$ & $-16,7$ & 7,1 \\
\hline \multicolumn{3}{|l|}{ élasticité e } & $-0,88$ & \multicolumn{3}{|l|}{ élasticité e } & $-0, \varepsilon$ \\
\hline \multicolumn{3}{|c|}{ taux marginal $t^{*}=1 /(1+e)(\%)$} & 100,0 & \multicolumn{3}{|c|}{ taux marginal $t^{*}=1 /(1+e)(\%)$} & 100 \\
\hline \multicolumn{3}{|c|}{ taux marginal $\mathrm{t}^{\prime *}=1 /(1+\mathrm{ae})(\%)$} & 100,0 & \multicolumn{3}{|c|}{ taux marginal $t^{\prime *}=1 /(1+a e)(\%)$} & 0 \\
\hline
\end{tabular}

\section{(b) 1985-1995}

\begin{tabular}{|c|c|c|c|c|c|c|c|}
\hline & P99-99,5 & P99,5-100 & ratio & & P99-99,5 & P99,5-100 & ratio \\
\hline y (1985) & 2,59 & 5,84 & 2,25 & y (1985) & 2,59 & 5,84 & 2,25 \\
\hline y (1989) & 2,72 & 6,24 & 2,29 & y (1995) & 2,47 & 5,27 & 2,13 \\
\hline dy/y (\%) & 5,2 & 6,9 & 1,6 & dy/y (\%) & $-4,5$ & $-9,6$ & $-5,3$ \\
\hline$t^{\prime}(1985)$ & 58 & 65 & 0,83 & t' (1985) & 58 & 65 & 0,83 \\
\hline$t^{\prime}(1989)$ & 56,2 & 56,8 & 0,99 & t' (1995) & 56,2 & 56,8 & 0,99 \\
\hline $\mathrm{dt}^{\prime} /\left(1-\mathrm{t}^{\prime}\right)(\%)$ & 4,2 & 23,4 & 18,4 & $\mathrm{dt}^{\prime} /\left(1-\mathrm{t}^{\prime}\right)(\%)$ & 4,2 & 23,4 & 18,4 \\
\hline \multicolumn{3}{|c|}{ élasticité e } & 0,09 & \multicolumn{3}{|c|}{ élasticité e } & $-0,29$ \\
\hline \multicolumn{3}{|c|}{ taux marginal $t^{*}=1 /(1+e)(\%)$} & 92,0 & \multicolumn{3}{|c|}{ taux marginal $t^{*}=1 /(1+e)(\%)$} & 100,0 \\
\hline \multicolumn{3}{|c|}{ taux marginal $\mathrm{t}^{\prime * *}=1 /(1+\mathrm{ae})(\%)$} & 83,3 & \multicolumn{3}{|c|}{ taux marginal $t^{\prime *}=1 /(1+a e)(\%)$} & 100,0 \\
\hline
\end{tabular}

Source: Calculs effectués à partir des résultats du tableau D-6 (cf. Annexe D.2). Lecture: cf. tableau 4-4. 
Tableau 4-6: Estimation de l'élasticité du revenu imposable à partir des foyers fiscaux ayant 2B parts de quotient familial

\section{(a) 1980-1985}

\begin{tabular}{|c|c|c|c|c|c|c|c|}
\hline & P99-99,5 & P99,5-100 & ratio & & P99-99,5 & P99,5-100 & ratio \\
\hline y (1980) & 2,14 & 4,60 & 2,15 & y (1980) & 2,14 & 4,60 & 2,15 \\
\hline y (1983) & 2,26 & 4,52 & 2,00 & y (1985) & 2,40 & 4,75 & 1,98 \\
\hline dy/y (\%) & 5,5 & $-1,8$ & $-7,0$ & dy/y (\%) & 11,8 & 3,1 & $-7,8$ \\
\hline$t^{\prime}(1980)$ & 40 & 51,5 & 0,81 & $t^{\prime}(1980)$ & 40 & 51,5 & 0,81 \\
\hline$t^{\prime}(1983)$ & 57 & 65 & 0,81 & $t^{\prime}(1985)$ & 57 & 65 & 0,81 \\
\hline $\mathrm{dt}^{\prime} /\left(1-\mathrm{t}^{\prime}\right)(\%)$ & $-28,3$ & $-27,8$ & 0,7 & $\mathrm{dt}^{\prime} /\left(1-\mathrm{t}^{\prime}\right)(\%)$ & $-28,3$ & $-27,8$ & 0,7 \\
\hline \multicolumn{3}{|l|}{ élasticité e } & $-10,00$ & \multicolumn{3}{|c|}{ élasticité e } & $-11,19$ \\
\hline \multicolumn{3}{|c|}{ taux marginal $t^{*}=1 /(1+e)(\%)$} & 100,0 & \multirow{2}{*}{\multicolumn{3}{|c|}{ taux marginal $t^{*}=1 /(1+e)(\%)$}} & 100,0 \\
\hline \multicolumn{3}{|c|}{ taux marginal $\mathrm{t}^{\prime *}=1 /(1+\mathrm{ae})(\%)$} & 100,0 & \multicolumn{2}{|c|}{ taux marginal $t^{\prime * *}=1 /(1+a e)(\%)$} & & 100,0 \\
\hline
\end{tabular}

\section{(b) 1985-1995}

\begin{tabular}{|c|c|c|c|c|c|c|c|}
\hline & P99-99,5 & P99,5-100 & ratio & & P99-99,5 & P99,5-100 & ratio \\
\hline у (1985) & 2,40 & 4,75 & 1,98 & y (1985) & 2,40 & 4,75 & 1,98 \\
\hline y (1989) & 2,50 & 5,27 & 2,11 & y (1995) & 2,47 & 4,99 & 2,02 \\
\hline dy/y (\%) & 4,2 & 11,0 & 6,5 & dy/y (\%) & 3,2 & 5,3 & 2,0 \\
\hline$t^{\prime}(1985)$ & 57 & 65 & 0,81 & $t^{\prime}(1985)$ & 57 & 65 & 0,8 \\
\hline$t^{\prime}(1989)$ & 56,8 & 56,8 & 1,00 & $t^{\prime}(1995)$ & 56,8 & 56,8 & 1,0 \\
\hline $\mathrm{dt}^{\prime} /\left(1-\mathrm{t}^{\prime}\right)(\%)$ & 0,5 & 23,4 & 22,9 & $\mathrm{dt}^{\prime} /\left(1-\mathrm{t}^{\prime}\right)(\%)$ & 0,5 & 23,4 & 22 \\
\hline \multicolumn{3}{|c|}{ élasticité e } & 0,29 & \multicolumn{3}{|c|}{ élasticité e } & 0,0 \\
\hline \multicolumn{3}{|c|}{ taux marginal $t^{*}=1 /(1+e)(\%)$} & 77,8 & \multicolumn{3}{|c|}{ taux marginal $t^{*}=1 /(1+e)(\%)$} & 91 \\
\hline \multicolumn{3}{|c|}{ taux marginal $t^{\prime *}=1 /(1+a e)(\%)$} & 60,4 & \multicolumn{3}{|c|}{ taux marginal $t^{\prime *}=1 /(1+a e)(\%)$} & 83, \\
\hline
\end{tabular}

Source: Calculs effectués à partir des résultats du tableau D-8 (cf. Annexe D.2). Lecture: cf. tableau 4-4. 
diminuée de moitié entre 1983 et 1985 pour les foyers ayant 1 part de quotient familial (cf. tableau 4-4), alors qu'elle est restée égale à environ 6\% jusqu'en 1985 pour les foyers ayant 1,5 parts de QF (cf. tableau 4-5), différence qui va dans le sens contraire à celle prédite par les variations de taux marginaux. Puisque les revenus de P99-99,5 ont progressé relativement à ceux de P99,5-100 malgré une hausse supérieure de leur taux marginal d'imposition, les élasticités estimées à partir de cet épisode sont négatives, et les taux $t^{*}$ et $t^{* *}$ correspondant sont donc égaux à $100 \%$ (cf. tableau 4-5).

De même, le tableau 4-6 indique que bien que les taux de rétention des foyers ayant 2B parts de quotient familial aient progressé d'environ $28 \%$ pour P99-99,5 comme pour $\mathrm{P} 99,5-100,{ }^{74}$ le ratio $\mathrm{P} 99,5-100 / \mathrm{P} 99-99,5$ a baissé d'environ $7-8 \%$, c'est-à-dire dans des proportions légèrement supérieures à celles observées pour les foyers ayant 1 part de quotient familial. Ces résultats montrent clairement que la baisse de $6 \%$ observée en 1981-1983 du ratio P99,5-100/P99-99,5 pour les foyers ayant 1 part de quotient familial n'a rien à voir avec le fait que seuls les foyers de P99,5-100 ont connu une hausse de leur taux marginal effectif d'imposition: on observe une baisse d'une ampleur similaire (voire légèrement supérieure) du ratio P99,5-100/P99-99,5 pour les foyers fiscaux ayant 1,5 et 2B parts de quotient familial, bien que pour ces foyers le taux marginal ait encore plus augmenté pour P99-99,5 que pour P99,5-100, du fait du mécanisme de plafonnement du quotient familial. Ces résultats n'impliquent pas nécessairement que la chute des hauts revenus de 19821983 ne soit pas due (en partie) aux phénomènes de reports volontaires et temporaires décrits dans la section 4.1: il est possible que les effets du plafonnement du quotient familial n'aient pas été immédiatement perçus correctement par les contribuables, et que tous les hauts revenus aient effectués de tels reports dans les mêmes proportions, indépendamment des hausses de taux marginaux qui les concernaient véritablement. ${ }^{75}$

4.2.2. La suppression de la tranche à $65 \%$ et l'abaissement du seuil de plafonnement du QF pour les foyers ayant 2B parts de quotient familial (1986-1987)

La réforme du barème de 1986-1987 conduit à des baisses de taux marginaux pour les foyers ayant 1,5 ou $2 \mathrm{~B}$ parts de quotient familial similaires à celles connues par les foyers ayant 1 part de quotient familial : les foyers de P99,5-100 voient leur taux marginal passer subitement de $65 \%$ à $56,8 \%$, alors que les autres foyers (et en particulier ceux de P99-99,5) ne connaissent que des baisses très faibles de leur

\footnotetext{
${ }^{74}$ Le taux marginal moyen des foyers de P99-99,5 est passé de 40\% en 1980 (tous ces foyers étaient dans la tranche à $40 \%$ ) à $57 \%$ à partir de 1981 (environ $60 \%$ se retrouvent dans la tranche à $55 \%$ et $40 \%$ dans la tranche à $60 \%$ ). Le taux marginal moyen des ménages de P99,5-100 est passé de $51,5 \%$ en 1980 ( $40 \%$ dans la tranche à $45 \%, 20 \%$ dans la tranche à $50 \%, 10 \%$ dans la tranche à $55 \%$ et $30 \%$ dans la tranche à $60 \%$ ) à $65 \%$ à partir de 1982 .

${ }^{75}$ Là encore, cette hypothèse de méconnaissance de la législation par les contribuables aisés nous semble relativement peu plausible : les débats sur la mise en place du plafonnement des effets du quotient familial ont été abondamment diffusés à l'époque, et quelques années devraient être suffisantes pour que les contribuables réalisent le nouveau sort qui leur est fait.
} 
taux marginal (cf. tableaux 4-5 et 4-6).$^{76}$ De la même façon que pour les foyers ayant 1 part de quotient familial, la hausse du ratio P99,5-100/P99-99,5 observée entre 1985 et 1989 s'annule entièrement entre 1989 et 1993. Pour les foyers ayant 2B parts de quotient familial, le ratio P99,5-100/P99-99,5 augmente de 6,5\% entre 1985 et 1989, mais la progression n'est plus que de 2,0\% si l'on compare les années 1985 et 1995, ce qui correspond à une élasticité inférieure à 0,1 et des taux marginaux optimaux de l'ordre de $80-90 \%$ (cf. tableau 4-6). ${ }^{77}$ L'élasticité estimée prend même une valeur négative pour les foyers fiscaux ayant 1,5 parts de quotient familial : le ratio P99,5-100/P99-99,5 progresse de seulement $1,6 \%$ entre 1985 et 1989 , et baisse de $5,3 \%$ entre 1985 et 1995 (cf. tableau 4-5). ${ }^{78}$ Ces résultats confirment donc la très faible élasticité estimée à partir des foyers ayant 1 part de quotient familial.

Une autre variation importante induite par l'alternance de 1986 est l'abaissement du seuil de plafonnement du quotient familial pour les foyers fiscaux ayant 2B parts de quotient familial, alors que les seuils de plafonnement pour tous les autres foyers fiscaux continuaient d'être relevés dans les mêmes proportions que l'inflation (cf. section 2.1.3 et annexe I). Alors que le seuil de plafonnement mis en place en 1981 concernait à peine $1 \%$ des foyers fiscaux ayant $2 \mathrm{~B}$ parts de $\mathrm{QF}$, le nouveau seuil applicable à compter des revenus de 1986 en concerne près de $5 \%$. La conséquence est que les foyers de P95-98 et P98-99 ont connu une forte augmentation de leur taux marginal d'imposition en 1986, alors que ceux de P9999,5 et P99,5-100 bénéficiaient des baisses des taux marginaux du barème. Le tableau 4-7 décrit quelles ont été les variations de taux marginal et de revenus enregistrés pour ces différents types de contribuables entre 1985-1995, en les comparant aux évolutions enregistrées pour les foyers fiscaux ayant 1 part ou 1,5 parts de quotient familial.

Insérer Tableau 4-7 : Comparaison entre les foyers de célibataires, 1985-1995

Pour les foyers ayant 1 part ou 1,5 parts de quotient familial, les taux marginaux ont légèrement baissé pour P95-98 comme pour P98-99. ${ }^{79} \mathrm{Par}$ contre, pour les foyers ayant 2B parts de quotient familial, le nouveau seuil de plafonnement du QF a fait passer les foyers de P95-98 de la tranche à $35 \%$ dans la tranche à $43,2 \%$ et les

\footnotetext{
${ }^{76}$ En 1989, pour ce qui des foyers ayant 1,5 parts de QF, environ $20 \%$ des foyers de P99-99,5 sont dans la tranche à $53,9 \%$ et $80 \%$ dans celle à $56,8 \%$; pour ce qui est foyers ayant $2 \mathrm{~B}$ parts de QF, tous les foyers de $\mathrm{P} 99-99,5$ sont dans la tranche à $56,8 \%$.

${ }^{77}$ La distribution du revenu imposable des foyers ayant 1,5 ou 2B parts de QF a été perturbée en 1995 par les nouvelles règles sur l'attribution d'une part complète pour le $1^{\text {er }}$ enfant à charge (cf. section 2.1.3). Mais les résultats détaillés de nos estimations pour les foyers ayant 1,5 ou $2 \mathrm{~B}$ parts de QF (cf. Annexe D.2, tableaux D-6 et D-8) montrent que les estimations des tableaux 4-5 à 4-7 ne seraient modifiées que de façon marginale si l'on utilisait l'année 1994 au lieu de l'année 1995.

${ }^{78}$ Cette particularité des foyers ayant 1,5 parts de quotient familial s'explique sans doute par le fait que ces derniers connaissent sur l'ensemble de la période 1970-1996 un trend de diminution de la concentration des très hauts revenus (cf. Annexe D.2, tableau D-6).

${ }^{79}$ Les foyers de P95-98 sont passés de la tranche à $40 \%$ à la tranche à $38,4 \%$, alors que ceux de P98-99 passaient de la tranche à $45 \%$ à celle à $43,2 \%$ (foyers ayant 1 part de QF) ou de celle à $50 \%$ à celle à $49 \%$ (foyers ayant 1,5 parts de QF).
} 
Tableau 4-7: Comparaison entre les foyers de célibataires, 1985-1995

\begin{tabular}{|c|c|c|c|c|c|}
\hline$Q F=1$ & P95-98 & P98-99 & P99-99,5 & P99,5-100 & P99,5-100/P95-98 \\
\hline$t^{\prime}(1985)$ & 40 & 45 & 52 & 63 & 0,62 \\
\hline$t^{\prime}(1989)$ & 38,4 & 43,2 & 51 & 56,8 & 0,70 \\
\hline $\mathrm{dt}^{\prime} /\left(1-\mathrm{t}^{\prime}\right)$ & 2,7 & 3,3 & 2,2 & 16,8 & 13,7 \\
\hline dy/y (85-89) & 3,1 & 5,1 & 6,0 & 16,1 & 12,6 \\
\hline dy/y (85-95) & 6,8 & 5,8 & 4,5 & 5,2 & $-1,5$ \\
\hline$Q F=1,5$ & P95-98 & P98-99 & P99-99,5 & P99,5-100 & P99,5-100/P95-98 \\
\hline$t^{\prime}(1985)$ & 40 & 50 & 57 & 65 & 0,58 \\
\hline$t^{\prime}(1989)$ & 38,4 & 49 & 56,8 & 56,8 & 0,70 \\
\hline $\mathrm{dt}^{\prime} /\left(1-\mathrm{tt}^{\prime}\right)$ & 2,7 & 2,0 & 0,5 & 23,4 & 20,2 \\
\hline dy/y (85-89) & $-2,0$ & 0,2 & 5,2 & 6,9 & 9,1 \\
\hline dy/y (85-95) & $-6,6$ & $-6,6$ & $-4,5$ & $-9,6$ & $-3,2$ \\
\hline$Q F=2 B$ & P95-98 & P98-99 & P99-99,5 & P99,5-100 & P99,5-100/P95-98 \\
\hline$t^{\prime}(1985)$ & 35 & 40 & 57 & 65 & 0,54 \\
\hline$t^{\prime}(1989)$ & 43,2 & 51,0 & 56,8 & 56,8 & 0,76 \\
\hline $\mathrm{dt}^{\prime} /\left(1-\mathrm{tt}^{\prime}\right)$ & $-12,6$ & $-18,3$ & 0,5 & 23,4 & 41,2 \\
\hline dy/y $(85$ & 1.2 & 2.0 & 4.2 & 11.0 & 9.7 \\
\hline dy/y (85-95) & 5,2 & 3,3 & 3,2 & 5,3 & 0,0 \\
\hline
\end{tabular}

Source: Calculs effectués à partir des résultats des tableaux 4-2, D-6 et D-8 (cf. Annexe D.2).

Lecture: Pour les foyers fiscaux ayant 1 part de quotient familial, le taux marginal moyen des foyers de P95-98 est passé de $40 \%$ en 1985 à $38,4 \%$ en 1989 , soit une hausse de $2,7 \%$ de leur taux de rétention; leur part dans le revenu imposable a progressé de 3,1\% entre 1985 et 1989, et de 6,8\% entre 1985 et 1995 . 
foyers de P98-99 de la tranche à $40 \%$ aux tranches à $50 \%$ et $53,9 \%{ }^{80} \mathrm{La}$ conséquence est que le ratio entre les taux de rétention de P99,5-100 et P95-98 a subitement progressé de plus de $40 \%$ pour les foyers fiscaux ayant $2 \mathrm{~B}$ parts de quotient familial (et d'avantage encore si l'on considérait le ratio P99,5-100/P98-99), alors que ce taux de progression n'était que d'environ $20 \%$ pour les foyers ayant 1,5 parts de quotient familial et de moins de $14 \%$ pour les foyers ayant 1 part de quotient familial. Si les taux marginaux avaient un impact sensible sur les comportements, on devrait donc s'attendre à ce qu'ils se manifestent ici, sous la forme d'une baisse du revenu relatif des revenus de P95-98 et P98-99 pour les foyers ayant 2B de quotient familial. Or, le tableau 4-7 indique que le ratio P99,5-100/P95-98 (tout comme le ratio P99,5-100/P98-99) a évolué exactement de la même façon pour tous les foyers de célibataires : quel que soit le nombre de parts de QF (1, 1,5 ou 2B), le ratio P99,5100/P95-98 a progressé d'environ 10\% entre 1985 et 1989, avant de retrouver en 1995 un niveau voisin (ou légèrement inférieur) de son niveau de 1985 (cf. tableau 47). Ce très haut degré de similarité entre les évolutions observées pour les différents types de foyers de célibataires est extrêmement frappant, compte tenu de l'ampleur des variations de taux marginaux induites par le nouveau seuil de plafonnement du QF pour les foyers ayant 2B parts de QF. Les élasticités impliqués par ces variations ne sont pas mentionnées sur le tableau 4-7, mais il va de soi qu'elles sont extrêmement faibles, voire négatives. Là encore, ces résultats suggèrent que les variations des taux marginaux effectifs d'imposition n'ont aucun effet significatif sur les comportements des contribuables, et que les effets "mécaniques" du cycle économique expliquent l'essentiel des fluctuations observées (à l'exception peut-être de phénomènes purement temporaires de reports de revenus, d'une ampleur relativement limitée).

\subsection{Estimations effectuées à partir des foyers de couples mariés ( $Q F=2 A, 2,5$ et 3 )}

4.3.1. La création de la tranche à $65 \%$ et la mise en place du plafonnement des effets du quotient familial (1981-1982)

Le plafonnement du quotient familial mis en place en 1981 conduit au mêmes variations de taux marginaux pour les foyers fiscaux de couples mariés que pour les foyers fiscaux de célibataires: alors que seuls les couples mariés sans enfant à charge concernés par la tranche à $65 \%$ connaissent une hausse de leur taux marginal d'imposition, les hausses de taux marginaux concernent une proportion sensiblement plus élevée des couples mariés ayant plus de 2 parts de quotient familial. Les tableaux 4-8, 4-9 et 4-10 montrent que les réactions des uns et des autres ont été sensiblement les mêmes pour les couples mariés que pour les célibataires, et ces résultats conduisent donc à confirmer des estimations

\footnotetext{
${ }^{80}$ En 1989 , environ $60 \%$ des foyers de P98-99 se retrouvent dans la tranche à $49 \%$, et $40 \%$ dans la tranche à $53,9 \%$.
} 
extrêmement faibles (voire négatives) de l'élasticité du revenu imposable vis-à-vis des taux marginaux effectifs d'imposition.

Insérer Tableau 4-8: Estimation de l'élasticité du revenu imposable à partir des foyers fiscaux ayant $2 \mathrm{~A}$ parts de quotient familial.

Insérer Tableau 4-9: Estimation de l'élasticité du revenu imposable à partir des foyers fiscaux ayant 2,5 parts de quotient familial.

Insérer Tableau 4-10: Estimation de l'élasticité du revenu imposable à partir des foyers fiscaux ayant 3 parts de quotient familial

Environ $0,5 \%$ des foyers ayant $2 \mathrm{~A}$ parts de quotient familial ont vu leur taux marginal passer de $60 \%$ à $65 \%$ en 1982 , alors que les foyers de P99-99,5 conservaient un taux marginal moyen de $57 \%$ (environ $60 \%$ se trouvaient dans la tranche à $55 \%$, et $40 \%$ dans la tranche à $60 \%$ ). Le ratio P99,5-100/P99-99,5 a diminué de $4,3 \%$ entre 1980 et 1983 , et de $3,3 \%$ entre 1980 et 1985 , soit une élasticité de 0,27 (cf. tableau 4-8). Pour ce qui est des foyers ayant 2,5 parts de quotient familial, les foyers de P99-99,5 ont connu une hausse de leur taux marginal moyen (de $50 \%$ à $57 \%$ ) supérieure à celle des foyers de P99,5-100 (de 60\% à $65 \%$ ). ${ }^{81}$ Or leur ratio $\mathrm{P} 99,5-$ 100/P99-99,5 a diminué de 5,0\% entre 1980 à 1983, et de $3,0 \%$ entre 1983 et 1985 , ce qui correspond à des élasticités négatives (cf. tableau 4-9). Cela montre clairement que la baisse de 3,3\% de P99,5-100/P99-99,5 pour les foyers ayant $2 \mathrm{~A}$ parts de quotient familial n'a rien à voir avec le fait que seuls les foyers de P99,5-100 ont connu une hausse de leur taux marginal, et donc que l'élasticité de 0,27 est très fortement surévaluée (de même que l'était l'élasticité de 0,26 estimée pour les foyers ayant 1 part de quotient familial). De même, pour ce qui est de foyers ayant 3 parts de quotient familial, bien que les foyers de P99-99,5 ont connu une hausse de leur taux marginal moyen (de $51 \%$ à $63 \%$ ) supérieure à celle des foyers de P99,5-100 (de $60 \%$ à $65 \%),{ }^{82}$ on observe que le ratio P99,5-100/P99-99,5 a baissé de $5,3 \%$ entre 1980 et 1983 et de 5,6\% entre 1980 et 1985 (cf. tableau 4-10). Ces résultats obtenus pour les couples mariés sont donc exactement les mêmes que ceux obtenus pour les célibataires, et ils montrent clairement que la chute des hauts revenus observée en 1982-1983 n'a pas été causée directement par les variations des taux marginaux effectifs.

\subsubsection{La suppression de la tranche à $65 \%$ (1986-1987)}

De la même façon que pour les foyers fiscaux de célibataires, la réforme du barème de 1986-1987 a conduit à des baisses sensibles de taux marginaux uniquement pour les couples mariés qui étaient concernés par la suppression de la

\footnotetext{
${ }^{81}$ En 1980 , environ $20 \%$ des foyers de P99-99,5 sont dans la tranche à $45 \%, 60 \%$ sont dans la tranche à $50 \%$, et $20 \%$ sont dans la tranche à $55 \%$. En 1983 , environ $60 \%$ sont la tranche à $55 \%$ et $40 \%$ sont dans la tranche à $60 \%$.

${ }^{82}$ En 1980 , environ $80 \%$ des foyers de P99-99,5 sont la tranche à $50 \%$ et $20 \%$ dans celle à $55 \%$. En $1983,40 \%$ sont dans la tranche à $60 \%$ et $60 \%$ dans celle à $65 \%$.
} 
Tableau 4-8: Estimation de l'élasticité du revenu imposable à partir des foyers fiscaux ayant $2 \mathrm{~A}$ parts de quotient familial

\section{(a) 1980-1985}

\begin{tabular}{|c|c|c|c|c|c|c|c|}
\hline & P99-99,5 & P99,5-100 & ratio & & P99-99,5 & P99,5-100 & ratio \\
\hline y (1980) & 2,63 & 5,75 & 2,19 & y (1980) & 2,63 & 5,75 & 2,19 \\
\hline y (1983) & 2,45 & 5,13 & 2,09 & y (1985) & 2,52 & 5,32 & 2,11 \\
\hline dy/y (\%) & $-6,8$ & $-10,8$ & $-4,3$ & dy/y (\%) & $-4,3$ & $-7,5$ & $-3,3$ \\
\hline$t^{\prime}(1980)$ & 57 & 60 & 0,93 & $t^{\prime}(1980)$ & 57 & 60 & 0,9 \\
\hline t' (1983) & 57 & 65 & 0,81 & $t^{\prime}(1985)$ & 57 & 65 & 0,8 \\
\hline $\mathrm{dt}^{\prime} /\left(1-\mathrm{t}^{\prime}\right)(\%)$ & 0,0 & $-12,5$ & $-12,5$ & $\mathrm{dt}^{\prime} /\left(1-\mathrm{t}^{\prime}\right)(\%)$ & 0,0 & $-12,5$ & \\
\hline \multicolumn{3}{|l|}{ élasticité e } & 0,34 & \multicolumn{3}{|l|}{ élasticité e } & \\
\hline \multirow{2}{*}{\multicolumn{3}{|c|}{ taux marginal $t^{*}=1 /(1+e)(\%)$}} & 74,5 & \multicolumn{3}{|c|}{ taux marginal $t^{*}=1 /(1+e)(\%)$} & \\
\hline & & & 56,0 & \multicolumn{3}{|c|}{ taux marginal $t^{\prime * *}=1 /(1+a e)(\%)$} & \\
\hline
\end{tabular}

\section{(b) 1985-1995}

\begin{tabular}{|c|c|c|c|c|c|c|c|}
\hline & P99-99,5 & P99,5-100 & ratio & & P99-99,5 & P99,5-100 & ratio \\
\hline у (1985) & 2,52 & 5,32 & 2,11 & у (1985) & 2,52 & 5,32 & 2,11 \\
\hline y (1989) & 2,66 & 6,24 & 2,35 & y (1995) & 2,42 & 5,40 & 2,23 \\
\hline dy/y (\%) & 5,6 & 17,3 & 11,1 & dy/y (\%) & $-3,7$ & 1,5 & 5,4 \\
\hline$t^{\prime}(1985)$ & 57 & 65 & 0,81 & $t^{\prime}(1985)$ & 57 & 65 & 0,8 \\
\hline$t^{\prime}(1989)$ & 56,2 & 56,8 & 0,99 & $t^{\prime}(1995)$ & 56,2 & 56,8 & 0,99 \\
\hline $\mathrm{dt}^{\prime} /\left(1-\mathrm{t}^{\prime}\right)(\%)$ & 1,8 & 23,4 & 21,2 & $\mathrm{dt}^{\prime} /\left(1-\mathrm{t}^{\prime}\right)(\%)$ & 1,8 & 23,4 & 21,2 \\
\hline \multicolumn{3}{|l|}{ élasticité e } & 0,52 & \multicolumn{3}{|l|}{ élasticité e } & 0,26 \\
\hline \multirow{2}{*}{\multicolumn{3}{|c|}{ taux marginal $t^{*}=1 /(1+e)(\%)$}} & 65,6 & \multicolumn{3}{|c|}{ taux marginal $t^{*}=1 /(1+e)(\%)$} & 79 , \\
\hline & & & 45,3 & \multicolumn{3}{|c|}{ taux marginal $t^{\prime *}=1 /(1+a e)(\%)$} & $63,($ \\
\hline
\end{tabular}

Source: Calculs effectués à partir des résultats du tableau D-10 (cf. Annexe D.2). Lecture: cf. tableau 4-4. 
Tableau 4-9: Estimation de l'élasticité du revenu imposable à partir des foyers fiscaux ayant 2,5 parts de quotient familial

\section{(a) 1980-1985}

\begin{tabular}{|c|c|c|c|c|c|c|c|}
\hline & P99-99,5 & P99,5-100 & ratio & & P99-99,5 & P99,5-100 & ratio \\
\hline y (1980) & 2,34 & 4,64 & 1,98 & y (1980) & 2,34 & 4,64 & 1,98 \\
\hline y (1983) & 2,21 & 4,16 & 1,88 & y (1985) & 2,24 & 4,27 & 1,91 \\
\hline dy/y (\%) & $-5,6$ & $-10,3$ & $-5,0$ & dy/y (\%) & $-4,3$ & $-8,1$ & $-4,0$ \\
\hline$t^{\prime}(1980)$ & 50 & 60 & 0,80 & $t^{\prime}(1980)$ & 50 & 60 & 0,8 \\
\hline$t^{\prime}(1983)$ & 57 & 65 & 0,81 & $t^{\prime}(1985)$ & 57 & 65 & 0,81 \\
\hline $\mathrm{dt}^{\prime} /\left(1-\mathrm{t}^{\prime}\right)(\%)$ & $-14,0$ & $-12,5$ & 1,7 & $\mathrm{dt}^{\prime} /\left(1-\mathrm{t}^{\prime}\right)(\%)$ & $-14,0$ & $-12,5$ & 1,7 \\
\hline \multicolumn{3}{|c|}{ élasticité e } & $-2,86$ & \multicolumn{3}{|l|}{ élasticité e } & $-2,2$ \\
\hline \multirow{2}{*}{\multicolumn{3}{|c|}{ taux marginal $t^{*}=1 /(1+e)(\%)$}} & 100,0 & \multicolumn{3}{|c|}{ taux marginal $t^{*}=1 /(1+e)(\%)$} & 100 \\
\hline & & & 100,0 & \multicolumn{3}{|c|}{ taux marginal $t^{\prime *}=1 /(1+a e)(\%)$} & 100 \\
\hline
\end{tabular}

\section{(b) 1985-1995}

\begin{tabular}{|c|c|c|c|c|c|c|c|}
\hline & P99-99,5 & P99,5-100 & ratio & & P99-99,5 & P99,5-100 & ratio \\
\hline у (1985) & 2,24 & 4,27 & 1,91 & у (1985) & 2,24 & 4,27 & 1,91 \\
\hline y (1989) & 2,46 & 5,26 & 2,14 & y (1995) & 2,32 & 4,71 & 2,03 \\
\hline dy/y (\%) & 9,7 & 23,4 & 12,5 & dy/y (\%) & 3,6 & 10,5 & 6,6 \\
\hline$t^{\prime}(1985)$ & 57 & 65 & 0,81 & $t^{\prime}(1985)$ & 57 & 65 & 0,8 \\
\hline$t^{\prime}(1989)$ & 55,6 & 56,8 & 0,97 & $t^{\prime}(1995)$ & 55,6 & 56,8 & 0,9 \\
\hline $\mathrm{dt}^{\prime} /\left(1-\mathrm{t}^{\prime}\right)(\%)$ & 3,2 & 23,4 & 19,6 & $\mathrm{dt}^{\prime} /\left(1-\mathrm{t}^{\prime}\right)(\%)$ & 3,2 & 23,4 & 19 \\
\hline \multicolumn{3}{|l|}{ élasticité e } & 0,64 & \multicolumn{3}{|c|}{ élasticité e } & 0,3 \\
\hline \multicolumn{3}{|c|}{ taux marginal $t^{*}=1 /(1+e)(\%)$} & 61,1 & \multicolumn{3}{|c|}{ taux marginal $t^{*}=1 /(1+e)(\%)$} & 74 \\
\hline \multicolumn{3}{|c|}{ taux marginal $\mathrm{t}^{\prime * *}=1 /(1+\mathrm{ae})(\%)$} & 40,6 & \multicolumn{3}{|c|}{ taux marginal $t^{\prime *}=1 /(1+a e)(\%)$} & 56, \\
\hline
\end{tabular}

Source: Calculs effectués à partir des résultats du tableau D-12 (cf. Annexe D.2). Lecture: cf. tableau 4-4. 
Tableau 4-10: Estimation de l'élasticité du revenu imposable à partir des foyers fiscaux ayant 3 parts de quotient familial

\section{(a) $1980-1985$}

\begin{tabular}{|c|c|c|c|c|c|c|c|}
\hline & P99-99,5 & P99,5-100 & ratio & & P99-99,5 & P99,5-100 & ratio \\
\hline y (1980) & 2,49 & 4,75 & 1,90 & y (1980) & 2,49 & 4,75 & 1,90 \\
\hline y (1983) & 2,30 & 4,14 & 1,80 & y (1985) & 2,34 & 4,20 & 1,80 \\
\hline dy/y (\%) & $-7,8$ & $-12,7$ & $-5,3$ & dy/y (\%) & $-6,2$ & $-11,5$ & $-5,6$ \\
\hline$t^{\prime}(1980)$ & 51 & 60 & 0,82 & t' (1980) & 51 & 60 & 0,8 \\
\hline$t^{\prime}(1983)$ & 63 & 65 & 0,95 & $t^{\prime}(1985)$ & 63 & 65 & 0,95 \\
\hline $\mathrm{dt}^{\prime} /\left(1-\mathrm{t}^{\prime}\right)(\%)$ & $-24,5$ & $-12,5$ & 15,9 & $\mathrm{dt}^{\prime} /\left(1-\mathrm{t}^{\prime}\right)(\%)$ & $-24,5$ & $-12,5$ & 15,9 \\
\hline \multicolumn{3}{|l|}{ élastic } & $-0,33$ & \multicolumn{3}{|l|}{ élasticité } & $-0,3$ \\
\hline \multicolumn{3}{|c|}{ taux marginal $t^{*}=1 /(1+e)(\%)$} & 100,0 & \multicolumn{3}{|c|}{ taux marginal $t^{*}=1 /(1+e)(\%)$} & 00 \\
\hline \multicolumn{3}{|c|}{ taux marginal $\mathrm{t}^{\prime *}=1 /(1+\mathrm{ae})(\%)$} & 100,0 & \multicolumn{3}{|c|}{ taux marginal $t^{\prime *}=1 /(1+a e)(\%)$} & \\
\hline
\end{tabular}

\section{(b) 1985-1995}

\begin{tabular}{|c|c|c|c|c|c|c|c|}
\hline & P99-99,5 & P99,5-100 & ratio & & P99-99,5 & P99,5-100 & ratio \\
\hline y (1985) & 2,34 & 4,20 & 1,80 & y (1985) & 2,34 & 4,20 & 1,80 \\
\hline y (1989) & 2,55 & 5,19 & 2,04 & y (1995) & 2,47 & 4,82 & 1,96 \\
\hline dy/y (\%) & 9,1 & 23,6 & 13,3 & dy/y (\%) & 5,5 & 14,8 & 8,8 \\
\hline$t^{\prime}(1985)$ & 63 & 65 & 0,95 & $t^{\prime}(1985)$ & 63 & 65 & \\
\hline$t^{\prime}(1989)$ & 56,8 & 56,8 & 1,00 & $t^{\prime}(1995)$ & 56,8 & 56,8 & \\
\hline $\mathrm{dt}^{\prime} /\left(1-\mathrm{t}^{\prime}\right)(\%)$ & 16,8 & 23,4 & 5,7 & $\mathrm{dt}^{\prime} /\left(1-\mathrm{t}^{\prime}\right)(\%)$ & 16,8 & 23,4 & 5, \\
\hline \multicolumn{3}{|c|}{ élasticité e } & 2,32 & \multicolumn{3}{|c|}{ élasticité e } & \\
\hline \multicolumn{3}{|c|}{ taux marginal $t^{*}=1 /(1+e)(\%)$} & 30,1 & \multicolumn{3}{|c|}{ taux marginal $t^{*}=1 /(1+e)(\%)$} & \\
\hline \multicolumn{3}{|c|}{ taux marginal $t^{\prime *}=1 /(1+a e)(\%)$} & 15,8 & \multicolumn{3}{|c|}{ taux marginal $\mathrm{t}^{\prime *}=1 /(1+\mathrm{ae})(\%)$} & \\
\hline
\end{tabular}

Source: Calculs effectués à partir des résultats du tableau D-14 (cf. Annexe D.2). Lecture: cf. tableau 4-4. 
tranche à $65 \%$. Pour ce qui est foyers ayant $2 \mathrm{~A}$ parts de quotient familial, les foyers de P99,5-100 ont vu leur taux marginal passer de $65 \%$ à $56,8 \%$, alors que le taux marginal moyen des foyers de P99-99,5 passe de $57 \%$ à $56,2 \%{ }^{83}$ Mais contrairement à ce que nous avions observé pour l'ensemble des foyers de célibataires, la hausse du ratio P99,5-100/P99-99,5 enregistrée entre 1985 et 1989 $(11,1 \%)$ ne s'annule pas entièrement pendant la récession de 1991-1993: entre 1985-1995, le ratio P99,5-100/P99-99,5 augmente de 5,4\% pour les foyers ayant $2 \mathrm{~A}$ parts de quotient familial, ce qui correspond à une élasticité $e=0,26$ et un taux marginal t'* maximisant les recettes fiscales de 63,0\% (cf. tableau 4-8). Cette élasticité reste extrêmement modeste, puisqu'elle implique en particulier que l'abaissement de $65 \%$ à $56,8 \%$ du taux marginal supérieur s'est traduit par une perte nette de recettes, mais elle est significativement plus élevés que les élasticités obtenues pour les foyers de célibataires. On observe le même phénomène pour les foyers fiscaux ayant 2,5 parts de quotient familial, dont le ratio P99,5-100/P99-99,5 a progressé de 6,6\% entre 1985 et 1995 (cf. tableau 4-9), ainsi que pour les foyers fiscaux ayant 3 parts de quotient familial, dont le ratio P99,5-100/P99-99,5 a progressé de 8,8\% entre 1985 et 1995 (cf. tableau 4-10). En l'absence d'une autre explication évidente pour un tel trend concernant l'ensemble des couples mariés, on pourrait donc attribuer cette hausse structurelle du ratio P99,5-100/P99-99,5 au fait que les foyers de P99,5-100 ont connu une hausse importante (près de $25 \%$ ) de leur taux de rétention. Le fait que l'élasticité du revenu imposable des couples soit plus élevée que l'élasticité du revenu imposable des célibataires $(0,2-0,3$ contre $0,0-0,1)$ serait d'ailleurs cohérent avec toutes les études économétriques traditionnelles sur l'élasticité de l'offre de travail : un des résultats les plus robustes de cette littérature est en effet que les comportements de participation au marché du travail des femmes mariées, de passage du temps partiel au temps plein, etc..., sont relativement élastiques vis-à-vis des incitations financières et des taux marginaux d'imposition. ${ }^{84}$

Cependant, le fait que le ratio P99,5-100/P99-99,5 augmente d'avantage pour les foyers fiscaux ayant 3 parts de quotient familial que pour les foyers ayant $2 \mathrm{~A}$ ou 2,5 parts de quotient familial invite à douter de cette interprétation. En effet, si environ $0,5 \%$ des foyers fiscaux ayant $2 \mathrm{~A}$ ou 2,5 parts de QF étaient concernés par la suppression de la tranche à $65 \%$, près de $0,8 \%$ des foyers ayant 3 parts de quotient familial étaient dans cette situation. Autrement dit, pour ce qui est des foyers ayant 3 parts de quotient familial, les foyers de P99-99,5 ont connu une baisse de taux marginal presque aussi importante que celle des foyers de P99,5-100. Cela se traduit sur le tableau 4-10 par une élasticité extrêmement élevée $(e=1,54)$, puisque l'on attribue une forte hausse du ratio P99,5-100/P99-99,5 à une très faible hausse du ratio des taux de rétention, mais cette estimation est totalement artificielle : ces résultats suggèrent surtout que la hausse du ratio $\mathrm{P99}, 5-100 / P 99-99,5$ observée pour l'ensemble des couples mariées n'a pas grand chose à voir avec les variations

\footnotetext{
${ }^{83}$ En 1989 , environ $20 \%$ des foyers de P99-99,5 sont dans la tranche à $53,9 \%$ et $80 \%$ dans celle à $56,8 \%$.

${ }^{84} \mathrm{Cf}$. par exemple les références données en introduction (section 1.2).
} 
de taux marginaux d'imposition. Afin de préciser cette intuition, le tableau 4-11 compare l'évolution des ratios P99,5-100/P99,2-99,5 pour les différents types de couples mariés sur la période 1985-1995.

Insérer Tableau 4-11 : Comparaison entre les foyers de couples mariés, 1985-1995

Pour ce qui est des foyers ayant 3 parts de quotient familial, les foyers de P99,2-99,5 et de P99,5-100 ont connu la même baisse de taux marginal : ils étaient tous dans la tranche à $65 \%$, et ils sont tous passés dans la tranche à $56,8 \%$. On ne peut donc pas attribuer une éventuelle hausse du ratio P99,5-100/P99,2-99,5 à des changements de comportements induits par des variations différentes de taux marginaux. Par contre, pour ce qui est des foyers ayant $2 \mathrm{~A}$ ou 2,5 parts de quotient familial, seul le taux de rétention des foyers de $\mathrm{P} 99,5-100$ a progressé de près de $25 \%$, alors que celui des foyers de P99-99,5 progressait de façon insignifiante. Si les fluctuations du ratio P99,5-100/P99,2-99,5 étaient dues (au moins en partie) aux variations des taux marginaux, on devrait donc s'attendre à que ce ratio ait progressé de façon sensiblement moins importante pour les foyers ayant 3 parts de quotient familial que pour les autres foyers de couples mariés. Or, le tableau 4-11 indique que le ratio P99-99,5/P99,2-99,5 a progressé de 5,1\% entre 1985 et 1995 pour les foyers ayant $2 \mathrm{~A}$ parts de $\mathrm{QF}$, de $6,2 \%$ pour les foyers ayant 2,5 parts de quotient familial, et de $8,2 \%$ pour les foyers ayant 3 parts de quotient familial. II semblerait donc que les fluctuations observées à l'intérieur du centile supérieur de la distribution de l'ensemble des couples mariés soient dues à des causes économiques communes (cycle économique, trend structurel,...) et non pas à des variations de taux marginaux.

Quoi qu'il en soit, les élasticités maximales que l'on peut raisonnablement inférer de cet épisode (0,2-0,3, d'après les tableaux 4-8 et 4-9) restent relativement faibles : en supposant que des élasticités de 0,2-0,3 caractérisent les couples mariés et que des élasticités de 0,0-0,1 caractérisent les célibataires, on aboutirait à la conclusion que l'élasticité moyenne des hauts revenus est de l'ordre de 0,1-0,2, c'est-à-dire un niveau très sensiblement inférieur au niveau requis pour que la suppression de la tranche à $65 \%$ ne conduise pas à des pertes nettes de recettes fiscales. 
Tableau 4-11: Comparaison entre les foyers de couples mariés, 1985-1995

\begin{tabular}{|c|c|c|c|c|c|c|c|}
\hline$Q F=2 A$ & P99,2-99,5 & P99,5-100 & ratio & & P99,2-99,5 & P99,5-100 & ratio \\
\hline y (1985) & 1,60 & 5,32 & 3,33 & y (1985) & 1,60 & 5,32 & 3,33 \\
\hline y (1989) & 1,70 & 6,24 & 3,68 & y (1995) & 1,54 & 5,40 & 3,50 \\
\hline dy/y (\%) & 6,2 & 17,3 & 10,5 & dy/y (\%) & $-3,4$ & 1,5 & 5,1 \\
\hline$t^{\prime}(1985)$ & 58 & 65 & 0,83 & $t^{\prime}(1985)$ & 58 & 65 & 0,83 \\
\hline$t^{\prime}(1989)$ & 56,8 & 56,8 & 1,00 & $t^{\prime}(1995)$ & 56,8 & 56,8 & 1,00 \\
\hline $\mathrm{dt}^{\prime} /\left(1-\mathrm{t}^{\prime}\right)(\%)$ & 2,9 & 23,4 & 20,0 & $\mathrm{dt}^{\prime} /\left(1-\mathrm{t}^{\prime}\right)(\%)$ & 2,9 & 23,4 & 20,0 \\
\hline$Q F=2,5$ & P99,2-99,5 & P99,5-100 & ratio & & P99,2-99,5 & P99,5-100 & ratio \\
\hline y (1985) & 1,41 & 4,27 & 3,02 & y (1985) & 1,41 & 4,27 & 3,02 \\
\hline y (1989) & 1,56 & 5,26 & 3,37 & y (1995) & 1,47 & 4,71 & 3,21 \\
\hline dy/y (\%) & 10,5 & 23,4 & 11,7 & dy/y (\%) & 4,0 & 10,5 & 6,2 \\
\hline$t^{\prime}(1985)$ & 58 & 65 & 0,83 & $t^{\prime}(1985)$ & 58 & 65 & 0,83 \\
\hline t' (1989) & 56,8 & 56,8 & 1,00 & $t^{\prime}(1995)$ & 56,8 & 56,8 & 1,00 \\
\hline $\mathrm{dt}^{\prime} /\left(1-\mathrm{t}^{\prime}\right)(\%)$ & 2,9 & 23,4 & 20,0 & $\mathrm{dt}^{\prime} /\left(1-\mathrm{t}^{\prime}\right)(\%)$ & 2,9 & 23,4 & 20,0 \\
\hline$Q F=3$ & P99,2-99,5 & P99,5-100 & ratio & & P99,2-99,5 & P99,5-100 & ratio \\
\hline y (1985) & 1,47 & 4,20 & 2,86 & y (1985) & 1,47 & 4,20 & 2,86 \\
\hline y (1989) & 1,62 & 5,19 & 3,21 & y (1995) & 1,56 & 4,82 & 3,09 \\
\hline dy/y (\%) & 10,0 & 23,6 & 12,4 & dy/y (\%) & 6,1 & 14,8 & 8,2 \\
\hline$t^{\prime}(1985)$ & 65 & 65 & 1,00 & $t^{\prime}(1985)$ & 65 & 65 & 1,00 \\
\hline$t^{\prime}(1989)$ & 56,8 & 56,8 & 1,00 & $t^{\prime}(1995)$ & 56,8 & 56,8 & 1,00 \\
\hline $\mathrm{dt}^{\prime} /\left(1-\mathrm{t}^{\prime}\right)(\%)$ & 23,4 & 23,4 & 0,0 & $\mathrm{dt}^{\prime} /\left(1-\mathrm{t}^{\prime}\right)(\%)$ & 23,4 & 23,4 & 0,0 \\
\hline
\end{tabular}

Source: Calculs effectués à partir des résultats des tableaux D-10, D-12 et D-14 (cf. Annexe D.2) Lecture: Pour les foyers ayant $2 \mathrm{~A}$ parts de quotient familial, la part du revenu imposable détenue par les foyers de P99,2-99,5 est passée de 1,60\% en 1985 à $1,70 \%$ en 1989, soit une hausse de $6,2 \%$, alors que celle de $\mathrm{P} 99,5-100$ passait de $5,32 \%$ à $6,24 \%$, soit une hausse de $17,3 \%$; le ratio entre la part de P99,5-100 et celle de P99,2-100 est passé de 3,33 à 3,68, soit une hausse de $10,5 \%$; le taux marginal moyen des foyers de $\mathrm{P} 99,2-99,5$ est passé de $58 \%$ à $56,8 \%$, soit une hausse de $2,9 \%$ du taux de rétention, alors que celui des foyers de P99,5-100 passait de $65 \%$ à $56,8 \%$, soit une hausse de $23,4 \%$ du taux de rétention; le ratio entre les taux de rétention de P99,5-100 et $\mathrm{P} 99,2-99,5$ est passé de 0,83 à 1 , soit une hausse de $20,0 \%$. 


\section{Conclusions et perspectives en termes de politique économique}

En conclusion, il convient de rappeler la fragilité des estimations obtenues. Compte tenu des très fortes fluctuations de court-terme auxquelles sont toujours soumis les hauts revenus, il est extrêmement difficile de déterminer avec certitude l'ampleur des fluctuations additionnelles éventuellement dues aux variations de taux marginaux et d'incitations au travail (au sens large). Mais tous les résultats obtenus, pour 6 groupes de quotient familial différents, vont cependant dans la même direction : la répartition des hauts et des très hauts revenus est extrêmement stable dans le moyen-terme et dans le long-terme, les fluctuations de court-terme s'expliquent principalement par les effets mécaniques du cycle économique sur cette répartition, et les modifications de taux marginaux ne semblent pas avoir causé de fluctuations additionnelles importantes. La seule fluctuation additionnelle que les données utilisées permettent éventuellement de détecter semble prendre la forme de reports purement temporaires, en 1982 comme en 1987-1988. Mais le fait est que les hauts revenus ont retrouvé leur niveau de 1980-1981 dès 1985, c'est-à-dire avant la suppression de la tranche à $65 \%$. De même, les hauts revenus ont retrouvé dès 1993 leur niveau de 1985, et se sont depuis lors stabilisés à ce niveau. Les estimations obtenues suggèrent des élasticités maximales de l'ordre de 0,1-0,2, c'est-à-dire des niveaux sensiblement inférieurs au niveau requis pour qu'une baisse du taux marginal supérieur de l'IR puisse être auto-financée.

En outre, tout laisse à penser que les élasticités caractérisant les revenus moyens et moyens-élevés, c'est-à-dire les revenus qui représentent des masses budgétaires importantes, sont sensiblement inférieures aux élasticités caractérisant les hauts et les très hauts revenus : les revenus moyens et moyens-élevés ont beaucoup moins d'opportunités pour faire varier leur niveau de revenu imposable, et leur évolution est caractérisée par une insensibilité au cycle économique et une très grande stabilité que rien ne semble pouvoir affecter. ${ }^{85}$

II convient également de rappeler que toutes les élasticités ont été estimées "localement ", c'est-à-dire à partir de variations de taux marginaux se situant aux alentours de 55-65\%. II serait donc extrêmement hasardeux d'utiliser ces élasticités pour prédire comment évolueraient les bases d'imposition si les taux marginaux atteignaient soudainement des niveaux substantiellement plus élevés, par exemple si l'on passait à des taux de l'ordre de $80-90 \%$. II est fort possible que les élasticités deviennent sensiblement plus élevées à ce niveau de taux marginaux. ${ }^{86}$ Par contre, tout semble indiquer que pour des modifications " raisonnables " des taux marginaux, se situant aux alentours des taux actuellement appliqués, toute baisse des taux se traduise inévitablement par des pertes nettes de recettes fiscales au bénéfice des contribuables concernés.

\footnotetext{
${ }^{85}$ Cf. la stabilité de la part de P90-95 sur les tableaux 3-3 à 3-5 (section 3.2).

${ }^{86}$ En particulier, les taux marginaux $\mathrm{t}^{*}$ et $\mathrm{t}^{\prime *}$ données dans les tableaux 4-4 à 4-10, qui ont été calculés mécaniquement en supposant une élasticité linéaire s'appliquant de façon proportionnelle à toute variation de taux marginaux, et qui atteignent souvent des niveaux supérieurs à $90 \%$, ne sont donnés qu'à titre illustratif.
} 
En particulier, ces élasticités " locales" de l'ordre de 0,1-0,2 semblent relativement faibles comparées aux estimations disponibles concernant les populations ayant de faibles revenus d'activité, pour lesquelles il n'est pas rare d'estimer "localement " des élasticités de l'offre de travail voisines ou supérieures à $0,5 .^{87}$ De plus, et surtout, toutes les estimations disponibles suggèrent que l'élasticité de la demande de travail de la part des entreprises vis-à-vis du coût du travail, et notamment du coût du travail peu qualifié, est significativement plus élevée que les élasticités de l'ordre de 0,1-0,2 estimées ici. ${ }^{88}$ Ces estimations suggèrent que l'impôt sur le revenu n'est pas le prélèvement obligatoire dont l'allégement aurait les effets positifs les plus importants sur l'activité économique et l'emploi.

\footnotetext{
${ }^{87} \mathrm{Cf}$. les références données en introduction (section 1.2). Rappelons que des élasticités de l'ordre de $0,1-0,2$ ne signifient pas que les hauts revenus soient totalement insensibles (ou presque) aux incitations financières : les élasticités "empiriques " estimées dans cette étude prennent en compte à la fois les effets de substitution (positifs) et les effets revenus (négatifs) (cf. notes 63 et 64), si bien qu'une élasticité faible peut dissimuler des effets de substitution et des effets revenus élevés allant en sens inverse (par exemple, si les hauts revenus se comportaient de façon à préserver quoi qu'il arrive leur niveau de revenu après impôt, alors l'élasticité "empirique" de leur revenu imposable serait négative, même si leur élasticité compensée était en fait relativement élevée).
}

${ }^{88}$ Cf. par exemple Hammermesh (1993) pour un survey des études internationales sur l'élasticité de la demande de travail, qui montre clairement que l'élasticité de la demande de travail peu qualifié est toujours sensiblement plus élevée que l'élasticité de la demande de travail qualifié. Cf. également Piketty (1997c), où nous montrons que le déficit français en emplois vis-à-vis des Etats-Unis s'est creusée principalement dans les secteurs intensifs en travail peu qualifié (comme le commerce de détail et l'hôtellerie-restauration), c'est-à-dire dans les secteurs où le différentiel de coût du travail est le plus important. 


\section{Annexe A: Les sources statistiques sur l'impôt sur le revenu en France}

II existe trois types de sources statistiques permettant d'étudier l'évolution de la répartition des revenus déclarés au titre de l'impôt sur le revenu en France. Tout d'abord, la DGI constitue chaque année des échantillons représentatifs de déclarations de revenus afin d'effectuer ses propres simulations. D'autre part, I'INSEE effectue tous les 5 ans environ des enquêtes sur les "Revenus fiscaux" des ménages, qui se fondent principalement sur des échantillons de déclarations de revenus transmis à I'INSEE par la DGI, suivant un mode d'échantillonnage différent de celui des échantillons constitués chaque année par la DGI. Enfin, la DGI produit chaque année un certain nombre de tableaux statistiques (les "Etats 1921 ") portant sur l'ensemble des impositions effectuées au titre de l'impôt sur le revenu de l'année précédente. Ces trois sources statistiques ont en commun de porter sur l'impôt émis sur la base des revenus spontanément déclarés par les contribuables, et non sur le recouvrement effectif, qui peut différer de l'impôt émis, notamment du fait du contrôle fiscal et d'éventuels redressements d'impôt. ${ }^{89} \mathrm{Au}$ moment du recouvrement, les recettes sont en effet encaissés de façon globale par le Trésor Public, si bien que les seules statistiques produites par le recouvrement concernent le montant total des recettes, sans aucune indication sur la répartition de ces recettes par niveau de revenu imposable ou par type de contribuable. ${ }^{90}$

\section{A.1. Les échantillons de déclarations de revenus constitués par la DGI}

Deux types d'échantillons de déclarations de revenus sont constitués chaque année par la DGI afin d'effectuer des simulations: l'échantillon dit "lourd" (" modèle lourd "), constitué d'environ 400000 déclarations, et l'échantillon dit "léger " (" modèle léger"), constitué d'environ 40000 déclarations issues de l'échantillon lourd. Ces échantillons sont notamment utilisés pour effectuer les prévisions de recettes et pour évaluer le coût des mesures nouvelles et des dépenses fiscales (réductions d'impôt, etc...) figurant dans les Projets de Loi de Finances. Ces échantillons sont constitués de façon à être représentatifs de l'ensemble des déclarations déposées par les foyers fiscaux résidant en France métropolitaine (foyers imposables et foyers non imposables). Ils contiennent plusieurs centaines de variables correspondant à l'ensemble des cases de la déclaration de revenus remplie par les contribuables (déclaration 2042), avec en outre un petit nombre de variables calculées directement par les services fiscaux (revenu imposable, nombre de part de quotient familial, montant de l'impôt figurant sur l'avis d'imposition, etc...).

Ces échantillons permettent de disposer d'une connaissance exceptionnellement fine des très hauts revenus, grâce à une très forte sur-représentation des contribuables fortunés: dans l'échantillon léger de 1995, bien que le taux de sondage moyen soit d'environ 1/740 (40442 observations pour environ 30 millions de foyers fiscaux), l'échantillon contient une déclaration sur 4 pour les foyers ayant plus de 1 million de francs de revenu imposable, et toutes les déclarations des foyers ayant plus de 3 millions de francs de revenu imposable (cf. Annexe F). II est cependant

\footnotetext{
${ }^{89}$ En pratique, l'impôt effectivement recouvré est toujours de l'ordre de 5 à $10 \%$ supérieur à l'impôt émis, principalement du fait du contrôle fiscal (cf. section 2.2, tableau 2.4).

90 Jusqu'en 1996, la procédure d'encaissement des recettes ne permettait même pas de distinguer les recettes encaissées au titre de l'impôt sur le revenu des recettes encaissés au titre des autres impôts perçus par voie de rôles (taxe d'habitation, etc..), si bien que la répartition des recettes se faisait de façon conventionnelle sur la base du montant des impôts émis.
} 
impossible de suivre les mêmes contribuables pendant plusieurs années consécutives, car aucun identifiant individuel n'est disponible (apparemment, la DGI n'a jamais constitué de panel représentatif de déclarations). Malgré cette imperfection, ces échantillons représentent une source statistique idéale pour étudier les réactions des très hauts revenus aux changements de législation.

Malheureusement, ces échantillons sont disponibles uniquement pour les années récentes. II s'est avéré difficile de déterminer avec certitude depuis quelle année ces échantillons existent-ils:

(i) Le Bureau des Etudes Fiscales (bureau D2) de la Direction de la Prévision utilise uniquement les échantillons légers. Le BEF possède les échantillons légers pour les revenus des années 1988-1995 (l'échantillon anticipé pour les revenus de 1996 venait d'arriver au BEF au moment de l'étude, et il n'a pas été utilisé). Les fichiers informatiques correspondants sont sous format SAS, et ils sont donc aisément utilisables sous programmation SAS ou après transfert sous Stata.

(ii) Le Service de la Législation Fiscale (bureau C1) possède les échantillons légers depuis 1985 et les échantillons lourds depuis 1988. Cependant, ces fichiers ne sont aisément convertibles sous format SAS qu'à compter de 1992. Pour les années 1985-1991, les fichiers informatiques ne sont utilisables que sous "Harry", un logiciel "maison" de simulation fiscale, relativement simple d'utilisation pour effectuer des simulations courantes, mais limité en termes d'applications statistiques ("Harry" ne doit pas être confondu avec "Casimir", l'autre logiciel maison de simulation fiscale, plus sophistiqué que "Harry" pour faire des projections de recettes fiscales (prise en compte automatique de trends sur les retraités, les BA, etc...), mais plus lourd d'utilisation, et fonctionnant uniquement avec les échantillons légers).

(iii) Le Bureau des Statistiques (bureau IIC) de la DGI, qui est le " producteur " de ces échantillons, devrait en principe posséder le plus grand nombre de fichiers. Malheureusement, de la même façon qu'au SLF, seuls les fichiers postérieurs à 1992 sont aisément exploitables sous format SAS, et les fichiers des années antérieures n'ont pas été utilisées depuis longtemps (ils ne sont d'aucune utilité pour les besoins courants du bureau IIC). II semblerait néanmoins que les premières utilisations de ce type d'échantillons annuels remontent à la fin des années 1970 (auparavant, les simulations étaient effectuées " à la main ", à partir des statistiques exhaustives issues des Etats 1921). D'une part, des dossiers papier édités par la DGI et contenant des tabulations effectuées à partir des échantillons lourds ont pu être retrouvés jusqu'en 1979 (cf. Annexe H). D'autre part, les études sur l'impôt sur le revenu réalisées par la DGI et publiées chaque année dans "Statistiques et études financières " (série rouge) jusqu'en 1984 (cf. Annexe B), mentionnent pour la première fois dans l'étude portant sur les revenus de 1979 que, outre les statistiques des émissions de rôles (Etats 1921) habituellement utilisées comme unique source statistique, l'étude utilisera un échantillon de déclaration: "Par ailleurs, des informations complémentaires sont extraites du " modèle statistique " de l'impôt sur le revenu que la DGI utilise depuis 1976. Reposant, pour l'année 1979, sur un échantillon de 330000 foyers (dont 46000 foyers non imposés), ce modèle fiscal permet d'obtenir sur certains points un degré de détail que la statistique des 
émissions de rôles ne fournit pas " (S\&EF "série rouge " n³90 (1983), p.57). ${ }^{91}$ Les échantillons lourds existent donc au moins depuis 1979, et vraisemblablement depuis 1976.

Cependant, compte tenu des difficultés techniques considérables que soulèverait l'utilisation des fichiers anciens (rappelons que les échantillons légers 1985-1987 du SLF ne sont pas exportables sous SAS ; il est probable que les difficultés techniques d'utilisation soient sensiblement supérieures pour les fichiers pour la période 19791985), il nous a semblé plus réaliste d'utiliser uniquement les fichiers informatiques présents au BEF, c'est-à-dire les échantillons légers pour les années 1988-1995, et d'établir notre étude sur longue période principalement à partir des tableaux statistiques issus des Etats 1921, qui sont disponibles pour toutes les années depuis que l'impôt sur le revenu existe. Les échantillons légers pour les années 1988-1995 ont donc été utilisés uniquement pour vérifier la fiabilité des estimations effectuées à partir des Etats 1921. Les principaux résultats des tests de fiabilité ainsi effectués sont présentés dans l'annexe $\mathrm{E}$. Les calculs réalisés à partir des échantillons légers, et en particulier l'ensemble des variables utilisés, sont décrites de façon détaillée dans l'annexe F. De la même façon, les dossiers papier contenant des tabulations effectuées chaque année à partir des échantillons lourds, disponibles pour la période 1979-1993, ont été utilisés uniquement pour s'assurer de la robustesse des résultats obtenus à partir des Etats 1921. Les principaux résultats de ces tests de fiabilité sont décrits dans l'annexe $\mathrm{E}$. La liste des tableaux contenus dans ces dossiers "Modèle Lourd" est décrite dans l'annexe $\mathrm{H}$.

\section{A.2. Les enquêtes "Revenus fiscaux" réalisées par l'INSEE}

L'INSEE a réalisé des enquêtes portant sur les "Revenus fiscaux" des ménages des années 1956, 1962, 1965, 1970, 1975, 1979, 1984 et 1990 (une nouvelle enquête portant sur les revenus fiscaux de 1996 est actuellement en cours). La méthodologie de ces enquêtes est pratiquement inchangée depuis 1956 : I'INSEE tire un échantillon représentatif de logements de France métropolitaine issu du dernier recensement, la DGI fournit à l'INSEE l'ensemble des déclarations de revenus déposées par les foyers fiscaux habitant ces logements et que les services fiscaux ont pu retrouver, puis l'INSEE se charge entièrement de l'exploitation des données. En particulier, l'INSEE effectue des redressements permettant de prendre en compte l'existence de logements pour lesquels la DGI n'est pas parvenu à retrouver de déclaration, regroupe en un seul ménage l'ensemble des déclarations des foyers fiscaux habitant dans le même logement, et impute aux ménages concernés un certain nombre de revenus non soumis à I'IR et ne figurant donc pas sur les déclarations de revenus (allocations familiales, minimas sociaux, etc..). Ainsi redressées, ces enquêtes permettent alors à I'INSEE d'obtenir des estimations de l'inégalité du revenu disponible des ménages, et non seulement de l'inégalité du revenu fiscal des foyers fiscaux (cf. Annexe $G$ pour la liste des publications de I'INSEE consacrées aux enquêtes “Revenus fiscaux" de 1970 à 1990).

\footnotetext{
${ }^{91}$ L'étude portant sur les revenus de 1978 contient également quelques tableaux issus de l'échantillon lourd (cf. S\&EF (série rouge) $n^{\circ} 380$, p.66). II en va de même de l'étude portant sur les revenus de 1976 : cf. S\&EF $n^{\circ} 363-364-365, p p .146-147$ (dans tous les cas, les tableaux issus des échantillons lourds et publiés dans "S\&EF" indiquent la répartition des différents types de RCM ("créances, dépôts", "avances, prêts", "valeurs mobilières avec abattement", "valeurs mobilières sans abattement"), avec les mêmes tranches de revenu imposable que les tableaux des Etats 1921.
} 
Comparées aux échantillons de déclarations constitués par la DGI, ces enquêtes "Revenus Fiscaux" ont l'immense mérite d'être disponibles pour une période beaucoup plus longue. Cependant, le fait que ces enquêtes ne soient effectuées que tous les 5 ans les rend totalement inadaptés à l'étude des réactions des très hauts revenus aux changements de législation : les très hauts revenus connaissent en effet des variations cycliques extrêmement prononcées, et il est donc impératif de disposer de données annuelles pour espérer pouvoir distinguer les effets éventuels des changements de législation de tous les autres effets en jeu. De plus, contrairement aux échantillons de déclarations constitués par la DGI, les enquêtes "Revenus fiscaux" ne surpondèrent pas les hauts revenus : il s'agit d'échantillons d'environ 40000 foyers, tout comme les échantillons légers de la DGI, mais contrairement ces derniers, le taux de sondage moyen (environ 1/700) est à peine plus précis pour les revenus élevés que pour les revenus moyens et faibles (cf. Annexe G). De plus, le mode d'échantillonnage des enquêtes "Revenus fiscaux" introduit des marges d'incertitude non négligeables lorsque l'on s'intéresse à l'évolution fine des hauts revenus : contrairement aux échantillons de la DGI, qui par définition sont constitués de façon à être représentatif de l'ensemble des déclarations déposées, les échantillons des enquêtes "Revenus fiscaux" sont constitués à partir d'un échantillon représentatif de logements, si bien par exemple que les foyers fiscaux de célibataires, qui ont tendance à déménager plus souvent que les autres, sont systématiquement sous-représentés dans ces enquêtes. ${ }^{92} \mathrm{Au}$ final, les enquêtes "Revenus fiscaux" sont parfaitement adaptés à l'étude des grandes tendances de l'inégalité des revenus si l'on s'intéresse à l'ensemble de la population, mais ils ne sont guère adaptés à l'étude des fluctuations fines des hauts revenus.

Nous avons donc décidé d'utiliser les enquêtes "Revenus fiscaux" uniquement pour tester la fiabilité des estimations effectuées à partir des Etats 1921. Les principaux résultats des tests de fiabilité effectués sont décrits dans l'annexe $\mathrm{E}$. Nous avons également utilisé les enquêtes "Revenus fiscaux" pour la construction d'une série continue de nombre de foyers fiscaux et de revenu imposable total sur l'ensemble de la période 1970-1996 (cf. Annexe C). Les calculs réalisés à partir des enquêtes "Revenus fiscaux", et en particulier l'ensemble des variables utilisées, sont décrites de façon détaillée dans l'annexe G. Le BEF ne disposant initialement que des fichiers informatiques des enquêtes "Revenus fiscaux" de 1979 et 1984 (sous format SAS), les fichiers informatiques de l'ensemble des enquêtes "Revenus fiscaux " de 1970, 1975, 1979, 1984 et 1990 (tous disponibles sous format SAS) ont été transmis au BEF par l'INSEE, après accord de la DGI.

\section{A.3. Les statistiques des émissions de rôles (Etats 1921)}

La DGI produit chaque année des tableaux statistiques portant sur l'ensemble des impositions établies au titre de l'impôt sur le revenu des années précédentes. Ces tableaux sont actuellement rassemblés chaque année par le Bureau des Statistiques de la DGI (bureau IIC) dans des brochures d'une cinquantaine de pages éditées à usage principalement interne au Ministère, qui constituent les "Etats 1921 ".

Un avantage de cette source est que, contrairement aux statistiques issues de l'exploitation des échantillons de déclarations de revenus, dont la fiabilité dépend de la représentativité présumée des échantillons utilisés, les tableaux statistiques des Etats 1921 ne souffrent d'aucune erreur d'échantillonnage, puisqu'ils sont le résultat

\footnotetext{
${ }^{92}$ Cf. Annexe C.3.
} 
du dépouillement exhaustif de l'ensemble des déclarations: ils sont d'abord dressés au niveau de chaque direction départementale des impôts à partir de l'exploitation de l'ensemble des déclarations déposées et des émissions de rôles effectuées, puis ils sont centralisés et agrégés au niveau régional puis national. De plus, et surtout, les Etats 1921 (ou des tableaux statistiques équivalents) existent sous une forme annuelle depuis que l'impôt sur le revenu existe. Le BEF a conservé les brochures des Etats 1921 éditées par le Bureau des Statistiques de la DGI depuis les revenus de 1979. Par ailleurs, jusqu'aux revenus de 1981 (inclus), les tableaux statistiques correspondant aux actuels Etats 1921 étaient intégralement reproduits dans la revue "Statistiques et Etudes Financières " publiée par le Ministère (cf. Annexe B.3).

Le désavantage de cette source est évidemment que seules des informations statistiques relativement sommaires peuvent être ainsi centralisées à l'échelon national. Les Etats 1921 nous informent du nombre et du revenu imposable total des foyers fiscaux dont le revenu imposable est compris dans un certain nombre de tranches de revenu imposable (de 100000 à 200000 francs, de 200000 à 400000 francs, plus de 400000 francs, etc...), mais l'inflation et la croissance générale des revenus réels font que ces chiffres bruts sont difficilement comparables d'une année sur l'autre. De plus, les Etats 1921 ne contiennent des informations statistiques portant sur l'ensemble des foyers fiscaux que depuis les revenus de 1985 : avant 1985, seuls les foyers fiscaux imposables faisaient l'objet de tableaux statistiques, et aucune information concernant les déclarations déposées par les foyers nonimposables n'étaient conservée. Ces inconvénients peuvent toutefois être surmontés. D'une part, le fait de ne pas disposer d'informations statistiques détaillées sur les foyers non-imposables avant 1985 ne pose pas véritablement problème dans le cadre d'une étude sur les hauts revenus: le nombre de foyers non-imposables dont le revenu imposable est compris dans les centiles supérieurs de la distribution du revenu imposable est en effet suffisamment faible pour être négligé. ${ }^{93}$ Pour étudier l'évolution de la part du revenu total détenu par les $10 \%$ les plus riches, les $1 \%$ les plus riches, etc..., il nous suffit donc de connaître le nombre total de foyers non-imposables et leur revenu moyen, série qui peut être obtenue pour la période 1970-1985 à partir des enquêtes "Revenus fiscaux" et d'estimations effectuées à l'époque par la DGI. D'autre part, et surtout, il est possible d'obtenir des estimations de l'ensemble de la distribution des hauts revenus à partir des chiffres bruts des Etats 1921, en utilisant le fait que la distribution des hauts revenus est très bien approximée par une loi de Pareto. Une procédure d'estimation similaire a déjà été utilisée avec succès à partir des statistiques fiscales américaines, ${ }^{94}$ et la comparaison entre nos estimations et les échantillons de déclarations disponibles confirment qu'il s'agit d'une procédure d'estimation extrêmement fiable.

C'est donc cette source statistique que nous avons utilisée pour étudier l'évolution de la distribution du revenu imposable sur la période 1970-1996, les autres sources statistiques (échantillons de déclarations constitués par la DGI et enquêtes "Revenus fiscaux" de l'INSEE) ayant été utilisées uniquement pour vérifier la fiabilité de la procédure d'estimation. L'annexe B décrit la liste des tableaux statistiques de ces Etats 1921, disponibles sous forme de brochures éditées par la DGI dans les années 1980 et 1990 ou publiés dans "Statistiques et études financières " dans les années 1970. L'annexe C présente la méthodologie utilisée pour construire une série continue portant sur le nombre total de foyers fiscaux et le revenu imposable moyen sur la période 1970-1996. L'annexe D décrit de façon détaillée la procédure utilisée

\footnotetext{
${ }^{93} \mathrm{Cf}$. les tests de fiabilité de l'annexe $\mathrm{E}$.

${ }^{94}$ Cf. section 1.2 .
} 
pour estimer les paramètres de la distribution des hauts revenus à partir des chiffres bruts des Etats 1921 sur la période 1970-1996. L'annexe E présente les résultats des principales comparaisons effectuées pour tester la fiabilité des estimations ainsi obtenues. 


\section{Annexe B : Les tableaux statistiques des Etats 1921}

\section{B.1. Les tableaux statistiques utilisés dans cette étude}

Depuis les revenus de 1987 (inclus), les Etats 1921 sont établis une première fois au 31/12/n+1 (c'est-à-dire au 31/12/1988 pour les revenus de l'année 1987, dont les déclarations ont en principe été déposées en mars 1988), et une dernière fois au $31 / 12 / n+2$ (c'est-à-dire au 31/12/1989 pour les revenus de l'année 1987). Par définition, l'Etat 1921 au 31/12/n+1 (resp. 31/12/n+2) concerne l'ensemble des émissions de rôles effectuées avant le $31 / 12 / n+1$ (resp. 31/12/n+2). Jusqu'aux revenus de 1986 (inclus), les Etats 1921 étaient établis une première fois au $31 / 12 / n+1$, et une dernière fois au $31 / 03 / n+2$. En pratique, l'immense majorité des impositions sont effectuées avant le 31/12/n+1. Les rares impositions effectuées durant l'année $\mathrm{n}+2$ (généralement au début de l'année) ne sont cependant pas choisis au hasard: elles concernent souvent des revenus d'activités non-salariaux (BA, BIC, BNC), dont les déclarations complètes ont été faites avec retard sur les autres. Afin de ne pas biaiser les résultats, nous avons toujours utilisé la version définitive des Etats 1921 (au 31/12/n+2 depuis les revenus de 1987, et au 31/03/n+2 jusqu'aux revenus de 1986), à l'exception des revenus de l'année 1996, pour lesquels seul l'Etat 1921 établi au 31/12/1997 était disponible au moment de cette étude..$^{95}$ Pour les revenus des années 1970-1981, nous avons utilisé les Etats 1921 reproduits dans "Statistiques et Etudes Financières" (cf. B.2. infra pour les références exactes des numéros de "S\&EF " utilisés) ; pour les revenus des années 1982-1996, nous avons utilisé les Etats 1921 reproduits dans les brochures édités par la DGI et conservées au BEF. ${ }^{96}$ Les Etats 1921 utilisés portent sur la métropole pour la période 1970-1984; ils incluent également les DOM/TOM depuis les revenus de 1985, ce qui n'introduit aucune discontinuité sensible, compte tenu du poids très faible des DOM/TOM. ${ }^{97}$

La liste exacte des tableaux statistiques figurant dans les Etats 1921 a eu tendance à s'allonger au cours du temps. Les deux tableaux de base que nous avons utilisés sont disponibles de façon continue pour l'ensemble de la période 1970-1996.

Le premier tableau utilisé, sur lequel repose l'essentiel de nos résultats, indique le nombre de contribuables, le montant du revenu imposable total et le montant du total des droits simples, en fonction du nombre de parts de quotient familial et de la

\footnotetext{
${ }^{95}$ Les résultats portant sur les revenus de 1996 sont donc susceptibles d'être légèrement révisés quand l'Etat 1921 (revenus 1996) établi au 31/12/1998 sera disponible (au printemps 1999). Cependant, l'écart sera vraissemblablement très faible : nous avons comparé les résultats obtenus à partir de l'Etat 1921 (revenus 1995) établi au 31/12/1996 avec les résultats obtenus à partir de l'Etat 1921 (revenus 1995) établi au 31/12/1997, et les chiffres obtenus pour la part du revenu imposable des différents fractiles ne varient jamais de plus de $0,1 \%$ (en pourcentage de l'estimation obtenu avec l'Etat 1921 définitif). L'écart entre les Etats 1921 au 31/3/n+2 et les Etats 1921 au 31/12/n+2 est encore plus faible.

${ }^{96}$ Suite à une demande faite auprès du SESDO, le BEF a également pu obtenir les Etats 1921 jusqu'aux revenus de 1975 (inclus) sous forme de brochures. Cependant, ces Etats 1921 consituent une série hétérogène (certains sont des Etats 1921 établis au 31/12/n+1, d'autres au 31/03/n+2 ; certains tableaux manquent à l'appel ; etc..), et nous avons donc choisi d'utiliser pour les revenus 1970-1981 les Etats 1921 reproduits dans "S\&EF " (Iorsque les mêmes tableaux sont disponibles à la fois dans "S\&EF" et dans les brochures conservées au BEF, les chiffres sont toujours rigoureusement identiques).

${ }^{97} \mathrm{Cf}$. les tests de fiabilité de l'annexe $\mathrm{E}$.
} 
tranche de revenu imposable. ${ }^{98}$ Ce tableau, intitulé "tableau IIA" dans les Etats 1921 reproduits sous forme de brochures édités par la DGI (revenus 1982-1996), et "tableau A" dans les Etats 1921 reproduits dans "S\&EF" (revenus 1970-1981), effectue toujours la décomposition entre les foyers de couples mariés ayant 2 parts de quotient familial (" $2 A$ ") et les foyers de célibataires ayant 2 parts de quotient familial ("2B "). ${ }^{99}$ Depuis les revenus de 1982 (inclus), ce tableau distingue les contribuables ayant 1 part de quotient familial, 1,5 parts, 2A parts, 2B parts, 2,5 parts, 3 parts, 3,5 parts, 4 parts, 4,5 parts, 5 parts et 5,5 parts, et les contribuables ayant 6 parts ou plus sont regroupés dans un même sous-tableau. Un sous-tableau récapitulatif additionne tous les sous-tableaux précédents et décrit ainsi la distribution du revenu imposable de l'ensemble des foyers fiscaux (tous nombres de parts confondus). Jusqu'aux revenus de 1981 (inclus), les tableaux IIA/A offrait des informations plus précises sur les contribuables ayant un nombre élevé de parts, puisque tous les nombres de parts jusqu'à 8 parts avaient droit à un sous-tableau séparé, et que seuls les contribuables ayant 8,5 parts ou plus étaient regroupés dans un même sous-tableau. ${ }^{100}$

Le second tableau utilisé indique le nombre et le montant des différents revenus catégoriels nets et du revenu imposable total, par tranche de revenu imposable, tous nombres de parts de quotient familial confondus. Ce tableau est intitulé " tableau IA " dans les Etats 1921 reproduits dans les brochures éditées par la DGI (revenus 19821996)), et " tableau B " dans les Etats 1921 reproduits dans "S\&EF " (revenus 19701981). Le tableau IA/B coïncide toujours exactement avec le sous-tableau récapitulatif (tous nombres de parts confondus) du tableau IIA/A pour ce qui est de la distribution du revenu imposable,$^{101}$ et il nous a donc été utile uniquement pour estimer l'évolution de la composition du revenu imposable pour l'ensemble des foyers fiscaux (aucun tableau des Etats 1921 ne donne d'informations sur la composition du revenu imposable en distinguant suivant le nombre de parts des contribuables).

Dans l'ensemble de ces tableaux, les "revenus catégoriels nets " désignent les revenus catégoriels déclarés par les contribuables, diminués des éventuelles déductions et abattements (comme par exemple les abattements de $10 \%$ et $20 \%$ pour les salaires). Le "revenu imposable" désigne le revenu soumis au barème progressif de l'impôt sur le revenu (avant division par le nombre de parts de quotient familial): il s'agit donc de la somme des revenus catégoriels nets (parfois appelée " revenu brut global "), diminuée des déficits et des charges venant en déduction du revenu global (comme par exemple les abattements en faveur des personnes

\footnotetext{
${ }^{98}$ Depuis les revenus de 1994 (inclus), les tableaux IIA indiquent également le montant du total de l'impôt net, par nombre de parts de quotient familial et par tranche de revenu imposable.

${ }_{99}$ Depuis les revenus de 1995 (inclus), suite aux changements de législation concernant la part entière accordée au $1^{\text {er }}$ enfant à charge des contribuables non mariés, les contribuables " $2 \mathrm{~A}$ " sont devenus " $2 \mathrm{M}$ " dans les tableaux IIA, et les contribuables " $2 \mathrm{~B}$ " sont devenus " $2 \mathrm{C}$ ". Sur l'évolution des règles de calcul du nombre de parts de quotient familial, cf. section 2.1.3.

${ }^{100}$ Ce découpage s'est appliqué des revenus de 1975 aux revenus de 1981. Jusqu'aux revenus de 1974 (inclus), tous les nombres de parts pour lesquels il existait au moins un foyer imposable étaient distingués : 12,5 parts en 1974, 12 parts en 1973, etc.. La rupture de 1982 n'est pas dûe au passage de "S\&EF " aux brochures éditées par la DGI : les brochures fournies par le SESDO pour les années 1970 utilisent le même découpage que les tableaux publiés dans S\&EF.

${ }^{101}$ Les seules années pour lesquelles les deux tableaux ne coïncident pas parfaitement sont les années 1976-1978, pour lesquelles le revenu imposable total mentionné dans les tableaux A (que nous avons retenus dans cette étude) est de l'ordre de $0,2 \%$ inférieur au revenu imposable total mentionné dans les tableaux $B$, probablement du fait d'une définition légèrement différente du revenu imposable (cf. Annexe D.3).
} 
âgées). Le revenu imposable exclut toujours les revenus qui ne sont pas soumis au barème progressif, comme les plus-values soumises à un taux proportionnel. Les "droits simples" désignent l'impôt brut obtenu après application du barème et du système du quotient familial au revenu imposable, par opposition à l'“ impôt net ", qui est égal aux droits simples diminués des éventuelles réductions d'impôt. Les tranches de revenu imposable (en francs courants) utilisées dans ces tableaux n'ont guère changé depuis 1970. En particulier, la tranche la plus élevée concerne les contribuables dont le revenu imposable est supérieur à 400000 francs pour la période 1970-1983, et les contribuables dont le revenu imposable est supérieur à 500000 francs pour la période 1984-1996. Le pourcentage de foyers fiscaux concernés par cette tranche supérieure est ainsi passée d'à peine $0,01 \%$ en 1970 à près de $0,7 \%$ en 1996, ce qui limite de façon importante la possibilité d'effectuer des estimations fiables pour les très hauts revenus à partir des Etats 1921 pour la période récente (cf. Annexes $D$ et $E$ ). II s'agit là d'une imperfection majeure, et il serait hautement souhaitable que de nouvelles tranches soient introduites. Depuis les revenus de 1985 (inclus), les tableaux IA et IIA, comme l'ensemble des tableaux des Etats 1921, sont dressés séparément pour les foyers imposables (y compris les foyers bénéficiant d'une restitution partielle d'avoir fiscal) et pour les foyers nonimposables (y compris les foyers bénéficiant d'une restitution totale d'avoir fiscal). Jusqu'aux revenus de 1984 (inclus), seuls les tableaux portant sur les foyers imposables figurent dans les Etats 1921 (aucune information concernant les déclarations déposées par les foyers non-imposables n’a été conservée).

\section{B.2. Les autres tableaux statistiques des Etats 1921}

Les Etats 1921 contiennent également des tableaux statistiques complémentaires qui sont apparus pendant les années 1980 , et que nous n'avons pas cherché à utiliser dans le cadre de cette étude (à l'exception du "tableau III"). Depuis les revenus de 1982 (inclus), les Etats 1921 comprennent un tableau ("tableau III") indiquant les "éléments de calculs participant à le détermination de l'impôt net à émettre" au niveau agrégé, c'est-à-dire le passage du montant total des droits simples à au montant total de l'impôt net à émettre, en passant par la décote, les éventuelles majorations/minorations, les réductions d'impôt, les plus-values taxées séparément à un taux proportionnel, etc... Ce tableau n'existait pas en tant que tel dans les Etats 1921 de la période 1970-1981, mais les numéros de "S\&EF" consacrés à "L'impôt sur le revenu en 19.." (cf. annexe B.3. infra) mentionnaient toujours une décomposition similaire dans le texte de l'étude, ce qui nous permet de disposer d'une série continue sur la période 1970-1996 concernant l'impôt net émis, série utilisée dans le tableau 2.4 (section 2.2).

Depuis les revenus de 1984 (inclus), les Etats 1921 incluent également un tableau indiquant le nombre de contribuables, le montant du revenu imposable total et de l'impôt net total, par nombre de parts de quotient familial et par tranche d'impôt net (" tableau IIB ") ; deux tableaux récapitulatifs indiquant le nombre, les bases et l'impôt émis au titre des plus-values, réparties suivant le régime d'imposition et la catégorie de plus-values ("tableau IB " et "tableau IC" de 1984 à 1987 ; "tableau IVA" et "tableau IVB" de 1988 à 1996); un tableau indiquant le montant des différentes charges ouvrant droit à des déductions du revenu global, par tranche de revenu imposable (" tableau ID " de 1984 à 1987 ; " tableau IB " de 1988 à 1996, décomposé en un " tableau IB1 " et un tableau "IB2 " depuis 1989, afin de distinguer les charges déclarées des charges effectivement retenues); et un tableau récapitulatif indiquant 
un certain nombre de "comptage supplémentaires" au niveau agrégé ("tableau IV ").

Depuis les revenus de 1985 (inclus), les Etats 1921 incluent aussi un tableau indiquant le montant des différentes charges ouvrant droit à des réductions d'impôt ("tableau IE" de 1985 à 1987 ; " tableau IC " de 1988 à 1996, décomposé en un tableau "IC1" et "IC2" depuis 1989, afin de distinguer les charges déclarées des charges effectivement retenues).

Depuis les revenus de 1989 (inclus), les Etats 1921 incluent un tableau indiquant le nombre de contribuables et le montant des différents revenus catégoriels déclarés dans les déclarations (avant tout abattement ou déduction), par tranche de revenu imposable (" tableau I "). ${ }^{102}$

Enfin, depuis les revenus de 1995, les Etats 1921 incluent pour la première fois un tableau indiquant le nombre de contribuables, le revenu fiscal déclaré, le revenu net imposable et l'impôt net par tranche de revenu fiscal déclaré (avant abattements et déductions), et non par tranche de revenu imposable.

\section{B.3. Références des numéros de "Statistiques et Etudes Financières " consacrés à l'impôt sur le revenu}

La revue "Statistiques et Etudes Financières " ("S\&EF ") publiée par le Ministère de l'Economie et des Finances de 1949 à 1985 contient un très grand nombre de statistiques et d'études portant sur l'impôt sur le revenu. A l'exception de la "série orange", qui est devenue "Economie et Prévision"," 103 la revue "S\&EF" a disparu pendant les années 1980, pour être progressivement remplacée par "Les Notes Bleues de Bercy ", qui tiennent désormais le rôle de diffusion à usage externe des statistiques et des études produites par le Ministère. ${ }^{104}$ Le passage de "S\&EF " aux "Notes Bleues" correspond à une transformation fondamentale du contenu des publications à usage externe, notamment pour tout ce qui concerne l'impôt sur le revenu et la fiscalité en général. Alors que "S\&EF" publiait chaque année des études statistiques sur l'évolution des revenus déclarés et de l'impôt payé en fonction du niveau de revenu des contribuables, et surtout l'intégralité des tableaux statistiques bruts des Etats 1921 , les “Notes Bleues" se contentent aujourd'hui de donner des informations statistiques au niveau agrégé sur les recettes globales de l'impôt sur le revenu, sans aucune indication sur la répartition des contribuables et de l'impôt payé par niveau de revenu. ${ }^{105}$

Les coûts d'impression et l'étroitesse du public intéressé par des statistiques détaillées ont vraisemblablement motivé cette évolution, mais il est tout de même permis de s'interroger sur le fait que les Etats 1921 ne sont plus désormais publiés

\footnotetext{
102 Ces tableaux sont précieux dans la mesure où il est impossible de reconstituer de façon parfaitement précise les revenus catégoriels nets à partir des revenus catégotiels (ou inversement) en utilisant les échantillons de déclarations, car ce passage dépend non seulement de règles mécaniques s'appliquant à tous (telles les abattements de $10 \%$ et $20 \%$ pour les salaires), mais également des pièces justificatives fournies par le contribuable pour bénéficier de certaines déductions supplémentaires (comme par exemple des frais professionnels supérieurs à la déduction forfaitaire de $10 \%$ ) et qui ne fugurent pas sur la déclaration de revenus.

${ }^{103}$ A partir de 1971, S\&EF a été séparée entre une "série bleue ", une "série rouge " et une "série orange".

${ }^{104}$ Les "Notes Bleues " existaient déjà sous ce titre dans les années 1970, mais étaient apparemment d'un usage essentiellement interne.

${ }^{105}$ Cf. par exemple "Les statistiques de la Direction générale des impôts, année 1996 ", Les Notes Bleues de Bercy n¹24, 1-15 décembre 1997.
} 
que sous la forme de brochures à usage interne au Ministère, alors que les Etats 1921 relatifs aux revenus des années 1948 à 1981 ont été intégralement publiés chaque année dans "S\&EF" (de 1878 à la Seconde Guerre Mondiale, des tableaux statistiques similaires aux "Etats 1921 " étaient également reproduits chaque année dans le "Bulletin de Statistiques et de Législation Comparée" publié par le Ministère). Le légitime respect du droit des personnes au secret statistique ne peut en aucun cas justifier cette absence de diffusion large et transparente: à de très rares exceptions près (qui peuvent aisément être effacés des documents diffusés à usage public), les chiffres contenus dans les Etats 1921 concernent un très grand nombre de contribuables, et en particulier respectent la règle selon laquelle aucun résultat relatif à un groupe de moins de 11 personnes ne peut être diffusé. De fait, quelques rares tableaux issus des "Etats 1921 " sont reproduits chaque année dans I' "Annuaire Statistique de la France " publié par l'INSEE, ${ }^{106}$ ce qui montre bien qu'il s'agit de données qui sont de nature à être rendues publiques. ${ }^{107}$ En principe, le SESDO (Service d'Enquêtes, de Statistiques et de Documentation de la DGI) est chargé de répondre aux demandes motivées du public intéressé par ce type de statistiques fiscales, mais une telle procédure peut difficilement se substituer à une politique de diffusion beaucoup plus ouverte et transparente, surtout pour ce qui est de statistiques de base telles que celles contenues dans les Etats 1921.

Les Etats 1921 relatifs aux revenus des années 1970 à 1981 que nous avons utilisés ont été publiés dans les numéros de "S\&EF" consacrés à "L'impôt sur le revenu en 19.." ". Ces études annuelles consacrés à l'IR se composent toujours de la même façon : d'une part une étude proprement dite, qui se réduit principalement à un commentaire sur l'évolution générale des chiffres bruts issus des Etats 1921 durant les 2-3 dernières années, ${ }^{108}$ mais qui fournit également des estimations du nombre total "théorique" de foyers fiscaux, estimations que nous utilisons dans l'annexe C.1; et d'autre part une annexe consacrée aux " Généralités sur l'assiette et le calcul de l'impôt ", qui constitue une source utile sur l'évolution de la législation, et surtout une annexe reproduisant l'intégralité des tableaux statistiques des Etats 1921 (annexe dont nous donnons les numéros de pages plus bas). ${ }^{109}$ Les références de ces numéros sont les suivantes :

\footnotetext{
${ }^{106}$ Par exemple, l' "Annuaire Statistique de la France 1994 " inclut un tableau de chiffres issus des tableaux I et IA de l'Etat 1921 portant sur les revenus de 1991 (situation au 31/03/93), indiquant le nombre de contribuables et le montant du revenu déclaré et du revenu imposable par tranche de revenu imposable, en distinguant foyers imposables et foyers non imposables (cf. Tableau P.02-8, p.869).

107 Les tableaux statistiques des Etats 1921 sont également reproduits chaque année dans I' “'Annuaire Statistique de la DGI ", qui existe sous sa forme actuelle depuis 1979.

${ }^{108}$ Pour la première (et dernière...) fois, le numéro consacré à "L'impôt sur le revenu en 1981 et 1982 " (S\&EF "série rouge" $\mathrm{n}^{\circ} 394$ ) comprend également une section consacrée à "l'étude de quelques dispositions récentes " (attribution d'une demi-part supplémentaire aux foyers ayant 3 ou 4 enfants à charge ; majorations exceptionnelles).

${ }^{109}$ Ces numéros consacrés à "L'impôt sur le revenu en 19.. " existaient également pour les revenus des années 1956-1969 (cf. S\&EF n¹16 (1958), "L'impôt sur le revenu en 1957 " (revenus de 1956)). Les Etats 1921 portant sur les revenus des années 1947-1972 ont également été publiés dans les numéros de "S\&EF" consacrés aux "Renseignements statistiques relatifs aux impôts directs en 19.. " (cf. S\&EF " série bleue " $n^{\circ} 315$ (1975), "Renseignements statistiques relatifs aux impôts directs en 1973 " (revenus de 1972), ..., et S\&EF "supplément Statistiques " n7 (1950), "Renseignements statistiques relatifs aux impôts directs en 1948 " (revenus de 1947)).
} 
S\&EF "série rouge " n³94 (1984), “L'impôt sur le revenu en 1981 et 1982 " (revenus de 1980, situation au 31/03/1982 (pp.40-47) ; revenus de 1981, situation au 31/03/1983 (pp.48-55))

S\&EF “ série rouge " n³90 (1983), “L’impôt sur le revenu en 1980 " (revenus de 1979, situation au 31/03/1981 (pp.97-105))

S\&EF “ série rouge " n³80 (1981), “L'impôt sur le revenu en 1979 " (revenus de 1978 , situation au 31/03/1980 (pp.80-89))

S\&EF “ série rouge " n³71 (1980), “L'impôt sur le revenu en 1978 ” (revenus de 1977, situation au 31/03/1979 (pp.95-109))

S\&EF "série rouge " n³63-364-365 (1980), "L'impôt sur le revenu en 1977 " (revenus de 1976, situation au 31/03/1978 (pp.159-174))

S\&EF “ série rouge " n³53 (1978), “L'impôt sur le revenu en 1976 ” (revenus de 1975, situation au 31/03/1977 (pp.27-40))

S\&EF " série rouge " n³37 (1977), “L'impôt sur le revenu en 1975 " (revenus de 1974, situation au 31/03/1976 (pp.27-40))

S\&EF " série rouge " n’328 (1976), “L'impôt sur le revenu en 1974 ” (revenus de 1973 , situation au 31/03/1975 (pp.25-38))

S\&EF " série rouge " n³19-320 (1975), " L’impôt sur le revenu en 1973 " (revenus de 1972, situation au 31/03/1974 (pp.97-107))

S\&EF " série rouge " n³09 (1974), "L'impôt sur le revenu en 1972 " (revenus de 1971, situation au 31/03/1973 (pp.23-33))

S\&EF " série rouge " n²93 (1973), "L'impôt sur le revenu en 1971" (revenus de 1970, situation au 31/03/1972 (pp.21-34))

Nous utilisons également dans l'Annexe C.1 les numéros de "S\&EF " consacrés aux "Statistiques de la DGI en 19..", qui contiennent des estimations du nombre total "théorique" de foyers fiscaux pour les années 1979-1983, ce qui nous permet de compléter la série issue des numéros consacrés à "L'impôt sur le revenu en 19..". ${ }^{110}$ Ces numéros reproduisent également le tableau IA issu de l'Etat 1921 portant sur les revenus de l'année considérée, mais ces tableaux nous sont inutiles, puisque nous disposons déjà de l'intégralité des tableaux des Etats 1921 sur la période 1970-1996 grâce aux numéros de "S\&EF" consacrés à "L'impôt sur le revenu en 19.." (revenus des années 1970-1981) et aux brochures conservées au BEF (revenus des années 1982-1996).

\footnotetext{
${ }^{110}$ Cf. S\&EF "série rouge " n³98 (1985) "Statistiques de la DGI en 1984" (revenus de 1983) ; S\&EF "série rouge " n³97 (1985) "Statistiques de la DGI en 1983" (revenus de 1982); S\&EF "série rouge " ${ }^{\circ} 396$ (1984) "Statistiques de la DGI en 1982" (revenus de 1981) ; S\&EF "série rouge" n`393 (1983) "Statistiques de la DGI en 1981" (revenus de 1980); S\&EF "série rouge " n³86 (1982) "Statistiques de la DGI en 1980" (revenus de 1979).
} 


\title{
Annexe C : Méthodologie de l'estimation d'une série continue portant sur le nombre de foyers fiscaux et le revenu imposable moyen sur la période 1970-1996
}

\section{C.1. Estimation d'une série continue du nombre total de foyers fiscaux, 1970-1996}

\author{
Les différentes étapes de l'estimation sont résumées sur le tableau C-1.
}

Insérer Tableau C-1: Estimation du nombre de foyers fiscaux imposables et nonimposables, 1970-1996

Les Etats 1921 nous donnent le nombre de foyers fiscaux imposables sur l'ensemble de la période 1970-1996 (colonne (1) du tableau C-1). Par contre, aucune information concernant les foyers non-imposables (pas même leur nombre) n'est disponible dans les Etats 1921 portant sur les revenus des années antérieures à 1985 (colonne (2)). Le tableau IV de l'Etat 1921 portant sur les revenus de 1984 indique cependant le nombre total de foyers fiscaux, dont on peut déduire le nombre de foyers non-imposables, ce qui explique pourquoi la colonne (2) du tableau C-1 commence en 1984 et non en 1985.

Pour les années antérieures à 1984, nous disposons des estimations du nombre total de foyers fiscaux non-imposables effectuées chaque année par la DGI (colonne (3)). Contrairement aux statistiques des Etats 1921, qui par définition concernent uniquement les foyers fiscaux ayant déposés une déclaration de revenus, il s'agit là d'estimations du nombre "théorique" de foyers non-imposables, c'est-à-dire du nombre de déclarations de revenus qui auraient dues être déposées si tous les foyers non-imposables avaient déposé une déclaration. Ces estimations ont été effectuées tous les ans pour les revenus de 1970 à 1986 par la DGI, en faisant différentes hypothèses à partir des statistiques socio-démographiques de l'INSEE (nombre de ménages, nombre de célibataires, nombre de concubins, etc...). Elles ont été publiées dans les différents numéros de "Statistiques et Etudes Financières" consacrés à l'impôt sur le revenu, ainsi que dans les rapports que Conseil des Impôts a consacré à l'impôt sur le revenu en 1984 et $1990 .{ }^{111}$ Pour les revenus des

\footnotetext{
${ }^{111}$ Pour les années 1975 à 1981, ces estimations ont été publiées dans les numéros de "Statistiques et Etudes Fiancières " consacrés à "L'impôt sur le revenu en 19.. " (cf. S\&EF (série rouge) n³63-364365 (p.135), $n^{\circ} 371$ (p.57), $n^{\circ} 380$ (p.87), n³90 (pp.61-62) et $n^{\circ} 394$ (p.6)). Pour les années 1972 à 1974 , ces estimations ont été obtenues en appliquant les pourcentages de foyers imposables pour 1972 et 1974 donnés dans S\&EF (série rouge) $n^{\circ} 390$ (p.62), qui sont cohérents avec les estimations d'un nombre total de foyers fiscaux de 22,1M en 1972, 22,4M en 1973, 22,6M en 1974 et 22,8M en 1975 données dans S\&EF (série rouge) $n^{\circ} 319-320$ (p.4), $n^{\circ} 328$ (p.4), $n^{\circ} 337$ (p.6), $n^{\circ} 353$ (p.4) et $n^{\circ} 363-364-365$ (p.131). Par contre, nous n'avons pas retenu les pourcentages de foyers imposables données dans S\&EF (série rouge) $n^{\circ} 390$ (p.62) pour les années 1970 et 1971, car ils ont manifestement été calculés à partir des estimations de nombre total de foyers de 22,2M en 1970 et $22,6 \mathrm{M}$ en 1971 donnés dans S\&EF (série rouge) $n^{\circ} 293$ (p.78) et $n^{\circ} 309$ (p.4), estimations qui présentent une forte discontinuité avec celles des années ultérieures. Nous avons donc retenu l'estimation d'un nombre total de foyers fiscaux de 21,486M en 1970 donnée dans les rapports du Conseil des Impôts de 1984 (cf. tableau n70, p.141) et de 1990 (cf. tableau n90, p.219) ; l'estimation du nombre théorique de foyers non-imposables pour 1971 a ensuite été obtenue à partir des chiffres de 1970 et 1972 en supposant une progression linéaire entre 1970 et 1972, ce qui nous donne un rythme de progression cohérent avec celui des années ultérieures. Pour les années 1982 et 1983, les estimations du nombre théorique de foyers non-imposables ont été publiés dans les numéros de S\&EF consacrées aux "Statistiques de la DGI en 19.. " (cf. S\&EF " série rouge " n³86 (p.14), n 393
} 
années 1977 à 1983, nous disposons également d'estimations séparées pour le nombre de foyers non-imposables ayant déposé une déclaration et du nombre de foyers non-imposables n'ayant pas déposé de déclaration (colonnes (4) et (5)), également publiées dans "Statistiques et Etudes Financières". ${ }^{112}$ Cette même décomposition peut être obtenue directement pour les années 1984-1986, puisque nous disposons pour ces années-là du nombre de foyers fiscaux ayant déposé une déclaration (grâce aux Etats 1921).

Ces statistiques indiquent que le nombre de foyers non-imposables ne déposant pas de déclaration de revenus a fortement baissé durant la fin des années 70 et le début des années 80, passant de près de 4 millions en 1977 à à peine plus de 500000 en 1986. Cette forte diminution s'explique principalement par le fait que les avis de non-imposition, qui par définition ne peuvent être obtenus que si une déclaration a été déposée, sont devenus de plus en plus utiles au cours du temps pour les foyers ayant des revenus modestes, notamment du fait du développement des prestations sociales sous conditions de ressources. Le "taux de déclaration" mentionné sur le tableau C-1 (colonne (6)), qui est défini comme le pourcentage de foyers fiscaux "théoriques" (imposables et non-imposables) ayant déposé une déclaration (colonne $(6)=[$ colonnes $(1)+(4)] /[$ colonnes $(1)+(3)]$, en $\%)$, est ainsi passé de $83,5 \%$ en 1977 à $97,9 \%$ en 1986 . On peut donc considérer que depuis le milieu des années 80 , la population des contribuables déposant une déclaration ne présente plus de biais significatif par rapport à la population des contribuables "théoriques". Ces estimations du nombre de foyers fiscaux théoriques n'ont apparemment plus été effectués par la DGI depuis 1986, vraisemblablement du fait que les foyers non-imposables sont désormais couverts dans leur immense majorité par les Etats 1921.

Afin d'éviter toute discontinuité en 1985-1986 et de ne pas avoir à corriger les séries issues des Etats 1921 pour la période récente, nous avons adopté la méthodologie suivante. Pour l'ensemble de la période 1970-1996, nous avons retenu les nombres de foyers imposables issus des Etats 1921 (colonne $(7)=$ colonne (1)). Pour les années 1986 à 1996, nous avons retenu les nombres de foyers non-imposables issus des Etats 1921 (pour les années 1986-1986, colonne $(7)=$ colonne (2)). Nous avons supposé que le taux de déclaration était resté stable à 97,9\% de 1986 à 1996, et nous avons donc retenu pour les années 1970-1985 un nombre de foyers nonimposables égal à $97,9 \%$ du nombre théorique de foyers fiscaux (pour les années 1970-1985, colonne $(8)=97,9 \%$ des colonnes $(1)+(3))$. Le nombre total de foyers fiscaux et le pourcentage de foyers imposables résultant de l'estimation de la colonne (8) sont mentionnés dans la colonne (9) (= colonnes $(7)+(8))$ et dans le colonne (10) (= colonne (7)/colonne (9), en \%).

(p.61), $n^{\circ} 396$ (p.51), $n^{\circ} 397$ (p.17) et $n^{\circ} 398$ (p.17)). Pour les années 1984 à 1986, ces estimations ont été publiés dans le rapport du Conseil des Impôts de 1990 (cf. tableau n 90 , p.219; les chiffres de ce tableau portent sur le champ "national" (métropole + DOM/TOM) pour les années 1980-1986 (ainsi que pour les années 1970-1974) (les chiffres du tableau $n^{\circ} 70$ du rapport de 1984 portent tous sur la métropole, mais la série s'arrête en 1982) ; nous avons donc minoré l'estimation pour l'année 1984 d'un coefficient égal à 0,9932 (en 1983, l'estimation du nombre de foyers fiscaux théoriques du champ métropole était égale à $99,32 \%$ de l'estimation du champ national ; ce pourcentage est relativement stable : $99,35 \%$ en $1982,99,40 \%$ en 1981 , etc..)).

${ }^{112}$ Ces estimations ont été publiés dans les numéros de S\&EF consacrées aux "Statistiques de la DGI en 19.." (cf. S\&EF "série rouge " $n^{\circ} 386$ (p.14), $n^{\circ} 393$ (p.61), n³96 (p.51), n³97 (p.17) et $n^{\circ} 398$ $($ p.17)). 
Tableau C-1: Estimation du nombre de foyers fiscaux imposables et non-imposables, 1970-1996

\begin{tabular}{|c|c|c|c|c|c|c|}
\hline & $\begin{array}{c}(1) \\
\mathrm{N} . \text { foyers } \\
\text { imposables } \\
\text { (Etats }\end{array}$ & $\begin{array}{l}\quad(2) \\
\text { N.foyers } \\
\text { non-impos. } \\
\text { 1921) }\end{array}$ & $\begin{array}{c}(3) \\
\text { N.foyers } \\
\text { non-impos. }\end{array}$ & $\begin{array}{c}\text { (4) } \\
\text { dt. N. décl. } \\
\text { déposées } \\
\text { Estimations }\end{array}$ & $\begin{array}{l}\text { (5) } \\
\text { dt. N. décl. } \\
\text { non dépos. } \\
\text { DGI) }\end{array}$ & $\begin{array}{c}\text { (6) } \\
\text { Taux } \\
\text { de décl. }\end{array}$ \\
\hline 1970 & 10513119 & & 10973000 & & & \\
\hline 1971 & 11019782 & & 10795000 & & & \\
\hline 1972 & 11502269 & & 10617000 & & & \\
\hline 1973 & 12092270 & & 10301000 & & & \\
\hline 1974 & 12767947 & & 9870000 & & & \\
\hline 1975 & 13494548 & & 9351000 & & & \\
\hline 1976 & 14242603 & & 8739000 & & & \\
\hline 1977 & 14007405 & & 9191000 & 5352000 & 3839000 & 83,5 \\
\hline 1978 & 14564035 & & 8869000 & 5575000 & 3294000 & 85,9 \\
\hline 1979 & 15000673 & & 8685000 & 5800000 & 2885000 & 87,8 \\
\hline 1980 & 15289641 & & 8673000 & 6632000 & 2041000 & 91,5 \\
\hline 1981 & 15056169 & & 9205000 & 7397000 & 1808000 & 92,5 \\
\hline 1982 & 15308540 & & 9252000 & 7744000 & 1508000 & 93,9 \\
\hline 1983 & 15242012 & & 9564000 & 8769000 & 795000 & 96,8 \\
\hline 1984 & 15209530 & 9249832 & 9892000 & 9250000 & 642000 & 97,4 \\
\hline 1985 & 15252320 & 9736114 & 10433000 & 9736000 & 697000 & 97,3 \\
\hline 1986 & 13314101 & 12220225 & 12770000 & 12220000 & 550000 & 97,9 \\
\hline 1987 & 13368628 & 12972674 & & & & \\
\hline 1988 & 13470354 & 13321014 & & & & \\
\hline 1989 & 13881932 & 13478101 & & & & \\
\hline 1990 & 14296524 & 13732940 & & & & \\
\hline 1991 & 14642747 & 13963896 & & & & \\
\hline 1992 & 14753713 & 14298409 & & & & \\
\hline 1993 & 14907267 & 14650903 & & & & \\
\hline 1994 & 14990137 & 15048099 & & & & \\
\hline 1995 & 15474244 & 15110886 & & & & \\
\hline $1996\left(^{*}\right)$ & 15007042 & 15717960 & & & & \\
\hline
\end{tabular}

\begin{tabular}{|c|c|c|c|c|c|}
\hline $\begin{array}{c}(7) \\
\text { N. foyers } \\
\text { mposables } \\
\text { (Estimation }\end{array}$ & $\begin{array}{c}(8) \\
\text { N.foyers } \\
\text { non-impos. } \\
\text { is retenues }\end{array}$ & $\begin{array}{c}(9) \\
N \text {. total } \\
\text { de foyers } \\
\text { dans cette é }\end{array}$ & $\begin{array}{l}(10) \\
\% \text { de } \\
\text { oy.imp. } \\
\text { de) }\end{array}$ & $\begin{array}{c}(11) \\
N . \text { total } \\
\text { de foyers } \\
\text { (Enquêtes }\end{array}$ & $\begin{array}{l}(12) \\
\text { n \% de } \\
1)+(3) \\
\text { 'RF") }\end{array}$ \\
\hline 10513119 & 10519951 & 21033070 & 50,0 & 20470258 & 95,3 \\
\hline 11019782 & 10335021 & 21354803 & 51,6 & & \\
\hline 11502269 & 10150601 & 21652870 & 53,1 & & \\
\hline 12092270 & 9828824 & 21921094 & 55,2 & & \\
\hline 12767947 & 9392664 & 22160611 & 57,6 & & \\
\hline 13494548 & 8869287 & 22363835 & 60,3 & 22451386 & 98,3 \\
\hline 14242603 & 8254418 & 22497021 & 63,3 & & \\
\hline 14007405 & 8701847 & 22709252 & 61,7 & & \\
\hline 14564035 & 8374899 & 22938934 & 63,5 & & \\
\hline 15000673 & 8185572 & 23186245 & 64,7 & 22431867 & 94,7 \\
\hline 15289641 & 8167732 & 23457373 & 65,2 & & \\
\hline 15056169 & 8693438 & 23749607 & 63,4 & & \\
\hline 15308540 & 8734125 & 24042665 & 63,7 & & \\
\hline 15242012 & 9040949 & 24282961 & 62,8 & & \\
\hline 15209530 & 9362718 & 24572248 & 61,9 & 24585220 & 97,9 \\
\hline 15252320 & 9891409 & 25143729 & 60,7 & & \\
\hline 13314101 & 12220225 & 25534326 & 52,1 & & \\
\hline 13368628 & 12972674 & 26341302 & 50,8 & & \\
\hline 13470354 & 13321014 & 26791368 & 50,3 & & \\
\hline 13881932 & 13478101 & 27360033 & 50,7 & & \\
\hline 14296524 & 13732940 & 28029464 & 51,0 & 27272048 & 97,3 \\
\hline 14642747 & 13963896 & 28606643 & 51,2 & & \\
\hline 14753713 & 14298409 & 29052122 & 50,8 & & \\
\hline 14907267 & 14650903 & 29558170 & 50,4 & & \\
\hline 14990137 & 15048099 & 30038236 & 49,9 & & \\
\hline 15474244 & 15110886 & 30585130 & 50,6 & & \\
\hline 15007042 & 15717960 & 30725002 & 48,8 & & \\
\hline
\end{tabular}

Sources et méthodologie : Cf. Annexe C.1.

Note : $1996\left(^{*}\right)$ : chiffres provisoires (rôles émis au 31/12/1997) 
Autrement dit, pour l'ensemble de la période 1970-1996, nous avons exclu de notre champ d'analyse les $2,1 \%$ de foyers fiscaux théoriques qui n'ont jamais rempli de déclaration, et nous nous concentrons sur les $97,9 \%$ restant. Le "décile supérieur de la distribution du revenu imposable " dont il est question dans cette étude regroupe donc en fait les $9,79 \%$ des foyers fiscaux théoriques ayant le revenu imposable le plus élevé, et le centile supérieur les $0,979 \%$ des foyers théoriques ayant le revenu imposable le plus élevé. L'hypothèse selon laquelle le taux de déclaration serait resté stable autour de $97,9 \%$ depuis 1986 semble raisonnable : dès 1983, on observe un certain plafonnement du taux de déclaration, le nombre résiduel de foyers théoriques ne déposant pas de déclaration ne baissant plus que marginalement. En outre, même si le taux de déclaration avait poursuivi son trend ascendant sur la période 1986-1996, les erreurs induites par nos hypothèses seraient d'une ampleur quantitative extrêmement faible. Supposons par exemple que le taux de déclaration ait atteint 100\% en 1996, c'est-à-dire que l'Etat 1921 pour les revenus de 1996 porte sur tous les foyers fiscaux théoriques, alors que l'Etat 1921 pour les revenus de 1986 portait sur seulement $97,9 \%$ d'entre eux. Cela impliquerait que quand nous comparons la part du revenu imposable total détenu par les $10 \%$ des foyers fiscaux ayant le revenu imposable le plus élevé (P90-100) en 1986 et 1996, nous comparons en fait la part des $9,79 \%$ des foyers fiscaux théoriques les plus riches de 1986 avec celle des $10 \%$ des foyers fiscaux théoriques les plus riches de 1996. En supposant que les foyers qui ne déposaient pas de déclaration en 1986 ont un revenu imposable nul, notre estimation de la part de P90-100 pour 1996 serait donc artificiellement surévalué au maximum de 2,1\% (comparée à l'estimation de 1986). En fait, cette "surévaluation maximale" est sensiblement inférieure (de l'ordre de $1,2 \%$ ), car les $0,21 \%$ des foyers les plus modestes du décile supérieur ont un revenu qui est de l'ordre de 1,7 fois plus faible que le revenu moyen du décile supérieur. ${ }^{113}$ En supposant que les foyers non-déclarants de 1986 ont un revenu faible mais positif, la surévaluation serait encore plus faible. ${ }^{114}$ Notre hypothèse de stabilité du taux de déclaration depuis 1986 engendre donc un biais maximal d'environ $1,2 \%$ sur les estimations de la part de P90-100, P99-100, etc..., de 1996 comparées celles de 1986, soit un trend positif artificiel de l'ordre de $0,1 \%$ par an pendant 10 ans. Comparé à l'amplitude des variations annuelles effectivement observées durant cette période, un tel biais éventuel est totalement négligeable. Enfin, l'effet d'une éventuelle erreur sur le nombre total de foyers fiscaux serait nul (au premier ordre) sur les ratios du type "P99-100/P90-100", puisque les deux termes de ce ratio seraient biaisés dans les mêmes proportions. Notre analyse des fluctuations des hauts revenus étant fondée principalement sur l'étude de tels ratios, on peut conclure que ce problème de l'estimation du nombre total de foyers fiscaux n'est aucunement susceptible de biaiser nos conclusions.

Le tableau C-1 mentionne également les estimations du nombre total de foyers fiscaux (imposables et non-imposables) issues des enquêtes "Revenus Fiscaux" de 1970, 1975, 1979, 1984 et 1990 (colonne (11)) (cf. annexe G), et exprime ces chiffres en pourcentage des estimations DGI du nombre théorique de foyers fiscaux (colonne $(12)=$ colonne $(11) /[$ colonne $(1)+(3)]$, en \%). Les taux obtenus sont de l'ordre de 95-98\% pour l'ensemble de la période 1970-1990. Ces taux élevés

\footnotetext{
${ }^{113}$ Ce ratio de 1,7 découle du fait que les foyers se trouvant à un percentile donné de la distribution ont un revenu qui est de l'ordre de 1,7 fois plus faible que le revenu moyen des foyers situés au-delà de ce percentile (cf. Annexe D.1, tableau D-1).

${ }^{114}$ Par exemple, si les foyers non-déclarants ont un revenu qui est de l'ordre de 1,7 fois inférieur au revenu moyen des foyers déclarants, alors l'erreur est nulle (au premier ordre).
} 
confirment la fiabilité de nos estimations, et montrent que la méthodologie adoptée par les enquêtes "RF" permet à ces dernières de comptabiliser une partie importante des foyers ne déposant pas de déclaration, et ce en particulier pendant les années 70. En effet, l'INSEE connaît la composition complète des logements issus du dernier recensement, ce qui permet de repérer l'existence de foyers fiscaux inconnus du fisc. Par exemple, si la DGI ne retrouve pas de déclarations de revenu pour une partie des membres du ménage, I'INSEE peut alors identifier cette souspartie du ménage comme un foyer fiscal non-déclarant (le cas typique est celui de personnes âgées vivant chez leurs enfants, disposant de très faibles allocations et ne déposant pas de déclaration, sans pour autant être rattachés au foyer fiscal de leurs enfants). Les estimations du nombre total de foyers fiscaux théoriques ainsi obtenues sont toujours légèrement inférieures aux estimations DGI, ce qui s'explique par le fait que l'INSEE connaît la composition complète des ménages et peut identifier les foyers non-déclarants uniquement dans le mesure où ces ménages n'ont pas déménagé depuis le dernier recensement. En cas de déménagement, la DGI transmet la déclaration des foyers fiscaux composant ce ménage au cours de l'année étudiée, et I'INSEE ne dispose d'aucune information lui permettant d'identifier d'éventuels foyers non-déclarants (à moins que la DGI dispose d'informations quand à leur existence, ce qui arrive parfois). Ce léger biais est d'autant plus important que l'année du dernier recensement et l'année de l'enquête sont éloignées, ce qui explique sans doute pourquoi l'enquête "RF" de 1975, dont l'échantillon de logements est issu du recensement de 1975, estime un nombre total de foyers égal à 98,3\% de l'estimation théorique, alors que les enquêtes de 1970 (échantillon issu du recensement de 1968) et de 1979 (échantillon issu du recensement de 1975) estiment un nombre total de foyers qui est de l'ordre de $95 \%$ de l'estimation théorique. Ce biais est devenu moins important depuis la stabilisation du nombre de foyers non-déclarants intervenu pendant les années $80 .{ }^{115}$

\section{C.2. Estimation d'une série continue de revenu imposable total, 1970-1996}

Les différentes étapes de l'estimation sont résumées sur le tableau C-2.

Insérer Tableau C-2 : Estimation du revenu imposable total, 1970-1996

De la même façon que pour le nombre de foyers fiscaux, les Etats 1921 nous donnent le revenu imposable des foyers imposables pour l'ensemble de la période 1970-1996 (colonne (1)), alors que le revenu imposable des foyers non-imposables est disponible uniquement depuis les revenus de 1985 (colonne (2)). Le revenu imposable total n'est donc connu avec certitude uniquement sur la période 19851996 (colonne (3)).

Le tableau C-2 indique également les estimations du revenu imposable total issues des enquêtes "Revenus Fiscaux" de 1970, 1975, 1979, 1984 et 1990 (colonne (4)) (cf. Annexe G), ainsi que la série annuelle de revenu primaire brut (RPB) des

\footnotetext{
${ }^{115}$ Cela explique sans doute pourquoi le taux obtenu pour l'enquête "RF" de 1984 est de près de $98 \%$, bien que l'échantillon soit issu du recensement de 1982. L'échantillon de l'enquête "RF" de 1990 est issu du recensement de 1990, et le taux obtenu serait également de l'ordre de $98 \%$ si l'on corrigeait le biais dû à l'inclusion des DOM/TOM (les enquêtes "RF" portent uniquement sur la métropole, alors que les Etats 1921 et les estimations DGI que nous utilisons portent sur le champ métropole + DOM/TOM depuis les revenus de 1985).
} 
Tableau C-2: Estimation du revenu imposable total, 1970-1996

$\begin{array}{rccc} & \begin{array}{c}(1) \\ \text { Rev.imp. } \\ \text { (foy.imp.) } \\ \text { (Etats } 1921 \text { ) } \\ \text { Rev.imp. }\end{array} & \begin{array}{c}\text { Rev.imp. } \\ \text { (f.i. }+ \text { f.ni.) }\end{array} \\ 1970 & 206268 & \\ 1971 & 235239 & & \\ 1972 & 265369 & & \\ 1973 & 313432 & & \\ 1974 & 374844 & & \\ 1975 & 448653 & & \\ 1976 & 527598 & & \\ 1977 & 576680 & & \\ 1978 & 663562 & & \\ 1979 & 755394 & & \\ 1980 & 866335 & & \\ 1981 & 995564 & & \\ 1982 & 1125249 & & \\ 1983 & 1262465 & & \\ 1984 & 1352028 & & \\ 1985 & 1447555 & 245070 & 1692625 \\ 1986 & 1409645 & 379927 & 1789572 \\ 1987 & 1466613 & 421592 & 1888205 \\ 1988 & 1536160 & 449022 & 1985182 \\ 1989 & 1647683 & 463781 & 2111464 \\ 1990 & 1767665 & 483177 & 2250842 \\ 1991 & 1857505 & 501000 & 2358505 \\ 1992 & 1911147 & 523717 & 2434864 \\ 1993 & 1956012 & 532973 & 2488985 \\ 1994 & 1998301 & 545998 & 2544299 \\ 1995 & 2081154 & 546349 & 2627503 \\ 1996\left(^{*}\right) & 2069451 & 617338 & 2686789\end{array}$

\begin{tabular}{|c|c|c|c|}
\hline $\begin{array}{c}(4) \\
\text { Rev.imp. } \\
\text { (f.i. + f.ni.) } \\
\text { (Enq."RF") }\end{array}$ & $\begin{array}{c}\text { (5) } \\
\text { RPB des } \\
\text { ménages } \\
\quad \text { (Con }\end{array}$ & $\begin{array}{c}(6) \\
\text { Rimp (i) } \\
\text { (\%RPB) } \\
\text { mptabilité }\end{array}$ & $\begin{array}{c}(7) \\
\text { Rimp(i+ni) } \\
\text { (\%RPB) } \\
\text { Nationale) }\end{array}$ \\
\hline \multirow[t]{5}{*}{258402} & 592332 & 34,8 & \\
\hline & 658383 & 35,7 & \\
\hline & 736561 & 36,0 & \\
\hline & 833744 & 37,6 & \\
\hline & 976261 & 38,4 & \\
\hline \multirow[t]{4}{*}{510468} & 1130484 & 39,7 & \\
\hline & 1293832 & 40,8 & \\
\hline & 1468296 & 39,3 & \\
\hline & 1667652 & 39,8 & \\
\hline \multirow[t]{5}{*}{850380} & 1888623 & 40,0 & \\
\hline & 2159630 & 40,1 & \\
\hline & 2472412 & 40,3 & \\
\hline & 2817659 & 39,9 & \\
\hline & 3101662 & 40,7 & \\
\hline \multirow[t]{6}{*}{1625165} & 3324436 & 40,7 & \\
\hline & 3550025 & 40,8 & 47,7 \\
\hline & 3728833 & 37,8 & 48,0 \\
\hline & 3900025 & 37,6 & 48,4 \\
\hline & 4106355 & 37,4 & 48,3 \\
\hline & 4443434 & 37,1 & 47,5 \\
\hline \multirow[t]{7}{*}{2137690} & 4731916 & 37,4 & 47,6 \\
\hline & 4993766 & 37,2 & 47,2 \\
\hline & 5178146 & 36,9 & 47,0 \\
\hline & 5276181 & 37,1 & 47,2 \\
\hline & 5436851 & 36,8 & 46,8 \\
\hline & 5693420 & 36,6 & 46,1 \\
\hline & 5856230 & 35,3 & 45,9 \\
\hline
\end{tabular}

\begin{tabular}{|c|c|c|}
\hline $\begin{array}{c}(8) \\
\text { Rimp(RF) } \\
\text { (\% RPB) }\end{array}$ & $\begin{array}{c}(9) \\
\text { Rimp(i+ni) } \\
\text { (\% RPB) } \\
\text { (Estimations }\end{array}$ & $\begin{array}{c}(10) \\
\text { Rev.imp. } \\
\text { (i.+ni.) } \\
\text { retenues) }\end{array}$ \\
\hline \multirow[t]{5}{*}{43,6} & 45,0 & 266545 \\
\hline & 45,0 & 296472 \\
\hline & 45,1 & 331905 \\
\hline & 45,1 & 375957 \\
\hline & 45,1 & 440525 \\
\hline \multirow[t]{4}{*}{45,2} & 45,2 & 510468 \\
\hline & 45,5 & 589300 \\
\hline & 45,9 & 674519 \\
\hline & 46,3 & 772638 \\
\hline \multirow[t]{5}{*}{45,0} & 46,7 & 882419 \\
\hline & 46,9 & 1012484 \\
\hline & 47,0 & 1163064 \\
\hline & 47,2 & 1329965 \\
\hline & 47,4 & 1468961 \\
\hline \multirow[t]{6}{*}{48,9} & 47,5 & 1579767 \\
\hline & 47,7 & 1692625 \\
\hline & 48,0 & 1789572 \\
\hline & 48,4 & 1888205 \\
\hline & 48,3 & 1985182 \\
\hline & 47,5 & 2111464 \\
\hline \multirow[t]{7}{*}{45,2} & 47,6 & 2250842 \\
\hline & 47,2 & 2358505 \\
\hline & 47,0 & 2434864 \\
\hline & 47,2 & 2488985 \\
\hline & 46,8 & 2544299 \\
\hline & 46,1 & 2627503 \\
\hline & 45,9 & 2686789 \\
\hline
\end{tabular}

Sources et méthodologie : Cf. Annexe C.2.

Notes : (i) 1996(*): chiffres provisoires (rôles émis au 31/12/1997)

(ii) Tous les revenus sont exprimés en millions de francs courants 
ménages issue de la Comptabilité Nationale (colonne (5)). ${ }^{116}$ On constate que le revenu imposable des foyers imposables, exprimé en pourcentage du RPB (colonne (6)), a évolué de la même façon que le pourcentage de foyers imposables (cf. tableau C-1) : en progression constante de $34,8 \%$ en 1970 à $40,8 \%$ en 1985, il s'est stabilisé autour de $37-38 \%$ du RPB depuis 1986. De fait, le revenu imposable total issu des Etats 1921, exprimé en pourcentage du RPB (colonne (7)), est relativement stable autour de $47-48 \%$ du RPB sur la période 1985-1996, avec cependant une légère baisse en fin de période.

Le revenu imposable total issu des enquêtes "Revenus Fiscaux", exprimé en pourcentage du RPB (colonne (8)), indique cependant une légère progression au cours des années 70 et au début des années 80 (le chiffre pour 1990 est artificiellement bas, du fait que le revenu imposable des foyers non identifiés par la DGI n'a pas été redressé lors de l'enquête de 1990, contrairement à ce qui a été fait pour toutes les autres enquêtes; ${ }^{117}$ cf. annexe F). Plusieurs facteurs structurels peuvent expliquer cette progression tendancielle du ratio entre le revenu imposable et le RPB. Tout d'abord, les revenus d'activité des non-salariés sont comptabilisés pour leur montant brut dans le RPB (sous la forme de l'EBE des entreprises individuelles), alors que le revenu imposable les retient pour leur montant net; l'importance des revenus d'activité des non-salariés (BA, BIC, BNC) ayant fortement diminué pendant les années 70 (relativement aux autres revenus), cela pourrait expliquer pourquoi le revenu imposable représentait un pourcentage plus faible du RPB en 1970 que dans les années $80-90 .^{118}$ On peut également imaginer que la fraude fiscale ait diminué pendant les années 70. Quoi qu'il en soit, il est difficile de retenir tels quels les chiffres bruts issus des enquêtes "Revenus Fiscaux", dans la mesure où les estimations issues des différentes enquêtes sont de qualité inégale. En particulier, l'estimation issue de l'enquête de 1975, qui porte sur plus de $98 \%$ des foyers fiscaux théoriques, est vraisemblablement d'une meilleure qualité que celles qui sont issues des enquêtes de 1970 et 1979, qui portent sur environ $95 \%$ des foyers fiscaux théoriques (cf. tableau C-1 supra). L'estimation du revenu imposable total issue de l'enquête de 1975 , qui est sans doute la plus fiable, montre que le trend de progression du ratio (revenu imposable)/RPB, en supposant qu'il existe, a été d'une ampleur limitée (de $45,2 \%$ en 1975 à $47,7 \%$ en 1985 , soit une progression de 2,2 points en 10 ans, c'est-à-dire moins de 0,2\% par an).

Nous avons donc suivi la méthodologie suivante. Pour la période 1985-1996, nous avons retenu le revenu imposable total issu des Etats 1921 (pour les années 19851996 , colonne $(9)=$ colonne $(7)$, et colonne $(10)=$ colonne $(3)$ ). Pour 1975 , nous avons retenu le revenu imposable total de 510 milliards de francs et le ratio (revenu imposable)/RPB de 45,2\% issus de l'enquête "Revenus fiscaux" (pour 1975, colonne $(9)=$ colonne $(8)$, et colonne $(10)=$ colonne $(4)$ ). Pour 1970 et 1979, nous avons calculé quelles auraient été les ratios (revenu imposable)/RPB si les enquêtes "RF" de 1970 et 1979 avaient porté sur le même champ que l'enquête de 1975 (pour 1970 , colonne $(9)=$ colonne $(8) \times 98,3 / 95,3$; pour 1979 , colonne $(9)=$ colonne

\footnotetext{
${ }^{116}$ Cf. INSEE-Résultats n547-548-549 (série "Economie générale " n¹45-146-147), " Rapport sur les Comptes de la Nation 1996 ", p.163.

${ }_{117}$ Inversement, le chiffre de $48,9 \%$ pour 1984 semble un peu élevé (comparé aux chiffres des Etats 1921 pour la période 1985-1996), ce qui peut s'expliquer par le fait que la méthodologie du redressement opéré par l'INSEE, qui consiste à attribuer aux foyers inconnus du fisc le même revenu imposable que les foyers connus à caractéristiques senblables, conduit sans doute à une légère surestimation du véritable revenu imposable de ces foyers.

${ }_{118}$ Le trend structurel de salarisation des revenus d'activité s'est cependant poursuivi entre 1985 et 1996, sans que cela ne se traduise par une hausse du ratio (revenu imposable)/RPB.
} 
(8)x98,3/94,7). ${ }^{119}$ Nous avons ensuite estimé les ratios (revenu imposable)/RPB pour les années 1971-1974, 1976-1978 et 1980-1984 en supposant des progressions linéaires entre 1970 et 1975, 1975 et 1979 , et 1979 et 1985 . Enfin, nous avons appliqué ces ratios (colonne (9)) au RPB (colonne (5)) pour obtenir notre estimation du revenu imposable total sur la période 1970-1984 (colonne (10)). Dans la série ainsi obtenue, le ratio (revenu imposable)/RPB passe de $45,0 \%$ en 1970 à $47,7 \%$ en 1985, soit une progression de 2,7 points en 15 ans. Cette estimation est cohérente avec la progression du ratio (revenu imposable des foyers imposables)/RPB de $34,8 \%$ en 1970 à $37-38 \%$ pendant les années 1986-1996, malgré un pourcentage de foyers imposables voisin de 50\% en 1970 comme en 1986-1996 (cf. tableau C-1).

De la même façon que pour le nombre total de foyers fiscaux, les erreurs éventuelles sur le revenu imposable total ne sont pas susceptibles de biaiser nos conclusions. Tout d'abord, en supposant que le ratio (revenu imposable)/RPB est passé de $45,0 \%$ en 1970 à $47,7 \%$ en 1985, nous avons choisi une hypothèse médiane. Les deux hypothèses polaires consisteraient soit à mettre en doute la qualité des enquêtes "RF" des années 70 et à supposer que le ratio est resté stable autour de $47,7 \%$ de 1970 à 1985, soit à retenir les chiffres bruts de l'enquête "RF" de 1970 et à supposer que le ratio est progressivement passé de 43,6\% en 1970 à $47,7 \%$ en 1985 . Notre hypothèse médiane suppose que le ratio est passé de $45,0 \%$ en 1970 à $47,7 \%$ en 1985 . Si la première hypothèse polaire était la bonne, alors les estimations de la part de P90-100, P99-100, etc.., pour 1970 obtenues à partir de I'hypothèse médiane serait de l'ordre de $6 \%$ trop élevées. Inversement, si la seconde hypothèse était la bonne, les estimations seraient de l'ordre de $3 \%$ trop faible. De telles marges d'erreur ne sont pas négligeables si l'on souhaite étudier de façon précise l'évolution à long-terme des niveaux de la part de P90-100, P99-100,..., sur la période 1970-1985. Mais si l'on s'intéresse aux fluctuations de court-terme, alors l'existence d'un trend biaisé de $6 \%$ ou de $3 \%$ sur 15 ans est relativement négligeable. De plus, et surtout, le niveau du revenu imposable total n'a par définition aucune conséquence sur les ratios du type P99-100/P90-100, etc..., et nos estimations de l'élasticité du revenu imposable reposent uniquement sur de tels ratios.

C.3. Estimations de séries continues pour le nombre de foyers fiscaux et le revenu imposable moyen en fonction du nombre de parts de quotient familial, 1970-1996

De la même façon que pour le nombre de foyers et le revenu imposable (tous nombres de parts confondus), les Etats 1921 nous permettent de connaître le nombre de foyers et le revenu imposable en fonction du nombre de parts de quotient familial pour l'ensemble des foyers fiscaux (imposables et non-imposables) uniquement depuis 1985 (cf. Tableaux C-3 et C-4).

Insérer Tableau C-3: L'évolution du nombre de foyers fiscaux en fonction du nombre de parts de quotient familial dans les Etats 1921 de 1970 à 1996 Insérer Tableau C-4: L'évolution du revenu imposable moyen en fonction du nombre de parts de quotient familial dans les Etats 1921 de 1970 à 1996

Le tableau C-3 montre à quel point il est difficile d'inférer les variations de la proportion de l'ensemble des foyers fiscaux (imposables et non-imposables) ayant

\footnotetext{
${ }^{119}$ Nous supposons implicitement que les foyers non identifiés par les enquêtes de 1970 et 1979 ont le même revenu imposable moyen que les autres foyers, ce qui est sans doute une sur-estimation.
} 
Tableau C-3: L'évolution du nombre de foyers fiscaux en fonction du nombre de parts de quotient familial dans les Etats 1921 de 1970 à 1996

(a) Foyers imposables (1970-1996)

\begin{tabular}{|c|c|c|c|c|c|c|c|c|c|c|c|c|c|}
\hline & $\mathrm{QF}=1$ & $Q F=1,5$ & $Q F=2 A$ & $Q F=2 B$ & $Q F=2,5$ & $Q F=3$ & $Q F=3,5$ & $\mathrm{QF}=4$ & $Q F=4,5$ & $Q F=5$ & $Q F=5,5$ & $Q F>=6$ & \\
\hline 1970 & 26,69 & 6,23 & 27,64 & 0,96 & 18,38 & 12,82 & 4,97 & 1,56 & 0,49 & 0,17 & 0,06 & 0,03 & 100,00 \\
\hline 1971 & 27,17 & 6,15 & 27,27 & 1,05 & 18,27 & 12,89 & 4,94 & 1,53 & 0,47 & 0,16 & 0,05 & 0,03 & 100,00 \\
\hline 1972 & 26,75 & 5,84 & 26,92 & 1,20 & 18,55 & 13,43 & 5,12 & 1,52 & 0,45 & 0,14 & 0,05 & 0,03 & 100,00 \\
\hline 1973 & 25,98 & 5,94 & 28,92 & 1,23 & 17,92 & 13,08 & 4,87 & 1,43 & 0,42 & 0,13 & 0,04 & 0,03 & 100,00 \\
\hline 1974 & 25,04 & 7,15 & 28,05 & 1,38 & 18,19 & 13,40 & 4,86 & 1,38 & 0,38 & 0,12 & 0,04 & 0,02 & 100,00 \\
\hline 1975 & 25,00 & 7,72 & 27,51 & 1,45 & 18,07 & 13,57 & 4,82 & 1,33 & 0,36 & 0,11 & 0,03 & 0,02 & 100,00 \\
\hline 1977 & 25,85 & 7,75 & 26,85 & 1,55 & 17,87 & 13,96 & 4,59 & 1,16 & 0,29 & 0,08 & 0,02 & 0,01 & 100,00 \\
\hline 1978 & 25,65 & 8,04 & 26,81 & 1,86 & 17,51 & 14,11 & 4,53 & 1,11 & 0,27 & 0,07 & 0,02 & 0,01 & 100,00 \\
\hline 1979 & 25,70 & 8,40 & 26,64 & 1,84 & 17,33 & 14,26 & 4,50 & 1,04 & 0,03 & 0,19 & 0,05 & 0,02 & 100,00 \\
\hline 1980 & 26,16 & 8,67 & 26,65 & 1,96 & 16,96 & 14,41 & 0,27 & 3,83 & 0,83 & 0,19 & 0,05 & 0,02 & 100,00 \\
\hline 1981 & 25,14 & 7,79 & 26,19 & 2,21 & 18,44 & 14,83 & 0,43 & 3,91 & 0,82 & 0,18 & 0,04 & 0,02 & 100,00 \\
\hline 1986 & 31,44 & 8,25 & 24,09 & 2,63 & 16,23 & 13,45 & 0,34 & 2,99 & 0,08 & 0,41 & 0,01 & 0,08 & 100,00 \\
\hline 1987 & 31,68 & 8,42 & 23,67 & 2,74 & 16,35 & 13,28 & 0,34 & 2,95 & 0,08 & 0,40 & 0,01 & 0,08 & 100,00 \\
\hline 1988 & 32,04 & 8,61 & 23,49 & 2,91 & 16,11 & 13,01 & 0,34 & 2,93 & 0,07 & 0,40 & 0,01 & 0,08 & 100,00 \\
\hline 1989 & 32,26 & 8,81 & 23,44 & 3,09 & 15,87 & 12,68 & 0,34 & 2,94 & 0,07 & 0,39 & 0,01 & 0,08 & 100,00 \\
\hline 1990 & 32,69 & 8,96 & 23,11 & 3,29 & 15,59 & 12,44 & 0,35 & 2,99 & 0,08 & 0,41 & 0,01 & 0,08 & 100,00 \\
\hline 1991 & 32,80 & 9,18 & 22,97 & 3,52 & 15,39 & 12,19 & 0,36 & 3,02 & 0,07 & 0,40 & 0,01 & 0,08 & 100,00 \\
\hline 1992 & 32,52 & 9,56 & 23,05 & 3,66 & 15,16 & 12,11 & 0,35 & 3,02 & 0,07 & 0,40 & 0,01 & 0,08 & 100,00 \\
\hline 1993 & 31,64 & 10,08 & 23,24 & 3,95 & 15,08 & 12,05 & 0,36 & 3,04 & 0,07 & 0,40 & 0,01 & 0,07 & 100,00 \\
\hline 1994 & 31,33 & 10,34 & 23,33 & 4,14 & 14,88 & 11,95 & 0,37 & 3,09 & 0,08 & 0,40 & 0,01 & 0,07 & 100,00 \\
\hline
\end{tabular}

(b) Ensemble des foyers fiscaux (imposables et non-imposables) (1985-1996)

\begin{tabular}{|c|c|c|c|c|c|c|c|c|c|c|c|c|c|}
\hline & $Q F=1$ & $Q F=1,5$ & $Q F=2 A$ & $Q F=2 B$ & $Q F=2,5$ & $Q F=3$ & $Q F=3,5$ & $\mathrm{QF}=4$ & $Q F=4,5$ & $Q F=5$ & $Q F=5,5$ & $\mathrm{QF}>=6$ & \\
\hline 1985 & 31,62 & 11,38 & 19,79 & 3,79 & 13,89 & 11,71 & 0,78 & 4,74 & 1,41 & 0,50 & 0,22 & 0,16 & 100,00 \\
\hline 1986 & 31,87 & 11,68 & 19,42 & 4,10 & 13,78 & 11,51 & 0,80 & 4,48 & 0,28 & 1,18 & 0,10 & 0,80 & 100,00 \\
\hline 1987 & 32,22 & 11,79 & 18,80 & 4,42 & 13,91 & 11,31 & 0,82 & 4,43 & 0,28 & 1,15 & 0,10 & 0,76 & 100,00 \\
\hline 1988 & 32,59 & 11,97 & 18,58 & 4,67 & 13,78 & 11,00 & 0,83 & 4,35 & 0,28 & 1,12 & 0,09 & 0,74 & 100,0 \\
\hline 1989 & 32,90 & 12,12 & 18,43 & 4,95 & 13,66 & 10,67 & 0,85 & 4,26 & 0,28 & 1,08 & 0,09 & 0,71 & 100, \\
\hline 1990 & 33,50 & 12,03 & 18,02 & 5,24 & 13,55 & 10,37 & 0,90 & 4,21 & 0,29 & 1,08 & 0,10 & 0,72 & 100, \\
\hline 1991 & 33,69 & 12,36 & 17,85 & 5,44 & 13,45 & 10,04 & 0,91 & 4,11 & 0,29 & 1,06 & 0,10 & 0,70 & $100, c$ \\
\hline 1992 & 33,65 & 12,72 & 17,62 & 5,68 & 13,31 & 9,90 & 0,93 & 4,07 & 0,30 & 1,04 & 0,10 & 0,69 & $100, c$ \\
\hline 1993 & 33,82 & 13,00 & 17,46 & 5,90 & 13,13 & 9,68 & 0,95 & 3,99 & 0,30 & 1,02 & 0,10 & 0,66 & 100,0 \\
\hline 1994 & 34,10 & 13,23 & 17,33 & 6,07 & 12,92 & 9,45 & 0,96 & 3,91 & 0,30 & 1,00 & 0,10 & 0,63 & 100,0 \\
\hline 1995 & 34,40 & 17,53 & 17,19 & 3,79 & 11,11 & 9,71 & 0,48 & 3,95 & 0,16 & 1,01 & 0,05 & 0,60 & 100,0 \\
\hline $1996\left(^{*}\right)$ & 34,72 & 17,69 & 17,11 & 3,90 & 10,91 & 9,55 & 0,49 & 3,87 & 0,16 & 0,98 & 0,05 & 0,56 & 100,0 \\
\hline
\end{tabular}

Source: Etats 1921

Lecture: En 1996, 32,44\% des foyers imposables avaient 1 part de quotient familial, 14,16\% avaient 1,5 parts, etc...; $34,72 \%$ de l'ensemble des foyers fiscaux (imposables et non-imposables) avaient 1 part de quotient familial, $17,69 \%$ avaient 1,5 parts, etc...

Note: $1996\left(^{*}\right)$ : chiffres provisoires (rôles émis au 31/12/97) 
Tableau C-4: L'évolution du revenu imposable moyen en fonction du nombre de parts de quotient familial dans les Etats 1921 de 1970 à 1996

\section{(a) Foyers imposables (1970-1996)}

\begin{tabular}{|c|c|c|c|c|c|c|c|c|c|c|c|c|c|}
\hline & $\mathrm{QF}=1$ & $\mathrm{QF}=1,5$ & $Q F=2 A$ & $Q F=2 B$ & $\mathrm{QF}=2,5$ & $Q F=3$ & $Q F=3,5$ & $\mathrm{QF}=4$ & $Q F=4,5$ & $Q F=5$ & $Q F=5,5$ & QF $>=6$ & \\
\hline 1970 & 0,56 & 0,80 & 1,07 & 0,77 & 1,15 & 1,32 & 1,51 & 1,74 & 1,93 & 2,08 & 2,30 & 2,36 & 1,00 \\
\hline 1971 & 0,56 & 0,79 & 1,07 & 0,76 & 1,15 & 1,33 & 1,52 & 1,74 & 1,92 & 2,09 & 2,27 & 2,29 & 1,00 \\
\hline 1972 & 0,57 & 0,79 & 1,08 & 0,75 & 1,14 & 1,30 & 1,48 & 1,70 & 1,89 & 2,08 & 2,26 & 2,30 & 1,00 \\
\hline 1973 & 0,58 & 0,74 & 1,05 & 0,74 & 1,16 & 1,32 & 1,52 & 1,74 & 1,95 & 2,11 & 2,32 & 2,23 & 1,00 \\
\hline 1974 & 0,58 & 0,72 & 1,08 & 0,73 & 1,16 & 1,30 & 1,45 & 1,64 & 1,80 & 1,97 & 2,07 & 2,11 & 1,0 \\
\hline 1975 & 0,58 & 0,71 & 1,10 & 0,72 & 1,16 & 1,30 & 1,44 & 1,61 & 1,76 & 1,92 & 1,95 & 2,09 & 1,0 \\
\hline 1976 & 0,59 & 0,69 & 1,11 & 0,72 & 1,17 & 1,31 & 1,44 & 1,59 & 1,74 & 1,83 & 1,88 & 2,04 & 1,0 \\
\hline 1977 & 0,58 & 0,70 & 1,11 & 0,73 & 1,18 & 1,32 & 1,46 & 1,63 & 1,73 & 1,83 & 1,92 & 2,00 & 1,00 \\
\hline 1978 & 0,58 & 0,70 & 1,11 & 0,74 & 1,18 & 1,32 & 1,46 & 1,61 & 1,72 & 1,79 & 1,96 & 1,97 & 1,00 \\
\hline 1979 & 0,58 & 0,69 & 1,09 & 0,74 & 1,19 & 1,34 & 1,49 & 1,67 & 1,61 & 2,01 & 2,08 & 2,20 & 1,00 \\
\hline 1980 & 0,58 & 0,69 & 1,09 & 0,74 & 1,20 & 1,34 & 1,26 & 1,62 & 1,83 & 1,97 & 2,03 & 2,22 & 1,00 \\
\hline 1981 & 0,61 & 0,72 & 1,08 & 0,72 & 1,15 & 1,31 & 1,25 & 1,58 & 1,77 & 1,91 & 2,00 & 2,12 & 1,0 \\
\hline 1982 & 0,61 & 0,72 & 1,08 & 0,72 & 1,15 & 1,31 & 1,25 & 1,57 & 1,76 & 1,89 & 1,96 & 2,20 & 1,0 \\
\hline 1983 & 0,61 & 0,72 & 1,08 & 0,72 & 1,17 & 1,36 & 1,29 & 1,67 & 1,88 & 2,01 & 2,08 & 2,23 & 1,0 \\
\hline 1984 & 0,61 & 0,73 & 1,08 & 0,73 & 1,17 & 1,37 & 1,29 & 1,68 & 1,90 & 2,05 & 2,09 & 2,24 & 1,00 \\
\hline 1985 & 0,62 & 0,73 & 1,08 & 0,73 & 1,17 & 1,37 & 1,27 & 1,69 & 1,92 & 2,07 & 2,20 & 2,18 & 1,0 \\
\hline 1986 & 0,58 & 0,77 & 1,13 & 0,83 & 1,22 & 1,42 & 1,41 & 1,81 & 1,81 & 2,27 & 2,21 & 2,65 & 1,00 \\
\hline 1987 & 0,58 & 0,77 & 1,13 & 0,84 & 1,23 & 1,43 & 1,43 & 1,83 & 1,83 & 2,30 & 2,22 & 2,67 & 1,00 \\
\hline 1988 & 0,57 & 0,77 & 1,14 & 0,84 & 1,23 & 1,44 & 1,47 & 1,86 & 1,86 & 2,43 & 2,27 & 2,73 & 1,00 \\
\hline 1989 & 0,58 & 0,77 & 1,13 & 0,85 & 1,24 & 1,46 & 1,43 & 1,86 & 1,85 & 2,38 & 2,21 & 2,75 & 1,0 \\
\hline 1990 & 0,58 & 0,77 & 1,14 & 0,85 & 1,25 & 1,47 & 1,43 & 1,86 & 1,86 & 2,39 & 2,17 & 2,76 & 1,0 \\
\hline 1991 & 0,58 & 0,77 & 1,14 & 0,85 & 1,25 & 1,47 & 1,42 & 1,84 & 1,85 & 2,35 & 2,21 & 2,73 & 1,0 \\
\hline 1992 & 0,59 & 0,77 & 1,14 & 0,85 & 1,25 & 1,47 & 1,44 & 1,85 & 1,86 & 2,37 & 2,26 & 2,76 & 1,0 \\
\hline 1993 & 0,59 & 0,76 & 1,13 & 0,84 & 1,25 & 1,46 & 1,43 & 1,84 & 1,85 & 2,38 & 2,25 & 2,78 & 1,0 \\
\hline 1994 & 0,60 & 0,76 & 1,13 & 0,84 & 1,25 & 1,46 & 1,41 & 1,83 & 1,82 & 2,38 & 2,31 & 2,80 & 1,0 \\
\hline 1995 & 0,60 & 0,76 & 1,15 & 0,87 & 1,27 & 1,48 & 1,43 & 1,84 & 1,84 & 2,39 & 2,35 & 2,81 & 1,0 \\
\hline $996\left(^{*}\right)$ & 0,60 & 0,76 & 1,16 & 0,89 & 1,30 & 1,51 & 1,50 & 1,94 & 1,97 & 2,56 & 2,63 & 3,04 & 1,0 \\
\hline
\end{tabular}

\section{(b) Ensemble des foyers fiscaux (imposables et non-imposables) (1985-1996)}

$\begin{array}{cccccccccccccc} & \text { QF=1 } & \text { QF=1,5 } & \text { QF=2A } & \text { QF=2B } & \text { QF=2,5 } & \text { QF=3 } & \text { QF=3,5 } & \text { QF=4 } & \text { QF=4,5 } & \text { QF=5 } & \text { QF=5,5 } & \text { QF >=6 } & \\ 1985 & 0,57 & 0,62 & 1,25 & 0,68 & 1,36 & 1,59 & 0,91 & 1,47 & 1,21 & 0,95 & 0,79 & 0,70 & 1,00 \\ 1986 & 0,57 & 0,63 & 1,27 & 0,69 & 1,36 & 1,60 & 0,91 & 1,50 & 0,91 & 1,26 & 0,78 & 0,85 & 1,00 \\ 1987 & 0,57 & 0,64 & 1,28 & 0,68 & 1,36 & 1,62 & 0,89 & 1,51 & 0,88 & 1,27 & 0,76 & 0,84 & 1,00 \\ 1988 & 0,56 & 0,64 & 1,29 & 0,69 & 1,37 & 1,64 & 0,89 & 1,54 & 0,87 & 1,31 & 0,75 & 0,84 & 1,00 \\ 1989 & 0,57 & 0,64 & 1,29 & 0,70 & 1,37 & 1,67 & 0,87 & 1,56 & 0,87 & 1,31 & 0,74 & 0,85 & 1,00 \\ 1990 & 0,57 & 0,66 & 1,31 & 0,70 & 1,37 & 1,69 & 0,86 & 1,58 & 0,85 & 1,33 & 0,72 & 0,85 & 1,00 \\ 1991 & 0,57 & 0,66 & 1,32 & 0,71 & 1,37 & 1,70 & 0,86 & 1,60 & 0,84 & 1,34 & 0,72 & 0,86 & 1,00 \\ 1992 & 0,57 & 0,67 & 1,33 & 0,72 & 1,36 & 1,71 & 0,86 & 1,62 & 0,84 & 1,35 & 0,71 & 0,86 & 1,00 \\ 1993 & 0,57 & 0,68 & 1,34 & 0,72 & 1,37 & 1,72 & 0,85 & 1,64 & 0,83 & 1,36 & 0,70 & 0,86 & 1,00 \\ 1994 & 0,57 & 0,68 & 1,36 & 0,73 & 1,37 & 1,74 & 0,85 & 1,66 & 0,82 & 1,39 & 0,70 & 0,87 & 1,00 \\ 1995 & 0,57 & 0,69 & 1,37 & 0,72 & 1,47 & 1,69 & 1,11 & 1,64 & 1,15 & 1,37 & 1,00 & 0,89 & 1,00 \\ 1996\left(^{*}\right) & 0,57 & 0,70 & 1,39 & 0,73 & 1,47 & 1,68 & 1,09 & 1,66 & 1,13 & 1,39 & 0,99 & 0,91 & 1,00\end{array}$

Source: Etats 1921

Lecture: En 1996, le revenu imposable moyen des foyers imposables ayant 1 part de quotient familial était égal à 0,60 fois le revenu imposable moyen des foyers imposables, celui des foyers ayant 1,5 parts de quotient familial était égal à 0,76 fois le revenu moyen, etc...; le revenu imposable moyen de l'ensemble des foyers fiscaux (imposables et nonimposables) ayant 1 part de quotient familial était égal à 0,57 fois le revenu imposable moyen de l'ensemble des foyers fiscaux (imposables et non-imposables), celui des foyers ayant 1,5 parts de quotient familial était égal à 0,70 fois le revenu moyen, etc...

Note: $1996\left(^{*}\right)$ : chiffres provisoires (rôles émis au 31/12/97) 
Tableau C-5: L'évolution du nombre de foyers et du revenu imposable moyen en fonction du nombre de parts de quotient familial dans les enquêtes "Revenus fiscaux" de 1970 à 1990

\begin{tabular}{|c|c|c|c|c|c|c|c|c|c|c|c|c|c|}
\hline 1970 & $Q F=1$ & $Q F=1,5$ & $Q F=2 A$ & $Q F=2 B$ & $Q F=2,5$ & $Q F=3$ & $\mathrm{QF}=3,5$ & $\mathrm{QF}=4$ & $\mathrm{QF}=4,5$ & $Q F=5$ & $\mathrm{QF}=5,5$ & $\mathrm{QF}>=6$ & \\
\hline RF70 & 23,70 & 6,95 & 28,19 & 1,03 & 18,74 & 13,69 & 5,21 & 1,63 & 0,55 & 0,16 & 0,09 & 0,07 & 100,00 \\
\hline E1921 & 26,69 & 6,23 & 27,64 & 0,96 & 18,38 & 12,82 & 4,97 & 1,56 & 0,49 & 0,17 & 0,06 & 0,03 & 100,00 \\
\hline F70(i-ni) & 22,92 & 13,87 & 24,90 & 1,09 & 13,90 & 11,91 & 6,25 & 2,75 & 1,28 & 0,56 & 0,28 & 0,30 & 100,00 \\
\hline estimation & 26,03 & 12,54 & 24,63 & 1,03 & 13,75 & 11,25 & 6,02 & 2,67 & 1,15 & 0,59 & 0,18 & 0,15 & 100,00 \\
\hline F70 & 0,56 & 0,75 & 1,06 & 0,82 & 1,14 & 1,29 & 1,46 & 1,69 & 1,76 & 2,33 & 1,68 & 1,81 & 1,00 \\
\hline E1921 & 0,56 & 0,80 & 1,07 & 0,77 & 1,15 & 1,32 & 1,51 & 1,74 & 1,93 & 2,08 & 2,30 & 2,36 & 1,00 \\
\hline F70(i-ni) & 0,57 & 0,51 & 1,13 & 0,84 & 1,38 & 1,43 & 1,34 & 1,27 & 1,16 & 1,18 & 1,00 & 1,10 & 1,00 \\
\hline estimation & 0,57 & 0,53 & 1,13 & 0,78 & 1,38 & 1,45 & 1,38 & 1,31 & 1,26 & 1,05 & 1,36 & 1,42 & 1,00 \\
\hline 1979 & $Q F=1$ & $Q F=1,5$ & $Q F=2 A$ & $Q F=2 B$ & $Q F=2,5$ & $Q F=3$ & $Q F=3,5$ & $Q F=4$ & $Q F=4,5$ & $Q F=5$ & $Q F=5,5$ & QF>=6 & \\
\hline RF79 & 21,54 & 9,30 & 28,84 & 1,98 & 17,69 & 14,45 & 4,65 & 1,14 & 0,08 & 0,27 & 0,04 & 0,03 & 100,00 \\
\hline E1921 & 25,70 & 8,40 & 26,64 & 1,84 & 17,33 & 14,26 & 4,50 & 1,04 & 0,03 & 0,19 & 0,05 & 0,02 & 100,00 \\
\hline F79(i-ni) & 24,61 & 12,17 & 24,90 & 2,10 & 14,52 & 12,98 & 5,27 & 1,89 & 0,19 & 0,81 & 0,31 & 0,25 & 100,00 \\
\hline estimation & 29,23 & 10,95 & 22,90 & 1,94 & 14,16 & 12,76 & 5,08 & 1,72 & 0,07 & 0,58 & 0,43 & 0,18 & 100,00 \\
\hline F79 & 0,58 & 0,66 & 1,07 & 0,90 & 1,18 & 1,30 & 1,43 & 1,50 & 1,41 & 1,71 & 1,80 & 1,71 & 1,00 \\
\hline E1921 & 0,58 & 0,69 & 1,09 & 0,74 & 1,19 & 1,34 & 1,49 & 1,67 & 1,61 & 2,01 & 2,08 & 2,20 & 1,00 \\
\hline F79(i-ni) & 0,51 & 0,55 & 1,19 & 0,87 & 1,36 & 1,44 & 1,38 & 1,17 & 0,83 & 1,03 & 0,82 & 0,77 & 1,00 \\
\hline estimation & 0,51 & 0,59 & 1,21 & 0,71 & 1,38 & 1,49 & 1,44 & 1,31 & 0,95 & 1,21 & 0,95 & 1,00 & 1,00 \\
\hline 1984 & $Q F=1$ & $Q F=1,5$ & $Q F=2 A$ & $Q F=2 B$ & $Q F=2,5$ & $Q F=3$ & $Q F=3,5$ & $\mathrm{QF}=4$ & $Q F=4,5$ & $Q F=5$ & $\mathrm{QF}=5,5$ & QF $>=6$ & \\
\hline RF84 & 22,52 & 8,77 & 26,69 & 2,69 & 18,98 & 15,31 & 0,49 & 3,62 & 0,72 & 0,16 & 0,04 & 0,00 & 100,00 \\
\hline E1921 & 26,54 & 8,62 & 25,16 & 3,07 & 17,69 & 14,33 & 0,41 & 3,43 & 0,61 & 0,11 & 0,02 & 0,01 & 100,00 \\
\hline RF84(i-ni) & 26,61 & 12,49 & 21,79 & 2,61 & 15,39 & 12,74 & 0,83 & 4,99 & 1,60 & 0,53 & 0,27 & 0,16 & 100,00 \\
\hline estimation & 30,85 & 12,07 & 20,21 & 2,93 & 14,11 & 11,73 & 0,69 & 4,65 & 1,33 & 0,37 & 0,17 & 0,89 & 100,00 \\
\hline RF84 & 0,60 & 0,70 & 1,05 & 0,78 & 1,13 & 1,34 & 1,28 & 1,62 & 1,85 & 1,79 & 1,74 & 3,69 & 1,00 \\
\hline E1921 & 0,61 & 0,73 & 1,08 & 0,73 & 1,17 & 1,37 & 1,29 & 1,68 & 1,90 & 2,05 & 2,09 & 2,24 & 1,00 \\
\hline RF84(i-ni) & 0,55 & 0,57 & 1,19 & 0,80 & 1,30 & 1,54 & 0,98 & 1,40 & 1,16 & 0,98 & 0,70 & 0,66 & 1,00 \\
\hline estimation & 0,57 & 0,60 & 1,23 & 0,76 & 1,37 & 1,59 & 1,01 & 1,47 & 1,21 & 1,14 & 0,85 & 0,40 & 1,00 \\
\hline 1990 & $\mathrm{QF}=1$ & $Q F=1,5$ & $Q F=2 A$ & $Q F=2 B$ & $Q F=2,5$ & $\mathrm{QF}=3$ & $\mathrm{QF}=3,5$ & $Q F=4$ & $Q F=4,5$ & $Q F=5$ & $\mathrm{QF}=5,5$ & QF $>=6$ & \\
\hline RF90 & 30,83 & 9,00 & 24,00 & 2,81 & 16,21 & 13,18 & 0,32 & 3,03 & 0,11 & 0,43 & 0,01 & 0,07 & 100,00 \\
\hline E1921 & 32,69 & 8,96 & 23,11 & 3,29 & 15,59 & 12,44 & 0,35 & 2,99 & 0,08 & 0,41 & 0,01 & 0,08 & 100,00 \\
\hline RF90(i-ni) & 30,62 & 12,21 & 19,39 & 4,47 & 14,51 & 11,2 & 0,84 & 4,69 & 0,27 & $0, \mathrm{c}$ & 0,11 & 0,73 & \\
\hline estimation & 32,24 & 12,06 & 18,54 & 5,20 & 13,86 & 10,53 & 0,92 & 4,61 & 0,19 & 0,88 & 0,16 & 0,83 & 100,00 \\
\hline RF90 & 0,56 & 0,75 & 1,11 & 0,87 & 1,2 & 1,4 & 1,36 & 1,86 & 1,9 & 2,3 & 3,43 & 2,63 & 1,00 \\
\hline E1921 & 0,58 & 0,77 & 1,14 & 0,85 & 1,25 & 1,47 & 1,43 & 1,86 & 1,86 & 2,39 & 2,17 & 2,76 & 1,00 \\
\hline F90(i-ni) & 0,56 & 0,64 & 1,25 & 0,73 & 1,34 & 1,61 & 0,80 & 1,48 & 1,02 & 1,42 & 0,77 & 0,95 & 1,00 \\
\hline estimation & 0,58 & 0,65 & 1,28 & 0,72 & 1,34 & 1,65 & 0,85 & 1,48 & 0,98 & 1,47 & 0,49 & 1,00 & 1,00 \\
\hline
\end{tabular}

Sources: Enquêtes "Revenus fiscaux" (INSEE) de 1970, 1979, 1984 et 1990 (cf. Annexe G)

Lecture : En 1970, l'enquête "RF" estime que $23,70 \%$ des foyers imposables ont 1 part de quotient familial, que $6,95 \%$ des foyers imposables ont 1,5 parts, etc..., alors que l'Etat 1921 estime ces chiffres à 26,69\%, 6,23\%, etc... L'enquête "RF" estime que 22,92\% de l'ensemble des foyers fiscaux (imposables et non-imposables) ont 1 part de quotient familial, que $13,87 \%$ ont 1,5 parts, etc.. (la ligne suivante ("estimation") redresse ces chiffres à partir de l'écart (en \% de l'estimation "RF") observé entre les estimations issues de l'enquête "RF" et de l'Etat 1921 pour les foyers imposables, puis en calant tous les pourcentages redressés de façon à ce que la somme soit égale à $100 \%$ ). L'enquête "RF" estime que le revenu imposable moyen des foyers imposables ayant 1 part de quotient familial est égal à 0,56 fois le revenu imposable moyen de l'ensemble des foyers imposables, que celui des foyers imposables ayant 1,5 parts de quotient familial est égal à 0,75 fois le revenu imposable moyen, etc..., alors que l'Etat 1921 estime ces chiffres à 0,56, 0,80, etc.. L'enquête "RF" estime que le revenu imposable moyen des foyers (imposables et non-imposables) ayant 1 part de quotient familial est égal à 0,57 fois le revenu imposable moyen de l'ensemble des foyers (imposables et non-imposables), que celui des foyers ayant 1,5 parts de quotient familial est égal à 0,51 fois le revenu moyen, etc... (la ligne suivante ("estimation") redresse ces chiffres de la même façon que précédemment). 
Tableau C-6: Estimation du nombre de foyers fiscaux et du revenu imposable moyen en fonction du nombre de parts de quotient familial, 1970-1996

\begin{tabular}{|c|c|c|c|c|c|c|c|c|c|c|c|c|c|c|c|c|}
\hline & $Q F=1$ & $Q F=1,5$ & $=24$ & סרתי & $=25$ & $Q F=3$ & $Q F>3$ & & $0-1$ & & $=-2 \Delta$ & ס2-5 & $=-25$ & $\mathrm{~F}=3$ & $Q F>3$ & \\
\hline 1970 & 27,86 & 10,73 & 24,45 & 1,73 & 13,89 & 11,71 & 9,63 & 100,00 & 0,57 & 0,54 & 1,15 & 0,68 & 1,36 & 1,49 & 1,33 & 1,00 \\
\hline 1971 & 28,10 & 10,77 & 24,11 & 1,82 & 13,89 & 11,71 & 9,60 & 100,00 & 0,57 & 0,54 & 1,16 & 0,68 & 1,36 & 1,49 & 1,32 & 1,00 \\
\hline 1972 & 28,34 & 10,81 & 23,77 & 1,92 & 3,89 & 11,71 & 9,56 & 100,00 & 0 & & 1,17 & 68 & 1,36 & 1,50 & 31 & 1,00 \\
\hline 1973 & 28,58 & 10,85 & 23,44 & 2,02 & 3,89 & 11,71 & 9,51 & 100,00 & 0,57 & & 1,17 & 0,68 & 1,36 & 1,51 & 1,31 & 1,00 \\
\hline 1974 & 28,82 & 10,90 & 23,11 & 2,13 & 13,89 & 11,71 & 9,44 & 100,00 & 0,57 & 0,56 & 1,18 & 0,68 & 1,36 & 1,51 & 1,30 & 1,00 \\
\hline 1975 & 29,06 & 10,94 & 22,79 & 2,25 & 13,89 & 11,71 & 9,3 & 100,00 & 0,57 & 56 & 1,19 & 0,68 & 1,36 & 1,52 & 1,30 & 1,00 \\
\hline 1976 & 29,31 & 10,98 & 22,47 & 2,37 & 3,89 & 11,71 & 9,27 & 100,00 & 0,57 & & 1,19 & 0,68 & 1,36 & 1,53 & 1,29 & 1,00 \\
\hline 1977 & 29,56 & 11,03 & 22,15 & 2,49 & 13,89 & 11,71 & 9,17 & 100,00 & 0,57 & 0,57 & 1,20 & 0,68 & 1,36 & 1,53 & 1,29 & 1,00 \\
\hline 1978 & 29,81 & 11,07 & 21,84 & 2,63 & 13,89 & 11,71 & 9,05 & 100,00 & 0,57 & 0,58 & 1,21 & 0,68 & 1,36 & 1,54 & 1,29 & 1,00 \\
\hline 1979 & 30,06 & 11,11 & 21,54 & 2,77 & 13,89 & 11,71 & 8,92 & 100,00 & 0,57 & & 1,21 & 0,68 & 1,36 & 1,55 & 1,29 & 1,00 \\
\hline 1980 & 30,32 & 11,16 & 21,24 & 2,92 & 13,89 & 11,71 & 8,77 & 100,00 & 0,57 & 59 & 1,22 & 0,68 & 1,36 & 1,55 & 1,28 & 1,00 \\
\hline 1981 & 30,57 & 11,20 & 20,94 & 3,07 & 13,89 & 11,71 & 8,61 & 100,00 & 0,57 & 0,60 & 1,23 & 0,68 & 1,36 & 1,56 & 1,29 & 1,00 \\
\hline 1982 & 30,83 & 11,25 & 20,64 & 3,24 & 3,89 & 11,71 & 8,44 & 00 & 0,57 & & 1,23 & 68 & ,36 &, 57 & 1,29 & ,00 \\
\hline 1983 & 31,09 & 11,29 & 20,36 & 3,41 & 13,89 & 11,71 & 8,25 & 100,00 & 0,57 & 0,61 & 1,24 & 0,68 & 1,36 & 1,57 & 1,29 & 1,00 \\
\hline 1984 & 31,36 & 11,34 & 20,07 & 3,60 & 13,89 & 11,71 & 8,04 & 100,00 & 0,57 & 0,61 & 1,25 & 0,68 & 1,36 & 1,58 & 1,29 & 1,00 \\
\hline 1985 & 31,62 & 11,38 & 9,79 & 3,79 & 89 & 11,71 & 7,82 & & 0 & & 1,25 & 68 & ,36 & 1,59 & 1,30 & 1,00 \\
\hline 1986 & 31,87 & 11,68 & 19,42 & 4,10 & 13,78 & 11,51 & 7,64 & & 0,57 & & 1,27 & & 1,36 & 1,60 & 1,30 & 1,00 \\
\hline 1987 & 32,22 & 11,79 & 18,80 & 4,42 & 13,91 & 11,31 & 7,55 & 100,00 & 0,57 & 0,64 & 1,28 & 0,68 & 1,36 & 1,62 & 1,31 & 1,00 \\
\hline 1988 & 32,59 & 11,97 & 18,58 & 4,67 & 13,78 & 11,00 & 7,41 & 100,00 & 0,56 & 0,64 & 1,29 & 0,69 & 1,37 & 1,64 & 1,32 & 1,00 \\
\hline 1989 & 32,90 & 12,12 & 18,43 & 4,95 & 13,66 & 10,67 & 7,27 & 10 & 0,57 & & 1,29 & & 1,37 & 1,67 & 1,34 & 1,00 \\
\hline 1990 & 33,50 & 12,03 & 18,02 & 5,24 & 13,55 & 10,37 & 7,29 & 100,00 & 0,57 & 0,66 & 1,31 & 0,70 & 1,37 & 1,69 & 1,34 & 1,00 \\
\hline 1991 & 33,69 & 12,36 & 17,85 & 5,44 & 13,45 & 10,04 & 7,16 & 100,00 & 0,57 & 0,66 & 1,32 & 0,71 & 1,37 & 1,70 & 1,35 & 1,00 \\
\hline 1992 & 33,65 & 12,72 & 17,62 & 5,68 & 13,31 & 9,90 & 7,13 & 100,00 & 0,57 & & 1,33 & 0,72 & 1,36 & 1,71 & 1,36 & 1,00 \\
\hline 1993 & 33,82 & 13,00 & 17,46 & 5,90 & 13,13 & 9,68 & 7,02 & 100,00 & 0,57 & 0,68 & 1,34 & 0,72 & 1,37 & 1,72 & 1,37 & 1,00 \\
\hline 1994 & 34,10 & 13,23 & 17,33 & 6,07 & 12,92 & 9,45 & 6,90 & 100,00 & 0,57 & 0,68 & 1,36 & 0,73 & 1,37 & 1,74 & 1,38 & 1,00 \\
\hline 1995 & 34,40 & 17,53 & 17,19 & 3,79 & 11,11 & 9,71 & 6,26 & 100,00 & 0,57 & 0,69 & 1,37 & 0,72 & 1,47 & 1,69 & 1,47 & 1,00 \\
\hline $996^{*}$ & 34,72 & 17,69 & 17,11 & 3,90 & 10,91 & 9,55 & 6,12 & 100,00 & 0,57 & 0,70 & 1,39 & 0,73 & 1,47 & 1,68 & 1,48 & 1,00 \\
\hline
\end{tabular}

Sources et méthodologie: cf. Annexe C.3.

Note: $1996\left(^{*}\right)$ : chiffres provisoires (rôles émis au 31/12/97) 
Tableau C-7: Estimation du nombre de foyers fiscaux et du revenu imposable moyen en fonction du nombre de parts de quotient familial, 1970-1996

$N . Q F=1 \quad R . Q F=1 \quad N \cdot Q F=1,5 R \cdot Q F=1,5 \quad N \cdot Q F=2 A \quad R \cdot Q F=2 A \quad N \cdot Q F=2 B \quad R \cdot Q F=2 B \quad N \cdot Q F=2,5 R \cdot Q F=2,5 \quad N \cdot Q F=3 \quad R \cdot Q F=3$

$\begin{array}{lllllllllllll}1970 & 5860452 & 42194 & 2256223 & 15305 & 5142446 & 75164 & 363533 & 3152 & 2922025 & 50241 & 2462802 & 46422\end{array}$

$\begin{array}{llllllllllllll}1971 & 6000497 & 47330 & 2299787 & 17261 & 5148036 & 82889 & 388922 & 3694 & 2966722 & 55882 & 2500474 & 51858\end{array}$

$\begin{array}{llllllllllllll}1972 & 6135788 & 53435 & 2341101 & 19593 & 5146837 & 92003 & 415535 & 4358 & 3008131 & 62561 & 2535376 & 58307\end{array}$

$\begin{array}{llllllllllllll}1973 & 6264413 & 61040 & 2379466 & 22502 & 5137668 & 103324 & 443282 & 5202 & 3045394 & 70864 & 2566782 & 66332\end{array}$

$\begin{array}{llllllllllllll}1974 & 6386503 & 72129 & 2414969 & 26734 & 5121114 & 120035 & 472199 & 6423 & 3078669 & 83035 & 2594828 & 78061\end{array}$

$\begin{array}{llllllllllllll}1975 & 6499664 & 84289 & 2446745 & 31411 & 5095747 & 137905 & 502129 & 7842 & 3106902 & 96218 & 2618624 & 90847\end{array}$

$\begin{array}{llllllllllllll}1976 & 6593757 & 98130 & 2471041 & 36766 & 5054352 & 157841 & 532255 & 9539 & 3125405 & 111077 & 2634219 & 105331\end{array}$

$\begin{array}{lllllllllllll}1977 & 6712340 & 113272 & 2504208 & 42670 & 5030628 & 179123 & 566139 & 11505 & 3154889 & 127140 & 2659069 & 121086\end{array}$

$\begin{array}{llllllllllllll}1978 & 6837662 & 130848 & 2539530 & 49557 & 5010390 & 203427 & 602587 & 13887 & 3186798 & 145634 & 2685963 & 139301\end{array}$

$\begin{array}{llllllllllllll}1979 & 6969924 & 150706 & 2577052 & 57387 & 4993530 & 230346 & 641804 & 16712 & 3221155 & 166327 & 2714921 & 159783\end{array}$

$\begin{array}{llllllllllllll}1980 & 7111157 & 174384 & 2617488 & 66763 & 4981217 & 262040 & 684190 & 20206 & 3258822 & 190843 & 2746668 & 184129\end{array}$

$\begin{array}{llllllllllllll}1981 & 7260734 & 202015 & 2660568 & 77761 & 4972691 & 298440 & 729927 & 24458 & 3299421 & 219226 & 2780886 & 212431\end{array}$

$\begin{array}{lllllllllllllll}1982 & 7412590 & 232962 & 2704040 & 90158 & 4963597 & 338351 & 778630 & 29470 & 3340134 & 250685 & 2815201 & 243968\end{array}$

$\begin{array}{llllllllllllll}1983 & 7550092 & 259488 & 2741857 & 100968 & 4943044 & 370520 & 828659 & 34299 & 3373517 & 276884 & 2843338 & 270633\end{array}$

$\begin{array}{lllllllllllllll}1984 & 7704753 & 281426 & 2785483 & 110097 & 4931927 & 395065 & 883578 & 38867 & 3413706 & 297770 & 2877211 & 292309\end{array}$

$\begin{array}{llllllllllllll}1985 & 7950726 & 304085 & 2861528 & 119606 & 4975999 & 419672 & 952698 & 43881 & 3493099 & 319042 & 2944127 & 314549\end{array}$

$\begin{array}{lllllllllllll}1986 & 8136737 & 324351 & 2982129 & 131427 & 4959579 & 440854 & 1047464 & 50310 & 3518923 & 334847 & 2938909 & 329685\end{array}$

$\begin{array}{llllllllllllll}1987 & 8486421 & 345775 & 3104835 & 141370 & 4953450 & 454315 & 1164346 & 57136 & 3664752 & 357794 & 2978382 & 345492\end{array}$

$\begin{array}{llllllllllllll}1988 & 8730297 & 364889 & 3206117 & 152387 & 4978967 & 477731 & 1252179 & 64270 & 3691267 & 373359 & 2947880 & 357846\end{array}$

$\begin{array}{llllllllllllll}1989 & 9002723 & 396227 & 3315912 & 164813 & 5041856 & 501097 & 1354108 & 73170 & 3737419 & 395147 & 2918050 & 375421\end{array}$

$\begin{array}{lllllllllllllll}1990 & 9390413 & 429573 & 3371801 & 177417 & 5051489 & 530371 & 1468496 & 82889 & 3796856 & 416455 & 2906695 & 393460\end{array}$

$\begin{array}{lllllllllllllll}1991 & 9637255 & 455106 & 3537160 & 192080 & 5107149 & 555100 & 1557541 & 91677 & 3846283 & 433621 & 2872531 & 402254\end{array}$

$\begin{array}{llllllllllllll}1992 & 9774905 & 470177 & 3695523 & 206137 & 5119457 & 569306 & 1651445 & 99512 & 3865616 & 442125 & 2875117 & 411384\end{array}$

$\begin{array}{lllllllllllllll}1993 & 9996852 & 480703 & 3841966 & 218869 & 5159932 & 583106 & 1742957 & 106187 & 3881742 & 446758 & 2859806 & 414029\end{array}$

$\begin{array}{llllllllllllll}1994 & 10243766 & 492241 & 3975214 & 229882 & 5205613 & 598439 & 1822765 & 112467 & 3881405 & 450698 & 2837553 & 417522\end{array}$

$\begin{array}{lllllllllllllll}1995 & 10522523 & 513358 & 5362660 & 319642 & 5257145 & 620346 & 1160612 & 72281 & 3398664 & 429487 & 2970279 & 431095\end{array}$

$\begin{array}{lllllllllllll}1996^{*} & 10666493 & 530822 & 5436287 & 332904 & 5256287 & 639997 & 1199338 & 76537 & 3352729 & 431074 & 2934411 & 432153\end{array}$

Source: Calculs effectués à partir des estimations du tableau C-6 et des estimations du nombre total de foyers et du revenu imposable total des tableaux C-1 et C-2.

Note: $1996\left(^{*}\right)$ : chiffres provisoires (rôles émis au 31/12/97) 
un nombre de parts donné à partir des variations de la proportion des foyers imposables ayant ce nombre de parts. Par exemple, suite à l'extension de la décote aux familles mise en place en 1986 (cf. section 2.1.2.), la proportion de foyers imposables ayant 1 part de quotient familial est passée subitement de $26,82 \%$ en 1985 à $31,44 \%$ en 1986, bien que dans le même temps la proportion de l'ensemble des foyers fiscaux ayant 1 part de quotient familial ait poursuivi tranquillement son trend séculaire, de $31,62 \%$ en 1985 à $31,87 \%$ en 1986. De même, la proportion de foyers imposables ayant 1 part de quotient familial est restée relativement stable depuis 1986, bien que pour l'ensemble des foyers fiscaux cette proportion ait suivi un trend croissant régulier, de $31,87 \%$ en 1986 à $34,72 \%$ en 1996 . Heureusement, les enquêtes "Revenus fiscaux " réalisées par l'INSEE nous fournissent des estimations pour l'ensemble des foyers fiscaux remontant jusqu'en 1970 (cf. Tableau C-5).

Insérer Tableau C-5: L'évolution du nombre de foyers et du revenu imposable moyen en fonction du nombre de parts de quotient familial dans les enquêtes "Revenus fiscaux" de 1970 à 1990

Le problème est que les estimations issues des enquêtes " Revenus fiscaux " ne sont pas entièrement fiables : outre qu'aucune variable sur le nombre de parts de quotient familial n'est disponible dans l'enquête de 1975 (cf. Annexe G), les enquêtes "Revenus fiscaux" sous-estiment systématiquement la proportion de foyers ayant 1 part de quotient familial, alors que la proportion de couples (avec ou sans enfants) est systématiquement surévaluée, ce qui s'explique assez bien par la méthodologie de ces enquêtes. ${ }^{120}$ Ces estimations doivent donc être redressés (cf. Tableau C-5). Les revenus imposables moyens en fonction du nombre de parts semblent par contre mieux estimés, et le redressement joue un rôle moins important. Plutôt que d'appliquer mécaniquement les chiffres bruts ou redressés issus des enquêtes "Revenus fiscaux", nous avons adopté les hypothèses simplificatrices suivantes afin d'estimer l'évolution du nombre de foyers en fonction du nombre de parts de quotient familial entre 1970 et 1985 (cf. Tableaux C-6 et C-7):

(i) Nous avons supposé que la proportion de foyers fiscaux ayant 1 part de quotient familial avait suivi le même trend sur la période 1970-1985 que sur la période 1985-1995, c'est-à-dire un taux de croissance annuel moyen de 0,847\%. De même, nous avons supposé que la proportion de foyers fiscaux ayant $2 \mathrm{~A}$ parts de quotient familial avait suivi le même trend sur la période 1970-1985 que sur la période 1985-1995, c'est-à-dire un taux de croissance annuel moyen de $-1,464 \%$. Ces hypothèses sont cohérentes à la fois avec les informations disponibles sur les foyers imposables (cf. Tableau C-3) et avec les estimations issues des enquêtes "Revenus fiscaux" (cf. Tableau C-5).

(ii) Pour la proportion de foyers fiscaux ayant 2B parts de quotient familial, nous avons supposé que la période 1970-1985 connaissait le même trend que la période 1985-1994 de 5,372\%. Le trend 1985-1994 a été utilisé de préférence au trend 1985-1995 car l'année 1995, du fait de la modification des règles pour l'attribution de part entière au premier enfant des couples non-mariés, est marquée par une chute brutale de la proportion de foyers ayant $2 \mathrm{~B}$ parts de

\footnotetext{
${ }^{120} \mathrm{Cf}$. Annexe A.2. Ce biais systématique est d'ailleurs reconnu dans les publications de I'INSEE consacrées aux enquêtes "Revenus fiscaux" (cf. Annexe G pour les références de ces publications), qui débutent toujours par une comparaison entre la distribution du nombre de parts estimée par l'enquête et la distribution issue des statistiques fiscales (c'est-à-dire des Etats 1921).
} 
quotient familial. Là encore, cette hypothèses sont cohérentes à la fois avec les informations disponibles sur les foyers imposables et avec les estimations issues des enquêtes "Revenus fiscaux".

(iii) Ni les informations disponibles sur les foyers imposables ni les estimations issues des enquêtes "Revenus fiscaux" révèlent le moindre trend substantiel pour la proportion de foyers fiscaux ayant 2,5 ou 3 parts de quotient familial sur la période 1970-1985. Plutôt que de faire des hypothèses hasardeuses sur les fluctuations de court-terme de ces proportions, nous avons donc supposé qu'elles avaient été constantes tout au long de la période 1970-1985.

(iv) La proportion de foyers ayant strictement plus de 3 parts de quotient familial en 1970 a été estimée en supposant que cette proportion avait suivi le même trend entre 1970 et 1985 qu'entre 1985 à 1994, c'est-à-dire un taux de croissance annuel moyen de $-1,381 \%$ (l'année 1994 a de nouveau été préféré à l'année 1995, car la forte chute de la proportion célibataires ayant droit à une part entière pour leur $1^{\mathrm{er}}$ enfant en 1995 a entraînée une baisse générale des proportions de foyers ayant un nombre élevé de parts). L'estimation ainsi obtenue (9,63\% en 1970 , contre $7,82 \%$ en 1985) est cohérente avec les estimations issues des enquêtes "Revenus fiscaux".

(v) La proportion de foyers ayant 1,5 parts de quotient familial en 1970 a été obtenue par différence avec les autres proportions pour 1970. Nous avons ensuite supposé que cette proportion avait suivi un trend linéaire entre cette estimation de 10,73\% pour 1970 et la valeur de $11,38 \%$ pour 1985. Les enquêtes "Revenus fiscaux" indiquent une légère baisse de cette proportion pendant les années 1970, suivie d'une légère hausse entre la fin des années 1970 et 1984, mais compte tenu de la médiocre fiabilité de ces estimations et de la faiblesse des fluctuations en jeu, nous avons préféré supposé un trend constant sur 1970-1985.

(vi) Enfin, la proportion de foyers ayant strictement plus de 3 parts pour les années 1971-1984 a été obtenue par différence avec toutes les autres proportions. La série ainsi obtenue est régulièrement décroissance, avec des taux de décroissance légèrement plus élevés depuis la fin des années 1970, ce qui est cohérent avec les estimations disponibles.

Insérer Tableau C-6: Estimations du nombre de foyers fiscaux et du revenu imposable moyen en fonction du nombre de parts de quotient familial, 1970-1996 Insérer Tableau C-7: Estimations du nombre de foyers fiscaux et du revenu imposable moyen en fonction du nombre de parts de quotient familial, 1970-1996

Ces hypothèses permettent d'obtenir la série de proportions reproduite dans la partie gauche du tableau C-6. Ces proportions ont ensuite été multipliées par la série portant sur le nombre total de foyers fiscaux obtenue dans le tableau C-1 pour obtenir les estimations de nombres absolus de foyers fiscaux en fonction du nombre de parts de quotient familial reproduites sur le tableau C-7.

Afin d'estimer l'évolution du revenu imposable moyen en fonction du nombre de parts de quotient familial sur la période 1970-1985, nous avons fait les hypothèses suivantes :

(i) Nous avons supposé que les ratio entre le revenu moyen des foyers ayant 1 part, 2B parts et 2,5 parts et le revenu moyen de l'ensemble des foyers étaient restés stables entre 1970 et 1985. La très grande stabilité de ces ratios pour les foyers à 1 part et 2,5 parts dans les enquêtes "Revenus fiscaux " justifie pleinement ces 
hypothèses. Pour les foyers à $2 \mathrm{~B}$ parts, les enquêtes "Revenus fiscaux " estiment en 1979 un ratio inférieur aux valeurs de 1970 et 1984, mais rien ne garantit la fiabilité de cette "courbe en $U$ ", et nous avons donc préféré supposer un ratio constant.

(ii) Pour les foyers ayant 1,5 parts , 2A parts ou 3 parts de quotient familial, nous avons retenu le ratio de 1970 issu de l'enquête "Revenus fiscaux", corrigé de l'écart observée en 1990 entre les ratios "RF" et les ratios issus de l'Etat 1921 pour l'ensemble des foyers fiscaux. Nous avons ensuite supposé que ces ratios avaient suivi un trend linéaire constant entre leur valeur de 1970 et leur valeur de 1985. Les trends ainsi obtenus sur la période 1970-1985 sont à peine plus faibles que les trends observés depuis 1985.

(iii) Enfin, nous avons calculé le ratio pour les foyers ayant strictement plus de 3 parts de quotient familial pour les années 1970-1984 par différence avec tous les autres ratios, de façon à ce que la moyenne des ratios (pondérée par les proportions de la partie gauche du tableau C-6) soit égale à 1,000. Les ratios ainsi obtenus sont relativement stables autour d'une valeur moyenne de 1,3 , ce qui est rassurant.

Ces hypothèses permettent d'obtenir la série de ratios reproduite dans la partie droite du tableau C-6. Ces ratios ont ensuite été multipliées par la série portant sur le revenu imposable total obtenue dans le tableau C-1 (et par la série de proportions de la partie gauche du tableau C-6) pour obtenir les estimations de revenu imposable total (en millions de francs courants) en fonction du nombre de parts de quotient familial reproduites sur le tableau C-7.

Les estimations du tableau C-7 pour la période 1970-1985 nous semblent relativement plausibles. En effet, même si nous ne disposons pas d'informations sur les éventuelles variations de court-terme du nombre et des revenus des foyers nonimposables en fonction de leur nombre de parts, ce qui nous a contraint à supposer des trends constants sur la période 1970-1985, le fait est que ces séries sont d'abord déterminées par des trends socio-démographiques lourds s'étalant sur de nombreuses années (si on fait exception des brusques variations dues aux changements de règles de calculs du nombre de parts), comme le montrent les séries issues des Etats 1921 pour la période 1985-1996. Quoi qu'il en soit, et de la même façon que pour les estimations du nombre total de foyers et le revenu imposable total (tous nombres de parts confondus) (cf. supra), les éventuelles erreurs des séries du tableau C-7 peuvent affecter légèrement nos estimations du niveau absolu de la part du revenu total détenu par P90-100, P99-100, etc..., et donc notre analyse de l'évolution générale de l'inégalité des revenus, mais ces éventuelles erreurs ne sont aucunement susceptibles de biaiser nos estimations de l'élasticité du revenu imposable. D'une part, tous les trends du tableau C-6 (à l'exception de celui concernant la proportion des foyers ayant 2B parts de quotient familial) sont des trends extrêmement faibles comparées aux variations annuelles observées pour la part de P90-100, P99-100, etc.., ce qui implique que ces variations annuelles sont robustes vis-à-vis d'éventuelles erreurs sur ces trends. D'autre part, l'effet d'une éventuelle erreur sur le nombre de foyers est nul (au premier ordre) sur les ratios du type P99-100/P90-100, etc.. , et notre analyse repose entièrement sur l'étude de tels ratios. Enfin, l'effet d'une éventuelle erreur sur le revenu imposable total d'un groupe de quotient familial donné est par définition nul sur les ratios du type P99-100/P90100. 


\title{
Annexe D : Méthodologie des estimations de la distribution du revenu imposable effectuées à partir des Etats 1921
}

\author{
D.1. L'estimation de la distribution du revenu imposable pour l'ensemble des foyers \\ fiscaux (tous nombres de parts de quotient familial confondus)
}

L'hypothèse de base de l'estimation est que la distribution du revenu imposable pour les hauts revenus suit une loi de Pareto, c'est à dire qu'à partir d'un certain niveau de revenu, la fonction de répartition $F(y)(F(y)$ est égal à la fraction de la population de foyers fiscaux étudiée dont le revenu est inférieur à y) peut être correctement approximée par une fonction du type:

$$
1-F(y)=(k / y)^{a} \quad(k>0, a>1)
$$

La densité $f(y)$ de la distribution a donc la forme $f(y)=a k^{a} / y^{(1+a)}$. Si la distribution du revenu imposable a cette forme, alors le revenu moyen $\mathrm{y}^{*}(\mathrm{y})$ des foyers fiscaux dont le revenu est supérieur à y est donné par :

$$
y^{*}(y)=\left[\int_{z>y} z f(z) d z\right] /\left[\int_{z>y} f(z) d z\right]=\left[\int_{z>y} d z / z^{a}\right] /\left[\int_{z>y} d z / z^{(1+a)}\right]=a y /(a-1)
$$

Autrement dit, la propriété remarquable de la loi de Pareto est que le ratio $\mathrm{y}^{*}(\mathrm{y}) / \mathrm{y}$ est égal à une constante $b=a /(a-1)$, indépendamment du niveau de revenu y. Pour estimer les paramètres $a$ et $b$, il suffit donc de connaître un niveau de revenu y et le revenu moyen $\mathrm{y}^{*}(\mathrm{y})$ des foyers dont le revenu est supérieur $\mathrm{y}$. II suffit alors de connaître le nombre de foyers fiscaux $\mathrm{N}(\mathrm{y})$ dont le revenu est supérieur à y pour estimer le paramètre $k$. La connaissance des paramètres a et $k$ permet alors d'estimer l'ensemble de la distribution des hauts revenus.

Les tableaux IIA (1982-1996) et les tableaux A (1970-1981) des Etats 1921 (cf. Annexe B) nous donnent pour chaque tranche de revenu imposable $\left[0 ; s_{1}\right],\left[s_{1} ; s_{2}\right], \ldots$, $\left[s_{i} ; s_{i+1}\right], \ldots,\left[s_{p} ;+\infty\right]$, le nombre total $N_{i}$ de foyers fiscaux imposables dont le revenu imposable est compris entre $s_{i}$ et $s_{i+1}$ et le revenu imposable total $Y_{i}$ de ces foyers fiscaux (tous nombres de parts de quotient familial confondus). ${ }^{121}$ Notons $\mathrm{N}_{\mathrm{i}}{ }^{*}=\mathrm{N}_{\mathrm{i}}+\mathrm{N}_{\mathrm{i}+1}+. .+\mathrm{N}_{\mathrm{p}}$ le nombre de foyers fiscaux imposables ayant un revenu imposable supérieur à $\mathrm{s}_{i} ; \mathrm{p}_{\mathrm{i}}=\mathrm{N}_{\mathrm{i}}^{*} / \mathrm{N}^{*}$ (où $\mathrm{N}^{*}$ est le nombre total de foyers fiscaux (imposables et non-imposables) estimé dans l'annexe C.1, tableau C-1) la proportion de foyers fiscaux ayant un revenu imposable supérieur à $s_{i}$. Notons $y_{i}=\left(Y_{i}+\ldots+Y_{p}\right) / N_{i}{ }^{*}$ le revenu imposable moyen de ces foyers, et $b_{i}=y_{i} / s_{i}$ le ratio entre le revenu moyen de ces foyers et leur revenu minimum. Notre objectif étant d'étudier l'évolution de la distribution des hauts revenus, nous avons utilisé uniquement les tranches qui concernent le $10^{\text {ème }}$ décile de la distribution du revenu imposable, c'est-à-dire les tranches $\left[\mathrm{s}_{\mathrm{i}^{*}} ; \mathrm{s}_{\mathrm{i}^{*}+1}\right], \ldots,\left[\mathrm{s}_{\mathrm{p}} ;+\infty\right]$, où $\left[\mathrm{s}_{\mathrm{i}^{*}} ; \mathrm{s}_{\mathrm{i}^{*}+1}\right]$ est la tranche la plus élevée telle que $p_{i^{*}}>0,1$. Le tableau D-1 décrit pour chaque année les seuils des tranches utilisées, ainsi que les valeurs de $p_{i}$ et $b_{i}$ pour chacune des tranches.

\footnotetext{
${ }^{121}$ Nous avons pris en compte uniquement les foyers imposables sur l'ensemble de la période 19701996, bien que les Etats 1921 portent également sur les foyers non-imposables sur la période 19851996. Ce choix est sans conséquence, car le nombre de foyers non-imposables dont le revenu imposable est compris dans les tranche supérieures des tableaux des Etats 1921 est suffisamment faible pour être négligé (cf. les tests de fiabilité de l'annexe E).
} 
Insérer Tableau D-1: Les seuils de revenu imposable et les ratios $\mathrm{y}^{*}(\mathrm{y}) / \mathrm{y}$ utilisés pour l'estimation de la distribution du revenu imposable de l'ensemble des foyers fiscaux (tous nombres de parts confondus)

Le tableau D-1 montre les conséquences du fait que les tranches de revenu imposable en francs courants utilisées dans les Etats 1921 n'ont pratiquement pas été indexées sur l'inflation sur la période 1970-1996: alors que les tranches des tableaux du début des années 1970 permettent de connaître dans le détail les revenus à l'intérieur du centile supérieur, et ce jusqu'au $0,01 \%$ les plus riches (c'està-dire les 2000 foyer les plus aisés), la tranche supérieure des tableaux des années 1990 regroupe près de $0,7 \%$ des foyers fiscaux. Mais le tableau D-1 montre surtout l'extraordinaire stabilité des ratios $b_{i}$. Sur l'ensemble de la période 1970-1996, le revenu moyen des foyers dont le revenu est supérieur à un seuil de revenu donné est toujours de l'ordre de 1,7-1,8 fois le niveau de ce seuil, et ce quel que soit le seuil de revenu considéré. Ces coefficients $b_{i}$ de l'ordre de 1,7-1,8 correspondent à des coefficients $a_{i}=b_{i} /\left(b_{i}-1\right)$ de l'ordre de 2,3-2,4. A titre de comparaison, ces coefficients a (estimés au niveau de P99,5) sont passés aux Etats-Unis de 2,3-2,4 à la fin des années 1970 à environ 1,6 à la fin des années 1980, ce qui correspond à un coefficient $b$ de l'ordre de $2,7 .{ }^{122}$ Remarquons également que sur l'ensemble de la période 1970-1996, les ratios $b_{i}$ ont tendance à croître légèrement avec le niveau de revenu (tout du moins à partir d'un certain niveau de revenu). ${ }^{123}$

Pour chaque année et pour chaque tranche $\left[s_{i} ; s_{i+1}\right]$, les coefficients $a_{i}=b_{i} /\left(b_{i}-1\right)$ permettent de calculer les constantes $k_{i}=s_{i} p_{i}^{(1 / a i)}$. Par exemple, pour l'année 1970, $k_{i}=$ $30000 \times\left(0,0640^{(1,77-1) / 1,77}\right)=9082$ francs pour $s_{i}=30000$, et $k_{i}=40000 \times\left(0,0331^{(1,76-1) / 1,76}\right)$ $=9126$ francs pour $s_{i}=40000 .^{124}$ Chaque paire $\left(a_{i}, k_{i}\right)$ permet ensuite d'estimer l'ensemble de la distribution des hauts revenus à l'aide de la formule $1-F(y)=(k / y)^{a}$. Par exemple, pour estimer le seuil P95 (c'est-à-dire le seuil de revenu imposable audelà duquel se trouve $5 \%$ des foyers fiscaux), il suffit d'appliquer la formule P95 = $\mathrm{K}_{\mathrm{i}} /(0,05)^{(1 / a i)}$. Pour l'année 1970 , on trouve ainsi $P 95=9082 /(0,05)^{(1,77-1) / 1,77)}=33395$ francs en utilisant les $\left(a_{i}, k_{i}\right)$ obtenus pour $s_{i}=30000$, et $P 95=9126 /(0,05)^{(1,76-}$ 1)/1,76) $=33432$ francs en utilisant les $\left(a_{i}, k_{i}\right)$ obtenus pour $s_{i}=40000$. Le tableau D-2 décrit les estimations des seuils P90, P95, P99, P99,5, P99,9 et P99,99 ainsi obtenues grâce à ces formules. Les estimations obtenus en utilisant différentes paires $\left(a_{i}, k_{i}\right)$ sont généralement extrêmement proches les unes des autres, pourvu que les seuils $s_{i}$ dont elles sont issues ne soient pas trop éloignées du seuil que l'on cherche à estimer. Dans le tableau D-2, comme dans l'ensemble de cette étude, nous avons retenu les estimations obtenues en utilisant toujours les coefficients $\left(a_{i}, k_{i}\right)$ correspondant au seuil $s_{i}$ le plus proche (en fractile) du seuil que l'on cherche à estimer. Par exemple, le seuil P95 indiqué sur le tableau D-2 pour l'année 1970 (33395 francs) a été calculé à partir des coefficients $\left(\mathrm{a}_{\mathrm{i}}, \mathrm{k}_{\mathrm{i}}\right)$ correspondant au seuil $s_{i}=30000$, car $p_{i}=6,40 \%$ est le $p_{i}$ le plus proche de $5 \%$ (parmi tous les $p_{i}$ disponibles pour l'année 1970).

\footnotetext{
${ }^{122}$ Cf. Feenberg et Poterba (1993, Table A-1, p.173).

${ }^{123}$ Les ratios $b_{i}$ diminuent à nouveau lorsqu'il ne reste plus que quelques contribuables au-delà du seuil considéré ; par définition, le ratio $\mathrm{y}^{*}(\mathrm{y}) / \mathrm{y}$ est en effet égal à 1 si y est le revenu les plus élevé de la distribution (cf. par exemple le tableau D-7 en 1970, année où seuls 15 contribuables ayant 2B parts de quotient familial ont un revenu imposable supérieur à 400000 francs).

${ }_{124}$ Les calculs décrits ici ne coïncident pas à l'unité près, du fait des erreurs d'arrondis (pour que les calculs coïncident à l'unité près, il faut utiliser les coefficients $p_{i}$ et $b_{i}$ avec d'avantage de chiffres après la virgule que ceux qui sont reproduits sur le tableau D-1).
} 
Tableau D-1: Les seuils de revenu imposable et les ratios $y^{*}(y) / y$ utilisés pour l'estimation de la distribution du revenu imposable de l'ensemble des foyers fiscaux (tous nombres de parts confondus)

\begin{tabular}{|c|c|c|c|c|c|c|c|c|c|c|c|c|c|c|}
\hline \multirow[b]{2}{*}{$\mathrm{s}_{\mathrm{i}}$} & \multicolumn{2}{|c|}{1970} & \multicolumn{2}{|c|}{1971} & \multicolumn{2}{|c|}{1972} & \multicolumn{2}{|c|}{1973} & \multicolumn{2}{|c|}{1974} & \multicolumn{2}{|c|}{1975} & \multicolumn{2}{|c|}{1976} \\
\hline & $\mathrm{p}_{\mathrm{i}}$ & $b_{i}$ & $\mathrm{p}_{\mathrm{i}}$ & $\mathrm{b}_{\mathrm{i}}$ & $\mathrm{p}_{\mathrm{i}}$ & $b_{i}$ & $\mathrm{p}_{\mathrm{i}}$ & $b_{i}$ & $\mathrm{p}_{\mathrm{i}}$ & $b_{i}$ & $\mathrm{p}_{\mathrm{i}}$ & $b_{i}$ & $\mathrm{p}_{\mathrm{i}}$ & $b_{i}$ \\
\hline 20000 & 15,08 & 1,82 & & & & & & & & & & & & \\
\hline 25000 & & & 11,76 & 1,80 & 14,11 & 1,81 & & & & & & & & \\
\hline 30000 & 6,40 & 1,77 & 7,91 & 1,78 & 9,58 & 1,80 & 12,77 & 1,81 & 17,59 & 1,80 & & & & \\
\hline 40000 & 3,31 & 1,76 & 4,09 & 1,79 & 4,97 & 1,80 & 6,70 & 1,82 & 9,43 & 1,77 & 13,29 & 1,76 & & \\
\hline 50000 & 1,98 & 1,76 & 2,46 & 1,79 & 2,98 & 1,81 & 4,01 & 1,83 & 5,57 & 1,79 & 7,92 & 1,76 & 10,82 & 1,75 \\
\hline 70000 & 0,92 & 1,75 & 1,16 & 1,77 & 1,41 & 1,80 & 1,91 & 1,83 & 2,57 & 1,79 & 3,54 & 1,78 & 4,81 & 1,78 \\
\hline 100000 & 0,41 & 1,73 & 0,53 & 1,75 & 0,65 & 1,78 & 0,88 & 1,81 & 1,17 & 1,78 & 1,58 & 1,78 & 2,10 & 1,79 \\
\hline 200000 & 0,078 & 1,72 & 0,10 & 1,72 & 0,13 & 1,74 & 0,19 & 1,79 & 0,25 & 1,73 & 0,34 & 1,73 & 0,45 & 1,74 \\
\hline \multirow[t]{2}{*}{400000} & 0,014 & 1,77 & 0,019 & 1,76 & 0,025 & 1,80 & 0,038 & 1,85 & 0,047 & 1,74 & 0,064 & 1,75 & 0,088 & 1,75 \\
\hline & \multicolumn{2}{|c|}{1977} & \multicolumn{2}{|c|}{1978} & \multicolumn{2}{|c|}{1979} & \multicolumn{2}{|c|}{1980} & \multicolumn{2}{|c|}{1981} & \multicolumn{2}{|c|}{1982} & \multicolumn{2}{|c|}{1983} \\
\hline $\mathrm{s}_{\mathrm{i}}$ & $\mathrm{p}_{\mathrm{i}}$ & $b_{i}$ & $\mathrm{p}_{\mathrm{i}}$ & $\mathrm{b}_{\mathrm{i}}$ & $\mathrm{p}_{\mathrm{i}}$ & $b_{i}$ & $\mathrm{p}_{\mathrm{i}}$ & $b_{i}$ & $\mathrm{p}_{\mathrm{i}}$ & $b_{i}$ & $\mathrm{p}_{\mathrm{i}}$ & $b_{i}$ & $\mathrm{p}_{\mathrm{i}}$ & $b_{i}$ \\
\hline 50000 & 13,57 & 1,73 & & & & & & & & & & & & \\
\hline 60000 & & & 11,65 & 1,72 & & & & & & & & & & \\
\hline 70000 & 6,05 & 1,73 & 8,03 & 1,73 & 10,33 & 1,74 & & & & & & & & \\
\hline 80000 & & & 5,75 & 1,74 & 7,45 & 1,74 & 10,15 & 1,72 & 14,01 & 1,71 & & & & \\
\hline 100000 & 2,53 & 1,75 & 3,32 & 1,76 & 4,29 & 1,77 & 5,80 & 1,74 & 8,16 & 1,71 & 10,77 & 1,67 & 14,60 & 1,65 \\
\hline 125000 & & & & & & & & & & & & & 8,43 & 1,64 \\
\hline 150000 & & & & & & & & & & & 3,84 & 1,69 & 5,20 & 1,65 \\
\hline 200000 & 0,50 & 1,74 & 0,68 & 1,74 & 0,88 & 1,77 & 1,12 & 1,76 & 1,50 & 1,75 & 1,88 & 1,70 & 2,46 & 1,66 \\
\hline \multirow[t]{2}{*}{400000} & 0,10 & 1,77 & 0,13 & 1,75 & 0,18 & 1,77 & 0,22 & 1,76 & 0,29 & 1,77 & 0,34 & 1,72 & 0,43 & 1,67 \\
\hline & \multicolumn{2}{|c|}{1984} & \multicolumn{2}{|c|}{1985} & \multicolumn{2}{|c|}{1986} & \multicolumn{2}{|c|}{1987} & \multicolumn{2}{|c|}{$1988\left(^{*}\right)$} & \multicolumn{2}{|c|}{1989} & \multicolumn{2}{|c|}{1990} \\
\hline $\mathrm{s}_{\mathrm{i}}$ & $\mathrm{p}_{\mathrm{i}}$ & $\mathrm{b}_{\mathrm{i}}$ & $\mathrm{p}_{\mathrm{i}}$ & $\mathrm{b}_{\mathrm{i}}$ & $\mathrm{p}_{\mathrm{i}}$ & $b_{i}$ & $\mathrm{p}_{\mathrm{i}}$ & $b_{i}$ & $\mathrm{p}_{\mathrm{i}}$ & $b_{i}$ & $\mathrm{p}_{\mathrm{i}}$ & $b_{i}$ & $\mathrm{p}_{\mathrm{i}}$ & $b_{i}$ \\
\hline 100000 & 16,77 & 1,67 & & & & & & & & & & & & \\
\hline 125000 & 9,93 & 1,64 & 11,40 & 1,65 & 12,46 & 1,68 & 12,95 & 1,70 & 13,94 & 1,72 & & & & \\
\hline 150000 & 6,17 & 1,64 & 7,20 & 1,65 & 8,00 & 1,67 & 8,41 & 1,70 & 9,19 & 1,71 & 10,10 & 1,73 & 11,21 & 1,74 \\
\hline 200000 & 2,89 & 1,66 & 3,38 & 1,67 & 3,80 & 1,69 & 4,05 & 1,72 & 4,50 & 1,73 & 4,98 & 1,76 & 5,62 & 1,75 \\
\hline 250000 & 1,64 & 1,66 & 1,92 & 1,68 & 2,17 & 1,70 & 2,32 & 1,74 & 2,63 & 1,74 & 2,88 & 1,78 & 3,25 & 1,77 \\
\hline 500000 & 0,28 & 1,68 & 0,34 & 1,69 & 0,40 & 1,72 & 0,45 & 1,78 & 0,54 & 1,72 & 0,57 & 1,84 & 0,63 & 1,84 \\
\hline & 195 & & 199 & & 199 & & 199 & & $19 s$ & & 1996 & & & \\
\hline $\mathrm{s}_{\mathrm{i}}$ & $\mathrm{p}_{\mathrm{i}}$ & $b_{i}$ & $\mathrm{p}_{\mathrm{i}}$ & $b_{i}$ & $\mathrm{p}_{\mathrm{i}}$ & $b_{i}$ & $\mathrm{p}_{\mathrm{i}}$ & $b_{i}$ & $\mathrm{p}_{\mathrm{i}}$ & $b_{i}$ & $\mathrm{p}_{\mathrm{i}}$ & $b_{i}$ & & \\
\hline 150000 & 12,05 & 1,72 & 12,65 & 1,70 & 12,94 & 1,69 & 13,23 & 1,70 & 13,70 & 1,70 & 14,05 & 1,70 & & \\
\hline 200000 & 6,07 & 1,71 & 6,39 & 1,69 & 6,56 & 1,67 & 6,74 & 1,67 & 7,04 & 1,67 & 7,30 & 1,66 & & \\
\hline 250000 & 3,48 & 1,73 & 3,63 & 1,70 & 3,70 & 1,68 & 3,80 & 1,68 & 3,97 & 1,68 & 4,14 & 1,67 & & \\
\hline 500000 & 0,64 & 1,80 & 0,64 & 1,77 & 0,63 & 1,76 & 0,64 & 1,78 & 0,67 & 1,76 & 0,68 & 1,76 & & \\
\hline
\end{tabular}

Source: Etats 1921 (cf. Annexe D.1.)

Lecture: En 1970, 15,08\% des foyers fiscaux ont un revenu imposable supérieur à 20000FF, et leur revenu imposable moyen est 1,82 fois supérieur à $20000 \mathrm{FF} ; 6,40 \%$ ont un revenu imposable supérieur à $30000 \mathrm{FF}$, et leur revenu imposable moyen est 1,77 fois supérieur à $30000 \mathrm{FF}$; etc...

Notes: (i) $1988\left(^{*}\right)$ : les chiffres ne sont pas homogènes aux chiffres des autres années (les tranches de revenu imposable incluent les plus-values à taux proportionnel).

(ii) $1996\left({ }^{* *}\right)$ : chiffres provisoires (rôles émis au 31/12/1997). 
Insérer Tableau D-2: Les seuils P90, P95, P99, P99,5, P99,9 et P99,99 de la distribution du revenu imposable de l'ensemble des foyers fiscaux (tous nombres de parts confondus)

A partir des estimations de seuils du tableau D-2, on peut estimer la part du revenu imposable total détenue par les $10 \%$ des foyers fiscaux ayant le revenu imposable le plus élevé (P90-100), la part des 5\% ayant le revenu imposable le plus élevé (P95100), etc... Par exemple, pour estimer la part de P95-100, il suffit d'appliquer la formule P95-100 $=5 b_{i} P 95 / y^{*}$, où $y^{*}=Y^{*} / N^{*}$ est le revenu imposable moyen $\left(Y^{*}\right.$ est le revenu imposable total estimé dans l'annexe C.2, tableau C-2). Pour l'année 1970, on trouve ainsi $\mathrm{P} 95-100=0,05 \times 1,77 \times 33395 / 12673=0,2331$, c'est-à-dire $23,31 \%$ du revenu total, en utilisant les coefficients $\left(a_{i}, k_{i}\right)$ obtenus pour $s_{i}=30000$, et P95$100=5 \times 1,76 \times 33432 / 12673=23,28(23,28 \%$ du revenu total $)$ en utilisant les coefficients $\left(\mathrm{a}_{\mathrm{i}}, \mathrm{k}_{\mathrm{i}}\right)$ obtenus pour $\mathrm{s}_{\mathrm{i}}=40000$. Les estimations de la part de P90-100, P95-100, P99100, P99,5-100, P99,9-100 et P99,99-100 obtenues grâce à ces formules sont reproduites sur le tableau $3-3$ (section 3.2). ${ }^{125}$

La seule correction que nous avons apportée à ces estimations concerne l'année 1988. En effet, pour la première et dernière fois sur la période 1970-1996, l'Etat 1921 portant sur les revenus de 1988 incluent les plus-values taxées à taux proportionnel dans le concept de "revenu imposable" utilisé dans les tranches de "revenu imposable " des différents tableaux. Les coefficients $b_{i}$ pour l'année 1988 ont été calculés en soustrayant les plus-values taxées à taux proportionnel du revenu imposable, faute de quoi les coefficients obtenus seraient beaucoup plus élevés, ${ }^{126}$ mais ces coefficients demeurent néanmoins fortement biaisés, car il est impossible d'effectuer la même correction pour les seuils des tranches de "revenu imposable " utilisées dans l'Etat 1921. Cela explique pourquoi les coefficients $b_{i}$ reportés sur le tableau D-1 pour l'année 1988 sont étrangement faibles (comparés aux coefficients des années 1987 et 1989) : par exemple, le revenu imposable moyen (hors plusvalues taxées à taux proportionnel) des contribuables dont le revenu imposable (y compris les plus-values à taux proportionnel) est supérieur à 500000 francs est relativement faible comparé à 500000 francs (puisque ce groupe inclut notamment des contribuables dont le revenu imposable (hors plus-values taxées à taux proportionnel) est en fait inférieur à 500000 francs). Nous avons donc décider de ne pas utiliser les estimations effectuées à partir de l'Etat 1921 pour l'année 1988. Les seuils indiqués sur le tableau D-2 pour l'année 1988, ainsi que les parts de P90-100, P95-100, etc..., indiqués sur le tableau 3-3 pour l'année 1988 ont été obtenus à partir de l'échantillon léger de déclarations de revenus 1988, en

\footnotetext{
${ }^{125}$ La technique d'extrapolation que nous avons utilisé est légèrement différente de celle utilisée par Feenberg et Poterba (1993) : pour estimer la part des revenus détenue par P99,5-100, Feenberg et Poterba utilisent les coefficients $\left(\mathrm{a}_{\mathrm{i}}, \mathrm{k}_{\mathrm{i}}\right)$ estimés à partir des $\left(\mathrm{s}_{\mathrm{i}}, \mathrm{p}_{\mathrm{i}}\right)$ et $\left(\mathrm{s}_{\mathrm{i}+1}, \mathrm{p}_{\mathrm{i}+1}\right)$ correspondant à la tranche de revenu imposable $\left[s_{i}, s_{i+1}\right]$ entourant le seuil P99,5 $\left(a_{i}=\log \left(p_{i} / p_{i+1}\right) / \log \left(s_{i+1} / s_{i}\right)\right.$ et $k_{i}=s_{i} p_{i}^{1 / a i} ; c f$. Feenberg et Poterba (1993, p.172)). Nous avons également testé cette méthode, et il est apparu que la fiabilité des estimations obtenues était sensiblement meilleure en utilisant notre technique d'extrapolation, probablement du fait que la méthode de Feenberg-Poterba revient à ne pas utiliser la moindre information sur le revenu moyen des contribuables dont le revenu est supérieur à $s_{i}$ (ou à $s_{i+1}$ ), bien que cette information soit disponible (et très "informative"). Pour d'autres techniques d'estimations des paramètres d'une loi de Pareto, cf. Quandt (1966).

${ }^{126}$ Les coefficients $b_{i}$ indiqués sur le tableau D-1 pour 1988 passeraient de $1,72-1,71-1,73-1,74-1,72$ à 1,79-1,79-1,85-1,91-2,09 si l'on incluait les plus-values taxées à taux proportionnel dans le revenu imposable, ce qui montre l'extrême concentration et l'importance des plus-values taxées à taux proportionnels pour les hauts revenus (le revenu imposable moyen des contribuables dont le revenu imposable est supérieur à 500000 francs augmenterait de plus de 20\%).
} 
Tableau D-2 : Les seuils P90, P95, P99, P99,5, P99,9 et P99,99 de la distribution du revenu imposable de l'ensemble des foyers fiscaux (tous nombres de parts confondus)

$\begin{array}{ccccccc} & \mathrm{P} 90 & \mathrm{P} 95 & \mathrm{P} 99 & \mathrm{P} 99,5 & \mathrm{P} 99,9 & \mathrm{P} 99,99 \\ 1970 & 24708 & 33395 & 67637 & 91884 & 180110 & 468546 \\ 1971 & 26869 & 36636 & 74690 & 102161 & 203641 & 532977 \\ 1972 & 29438 & 39889 & 82700 & 111995 & 227124 & 605313 \\ 1973 & 33482 & 45242 & 94489 & 130540 & 254964 & 735862 \\ 1974 & 38981 & 52435 & 106941 & 148388 & 290304 & 774052 \\ 1975 & 45218 & 60143 & 122169 & 169103 & 329353 & 884805 \\ 1976 & 51714 & 68813 & 142710 & 191747 & 378813 & 1017347 \\ 1977 & 56844 & 75869 & 149207 & 200576 & 394434 & 1076499 \\ 1978 & 63962 & 84895 & 169459 & 227679 & 450665 & 1208689 \\ 1979 & 70984 & 93526 & 189298 & 255453 & 514351 & 1399950 \\ 1980 & 80485 & 106476 & 210236 & 283343 & 567648 & 1533941 \\ 1981 & 91940 & 122481 & 237885 & 315874 & 634993 & 1724360 \\ 1982 & 103040 & 134672 & 255357 & 341322 & 669523 & 1755428 \\ 1983 & 116975 & 152279 & 284346 & 375366 & 715319 & 1799504 \\ 1984 & 124677 & 162869 & 304528 & 396369 & 761507 & 1938104 \\ 1985 & 131608 & 173131 & 322436 & 427416 & 822383 & 2097518 \\ 1986 & 137203 & 178891 & 340949 & 455229 & 890688 & 2326814 \\ 1987 & 139690 & 183093 & 350926 & 475365 & 961800 & 2636056 \\ 1988 & 142733 & 192530 & 364626 & 493932 & 1009568 & 2854054 \\ 1989 & 150625 & 199654 & 386580 & 530763 & 1108003 & 3175723 \\ 1990 & 157427 & 210188 & 403703 & 553934 & 1154731 & 3302929 \\ 1991 & 162153 & 216876 & 410094 & 558022 & 1140909 & 3174090 \\ 1992 & 165270 & 219057 & 411534 & 556323 & 1120257 & 3049507 \\ 1993 & 166697 & 221362 & 409619 & 552927 & 1109647 & 3006000 \\ 1994 & 168251 & 223547 & 411513 & 557325 & 1127108 & 3087081 \\ 1995 & 173848 & 227778 & 419665 & 566731 & 1138489 & 3088547 \\ 1996\left(^{*}\right) & 176486 & 231774 & 422628 & 569903 & 1140998 & 3080430\end{array}$

Source: Estimations de l'auteur à partir des Etats 1921 (cf. Annexe D.1.)

Lecture: En 1970, le seuil inférieur du 90ème centile ("P90") de la distribution du revenu imposable (tous foyers fiscaux condondus) est de 24708FF; le seuil inférieur du 95ème centile ("P95") est de 33395FF; etc...

Note: $1996\left(^{*}\right)$ : chiffres provisoires (rôles émis au 31/12/97). 
corrigeant les estimations issues de l'échantillon léger d'un même coefficient d'erreur que celui observé en 1989 (cf. annexe E, tableau E-1).

\section{D.2. L'estimation de la distribution du revenu imposable en fonction du nombre de part de quotient familial}

Pour estimer la distribution du revenu imposable des foyers ayant un nombre donné de parts de quotient familial (1 part, 1,5 parts, 2A parts, 2B parts, 2,5 parts et 3 parts), nous avons suivi exactement la même méthodologie que pour la distribution de l'ensemble des foyers fiscaux (tous nombres de parts confondus), en utilisant les différents sous-tableaux des tableaux IIA/A des Etats 1921 et les estimations du nombre de foyers et du revenu imposable total en fonction du nombre de parts de quotient familial obtenues dans l'annexe C-3 (tableau C-7). Le tableau D-3 indique pour chaque année les seuils des tranches utilisées, ainsi que les valeurs de $p_{i}$ et $b_{i}$ obtenues pour chacune des tranches pour les foyers fiscaux ayant 1 part de quotient familial. Le tableau D-4 donne les résultats des estimations des seuils P95, P98, P99, P99,5, P99,9 et P99,99 de la distribution des foyers ayant 1 part de quotient familial. .... pour les célibataires, uniquement les top $5 \%$..

Insérer Tableau D-3: Les seuils de revenu imposable et les ratios $y^{*}(y) / y$ utilisés pour l'estimation de la distribution du revenu imposable des foyers fiscaux ayant 1 part de quotient familial.

Insérer Tableau D-4: Les seuils P90, P95, P99, P99,5, P99,9 et P99,99 de la distribution du revenu imposable des foyers fiscaux ayant 1 part de quotient familial

Les estimations de la part du revenu imposable des foyers ayant 1 part de quotient familial détenue par P95-100, P98-100, P99-100, P99,5-100, P99,9-100 et P99,99100 sont données dans le tableau 4-1 (section 4). Pour les foyers ayant 1 part de quotient familial, de même que pour les 5 autres groupes de quotient familial étudiés, nous avons également estimé les seuils P99,1, P99,2, P99,3,..,P99,8, P99, 9 (ainsi que les parts $\mathrm{P} 99,1-100, \ldots, \mathrm{P} 99,9-100$ correspondantes), afin de pouvoir déterminer avec précision les seuils des tranches d'imposition du barème de l'IR et d'estimer ainsi les taux marginaux moyens des foyers de P99-99,5, P99,5-100, etc..., utilisés dans la section 4. Les différentes étapes de l'estimation et les résultats détaillés concernant la distribution du revenu imposable des 5 autres groupes de quotient familial étudiés sont décrits dans les tableaux D-5 à D-14.

\section{Insérer Tableaux D-5 à D-14}

De la même façon que pour la distribution du revenu imposable de l'ensemble des foyers fiscaux, la seule correction que nous avons apportée à ces estimations concerne l'année 1988, qui est la seule année pour laquelle les tranches de revenu imposable utilisées dans l'Etat 1921 incluent les plus-values taxées à taux proportionnel, ce qui explique les ratios $\mathrm{y}^{*}(\mathrm{y}) / \mathrm{y}$ extrêmement élevés obtenus pour les hauts revenus de l'année 1988 sur les tableaux D-3, D-5, D-7, D-9, D-11 et D-13. ${ }^{127}$

\footnotetext{
${ }^{127}$ La composition du revenu imposable n'étant disponible dans les Etats 1921 uniquement pour l'ensemble des foyers fiscaux (tableaux IA/B), les ratios $\mathrm{y}^{*}(\mathrm{y}) / \mathrm{y}$ indiqués sur les tableaux D-3 à D-13 pour l'année 1988 incluent les plus-values à la fois au numérateur et au dénominateur (alors que les ratios $\mathrm{y}^{*}(\mathrm{y}) / \mathrm{y}$ indiqué sur le tableau D-1 pour l'année 1988 incluent les plus-values uniquement au dénominateur, le tableau IA de l'Etat 1921 nous permettant de soustraire les plus-values du revenu
} 


\section{D.3. L'estimation de la composition du revenu imposable}

L'estimation de la composition du revenu imposable des foyers de P90-100, P99100, P99,9-100, etc.., à partir des données en tranche des Etats 1921 pose beaucoup plus de difficultés que l'estimation de la distribution du revenu imposable. En effet, si la distribution suit une loi de Pareto dont il suffit d'estimer les paramètres structurels, la composition ne suit aucune loi évidente. Les échantillons légers de déclaration de revenus des années 1988-1995 montrent que la composition du revenu imposable peut varier de façon extrêmement rapide, et surtout de façon nonlinéaire et même non-monotone, à l'intérieur du centile supérieur de la distribution des revenus (cf. tableau 3-2, section 3.1, ainsi que les tableaux de l'annexe F), et il est extrêmement difficile d'inférer de telles variations à partir d'informations portant sur quelques tranches de revenu imposable. Cela est particulièrement vrai pour les années 1980-1990, du fait du nombre limité de tranches de revenu imposable concernant les hauts revenus dans les Etats 1921 (cf. tableau D-1, annexe D.1). Feenberg et Poterba (1993) font l'hypothèse que le montant moyen des différents types de revenus catégoriels peut être approximé (entre deux seuils de revenu imposable) par une fonction puissance du niveau de revenu imposable total. Cette technique semble donner de bons résultats pour le cas américain, vraisemblablement du fait que l'équivalent américain des Etats 1921 fournit la composition du revenu imposable pour un nombre de tranches sensiblement plus élevé, mais elle ne nous a pas semblé très fiable pour le cas français, tout du moins si l'on cherche à remonter au-delà de P99,5-100. Une solution pourrait consister à utiliser les échantillons légers de déclarations de revenus des années 1988-1995 pour estimer de façon fine la forme de la loi non-linéaire suivie par la composition des hauts revenus, puis à utiliser les tableaux IA/B des Etats 1921 pour estimer les paramètres de cette loi pour les autres années. ${ }^{128}$

Dans le cadre de cette étude, nous nous sommes limités à une estimation plus modeste, dont le tableau 3-6 (section 3-3) donne les résultats. Ces résultats ont été obtenus en utilisant uniquement les tableaux statistiques des Etats 1921 (tableaux IA/B) et en faisant l'hypothèse que la composition du revenu imposable évolue linéairement entre deux seuils de revenu imposable. Par exemple, pour l'année 1970, le tableau B de l'Etat 1921 nous fournit la composition du revenu imposable des foyers fiscaux dont le revenu imposable est supérieur à 70000 francs $(0,92 \%$ des foyers fiscaux) et de ceux dont le revenu imposable est supérieur à 100000 francs $(0,41 \%$ des foyers fiscaux ; cf. tableau D-1) ; pour estimer la composition des $0,5 \%$ des foyers fiscaux ayant le revenu imposable le plus élevé (P99,5-100), c'est-à-dire des foyers fiscaux dont le revenu imposable est supérieur à 91884 francs (cf. tableau D-2), nous avons supposé que la composition du revenu imposable des

imposable pour ce qui est du numérateur). Pour l'année 1988, nous avons retenu sur les tableaux D4, 4-1, D-6, D-8, D-10, D-12 et D-14 une estimation égale à la moyenne des estimations obtenues pour l'année 1987 et 1989, hypothèse simple qui est cohérente avec les estimations issues des échantillons légers de déclarations de revenu. Les estimations de l'élasticité du revenu imposable présentées dans la section 4 n'utilisant jamais directement les résultats de l'année 1988 (tous les estimations comparent les années 1985 et 1989, 1985 et 1995, etc..), cette correction est de toute façon sans conséquence.

${ }^{128}$ Sur la période 1983-1993, les tableaux IA des Etats 1921 pourraient également être complétés par les tableaux 20 des dossiers "Modèle lourd " (cf. annexe $\mathrm{H}$ ), qui fournissent la composition du revenu imposable des foyers dont le revenu imposable est supérieur à 1 million de francs, et dont les résultats coïncident parfaitement avec ceux des tableaux IA des Etats 1921 pour les tranches inférieures. 
Tableau D-3: Les seuils de revenu imposable et les ratios $y^{*}(y) / y$ utilisés pour l'estimation de la distribution du revenu imposable des foyers fiscaux ayant 1 part de quotient familial

\begin{tabular}{|c|c|c|c|c|c|c|c|c|c|c|c|c|c|c|}
\hline & \multicolumn{2}{|c|}{1970} & \multicolumn{2}{|c|}{1971} & \multicolumn{2}{|c|}{1972} & \multicolumn{2}{|c|}{1973} & \multicolumn{2}{|c|}{1974} & \multicolumn{2}{|c|}{1975} & \multicolumn{2}{|c|}{1976} \\
\hline $\mathrm{s}_{\mathrm{i}}$ & $\mathrm{p}_{\mathrm{i}}$ & $b_{i}$ & $\mathrm{p}_{\mathrm{i}}$ & $b_{i}$ & $\mathrm{p}_{\mathrm{i}}$ & $b_{i}$ & $\mathrm{p}_{\mathrm{i}}$ & $b_{i}$ & $\mathrm{p}_{\mathrm{i}}$ & $b_{i}$ & $\mathrm{p}_{\mathrm{i}}$ & $b_{i}$ & $\mathrm{p}_{\mathrm{i}}$ & $b_{i}$ \\
\hline 15000 & 6,38 & 1,61 & 8,34 & 1,59 & 10,85 & 1,59 & & & & & & & & \\
\hline 20000 & 2,87 & 1,63 & 3,75 & 1,63 & 4,85 & 1,63 & 6,94 & 1,64 & & & & & & \\
\hline 25000 & & & 2,00 & 1,66 & 2,60 & 1,66 & 3,73 & 1,68 & 5,38 & 1,60 & 8,12 & 1,58 & & \\
\hline 30000 & 0,94 & 1,69 & 1,21 & 1,69 & 1,58 & 1,69 & 2,24 & 1,72 & 3,14 & 1,64 & 4,81 & 1,60 & 6,96 & 1,58 \\
\hline 40000 & 0,44 & 1,72 & 0,57 & 1,73 & 0,74 & 1,74 & 1,05 & 1,79 & 1,40 & 1,70 & 2,04 & 1,67 & 2,94 & 1,63 \\
\hline 50000 & 0,26 & 1,74 & 0,33 & 1,75 & 0,42 & 1,76 & 0,60 & 1,84 & 0,78 & 1,74 & 1,10 & 1,72 & 1,54 & 1,68 \\
\hline 70000 & 0,11 & 1,75 & 0,15 & 1,77 & 0,19 & 1,78 & 0,27 & 1,89 & 0,34 & 1,78 & 0,46 & 1,79 & 0,62 & 1,76 \\
\hline 100000 & 0,049 & 1,77 & 0,064 & 1,78 & 0,083 & 1,79 & 0,12 & 1,94 & 0,14 & 1,82 & 0,19 & 1,84 & 0,26 & 1,81 \\
\hline 200000 & 0,010 & 1,76 & 0,013 & 1,78 & 0,018 & 1,75 & 0,027 & 2,08 & 0,029 & 1,90 & 0,038 & 1,99 & 0,052 & 1,88 \\
\hline \multirow[t]{2}{*}{400000} & 0,002 & 1,82 & 0,003 & 1,79 & 0,004 & 1,70 & 0,007 & 2,17 & 0,007 & 1,91 & 0,008 & 2,24 & 0,011 & 2,04 \\
\hline & \multicolumn{2}{|c|}{1977} & \multicolumn{2}{|c|}{1978} & \multicolumn{2}{|c|}{1979} & \multicolumn{2}{|c|}{1980} & \multicolumn{2}{|c|}{1981} & \multicolumn{2}{|c|}{1982} & \multicolumn{2}{|c|}{1983} \\
\hline $\mathrm{s}_{\mathrm{i}}$ & $\mathrm{p}_{\mathrm{i}}$ & $b_{i}$ & $\mathrm{p}_{\mathrm{i}}$ & $b_{i}$ & $\mathrm{p}_{\mathrm{i}}$ & $b_{i}$ & $\mathrm{p}_{\mathrm{i}}$ & $b_{i}$ & $\mathrm{p}_{\mathrm{i}}$ & $b_{i}$ & $\mathrm{p}_{\mathrm{i}}$ & $b_{i}$ & $\mathrm{p}_{\mathrm{i}}$ & $b_{i}$ \\
\hline 30000 & 9,19 & 1,55 & & & & & & & & & & & & \\
\hline 40000 & 3,87 & 1,59 & 5,42 & 1,57 & 7,33 & 1,57 & & & & & & & & \\
\hline 50000 & & & 2,72 & 1,62 & 3,70 & 1,62 & 5,43 & 1,57 & 8,56 & 1,55 & & & & \\
\hline 60000 & 1,97 & 1,64 & 1,59 & 1,67 & 2,14 & 1,67 & 3,07 & 1,62 & 4,93 & 1,58 & 6,96 & 1,53 & & \\
\hline 70000 & 0,76 & 1,72 & 1,02 & 1,71 & 1,38 & 1,72 & 1,93 & 1,66 & 3,05 & 1,62 & 4,28 & 1,55 & 6,43 & 1,52 \\
\hline 80000 & & & 0,71 & 1,74 & 0,96 & 1,75 & 1,32 & 1,70 & 2,04 & 1,65 & 2,83 & 1,58 & 4,21 & 1,54 \\
\hline 90000 & & & & & & & & & & & 1,97 & 1,60 & 2,92 & 1,56 \\
\hline 100000 & 0,30 & 1,80 & 0,41 & 1,78 & 0,54 & 1,79 & 0,72 & 1,75 & 1,08 & 1,71 & 1,44 & 1,63 & 2,11 & 1,58 \\
\hline 125000 & & & & & & & & & & & & & 1,08 & 1,63 \\
\hline 150000 & & & & & & & & & & & 0,47 & 1,689 & 0,65 & 1,65 \\
\hline 200000 & 0,058 & 1,93 & 0,081 & 1,84 & 0,11 & 1,83 & 0,14 & 1,83 & 0,19 & 1,80 & 0,22 & 1,72 & 0,30 & 1,69 \\
\hline \multirow[t]{2}{*}{400000} & 0,013 & 2,07 & 0,017 & 1,93 & 0,024 & 1,85 & 0,029 & 1,89 & 0,039 & 1,85 & 0,042 & 1,75 & 0,052 & 1,75 \\
\hline & \multicolumn{2}{|c|}{1984} & \multicolumn{2}{|c|}{1985} & \multicolumn{2}{|c|}{1986} & \multicolumn{2}{|c|}{1987} & 198 & & 198 & & 199 & \\
\hline $\mathrm{s}_{\mathrm{i}}$ & $\mathrm{p}_{\mathrm{i}}$ & $b_{i}$ & $\mathrm{p}_{\mathrm{i}}$ & $b_{i}$ & $\mathrm{p}_{\mathrm{i}}$ & $b_{i}$ & $\mathrm{p}_{\mathrm{i}}$ & $b_{i}$ & $\mathrm{p}_{\mathrm{i}}$ & $b_{i}$ & $\mathrm{p}_{\mathrm{i}}$ & $b_{i}$ & $\mathrm{p}_{\mathrm{i}}$ & $b_{i}$ \\
\hline 80000 & 5,33 & 1,53 & 6,55 & 1,54 & & & & & & & & & & \\
\hline 90000 & 3,67 & 1,55 & 4,54 & 1,56 & 5,26 & 1,57 & 5,68 & 1,58 & 6,37 & 1,65 & & & & \\
\hline 100000 & 2,66 & 1,57 & 3,30 & 1,58 & 3,83 & 1,59 & 4,15 & 1,60 & 4,69 & 1,68 & 5,39 & 1,62 & 6,23 & 1,60 \\
\hline 125000 & 1,35 & 1,61 & 1,68 & 1,62 & 1,96 & 1,63 & 2,15 & 1,65 & 2,46 & 1,76 & 2,82 & 1,67 & 3,25 & 1,63 \\
\hline 150000 & 0,80 & 1,65 & 0,99 & 1,66 & 1,16 & 1,67 & 1,27 & 1,69 & 1,48 & 1,84 & 1,68 & 1,72 & 1,92 & 1,68 \\
\hline 200000 & 0,37 & 1,69 & 0,45 & 1,71 & 0,53 & 1,73 & 0,59 & 1,76 & 0,70 & 1,97 & 0,77 & 1,80 & 0,86 & 1,76 \\
\hline 250000 & 0,21 & 1,71 & 0,26 & 1,74 & 0,30 & 1,77 & 0,33 & 1,80 & 0,41 & 2,06 & 0,44 & 1,87 & 0,48 & 1,82 \\
\hline 500000 & 0,037 & 1,76 & 0,049 & 1,80 & 0,059 & 1,83 & 0,068 & 1,85 & 0,094 & 2,30 & 0,09 & 1,99 & 0,10 & 1,95 \\
\hline & 19 & & 199 & & 195 & & 195 & & 199 & & 1996 & $\left.b^{* *}\right)$ & & \\
\hline $\mathrm{s}_{\mathrm{i}}$ & $\mathrm{p}_{\mathrm{i}}$ & $b_{i}$ & $\mathrm{p}_{\mathrm{i}}$ & $b_{i}$ & $\mathrm{p}_{\mathrm{i}}$ & $b_{i}$ & $\mathrm{p}_{\mathrm{i}}$ & $b_{i}$ & $\mathrm{p}_{\mathrm{i}}$ & $b_{i}$ & $\mathrm{p}_{\mathrm{i}}$ & $b_{i}$ & & \\
\hline 100000 & 6,94 & 1,57 & 7,48 & 1,55 & 7,77 & 1,53 & 8,02 & 1,53 & 8,50 & 1,52 & 8,90 & 1,52 & & \\
\hline 125000 & 3,59 & 1,60 & 3,82 & 1,57 & 3,95 & 1,56 & 4,08 & 1,55 & 4,33 & 1,54 & 4,52 & 1,54 & & \\
\hline 150000 & 2,09 & 1,64 & 2,19 & 1,61 & 2,24 & 1,60 & 2,30 & 1,59 & 2,43 & 1,58 & 2,53 & 1,57 & & \\
\hline 200000 & 0,91 & 1,72 & 0,94 & 1,69 & 0,94 & 1,68 & 0,96 & 1,68 & 1,01 & 1,66 & 1,05 & 1,65 & & \\
\hline 250000 & 0,50 & 1,78 & 0,50 & 1,76 & 0,50 & 1,74 & 0,50 & 1,75 & 0,53 & 1,73 & 0,55 & 1,72 & & \\
\hline 500000 & 0,09 & 1,92 & 0,09 & 1,91 & 0,09 & 1,90 & 0,09 & 1,91 & 0,09 & 1,91 & 0,09 & 1,91 & & \\
\hline
\end{tabular}

Source: Etats 1921 (cf. annexes D.1 et D.2)

Lecture: cf. tableau D-1.

Notes: (i) 1988( ${ }^{*}$ ): les chiffres ne sont pas homogènes aux chiffres des autres années (les tranches de revenu imposable et les revenus imposables incluent les plus-values taxées à taux proportionnel) (cf. annexes D-1 et D-2).

(ii) $1996\left(^{* *}\right)$ : chiffres provisoires (rôles émis au 31/12/1997). 
Tableau D-4 : Les seuils P90, P95, P99, P99,5, P99,7, P99,9 et P99,99 de la distribution du revenu imposable des foyers fiscaux ayant 1 part de quotient familial

$\begin{array}{cccccccc} & \mathrm{P} 95 & \mathrm{P} 98 & \mathrm{P} 99 & \mathrm{P} 99,5 & \mathrm{P} 99,7 & \mathrm{P} 99,9-100 & \mathrm{P} 99,99-100 \\ 1970 & 16442 & 22983 & 29190 & 38021 & 46704 & 73963 & 200726 \\ 1971 & 17898 & 25001 & 32475 & 42358 & 52027 & 82336 & 226848 \\ 1972 & 19766 & 27246 & 35168 & 46487 & 57188 & 92251 & 265699 \\ 1973 & 22195 & 31480 & 40886 & 54483 & 67082 & 110061 & 322157 \\ 1974 & 25691 & 34533 & 44949 & 58898 & 73699 & 117842 & 334576 \\ 1975 & 29557 & 40353 & 52000 & 67313 & 81616 & 123972 & 357280 \\ 1976 & 33848 & 44923 & 57015 & 76830 & 93088 & 146877 & 410295 \\ 1977 & 36396 & 49694 & 62489 & 80082 & 100428 & 154198 & 450323 \\ 1978 & 41180 & 54664 & 70706 & 91192 & 114114 & 181409 & 511483 \\ 1979 & 44535 & 61691 & 78567 & 103775 & 127626 & 209729 & 593462 \\ 1980 & 51512 & 69043 & 86802 & 116828 & 139330 & 229609 & 655000 \\ 1981 & 59676 & 80670 & 103304 & 130555 & 163942 & 260725 & 749107 \\ 1982 & 66239 & 89550 & 114962 & 146240 & 177217 & 274467 & 737852 \\ 1983 & 75297 & 101928 & 128920 & 166462 & 200716 & 303405 & 812203 \\ 1984 & 81810 & 107476 & 137203 & 176030 & 216803 & 327369 & 883764 \\ 1985 & 86962 & 116800 & 149238 & 192238 & 233828 & 362838 & 1010966 \\ 1986 & 91679 & 124054 & 159319 & 205419 & 250479 & 394471 & 1117424 \\ 1987 & 94310 & 128506 & 165659 & 214292 & 262062 & 419603 & 1208268 \\ 1988 & 98625 & 134014 & 172059 & 224928 & 280331 & 449732 & 1357873 \\ 1989 & 102940 & 139523 & 178458 & 235564 & 298600 & 479860 & 1507478 \\ 1990 & 108570 & 147490 & 187582 & 245962 & 309509 & 489113 & 1499544 \\ 1991 & 110363 & 152606 & 192455 & 249558 & 312184 & 486114 & 1464923 \\ 1992 & 113306 & 155362 & 194795 & 250329 & 311895 & 478662 & 1434073 \\ 1993 & 114937 & 156570 & 195132 & 249895 & 310747 & 473745 & 1410170 \\ 1994 & 116256 & 158041 & 196640 & 250993 & 312558 & 479595 & 1434644 \\ 1995 & 118799 & 161030 & 200572 & 255668 & 317018 & 477544 & 1430334 \\ 1996\left(^{*}\right) & 120689 & 163536 & 204089 & 259343 & 321125 & 480824 & 1444229\end{array}$

Source: Estimations de l'auteur à partir des Etats 1921 (cf. annexes D.1. et D.2.) Lecture: Cf. tableau D-2.

Note: $1996\left(^{*}\right)$ : chiffres provisoires (rôles émis au 31/12/97). 
Tableau D-5: Les seuils de revenu imposable et les ratios $y^{*}(y) / y$ utilisés pour l'estimation de la distribution du revenu imposable des foyers fiscaux ayant 1,5 parts de quotient familial

\begin{tabular}{|c|c|c|c|c|c|c|c|c|c|c|c|c|c|c|}
\hline \multirow[b]{2}{*}{$\mathrm{s}_{\mathrm{i}}$} & \multicolumn{2}{|c|}{1970} & \multicolumn{2}{|c|}{1971} & \multicolumn{2}{|c|}{1972} & \multicolumn{2}{|c|}{1973} & \multicolumn{2}{|c|}{1974} & \multicolumn{2}{|c|}{1975} & \multicolumn{2}{|c|}{1976} \\
\hline & $\mathrm{p}_{\mathrm{i}}$ & $b_{i}$ & $\mathrm{p}_{\mathrm{i}}$ & $\mathrm{b}_{\mathrm{i}}$ & $\mathrm{p}_{\mathrm{i}}$ & $\mathrm{b}_{\mathrm{i}}$ & $\mathrm{p}_{\mathrm{i}}$ & $b_{i}$ & $\mathrm{p}_{\mathrm{i}}$ & $b_{i}$ & $\mathrm{p}_{\mathrm{i}}$ & $b_{i}$ & $\mathrm{p}_{\mathrm{i}}$ & $\mathrm{b}_{\mathrm{i}}$ \\
\hline 15000 & 8,62 & 1,91 & & & & & & & & & & & & \\
\hline 20000 & 4,75 & 1,89 & 5,67 & 1,90 & 6,48 & 1,89 & 7,94 & 1,91 & & & & & & \\
\hline 25000 & & & 3,38 & 1,95 & 3,92 & 1,92 & 4,96 & 1,91 & & & & & & \\
\hline 30000 & 1,90 & 1,96 & 2,27 & 1,97 & 2,57 & 1,97 & 3,21 & 1,97 & 5,20 & 1,88 & 7,66 & 1,82 & & \\
\hline 40000 & 1,04 & 1,97 & 1,25 & 1,99 & 1,39 & 2,00 & 1,71 & 2,02 & 2,61 & 1,97 & 3,77 & 1,88 & 5,43 & 1,81 \\
\hline 50000 & 0,65 & 1,99 & 0,79 & 1,99 & 0,88 & 2,01 & 1,09 & 2,03 & 1,62 & 2,00 & 2,23 & 1,93 & 3,06 & 1,89 \\
\hline 70000 & 0,32 & 2,02 & 0,40 & 2,01 & 0,44 & 2,03 & 0,55 & 2,04 & 0,81 & 2,01 & 1,08 & 1,97 & 1,42 & 1,95 \\
\hline 100000 & 0,15 & 2,06 & 0,19 & 2,04 & 0,21 & 2,07 & 0,27 & 2,07 & 0,40 & 2,03 & 0,51 & 1,99 & 0,66 & 1,99 \\
\hline 200000 & 0,037 & 2,17 & 0,045 & 2,15 & 0,050 & 2,20 & 0,068 & 2,13 & 0,10 & 2,06 & 0,12 & 2,03 & 0,16 & 2,03 \\
\hline \multirow[t]{2}{*}{400000} & 0,010 & 2,14 & 0,012 & 2,16 & 0,013 & 2,28 & 0,017 & 2,25 & 0,024 & 2,17 & 0,030 & 2,12 & 0,038 & 2,15 \\
\hline & \multicolumn{2}{|c|}{1977} & \multicolumn{2}{|c|}{1978} & \multicolumn{2}{|c|}{1979} & \multicolumn{2}{|c|}{1980} & \multicolumn{2}{|c|}{1981} & \multicolumn{2}{|c|}{1982} & \multicolumn{2}{|c|}{1983} \\
\hline$s_{i}$ & $\mathrm{p}_{\mathrm{i}}$ & $b_{i}$ & $\mathrm{p}_{\mathrm{i}}$ & $b_{i}$ & $\mathrm{p}_{\mathrm{i}}$ & $b_{i}$ & $\mathrm{p}_{\mathrm{i}}$ & $b_{i}$ & $\mathrm{p}_{\mathrm{i}}$ & $b_{i}$ & $\mathrm{p}_{\mathrm{i}}$ & $b_{i}$ & $\mathrm{p}_{\mathrm{i}}$ & $\mathrm{b}_{\mathrm{i}}$ \\
\hline \multicolumn{15}{|l|}{30000} \\
\hline 40000 & 6,27 & 1,81 & 8,29 & 1,80 & & & & & & & & & & \\
\hline 50000 & 3,54 & 1,88 & 4,70 & 1,86 & 6,38 & 1,81 & & & & & & & & \\
\hline 60000 & & & 2,99 & 1,92 & 3,97 & 1,88 & 5,50 & 1,80 & & & & & & \\
\hline 70000 & 1,63 & 1,94 & 2,10 & 1,95 & 2,73 & 1,92 & 3,68 & 1,86 & 5,11 & 1,81 & & & & \\
\hline 80000 & & & 1,56 & 1,97 & 2,02 & 1,94 & 2,65 & 1,89 & 3,62 & 1,85 & 5,13 & 1,74 & & \\
\hline 90000 & & & & & & & & & & & 3,73 & 1,77 & 5,08 & 1,70 \\
\hline 100000 & 0,75 & 1,99 & 0,97 & 1,99 & 1,23 & 1,98 & 1,59 & 1,93 & 2,11 & 1,91 & 2,83 & 1,80 & 3,78 & 1,73 \\
\hline 125000 & & & & & & & & & & & & & 2,09 & 1,79 \\
\hline 150000 & & & & & & & & & & & 1,05 & 1,88 & 1,33 & 1,83 \\
\hline 200000 & 0,18 & 2,07 & 0,24 & 2,01 & 0,28 & 2,08 & 0,35 & 2,05 & 0,45 & 2,04 & 0,54 & 1,95 & 0,66 & 1,89 \\
\hline \multirow[t]{2}{*}{400000} & 0,042 & 2,23 & 0,057 & 2,12 & 0,070 & 2,20 & 0,084 & 2,157 & 0,11 & 2,188 & 0,12 & 2,08 & 0,14 & 2,01 \\
\hline & \multicolumn{2}{|c|}{1984} & \multicolumn{2}{|c|}{1985} & \multicolumn{2}{|c|}{1986} & 198 & & 1988 & $\left({ }^{*}\right)$ & 198 & & 199 & \\
\hline$s_{i}$ & $\mathrm{p}_{\mathrm{i}}$ & $b_{i}$ & $\mathrm{p}_{\mathrm{i}}$ & $b_{i}$ & $\mathrm{p}_{\mathrm{i}}$ & $b_{i}$ & $\mathrm{p}_{\mathrm{i}}$ & $\mathrm{b}_{\mathrm{i}}$ & $\mathrm{p}_{\mathrm{i}}$ & $b_{i}$ & $\mathrm{p}_{\mathrm{i}}$ & $b_{i}$ & $\mathrm{p}_{\mathrm{i}}$ & $b_{i}$ \\
\hline 90000 & 6,43 & 1,66 & & & & & & & & & & & & \\
\hline 100000 & 4,72 & 1,69 & 5,63 & 1,68 & 6,48 & 1,67 & 6,96 & 1,69 & 7,75 & 1,80 & 8,71 & 1,70 & & \\
\hline 125000 & 2,54 & 1,76 & 3,01 & 1,74 & 3,45 & 1,74 & 3,73 & 1,75 & 4,21 & 1,90 & 4,75 & 1,76 & 5,50 & 1,74 \\
\hline 150000 & 1,58 & 1,81 & 1,86 & 1,79 & 2,13 & 1,78 & 2,31 & 1,80 & 2,64 & 1,98 & 2,94 & 1,81 & 3,38 & 1,79 \\
\hline 200000 & 0,77 & 1,88 & 0,90 & 1,86 & 1,02 & 1,86 & 1,12 & 1,88 & 1,33 & 2,11 & 1,43 & 1,88 & 1,61 & 1,87 \\
\hline 250000 & 0,45 & 1,94 & 0,52 & 1,92 & 0,60 & 1,91 & 0,65 & 1,94 & 0,81 & 2,21 & 0,84 & 1,94 & 0,94 & 1,94 \\
\hline 500000 & 0,10 & 2,11 & 0,11 & 2,08 & 0,13 & 2,05 & 0,14 & 2,08 & 0,20 & 2,41 & 0,19 & 2,08 & 0,20 & 2,09 \\
\hline & $19 s$ & & 199 & & 199 & & 199 & & 199 & & 199 & $\left.{ }^{* *}\right)$ & & \\
\hline $\mathrm{s}_{\mathrm{i}}$ & $\mathrm{p}_{\mathrm{i}}$ & $b_{i}$ & $\mathrm{p}_{\mathrm{i}}$ & $b_{i}$ & $\mathrm{p}_{\mathrm{i}}$ & $b_{i}$ & $\mathrm{p}_{\mathrm{i}}$ & $b_{i}$ & $\mathrm{p}_{\mathrm{i}}$ & $b_{i}$ & $\mathrm{p}_{\mathrm{i}}$ & $b_{i}$ & & \\
\hline 125000 & 5,97 & 1,70 & 6,41 & 1,67 & 6,81 & 1,64 & 7,07 & 1,64 & 7,30 & 1,62 & 7,63 & 1,61 & & \\
\hline 150000 & 3,62 & 1,75 & 3,83 & 1,72 & 4,02 & 1,69 & 4,16 & 1,68 & 4,28 & 1,66 & 4,44 & 1,65 & & \\
\hline 200000 & 1,72 & 1,83 & 1,79 & 1,79 & 1,84 & 1,76 & 1,89 & 1,76 & 1,92 & 1,74 & 1,97 & 1,72 & & \\
\hline 250000 & 0,98 & 1,89 & 1,01 & 1,86 & 1,02 & 1,83 & 1,04 & 1,83 & 1,04 & 1,81 & 1,08 & 1,78 & & \\
\hline 500000 & 0,20 & 2,05 & 0,20 & 2,03 & 0,20 & 2,01 & 0,20 & 2,01 & 0,20 & 2,01 & 0,19 & 2,00 & & \\
\hline
\end{tabular}

Source: Etats 1921 (cf. annexes D.1 et D.2)

Lecture: cf. tableau D-1.

Notes: (i) 1988( ${ }^{*}$ ): les chiffres ne sont pas homogènes aux chiffres des autres années (les tranches de revenu imposable et les revenus imposables incluent les plus-values taxées à taux proportionnel) (cf. annexes D-1 et D-2).

(ii) $1996\left(^{* *}\right)$ : chiffres provisoires (rôles émis au 31/12/1997). 
Tableau D-6: Résultats de l'estimation de la distribution du revenu imposable des foyers fiscaux ayant 1,5 parts de quotient familial

\begin{tabular}{|c|c|c|c|c|c|c|c|c|c|c|c|c|}
\hline & P95 & P98 & P99 & P99,5 & P99,9 & P99,99 & P95-100 & P98-100 & P99-100 & P99,5-100 & P99,9-10 & $99,99-100$ \\
\hline 1970 & 19530 & 29256 & 40793 & 57024 & 124934 & 409696 & 27,22 & 16,89 & 11,86 & 8,36 & 3,78 & 1,29 \\
\hline 1971 & 20680 & 31943 & 44460 & 62252 & 131268 & 448740 & 26,82 & 16,78 & 11,82 & 8,35 & 3,76 & 1,29 \\
\hline 1972 & 22247 & 33934 & 46906 & 65831 & 137757 & 473395 & 25,55 & 15,94 & 11,25 & 7,97 & 3,62 & 1,29 \\
\hline 1973 & 24899 & 36966 & 52266 & 73801 & 163055 & 541406 & 25,19 & 15,78 & 11,20 & 7,96 & 3,66 & 1,29 \\
\hline 1974 & 30565 & 44938 & 63018 & 88761 & 197904 & 642302 & 26,00 & 16,21 & 11,46 & 8,13 & 3,68 & 1,26 \\
\hline 1975 & 35052 & 52659 & 72587 & 100969 & 221920 & 709299 & 25,68 & 15,86 & 11,12 & 7,83 & 3,52 & 1,17 \\
\hline 1976 & 41510 & 59333 & 81399 & 114853 & 252928 & 814704 & 25,31 & 15,52 & 10,87 & 7,67 & 3,45 & 1,18 \\
\hline 1977 & 44272 & 63356 & 86708 & 122409 & 268286 & 886584 & 23,49 & 14,45 & 10,13 & 7,15 & 3,26 & 1,16 \\
\hline 1978 & 48615 & 71648 & 98612 & 138151 & 295847 & 998790 & 23,14 & 14,30 & 10,06 & 7,12 & 3,21 & 1,09 \\
\hline 1979 & 53882 & 80387 & 110931 & 149227 & 328597 & 1155875 & 22,72 & 14,04 & 9,87 & 6,98 & 3,25 & 1,14 \\
\hline 1980 & 62611 & 89528 & 125061 & 166041 & 364583 & 1253597 & 22,15 & 13,56 & 9,47 & 6,68 & 3,08 & 1,06 \\
\hline 1981 & 70682 & 102536 & 133362 & 189849 & 411807 & 1437722 & 21,88 & 13,39 & 9,30 & 6,62 & 3,08 & 1,08 \\
\hline 1982 & 80882 & 116746 & 153773 & 207332 & 438342 & 1449925 & 21,08 & 12,60 & 8,68 & 6,05 & 2,74 & 0,91 \\
\hline 1983 & 90613 & 127556 & 170644 & 228244 & 476527 & 1513775 & 20,93 & 12,39 & 8,46 & 5,86 & 2,60 & 0,83 \\
\hline 1984 & 97708 & 134991 & 177050 & 238111 & 492633 & 1654538 & 20,95 & 12,34 & 8,43 & 5,84 & 2,63 & 0,88 \\
\hline 1985 & 104937 & 145392 & 190552 & 255234 & 529088 & 1747433 & 21,21 & 12,39 & 8,43 & 5,84 & 2,63 & 0,87 \\
\hline 1986 & 106825 & 154222 & 202232 & 272508 & 567067 & 1848657 & 21,06 & 12,47 & 8,52 & 5,91 & 2,64 & 0,86 \\
\hline 1987 & 110160 & 159798 & 211153 & 284872 & 606447 & 2007048 & 21,22 & 12,66 & 8,71 & 6,08 & 2,77 & 0,92 \\
\hline 1988 & 116200 & 165409 & 220287 & 291752 & 647294 & 2139391 & 21,40 & 12,80 & 8,84 & 6,16 & 2,82 & 0,93 \\
\hline 1989 & 122241 & 171020 & 229421 & 298631 & 688141 & 2271735 & 21,58 & 12,94 & 8,97 & 6,24 & 2,88 & 0,95 \\
\hline 1990 & 130113 & 180894 & 242550 & 313336 & 726106 & 2416471 & 21,47 & 12,88 & 8,93 & 6,23 & 2,89 & 0,96 \\
\hline 1991 & 134514 & 186600 & 247847 & 315459 & 720464 & 2348296 & 21,09 & 12,55 & 8,63 & 5,97 & 2,73 & 0,89 \\
\hline 1992 & 134183 & 190529 & 251490 & 316478 & 715612 & 2299398 & 20,73 & 12,25 & 8,37 & 5,75 & 2,60 & 0,84 \\
\hline 1993 & 137120 & 192797 & 252634 & 314333 & 706007 & 2246862 & 20,38 & 11,94 & 8,12 & 5,55 & 2,49 & 0,79 \\
\hline 1994 & 139184 & 195046 & 254537 & 317726 & 712511 & 2262479 & 20,26 & 11,85 & 8,05 & 5,51 & 2,47 & 0,79 \\
\hline 1995 & 141027 & 196535 & 254881 & 312936 & 702321 & 2232633 & 19,69 & 11,45 & 7,75 & 5,27 & 2,37 & 0,75 \\
\hline \multirow[t]{2}{*}{$1996^{*}$} & 143195 & 198658 & 258227 & 311503 & 696479 & 2202174 & 19,28 & 11,17 & 7,52 & 5,09 & 2,27 & 0,72 \\
\hline & P95-98 & P98-99 & P99-99,5 & P99,5-99,9 & P99,9-100 & & P98-99 & P99-99,5 & P99,5-99,9 & P99,9-100 & & $g(P \mid B)$ \\
\hline 1970 & 10,33 & 5,03 & 3,50 & 4,57 & 3,78 & & & & & & & \\
\hline 1971 & 10,04 & 4,96 & 3,47 & 4,59 & 3,76 & & 1,4 & 1,9 & 3,3 & 2,1 & & 5,6 \\
\hline 1972 & 9,61 & 4,69 & 3,28 & 4,35 & 3,62 & & $-1,2$ & $-1,1$ & $-0,9$ & 0,6 & & 5,2 \\
\hline 1973 & 9,41 & 4,58 & 3,24 & 4,29 & 3,66 & & $-0,2$ & 0,7 & 0,7 & 3,4 & & 6,7 \\
\hline 1974 & 9,79 & 4,75 & 3,34 & 4,44 & 3,68 & & $-0,3$ & $-1,0$ & $-0,5$ & $-3,4$ & & 1,3 \\
\hline 1975 & 9,82 & 4,74 & 3,29 & 4,32 & 3,52 & & $-0,7$ & $-1,8$ & $-3,2$ & $-4,8$ & & 0,8 \\
\hline 1976 & 9,78 & 4,65 & 3,20 & 4,22 & 3,45 & & $-1,4$ & $-2,3$ & $-1,9$ & $-1,5$ & & 5,6 \\
\hline 1977 & 9,04 & 4,32 & 2,98 & 3,89 & 3,26 & & 0,5 & 0,7 & $-0,2$ & 2,3 & & 3,2 \\
\hline 1978 & 8,84 & 4,24 & 2,94 & 3,91 & 3,21 & & 0,3 & 0,8 & 2,8 & 0,8 & & 4,3 \\
\hline 1979 & 8,68 & 4,17 & 2,89 & 3,73 & 3,25 & & 0,1 & 0,3 & $-2,9$ & 3,0 & & 2,6 \\
\hline 1980 & 8,59 & 4,09 & 2,79 & 3,60 & 3,08 & & $-0,9$ & $-2,7$ & $-2,4$ & $-4,2$ & & $-0,3$ \\
\hline 1981 & 8,49 & 4,09 & 2,68 & 3,54 & 3,08 & & 1,1 & $-2,6$ & $-0,6$ & 1,1 & & $-0,6$ \\
\hline 1982 & 8,48 & 3,92 & 2,63 & 3,31 & 2,74 & & $-3,9$ & $-1,5$ & $-6,2$ & $-11,1$ & & 2,5 \\
\hline 1983 & 8,54 & 3,93 & 2,60 & 3,26 & 2,60 & & $-0,3$ & $-1,9$ & $-2,3$ & $-5,7$ & & 0,8 \\
\hline 1984 & 8,60 & 3,92 & 2,58 & 3,21 & 2,63 & & $-1,2$ & $-1,6$ & $-2,1$ & 0,5 & & 1,4 \\
\hline 1985 & 8,82 & 3,97 & 2,59 & 3,21 & 2,63 & & $-1,3$ & $-2,2$ & $-2,7$ & $-2,4$ & & 1,8 \\
\hline 1986 & 8,60 & 3,94 & 2,61 & 3,27 & 2,64 & & 2,0 & 3,6 & 4,5 & 3,1 & & 5,0 \\
\hline 1987 & 8,56 & 3,95 & 2,63 & 3,31 & 2,77 & & 0,7 & 1,0 & 1,9 & 5,4 & & 2,1 \\
\hline 1988 & 8,60 & 3,96 & 2,68 & 3,34 & 2,82 & & $-0,2$ & 1,3 & 0,3 & 1,4 & & 4,6 \\
\hline 1989 & 8,64 & 3,97 & 2,72 & 3,36 & 2,88 & & $-0,2$ & 1,3 & 0,3 & 1,3 & & 3,7 \\
\hline 1990 & 8,60 & 3,95 & 2,69 & 3,34 & 2,89 & & $-0,1$ & $-0,6$ & $-0,1$ & 0,9 & & 2,2 \\
\hline 1991 & 8,54 & 3,92 & 2,66 & 3,24 & 2,73 & & $-0,1$ & $-0,6$ & $-2,5$ & $-5,0$ & & 0,9 \\
\hline 1992 & 8,47 & 3,88 & 2,62 & 3,15 & 2,60 & & $-0,3$ & $-0,7$ & $-2,0$ & $-3,7$ & & 0,9 \\
\hline 1993 & 8,43 & 3,83 & 2,57 & 3,06 & 2,49 & & $-0,8$ & $-1,6$ & $-2,5$ & $-3,7$ & & $-1,0$ \\
\hline 1994 & 8,41 & 3,80 & 2,54 & 3,04 & 2,47 & & $-0,4$ & $-0,8$ & $-0,2$ & $-0,5$ & & 2,7 \\
\hline 1995 & 8,24 & 3,71 & 2,47 & 2,91 & 2,37 & & $-0,5$ & $-0,5$ & $-2,4$ & $-2,3$ & & 2,0 \\
\hline $1996^{*}$ & 8,11 & 3,65 & 2,44 & 2,81 & 2,27 & & $-0,1$ & 0,0 & $-1,8$ & $-2,4$ & & 0,6 \\
\hline & & & & & & 1970-1995 & $-0,3$ & $-0,5$ & $-1,0$ & $-1,1$ & & 2,5 \\
\hline
\end{tabular}

Source: Estimations de l'auteur à partir des Etats 1921 (cf. annexes D-1 et D-2)

Lecture: En 1970, le seuil inférieur du demi-décile supérieur (P95) de la distribution du revenu imposable des foyers fiscaux ayant 1,5 parts de quotient familial est de 19530 francs; la part du revenu imposable du demi-centile supérieur (P95-100) est de 27,22\%; la part de P95-98 est de 10,33\% (=P95-100 - P98-100); entre 1970 et 1971, la part de P98-99 progresse de 1,4\% relativement à celle de P95-98, la part de P99-99,5 progresse de 1,9\% relativement à celle de P95-98, etc.. (cf. tableau 3-5). Note: $1996\left(^{*}\right)$ : chiffres provisoires (rôles émis au 31/12/97). 
Tableau D-7: Les seuils de revenu imposable et les ratios $y^{*}(y) / y$ utilisés pour l'estimation de la distribution du revenu imposable des foyers fiscaux ayant 2B parts de quotient familial

\begin{tabular}{|c|c|c|c|c|c|c|c|c|c|c|c|c|c|c|}
\hline \multirow[b]{2}{*}{$\mathrm{s}_{\mathrm{i}}$} & \multicolumn{2}{|c|}{1970} & \multicolumn{2}{|c|}{1971} & \multicolumn{2}{|c|}{1972} & \multicolumn{2}{|c|}{1973} & \multicolumn{2}{|c|}{1974} & \multicolumn{2}{|c|}{1975} & \multicolumn{2}{|c|}{1976} \\
\hline & $\mathrm{p}_{\mathrm{i}}$ & $b_{i}$ & $\mathrm{p}_{\mathrm{i}}$ & $b_{i}$ & $\mathrm{p}_{\mathrm{i}}$ & $b_{i}$ & $\mathrm{p}_{\mathrm{i}}$ & $b_{i}$ & $\mathrm{p}_{\mathrm{i}}$ & $b_{i}$ & $\mathrm{p}_{\mathrm{i}}$ & $b_{i}$ & $\mathrm{p}_{\mathrm{i}}$ & $b_{i}$ \\
\hline 15000 & 8,57 & 1,63 & & & & & & & & & & & & \\
\hline 20000 & 3,92 & 1,65 & 5,03 & 1,67 & 6,63 & 1,66 & 8,56 & 1,68 & & & & & & \\
\hline 25000 & & & 2,70 & 1,73 & 3,57 & 1,70 & 4,65 & 1,72 & 7,16 & 1,70 & & & & \\
\hline 30000 & 1,32 & 1,70 & 1,67 & 1,76 & 2,18 & 1,74 & 2,83 & 1,77 & 4,24 & 1,77 & 6,30 & 1,68 & 8,87 & 1,66 \\
\hline 40000 & 0,64 & 1,72 & 0,80 & 1,83 & 1,06 & 1,78 & 1,37 & 1,83 & 1,97 & 1,88 & 2,77 & 1,78 & 3,88 & 1,74 \\
\hline 50000 & 0,36 & 1,74 & 0,47 & 1,86 & 0,63 & 1,79 & 0,81 & 1,86 & 1,15 & 1,94 & 1,57 & 1,83 & 2,13 & 1,81 \\
\hline 70000 & 0,16 & 1,76 & 0,22 & 1,91 & 0,29 & 1,82 & 0,38 & 1,89 & 0,53 & 2,04 & 0,72 & 1,88 & 0,95 & 1,87 \\
\hline 100000 & 0,071 & 1,75 & 0,098 & 1,95 & 0,12 & 1,86 & 0,17 & 1,92 & 0,25 & 2,11 & 0,32 & 1,94 & 0,43 & 1,90 \\
\hline 200000 & 0,011 & 2,05 & 0,022 & 2,03 & 0,024 & 2,06 & 0,040 & 1,95 & 0,078 & 1,89 & 0,091 & 1,75 & 0,11 & 1,82 \\
\hline \multirow[t]{2}{*}{400000} & 0,004 & 1,69 & 0,006 & 1,94 & 0,008 & 1,77 & 0,012 & 1,80 & 0,018 & 1,92 & 0,019 & 1,74 & 0,027 & 1,67 \\
\hline & \multicolumn{2}{|c|}{1977} & \multicolumn{2}{|c|}{1978} & \multicolumn{2}{|c|}{1979} & \multicolumn{2}{|c|}{1980} & \multicolumn{2}{|c|}{1981} & \multicolumn{2}{|c|}{1982} & \multicolumn{2}{|c|}{1983} \\
\hline $\begin{array}{c}s_{i} \\
30000\end{array}$ & $\mathrm{p}_{\mathrm{i}}$ & $b_{i}$ & $\mathrm{p}_{\mathrm{i}}$ & $b_{i}$ & $\mathrm{p}_{\mathrm{i}}$ & $b_{i}$ & $\mathrm{p}_{\mathrm{i}}$ & $b_{i}$ & $\mathrm{p}_{\mathrm{i}}$ & $b_{i}$ & $\mathrm{p}_{\mathrm{i}}$ & $b_{i}$ & $\mathrm{p}_{\mathrm{i}}$ & $b_{i}$ \\
\hline 40000 & 5,16 & 1,65 & 8,52 & 1,64 & & & & & & & & & & \\
\hline 50000 & 2,71 & 1,72 & 4,56 & 1,69 & 5,45 & 1,71 & 7,89 & 1,65 & & & & & & \\
\hline 60000 & & & 2,80 & 1,72 & 3,29 & 1,77 & 4,61 & 1,71 & 7,14 & 1,65 & & & & \\
\hline 70000 & 1,15 & 1,77 & 1,90 & 1,73 & 2,22 & 1,80 & 3,01 & 1,75 & 4,52 & 1,70 & 6,42 & 1,62 & & \\
\hline 80000 & & & 1,36 & 1,75 & 1,60 & 1,83 & 2,11 & 1,79 & 3,11 & 1,74 & 4,31 & 1,65 & & \\
\hline 90000 & & & & & & & & & & & 3,10 & 1,68 & 5,02 & 1,63 \\
\hline 100000 & 0,50 & 1,78 & 0,81 & 1,75 & 0,96 & 1,85 & 1,24 & 1,81 & 1,72 & 1,80 & 2,33 & 1,69 & 3,72 & 1,65 \\
\hline 125000 & & & & & & & & & & & & & 2,03 & 1,68 \\
\hline 150000 & & & & & & & & & & & 0,83 & 1,73 & 1,27 & 1,70 \\
\hline 200000 & 0,11 & 1,78 & 0,15 & 1,85 & 0,21 & 1,86 & 0,25 & 1,85 & 0,35 & 1,88 & 0,41 & 1,76 & 0,62 & 1,71 \\
\hline \multirow[t]{2}{*}{400000} & 0,025 & 1,60 & 0,032 & 1,83 & 0,043 & 1,93 & 0,052 & 1,97 & 0,072 & 2,01 & 0,080 & 1,80 & 0,11 & 1,76 \\
\hline & \multicolumn{2}{|c|}{1984} & \multicolumn{2}{|c|}{1985} & \multicolumn{2}{|c|}{1986} & \multicolumn{2}{|c|}{1987} & 198 & & 198 & & 199 & \\
\hline $\mathrm{s}_{\mathrm{i}}$ & $\mathrm{p}_{\mathrm{i}}$ & $b_{i}$ & $\mathrm{p}_{\mathrm{i}}$ & $b_{i}$ & $\mathrm{p}_{\mathrm{i}}$ & $b_{i}$ & $\mathrm{p}_{\mathrm{i}}$ & $b_{i}$ & $\mathrm{p}_{\mathrm{i}}$ & $b_{i}$ & $\mathrm{p}_{\mathrm{i}}$ & $b_{i}$ & $\mathrm{p}_{\mathrm{i}}$ & $b_{i}$ \\
\hline 90000 & 6,38 & 1,60 & & & & & & & & & & & & \\
\hline 100000 & 4,71 & 1,62 & 5,98 & 1,62 & 6,72 & 1,62 & 7,13 & 1,64 & 8,15 & 1,71 & & & & \\
\hline 125000 & 2,52 & 1,65 & 3,17 & 1,65 & 3,57 & 1,65 & 3,84 & 1,68 & 4,45 & 1,77 & 5,04 & 1,69 & 5,75 & 1,68 \\
\hline 150000 & 1,55 & 1,67 & 1,94 & 1,68 & 2,18 & 1,68 & 2,34 & 1,71 & 2,71 & 1,84 & 3,08 & 1,72 & 3,51 & 1,72 \\
\hline 200000 & 0,75 & 1,67 & 0,93 & 1,69 & 1,05 & 1,69 & 1,13 & 1,75 & 1,33 & 1,93 & 1,48 & 1,77 & 1,64 & 1,79 \\
\hline 250000 & 0,43 & 1,66 & 0,54 & 1,70 & 0,60 & 1,70 & 0,65 & 1,78 & 0,78 & 2,00 & 0,86 & 1,80 & 0,94 & 1,83 \\
\hline 500000 & 0,074 & 1,67 & 0,10 & 1,71 & 0,11 & 1,70 & 0,13 & 1,79 & 0,18 & 2,18 & 0,18 & 1,84 & 0,20 & 1,91 \\
\hline & 19 & & 199 & & 199 & & 199 & & 199 & & 1996 & $\left.{ }^{* *}\right)$ & & \\
\hline $\mathrm{s}_{\mathrm{i}}$ & $\mathrm{p}_{\mathrm{i}}$ & $b_{i}$ & $\mathrm{p}_{\mathrm{i}}$ & $b_{i}$ & $\mathrm{p}_{\mathrm{i}}$ & $b_{i}$ & $\mathrm{p}_{\mathrm{i}}$ & $b_{i}$ & $\mathrm{p}_{\mathrm{i}}$ & $b_{i}$ & $\mathrm{p}_{\mathrm{i}}$ & $b_{i}$ & & \\
\hline 125000 & 6,37 & 1,64 & 6,80 & 1,61 & 7,17 & 1,59 & 7,56 & 1,57 & & & & & & \\
\hline 150000 & 3,83 & 1,67 & 4,02 & 1,64 & 4,19 & 1,62 & 4,38 & 1,60 & 5,25 & 1,60 & 5,44 & 1,59 & & \\
\hline 200000 & 1,76 & 1,73 & 1,77 & 1,71 & 1,81 & 1,69 & 1,84 & 1,69 & 2,25 & 1,67 & 2,32 & 1,66 & & \\
\hline 250000 & 0,99 & 1,77 & 0,98 & 1,76 & 0,97 & 1,75 & 0,98 & 1,77 & 1,22 & 1,71 & 1,24 & 1,71 & & \\
\hline 500000 & 0,20 & 1,83 & 0,18 & 1,85 & 0,18 & 1,88 & 0,18 & 1,95 & 0,21 & 1,89 & 0,20 & 1,91 & & \\
\hline
\end{tabular}

Source: Etats 1921 (cf. annexes D.1 et D.2)

Lecture: cf. tableau D-1.

Notes: (i) 1988( ${ }^{*}$ ): les chiffres ne sont pas homogènes aux chiffres des autres années (les tranches de revenu imposable et les revenus imposables incluent les plus-values taxées à taux proportionnel) (cf. annexes D-1 et D-2).

(ii) $1996\left({ }^{* *}\right)$ : chiffres provisoires (rôles émis au 31/12/1997). 
Tableau D-8: Résultats de l'estimation de la distribution du revenu imposable des foyers fiscaux ayant $2 B$ parts de quotient familial

\begin{tabular}{|c|c|c|c|c|c|c|c|c|c|c|c|c|}
\hline & P95 & P98 & P99 & P99,5 & P99,9 & P99,99 & P95-100 & P98-100 & P99-100 & P99,5-100 & $P 99,9-100$ & $99,99-10$ \\
\hline 1970 & 18179 & 25269 & 33580 & 44234 & 86467 & 212720 & 17,26 & 9,88 & 6,57 & 4,38 & 1,75 & 0,50 \\
\hline 1971 & 20047 & 27759 & 36211 & 48737 & 99254 & 303516 & 17,65 & 10,30 & 6,97 & 4,77 & 2,04 & 0,62 \\
\hline 1972 & 21760 & 31147 & 40984 & 55229 & 110349 & 357026 & 17,64 & 10,33 & 6,96 & 4,73 & 1,96 & 0,60 \\
\hline 1973 & 24252 & 33727 & 45466 & 61479 & 128719 & 429458 & 17,77 & 10,51 & 7,19 & 4,95 & 2,13 & 0,66 \\
\hline 1974 & 27926 & 39756 & 53518 & 72097 & 175666 & 530360 & 18,19 & 10,97 & 7,64 & 5,40 & 2,48 & 0,75 \\
\hline 1975 & 32935 & 44774 & 59885 & 80159 & 192427 & 521921 & 17,71 & 10,51 & 7,20 & 4,97 & 2,16 & 0,58 \\
\hline 1976 & 35922 & 51416 & 68360 & 92863 & 208081 & 594350 & 17,45 & 10,39 & 7,13 & 4,93 & 2,12 & 0,55 \\
\hline 1977 & 40508 & 56750 & 74329 & 99865 & 206476 & 561187 & 16,47 & 9,59 & 6,47 & 4,38 & 1,80 & 0,44 \\
\hline 1978 & 48176 & 68431 & 91153 & 122629 & 240337 & 678093 & 17,61 & 10,29 & 6,91 & 4,65 & 1,93 & 0,54 \\
\hline 1979 & 51843 & 73276 & 97937 & 133668 & 266386 & 807673 & 17,05 & 10,15 & 6,95 & 4,76 & 1,97 & 0,60 \\
\hline 1980 & 58024 & 81945 & 109960 & 146653 & 291155 & 904910 & 16,76 & 9,91 & 6,75 & 4,60 & 1,94 & 0,60 \\
\hline 1981 & 67145 & 93624 & 121906 & 168571 & 338102 & 1072685 & 17,06 & 10,07 & 6,83 & 4,72 & 2,02 & 0,64 \\
\hline 1982 & 75434 & 106508 & 138706 & 183550 & 362612 & 1006230 & 16,46 & 9,51 & 6,36 & 4,27 & 1,72 & 0,48 \\
\hline 1983 & 90115 & 125651 & 165299 & 218417 & 419298 & 1135475 & 17,75 & 10,21 & 6,78 & 4,52 & 1,79 & 0,48 \\
\hline 1984 & 97749 & 135335 & 178560 & 235801 & 444007 & 1122797 & 17,96 & 10,25 & 6,76 & 4,46 & 1,69 & 0,43 \\
\hline 1985 & 107048 & 148068 & 194568 & 257326 & 495895 & 1291965 & 18,78 & 10,79 & 7,14 & 4,75 & 1,84 & 0,48 \\
\hline 1986 & 109414 & 155342 & 203753 & 270348 & 526190 & 1355670 & 18,82 & 10,84 & 7,19 & 4,78 & 1,86 & 0,48 \\
\hline 1987 & 112385 & 160008 & 210413 & 280203 & 564721 & 1564435 & 19,20 & 11,17 & 7,50 & 5,07 & 2,06 & 0,57 \\
\hline 1988 & 118906 & 167686 & 221964 & 295234 & 605086 & 1702617 & 19,39 & 11,33 & 7,63 & 5,17 & 2,13 & 0,60 \\
\hline 1989 & 125427 & 175364 & 233514 & 310264 & 645451 & 1840799 & 19,58 & 11,48 & 7,77 & 5,27 & 2,19 & 0,63 \\
\hline 1990 & 132322 & 183242 & 243578 & 320174 & 688331 & 2057643 & 19,72 & 11,60 & 7,89 & 5,41 & 2,33 & 0,70 \\
\hline 1991 & 134753 & 189470 & 248564 & 327280 & 678550 & 1925856 & 19,16 & 11,14 & 7,49 & 5,08 & 2,11 & 0,60 \\
\hline 1992 & 137803 & 190203 & 247584 & 315329 & 661438 & 1908858 & 18,73 & 10,78 & 7,21 & 4,85 & 2,03 & 0,59 \\
\hline 1993 & 140240 & 191898 & 247148 & 308482 & 654817 & 1922175 & 18,59 & 10,63 & 7,09 & 4,76 & 2,02 & 0,59 \\
\hline 1994 & 142708 & 193371 & 247536 & 301478 & 661391 & 2035207 & 18,56 & 10,60 & 7,08 & 4,77 & 2,09 & 0,64 \\
\hline 1995 & 152769 & 209682 & 271423 & 329491 & 702447 & 2074806 & 19,65 & 11,23 & 7,47 & 4,99 & 2,13 & 0,63 \\
\hline \multirow[t]{2}{*}{$1996^{*}$} & 154821 & 212224 & 273521 & 325582 & 701166 & 2101195 & 19,33 & 11,02 & 7,32 & 4,87 & 2,10 & 0,63 \\
\hline & P95-98 & P98-99 & P99-99,5 & P99,5-99,9 & P99,9-100 & & P98-99 & P99-99,5 & P99,5-99,9 & P99,9-100 & & $g(P \mid B)$ \\
\hline 1970 & 7,38 & 3,32 & 2,19 & 2,63 & 1,75 & & & & & & & \\
\hline 1971 & 7,35 & 3,34 & 2,19 & 2,73 & 2,04 & & 1,0 & 0,7 & 4,2 & 17,3 & & 5,6 \\
\hline 1972 & 7,31 & 3,36 & 2,24 & 2,77 & 1,96 & & 1,3 & 2,5 & 1,7 & $-3,4$ & & 5,2 \\
\hline 1973 & 7,27 & 3,32 & 2,24 & 2,81 & 2,13 & & $-0,8$ & 0,8 & 2,3 & 9,5 & & 6,7 \\
\hline 1974 & 7,22 & 3,33 & 2,24 & 2,91 & 2,48 & & 1,2 & 0,5 & 4,3 & 17,1 & & 1,3 \\
\hline 1975 & 7,21 & 3,31 & 2,23 & 2,81 & 2,16 & & $-0,7$ & $-0,5$ & $-3,2$ & $-12,8$ & & 0,8 \\
\hline 1976 & 7,06 & 3,26 & 2,20 & 2,81 & 2,12 & & 0,7 & 0,8 & 2,1 & 0,1 & & 5,6 \\
\hline 1977 & 6,88 & 3,12 & 2,09 & 2,58 & 1,80 & & $-1,8$ & $-2,5$ & $-6,1$ & $-12,7$ & & 3,2 \\
\hline 1978 & 7,32 & 3,38 & 2,26 & 2,72 & 1,93 & & 1,8 & 1,9 & $-0,6$ & 0,5 & & 4,3 \\
\hline 1979 & 6,90 & 3,20 & 2,19 & 2,79 & 1,97 & & 0,5 & 2,6 & 8,6 & 8,6 & & 2,6 \\
\hline 1980 & 6,85 & 3,16 & 2,14 & 2,66 & 1,94 & & $-0,7$ & $-1,4$ & $-4,0$ & $-0,9$ & & $-0,3$ \\
\hline 1981 & 6,99 & 3,24 & 2,11 & 2,70 & 2,02 & & 0,4 & $-3,5$ & $-0,4$ & 2,2 & & $-0,6$ \\
\hline 1982 & 6,94 & 3,16 & 2,09 & 2,54 & 1,72 & & $-1,8$ & $-0,3$ & $-5,2$ & $-14,4$ & & 2,5 \\
\hline 1983 & 7,54 & 3,43 & 2,26 & 2,73 & 1,79 & & 0,1 & $-0,3$ & $-1,0$ & $-4,4$ & & 0,8 \\
\hline 1984 & 7,71 & 3,49 & 2,30 & 2,77 & 1,69 & & $-0,6$ & $-0,5$ & $-0,9$ & $-7,5$ & & 1,4 \\
\hline 1985 & 8,00 & 3,64 & 2,40 & 2,90 & 1,84 & & 0,7 & 0,4 & 1,0 & 5,1 & & 1,8 \\
\hline 1986 & 7,98 & 3,65 & 2,41 & 2,92 & 1,86 & & 0,5 & 0,7 & 0,7 & 1,1 & & 5,0 \\
\hline 1987 & 8,02 & 3,67 & 2,43 & 3,01 & 2,06 & & 0,0 & 0,3 & 2,6 & 10,4 & & 2,1 \\
\hline 1988 & 8,06 & 3,69 & 2,46 & 3,04 & 2,13 & & 0,1 & 1,0 & 0,7 & 2,6 & & 4,6 \\
\hline 1989 & 8,10 & 3,72 & 2,50 & 3,08 & 2,19 & & 0,1 & 1,0 & 0,7 & 2,5 & & 3,7 \\
\hline 1990 & 8,12 & 3,71 & 2,48 & 3,08 & 2,33 & & $-0,6$ & $-1,0$ & $-0,1$ & 5,7 & & 2,2 \\
\hline 1991 & 8,02 & 3,65 & 2,41 & 2,98 & 2,11 & & $-0,4$ & $-1,7$ & $-2,3$ & $-8,2$ & & 0,9 \\
\hline 1992 & 7,95 & 3,56 & 2,36 & 2,81 & 2,03 & & $-1,4$ & $-0,9$ & $-4,6$ & $-2,7$ & & 0,9 \\
\hline 1993 & 7,96 & 3,54 & 2,33 & 2,74 & 2,02 & & $-0,8$ & $-1,6$ & $-2,9$ & $-0,9$ & & $-1,0$ \\
\hline 1994 & 7,96 & 3,51 & 2,31 & 2,68 & 2,09 & & $-0,7$ & $-0,9$ & $-2,1$ & 3,7 & & 2,7 \\
\hline 1995 & 8,41 & 3,76 & 2,47 & 2,86 & 2,13 & & 1,3 & 1,3 & 1,2 & $-3,8$ & & 2,0 \\
\hline $1996^{*}$ & 8,31 & 3,70 & 2,45 & 2,77 & 2,10 & & $-0,6$ & 0,2 & $-1,9$ & $-0,2$ & & 0,6 \\
\hline & & & & & & 1970-1995 & 0,0 & 0,0 & $-0,3$ & 0,3 & & 2,5 \\
\hline
\end{tabular}

Source: Estimations de l'auteur à partir des Etats 1921 (cf. annexes D-1 et D-2) Lecture: Cf. tableau D-6.

Note: $1996\left(^{*}\right)$ : chiffres provisoires (rôles émis au 31/12/97). 
Tableau D-9: Les seuils de revenu imposable et les ratios $y^{*}(y) / y$ utilisés pour l'estimation de la distribution du revenu imposable des foyers fiscaux ayant 2A parts de quotient familial

\begin{tabular}{|c|c|c|c|c|c|c|c|c|c|c|c|c|c|c|}
\hline \multirow[b]{2}{*}{$\mathrm{s}_{\mathrm{i}}$} & \multicolumn{2}{|c|}{1970} & \multicolumn{2}{|c|}{1971} & \multicolumn{2}{|c|}{1972} & \multicolumn{2}{|c|}{1973} & \multicolumn{2}{|c|}{1974} & \multicolumn{2}{|c|}{1975} & \multicolumn{2}{|c|}{1976} \\
\hline & $\mathrm{p}_{\mathrm{i}}$ & $b_{i}$ & $\mathrm{p}_{\mathrm{i}}$ & $b_{i}$ & $\mathrm{p}_{\mathrm{i}}$ & $b_{i}$ & $\mathrm{p}_{\mathrm{i}}$ & $b_{i}$ & $\mathrm{p}_{\mathrm{i}}$ & $b_{i}$ & $\mathrm{p}_{\mathrm{i}}$ & $b_{i}$ & $\mathrm{p}_{\mathrm{i}}$ & $b_{i}$ \\
\hline 20000 & 18,93 & 1,79 & & & & & & & & & & & & \\
\hline 25000 & & & 14,82 & 1,77 & & & & & & & & & & \\
\hline 30000 & 7,68 & 1,76 & 9,78 & 1,77 & 12,21 & 1,77 & 17,24 & 1,78 & & & & & & \\
\hline 40000 & 3,84 & 1,79 & 4,88 & 1,80 & 6,13 & 1,80 & 8,73 & 1,80 & 12,76 & 1,82 & & & & \\
\hline 50000 & 2,26 & 1,81 & 2,87 & 1,82 & 3,59 & 1,83 & 5,08 & 1,83 & 7,43 & 1,87 & 10,93 & 1,81 & 15,29 & 1,80 \\
\hline 70000 & 1,05 & 1,81 & 1,34 & 1,83 & 1,68 & 1,85 & 2,34 & 1,86 & 3,41 & 1,93 & 4,81 & 1,89 & 6,70 & 1,86 \\
\hline 100000 & 0,48 & 1,81 & 0,62 & 1,82 & 0,78 & 1,85 & 1,07 & 1,88 & 1,61 & 1,94 & 2,17 & 1,93 & 2,93 & 1,93 \\
\hline 200000 & 0,10 & 1,81 & 0,13 & 1,81 & 0,17 & 1,83 & 0,24 & 1,89 & 0,43 & 1,79 & 0,57 & 1,80 & 0,76 & 1,83 \\
\hline \multirow[t]{2}{*}{400000} & 0,021 & 1,83 & 0,028 & 1,83 & 0,036 & 1,87 & 0,052 & 1,96 & 0,089 & 1,80 & 0,12 & 1,80 & 0,17 & 1,81 \\
\hline & \multicolumn{2}{|c|}{1977} & \multicolumn{2}{|c|}{1978} & \multicolumn{2}{|c|}{1979} & \multicolumn{2}{|c|}{1980} & \multicolumn{2}{|c|}{1981} & \multicolumn{2}{|c|}{1982} & \multicolumn{2}{|c|}{1983} \\
\hline $\mathrm{s}_{\mathrm{i}}$ & $\mathrm{p}_{\mathrm{i}}$ & $b_{i}$ & $\mathrm{p}_{\mathrm{i}}$ & $b_{i}$ & $\mathrm{p}_{\mathrm{i}}$ & $b_{i}$ & $\mathrm{p}_{\mathrm{i}}$ & $b_{i}$ & $\mathrm{p}_{\mathrm{i}}$ & $b_{i}$ & $\mathrm{p}_{\mathrm{i}}$ & $b_{i}$ & $\mathrm{p}_{\mathrm{i}}$ & $b_{i}$ \\
\hline 50000 & 19,05 & 1,77 & & & & & & & & & & & & \\
\hline 70000 & 8,41 & 1,81 & 11,36 & 1,79 & & & & & & & & & & \\
\hline 80000 & & & 8,12 & 1,82 & 10,48 & 1,74 & 14,54 & 1,71 & & & & & & \\
\hline 100000 & 3,53 & 1,88 & 4,71 & 1,87 & 5,98 & 1,77 & 8,23 & 1,74 & 11,42 & 1,72 & 15,48 & 1,68 & & \\
\hline 125000 & & & & & & & & & & & & & 11,44 & 1,66 \\
\hline 150000 & & & & & & & & & & & 5,42 & 1,72 & 7,02 & 1,68 \\
\hline 200000 & 0,84 & 1,85 & 1,09 & 1,86 & 1,17 & 1,84 & 1,52 & 1,83 & 2,05 & 1,82 & 2,62 & 1,76 & 3,30 & 1,72 \\
\hline \multirow[t]{2}{*}{400000} & 0,18 & 1,83 & 0,25 & 1,80 & 0,25 & 1,87 & 0,32 & 1,85 & 0,42 & 1,87 & 0,51 & 1,83 & 0,60 & 1,77 \\
\hline & \multicolumn{2}{|c|}{1984} & \multicolumn{2}{|c|}{1985} & \multicolumn{2}{|c|}{1986} & \multicolumn{2}{|c|}{1987} & \multicolumn{2}{|c|}{$1988\left(^{*}\right)$} & \multicolumn{2}{|c|}{1989} & \multicolumn{2}{|c|}{1990} \\
\hline $\mathrm{s}_{\mathrm{i}}$ & $\mathrm{p}_{\mathrm{i}}$ & $b_{i}$ & $\mathrm{p}_{\mathrm{i}}$ & $b_{i}$ & $\mathrm{p}_{\mathrm{i}}$ & $b_{i}$ & $\mathrm{p}_{\mathrm{i}}$ & $b_{i}$ & $\mathrm{p}_{\mathrm{i}}$ & $b_{i}$ & $\mathrm{p}_{\mathrm{i}}$ & $b_{i}$ & $\mathrm{p}_{\mathrm{i}}$ & $b_{i}$ \\
\hline 125000 & 13,85 & 1,65 & & & & & & & & & & & & \\
\hline 150000 & 8,51 & 1,67 & 10,14 & 1,67 & 11,56 & 1,68 & 12,28 & 1,71 & 13,45 & 1,83 & 15,00 & 1,73 & 17,28 & 1,72 \\
\hline 200000 & 3,97 & 1,70 & 4,74 & 1,71 & 5,44 & 1,72 & 5,81 & 1,76 & 6,44 & 1,94 & 7,19 & 1,78 & 8,36 & 1,75 \\
\hline 250000 & 2,25 & 1,73 & 2,67 & 1,74 & 3,07 & 1,76 & 3,29 & 1,80 & 3,70 & 2,04 & 4,09 & 1,82 & 4,72 & 1,80 \\
\hline \multirow[t]{2}{*}{500000} & 0,41 & 1,80 & 0,50 & 1,79 & 0,58 & 1,83 & 0,65 & 1,90 & 0,80 & 2,36 & 0,82 & 1,95 & 0,90 & 1,95 \\
\hline & 19 & & 199 & & 199 & & 199 & & 199 & & 199 & (**) & & \\
\hline $\mathrm{s}_{\mathrm{i}}$ & $\mathrm{p}_{\mathrm{i}}$ & $\mathrm{b}_{\mathrm{i}}$ & $\mathrm{p}_{\mathrm{i}}$ & $\mathrm{b}_{\mathrm{i}}$ & $\mathrm{p}_{\mathrm{i}}$ & $b_{i}$ & $\mathrm{p}_{\mathrm{i}}$ & $b_{i}$ & $\mathrm{p}_{\mathrm{i}}$ & $b_{i}$ & $\mathrm{p}_{\mathrm{i}}$ & $b_{i}$ & & \\
\hline 150000 & 18,99 & 1,70 & 20,25 & 1,68 & & & & & & & & & & \\
\hline 200000 & 9,20 & 1,71 & 9,84 & 1,68 & 10,34 & 1,65 & 10,81 & 1,65 & 11,59 & 1,64 & 12,39 & 1,63 & & \\
\hline 250000 & 5,14 & 1,75 & 5,42 & 1,72 & 5,63 & 1,69 & 5,86 & 1,69 & 6,26 & 1,67 & 6,72 & 1,66 & & \\
\hline 500000 & 0,93 & 1,91 & 0,93 & 1,88 & 0,91 & 1,87 & 0,93 & 1,88 & 0,97 & 1,87 & 1,01 & 1,87 & & \\
\hline
\end{tabular}

Source: Etats 1921 (cf. annexes D.1 et D.2)

Lecture: cf. tableau D-1.

Notes: (i) 1988(*): les chiffres ne sont pas homogènes aux chiffres des autres années (les tranches de revenu imposable et les revenus imposables incluent les plus-values taxées à taux proportionnel) (cf. annexes D-1 et D-2).

(ii) $1996\left(^{* *}\right)$ : chiffres provisoires (rôles émis au 31/12/1997). 
Tableau D-10: Résultats de l'estimation de la distribution du revenu imposable des foyers fiscaux ayant $2 A$ parts de quotient familial

\begin{tabular}{|c|c|c|c|c|c|c|c|c|c|c|c|c|}
\hline & P90 & P95 & P99 & P99,5 & P99,9 & P99,99 & P90-100 & P95-100 & P99-100 & P99,5-100 & P99,9-100 & $99,99-10$ \\
\hline 1970 & 26768 & 35599 & 71619 & 97953 & 201065 & 559832 & 32,25 & 21,74 & 8,89 & 6,06 & 2,48 & 0,70 \\
\hline 1971 & 29708 & 39561 & 80056 & 110103 & 228309 & 637410 & 32,61 & 22,08 & 9,12 & 6,24 & 2,56 & 0,73 \\
\hline 1972 & 32729 & 43790 & 89023 & 122334 & 249916 & 729194 & 32,49 & 22,06 & 9,20 & 6,32 & 2,61 & 0,76 \\
\hline 1973 & 37664 & 50380 & 103370 & 141456 & 291446 & 900382 & 33,62 & 22,91 & 9,64 & 6,65 & 2,84 & 0,88 \\
\hline 1974 & 43539 & 57639 & 137742 & 187211 & 380157 & 1055415 & 34,71 & 23,89 & 10,54 & 7,17 & 2,91 & 0,81 \\
\hline 1975 & 52025 & 68714 & 156408 & 212761 & 432857 & 1205831 & 34,83 & 23,93 & 10,39 & 7,07 & 2,88 & 0,80 \\
\hline 1976 & 58136 & 80152 & 176771 & 242154 & 502730 & 1406671 & 34,69 & 23,91 & 10,37 & 7,10 & 2,91 & 0,81 \\
\hline 1977 & 64780 & 84946 & 184402 & 254036 & 527941 & 1503476 & 32,85 & 22,42 & 9,59 & 6,54 & 2,72 & 0,77 \\
\hline 1978 & 74058 & 97240 & 208066 & 292692 & 599535 & 1672368 & 32,64 & 22,35 & 9,53 & 6,50 & 2,66 & 0,74 \\
\hline 1979 & 81612 & 108093 & 215258 & 291361 & 615658 & 1795470 & 30,82 & 20,79 & 8,59 & 5,90 & 2,49 & 0,73 \\
\hline 1980 & 92052 & 123553 & 241504 & 327229 & 685425 & 1974066 & 30,41 & 20,41 & 8,38 & 5,75 & 2,41 & 0,69 \\
\hline 1981 & 105700 & 133958 & 266819 & 368618 & 780695 & 2284263 & 30,29 & 20,26 & 8,33 & 5,75 & 2,44 & 0,71 \\
\hline 1982 & 115989 & 155197 & 293979 & 402297 & 833423 & 2362738 & 29,34 & 19,63 & 7,88 & 5,39 & 2,23 & 0,63 \\
\hline 1983 & 131848 & 168164 & 320458 & 433698 & 875642 & 2392669 & 29,14 & 19,25 & 7,59 & 5,13 & 2,07 & 0,57 \\
\hline 1984 & 140647 & 181719 & 338232 & 459802 & 938026 & 2601448 & 29,25 & 19,34 & 7,58 & 5,15 & 2,10 & 0,58 \\
\hline 1985 & 150865 & 195635 & 369582 & 501760 & 1020501 & 2817834 & 29,87 & 19,81 & 7,84 & 5,32 & 2,16 & 0,60 \\
\hline 1986 & 159070 & 207138 & 392053 & 536817 & 1113592 & 3163059 & 30,11 & 20,07 & 8,07 & 5,52 & 2,29 & 0,65 \\
\hline 1987 & 163342 & 213370 & 408395 & 566782 & 1213137 & 3603619 & 30,40 & 20,42 & 8,45 & 5,86 & 2,51 & 0,75 \\
\hline 1988 & 168241 & 220863 & 430549 & 600919 & 1303357 & 3946767 & 30,67 & 20,69 & 8,67 & 6,05 & 2,62 & 0,79 \\
\hline 1989 & 173140 & 228355 & 452703 & 635055 & 1393577 & 4289915 & 30,94 & 20,96 & 8,90 & 6,24 & 2,74 & 0,84 \\
\hline 1990 & 185175 & 243597 & 474887 & 666187 & 1461954 & 4500672 & 30,92 & 20,89 & 8,84 & 6,20 & 2,72 & 0,84 \\
\hline 1991 & 193181 & 252956 & 482270 & 670904 & 1443967 & 4323389 & 30,47 & 20,42 & 8,47 & 5,89 & 2,54 & 0,76 \\
\hline 1992 & 198675 & 258635 & 482161 & 667053 & 1417355 & 4166444 & 30,03 & 19,98 & 8,15 & 5,64 & 2,40 & 0,70 \\
\hline 1993 & 202635 & 262444 & 478677 & 660246 & 1393135 & 4054560 & 29,66 & 19,60 & 7,90 & 5,45 & 2,30 & 0,67 \\
\hline 1994 & 206207 & 266754 & 484002 & 670064 & 1426046 & 4201652 & 29,62 & 19,57 & 7,93 & 5,49 & 2,34 & 0,69 \\
\hline 1995 & 211839 & 273625 & 493922 & 681819 & 1441331 & 4206002 & 29,42 & 19,38 & 7,83 & 5,40 & 2,28 & 0,67 \\
\hline \multirow[t]{2}{*}{$1996^{*}$} & 217294 & 281186 & 503071 & 693985 & 1464778 & 4264950 & 29,12 & 19,14 & 7,71 & 5,32 & 2,25 & 0,65 \\
\hline & P90-95 & P95-99 & P99-99,5 & P99,5-99,9 & P99,9-100 & & P95-99 & P99-99,5 & P99,5-99,9 & P99,9-100 & & $g(P \mid B)$ \\
\hline 1970 & 10,51 & 12,85 & 2,83 & 3,58 & 2,48 & & & & & & & \\
\hline 1971 & 10,54 & 12,95 & 2,88 & 3,68 & 2,56 & & 0,5 & 1,8 & 2,4 & 2,8 & & 5,6 \\
\hline 1972 & 10,44 & 12,86 & 2,88 & 3,71 & 2,61 & & 0,2 & 0,8 & 1,8 & 3,0 & & 5,2 \\
\hline 1973 & 10,71 & 13,27 & 2,99 & 3,81 & 2,84 & & 0,5 & 1,3 & 0,0 & 5,9 & & 6,7 \\
\hline 1974 & 10,81 & 13,35 & 3,38 & 4,25 & 2,91 & & $-0,3$ & 11,9 & 10,7 & 1,7 & & 1,3 \\
\hline 1975 & 10,89 & 13,54 & 3,32 & 4,19 & 2,88 & & 0,7 & $-2,3$ & $-2,2$ & $-1,8$ & & 0,8 \\
\hline 1976 & 10,78 & 13,54 & 3,27 & 4,19 & 2,91 & & 1,1 & $-0,7$ & 1,2 & 2,1 & & 5,6 \\
\hline 1977 & 10,43 & 12,84 & 3,05 & 3,82 & 2,72 & & $-2,1$ & $-3,6$ & $-5,8$ & $-3,5$ & & 3,2 \\
\hline 1978 & 10,29 & 12,82 & 3,03 & 3,84 & 2,66 & & 1,2 & 0,7 & 1,8 & $-0,7$ & & 4,3 \\
\hline 1979 & 10,03 & 12,21 & 2,68 & 3,41 & 2,49 & & $-2,2$ & $-9,0$ & $-8,8$ & $-3,9$ & & 2,6 \\
\hline 1980 & 10,00 & 12,02 & 2,63 & 3,34 & 2,41 & & $-1,3$ & $-1,7$ & $-1,7$ & $-3,1$ & & $-0,3$ \\
\hline 1981 & 10,03 & 11,93 & 2,58 & 3,32 & 2,44 & & $-1,0$ & $-2,4$ & $-1,1$ & 0,9 & & $-0,6$ \\
\hline 1982 & 9,71 & 11,75 & 2,49 & 3,16 & 2,23 & & 1,7 & $-0,3$ & $-1,7$ & $-5,4$ & & 2,5 \\
\hline 1983 & 9,89 & 11,66 & 2,45 & 3,06 & 2,07 & & $-2,6$ & $-3,2$ & $-4,8$ & $-8,8$ & & 0,8 \\
\hline 1984 & 9,92 & 11,76 & 2,43 & 3,05 & 2,10 & & 0,5 & $-1,3$ & $-0,6$ & 1,1 & & 1,4 \\
\hline 1985 & 10,06 & 11,97 & 2,52 & 3,16 & 2,16 & & 0,4 & 2,2 & 2,0 & 1,5 & & 1,8 \\
\hline 1986 & 10,05 & 12,00 & 2,54 & 3,23 & 2,29 & & 0,3 & 1,2 & 2,5 & 6,0 & & 5,0 \\
\hline 1987 & 9,98 & 11,97 & 2,59 & 3,35 & 2,51 & & 0,5 & 2,3 & 4,4 & 10,2 & & 2,1 \\
\hline 1988 & 9,98 & 12,02 & 2,62 & 3,43 & 2,62 & & 0,4 & 1,4 & 2,3 & 4,7 & & 4,6 \\
\hline 1989 & 9,97 & 12,06 & 2,66 & 3,50 & 2,74 & & 0,4 & 1,4 & 2,2 & 4,4 & & 3,7 \\
\hline 1990 & 10,03 & 12,05 & 2,64 & 3,48 & 2,72 & & $-0,6$ & $-1,2$ & $-1,2$ & $-1,2$ & & 2,2 \\
\hline 1991 & 10,05 & 11,95 & 2,58 & 3,36 & 2,54 & & $-1,1$ & $-2,5$ & $-3,8$ & $-7,0$ & & 0,9 \\
\hline 1992 & 10,06 & 11,82 & 2,51 & 3,24 & 2,40 & & $-1,1$ & $-2,6$ & $-3,4$ & $-5,6$ & & 0,9 \\
\hline 1993 & 10,06 & 11,70 & 2,45 & 3,15 & 2,30 & & $-1,1$ & $-2,5$ & $-2,9$ & $-4,1$ & & $-1,0$ \\
\hline 1994 & 10,04 & 11,64 & 2,44 & 3,15 & 2,34 & & $-0,4$ & $-0,3$ & 0,3 & 1,8 & & 2,7 \\
\hline 1995 & 10,03 & 11,56 & 2,42 & 3,12 & 2,28 & & $-0,6$ & $-0,6$ & $-1,0$ & $-2,2$ & & 2,0 \\
\hline $1996^{*}$ & 9,98 & 11,43 & 2,39 & 3,07 & 2,25 & & $-0,6$ & $-0,8$ & $-0,9$ & $-1,2$ & & 0,6 \\
\hline & & & & & & 1970-1995 & $-0,3$ & $-0,5$ & $-0,4$ & $-0,2$ & & 2,5 \\
\hline
\end{tabular}

Source: Estimations de l'auteur à partir des Etats 1921 (cf. annexes D-1 et D-2) Lecture: Cf. tableau D-6.

Note: 1996(*): chiffres provisoires (rôles émis au 31/12/97). 
Tableau D-11: Les seuils de revenu imposable et les ratios $y^{\star}(y) / y$ utilisés pour l'estimation de la distribution du revenu imposable des foyers fiscaux ayant 2,5 parts de quotient familial

\begin{tabular}{|c|c|c|c|c|c|c|c|c|c|c|c|c|c|c|}
\hline \multirow[b]{2}{*}{$\mathrm{s}_{\mathrm{i}}$} & \multicolumn{2}{|c|}{1970} & \multicolumn{2}{|c|}{1971} & \multicolumn{2}{|c|}{1972} & \multicolumn{2}{|c|}{1973} & \multicolumn{2}{|c|}{1974} & \multicolumn{2}{|c|}{1975} & \multicolumn{2}{|c|}{1976} \\
\hline & $\mathrm{p}_{\mathrm{i}}$ & $b_{i}$ & $\mathrm{p}_{\mathrm{i}}$ & $\mathrm{b}_{\mathrm{i}}$ & $\mathrm{p}_{\mathrm{i}}$ & $\mathrm{b}_{\mathrm{i}}$ & $\mathrm{p}_{\mathrm{i}}$ & $b_{i}$ & $\mathrm{p}_{\mathrm{i}}$ & $b_{i}$ & $\mathrm{p}_{\mathrm{i}}$ & $\mathrm{b}_{\mathrm{i}}$ & $\mathrm{p}_{\mathrm{i}}$ & $\mathrm{b}_{\mathrm{i}}$ \\
\hline 30000 & 10,69 & 1,65 & 13,56 & 1,66 & 16,81 & 1,67 & & & & & & & & \\
\hline 40000 & 5,00 & 1,67 & 6,39 & 1,68 & 7,95 & 1,69 & 10,91 & 1,70 & 16,29 & 1,64 & & & & \\
\hline 50000 & 2,79 & 1,69 & 3,56 & 1,70 & 4,43 & 1,72 & 6,08 & 1,73 & 8,94 & 1,66 & 13,35 & 1,63 & 18,85 & 1,62 \\
\hline 70000 & 1,22 & 1,69 & 1,55 & 1,72 & 1,94 & 1,74 & 2,65 & 1,77 & 3,67 & 1,69 & 5,31 & 1,68 & 7,52 & 1,66 \\
\hline 100000 & 0,52 & 1,68 & 0,68 & 1,70 & 0,85 & 1,73 & 1,17 & 1,76 & 1,53 & 1,70 & 2,15 & 1,70 & 2,94 & 1,70 \\
\hline 200000 & 0,090 & 1,68 & 0,13 & 1,68 & 0,17 & 1,71 & 0,24 & 1,73 & 0,29 & 1,70 & 0,37 & 1,77 & 0,51 & 1,76 \\
\hline \multirow[t]{2}{*}{400000} & 0,016 & 1,73 & 0,022 & 1,75 & 0,031 & 1,75 & 0,045 & 1,78 & 0,055 & 1,61 & 0,078 & 1,65 & 0,11 & 1,64 \\
\hline & \multicolumn{2}{|c|}{1977} & \multicolumn{2}{|c|}{1978} & \multicolumn{2}{|c|}{1979} & \multicolumn{2}{|c|}{1980} & \multicolumn{2}{|c|}{1981} & \multicolumn{2}{|c|}{1982} & \multicolumn{2}{|c|}{1983} \\
\hline $\mathrm{s}_{\mathrm{i}}$ & $\mathrm{p}_{\mathrm{i}}$ & $b_{i}$ & $\mathrm{p}_{\mathrm{i}}$ & $b_{i}$ & $\mathrm{p}_{\mathrm{i}}$ & $b_{i}$ & $\mathrm{p}_{\mathrm{i}}$ & $b_{i}$ & $\mathrm{p}_{\mathrm{i}}$ & $b_{i}$ & $\mathrm{p}_{\mathrm{i}}$ & $b_{i}$ & $\mathrm{p}_{\mathrm{i}}$ & $\mathrm{b}_{\mathrm{i}}$ \\
\hline 50000 & 24,63 & 1,60 & & & & & & & & & & & & \\
\hline 70000 & 9,91 & 1,61 & 13,52 & 1,61 & & & & & & & & & & \\
\hline 80000 & & & 9,26 & 1,63 & 12,43 & 1,64 & 17,33 & 1,62 & & & & & & \\
\hline 100000 & 3,69 & 1,65 & 4,94 & 1,66 & 6,66 & 1,69 & 9,26 & 1,65 & 13,97 & 1,61 & 18,95 & 1,58 & & \\
\hline 125000 & & & & & & & & & & & & & 14,57 & 1,55 \\
\hline 150000 & & & & & & & & & & & 6,01 & 1,62 & 8,47 & 1,57 \\
\hline 200000 & 0,59 & 1,75 & 0,82 & 1,74 & 1,22 & 1,71 & 1,56 & 1,70 & 2,16 & 1,68 & 2,76 & 1,64 & 3,69 & 1,60 \\
\hline \multirow[t]{2}{*}{400000} & 0,12 & 1,69 & 0,17 & 1,71 & 0,23 & 1,72 & 0,29 & 1,70 & 0,38 & 1,71 & 0,45 & 1,68 & 0,57 & 1,63 \\
\hline & \multicolumn{2}{|c|}{1984} & \multicolumn{2}{|c|}{1985} & \multicolumn{2}{|c|}{1986} & \multicolumn{2}{|c|}{1987} & \multicolumn{2}{|c|}{$1988\left(^{*}\right)$} & \multicolumn{2}{|c|}{1989} & \multicolumn{2}{|c|}{1990} \\
\hline $\mathrm{s}_{\mathrm{i}}$ & $\mathrm{p}_{\mathrm{i}}$ & $b_{i}$ & $\mathrm{p}_{\mathrm{i}}$ & $\mathrm{b}_{\mathrm{i}}$ & $\mathrm{p}_{\mathrm{i}}$ & $\mathrm{b}_{\mathrm{i}}$ & $\mathrm{p}_{\mathrm{i}}$ & $b_{i}$ & $\mathrm{p}_{\mathrm{i}}$ & $b_{i}$ & $\mathrm{p}_{\mathrm{i}}$ & $\mathrm{b}_{\mathrm{i}}$ & $\mathrm{p}_{\mathrm{i}}$ & $\mathrm{b}_{\mathrm{i}}$ \\
\hline 150000 & 10,18 & 1,56 & 11,86 & 1,56 & 13,40 & 1,58 & 14,28 & 1,61 & 15,85 & 1,68 & 17,64 & 1,65 & 19,63 & 1,65 \\
\hline 200000 & 4,36 & 1,60 & 5,11 & 1,60 & 5,88 & 1,62 & 6,40 & 1,65 & 7,25 & 1,74 & 8,18 & 1,67 & 9,35 & 1,66 \\
\hline 250000 & 2,36 & 1,61 & 2,76 & 1,61 & 3,20 & 1,64 & 3,50 & 1,68 & 4,02 & 1,80 & 4,51 & 1,71 & 5,14 & 1,69 \\
\hline \multirow[t]{2}{*}{500000} & 0,37 & 1,65 & 0,44 & 1,64 & 0,53 & 1,66 & 0,60 & 1,74 & 0,74 & 2,01 & 0,79 & 1,81 & 0,88 & 1,80 \\
\hline & \multicolumn{2}{|c|}{1991} & \multicolumn{2}{|c|}{1992} & 199 & & 199 & & 199 & & 199 & $\left.{ }^{* *}\right)$ & & \\
\hline $\mathrm{s}_{\mathrm{i}}$ & $\mathrm{p}_{\mathrm{i}}$ & $b_{i}$ & $\mathrm{p}_{\mathrm{i}}$ & $b_{i}$ & $\mathrm{p}_{\mathrm{i}}$ & $b_{i}$ & $\mathrm{p}_{\mathrm{i}}$ & $b_{i}$ & $\mathrm{p}_{\mathrm{i}}$ & $b_{i}$ & $\mathrm{p}_{\mathrm{i}}$ & $b_{i}$ & & \\
\hline 200000 & 10,30 & 1,64 & 10,93 & 1,61 & 11,35 & 1,59 & 11,76 & 1,59 & 13,84 & 1,59 & 14,50 & 1,59 & & \\
\hline 250000 & 5,61 & 1,66 & 5,88 & 1,63 & 6,08 & 1,61 & 6,30 & 1,61 & 7,45 & 1,60 & 7,85 & 1,59 & & \\
\hline 500000 & 0,91 & 1,77 & 0,91 & 1,73 & 0,89 & 1,72 & 0,91 & 1,74 & 1,06 & 1,73 & 1,09 & 1,72 & & \\
\hline
\end{tabular}

Source: Etats 1921 (cf. annexes D.1 et D.2)

Lecture: cf. tableau D-1.

Notes: (i) 1988(*): les chiffres ne sont pas homogènes aux chiffres des autres années (les tranches de revenu imposable et les revenus imposables incluent les plus-values taxées à taux proportionnel) (cf. annexes D-1 et D-2).

(ii) 1996 $\left({ }^{* *}\right)$ : chiffres provisoires (rôles émis au 31/12/1997). 
Tableau D-12: Résultats de l'estimation de la distribution du revenu imposable des foyers fiscaux ayant 2,5 parts de quotient familial

\begin{tabular}{|c|c|c|c|c|c|c|c|c|c|c|c|c|}
\hline & P90 & P95 & P99 & P99,5 & P99,9 & P99,99 & P90-100 & P95-100 & P99-100 & P99,5-100 & P99,9-10 & $99,99-100$ \\
\hline 1970 & 30795 & 39990 & 75865 & 101592 & 191717 & 485107 & 29,52 & 19,41 & 7,48 & 4,95 & 1,88 & 0,49 \\
\hline 1971 & 33860 & 44165 & 85107 & 113208 & 219262 & 559919 & 29,81 & 19,68 & 7,68 & 5,11 & 1,96 & 0,52 \\
\hline 1972 & 36423 & 47545 & 93240 & 124992 & 246771 & 647590 & 29,58 & 19,65 & 7,77 & 5,21 & 2,03 & 0,54 \\
\hline 1973 & 41458 & 54313 & 106877 & 145931 & 282065 & 775636 & 30,24 & 20,20 & 8,09 & 5,44 & 2,16 & 0,59 \\
\hline 1974 & 47836 & 61689 & 119119 & 159252 & 319403 & 766893 & 29,37 & 19,36 & 7,50 & 5,02 & 1,91 & 0,46 \\
\hline 1975 & 55910 & 71711 & 129973 & 175566 & 363714 & 897417 & 29,46 & 19,41 & 7,41 & 5,01 & 1,93 & 0,48 \\
\hline 1976 & 62502 & 80426 & 149316 & 201363 & 413343 & 1013845 & 29,17 & 19,20 & 7,39 & 4,98 & 1,91 & 0,47 \\
\hline 1977 & 69773 & 88655 & 159301 & 214175 & 431462 & 1102761 & 27,90 & 18,20 & 6,90 & 4,64 & 1,81 & 0,46 \\
\hline 1978 & 77669 & 99549 & 183686 & 246768 & 494136 & 1287597 & 27,66 & 18,13 & 7,00 & 4,70 & 1,85 & 0,48 \\
\hline 1979 & 87108 & 112404 & 216929 & 288181 & 564155 & 1474939 & 27,74 & 18,36 & 7,19 & 4,79 & 1,88 & 0,49 \\
\hline 1980 & 97046 & 123472 & 240045 & 319460 & 620535 & 1604360 & 27,28 & 17,96 & 6,98 & 4,64 & 1,80 & 0,47 \\
\hline 1981 & 113487 & 142298 & 268361 & 357848 & 698055 & 1815765 & 27,48 & 18,02 & 6,91 & 4,60 & 1,80 & 0,47 \\
\hline 1982 & 123417 & 160958 & 289979 & 384180 & 738247 & 1879473 & 26,66 & 17,38 & 6,50 & 4,31 & 1,66 & 0,42 \\
\hline 1983 & 141263 & 178379 & 321245 & 419743 & 781034 & 1898907 & 27,00 & 17,39 & 6,37 & 4,16 & 1,55 & 0,38 \\
\hline 1984 & 150965 & 190047 & 336272 & 441824 & 832791 & 2062442 & 26,99 & 17,39 & 6,36 & 4,18 & 1,58 & 0,39 \\
\hline 1985 & 159515 & 201626 & 361674 & 474350 & 890385 & 2191987 & 27,32 & 17,64 & 6,51 & 4,27 & 1,60 & 0,39 \\
\hline 1986 & 167099 & 212756 & 387620 & 511135 & 971551 & 2435142 & 27,82 & 18,08 & 6,78 & 4,47 & 1,70 & 0,43 \\
\hline 1987 & 167822 & 220335 & 402517 & 540487 & 1071504 & 2852352 & 28,31 & 18,58 & 7,17 & 4,82 & 1,91 & 0,51 \\
\hline 1988 & 176148 & 229911 & 426720 & 577722 & 1167689 & 3197403 & 28,76 & 18,97 & 7,45 & 5,04 & 2,04 & 0,56 \\
\hline 1989 & 184475 & 239488 & 450923 & 614956 & 1263873 & 3542453 & 29,21 & 19,35 & 7,72 & 5,26 & 2,16 & 0,61 \\
\hline 1990 & 194717 & 252876 & 471360 & 640774 & 1307193 & 3625156 & 29,48 & 19,48 & 7,72 & 5,24 & 2,14 & 0,59 \\
\hline 1991 & 202339 & 261767 & 480336 & 649073 & 1305815 & 3549914 & 29,35 & 19,29 & 7,53 & 5,09 & 2,05 & 0,56 \\
\hline 1992 & 206843 & 266131 & 479758 & 642281 & 1264506 & 3332880 & 29,05 & 18,94 & 7,24 & 4,85 & 1,91 & 0,50 \\
\hline 1993 & 209627 & 269233 & 476443 & 636943 & 1249892 & 3278943 & 28,97 & 18,79 & 7,12 & 4,76 & 1,87 & 0,49 \\
\hline 1994 & 212426 & 272741 & 480740 & 645176 & 1277456 & 3394547 & 29,09 & 18,87 & 7,19 & 4,83 & 1,91 & 0,51 \\
\hline 1995 & 223827 & 290313 & 513202 & 687823 & 1357685 & 3591782 & 28,35 & 18,39 & 7,03 & 4,71 & 1,86 & 0,49 \\
\hline \multirow[t]{2}{*}{$1996^{*}$} & 228575 & 295606 & 519085 & 693387 & 1358090 & 3553196 & 28,26 & 18,28 & 6,93 & 4,63 & 1,81 & 0,47 \\
\hline & P90-95 & P95-99 & P99-99,5 & P99,5-99,9 & P99,9-100 & & P95-99 & P99-99,5 & P99,5-99,9 & P99,9-100 & & $g(\mathrm{PIB})$ \\
\hline 1970 & 10,51 & 12,85 & 2,83 & 3,58 & 2,48 & & & & & & & \\
\hline 1971 & 10,54 & 12,95 & 2,88 & 3,68 & 2,56 & & 0,5 & 1,8 & 2,4 & 2,8 & & 5,6 \\
\hline 1972 & 10,44 & 12,86 & 2,88 & 3,71 & 2,61 & & 0,2 & 0,8 & 1,8 & 3,0 & & 5,2 \\
\hline 1973 & 10,71 & 13,27 & 2,99 & 3,81 & 2,84 & & 0,5 & 1,3 & 0,0 & 5,9 & & 6,7 \\
\hline 1974 & 10,81 & 13,35 & 3,38 & 4,25 & 2,91 & & $-0,3$ & 11,9 & 10,7 & 1,7 & & 1,3 \\
\hline 1975 & 10,89 & 13,54 & 3,32 & 4,19 & 2,88 & & 0,7 & $-2,3$ & $-2,2$ & $-1,8$ & & 0,8 \\
\hline 1976 & 10,78 & 13,54 & 3,27 & 4,19 & 2,91 & & 1,1 & $-0,7$ & 1,2 & 2,1 & & 5,6 \\
\hline 1977 & 10,43 & 12,84 & 3,05 & 3,82 & 2,72 & & $-2,1$ & $-3,6$ & $-5,8$ & $-3,5$ & & 3,2 \\
\hline 1978 & 10,29 & 12,82 & 3,03 & 3,84 & 2,66 & & 1,2 & 0,7 & 1,8 & $-0,7$ & & 4,3 \\
\hline 1979 & 10,03 & 12,21 & 2,68 & 3,41 & 2,49 & & $-2,2$ & $-9,0$ & $-8,8$ & $-3,9$ & & 2,6 \\
\hline 1980 & 10,00 & 12,02 & 2,63 & 3,34 & 2,41 & & $-1,3$ & $-1,7$ & $-1,7$ & $-3,1$ & & $-0,3$ \\
\hline 1981 & 10,03 & 11,93 & 2,58 & 3,32 & 2,44 & & $-1,0$ & $-2,4$ & $-1,1$ & 0,9 & & $-0,6$ \\
\hline 1982 & 9,71 & 11,75 & 2,49 & 3,16 & 2,23 & & 1,7 & $-0,3$ & $-1,7$ & $-5,4$ & & 2,5 \\
\hline 1983 & 9,89 & 11,66 & 2,45 & 3,06 & 2,07 & & $-2,6$ & $-3,2$ & $-4,8$ & $-8,8$ & & 0,8 \\
\hline 1984 & 9,92 & 11,76 & 2,43 & 3,05 & 2,10 & & 0,5 & $-1,3$ & $-0,6$ & 1,1 & & 1,4 \\
\hline 1985 & 10,06 & 11,97 & 2,52 & 3,16 & 2,16 & & 0,4 & 2,2 & 2,0 & 1,5 & & 1,8 \\
\hline 1986 & 10,05 & 12,00 & 2,54 & 3,23 & 2,29 & & 0,3 & 1,2 & 2,5 & 6,0 & & 5,0 \\
\hline 1987 & 9,98 & 11,97 & 2,59 & 3,35 & 2,51 & & 0,5 & 2,3 & 4,4 & 10,2 & & 2,1 \\
\hline 1988 & 9,98 & 12,02 & 2,62 & 3,43 & 2,62 & & 0,4 & 1,4 & 2,3 & 4,7 & & 4,6 \\
\hline 1989 & 9,97 & 12,06 & 2,66 & 3,50 & 2,74 & & 0,4 & 1,4 & 2,2 & 4,4 & & 3,7 \\
\hline 1990 & 10,03 & 12,05 & 2,64 & 3,48 & 2,72 & & $-0,6$ & $-1,2$ & $-1,2$ & $-1,2$ & & 2,2 \\
\hline 1991 & 10,05 & 11,95 & 2,58 & 3,36 & 2,54 & & $-1,1$ & $-2,5$ & $-3,8$ & $-7,0$ & & 0,9 \\
\hline 1992 & 10,06 & 11,82 & 2,51 & 3,24 & 2,40 & & $-1,1$ & $-2,6$ & $-3,4$ & $-5,6$ & & 0,9 \\
\hline 1993 & 10,06 & 11,70 & 2,45 & 3,15 & 2,30 & & $-1,1$ & $-2,5$ & $-2,9$ & $-4,1$ & & $-1,0$ \\
\hline 1994 & 10,04 & 11,64 & 2,44 & 3,15 & 2,34 & & $-0,4$ & $-0,3$ & 0,3 & 1,8 & & 2,7 \\
\hline 1995 & 10,03 & 11,56 & 2,42 & 3,12 & 2,28 & & $-0,6$ & $-0,6$ & $-1,0$ & $-2,2$ & & 2,0 \\
\hline $1996^{*}$ & 9,98 & 11,43 & 2,39 & 3,07 & 2,25 & & $-0,6$ & $-0,8$ & $-0,9$ & $-1,2$ & & 0,6 \\
\hline & & & & & & 1970-1995 & $-0,3$ & $-0,5$ & $-0,4$ & $-0,2$ & & 2,5 \\
\hline
\end{tabular}

Source: Estimations de l'auteur à partir des Etats 1921 (cf. annexes D-1 et D-2) Lecture: Cf. tableau D-6.

Note: 1996(*): chiffres provisoires (rôles émis au 31/12/97). 
Tableau D-13: Les seuils de revenu imposable et les ratios $y^{\star}(y) / y$ utilisés pour l'estimation de la distribution du revenu imposable des foyers fiscaux ayant 3 parts de quotient familial

\begin{tabular}{|c|c|c|c|c|c|c|c|c|c|c|c|c|c|c|}
\hline \multirow[b]{2}{*}{$\mathrm{s}_{\mathrm{i}}$} & \multicolumn{2}{|c|}{1970} & \multicolumn{2}{|c|}{1971} & \multicolumn{2}{|c|}{1972} & \multicolumn{2}{|c|}{1973} & \multicolumn{2}{|c|}{1974} & \multicolumn{2}{|c|}{1975} & \multicolumn{2}{|c|}{1976} \\
\hline & $\mathrm{p}_{\mathrm{i}}$ & $b_{i}$ & $\mathrm{p}_{\mathrm{i}}$ & $b_{i}$ & $\mathrm{p}_{\mathrm{i}}$ & $b_{i}$ & $\mathrm{p}_{\mathrm{i}}$ & $b_{i}$ & $\mathrm{p}_{\mathrm{i}}$ & $b_{i}$ & $\mathrm{p}_{\mathrm{i}}$ & $b_{i}$ & $\mathrm{p}_{\mathrm{i}}$ & $b_{i}$ \\
\hline 30000 & 12,21 & 1,75 & 15,08 & 1,77 & 18,23 & 1,80 & & & & & & & & \\
\hline 40000 & 6,40 & 1,72 & 7,97 & 1,75 & 9,76 & 1,77 & 13,04 & 1,79 & & & & & & \\
\hline 50000 & 3,82 & 1,71 & 4,79 & 1,74 & 5,87 & 1,76 & 7,89 & 1,79 & 11,02 & 1,71 & 15,80 & 1,71 & 21,69 & 1,72 \\
\hline 70000 & 1,75 & 1,68 & 2,23 & 1,70 & 2,76 & 1,74 & 3,76 & 1,76 & 5,04 & 1,69 & 7,10 & 1,70 & 9,80 & 1,71 \\
\hline 100000 & 0,74 & 1,65 & 0,99 & 1,66 & 1,24 & 1,70 & 1,72 & 1,72 & 2,21 & 1,65 & 3,11 & 1,67 & 4,25 & 1,69 \\
\hline 200000 & 0,13 & 1,63 & 0,17 & 1,64 & 0,24 & 1,65 & 0,34 & 1,66 & 0,36 & 1,65 & 0,55 & 1,62 & 0,80 & 1,62 \\
\hline \multirow[t]{2}{*}{400000} & 0,020 & 1,71 & 0,029 & 1,68 & 0,038 & 1,76 & 0,055 & 1,73 & 0,06 & 1,63 & 0,091 & 1,64 & 0,13 & 1,64 \\
\hline & \multicolumn{2}{|c|}{1977} & \multicolumn{2}{|c|}{1978} & \multicolumn{2}{|c|}{1979} & \multicolumn{2}{|c|}{1980} & \multicolumn{2}{|c|}{1981} & \multicolumn{2}{|c|}{1982} & \multicolumn{2}{|c|}{1983} \\
\hline $\mathrm{s}_{\mathrm{i}}$ & $\mathrm{p}_{\mathrm{i}}$ & $b_{i}$ & $\mathrm{p}_{\mathrm{i}}$ & $b_{i}$ & $\mathrm{p}_{\mathrm{i}}$ & $b_{i}$ & $\mathrm{p}_{\mathrm{i}}$ & $b_{i}$ & $\mathrm{p}_{\mathrm{i}}$ & $b_{i}$ & $\mathrm{p}_{\mathrm{i}}$ & $b_{i}$ & $\mathrm{p}_{\mathrm{i}}$ & $b_{i}$ \\
\hline 70000 & 12,80 & 1,66 & & & & & & & & & & & & \\
\hline 80000 & & & 12,36 & 1,67 & 16,38 & 1,72 & & & & & & & & \\
\hline 100000 & 5,28 & 1,65 & 7,04 & 1,67 & 9,38 & 1,74 & 12,80 & 1,70 & 18,23 & 1,67 & 24,02 & 1,65 & & \\
\hline \multicolumn{15}{|l|}{125000} \\
\hline 150000 & & & & & & & & & & & 8,52 & 1,65 & 12,15 & 1,61 \\
\hline 200000 & 0,92 & 1,61 & 1,31 & 1,60 & 1,92 & 1,69 & 2,47 & 1,69 & 3,26 & 1,68 & 4,16 & 1,64 & 5,65 & 1,61 \\
\hline \multirow[t]{2}{*}{400000} & 0,15 & 1,63 & 0,22 & 1,60 & 0,36 & 1,65 & 0,46 & 1,64 & 0,59 & 1,65 & 0,72 & 1,61 & 0,93 & 1,57 \\
\hline & \multicolumn{2}{|c|}{1984} & \multicolumn{2}{|c|}{1985} & \multicolumn{2}{|c|}{1986} & \multicolumn{2}{|c|}{1987} & \multicolumn{2}{|c|}{$1988\left(^{*}\right)$} & \multicolumn{2}{|c|}{1989} & \multicolumn{2}{|c|}{1990} \\
\hline $\mathrm{s}_{\mathrm{i}}$ & $\mathrm{p}_{\mathrm{i}}$ & $b_{i}$ & $\mathrm{p}_{\mathrm{i}}$ & $b_{i}$ & $\mathrm{p}_{\mathrm{i}}$ & $b_{i}$ & $\mathrm{p}_{\mathrm{i}}$ & $b_{i}$ & $\mathrm{p}_{\mathrm{i}}$ & $b_{i}$ & $\mathrm{p}_{\mathrm{i}}$ & $b_{i}$ & $\mathrm{p}_{\mathrm{i}}$ & $b_{i}$ \\
\hline 150000 & 14,56 & 7,13 & 16,95 & 1,61 & 19,00 & 1,64 & 20,21 & 1,67 & & & & & & \\
\hline 200000 & 6,68 & 1,61 & 7,83 & 1,62 & 8,93 & 1,65 & 9,69 & 1,68 & 10,99 & 1,76 & 12,49 & 1,72 & 14,40 & 1,72 \\
\hline 250000 & 3,76 & 1,60 & 4,41 & 1,61 & 5,06 & 1,65 & 5,51 & 1,69 & 6,35 & 1,78 & 7,23 & 1,73 & 8,37 & 1,72 \\
\hline \multirow[t]{2}{*}{500000} & 0,60 & 1,56 & 0,73 & 1,57 & 0,88 & 1,61 & 1,01 & 1,68 & 1,23 & 1,87 & 1,39 & 1,73 & 1,57 & 1,75 \\
\hline & \multicolumn{2}{|c|}{1991} & 199 & & 199 & & 199 & & 199 & & 199 & & & \\
\hline $\mathrm{s}_{\mathrm{i}}$ & $\mathrm{p}_{\mathrm{i}}$ & $b_{i}$ & $\mathrm{p}_{\mathrm{i}}$ & $b_{i}$ & $\mathrm{p}_{\mathrm{i}}$ & $b_{i}$ & $\mathrm{p}_{\mathrm{i}}$ & $b_{i}$ & $\mathrm{p}_{\mathrm{i}}$ & $b_{i}$ & $\mathrm{p}_{\mathrm{i}}$ & $b_{i}$ & & \\
\hline 200000 & 15,95 & 1,69 & 17,09 & 1,66 & & & & & & & & & & \\
\hline 250000 & 9,21 & 1,69 & 9,77 & 1,66 & 10,17 & 1,65 & 10,62 & 1,65 & 10,78 & 1,64 & 11,26 & 1,63 & & \\
\hline 500000 & 1,64 & 1,70 & 1,67 & 1,67 & 1,69 & 1,68 & 1,74 & 1,69 & 1,77 & 1,68 & 1,81 & 1,66 & & \\
\hline
\end{tabular}

Source: Etats 1921 (cf. annexes D.1 et D.2)

Lecture: cf. tableau D-1.

Notes: (i) $1988\left(^{*}\right)$ : les chiffres ne sont pas homogènes aux chiffres des autres années (les tranches de revenu imposable et les revenus imposables incluent les plus-values taxées à taux proportionnel) (cf. annexes D-1 et D-2).

(ii) $1996\left(^{* *}\right)$ : chiffres provisoires (rôles émis au 31/12/1997). 
Tableau D-14: Résultats de l'estimation de la distribution du revenu imposable des foyers fiscaux ayant 3 parts de quotient familial

\begin{tabular}{|c|c|c|c|c|c|c|c|c|c|c|c|c|}
\hline & P90 & P95 & P99 & P99,5 & P99,9 & P99,99 & P90-100 & P95-100 & P99-100 & P99,5-100 & P99,9-100 & $99,99-100$ \\
\hline 1970 & 32676 & 44743 & 89033 & 116889 & 218747 & 532794 & 30,31 & 20,25 & 7,78 & 5,11 & 1,90 & 0,48 \\
\hline 1971 & 36293 & 49086 & 99417 & 132164 & 241468 & 611592 & 30,58 & 20,54 & 7,98 & 5,23 & 1,95 & 0,49 \\
\hline 1972 & 39579 & 53607 & 109313 & 148591 & 262607 & 711446 & 30,45 & 20,55 & 8,09 & 5,35 & 2,01 & 0,55 \\
\hline 1973 & 45039 & 61852 & 129773 & 171070 & 310641 & 820909 & 31,12 & 21,09 & 8,35 & 5,50 & 2,08 & 0,55 \\
\hline 1974 & 52058 & 70231 & 133897 & 176082 & 336260 & 816825 & 29,68 & 19,74 & 7,36 & 4,84 & 1,82 & 0,44 \\
\hline 1975 & 60758 & 82652 & 159171 & 207745 & 385538 & 943800 & 29,85 & 19,90 & 7,45 & 4,86 & 1,82 & 0,45 \\
\hline 1976 & 69412 & 93536 & 183833 & 239872 & 446848 & 1097201 & 29,70 & 19,78 & 7,46 & 4,87 & 1,83 & 0,45 \\
\hline 1977 & 77217 & 102185 & 193872 & 251940 & 467530 & 1134208 & 28,13 & 18,50 & 6,84 & 4,45 & 1,67 & 0,40 \\
\hline 1978 & 87059 & 114720 & 221578 & 291869 & 533949 & 1267023 & 27,96 & 18,46 & 6,85 & 4,50 & 1,65 & 0,39 \\
\hline 1979 & 97307 & 135360 & 267498 & 351413 & 662155 & 1639075 & 28,72 & 19,44 & 7,50 & 4,92 & 1,86 & 0,46 \\
\hline 1980 & 110744 & 150003 & 295402 & 387418 & 727151 & 1789930 & 28,15 & 18,90 & 7,24 & 4,75 & 1,78 & 0,44 \\
\hline 1981 & 127191 & 168302 & 324446 & 426445 & 804492 & 1994808 & 27,94 & 18,48 & 7,01 & 4,61 & 1,74 & 0,43 \\
\hline 1982 & 140800 & 186062 & 352860 & 458948 & 844961 & 2023348 & 26,84 & 17,65 & 6,56 & 4,27 & 1,57 & 0,38 \\
\hline 1983 & 161459 & 209490 & 389936 & 501779 & 901175 & 2082691 & 27,27 & 17,76 & 6,44 & 4,14 & 1,49 & 0,34 \\
\hline 1984 & 171659 & 224542 & 416344 & 534442 & 954355 & 2187600 & 27,20 & 17,69 & 6,41 & 4,11 & 1,47 & 0,34 \\
\hline 1985 & 182151 & 238290 & 445790 & 572898 & 1025757 & 2360252 & 27,62 & 18,00 & 6,54 & 4,20 & 1,50 & 0,35 \\
\hline 1986 & 191286 & 251117 & 476672 & 619828 & 1140489 & 2728745 & 28,07 & 18,43 & 6,84 & 4,45 & 1,64 & 0,39 \\
\hline 1987 & 197445 & 260067 & 502411 & 664558 & 1272287 & 3221894 & 28,55 & 18,91 & 7,26 & 4,80 & 1,84 & 0,47 \\
\hline 1988 & 208453 & 275990 & 538402 & 717474 & 1397754 & 3630646 & 28,91 & 19,25 & 7,50 & 5,00 & 1,95 & 0,51 \\
\hline 1989 & 219462 & 291912 & 574393 & 770391 & 1523221 & 4039398 & 29,27 & 19,59 & 7,75 & 5,19 & 2,05 & 0,54 \\
\hline 1990 & 232060 & 310336 & 606519 & 815807 & 1623752 & 4347052 & 29,52 & 19,74 & 7,83 & 5,27 & 2,10 & 0,56 \\
\hline 1991 & 241810 & 315735 & 614073 & 817789 & 1590518 & 4119620 & 29,11 & 19,22 & 7,47 & 4,98 & 1,94 & 0,50 \\
\hline 1992 & 247702 & 321653 & 614947 & 812927 & 1554180 & 3927942 & 28,68 & 18,82 & 7,19 & 4,76 & 1,82 & 0,46 \\
\hline 1993 & 251638 & 322676 & 617547 & 816731 & 1563084 & 3956348 & 28,60 & 18,68 & 7,15 & 4,73 & 1,81 & 0,46 \\
\hline 1994 & 256004 & 324285 & 627316 & 833490 & 1612352 & 4144128 & 28,63 & 18,68 & 7,23 & 4,80 & 1,86 & 0,48 \\
\hline 1995 & 257433 & 328963 & 630428 & 834250 & 1598764 & 4054465 & 29,15 & 19,02 & 7,29 & 4,82 & 1,85 & 0,47 \\
\hline \multirow[t]{2}{*}{$1996^{*}$} & 261730 & 333548 & 632437 & 833077 & 1579591 & 3945225 & 28,96 & 18,80 & 7,13 & 4,69 & 1,78 & 0,44 \\
\hline & P90-95 & P95-99 & P99-99,5 & P99,5-99,9 & P99,9-100 & & P95-99 & P99-99,5 & P99,5-99,9 & P99,9-100 & & $g(\mathrm{PIB})$ \\
\hline 1970 & 10,51 & 12,85 & 2,83 & 3,58 & 2,48 & & & & & & & \\
\hline 1971 & 10,54 & 12,95 & 2,88 & 3,68 & 2,56 & & 0,5 & 1,8 & 2,4 & 2,8 & & 5,6 \\
\hline 1972 & 10,44 & 12,86 & 2,88 & 3,71 & 2,61 & & 0,2 & 0,8 & 1,8 & 3,0 & & 5,2 \\
\hline 1973 & 10,71 & 13,27 & 2,99 & 3,81 & 2,84 & & 0,5 & 1,3 & 0,0 & 5,9 & & 6,7 \\
\hline 1974 & 10,81 & 13,35 & 3,38 & 4,25 & 2,91 & & $-0,3$ & 11,9 & 10,7 & 1,7 & & 1,3 \\
\hline 1975 & 10,89 & 13,54 & 3,32 & 4,19 & 2,88 & & 0,7 & $-2,3$ & $-2,2$ & $-1,8$ & & 0,8 \\
\hline 1976 & 10,78 & 13,54 & 3,27 & 4,19 & 2,91 & & 1,1 & $-0,7$ & 1,2 & 2,1 & & 5,6 \\
\hline 1977 & 10,43 & 12,84 & 3,05 & 3,82 & 2,72 & & $-2,1$ & $-3,6$ & $-5,8$ & $-3,5$ & & 3,2 \\
\hline 1978 & 10,29 & 12,82 & 3,03 & 3,84 & 2,66 & & 1,2 & 0,7 & 1,8 & $-0,7$ & & 4,3 \\
\hline 1979 & 10,03 & 12,21 & 2,68 & 3,41 & 2,49 & & $-2,2$ & $-9,0$ & $-8,8$ & $-3,9$ & & 2,6 \\
\hline 1980 & 10,00 & 12,02 & 2,63 & 3,34 & 2,41 & & $-1,3$ & $-1,7$ & $-1,7$ & $-3,1$ & & $-0,3$ \\
\hline 1981 & 10,03 & 11,93 & 2,58 & 3,32 & 2,44 & & $-1,0$ & $-2,4$ & $-1,1$ & 0,9 & & $-0,6$ \\
\hline 1982 & 9,71 & 11,75 & 2,49 & 3,16 & 2,23 & & 1,7 & $-0,3$ & $-1,7$ & $-5,4$ & & 2,5 \\
\hline 1983 & 9,89 & 11,66 & 2,45 & 3,06 & 2,07 & & $-2,6$ & $-3,2$ & $-4,8$ & $-8,8$ & & 0,8 \\
\hline 1984 & 9,92 & 11,76 & 2,43 & 3,05 & 2,10 & & 0,5 & $-1,3$ & $-0,6$ & 1,1 & & 1,4 \\
\hline 1985 & 10,06 & 11,97 & 2,52 & 3,16 & 2,16 & & 0,4 & 2,2 & 2,0 & 1,5 & & 1,8 \\
\hline 1986 & 10,05 & 12,00 & 2,54 & 3,23 & 2,29 & & 0,3 & 1,2 & 2,5 & 6,0 & & 5,0 \\
\hline 1987 & 9,98 & 11,97 & 2,59 & 3,35 & 2,51 & & 0,5 & 2,3 & 4,4 & 10,2 & & 2,1 \\
\hline 1988 & 9,98 & 12,02 & 2,62 & 3,43 & 2,62 & & 0,4 & 1,4 & 2,3 & 4,7 & & 4,6 \\
\hline 1989 & 9,97 & 12,06 & 2,66 & 3,50 & 2,74 & & 0,4 & 1,4 & 2,2 & 4,4 & & 3,7 \\
\hline 1990 & 10,03 & 12,05 & 2,64 & 3,48 & 2,72 & & $-0,6$ & $-1,2$ & $-1,2$ & $-1,2$ & & 2,2 \\
\hline 1991 & 10,05 & 11,95 & 2,58 & 3,36 & 2,54 & & $-1,1$ & $-2,5$ & $-3,8$ & $-7,0$ & & 0,9 \\
\hline 1992 & 10,06 & 11,82 & 2,51 & 3,24 & 2,40 & & $-1,1$ & $-2,6$ & $-3,4$ & $-5,6$ & & 0,9 \\
\hline 1993 & 10,06 & 11,70 & 2,45 & 3,15 & 2,30 & & $-1,1$ & $-2,5$ & $-2,9$ & $-4,1$ & & $-1,0$ \\
\hline 1994 & 10,04 & 11,64 & 2,44 & 3,15 & 2,34 & & $-0,4$ & $-0,3$ & 0,3 & 1,8 & & 2,7 \\
\hline 1995 & 10,03 & 11,56 & 2,42 & 3,12 & 2,28 & & $-0,6$ & $-0,6$ & $-1,0$ & $-2,2$ & & 2,0 \\
\hline $1996^{*}$ & 9,98 & 11,43 & 2,39 & 3,07 & 2,25 & & $-0,6$ & $-0,8$ & $-0,9$ & $-1,2$ & & 0,6 \\
\hline & & & & & & 1970-1995 & $-0,3$ & $-0,5$ & $-0,4$ & $-0,2$ & & 2,5 \\
\hline
\end{tabular}

Source: Estimations de l'auteur à partir des Etats 1921 (cf. annexes D-1 et D-2) Lecture: Cf. tableau D-6.

Note: 1996(*): chiffres provisoires (rôles émis au 31/12/97). 
foyers dont le revenu imposable est supérieur à $x$ francs avait évolué linéairement entre $x=70000$ francs et $x=100000$ francs. Nous avons comparé les estimations ainsi obtenues pour les années 1988-1995 avec les estimations issues des échantillons légers, et il apparaît que cette hypothèse de linéarité fournit des estimations acceptables, pourvu que l'on se limite à la composition des foyers de P90-100, P95100 , P99-100 et P99,5-100 et que l'on ne cherche pas à aller au-delà de P99,5.

Afin de rendre plus lisible les résultats du tableau 3-6, nous avons regroupé les bénéfices agricoles imposés au forfait et les bénéfices agricoles imposés au régime du bénéfice réel, catégories distinguées dans les tableaux IA/B des Etats 1921, en une seule catégorie de "bénéfices agricoles", de même que pour les bénéfices industriels et commerciaux et les bénéfices non-commerciaux. Nous avons inclus la catégorie des "revenus accessoires", qui apparaît dans les tableaux IA/B à partir des revenus de 1975 , et qui ne représentent jamais plus de $0,1 \%$ du revenu total, dans les bénéfices industriels et commerciaux. Les plus-values soumises au barème apparaissent dans la liste des revenus catégoriels pris en compte dans les tableaux IA/B à partir des revenus de 1979 ; des revenus de 1970 aux revenus de 1976, ces revenus apparaissaient dans la catégorie des "revenus divers". Leur importance pour l'ensemble des foyers imposables est faible (environ $0,2-0,3 \%$ du revenu total), mais elles peuvent atteindre $2 \%$ du revenu total pour la tranche la plus élevée, et afin de ne pas biaiser les évolutions nous les avons exclu des résultats du tableau 3-6. Le tableau 3-6 indique donc la composition du revenu imposable diminué des plusvalues soumises au barème (1979-1996) et des revenus divers (1970-1976). ${ }^{129}$ Les deux seules années pour lesquelles nous avons du apporter des corrections aux estimations issues des Etats 1921 sont l'année 1988 (pour les mêmes raisons que lors de l'estimation de la distribution du revenu imposable) et l'année 1991. En effet, la page introductive de l'Etat 1921 portant sur les revenus de 1991 précise que " pour des raisons techniques, la tableau IA n'a pu être éditée cette année ", et nous disposons donc d'aucune donnée sur la composition du revenu imposable pour cette année-là. Sur le tableau 3-6, nous avons retenu comme estimations pour la composition de P90-95, P95-99, P99-99,5 et P99,5-100 des années 1988 et 1991 la moyenne des estimations obtenues pour 1987-1989 et 1990-1992, hypothèse qui est cohérente avec les estimations de composition issues des échantillons légers de déclarations de revenus.

\footnotetext{
${ }^{129}$ Plus précisément, le tableau 3-6 indique la composition de la somme des revenus catégoriels (ou "revenu brut global") diminuée des plus-values soumises au barême ou des revenus divers. Pour l'ensemble des foyers imposables, le revenu imposable représente environ $96 \%$ du revenu brut global au début des années 1970, avant de décrôitre régulièrement jusqu'à environ $94 \%$ au début des années 1980 ; à compter des revenus de 1983, un certain nombre de charges déductibles du revenu brut global (comme les charges afférentes à l'habitation principale et les primes d'assurance-vie) ont été transformées en réductions d'impôt, si bien que le ratio (revenu imposable)/(revenu brut global) est subitement passé à près de $98 \%$ en 1983, et de se stabiliser autour de $97-98 \%$ depuis lors.
} 


\section{Annexe E : Fiabilité des estimations de la distribution du revenu imposable effectuées à partir des Etats 1921}

Cette annexe décrit les résultats des tests de fiabilité que nous avons réalisés pour les estimations de la distribution du revenu imposable de l'ensemble des foyers fiscaux (tous nombres de parts de quotient familial confondus). Nous avons réalisé le même type de tests pour les estimations de la distribution du revenu imposable des foyers fiscaux ayant 1, 1,5, 2B, 2A, 2,5 ou 3 parts de quotient familial, et nous avons obtenu des marges d'erreur similaires. Les coefficients $b_{i}$ des tableaux D-3, D-5, D-7, D-9, D-11 et D-13 attestent d'ailleurs du fait que les hauts revenus pour un nombre de parts donné suivent une loi de Pareto du même type que celle des hauts revenus en général (tous nombres de parts confondus)

E.1. Comparaison avec les estimations issues des échantillons légers de déclarations de revenus, 1988-1995

Le tableau E-1 compare les estimations de la distribution du revenu imposable de l'ensemble des foyers fiscaux (tous nombres de parts confondus) obtenues à partir des Etats 1921 en supposant que la distribution suit une loi de Pareto (cf. Annexe D.1) aux chiffres bruts obtenus en utilisant la variable " rimp " des échantillons légers de déclarations de revenus des années 1988 à 1995 (cf. Annexe F).

Insérer Tableau E-1: Comparaison entre les chiffres bruts issus des échantillons légers et les estimations effectuées à partir des Etats 1921.

Le tableau E-1 indique que les écarts entre les deux estimations ne dépassent jamais les $0,5 \%$ pour les parts de $\mathrm{P} 90-100$ et $\mathrm{P} 95-100$, et sont généralement très inférieurs à $1 \%$ pour les parts de P99-100 et P99,5-100. II s'agit d'écarts extrêmement faibles, comparés aux fluctuations observées d'une année sur l'autre sur la période 1970-1996 (cf. tableaux 3-1 à 3-3, section 3.2). En particulier, les estimations obtenues à partir des Etats 1921 permettent de prédire de façon très satisfaisante les évolutions enregistrées dans les échantillons légers de déclarations de revenu entre 1989 et $1995 .{ }^{130}$ Entre 1989 et 1995, la part de P90-100 a diminué de $0,4 \%$ selon les échantillons légers, et de $0,3 \%$ selon nos estimations ; la part de P95-100 a diminué de 2,7\% selon les échantillons légers, et de $2,1 \%$ selon nos estimations ; la part de P99-100 a diminué de 6,8\% selon les échantillons légers, et de $6,6 \%$ selon nos estimations ; la part de P99,5-100 a diminué de $8,4 \%$ selon les échantillons légers, et de $8,1 \%$ selon nos estimations.

Les écarts entre les deux estimations deviennent plus importantes pour P99,9-100 et surtout pour P99,99-100 : les estimations obtenues à partir des Etats 1921 sousévaluent systématiquement la part de P99,9-100 et P99,99-100, et l'ampleur de cette sous-évaluation devient de plus en plus importante au cours du temps. Cette sousévaluation systématique de la part des très hauts revenus s'expliquent aisément. Les estimations obtenues à partir des Etats 1921 pour les très hauts revenus des années 1989-1995 ont en effet été calculées à partir du ratio $\mathrm{y}^{*}(\mathrm{y}) / \mathrm{y}$ des contribuables dont le revenu imposable est supérieur à 500000 francs, tranche qui

\footnotetext{
${ }^{130}$ Nous comparons les évolutions observées sur la période 1989-1995 et non sur la période 19881995, car les estimations "Etats 1921 " pour l'année 1988 ont par construction le même écart avec les estimations " échantillons légers " que celles de l'année 1989 (cf. Annexe D.1).
} 
Tableau E-1: Comparaison entre les chiffres bruts issus des échantillons légers et les estimations effectuées à partir des Etats 1921

$\begin{array}{lrrrrrrrr} & 1988 & 1989 & 1990 & 1991 & 1992 & 1993 & 1994 & 1995 \\ \text { P90-100 (EL) } & 33,39 & 33,76 & 34,07 & 33,97 & 33,53 & 33,58 & 33,84 & 33,63 \\ \text { P90-100 (E1921) } & 33,44 & 33,82 & 34,04 & 33,79 & 33,55 & 33,52 & 33,67 & 33,71 \\ \text { écart absolu } & 0,06 & 0,06 & -0,03 & -0,17 & 0,02 & -0,06 & -0,16 & 0,08 \\ \text { écart en \% } & \mathbf{0 , 1 7} & \mathbf{0 , 1 7} & \mathbf{- 0 , 0 9} & \mathbf{- 0 , 5 0} & \mathbf{0 , 0 6} & \mathbf{- 0 , 1 7} & \mathbf{- 0 , 4 9} & \mathbf{0 , 2 4} \\ & & & & & & & & \\ \text { P95-100 (EL) } & 22,24 & 22,69 & 22,87 & 22,66 & 22,28 & 22,09 & 22,31 & 22,07 \\ \text { P95-100 (E1921) } & 22,25 & 22,71 & 22,84 & 22,53 & 22,21 & 22,11 & 22,21 & 22,23 \\ \text { écart absolu } & 0,01 & 0,02 & -0,03 & -0,13 & -0,07 & 0,03 & -0,10 & 0,16 \\ \text { écart en \% } & \mathbf{0 , 0 7} & \mathbf{0 , 0 7} & \mathbf{- 0 , 1 4} & \mathbf{- 0 , 5 6} & \mathbf{- 0 , 2 9} & \mathbf{0 , 1 1} & \mathbf{- 0 , 4 5} & \mathbf{0 , 7 2} \\ & & & & & & & & \\ \text { P99-100 (EL) } & 8,88 & 9,20 & 9,26 & 9,12 & 8,68 & 8,43 & 8,69 & 8,58 \\ \text { P99-100 (E1921) } & \mathbf{8 , 9 0} & \mathbf{9 , 2 3} & \mathbf{9 , 2 5} & \mathbf{8 , 9 5} & 8,69 & 8,58 & 8,64 & 8,62 \\ \text { écart absolu } & 0,03 & 0,03 & -0,01 & -0,17 & 0,01 & 0,14 & -0,05 & 0,04 \\ \text { écart en \% } & \mathbf{0 , 2 9} & \mathbf{0 , 2 9} & \mathbf{- 0 , 1 2} & \mathbf{- 1 , 8 3} & \mathbf{0 , 0 9} & \mathbf{1 , 7 2} & \mathbf{- 0 , 6 3} & \mathbf{0 , 5 1} \\ & & & & & & & & \\ \text { P99,5-100 (EL) } & 6,04 & 6,30 & 6,36 & 6,17 & 5,91 & 5,67 & 5,87 & 5,77 \\ \text { P99,5-100 (E1921) } & \mathbf{6 , 0 7} & 6,34 & 6,35 & 6,09 & 5,87 & 5,79 & 5,85 & 5,82 \\ \text { écart absolu } & 0,03 & 0,03 & -0,02 & -0,08 & -0,04 & 0,12 & -0,02 & 0,05 \\ \text { écart en \% } & \mathbf{0 , 5 4} & \mathbf{0 , 5 4} & \mathbf{- 0 , 3 0} & \mathbf{- 1 , 2 7} & \mathbf{- 0 , 6 9} & \mathbf{2 , 1 7} & \mathbf{- 0 , 4 1} & \mathbf{0 , 8 4} \\ & & & & & & & & \\ \text { P99,9-100 (EL) } & 2,51 & 2,66 & 2,67 & 2,53 & 2,39 & 2,36 & 2,43 & 2,40 \\ \text { P99,9-100 (E1921) } & 2,49 & 2,65 & 2,65 & 2,49 & 2,37 & 2,32 & 2,37 & 2,34 \\ \text { écart absolu } & -0,02 & -0,02 & -0,02 & -0,04 & -0,03 & -0,04 & -0,07 & -0,06 \\ \text { écart en \% } & \mathbf{- 0 , 6 7} & \mathbf{- 0 , 6 7} & \mathbf{- 0 , 7 6} & \mathbf{- 1 , 4 5} & \mathbf{- 1 , 1 0} & \mathbf{- 1 , 5 0} & \mathbf{- 2 , 6 9} & \mathbf{- 2 , 5 2} \\ & & & & & & & & \\ \text { P99,99-100 (EL) } & \mathbf{0 , 7 3} & \mathbf{0 , 7 9} & \mathbf{0 , 8 1} & \mathbf{0 , 7 3} & \mathbf{0 , 6 9} & \mathbf{0 , 6 7} & \mathbf{0 , 7 0} & \mathbf{0 , 7 0} \\ \text { P99,99-100(E1921) } & \mathbf{0 , 7 0} & \mathbf{0 , 7 6} & \mathbf{0 , 7 6} & \mathbf{0 , 6 9} & \mathbf{0 , 6 4} & \mathbf{0 , 6 3} & \mathbf{0 , 6 5} & \mathbf{0 , 6 3} \\ \text { écart absolu } & -0,03 & -0,03 & -0,05 & -0,04 & -0,04 & -0,04 & -0,06 & -0,07 \\ \text { écart en \% } & \mathbf{- 4 , 2 2} & \mathbf{- 4 , 2 2} & \mathbf{- 6 , 4 7} & \mathbf{- 5 , 5 0} & \mathbf{- 6 , 3 2} & \mathbf{- 6 , 4 2} & \mathbf{- 7 , 8 6} & \mathbf{- 9 , 9 7} \\ & & & & & & & & \end{array}$

Sources: Calculs de l'auteur à partir des échantillons légers de déclarations de revenus (cf. Annexe F) et estimations de l'auteur à partir des Etats 1921 (cf. Annexe D.1).

Lecture: En 1995, la part du revenu imposable détenue par les 10\% des foyers fiscaux ayant le revenu imposable le plus élevé est de $33,63 \%$ d'après l'échantillon léger et de $33,71 \%$ d'après les estimations effectuées à partir des Etats 1921; l'écart absolu est de 0,08 $(=33,71-33,63)$, et l'écart en $\%$ est de $0,24 \%(=(33,71-33,63) / 33,63)$. 
regroupe plus de $0,5 \%$ des contribuables durant cette période. Or ce ratio $\mathrm{y}^{*}(\mathrm{y}) / \mathrm{y}$ a toujours tendance à augmenter à l'intérieur du centile supérieur (cf. tableau D-1, annexe D.1), si bien que toute estimation de la part de P99,9-100 ou P99,99-100 obtenue à partir d'une loi de Pareto estimée au niveau de P99,5 est nécessairement sous-évaluée. Le fait que la sous-évaluation tend à s'aggraver au cours du temps s'explique par le fait que les seuils P99,9 et P99,99 (cf. tableau D-2) tendent à s'éloigner du seuil de 500000 francs. Autrement dit, si le niveau des tranches de revenu imposable utilisées dans les Etats 1921 n'est pas ajusté suffisamment souvent, alors la qualité des estimations se dégrade. La conséquence est que les estimations obtenues à partir des Etats 1921 surévaluent l'ampleur de la diminution des parts de P99,9-100 et P99,99-100 entre 1989 et 1995: la part de P99,9-100 aurait diminué de $11,6 \%$ entre 1989 et 1995 d'après ces estimations, contre 9,9\% d'après les échantillons légers, et la part de P99,99-100 aurait diminué de 16,3\% d'après ces estimations, et de $11,0 \%$ d'après les échantillons légers.

Cependant, l'importance pratique de ces écarts observés pour les très hauts revenus ne doit pas être surestimée. Tout d'abord, les estimations obtenues à partir des Etats 1921 permettent toujours de bien prédire les trends généraux. Ensuite, ce problème ne se pose vraiment que pour les Etats 1921 des années récentes (pour lesquelles nous disposons des échantillons légers): les Etats 1921 des années 1970 et du début des années 1980 permettent d'obtenir d'excellentes estimations de la part de P99,9-100 et P99,99-100, car les tranches de revenu imposable utilisées pendant toutes ces années permettent d'obtenir des informations fines sur les $0,1 \%$ les plus riches, les $0,01 \%$ les plus riches, etc.., et non seulement pour les $0,5 \%$ les plus riches (cf. tableau D-1, annexe D.1; cf. annexes E.2 et E.3 infra). Enfin, et surtout, les estimations de l'élasticité du revenu imposable proposées dans cette étude n'utilisent pas les estimations de la part de P99,9-100 et P99,99-100 : seul compte le fait que les écarts constatés pour les estimations de P90-100, P95-100, P99-100 et P99,5-100 soient de l'ordre de $0,5 \%$ ou $1 \%$.

De plus, ces écarts de $0,5 \%$ ou $1 \%$ ne sont pas seulement dus au fait que l'approximation de la distribution par une loi de Pareto entraîne inévitablement de légères erreurs d'approximation. Ces écarts reflètent également les légères erreurs d'échantillonnages des échantillons légers de déclarations de revenus : par exemple, le nombre et le revenu moyen des contribuables dont le revenu imposable est supérieur à 500000 francs, tels qu'ils peuvent être estimés à partir des échantillons légers, sont toujours extrêmement proches des chiffres des Etats 1921, mais il existe toujours de légers écarts. ${ }^{131}$ Par définition, les chiffres de l'Etat 1921 sont les bons, puisqu'ils sont obtenus grâce au dépouillement exhaustif de l'ensemble des déclarations de revenus. Afin d'évaluer quelle partie des écarts mesurés sur le tableau E-1 est due à ces erreurs d'échantillonnage et quelle partie est véritablement due à la technique d'approximation par une loi de Pareto, nous avons utilisé les échantillons légers sur la période 1988-1995 pour calculer les mêmes informations que celles contenues dans les tableaux des Etats 1921 (nombre et revenu moyen des contribuables dont le revenu imposable est supérieur à 500000 francs, nombre

\footnotetext{
${ }^{131}$ Ces écarts ne sont pas seulement dûs à des "erreurs" d'échantillonage au sens strict : ils reflètent également le fait que les échantillons légers portent uniquement sur les émissions de rôles effectuées au 31/03/n+2 (alors que les Etats 1921 utilisés portent sur les émissions effectuées au 31/12/n+2 depuis les revenus de 1987), que les échantillons légers portent uniquement sur la métropole (alors que les Etats 1921 utilisés portent sur le champ national (métropole + DOM/TOM) depuis les revenus de 1985), et que seuls les foyers imposables ont été retenus pour nos estimations établies à partir des Etats 1921. La faiblesse des écarts constatés montre que toutes ces légères variations de champ ont des conséquences relativement mineures.
} 
et revenu moyen des contribuables dont le revenu imposable est compris entre 250000 et 500000 francs, etc...), puis nous avons utilisé ces chiffres bruts pour appliquer la même technique d'approximation par une loi de Pareto et d'estimation de la part de P90-100, P95-100, etc..., que celle que nous avons appliqué aux chiffres bruts des Etats 1921. Le tableau D-2 compare les résultats obtenus aux chiffres bruts issus des échantillons légers.

Insérer Tableau E-2: Comparaison entre les chiffres bruts issus des échantillons légers et les estimations effectuées à partir des échantillons légers

On constate que les écarts entre les deux estimations sont pratiquement nuls pour les parts de P90-100, P95-100, P99-100 et P99,5-100. Sur l'ensemble de la période 1988-1995, les écarts pour ces 4 parts ne dépassent jamais les 0,44\%, et sont généralement inférieurs à $0,1-0,2 \%$. Autrement dit, l'essentiel des écarts mesurés sur le tableau E-1 étaient dus à des erreurs d'échantillonnages des échantillons légers et non pas à des erreurs d'approximation dues à la technique d'estimation utilisée. Les marges d'erreurs résiduelles, qui sont les seules que l'on puisse imputer à la technique d'approximation par une loi de Pareto, sont totalement négligeables comparées aux fluctuations observées d'une année sur l'autre, et en particulier ne sont aucunement susceptibles de biaiser nos conclusions. Par contre, pour ce qui est de la part de P99,9-100 et P99,99-100, les écarts mesurés sur le tableau E-2 ont le même niveau que les écarts mesurés sur le tableau $\mathrm{E}-1$, ce qui montre que les écarts pour ces 2 parts sont véritablement dues au fait qu'il est difficile d'approximer la loi de Pareto suivie par les $0,1 \%$ ou les $0,01 \%$ les plus riches à partir d'informations portant uniquement sur les $0,5 \%$ les plus riches, et non pas à des erreurs d'échantillonnage des échantillons légers.

\section{E.2. Comparaison avec les estimations issues des enquêtes "Revenus fiscaux", 1970-1990}

Les tableaux E-3 et E-4 effectuent les mêmes comparaisons en utilisant les chiffres bruts issus des enquêtes "Revenus fiscaux" (cf. Annexe G).

Insérer Tableau E-3: Comparaison entre les chiffres bruts issus des enquêtes "Revenus fiscaux" et les estimations effectuées à partir des Etats 1921

Insérer Tableau E-4: Comparaison entre les chiffres bruts issus des enquêtes "Revenus fiscaux" et les estimations effectuées à partir des enquêtes "RF "

Le tableau E-3 montre que les écarts entre les chiffres bruts issus des enquêtes "RF" et les estimations obtenues à partir des Etats 1921 sont sensiblement plus élevés que les écarts entre ces dernières et les chiffres bruts issus des échantillons légers. Cela s'explique par le fait que si les enquêtes "RF" sont globalement représentatives de l'ensemble des foyers fiscaux, leur représentativité devient beaucoup plus mauvaise lorsque l'on s'intéresse spécifiquement aux hauts revenus. En général (mais pas toujours), les enquêtes "RF" tendent à surévaluer la part de P90-100, ce qui doit sans doute être mis en parallèle avec le fait qu'elles sousestiment la proportion de contribuables célibataires (cf. Annexe C.3), et à sousévaluer la part des très hauts revenus. ${ }^{132}$ L'ampleur de ces erreurs, qui sont du

\footnotetext{
${ }^{132}$ Ces erreurs d'échantillonage, variables suivant les années, sont d'ailleurs reconnues dans les publications de l'INSEE consacrées aux enquêtes "Revenus fiscaux" (cf. Annexe G pour les
} 
même ordre de grandeur que les fluctuations de la distribution observées d'une année sur l'autre (cf. tableaux 3-1 à 3-3, section 3.2), montre qu'il est tout à fait impossible d'utiliser les enquêtes "RF" pour étudier de façon fine les fluctuations des hauts revenus.

Par contre, les enquêtes "RF" nous permettent de constater que la forme structurelle de la distribution des hauts revenus est bien approximée par une loi de Pareto sur l'ensemble de la période 1970-1996, et non seulement sur la période 1988-1995. Les écarts mesurés sur le tableau E-4 sont en effet tout aussi faibles que ceux du tableau E-2 : sur l'ensemble de la période 1970-1990, les écarts pour les parts de P90-100, P95-100, P99-100 et P99,5-100 ne dépassent jamais 0,48\%, et ils sont généralement inférieurs à $0,1 \%$. Cela montre de façon claire que les écarts importants mesurées sur le tableau E-3 sont dus aux erreurs d'échantillonnage des enquêtes "Revenus fiscaux", et non à un défaut de la procédure d'approximation par une loi de Pareto. La faiblesse des écarts mesurées sur le tableau E-4 montre qu'en utilisant les Etats 1921 pour estimer la part de P90-100, P95-100, P99-100 et P99,5-100 sur la période 1970-1996, on commet des erreurs maximales de 0,5\% par rapport aux vrais parts, c'est-à-dire des erreurs négligeables comparées à l'ampleur des fluctuations observées. Le tableau D-6 confirme également qu'il suffit de disposer d'informations portant sur des tranches de revenu imposable pas trop éloignées du seuil que l'on cherche à estimer pour que l'approximation par une loi de Pareto donne d'excellents résultats, y compris pour la part de P99,9-100 : environ $0,078 \%$ des foyers fiscaux ont un revenu imposable supérieur à 200000 francs en 1970 (cf. tableau D-1, annexe D.1), si bien qu'en connaissant uniquement le nombre et le revenu moyen des contribuables dont le revenu imposable est supérieur à 200000 francs, on parvient à estimer à $0,02 \%$ près la part de P99,9-100. On peut donc considérer que les estimations de la part de P99,9-100 obtenues pour les années 1970 sont tout aussi précises que les estimations des parts de P90-100, P95-100, P99-100 et P99,5-100 pour l'ensemble de la période 1970-1996.

\section{E.3. Comparaison avec les estimations issues des dossiers "Modèle lourd", 1983-} 1993

Les tableaux 10 des dossiers "Modèle lourd" contiennent le même type d'informations que les tableaux des Etats 1921, mais ils comptent une tranche de plus, à savoir celle concernant les contribuables dont le revenu imposable est supérieur à 1 million de francs (cf. Annexe $\mathrm{H}$ ). En appliquant aux chiffres bruts issus de ces tableaux la même technique d'approximation par une loi de Pareto que celle que nous avons appliqué aux chiffres bruts des Etats 1921 (cf. Annexe D-1), et en comparant les résultats obtenus, on obtient des écarts qui confirment les ordres de grandeur obtenus lors des comparaisons précédentes.

Insérer Tableau E-5 : Les seuils de revenu imposable et les ratios $\mathrm{y}^{*}(\mathrm{y}) / \mathrm{y}$ utilisés pour les estimations effectuées à partir des dossiers "Modèle lourd"

Insérer Tableau E-6: Comparaison entre les estimations effectuées à partir des dossiers "Modèle lourd" et les estimations effectuées à partir des Etats 1921

références de ces publications), qui débutent toujours par une comparaison entre la répartition du revenu imposable estimée par l'enquête et la répartition du revenu imposable issue des statistiques fiscales (c'est-à-dire des Etats 1921). 
Tableau E-2: Comparaison entre les chiffres bruts issus des échantillons légers et les estimations effectuées à partir des échantillons légers

\begin{tabular}{|c|c|c|c|c|c|c|c|c|}
\hline & 1988 & 1989 & 1990 & 1991 & 1992 & 1993 & 1994 & 1995 \\
\hline P90-100 (EL) & 33,39 & 33,76 & 34,07 & 33,97 & 33,53 & 33,58 & 33,84 & 33,63 \\
\hline P90-100 (Ext.EL) & 33,39 & 33,76 & 34,07 & 33,98 & 33,47 & 33,55 & 33,80 & 33,53 \\
\hline écart absolu & 0,00 & 0,00 & 0,00 & 0,01 & $-0,05$ & $-0,03$ & $-0,04$ & $-0,10$ \\
\hline écart en \% & 0,00 & $-0,01$ & 0,01 & 0,03 & $-0,16$ & $-0,09$ & $-0,11$ & $-0,30$ \\
\hline P95-100 (EL) & 22,24 & 22,69 & 22,87 & 22,66 & 22,28 & 22,09 & 22,31 & 22,07 \\
\hline P95-100 (Ext.EL) & 22,22 & 22,69 & 22,87 & 22,64 & 22,25 & 22,06 & 22,29 & 22,06 \\
\hline écart absolu & $-0,01$ & 0,00 & $-0,01$ & $-0,02$ & $-0,02$ & $-0,03$ & $-0,02$ & $-0,01$ \\
\hline écart en \% & $-0,07$ & $-0,01$ & $-0,04$ & $-0,08$ & $-0,11$ & $-0,14$ & $-0,09$ & $-0,06$ \\
\hline P99-100 (EL) & 8,88 & 9,20 & 9,26 & 9,12 & 8,68 & 8,43 & 8,69 & 8,58 \\
\hline P99-100 (Ext.EL) & 8,85 & 9,17 & 9,22 & 9,12 & 8,64 & 8,40 & 8,67 & 8,55 \\
\hline écart absolu & $-0,03$ & $-0,03$ & $-0,04$ & 0,00 & $-0,04$ & $-0,03$ & $-0,02$ & $-0,03$ \\
\hline écart en \% & $-0,35$ & $-0,34$ & $-0,39$ & $-0,03$ & $-0,44$ & $-0,33$ & $-0,22$ & $-0,32$ \\
\hline P99,5-100 (EL) & 6,04 & 6,30 & 6,36 & 6,17 & 5,91 & 5,67 & 5,87 & 5,77 \\
\hline P99,5-100 (Ext.EL) & 6,04 & 6,30 & 6,36 & 6,17 & 5,91 & 5,65 & 5,87 & 5,77 \\
\hline écart absolu & 0,00 & 0,00 & $-0,01$ & 0,00 & $-0,01$ & $-0,02$ & 0,00 & $-0,01$ \\
\hline écart en \% & $-0,02$ & $-0,05$ & $-0,10$ & 0,02 & $-0,11$ & $-0,35$ & $-0,06$ & $-0,12$ \\
\hline P99,9-100 (EL) & 2,51 & 2,66 & 2,67 & 2,53 & 2,39 & 2,36 & 2,43 & 2,40 \\
\hline P99,9-100 (Ext.EL) & 2,49 & 2,63 & 2,68 & 2,49 & 2,44 & 2,24 & 2,37 & 2,31 \\
\hline écart absolu & $-0,02$ & $-0,03$ & 0,01 & $-0,03$ & 0,05 & $-0,12$ & $-0,06$ & $-0,09$ \\
\hline écart en \% & $-0,81$ & $-1,16$ & 0,54 & $-1,37$ & 2,08 & $-4,98$ & $-2,48$ & $-3,76$ \\
\hline P99,99-100 (EL) & 0,73 & 0,79 & 0,81 & 0,73 & 0,69 & 0,67 & 0,70 & 0,70 \\
\hline P99,99-100(Ext.EL) & 0,70 & 0,76 & 0,78 & 0,68 & 0,69 & 0,60 & 0,65 & 0,62 \\
\hline écart absolu & $-0,04$ & $-0,04$ & $-0,03$ & $-0,05$ & 0,00 & $-0,07$ & $-0,06$ & $-0,08$ \\
\hline écart en \% & $-4,81$ & $-4,56$ & $-3,74$ & $-7,06$ & 0,32 & $-11,14$ & $-7,82$ & $-11,52$ \\
\hline
\end{tabular}

Sources: Calculs de l'auteur à partir des échantillons légers de déclarations de revenus (cf. Annexes E.1 et F).

Lecture: En 1988, la part du revenu imposable détenue par les 10\% des foyers fiscaux ayant le revenu imposable le plus élevé est de 33,39\% d'après l'échantillon léger et de 33,39\% d'après les estimations effectuées à partir des tabulations par tranche issues des échantillons légers. 
Tableau E-3: Comparaison entre les chiffres bruts issus des enquêtes "Revenus fiscaux" et les estimations effectuées à partir des Etats 1921

$\begin{array}{lrrrrr} & 1970 & 1975 & 1979 & 1984 & 1990 \\ \text { P90-100 (RF) } & 35,51 & 34,75 & 33,32 & 32,83 & 34,45 \\ \text { P90-100 (E1921) } & 34,49 & 34,86 & 32,37 & 31,73 & 34,04 \\ \text { écart absolu } & -1,02 & 0,11 & -0,95 & -1,10 & -0,41 \\ \text { écart en \% } & \mathbf{- 2 , 8 7} & \mathbf{0 , 3 1} & \mathbf{- 2 , 8 6} & \mathbf{- 3 , 3 5} & \mathbf{- 1 , 2 0} \\ & & & & & \\ \text { P95-100 (RF) } & 23,72 & 23,21 & 22,45 & 21,58 & 22,96 \\ \text { P95-100 (E1921) } & 23,31 & 23,51 & 21,75 & 20,78 & 22,84 \\ \text { écart absolu } & -0,42 & 0,30 & -0,70 & -0,80 & -0,12 \\ \text { écart en \% } & \mathbf{- 1 , 7 5} & \mathbf{1 , 2 9} & \mathbf{- 3 , 1 1} & \mathbf{- 3 , 7 1} & \mathbf{- 0 , 5 2} \\ & & & & & \\ \text { P99-100 (RF) } & 9,19 & 9,00 & 9,28 & 8,12 & 9,07 \\ \text { P99-100 (E1921) } & 9,32 & 9,54 & 8,80 & 7,88 & 9,25 \\ \text { écart absolu } & 0,13 & 0,54 & -0,48 & -0,24 & 0,17 \\ \text { écart en \% } & \mathbf{1 , 4 6} & \mathbf{6 , 0 0} & \mathbf{- 5 , 1 3} & \mathbf{- 2 , 9 9} & \mathbf{1 , 9 2} \\ & & & & & \\ \text { P99,5-100 (RF) } & 6,04 & 5,89 & 6,40 & 5,27 & 6,10 \\ \text { P99,5-100 (E1921) } & 6,26 & 6,41 & 5,94 & 5,19 & 6,35 \\ \text { écart absolu } & 0,22 & 0,52 & -0,46 & -0,09 & 0,24 \\ \text { écart en \% } & \mathbf{3 , 5 9} & \mathbf{8 , 8 7} & \mathbf{- 7 , 2 4} & \mathbf{- 1 , 6 4} & \mathbf{3 , 9 9} \\ & & & & & \\ \text { P99,9-100 (RF) } & 2,19 & 2,07 & 2,65 & 1,93 & 2,32 \\ \text { P99,9-100 (E1921) } & 2,45 & 2,53 & 2,39 & 1,99 & 2,65 \\ \text { écart absolu } & 0,25 & 0,46 & -0,26 & 0,07 & 0,32 \\ \text { écart en \% } & \mathbf{1 1 , 5 2} & \mathbf{2 2 , 2 0} & \mathbf{- 9 , 6 6} & \mathbf{3 , 3 7} & \mathbf{1 3 , 7 8} \\ & & & & & \end{array}$

Sources: Calculs de l'auteur à partir des enquêtes "Revenus fiscaux" (cf. Annexe G) et estimations de l'auteur à partir des Etats 1921 (cf. Annexe A.1)

Lecture: En 1970, la part du revenu imposable détenue par les $10 \%$ des foyers fiscaux ayant le revenu imposable le plus élevé est de $35,51 \%$ d'après l'enquête "Revenus fiscaux", et de $34,49 \%$ d'après les estimations effectuées à partir des Etats 1921. 
Tableau E-4: Comparaison entre les chiffres bruts issus des enquêtes "Revenus fiscaux" et les estimations effectuées à partir des enquêtes "RF"

$\begin{array}{lrrrrr} & 1970 & 1975 & 1979 & 1984 & 1990 \\ \text { P90-100 (RF) } & 35,51 & 34,75 & 33,32 & 32,83 & 34,45 \\ \text { P90-100 (Ext.RF) } & 35,51 & 34,76 & 33,31 & 32,83 & 34,45 \\ \text { écart absolu } & 0,00 & 0,01 & -0,01 & 0,00 & 0,00 \\ \text { écart en \% } & \mathbf{0 , 0 1} & \mathbf{0 , 0 2} & \mathbf{- 0 , 0 2} & \mathbf{0 , 0 1} & \mathbf{- 0 , 0 1} \\ & & & & & \\ \text { P95-100 (RF) } & 23,72 & 23,21 & 22,45 & 21,58 & 22,96 \\ \text { P95-100 (Ext.RF) } & 23,71 & 23,21 & 22,43 & 21,59 & 22,96 \\ \text { écart absolu } & -0,01 & 0,00 & -0,02 & 0,02 & 0,00 \\ \text { écart en \% } & \mathbf{- 0 , 0 3} & \mathbf{0 , 0 0} & -\mathbf{0 , 0 8} & \mathbf{0 , 0 9} & \mathbf{- 0 , 0 2} \\ & & & & & \\ \text { P99-100 (RF) } & 9,19 & 9,00 & 9,28 & 8,12 & 9,07 \\ \text { P99-100 (Ext.RF) } & 9,19 & 9,00 & 9,28 & 8,15 & 9,07 \\ \text { écart absolu } & 0,00 & 0,00 & 0,00 & 0,02 & -0,01 \\ \text { écart en \% } & \mathbf{- 0 , 0 2} & \mathbf{0 , 0 0} & \mathbf{0 , 0 3} & \mathbf{0 , 3 0} & \mathbf{- 0 , 1 0} \\ & & & & & \\ \text { P99,5-100 (RF) } & 6,04 & 5,89 & 6,40 & 5,27 & 6,10 \\ \text { P99,5-100 (Ext.RF) } & 6,04 & 5,91 & 6,43 & 5,29 & 6,10 \\ \text { écart absolu } & 0,00 & 0,02 & 0,03 & 0,02 & -0,01 \\ \text { écart en \% } & \mathbf{0 , 0 5} & \mathbf{0 , 3 7} & \mathbf{0 , 4 8} & \mathbf{0 , 3 9} & \mathbf{- 0 , 0 8} \\ & & & & & \\ \text { P99,9-100 (RF) } & 2,19 & 2,07 & 2,65 & 1,93 & 2,32 \\ \text { P99,9-100 (Ext.RF) } & 2,19 & 2,09 & 2,67 & 1,92 & 2,43 \\ \text { écart absolu } & 0,00 & 0,02 & 0,02 & -0,01 & 0,10 \\ \text { écart en \% } & \mathbf{0 , 0 2} & \mathbf{1 , 0 9} & \mathbf{0 , 8 0} & \mathbf{- 0 , 6 7} & \mathbf{4 , 3 6} \\ & & & & & \end{array}$

Sources: Calculs de l'auteur à partir des enquêtes "Revenus fiscaux" (cf. Annexes G et E.2).

Lecture: En 1970, la part du revenu imposable détenue par les $10 \%$ des foyers fiscaux ayant le revenu imposable le plus élevé est de $35,51 \%$ d'après l'enquête "Revenus fiscaux", et de $33,51 \%$ d'après les estimations effectuées à partir des tabulations par tranches issues des enquêtes "Revenus fiscaux". 
Tableau E-5: Les seuils de revenu imposable et les ratios $y^{*}(y) / y$ utilisés pour les estimations effectuées à partir des dossiers "Modèle lourd"

\begin{tabular}{rrrrrrrrrrrrr} 
& \multicolumn{2}{c}{1983} & \multicolumn{1}{c}{1984} & \multicolumn{2}{c}{1985} & \multicolumn{2}{c}{1986} & \multicolumn{2}{c}{1987} & \multicolumn{2}{c}{1988} \\
$\mathrm{~s}_{\mathrm{i}}$ & $\mathrm{p}_{\mathrm{i}}$ & $\mathrm{b}_{\mathrm{i}}$ & \multicolumn{1}{c}{$\mathrm{p}_{\mathrm{i}}$} & $\mathrm{b}_{\mathrm{i}}$ & $\mathrm{p}_{\mathrm{i}}$ & $\mathrm{b}_{\mathrm{i}}$ & $\mathrm{p}_{\mathrm{i}}$ & $\mathrm{b}_{\mathrm{i}}$ & $\mathrm{p}_{\mathrm{i}}$ & $\mathrm{b}_{\mathrm{i}}$ & $\mathrm{p}_{\mathrm{i}}$ & $\mathrm{b}_{\mathrm{i}}$ \\
100000 & 14,72 & 1,65 & 16,80 & 1,66 & 18,78 & 1,69 & 20,10 & 1,72 & 20,79 & 1,74 & 21,95 & 1,77 \\
150000 & 5,22 & 1,65 & 6,15 & 1,64 & 7,15 & 1,65 & 7,93 & 1,66 & 8,38 & 1,69 & 9,10 & 1,71 \\
200000 & 2,46 & 1,66 & 2,87 & 1,66 & 3,35 & 1,67 & 3,75 & 1,69 & 4,01 & 1,72 & 4,40 & 1,74 \\
300000 & 0,89 & 1,66 & 1,03 & 1,66 & 1,21 & 1,68 & 1,37 & 1,70 & 1,47 & 1,75 & 1,63 & 1,77 \\
400000 & 0,43 & 1,67 & 0,49 & 1,67 & 0,59 & 1,68 & 0,68 & 1,71 & 0,74 & 1,77 & 0,82 & 1,80 \\
1000000 & 0,04 & 1,80 & 0,05 & 1,78 & 0,06 & 1,78 & 0,07 & 1,77 & 0,09 & 1,82 & 0,10 & 1,84
\end{tabular}

\begin{tabular}{rrrrrrrrrrr} 
& \multicolumn{2}{c}{1989} & \multicolumn{2}{c}{1990} & \multicolumn{2}{c}{1991} & \multicolumn{2}{c}{1992} & \multicolumn{2}{c}{1993} \\
$\mathrm{~s}_{\mathrm{i}}$ & $\mathrm{p}_{\mathrm{i}}$ & $\mathrm{b}_{\mathrm{i}}$ & \multicolumn{1}{c}{$\mathrm{p}_{\mathrm{i}}$} & \multicolumn{1}{l}{$\mathrm{b}_{\mathrm{i}}$} & \multicolumn{1}{c}{$\mathrm{p}_{\mathrm{i}}$} & $\mathrm{b}_{\mathrm{i}}$ & \multicolumn{1}{c}{$\mathrm{p}_{\mathrm{i}}$} & $\mathrm{b}_{\mathrm{i}}$ & \multicolumn{1}{c}{$\mathrm{p}_{\mathrm{i}}$} & $\mathrm{b}_{\mathrm{i}}$ \\
100000 & 23,54 & 1,81 & 25,18 & 1,83 & 26,48 & 1,84 & 27,47 & 1,83 & 27,92 & 1,84 \\
150000 & 10,16 & 1,73 & 11,27 & 1,73 & 12,15 & 1,71 & 12,78 & 1,70 & 13,14 & 1,69 \\
200000 & 4,99 & 1,76 & 5,62 & 1,75 & 6,08 & 1,71 & 6,42 & 1,68 & 6,61 & 1,67 \\
300000 & 1,87 & 1,80 & 2,09 & 1,79 & 2,22 & 1,75 & 2,29 & 1,72 & 2,32 & 1,70 \\
400000 & 0,95 & 1,82 & 1,05 & 1,82 & 1,10 & 1,78 & 1,12 & 1,74 & 1,11 & 1,73 \\
1000000 & 0,12 & 1,87 & 0,13 & 1,87 & 0,13 & 1,83 & 0,13 & 1,81 & 0,12 & 1,81
\end{tabular}

Source: Dossiers "Modèle lourd" (cf. Annexe H)

Lecture: En 1983, 14,72\% des foyers fiscaux ont un revenu imposable supérieur à $100000 \mathrm{FF}$, et leur revenu imposable moyen 1,65 fois supérieur à $100000 \mathrm{FF} ; 5,22 \%$ ont un revenu supérieur à $150000 \mathrm{FF}$, et leur revenu imposable moyen est 1,65 fois supérieur à $150000 \mathrm{FF}$; etc... 
Tableau E-6: Comparaison entre les estimations effectuées à partir des dossiers "Modèle lourd" et les estimations effectuées à partir des Etats 1921

\begin{tabular}{|c|c|c|c|c|c|c|c|c|c|c|c|}
\hline & 1983 & 1984 & 1985 & 1986 & 1987 & 1988 & 1989 & 1990 & 1991 & 1992 & 1993 \\
\hline P90-100 (ML) & 31,65 & 31,68 & 32,08 & 32,44 & 32,93 & 33,31 & 33,76 & 33,96 & 33,73 & 33,46 & 33,44 \\
\hline P90-100 (E1921) & 31,64 & 31,73 & 32,29 & 32,67 & 33,05 & 33,44 & 33,82 & 34,04 & 33,79 & 33,55 & 33,52 \\
\hline écart absolu & $-0,01$ & 0,05 & 0,21 & 0,23 & 0,13 & 0,13 & 0,06 & 0,08 & 0,07 & 0,09 & 0,07 \\
\hline cart en \% & $-0,04$ & 0,15 & 0,65 & 0,71 & 0,39 & 0,39 & 0,18 & 0,22 & 0,20 & 0,27 & 0,22 \\
\hline 95-100 (ML) & 20,70 & 20,73 & 21,05 & 21,41 & 21,87 & 22,23 & 22,67 & 22,80 & 22,49 & 22,16 & 22,05 \\
\hline P95-100 (E1921) & 20,73 & 20,78 & 21,20 & 21,57 & 21,99 & 22,25 & 22,71 & 22,84 & 22,53 & 22,21 & 22,11 \\
\hline écart absolu & 0,04 & 0,04 & 0,15 & 0,17 & 0,11 & 0,02 & 0,04 & 0,04 & 0,04 & 0,06 & 0,06 \\
\hline écart en \% & 0,18 & 0,20 & 0,70 & 0,77 & 0,52 & 0,09 & 0,17 & 0,20 & 0,18 & 0,25 & 0,28 \\
\hline 99-100 (ML) & 7,84 & 7,85 & 8,04 & 8,29 & 8,66 & 8,92 & 9,23 & 9,25 & 8,96 & 8,69 & 8,57 \\
\hline 299-100 (E1921) & 7,84 & 7,88 & 8,07 & 8,35 & 8,71 & 8,90 & 9,23 & 9,25 & 8,95 & 8,69 & 8,58 \\
\hline écart absolu & 0,00 & 0,03 & 0,03 & 0,05 & 0,05 & $-0,02$ & 0,00 & $-0,01$ & $-0,01$ & 0,00 & 0,01 \\
\hline écart en \% & $-0,01$ & 0,37 & 0,38 & 0,63 & 0,56 & $-0,20$ & $-0,03$ & $-0,06$ & $-0,09$ & $-0,01$ & 0,08 \\
\hline 999,5-100 (ML) & 5,17 & 5,17 & 5,32 & 5,53 & 5,85 & 6,07 & 6,30 & 6,32 & 6,06 & 5,84 & 5,75 \\
\hline P99,5-100 (E1921) & 5,18 & 5,19 & 5,35 & 5,57 & 5,90 & 6,07 & 6,34 & 6,35 & 6,09 & 5,87 & 5,79 \\
\hline écart absolu & 0,01 & 0,01 & 0,03 & 0,04 & 0,05 & 0,01 & 0,03 & 0,02 & 0,03 & 0,04 & 0,04 \\
\hline écart en \% & 0,19 & 0,28 & 0,64 & 0,76 & 0,87 & 0,09 & 0,55 & 0,38 & 0,43 & 0,62 & 0,71 \\
\hline P99,9-100 (ML) & 1,98 & 1,99 & 2,06 & 2,17 & 2,38 & 2,50 & 2,66 & 2,67 & 2,51 & 2,40 & 2,37 \\
\hline P99,9-100 (E1921) & 1,97 & 1,99 & 2,06 & 2,18 & 2,39 & 2,49 & 2,65 & 2,65 & 2,49 & 2,37 & 2,32 \\
\hline écart absolu & $-0,01$ & 0,00 & 0,00 & 0,01 & 0,00 & $-0,01$ & $-0,01$ & $-0,02$ & $-0,02$ & $-0,03$ & $-0,04$ \\
\hline écart en \% & $-0,29$ & 0,18 & 0,07 & 0,36 & 0,08 & $-0,58$ & $-0,51$ & $-0,84$ & $-0,78$ & $-1,33$ & $-1,81$ \\
\hline 299,9 & 0,55 & 0,55 & 0,57 & 0,59 & 0,67 & 0,71 & 0,77 & 0,78 & 0,71 & 0,67 & 0,66 \\
\hline P99,99-100(E1921) & 0,50 & 0,51 & 0,53 & 0,57 & 0,65 & 0,70 & 0,76 & 0,76 & 0,69 & 0,64 & 0,63 \\
\hline écart absolu & $-0,05$ & $-0,04$ & $-0,04$ & $-0,02$ & $-0,02$ & $-0,01$ & $-0,02$ & $-0,02$ & $-0,02$ & $-0,03$ & $-0,04$ \\
\hline écart en \% & $-9,61$ & $-7,30$ & $-7,12$ & $-3,91$ & $-2,74$ & $-1,61$ & $-2,00$ & $-2,86$ & $-2,79$ & $-4,06$ & $-5,32$ \\
\hline
\end{tabular}

Sources: Estimations de l'auteur à partir des dossiers "Modèle lourd" (cf. Annexe G) et des Etats 1921 (cf. Annexe D.1)

Lecture: En 1983, la part du revenu imposable détenue par les 10\% des foyers fiscaux ayant le revenu imposable le plus élevé est de $31,65 \%$ d'après les estimations effectuées à partir des dossiers "Modèle Lourd", et de 31,64\% d'après les estimations effectuées à partir des Etats 1921. 
Tableau E-7: Comparaison entre les chiffres bruts issus des échantillons légers et les estimations effectuées à partir des dossiers "Modèle lourd"

\begin{tabular}{|c|c|c|c|c|c|c|}
\hline & 1988 & 1989 & 1990 & 1991 & 1992 & 1993 \\
\hline P90-100 (EL) & 33,39 & 33,76 & 34,07 & 33,97 & 33,53 & 33,58 \\
\hline P90-100 (ML) & 33,31 & 33,76 & 33,96 & 33,73 & 33,46 & 33,44 \\
\hline écart absolu & $-0,07$ & 0,00 & $-0,11$ & $-0,24$ & $-0,07$ & $-0,13$ \\
\hline écart en \% & $-0,22$ & $-0,01$ & $-0,31$ & $-0,70$ & $-0,20$ & $-0,40$ \\
\hline P95-100 (EL) & 22,24 & 22,69 & 22,87 & 22,66 & 22,28 & 22,09 \\
\hline P95-100 (ML) & 22,23 & 22,67 & 22,80 & 22,49 & 22,16 & 22,05 \\
\hline écart absolu & $-0,01$ & $-0,02$ & $-0,08$ & $-0,17$ & $-0,12$ & $-0,04$ \\
\hline écart en \% & $-0,02$ & $-0,11$ & $-0,33$ & $-0,74$ & $-0,54$ & $-0,16$ \\
\hline P99-100 (EL) & 8,88 & 9,20 & 9,26 & 9,12 & 8,68 & 8,43 \\
\hline P99-100 (ML) & 8,92 & 9,23 & 9,25 & 8,96 & 8,69 & 8,57 \\
\hline écart absolu & 0,04 & 0,03 & $-0,01$ & $-0,16$ & 0,01 & 0,14 \\
\hline écart en \% & 0,48 & 0,32 & $-0,06$ & $-1,75$ & 0,10 & 1,63 \\
\hline P99,5-100 (EL) & 6,04 & 6,30 & 6,36 & 6,17 & 5,91 & 5,67 \\
\hline P99,5-100 (ML) & 6,07 & 6,30 & 6,32 & 6,06 & 5,84 & 5,75 \\
\hline écart absolu & 0,03 & 0,00 & $-0,04$ & $-0,10$ & $-0,08$ & 0,08 \\
\hline écart en \% & 0,44 & $-0,01$ & $-0,68$ & $-1,69$ & $-1,30$ & 1,45 \\
\hline P99,9-100 (EL) & 2,51 & 2,66 & 2,67 & 2,53 & 2,39 & 2,36 \\
\hline P99,9-100 (ML) & 2,50 & 2,66 & 2,67 & 2,51 & 2,40 & 2,37 \\
\hline écart absolu & 0,00 & 0,00 & 0,00 & $-0,02$ & 0,01 & 0,01 \\
\hline écart en \% & $-0,09$ & $-0,17$ & 0,08 & $-0,67$ & 0,24 & 0,32 \\
\hline P99,99-100 (EL) & 0,73 & 0,79 & 0,81 & 0,73 & 0,69 & 0,67 \\
\hline P99,99-100 (ML) & 0,71 & 0,77 & 0,78 & 0,71 & 0,67 & 0,66 \\
\hline écart absolu & $-0,02$ & $-0,02$ & $-0,03$ & $-0,02$ & $-0,02$ & $-0,01$ \\
\hline écart en \% & $-2,65$ & $-2,27$ & $-3,72$ & $-2,79$ & $-2,36$ & $-1,16$ \\
\hline
\end{tabular}

Sources: Calculs de l'auteur à partir des échantillons légers de déclarations de revenus (cf. Annexe F) et estimations de l'auteur à partir des dossiers "Modèle lourd" (cf. Annexe G). Lecture: En 1988, la part du revenu imposable détenue par les 10\% des foyers fiscaux ayant le revenu imposable le plus élevé est de $33,39 \%$ d'après l'échantillon léger et de $33,31 \%$ d'après les estimations effectuées à partir des dossiers "Modèle lourd". 
Insérer Tableau E-7: Comparaison entre les chiffres bruts issus des échantillons légers et les estimations effectuées à partir des dossiers "Modèle lourd"

Le tableau E-6 indique des écarts de l'ordre de 0,5\% pour les parts de P90-100, P95$100, P 99-100$ et $P 99,5-100$. Le fait que ces écarts soient légèrement inférieurs aux écarts obtenus sur le tableau E-1 n'est pas véritablement étonnant: les erreurs d'échantillonnage sont plus faibles pour les échantillons lourds que pour les échantillons légers. ${ }^{133}$ Le tableau E-7 indique des écarts généralement inférieurs à $0,3 \%$ pour la part de P99,9-100. Cela confirme que l'approximation par une loi de Pareto conduit à d'excellentes estimations, y compris pour les très hauts revenus de P99,9-100, pourvu que l'on dispose d'informations en tranche suffisamment proches : la proportion de contribuables ayant un revenu imposable supérieur à 1 million de francs varie entre $0,04 \%$ en 1983 à $0,12 \%$ en 1993 (cf. tableau E-5), et cette tranche permet donc une très bonne approximation de la part de P99,9-100. Par contre, cette tranche permet seulement d'atténuer la sous-évaluation de la part de P99,99-100, dont l'évolution est cependant très bien prédite, mais pas de l'éliminer complètement (cf. tableau E-7).

\footnotetext{
${ }^{133}$ Les extrapolations effectuées à partir des tableaux 10 des dossiers "Modèle lourd " ont été faites en additionnant les nombres de foyers et les revenus imposables des foyers imposables et des foyers non-imposables des tranches utilisées, alors que seuls les foyers imposables ont été pris en compte pour les estimations effectués à partir des Etats 1921. La faiblesse des écarts obtenus confirme que les foyers non-imposables sont suffisamment peu nombreux dans les tranches supérieures pour pouvoir être négligés. En outre, les dossiers "Modèle lourd" portent toujours sur la situation des impositions émises en métropole au 31/3/n+2 (alors que les Etats 1921 utilisés portent sur le champ métropole + DOM/TOM depuis 1985 , et sur la situation au $31 / 12 / n+2$ depuis 1987 ), ce qui confirme que ces petites différences n'ont pas de conséquence importante.
} 


\section{Annexe F : Calculs effectués à partir des “ échantillons légers ”, 1988-1995}

Tous les calculs effectués à partir des échantillons légers ont été réalisés en utilisant le logiciel Stata (sous Windows). Les échantillons légers sont constitués à l'issue des émissions de rôles au 31/12/n+1 ("échantillon anticipé") et une dernière fois à l'issue des émissions de rôles au $31 / 03 / n+2$ ("échantillon semi-définitif"), et nous avons toujours utilisés les échantillons semi-définitifs. Les échantillons se présentent initialement sous la forme de bases SAS à plusieurs niveaux : un niveau "DEC", qui contient toutes les variables générales concernant le foyer déclarant, et notamment les variables recalculés par la DGI pour le calcul de l'impôt (revenu imposable (" rimp "), nombre de parts de quotient familial ("nbp"); droits simples ("drs"); impôt net ("inet"); etc..) ; et des niveaux "SAL", "BA ", "BIC ", etc.., contenant l'ensemble des variables correspondant aux cases de la déclaration 2042 remplie par les contribuables concernant les traitements et salaires, les bénéfices agricoles, les bénéfices industriels et commerciaux, etc... Une variable de pondération ("pond") permet de redresser l'échantillon et de le caler sur la population des foyers fiscaux de France métropolitaine. Pour calculer les parts du revenu imposable détenus par les foyers de P90-100, P95-100, etc.., que nous utilisons dans l'annexe E.1 pour tester la fiabilité des estimations obtenues à partir des Etats 1921, nous avons utilisé la variable " rimp" (ce qui est sans doute plus fiable que de chercher à recalculer le revenu imposable à partir des cases de la déclaration). Nous avons classé les foyers fiscaux (tous nombres de parts confondus) par ordre de rimp croissant, et nous avons calculé la part du rimp total détenu par les $10 \%$ ayant le rimp le plus élevé, par les $5 \%$ ayant le rimp le plus élevé, etc... Les résultats détaillés de ces calculs pour les échantillons légers de déclarations de revenus 1988 à 1994 sont donnés dans les tableaux F-1 à F-14 (les résultats pour 1995, sont donnés dans les tableaux 3-1 et 32, section 3.1).

\section{Insérer Tableaux F-1 à F-14}

Les chiffres utilisés dans les comparaisons des tableaux E-1 et E-2 (annexe E.1) sont issus des résultats des tableaux F-1 à F-14. Par exemple, la part de 33,39\% donnée pour P90-100 en 1988 sur le tableau E-1 correspond au revenu imposable moyen de 247362 francs pour les foyers de P90-100 donné sur le tableau F-1 $(247362 / 74093=3,339)$. Les tableaux F-1 à F-14 décrivent également la distribution et la composition du revenu fiscal déclaré. Ces résultats ont été obtenus en additionnant les cases de la déclaration 2042 correspondant aux différents types de revenu déclaré (avant tout abattement ou déduction, et à l'exclusion des plus-values soumises à un taux proportionnel), ce qui nous a permis d'obtenir des variables "sal", " pen ", "ba", " bic", bnc", " rga ", "rcm " et "rf"; ;34 puis ces variables ont été additionnés pour obtenir une variable de "revenu fiscal déclaré" (rfisc=sal+pen+ba+bic+bnc+rga+rcm+rf); enfin, nous avons classé les foyers fiscaux

\footnotetext{
${ }^{134}$ La variable "sal " est définie comme la somme de toutes les cases de revenu déclaré concernant les traitements et salaires, "pen" comme la somme de toutes les cases de revenu déclaré concernant les pensions et rentes viagères; "ba" comme la somme de toutes les cases de revenu déclaré concernant les bénéfices agricoles ; etc.. Tous les revenus accessoires ont été inclus dans la variable "bic" (à l'exception des bénéfices non-commerciaux accessoires, qui ont été inclus dans la variable " bnc"). Les cases concernant les déficits n'ont pas été prises en compte. La variable "rcm " inclut les avoirs fiscaux (mais ne prend pas en compte les frais).
} 
Tableau F-1: La distribution du revenu fiscal déclaré et du revenu imposable en 1988

Revenu fiscal déclaré

\begin{tabular}{lrc} 
N.foyers fisc. & 26112357 \\
rfisc.moyen & 106107 & \\
rfisc.médian & 79880 \\
& \multicolumn{3}{c}{ rfisc.moy. } & lim.inf. \\
P90-100 & 338024 & 203577 \\
P90-95 & 231930 & \\
P95-100 & 443554 & 269344 \\
P95-99 & 344708 & \\
P99-100 & 838985 & 510526 \\
P99-99,5 & 578067 & \\
P99,5-100 & 1100603 & 671521 \\
P99,5-99,9 & 845187 & \\
P99,9-100 & 2120967 & 1219370 \\
P99,9-99,99 & 1708031 & \\
P99,99-100 & 5835809 & 3153583 \\
P99,99-99,999 & 4641685 & \\
P99,999-100 & 16588400 & 9524813
\end{tabular}

Revenu imposable

N.foyers fisc. 26112357

rimp.moyen $\quad 74093 \quad 69,8$

rimp.médian $\quad 54950$

$\begin{array}{lrcc}\text { P90-100 } & 247362 & 143420 & \\ \text { P90-95 } & 165195 & & 71,2 \\ \text { P95-100 } & 329607 & 192440 & \\ \text { P95-99 } & 247473 & & 71,8 \\ \text { P99-100 } & 657817 & 369040 & \\ \text { P99-99,5 } & 420971 & & 72,8 \\ \text { P99,5-100 } & 894941 & 491910 & \\ \text { P99,5-99,9 } & 654316 & & 77,4 \\ \text { P99,9-100 } & 1856998 & 1007670 & \\ \text { P99,9-99,99 } & 1459070 & & 85,4 \\ \text { P99,99-100 } & 5437282 & 2866040 & \\ \text { P99,99-99,999 } & 4274437 & & 92,1 \\ \text { P99,999-100 } & 15907340 & 8837690 & 95,9\end{array}$

Source: Calculs de l'auteur à partir de l'échantillon léger de déclarations de revenus 1988 (cf. Annexe F). Le taux de sondage moyen de l'échantillon est de 1/516 (50621 observations pour 26112357 foyers), mais il passe de $1 / 889$ sur P0-90 (26438 observations pour 23501121 foyers) à $1 / 322$ sur P90-95 (4059 observations pour 1305618 foyers), $1 / 211$ sur P95-99 (4944 observations pour 1044494 foyers), 1/97 sur P99-99,5 (1345 observations pour 130562 foyers), 1/85 sur P99,5-99,9 (1225 observations pour 104494 foyers), 1/2 sur P99,9-99,99 (9999 observations pour 23501 foyers), et 1/1 sur P99,99-100 (2611 observations pour 2611 foyers).

Lecture: cf. Tableau 3-1 (section 3.1)

Tableau F-2 : La composition du revenu fiscal déclaré en 1988

$\begin{array}{lccccccccc}\text { En \%: } & \text { BA } & \text { BIC } & \text { BNC } & \text { RGA } & \text { RF } & \text { RCM } & \text { SAL } & \text { PEN } & \\ \text { P0-100 } & 1,4 & 5,0 & 3,5 & 0,1 & 2,1 & 3,2 & 64,5 & 20,3 & 100,0 \\ \text { P90-100 } & 1,4 & 8,9 & 9,5 & 0,2 & 3,5 & 4,5 & 61,6 & 10,3 & 100,0 \\ \text { P95-100 } & 1,6 & 10,3 & 13,0 & 0,2 & 4,1 & 5,6 & 56,9 & 8,2 & 100,0 \\ \text { P99-100 } & 1,9 & 12,5 & 21,4 & 0,4 & 5,6 & 9,5 & 45,4 & 3,4 & 100,0 \\ \text { P99,5-100 } & 2,0 & 12,4 & 22,6 & 0,4 & 5,7 & 12,4 & 41,8 & 2,6 & 100,0 \\ \text { P99,9-100 } & 2,2 & 13,1 & 22,6 & 0,7 & 6,2 & 22,3 & 31,0 & 1,9 & 100,0 \\ \text { P99,99-100 } & 2,4 & 14,6 & 14,9 & 0,7 & 5,7 & 39,1 & 21,2 & 1,6 & 100,0 \\ \text { P99,999-100 } & 2,8 & 17,8 & 10,8 & 0,1 & 4,5 & 47,4 & 14,0 & 2,4 & 100,0 \\ & & & & & & & & & \\ \text { P0-90 } & \text { BA } & \text { BIC } & \text { BNC } & \text { RGA } & \text { RF } & \text { RCM } & \text { SAL } & \text { PEN } & \\ \text { P90-95 } & 1,4 & 3,1 & 0,7 & 0,0 & 1,4 & 2,5 & 65,8 & 25,0 & 100,0 \\ \text { P95-99 } & 1,2 & 6,4 & 2,7 & 0,1 & 2,2 & 2,5 & 70,7 & 14,3 & 100,0 \\ \text { P99-99,5 } & 1,4 & 8,9 & 7,9 & 0,1 & 3,3 & 3,3 & 63,9 & 11,2 & 100,0 \\ \text { P99,5-99,9 } & 1,7 & 12,7 & 19,1 & 0,3 & 5,3 & 3,9 & 52,3 & 4,7 & 100,0 \\ \text { P99,9-99,99 } & 1,9 & 12,0 & 22,6 & 0,2 & 5,5 & 6,2 & 48,5 & 3,1 & 100,0 \\ \text { P99,99-99,999 } & 2,1 & 12,6 & 25,5 & 0,7 & 6,3 & 15,9 & 34,8 & 2,1 & 100,0 \\ \text { P99,999-100 } & 2,3 & 13,3 & 16,5 & 0,9 & 6,1 & 35,7 & 24,0 & 1,3 & 100,0 \\ & 2,8 & 17,8 & 10,8 & 0,1 & 4,5 & 47,4 & 14,0 & 2,4 & 100,0\end{array}$

Source: cf. Tableau F-1.

Lecture: cf. Tableau 3-2 (section 3.1) 
Tableau F-3: La distribution du revenu fiscal déclaré et du revenu imposable en 1989

Revenu fiscal déclaré

\begin{tabular}{lrl} 
N.foyers fisc. & 26780723 \\
rfisc.moyen & 110889 & \\
rfisc.médian & 82590 \\
& \multicolumn{3}{c}{ rfisc. moy. } & lim.inf. \\
P90-100 & 354836 & 214159 \\
P90-95 & 242945 & \\
P95-100 & 466376 & 278279 \\
P95-99 & 361768 & \\
P99-100 & 885111 & 523960 \\
P99-99,5 & 598025 & \\
P99,5-100 & 1172401 & 688145 \\
P99,5-99,9 & 886412 & \\
P99,9-100 & 2314852 & 1310352 \\
P99,9-99,99 & 1840644 & \\
P99,99-100 & 6582548 & 3527887 \\
P99,99-99,999 & 5241229 & \\
P99,999-100 & 18694610 & 10654250
\end{tabular}

\begin{tabular}{|c|c|c|}
\hline \multicolumn{3}{|c|}{ Revenu imposable } \\
\hline \multicolumn{3}{|c|}{26780723} \\
\hline 77490 & & 69,9 \\
\hline 56620 & & \\
\hline rimp.moy. & lim.inf. & \%rimp/rfisc \\
\hline 261590 & 151350 & \\
\hline 171497 & & 70,6 \\
\hline 351665 & 199560 & \\
\hline 261315 & & 72,2 \\
\hline 713221 & 391260 & \\
\hline 449706 & & 75,2 \\
\hline 977021 & 528590 & \\
\hline 704998 & & 79,5 \\
\hline 2063959 & 1105920 & \\
\hline 1611584 & & 87,6 \\
\hline 6134653 & 3189060 & \\
\hline 4837457 & & 92,3 \\
\hline 17848290 & 10079210 & 95,5 \\
\hline
\end{tabular}

Source: Calculs de l'auteur à partir de l'échantillon léger de déclarations de revenus 1989 (cf. Annexe F). Le taux de sondage moyen de l'échantillon est de 1/656 (40809 observations pour 26780723 foyers), mais il passe de $1 / 1282$ sur P0-90 (18807 observations pour 24102651 foyers) à $1 / 553$ sur P90-95 (2423 observations pour 1339036 foyers), $1 / 323$ sur P95-99 (3316 observations pour 1071229 foyers), 1/159 sur P99-99,5 (844 observations pour 133904 foyers), 1/98 sur P99,5-99,9 (1092 observations pour 107123 foyers), 1/2 sur P99,9-99,99 (11649 observations pour 24103 foyers), et 1/1 sur P99,99-100 (2678 observations pour 2678 foyers).

Tableau F-4 : La composition du revenu fiscal déclaré en 1989

$\begin{array}{lccccccccc}\text { En \%: } & \text { BA } & \text { BIC } & \text { BNC } & \text { RGA } & \text { RF } & \text { RCM } & \text { SAL } & \text { PEN } & \\ \text { P0-100 } & 1,5 & 4,8 & 3,7 & 0,1 & 2,4 & 3,3 & 63,6 & 20,7 & 100,0 \\ \text { P90-100 } & 1,8 & 8,1 & 10,0 & 0,2 & 4,0 & 4,8 & 60,5 & 10,6 & 100,0 \\ \text { P95-100 } & 2,0 & 9,9 & 13,8 & 0,3 & 4,7 & 6,0 & 54,5 & 8,6 & 100,0 \\ \text { P99-100 } & 2,4 & 12,5 & 22,7 & 0,4 & 6,0 & 10,6 & 41,7 & 3,9 & 100,0 \\ \text { P99,5-100 } & 2,6 & 12,4 & 23,6 & 0,5 & 6,4 & 13,4 & 38,0 & 3,0 & 100,0 \\ \text { P99,9-100 } & 2,2 & 13,2 & 22,4 & 0,6 & 6,9 & 23,4 & 29,8 & 1,6 & 100,0 \\ \text { P99,99-100 } & 2,0 & 14,6 & 17,3 & 0,5 & 5,7 & 38,3 & 20,4 & 1,1 & 100,0 \\ \text { P99,999-100 } & 2,1 & 20,4 & 12,5 & 0,2 & 3,3 & 43,8 & 16,3 & 1,3 & 100,0 \\ & & & & & & & & & \\ \text { P0-90 } & \text { BA } & \text { BIC } & \text { BNC } & \text { RGA } & \text { RF } & \text { RCM } & \text { SAL } & \text { PEN } & \\ \text { P90-95 } & 1,4 & 3,2 & 0,7 & 0,0 & 1,6 & 2,5 & 65,1 & 25,5 & 100,0 \\ \text { P95-99 } & 1,4 & 4,7 & 2,5 & 0,0 & 2,5 & 2,5 & 72,2 & 14,3 & 100,0 \\ \text { P99-99,5 } & 1,8 & 8,4 & 8,4 & 0,3 & 4,0 & 3,3 & 62,3 & 11,5 & 100,0 \\ \text { P99,5-99,9 } & 2,0 & 12,5 & 20,8 & 0,1 & 5,3 & 4,9 & 48,8 & 5,5 & 100,0 \\ \text { P99,9-99,99 } & 2,8 & 12,0 & 24,5 & 0,5 & 6,1 & 6,9 & 43,4 & 4,0 & 100,0 \\ \text { P99,99-99,999 } & 2,3 & 12,6 & 24,3 & 0,6 & 7,3 & 17,4 & 33,5 & 1,8 & 100,0 \\ \text { P99,999-100 } & 2,0 & 12,2 & 19,3 & 0,7 & 6,7 & 36,1 & 22,0 & 1,1 & 100,0 \\ & 2,1 & 20,4 & 12,5 & 0,2 & 3,3 & 43,8 & 16,3 & 1,3 & 100,0\end{array}$

Source: cf. Tableau F-3. 
Tableau F-5: La distribution du revenu fiscal déclaré et du revenu imposable en 1990

Revenu fiscal déclaré

\begin{tabular}{lrc} 
N.foyers fisc. & 27397921 \\
rfisc.moyen & 115012 & \\
rfisc.médian & 85620 \\
& \multicolumn{3}{c}{ rfisc.moy. } & lim.inf. \\
P90-100 & 371400 & 226379 \\
P90-95 & 255982 & \\
P95-100 & 486848 & 294773 \\
P95-99 & 374674 & \\
P99-100 & 935438 & 554974 \\
P99-99,5 & 626315 & \\
P99,5-100 & 1245606 & 724445 \\
P99,5-99,9 & 939201 & \\
P99,9-100 & 2468597 & 1414538 \\
P99,9-99,99 & 1953655 & \\
P99,99-100 & 7104100 & 3776373 \\
P99,99-99,999 & 5700256 & \\
P99,999-100 & 19784970 & 11299880
\end{tabular}

Revenu imposable

N.foyers fisc. $\quad 27397921$

rimp.moyen $\quad 80636 \quad 70,1$

rimp.médian $\quad 58294$

rimp.moy. lim.inf. \%rimp/rfisc

P90-100

P90-95

P95-100

P95-99

P99-100

P99-99,5

P99,5-100

P99,5-99,9

P99,9-100

P99,9-99,99

P99,99-100

P99,99-99,999

P99,999-100
$274706 \quad 158546$

180510

368904

274478

746634

468864

1026807

745588

2149500

1663526

6523617

5169012

1875972010499930
70,5

208064

73,3

408145

74,9

558114

79,4

85,1

90,7

94,8

Source: Calculs de l'auteur à partir de l'échantillon léger de déclarations de revenus 1990 (cf. Annexe F). Le taux de sondage moyen de l'échantillon est de 1/684 (40053 observations pour 27397921 foyers), mais il passe de 1/1401 sur P0-90 (17597 observations pour 24658129 foyers) à 1/657 sur P90-95 (2085 observations pour 1369896 foyers), 1/370 sur P95-99 (2985 observations pour 1095917 foyers), 1/203 sur P99-99,5 (674 observations pour 136990 foyers), 1/101 sur P99,5-99,9 (1086 observations pour 109592 foyers), $1 / 2$ sur P99,9-99,99 (12907 observations pour 24658 foyers), et 1/1 sur P99,99-100 (2739 observations pour 2739 foyers).

Tableau F-6 : La composition du revenu fiscal déclaré en 1990

$\begin{array}{lccccccccc}\text { En \%: } & \text { BA } & \text { BIC } & \text { BNC } & \text { RGA } & \text { RF } & \text { RCM } & \text { SAL } & \text { PEN } \\ \text { P0-100 } & 1,5 & 4,6 & 3,6 & 0,1 & 2,4 & 3,1 & 64,1 & 20,5 & 100,0 \\ \text { P90-100 } & 1,8 & 8,2 & 9,5 & 0,3 & 4,2 & 4,3 & 61,9 & 9,8 & 100,0 \\ \text { P95-100 } & 2,1 & 9,8 & 13,3 & 0,4 & 4,7 & 5,6 & 56,4 & 7,7 & 100,0 \\ \text { P99-100 } & 2,7 & 12,5 & 21,2 & 0,7 & 6,1 & 10,2 & 43,0 & 3,7 & 100,0 \\ \text { P99,5-100 } & 2,9 & 12,8 & 23,7 & 0,8 & 6,7 & 13,2 & 37,6 & 2,4 & 100,0 \\ \text { P99,9-100 } & 2,8 & 12,1 & 19,4 & 0,6 & 6,5 & 22,6 & 34,4 & 1,5 & 100,0 \\ \text { P99,99-100 } & 3,0 & 13,6 & 17,2 & 0,6 & 5,4 & 39,1 & 20,2 & 0,8 & 100,0 \\ \text { P99,999-100 } & 1,9 & 20,2 & 15,7 & 0,2 & 3,1 & 45,4 & 12,8 & 0,6 & 100,0 \\ & & & & & & & & & \\ \text { P0-90 } & \text { BA } & B I C & B N C & R G A & R F & R C M & S A L & P E N & \\ \text { P90-95 } & 1,4 & 2,9 & 0,8 & 0,1 & 1,6 & 2,5 & 65,2 & 25,6 & 100,0 \\ \text { P95-99 } & 1,3 & 5,0 & 2,4 & 0,1 & 3,1 & 1,8 & 72,3 & 13,9 & 100,0 \\ \text { P99-99,5 } & 1,7 & 8,2 & 8,3 & 0,3 & 3,8 & 2,7 & 64,8 & 10,1 & 100,0 \\ \text { P99,5-99,9 } & 2,3 & 11,7 & 16,3 & 0,4 & 5,0 & 4,3 & 53,6 & 6,3 & 100,0 \\ \text { P99,9-99,99 } & 2,9 & 13,3 & 26,5 & 1,0 & 6,7 & 6,9 & 39,7 & 2,9 & 100,0 \\ \text { P99,99-99,999 } & 2,8 & 11,5 & 20,3 & 0,6 & 7,0 & 15,9 & 40,1 & 1,8 & 100,0 \\ \text { P99,999-100 } & 3,5 & 11,0 & 17,8 & 0,7 & 6,3 & 36,7 & 23,1 & 1,0 & 100,0 \\ & 1,9 & 20,2 & 15,7 & 0,2 & 3,1 & 45,4 & 12,8 & 0,6 & 100,0\end{array}$

Source: cf. Tableau F-5. 
Tableau F-7: La distribution du revenu fiscal déclaré et du revenu imposable en 1991

Revenu fiscal déclaré

\begin{tabular}{lrc} 
N.foyers fisc. & 28196553 \\
rfisc.moyen & 117513 \\
rfisc.médian & 86721 \\
& \multicolumn{3}{c}{ rfisc. moy. } & lim.inf. \\
P90-100 & 379547 & 229907 \\
P90-95 & 264509 & \\
P95-100 & 496280 & 303799 \\
P95-99 & 382406 & \\
P99-100 & 947765 & 555040 \\
P99-99,5 & 640348 & \\
P99,5-100 & 1257123 & 757236 \\
P99,5-99,9 & 962203 & \\
P99,9-100 & 2431345 & 1382384 \\
P99,9-99,99 & 1946294 & \\
P99,99-100 & 6791639 & 3989299 \\
P99,99-99,999 & 5535272 & \\
P99,999-100 & 18139180 & 10444570
\end{tabular}

Revenu imposable

N.foyers fisc. 28196553

rimp.moyen $\quad 82114 \quad 69,9$

rimp.médian 60193

rimp.moy. lim.inf. \%rimp/rfisc

P90-100

$\mathrm{P} 90-95$

P95-100

P95-99

P99-100

P99-99,5

P99,5-100

$\mathrm{P} 99,5-99,9$

$\mathrm{P} 99,9-100$

P99,9-99, 99

$\mathrm{P} 99,99-100$

P99,99-99,999

P99,999-100
$278904 \quad 160561$

$185735 \quad 70,2$

372294

277962

748829

485603

1013338

747576

2075197

6020603

4813161

16926250
1636178
214715

72,7

422211

75,8

570430

77,7

84,1

3211765

87,0

87,0

Source: Calculs de l'auteur à partir de l'échantillon léger de déclarations de revenus 1991 (cf. Annexe F). Le taux de sondage moyen de l'échantillon est de 1/709 (39775 observations pour 28196553 foyers), mais il passe de 1/1732 sur P0-90 (14653 observations pour 25376897 foyers) à $1 / 944$ sur P90-95 (1493 observations pour 1409828 foyers), $1 / 374$ sur P95-99 (3018 observations pour 1127862 foyers), 1/105 sur P99-99,5 (1346 observations pour 140983 foyers), $1 / 40$ sur P99,5-99,9 (2845 observations pour 112786 foyers), $1 / 2$ sur P99,9-99,99 (13601 observations pour 25377 foyers), et 1/1 sur P99,99-100 (2819 observations pour 2819 foyers).

Tableau F-8 : La composition du revenu fiscal déclaré en 1991

$\begin{array}{lccccccccc}\text { En \%: } & \text { BA } & \text { BIC } & \text { BNC } & \text { RGA } & \text { RF } & \text { RCM } & \text { SAL } & \text { PEN } & \\ \text { P0-100 } & 1,3 & 4,1 & 3,6 & 0,1 & 2,5 & 2,7 & 64,2 & 21,4 & 100,0 \\ \text { P90-100 } & 1,6 & 7,1 & 9,6 & 0,4 & 4,7 & 4,0 & 61,6 & 11,0 & 100,0 \\ \text { P95-100 } & 2,0 & 8,2 & 13,5 & 0,6 & 5,5 & 5,1 & 57,2 & 8,2 & 100,0 \\ \text { P99-100 } & 2,3 & 11,0 & 22,0 & 1,0 & 6,9 & 9,8 & 42,6 & 4,4 & 100,0 \\ \text { P99,5-100 } & 2,3 & 12,3 & 22,0 & 0,8 & 7,8 & 12,7 & 39,0 & 3,1 & 100,0 \\ \text { P99,9-100 } & 2,6 & 11,4 & 22,5 & 0,5 & 8,9 & 25,2 & 26,9 & 1,9 & 100,0 \\ \text { P99,99-100 } & 1,9 & 13,9 & 11,3 & 0,5 & 10,7 & 42,3 & 17,9 & 1,4 & 100,0 \\ \text { P99,999-100 } & 2,2 & 14,2 & 10,3 & 0,4 & 3,9 & 52,4 & 16,1 & 0,4 & 100,0 \\ & & & & & & & & & \\ \text { P0-90 } & \text { BA } & \text { BIC } & \text { BNC } & \text { RGA } & \text { RF } & \text { RCM } & \text { SAL } & \text { PEN } & \\ \text { P90-95 } & 1,2 & 2,7 & 0,7 & 0,0 & 1,5 & 2,1 & 65,5 & 26,3 & 100,0 \\ \text { P95-99 } & 0,9 & 5,0 & 2,3 & 0,1 & 3,4 & 2,0 & 70,1 & 16,2 & 100,0 \\ \text { P99-99,5 } & 1,8 & 6,4 & 8,2 & 0,3 & 4,6 & 2,1 & 66,0 & 10,5 & 100,0 \\ \text { P99,5-99,9 } & 2,2 & 8,5 & 22,2 & 1,4 & 5,1 & 4,1 & 49,5 & 7,0 & 100,0 \\ \text { P99,9-99,99 } & 2,1 & 12,9 & 21,7 & 1,0 & 7,1 & 4,8 & 46,8 & 3,8 & 100,0 \\ \text { P99,99-99,999 } & 2,9 & 10,5 & 26,9 & 0,5 & 8,2 & 18,6 & 30,4 & 2,1 & 100,0 \\ \text { P99,999-100 } & 1,8 & 13,8 & 11,7 & 0,6 & 13,2 & 38,5 & 18,6 & 1,8 & 100,0 \\ & 2,2 & 14,2 & 10,3 & 0,4 & 3,9 & 52,4 & 16,1 & 0,4 & 100,0\end{array}$

Source: cf. Tableau F-7. 
Tableau F-9: La distribution du revenu fiscal déclaré et du revenu imposable en 1992

Revenu fiscal déclaré

\begin{tabular}{lrc} 
N.foyers fisc. & 28356371 \\
rfisc.moyen & 121655 \\
rfisc.médian & 90434 \\
& \multicolumn{3}{c}{ rfisc.moy. } & lim.inf. \\
P90-100 & 387858 & 235604 \\
P90-95 & 268131 & \\
P95-100 & 508941 & 310884 \\
P95-99 & 397293 & \\
P99-100 & 950976 & 560421 \\
P99-99,5 & 632643 & \\
P99,5-100 & 1274578 & 724186 \\
P99,5-99,9 & 968902 & \\
P99,9-100 & 2484603 & 1388130 \\
P99,9-99,99 & 2043920 & \\
P99,99-100 & 6449655 & 3542391 \\
P99,99-99,999 & 5200420 \\
P99,999-100 & 17714840 & 10113990
\end{tabular}

Revenu imposable

N.foyers fisc. 28356371

rimp.moyen $\quad 84503 \quad 69,5$

rimp.médian 62072

rimp.moy. lim.inf. \%rimp/rfisc

P90-100

$\mathrm{P} 90-95$

P95-100

P95-99

P99-100

P99-99,5

P99,5-100

P99,5-99,9

P99,9-100

P99,9-99, 99

P99,99-100

P99,99-99,999

P99,999-100

\section{$283312 \quad 165789$}

189844

376875

287228

733628

468422

999790

744124

2021009

1599901

5808157

4634032

16396020
$225640 \quad 70,8$

225640

72,3

420177

74,0

547999

76,8

1121582

78,3

3137208

89,1

92,6

Source: Calculs de l'auteur à partir de l'échantillon léger de déclarations de revenus 1992 (cf. Annexe F). Le taux de sondage moyen de l'échantillon est de 1/713 (39794 observations pour 28356371 foyers), mais il passe de 1/1694 sur P0-90 (15065 observations pour 25520734 foyers) à $1 / 871$ sur P90-95 (1628 observations pour 1417819 foyers), 1/386 sur P95-99 (2941 observations pour 1134255 foyers), 1/127 sur P99-99,5 (1120 observations pour 141782 foyers), 1/38 sur P99,5-99,9 (2965 observations pour 113426 foyers), 1/2 sur P99,9-99,99 (13240 observations pour 25521 foyers), et 1/1 sur P99,99-100 (2835 observations pour 2835 foyers).

Tableau F-10 : La composition du revenu fiscal déclaré en 1992

$\begin{array}{lccccccccc}\text { En \%: } & \text { BA } & \text { BIC } & \text { BNC } & \text { RGA } & \text { RF } & \text { RCM } & \text { SAL } & \text { PEN } & \\ \text { P0-100 } & 1,2 & 3,9 & 3,5 & 0,2 & 2,5 & 2,7 & 64,8 & 21,1 & 100,0 \\ \text { P90-100 } & 1,4 & 6,6 & 9,5 & 0,4 & 4,6 & 4,3 & 61,6 & 11,6 & 100,0 \\ \text { P95-100 } & 1,6 & 7,6 & 13,5 & 0,5 & 5,1 & 5,3 & 58,3 & 8,3 & 100,0 \\ \text { P99-100 } & 1,8 & 8,6 & 22,3 & 0,7 & 6,7 & 10,2 & 44,8 & 5,0 & 100,0 \\ \text { P99,5-100 } & 2,1 & 9,8 & 21,9 & 0,8 & 7,7 & 13,6 & 40,2 & 4,0 & 100,0 \\ \text { P99,9-100 } & 1,6 & 7,8 & 19,4 & 0,6 & 7,9 & 22,8 & 37,5 & 2,4 & 100,0 \\ \text { P99,99-100 } & 1,1 & 11,4 & 13,9 & 0,7 & 7,5 & 42,4 & 21,7 & 1,2 & 100,0 \\ \text { P99,999-100 } & 0,5 & 13,9 & 9,3 & 0,6 & 5,2 & 54,1 & 15,9 & 0,5 & 100,0 \\ & & & & & & & & & \\ \text { P0-90 } & \text { BA } & \text { BIC } & \text { BNC } & \text { RGA } & \text { RF } & \text { RCM } & \text { SAL } & \text { PEN } & \\ \text { P90-95 } & 1,2 & 2,6 & 0,7 & 0,1 & 1,6 & 2,0 & 66,3 & 25,6 & 100,0 \\ \text { P95-99 } & 1,0 & 4,7 & 1,9 & 0,3 & 3,4 & 2,6 & 68,0 & 18,0 & 100,0 \\ \text { P99-99,5 } & 1,5 & 7,0 & 8,2 & 0,4 & 4,2 & 2,3 & 66,1 & 10,2 & 100,0 \\ \text { P99,5-99,9 } & 1,3 & 6,2 & 23,1 & 0,3 & 4,7 & 3,2 & 54,1 & 7,1 & 100,0 \\ \text { P99,9-99,99 } & 2,3 & 11,0 & 23,4 & 1,0 & 7,6 & 7,7 & 41,9 & 5,0 & 100,0 \\ \text { P99,99-99,999 } & 1,8 & 6,5 & 21,4 & 0,6 & 8,0 & 15,9 & 43,1 & 2,8 & 100,0 \\ \text { P99,999-100 } & 1,3 & 10,5 & 15,6 & 0,7 & 8,4 & 38,0 & 23,9 & 1,5 & 100,0 \\ & 0,5 & 13,9 & 9,3 & 0,6 & 5,2 & 54,1 & 15,9 & 0,5 & 100,0\end{array}$

Source: cf. Tableau F-9. 
Tableau F-11: La distribution du revenu fiscal déclaré et du revenu imposable en 1993

Revenu fiscal déclaré

\begin{tabular}{lrc} 
N.foyers fisc. & 29075122 & \\
rfisc.moyen & 122485 & \\
rfisc.médian & 91112 & \\
& \multicolumn{3}{c}{ rfisc.moy. } & lim.inf. \\
P90-100 & 389158 & 241511 \\
P90-95 & 276120 & \\
P95-100 & 503094 & 323283 \\
P95-99 & 402175 & \\
P99-100 & 907434 & 570444 \\
P99-99,5 & 632543 & \\
P99,5-100 & 1186736 & 706352 \\
P99,5-99,9 & 892604 & \\
P99,9-100 & 2346059 & 1341273 \\
P99,9-99,99 & 1903559 & \\
P99,99-100 & 6315016 & 3549383 \\
P99,99-99,999 & 5161170 & \\
P99,999-100 & 16727490 & 9974616
\end{tabular}

Revenu imposable

N.foyers fisc. 29075122

rimp.moyen $\quad 84654 \quad 69,1$

rimp.médian $\quad 62118$

rimp.moy. lim.inf. \%rimp/rfisc

P90-100

P90-95

P95-100

P95-99

P99-100

P99-99,5

P99,5-100

P99,5-99,9

P99,9-100

P99,9-99,99

P99,99-100

P99,99-99,999

P99,999-100
284242

193384

374043

289052

713779

469950

960828

699786

1996750

1585838

5693688

4617232

15407780
170499

230221

70,0

71,9

420522

74,3

538626

78,4

1083916

83,3

3140526

89,5

92,1

Source: Calculs de l'auteur à partir de l'échantillon léger de déclarations de revenus 1993 (cf. Annexe F). Le taux de sondage moyen de l'échantillon est de 1/656 (44337 observations pour 29075122 foyers), mais il passe de 1/1408 sur P0-90 (18584 observations pour 26167610 foyers) à $1 / 860$ sur P90-95 (1690 observations pour 1453756 foyers), 1/378 sur P95-99 (3078 observations pour 1163005 foyers), 1/120 sur P99-99,5 (1212 observations pour 145376 foyers), 1/37 sur P99,5-99,9 (3185 observations pour 116301 foyers), 1/2 sur P99,9-99,99 (13681 observations pour 26168 foyers), et 1/1 sur P99,99-100 (2907 observations pour 2907 foyers).

Tableau F-12 : La composition du revenu fiscal déclaré en 1993

$\begin{array}{lccccccccc}\text { En \%: } & \text { BA } & \text { BIC } & \text { BNC } & \text { RGA } & \text { RF } & \text { RCM } & \text { SAL } & \text { PEN } & \\ \text { P0-100 } & 1,1 & 3,8 & 3,4 & 0,2 & 2,4 & 2,5 & 64,4 & 22,2 & 100,0 \\ \text { P90-100 } & 1,0 & 6,0 & 9,1 & 0,6 & 4,3 & 3,9 & 63,6 & 11,5 & 100,0 \\ \text { P95-100 } & 1,1 & 6,9 & 12,7 & 0,8 & 5,0 & 5,1 & 59,7 & 8,7 & 100,0 \\ \text { P99-100 } & 1,3 & 8,9 & 22,0 & 0,9 & 7,0 & 10,7 & 44,1 & 5,0 & 100,0 \\ \text { P99,5-100 } & 1,3 & 9,6 & 25,7 & 0,8 & 7,6 & 14,8 & 35,7 & 4,6 & 100,0 \\ \text { P99,9-100 } & 1,2 & 10,2 & 20,5 & 0,9 & 8,3 & 27,0 & 29,4 & 2,5 & 100,0 \\ \text { P99,99-100 } & 1,1 & 9,1 & 13,8 & 0,8 & 6,8 & 45,4 & 21,6 & 1,5 & 100,0 \\ \text { P99,999-100 } & 0,7 & 14,9 & 9,0 & 0,9 & 3,6 & 52,9 & 16,1 & 1,8 & 100,0 \\ & & & & & & & & & \\ \text { P0-90 } & \text { BA } & \text { BIC } & \text { BNC } & \text { RGA } & \text { RF } & \text { RCM } & \text { SAL } & \text { PEN } & \\ \text { P90-95 } & 1,2 & 2,7 & 0,7 & 0,1 & 1,5 & 1,9 & 64,8 & 27,1 & 100,0 \\ \text { P95-99 } & 0,8 & 4,4 & 2,6 & 0,1 & 3,1 & 1,8 & 70,6 & 16,5 & 100,0 \\ \text { P99-99,5 } & 1,1 & 5,7 & 7,4 & 0,7 & 3,8 & 2,0 & 68,5 & 10,8 & 100,0 \\ \text { P99,5-99,9 } & 1,2 & 7,7 & 15,1 & 1,2 & 6,0 & 3,0 & 60,0 & 5,7 & 100,0 \\ \text { P99,9-99,99 } & 1,4 & 9,2 & 29,1 & 0,7 & 7,1 & 6,9 & 39,8 & 6,0 & 100,0 \\ \text { P99,99-99,999 } & 1,3 & 10,6 & 23,0 & 1,0 & 8,9 & 20,2 & 32,3 & 2,8 & 100,0 \\ \text { P99,999-100 } & 1,2 & 7,0 & 15,5 & 0,7 & 7,9 & 42,7 & 23,6 & 1,4 & 100,0 \\ & 0,7 & 14,9 & 9,0 & 0,9 & 3,6 & 52,9 & 16,1 & 1,8 & 100,0\end{array}$

Source: cf. Tableau F-11. 
Tableau F-13: La distribution du revenu fiscal déclaré et du revenu imposable en 1994

Revenu fiscal déclaré

\begin{tabular}{lrc} 
N.foyers fisc. & 29775046 \\
rfisc.moyen & 123405 & \\
rfisc.médian & 90837 & \\
& \multicolumn{3}{c}{ rfisc.moy. } & lim.inf. \\
P90-100 & 399176 & 248503 \\
P90-95 & 281706 & \\
P95-100 & 515943 & 323864 \\
P95-99 & 405657 & \\
P99-100 & 956629 & 570359 \\
P99-99,5 & 644878 & \\
P99,5-100 & 1267695 & 745874 \\
P99,5-99,9 & 966659 & \\
P99,9-100 & 2585452 & 1458510 \\
P99,9-99,99 & 2060367 & \\
P99,99-100 & 6778550 & 3853943 \\
P99,99-99,999 & 5603941 & \\
P99,999-100 & 17377720 & 10408690
\end{tabular}

Revenu imposable

N.foyers fisc. 29775046

rimp.moyen $\quad 84181 \quad 68,2$

rimp.médian 61849

rimp.moy. lim.inf. \%rimp/rfisc

$\begin{array}{lrcc}\text { P90-100 } & 284867 & 173161 & \\ \text { P90-95 } & 194204 & & 68,9 \\ \text { P95-100 } & 375627 & 227031 & \\ \text { P95-99 } & 286536 & & 70,6 \\ \text { P99-100 } & 731767 & 418906 & \\ \text { P99-99,5 } & 474589 & & 73,6 \\ \text { P99,5-100 } & 988936 & 552670 & \\ \text { P99,5-99,9 } & 724455 & & 74,9 \\ \text { P99,9-100 } & 2046845 & 1112697 & \\ \text { P99,9-99,99 } & 1616417 & & 78,5 \\ \text { P99,99-100 } & 5920692 & 3215893 & \\ \text { P99,99-99,999 } & 4792478 & & 85,5 \\ \text { P99,999-100 } & 16101210 & 9624691 & 92,7\end{array}$

Source: Calculs de l'auteur à partir de l'échantillon léger de déclarations de revenus 1994 (cf. Annexe F). Le taux de sondage moyen de l'échantillon est de 1/725 (41068 observations pour 29775046 foyers), mais il passe de 1/1862 sur P0-90 (14393 observations pour 26797541 foyers) à $1 / 917$ sur P90-95 (1624 observations pour 1488752 foyers), 1/435 sur P95-99 (2741 observations pour 1191002 foyers), 1/93 sur P99-99,5 (1594 observations pour 148875 foyers), 1/37 sur P99,5-99,9 (3258 observations pour 119100 foyers), 1/2 sur P99,9-99,99 (14569 observations pour 26798 foyers), et 1/1 sur P99,99-100 (2889 observations pour 2977 foyers).

Tableau F-14 : La composition du revenu fiscal déclaré en 1994

$\begin{array}{lccccccccc}\text { En \%: } & \text { BA } & \text { BIC } & \text { BNC } & \text { RGA } & \text { RF } & \text { RCM } & \text { SAL } & \text { PEN } \\ \text { P0-100 } & 1,2 & 3,6 & 3,6 & 0,2 & 2,6 & 2,7 & 62,8 & 23,3 & 100,0 \\ \text { P90-100 } & 1,1 & 5,8 & 9,3 & 0,5 & 4,5 & 4,8 & 61,1 & 12,8 & 100,0 \\ \text { P95-100 } & 1,3 & 6,4 & 12,4 & 0,6 & 5,7 & 6,5 & 57,7 & 9,5 & 100,0 \\ \text { P99-100 } & 1,3 & 8,7 & 21,7 & 0,6 & 7,0 & 12,4 & 43,1 & 5,2 & 100,0 \\ \text { P99,5-100 } & 1,3 & 8,6 & 22,6 & 0,5 & 7,0 & 16,2 & 40,0 & 3,8 & 100,0 \\ \text { P99,9-100 } & 1,2 & 7,1 & 22,7 & 0,8 & 7,4 & 29,8 & 28,8 & 2,1 & 100,0 \\ \text { P99,99-100 } & 0,6 & 7,3 & 14,8 & 0,6 & 5,5 & 50,5 & 19,5 & 1,2 & 100,0 \\ \text { P99,999-100 } & 0,5 & 12,1 & 8,5 & 0,6 & 3,6 & 59,2 & 14,9 & 0,7 & 100,0 \\ & & & & & & & & & \\ \text { P0-90 } & \text { BA } & \text { BIC } & \text { BNC } & \text { RGA } & \text { RF } & \text { RCM } & \text { SAL } & \text { PEN } \\ \text { P90-95 } & 1,2 & 2,5 & 0,9 & 0,0 & 1,7 & 1,7 & 63,5 & 28,3 & 100,0 \\ \text { P95-99 } & 0,9 & 4,6 & 3,6 & 0,5 & 2,5 & 1,9 & 67,3 & 18,6 & 100,0 \\ \text { P99-99,5 } & 1,2 & 5,1 & 6,9 & 0,6 & 4,9 & 2,9 & 66,3 & 12,1 & 100,0 \\ \text { P99,5-99,9 } & 1,1 & 9,0 & 19,8 & 0,7 & 7,0 & 5,0 & 49,4 & 8,1 & 100,0 \\ \text { P99,9-99,99 } & 1,4 & 9,6 & 22,5 & 0,3 & 6,7 & 6,8 & 47,6 & 5,0 & 100,0 \\ \text { P99,99-99,999 } & 1,5 & 7,0 & 25,5 & 0,9 & 8,1 & 22,5 & 32,1 & 2,4 & 100,0 \\ \text { P99,999-100 } & 0,5 & 5,6 & 17,0 & 0,5 & 6,2 & 47,5 & 21,0 & 1,4 & 100,0 \\ & & 12,1 & 8,5 & 0,6 & 3,6 & 59,2 & 14,9 & 0,7 & 100,0\end{array}$

Source: cf. Tableau F-13. 
(tous nombres de parts confondus) par ordre de rfisc croissant pour obtenir les résultats bruts décrits sur les tableaux F-1 à F-14. Ces tableaux permettent d'observer les très fortes fluctuations de niveau et de composition auxquelles sont soumis les très hauts revenus au cours d'une période relativement courte. Enfin, les tableaux F-1 et F-14 indiquent les taux de sondage des échantillons légers, ce qui permet de constater l'excellente précision fournie par ces échantillons pour étudier les hauts et les très hauts revenus. ${ }^{135}$

\footnotetext{
${ }^{135}$ Les taux de sondage indiqués sur les tableaux 3-1 à 3-2 et F-1 à F-14 ont été calculés à partir de la centilisation en rimp ; les deux hiérarchies de revenu ne coïncidant qu'imparfaitement, les taux de sondage obtenus à partir de la centilisation en rfisc seraient (très) légèrement différents.
} 


\section{Annexe G: Calculs effectués à partir des enquêtes " Revenus fiscaux", 1970-1990}

Tous les calculs effectués à partir des enquêtes "Revenus fiscaux" ont été réalisés en utilisant le logiciel Stata (sous Windows). De la même façon que les échantillons légers, les fichiers des enquêtes "Revenus fiscaux" se présentent initialement sous la forme de bases SAS à plusieurs niveaux : un niveau "foyer " contenant toutes les variables générales concernant le foyer déclarant, et notamment les variables recalculées par l'INSEE (cf. infra); un niveau " ménage ", où l'INSEE a agrégé les variables des différents foyers composant chaque ménage, et qui contient également les variables concernant les revenus non-imposables (prestations familiales, etc..) imputés sur barème par l'INSEE ; et des niveaux "salaire ", "pension ", " ba ", " bic ",..., qui contiennent l'ensemble des variables correspondant aux cases de la déclaration 2042 remplie par les contribuables concernant les traitements et salaires, les bénéfices agricoles, les bénéfices industriels et commerciaux, etc... Dans le cadre de cette étude, nous n'avons pas cherché à utiliser directement les variables correspondant aux cases de la déclaration, et nous nous sommes contenté d'utiliser les variables recalculés par l'INSEE figurant au niveau "foyer". Compte tenu des multiples problèmes de déclarations manquantes et de redressement, il nous a paru plus sur de faire confiance au travail effectué à l'époque par l'INSEE. Pour le revenu imposable, nous avons utilisé la variable " zfisf " (" rif " pour l'enquête de 1970). ${ }^{136}$ La variable de pondération permettant de redresser l'échantillon et de la caler sur la population des foyers fiscaux de France métropolitaine est la variable " prf ". 137 Pour calculer les nombres de foyers et les revenus imposables moyens en fonction du nombre de parts de quotient familial rapportés sur le tableau C-5 (annexe C.3), nous avons utilisé la variable "npart" ("npartsf" pour l'enquête de 1970). ${ }^{138}$ Outre qu'aucune variable "npart" n'est disponible dans le fichier de l'enquête de 1975, cette variable "npart" doit être utilisée avec précaution : elle a été reconstruite par I'INSEE à partir des informations disponibles sur le foyer déclarant, et elle est généralement manquante pour une proportion non négligeable de foyers fiscaux (jusqu'à $6 \%$ en 1970). ${ }^{139}$

Pour calculer les parts du revenu imposable détenus par les foyers de P90-100, P95-100, etc.., que nous utilisons dans l'annexe E.2 pour tester la fiabilité des estimations obtenues à partir des Etats 1921, nous avons classé les foyers fiscaux (tous nombres de parts confondus) par ordre de zfisf croissant, et nous avons calculé la part du zfisf total détenu par les $10 \%$ ayant le zfisf le plus élevé, par les $5 \%$ ayant

\footnotetext{
${ }^{136}$ La variable zfisf ne semble pas avoir été complètement redressée dans l'enquête "Revenus fiscaux " de 1990 : zfisf est nulle pour plus de 10\% des foyers fiscaux, soit plus de 3 fois plus que lors des enquêtes précécentes. Cela explique la faiblesse du taux d'imposabilité moyen indiqué sur le tableau G-9. Ce problème de redressement incomplet est peut-être lié au fait que des "ménages fictifs" ont été introduit ex post dans le fichier de l'enquête de 1990 (afin de pouvoir imputer un nombre correct de RMI).

${ }^{137}$ Pour l'enquête de 1990, la variable de pondération au niveau " foyer " esr intitulée " prm ", bien que ce code soit généralement réservé à la variable de pondération au niveau "ménage" dans les enquêtes précédentes.

${ }^{138}$ Pour distinguer les foyers imposables et les foyers non-imposables, nous avons utilisé la variable " fi " (" imposab " pour l'enquête de 1990).

${ }^{139}$ Les chiffres du tableau C-5 ont été obtenus en excluant les observations pour lesquelles la variable "npart" n'est pas renseignée.
} 
le zfisf le plus élevé, etc... Les résultats détaillés de ces calculs pour les enquêtes "Revenus fiscaux" de 1970 à 1990 sont donnés dans les tableaux G-1 à G-10.

Insérer Tableau G-1 : La distribution du revenu fiscal déclaré et du revenu imposable en 1970

Insérer Tableau G-2 : La composition du revenu fiscal déclaré en 1970

Insérer Tableau G-3 : La distribution du revenu fiscal déclaré et du revenu imposable en 1975

Insérer Tableau G-4 : La composition du revenu fiscal déclaré en 1975

Insérer Tableau G-5 : La distribution du revenu fiscal déclaré et du revenu imposable en 1979

Insérer Tableau G-6 : La composition du revenu fiscal déclaré en 1979

Insérer Tableau G-7 : La distribution du revenu fiscal déclaré et du revenu imposable en 1984

Insérer Tableau G-8 : La composition du revenu fiscal déclaré en 1984

Insérer Tableau G-9 : La distribution du revenu fiscal déclaré et du revenu imposable en 1990

Insérer Tableau G-10 : La composition du revenu fiscal déclaré en 1990

Les chiffres utilisés dans les comparaisons des tableaux E-3 et E-4 (annexe E.2) sont issus des résultats des tableaux G-1 à G-10. Par exemple, la part de 35,51\% donnée pour P90-100 en 1970 sur le tableau E-3 correspond au revenu imposable moyen de 44822 francs pour les foyers de P90-100 donné sur le tableau G-1 $(44822 / 12623=3,551)$. Les tableaux G-1 à G-10 décrivent également la distribution et la composition du revenu fiscal déclaré (avant tout abattement ou déduction). Ces résultats obtenus en utilisant pour le revenu fiscal déclaré la variable "zrecf" et pour les revenus catégoriels bruts les variables "zbagf", "zbicf”, "zaccf", "zbncf ", "zrgaf", "zforf ", "zfouf ", "zvamf ", "ztsaf ", "zperf” et "zdivf”. Toutes ces variables sont des variables recalculées et redressées par l'INSEE à partir des cases de la déclaration 2042. ${ }^{140}$ Par construction, la variable "zrecf" est égale à la somme des 11 variables de revenus catégoriels. Le revenu fiscal "zrecf" inclut notamment "zdivf", c'est-à-dire les plus values taxées à taux proportionnel, que nous avons exclu afin que les résultats soient comparables aux autres résultats de cette étude : nous avons utilisé la variable zrecf2=zrecf-zdivf. Nous avons classé les foyers fiscaux (tous nombres de parts confondus) par ordre de zrecf2 croissant pour obtenir les résultats bruts décrits sur les tableaux G-1 à G-10. Nous avons regroupé les revenus accessoires ("zaccf") avec les BIC ("zbicf "), ${ }^{141}$ et le foncier rural (" zforf ") avec le foncier urbain (" zfouf "). ${ }^{142}$

\footnotetext{
${ }^{140}$ Contrairement à ce que nous avons fait avec les échantillons légers (cf. annexe F), l'INSEE a toujours choisi d'utiliser les cases de la déclaration correspondant aux déficits, si bien que les variables de revenus catégoriels mesurent des revenus catégoriels nets de déficits et peuvent donc être négatives (de même que les variables "zfisf" et "zrecf "). Le biais introduit n'est cependant pas énorme : en ramenant tous les zfisf négatifs à 0 , le zfisf total n'augmente jamais de plus de $0,4 \%$ (en ramenant tous les les zrecf négtifs à 0 , le zrecf total n'augmente jamais de plus de $0,2 \%$ ).

${ }^{141}$ La variable "zaccf" est manquante dans l'enquête de 1979.

142 Cette distinction a été abandonné dans l'enquête de 1990, qui utilise une unique variable ("zfonf") pour les revenus fonciers.
} 
Tableau G-1:

La distribution du revenu fiscal déclaré et du revenu imposable en 1970

Revenu fiscal déclaré

$\begin{array}{lrc}\begin{array}{l}\text { N.foyers fisc. } \\ \text { rfisc.moyen }\end{array} & \begin{array}{r}20470258 \\ 17356\end{array} \\ \text { rfisc.médian } & \begin{array}{r}12739 \\ \text { rfisc.moy. }\end{array} & \text { lim.inf. } \\ \text { P90-100 } & 59898 & 35698 \\ \text { P90-95 } & 40837 & \\ \text { P95-100 } & 78956 & 47875 \\ \text { P95-99 } & 62011 & \\ \text { P99-100 } & 146740 & 92175 \\ \text { P99-99,5 } & 103286 & \\ \text { P99,5-100 } & 190339 & 117703 \\ \text { P99,5-99,9 } & 153620 & \\ \text { P99,9-100 } & 338252 & 221692 \\ \text { P99,9-99,99 } & 297222 & \\ \text { P99,99-100 } & 716794 & 484245\end{array}$

Revenu imposable

$\begin{array}{lrrr}\begin{array}{l}\text { N.foyers fisc. } \\ \text { rimp.moyen } \\ \text { rimp.médian }\end{array} & \begin{array}{r}20470258 \\ 12623 \\ 9154\end{array} & & 72,7 \\ & \begin{array}{r}\text { rimp.moy. } \\ \text { P90-100 }\end{array} & \begin{array}{l}\text { lim.inf. } \\ \text { \%4822 }\end{array} & \text { \%rimp/rfisc } \\ \text { P90-95 } & 29750 & & 72,9 \\ \text { P95-100 } & 59890 & 34983 & \\ \text { P95-99 } & 45873 & & 74,0 \\ \text { P99-100 } & 115991 & 69535 & \\ \text { P99-99,5 } & 79437 & & 76,9 \\ \text { P99,5-100 } & 152505 & 93404 & \\ \text { P99,5-99,9 } & 121433 & & 79,0 \\ \text { P99,9-100 } & 277011 & 176116 & \\ \text { P99,9-99,99 } & 242264 & & 81,5 \\ \text { P99,99-100 } & 605553 & 421625 & 84,5\end{array}$

Source: Calculs de l'auteur à partir de l'enquête "Revenus fiscaux" de 1970 (INSEE) (cf. Annexe G) Note: Le taux de sondage moyen est de $1 / 422$ (48490 observations pour 20470258 foyers fiscaux). Le taux de sondage passe de $1 / 471$ sur $\mathrm{P} 0-90$ (39131 observations pour 18423232 foyers) à $1 / 314$ sur P90-95 (3255 observations pour 1023513 foyers), 1/202 sur P95-99 (4061 observations pour 818810 foyers), $1 / 110$ sur P99-99,5 (928 observations pour 102036 foyers), 1/94 sur P99,5-99,9 (873 observations pour 81881 foyers), 1/86 sur P99,9-99,99 (213 observations pour 18423 foyers), et 1/71 sur P99,99-100 (29 observations pour 2047 foyers).

Tableau G-2 : La composition du revenu fiscal déclaré en 1970

$\begin{array}{lccccccccc}\text { En \%: } & \text { BA } & \text { BIC } & \text { BNC } & \text { RGA } & \text { RF } & \text { RCM } & \text { SAL } & \text { PEN } & \\ \text { P0-100 } & 2,3 & 8,9 & 2,9 & 0,2 & 2,8 & 2,1 & 67,2 & 13,6 & 100,0 \\ \text { P90-100 } & 1,5 & 12,6 & 7,3 & 0,4 & 4,3 & 3,2 & 65,0 & 5,9 & 100,0 \\ \text { P95-100 } & 1,7 & 13,0 & 9,8 & 0,4 & 4,8 & 4,1 & 61,8 & 4,5 & 100,0 \\ \text { P99-100 } & 1,5 & 15,2 & 14,9 & 0,4 & 5,3 & 7,0 & 52,4 & 3,3 & 100,0 \\ \text { P99,5-100 } & 0,7 & 16,3 & 16,7 & 0,4 & 5,8 & 8,7 & 48,7 & 2,6 & 100,0 \\ \text { P99,9-100 } & 0,1 & 14,6 & 17,9 & 0,2 & 3,7 & 11,9 & 49,7 & 2,0 & 100,0 \\ \text { P99,99-100 } & 0,1 & 14,1 & 10,1 & 0,0 & 7,4 & 26,5 & 39,6 & 2,2 & 100,0 \\ & & & & & & & & & \\ \text { P0-90 } & \text { BA } & \text { BIC } & \text { BNC } & \text { RGA } & \text { RF } & \text { RCM } & \text { SAL } & \text { PEN } & \\ \text { P90-95 } & 2,7 & 7,0 & 0,7 & 0,1 & 2,0 & 1,5 & 68,4 & 17,7 & 100,0 \\ \text { P95-99 } & 1,1 & 11,8 & 2,3 & 0,3 & 3,3 & 1,6 & 71,1 & 8,5 & 100,0 \\ \text { P99-99,5 } & 1,7 & 11,7 & 6,9 & 0,4 & 4,4 & 2,4 & 67,3 & 5,2 & 100,0 \\ \text { P99,5-99,9 } & 3,1 & 13,1 & 11,5 & 0,4 & 4,5 & 3,8 & 59,2 & 4,5 & 100,0 \\ \text { P99,9-100 } & 1,0 & 17,3 & 16,0 & 0,6 & 7,0 & 7,0 & 48,2 & 2,9 & 100,0 \\ & 0,1 & 14,6 & 17,9 & 0,2 & 3,7 & 11,9 & 49,7 & 2,0 & 100,0\end{array}$

Source: Cf. Tableau G-1. 
Tableau G-3:

La distribution du revenu fiscal déclaré et du revenu imposable en 1975

Revenu fiscal déclaré

\begin{tabular}{|c|c|c|}
\hline N.foyers fisc. & 22451386 & \\
\hline rfisc.moyen & 32488 & \\
\hline rfisc.médian & 24588 & \\
\hline & rfisc.moy. & lim.inf. \\
\hline P90-100 & 107737 & 65218 \\
\hline P90-95 & 74194 & \\
\hline P95-100 & 141271 & 86683 \\
\hline P95-99 & 111199 & \\
\hline P99-100 & 261665 & 165164 \\
\hline P99-99,5 & 186073 & \\
\hline P99,5-100 & 337335 & 212845 \\
\hline P99,5-99,9 & 276323 & \\
\hline P99,9-100 & 581703 & 389048 \\
\hline P99,9-99,99 & 531795 & \\
\hline P99,99-100 & 1066958 & 987891 \\
\hline
\end{tabular}

Revenu imposable

\begin{tabular}{|c|c|c|c|}
\hline N.foyers fisc. & 22451386 & & \\
\hline rimp.moyen & 22737 & & 70,0 \\
\hline rimp.médian & $\begin{array}{r}17191 \\
\text { rimp mov }\end{array}$ & & \\
\hline P90-100 & $\begin{array}{r}\text { rimp.moy. } \\
79009\end{array}$ & $\begin{array}{l}\lim . \text { inf. } \\
46160\end{array}$ & \%rimp/rfisc \\
\hline P90-95 & 52478 & & 70,7 \\
\hline P95-100 & 105541 & 61351 & \\
\hline P95-99 & 80789 & & 72,7 \\
\hline P99-100 & 204531 & 122902 & \\
\hline P99-99,5 & 141455 & & 76,0 \\
\hline P99,5-100 & 267690 & 166915 & \\
\hline P99,5-99,9 & 217005 & & 78,5 \\
\hline P99,9-100 & 470320 & 310667 & \\
\hline P99,9-99,99 & 419541 & & 78,9 \\
\hline P99,99-100 & 927890 & 730885 & 87,0 \\
\hline
\end{tabular}

Source: Calculs de l'auteur à partir de l'enquête "Revenus fiscaux" de 1975 (INSEE) (cf. Annexe G) Note: Le taux de sondage moyen est de $1 / 505$ (44490 observations pour 22451386 foyers fiscaux). Le taux de sondage passe de $1 / 562$ sur P0-90 (35933 observations pour 20206247 foyers) à $1 / 387$ sur P90-95 (2903 observations pour 1122569 foyers), 1/242 sur P95-99 (3710 observations pour 898055 foyers), 1/130 sur P99-99,5 (863 observations pour 112257 foyers), 1/108 sur P99,5-99,9 (829 observations pour 89806 foyers), 1/88 sur P99,9-99,99 (230 observations pour 20206 foyers), et 1/102 sur P99,99-100 (22 observations pour 2245 foyers).

\section{Tableau G-4 : La composition du revenu fiscal déclaré en 1975}

$\begin{array}{lccccccccc}\text { En \%: } & \text { BA } & \text { BIC } & \text { BNC } & \text { RGA } & \text { RF } & \text { RCM } & \text { SAL } & \text { PEN } & \\ \text { P0-100 } & 1,9 & 7,6 & 2,5 & 0,1 & 2,7 & 1,9 & 68,5 & 14,7 & 100,0 \\ \text { P90-100 } & 0,8 & 11,5 & 6,3 & 0,3 & 4,8 & 2,7 & 66,9 & 6,7 & 100,0 \\ \text { P95-100 } & 0,7 & 13,3 & 8,8 & 0,3 & 5,8 & 3,3 & 62,3 & 5,4 & 100,0 \\ \text { P99-100 } & 0,4 & 17,4 & 13,0 & 0,4 & 8,5 & 5,2 & 51,9 & 3,1 & 100,0 \\ \text { P99,5-100 } & 0,2 & 18,3 & 14,3 & 0,4 & 9,4 & 6,6 & 48,1 & 2,6 & 100,0 \\ \text { P99,9-100 } & 0,2 & 19,5 & 15,2 & 0,2 & 7,9 & 8,1 & 48,2 & 0,7 & 100,0 \\ \text { P99,99-100 } & 0,0 & 24,6 & 12,2 & 0,0 & 9,5 & 18,0 & 35,0 & 0,8 & 100,0 \\ & & & & & & & & & \\ & & & & & & & & & \\ \text { P0-90 } & \text { BA } & \text { BIC } & \text { BNC } & \text { RGA } & \text { RF } & \text { RCM } & \text { SAL } & \text { PEN } & \\ \text { P90-95 } & 2,5 & 5,7 & 0,6 & 0,1 & 1,6 & 1,5 & 69,3 & 18,7 & 100,0 \\ \text { P95-99 } & 0,9 & 8,2 & 1,6 & 0,1 & 2,9 & 1,5 & 75,7 & 9,1 & 100,0 \\ \text { P99-99,5 } & 0,8 & 10,8 & 6,4 & 0,3 & 4,3 & 2,2 & 68,4 & 6,8 & 100,0 \\ \text { P99,5-99,9 } & 0,9 & 15,8 & 10,6 & 0,4 & 6,8 & 2,7 & 58,8 & 4,0 & 100,0 \\ \text { P99,9-100 } & 0,2 & 17,7 & 13,9 & 0,6 & 10,2 & 5,8 & 48,1 & 3,6 & 100,0 \\ & 0,2 & 19,5 & 15,2 & 0,2 & 7,9 & 8,1 & 48,2 & 0,7 & 100,0\end{array}$


Tableau G-5:

La distribution du revenu fiscal déclaré et du revenu imposable en 1979

Revenu fiscal déclaré

$\begin{array}{lrc}\begin{array}{l}\text { N.foyers fisc. } \\ \text { rfisc.moyen }\end{array} & \begin{array}{r}22431867 \\ \text { rfisc.médian }\end{array} & \begin{array}{r}42765 \\ \text { rfisc.moy. }\end{array} \\ & \text { lim.inf. } \\ \text { P90-100 } & 173096 & 106136 \\ \text { P90-95 } & 119143 & \\ \text { P95-100 } & 227060 & 136813 \\ \text { P95-99 } & 174967 & \\ \text { P99-100 } & 435651 & 261012 \\ \text { P99-99,5 } & 294407 & \\ \text { P99,5-100 } & 576608 & 338659 \\ \text { P99,5-99,9 } & 440207 & \\ \text { P99,9-100 } & 1122388 & 644919 \\ \text { P99,9-99,99 } & 868130 & \\ \text { P99,99-100 } & 3987380 & 1473528\end{array}$

Revenu imposable

\begin{tabular}{lrrr}
$\begin{array}{l}\text { N.foyers fisc. } \\
\text { rimp.moyen }\end{array}$ & $\begin{array}{r}22431867 \\
37909\end{array}$ & & 68,5 \\
rimp.médian & 29100 & & \\
& \multicolumn{1}{l}{ rimp.moy. } & lim.inf. & \%rimp/rfisc \\
P90-100 & 126323 & 73500 & \\
P90-95 & 82501 & & 69,2 \\
P95-100 & 170226 & 95500 & \\
P95-99 & 124790 & & 71,3 \\
P99-100 & 351700 & 189800 & \\
P99-99,5 & 218743 & & 74,3 \\
P99,5-100 & 485407 & 259700 & \\
P99,5-99,9 & 355468 & & 80,8 \\
P99,9-100 & 1003487 & 533000 & \\
P99,9-99,99 & 749268 & & 86,3 \\
P99,99-100 & 3293273 & 1240600 & 82,6
\end{tabular}

Source: Calculs de l'auteur à partir de l'enquête "Revenus fiscaux" de 1979 (INSEE) (cf. Annexe G) Note: Le taux de sondage moyen est de 1/571 (39301 observations pour 22431867 foyers fiscaux). Le taux de sondage passe de $1 / 632$ sur P0-90 (31965 observations pour 20188680 foyers) à 1/448 sur P90-95 (2505 observations pour 1121593 foyers), 1/287 sur P95-99 (3121 observations pour 897275 foyers), 1/149 sur P99-99,5 (754 observations pour 112159 foyers), 1/119 sur P99,5-99,9 (753 obser. pour 89727 foyers), 1/110 sur P99,9-99,99 (184 observations pour 20189 foyers), et 1/118 sur P99,99-100 (19 observations pour 2243 foyers).

Tableau G-6 : La composition du revenu fiscal déclaré en 1979

$\begin{array}{lccccccccc}\text { En \%: } & \text { BA } & \text { BIC } & \text { BNC } & \text { RGA } & \text { RF } & \text { RCM } & \text { SAL } & \text { PEN } & \\ \text { P0-100 } & 2,2 & 6,9 & 2,8 & 0,1 & 2,1 & 2,0 & 66,5 & 17,4 & 100,0 \\ \text { P90-100 } & 1,9 & 10,7 & 7,4 & 0,2 & 3,5 & 3,7 & 64,5 & 8,1 & 100,0 \\ \text { P95-100 } & 2,0 & 12,6 & 10,4 & 0,3 & 4,1 & 4,9 & 59,0 & 6,6 & 100,0 \\ \text { P99-100 } & 2,7 & 14,0 & 18,8 & 0,3 & 5,4 & 9,5 & 46,1 & 3,3 & 100,0 \\ \text { P99,5-100 } & 2,6 & 13,7 & 20,9 & 0,3 & 6,4 & 12,3 & 40,9 & 2,8 & 100,0 \\ \text { P99,9-100 } & 3,1 & 15,1 & 26,3 & 0,3 & 5,0 & 21,6 & 25,4 & 3,1 & 100,0 \\ \text { P99,99-100 } & \text { 0,0 } & 14,9 & 42,5 & 0,3 & 1,8 & 22,5 & 18,0 & 0,1 & 100,0 \\ & & & & & & & & & \\ & \text { BA } 1979 & \text { BIC } & \text { BNC } & \text { RGA } & \text { RF } & \text { RCM } & \text { SAL } & \text { PEN } & \\ \text { P0-90 } & 2,4 & 5,2 & 0,6 & 0,0 & 1,5 & 1,3 & 67,4 & 21,6 & 100,0 \\ \text { P90-95 } & 1,6 & 6,9 & 1,8 & 0,2 & 2,4 & 1,3 & 75,0 & 10,9 & 100,0 \\ \text { P95-99 } & 1,7 & 11,8 & 5,2 & 0,2 & 3,4 & 2,1 & 67,0 & 8,8 & 100,0 \\ \text { P99-99,5 } & 2,8 & 14,7 & 14,6 & 0,3 & 3,3 & 3,9 & 56,3 & 4,2 & 100,0 \\ \text { P99,5-99,9 } & 2,3 & 12,8 & 17,5 & 0,3 & 7,3 & 6,3 & 50,7 & 2,6 & 100,0 \\ \text { P99,9-100 } & 3,1 & 15,1 & 26,3 & 0,3 & 5,0 & 21,6 & 25,4 & 3,1 & 100,0\end{array}$

Source: Cf. Tableau G-5 
Tableau G-7:

La distribution du revenu fiscal déclaré et du revenu imposable en 1984

Revenu fiscal déclaré

$\begin{array}{lrc}\begin{array}{l}\text { N.foyers fisc. } \\ \text { rfisc.moyen } \\ \text { rfisc.médian }\end{array} & \begin{array}{r}24585220 \\ 93006\end{array} \\ & \begin{array}{r}69687 \\ \text { rfisc.moy. }\end{array} & \\ \text { lim.inf. } \\ \text { P90-100 } & 294686 & 183473 \\ \text { P90-95 } & 208517 & \\ \text { P95-100 } & 380849 & 240153 \\ \text { P95-99 } & 305192 & \\ \text { P99-100 } & 683675 & 442339 \\ \text { P99-99,5 } & 495511 & \\ \text { P99,5-100 } & 871680 & 570940 \\ \text { P99,5-99,9 } & 701706 & \\ \text { P99,9-100 } & 1552509 & 958970 \\ \text { P99,9-99,99 } & 1331784 & \\ \text { P99,99-100 } & 3677043 & 2444899\end{array}$

Revenu imposable

$\begin{array}{lrrr}\begin{array}{l}\text { N.foyers fisc. } \\ \text { rimp.moyen } \\ \text { rimp.médian }\end{array} & \begin{array}{r}24585220 \\ 66103\end{array} & & 71,1 \\ & 49283 & & \\ \text { rimp.moy. } & \text { lim.inf. } & \text { \%rimp/rfisc } \\ \text { P90-100 } & 216994 & 131476 & \\ \text { P90-95 } & 148735 & & 71,3 \\ \text { P95-100 } & 285248 & 171707 & \\ \text { P95-99 } & 225519 & & 73,9 \\ \text { P99-100 } & 536957 & 336885 & \\ \text { P99-99,5 } & 376136 & & 75,9 \\ \text { P99,5-100 } & 697196 & 428673 & \\ \text { P99,5-99,9 } & 553313 & & 78,9 \\ \text { P99,9-100 } & 1274487 & 763762 & \\ \text { P99,9-99,99 } & 1062992 & & 79,8 \\ \text { P99,99-100 } & 3200359 & 1967279 & 87,0\end{array}$

Source: Calculs de l'auteur à partir de l'enquête "Revenus fiscaux" de 1984 (INSEE) (cf. Annexe G) Note: Le taux de sondage moyen est de $1 / 532$ (46216 observations pour 24585220 foyers fiscaux). Le taux de sondage passe de $1 / 583$ sur P0-90 (37976 observations pour 22126698 foyers) à $1 / 417$ sur P90-95 (2950 observations pour 1229261 foyers), 1/268 sur P95-99 (3668 observations pour 983409 foyers), 1/184 sur P99-99,5 (675 observations pour 122926 foyers), 1/134 sur P99,5-99,9 (741 obser. pour 99433 foyers), 1/123 sur P99,9-99,99 (180 observations pour 22127 foyers), et 1/95 sur P99,99-100 (26 observations pour 2459 foyers).

\section{Tableau G-8 : La composition du revenu fiscal déclaré en 1984}

$\begin{array}{lccccccccc}\text { En \%: } & \text { BA } & \text { BIC } & \text { BNC } & \text { RGA } & \text { RF } & \text { RCM } & \text { SAL } & \text { PEN } & \\ \text { P0-100 } & 1,9 & 5,1 & 3,3 & 0,1 & 2,6 & 2,4 & 64,7 & 19,9 & 100,0 \\ \text { P90-100 } & 1,9 & 8,0 & 8,8 & 0,2 & 4,7 & 3,3 & 64,0 & 9,1 & 100,0 \\ \text { P95-100 } & 2,3 & 9,4 & 12,4 & 0,2 & 5,5 & 4,1 & 58,0 & 8,1 & 100,0 \\ \text { P99-100 } & 1,6 & 11,1 & 21,7 & 0,3 & 7,9 & 7,1 & 44,0 & 6,3 & 100,0 \\ \text { P99,5-100 } & 1,6 & 12,0 & 21,7 & 0,4 & 9,6 & 8,7 & 39,9 & 6,2 & 100,0 \\ \text { P99,9-100 } & 2,3 & 11,9 & 15,8 & 0,5 & 11,7 & 15,9 & 36,6 & 5,3 & 100,0 \\ \text { P99,99-100 } & \text { 0,4 } & 11,9 & 12,6 & 0,3 & 7,3 & 27,8 & 39,1 & 0,6 & 100,0 \\ & & & & & & & & & \\ \text { P0-90 } & \text { BA } & \text { BIC } & \text { BNC } & \text { RGA } & \text { RF } & \text { RCM } & \text { SAL } & \text { PEN } & \\ \text { P90-95 } & 2,0 & 3,8 & 0,7 & 0,1 & 1,7 & 1,9 & 65,0 & 24,9 & 100,0 \\ \text { P95-99 } & 1,1 & 5,5 & 2,2 & 0,1 & 3,4 & 1,8 & 74,9 & 11,0 & 100,0 \\ \text { P99-99,5 } & 2,7 & 8,4 & 7,2 & 0,1 & 4,1 & 2,5 & 65,8 & 9,1 & 100,0 \\ \text { P99,5-99,9 } & 1,4 & 10,8 & 23,4 & 0,3 & 6,8 & 4,5 & 46,2 & 6,6 & 100,0 \\ \text { P99,9-100 } & 1,2 & 12,0 & 25,0 & 0,3 & 8,4 & 4,7 & 41,7 & 6,7 & 100,0 \\ & 2,3 & 11,9 & 15,8 & 0,5 & 11,7 & 15,9 & 36,6 & 5,3 & 100,0\end{array}$

Source: Cf. Tableau G-7 
Tableau G-9:

La distribution du revenu fiscal déclaré et du revenu imposable en 1990

Revenu fiscal déclaré

$\begin{array}{lrc}\begin{array}{l}\text { N.foyers fisc. } \\ \text { rfisc.moyen }\end{array} & \begin{array}{r}27272048 \\ 117271\end{array} \\ \text { rfisc.médian } & 88532 & \\ & \begin{array}{r}8 \\ \text { rfisc.moy. }\end{array} & \text { lim.inf. } \\ \text { P90-100 } & 368526 & 226958 \\ \text { P90-95 } & 256686 & \\ \text { P95-100 } & 480279 & 299068 \\ \text { P95-99 } & 380432 & \\ \text { P99-100 } & 879726 & 553491 \\ \text { P99-99,5 } & 629255 & \\ \text { P99,5-100 } & 1130301 & 734089 \\ \text { P99,5-99,9 } & 919041 & \\ \text { P99,9-100 } & 1976877 & 1332021 \\ \text { P99,9-99,99 } & 1803387 & \\ \text { P99,99-100 } & 4086090 & 3172339\end{array}$

Revenu imposable

$\begin{array}{lrrr}\begin{array}{l}\text { N.foyers fisc. } \\ \text { rimp.moyen } \\ \text { rimp.médian }\end{array} & \begin{array}{r}27272048 \\ 58384\end{array} & & 66,8 \\ & \begin{array}{r}58415 \\ \text { rimp.moy. }\end{array} & \text { lim.inf. } & \% \text { rimp/rfisc } \\ \text { P90-100 } & 270035 & 158075 & \\ \text { P90-95 } & 180098 & & 70,2 \\ \text { P95-100 } & 359975 & 210023 & \\ \text { P95-99 } & 272233 & & 71,6 \\ \text { P99-100 } & 711270 & 406217 & \\ \text { P99-99,5 } & 467901 & & 74,4 \\ \text { P99,5-100 } & 956512 & 554764 & \\ \text { P99,5-99,9 } & 738972 & & 80,4 \\ \text { P99,9-100 } & 1822407 & 1143052 & \\ \text { P99,9-99,99 } & 1594901 & & 88,4 \\ \text { P99,99-100 } & 3926087 & 3176104 & 96,1\end{array}$

Source: Calculs de l'auteur à partir de l'enquête "Revenus fiscaux" de 1990 (INSEE) (cf. Annexe G) Note: Le taux de sondage moyen est de 1/809 (33709 observations pour 27272048 foyers fiscaux). Le taux de sondage passe de $1 / 926$ sur P0-90 (26514 observations pour 24544843 foyers) à $1 / 581$ sur P90-95 (2347 observations pour 1363602 foyers), 1/346 sur P95-99 (3149 observations pour 1090882 foyers), $1 / 190$ sur P99-99,5 (717 observations pour 136360 foyers), 1/143 sur P99,5-99,9 (762 observations pour 109088 foyers), 1/124 sur P99,9-99,99 (198 observations pour 24545 foyers), et $1 / 124$ sur $\mathrm{P} 99,99-100$ (22 observations pour 2727 foyers).

Tableau G-10 : La composition du revenu fiscal déclaré en 1990

$\begin{array}{lccccccccc}\text { En \%: } & \text { BA } & \text { BIC } & \text { BNC } & \text { RGA } & \text { RF } & \text { RCM } & \text { SAL } & \text { PEN } & \\ \text { P0-100 } & 1,9 & 5,0 & 3,4 & 0,1 & 2,1 & 2,3 & 64,8 & 20,4 & 100,0 \\ \text { P90-100 } & 2,5 & 9,0 & 9,4 & 0,3 & 3,5 & 2,9 & 62,3 & 10,0 & 100,0 \\ \text { P95-100 } & 3,0 & 10,8 & 13,4 & 0,3 & 4,2 & 3,5 & 56,8 & 8,0 & 100,0 \\ \text { P99-100 } & 3,5 & 14,4 & 22,3 & 0,4 & 5,8 & 5,9 & 43,5 & 4,2 & 100,0 \\ \text { P99,5-100 } & 3,6 & 15,4 & 22,8 & 0,2 & 6,4 & 7,6 & 41,0 & 3,0 & 100,0 \\ \text { P99,9-100 } & 2,1 & 24,4 & 21,5 & 0,4 & 6,5 & 11,0 & 32,5 & 1,6 & 100,0 \\ \text { P99,99-100 } & 0,0 & 26,7 & 24,8 & -0,2 & 3,9 & 16,1 & 28,4 & 0,2 & 100,0 \\ & & & & & & & & & \\ & \text { BA } 1990 & \text { BIC } & \text { BNC } & \text { RGA } & \text { RF } & \text { RCM } & \text { SAL } & \text { PEN } & \\ \text { P0-90 } & 1,6 & 3,1 & 0,6 & 0,1 & 1,5 & 2,0 & 65,9 & 25,1 & 100,0 \\ \text { P90-95 } & 1,5 & 5,6 & 2,1 & 0,2 & 2,3 & 1,8 & 72,7 & 13,9 & 100,0 \\ \text { P95-99 } & 2,7 & 8,8 & 8,2 & 0,3 & 3,3 & 2,1 & 64,5 & 10,1 & 100,0 \\ \text { P99-99,5 } & 3,4 & 12,6 & 21,4 & 0,6 & 4,7 & 2,8 & 48,0 & 6,5 & 100,0 \\ \text { P99,5-99,9 } & 4,4 & 10,6 & 23,5 & 0,2 & 6,3 & 5,8 & 45,6 & 3,7 & 100,0 \\ \text { P99,9-100 } & 2,1 & 24,4 & 21,5 & 0,4 & 6,5 & 11,0 & 32,5 & 1,6 & 100,0\end{array}$

Source: cf. Tableau G-9 
Enfin, les tableaux G-1 et G-10 indiquent les taux de sondage des échantillons légers. ${ }^{143}$ Ces taux de sondage permettent de comprendre pourquoi les enquêtes "Revenus fiscaux" ne permettent pas d'étudier de façon fine les hauts revenus, et en particulier les revenus du centile supérieur, contrairement aux échantillons légers: alors que le taux de sondage atteint 1/1 à l'intérieur de P99,9-100 dans les échantillons légers, les hauts revenus ne sont que légèrement sur-représentés dans les enquêtes "Revenus fiscaux". De plus, et surtout, les enquêtes "Revenus fiscaux" souffrent de multiples problèmes d'échantillonnage, impliquant que les chiffres bruts des tableaux C-5 et G-1 à G-10 doivent être utilisées avec beaucoup de précaution: la proportion de foyers fiscaux ayant 1 part de quotient familial est systématiquement sous-évaluée (cf. annexe C.3), et la part du revenu imposable détenue par les hauts revenus est fortement surévalué ou sous-évalué suivant les années (cf. annexe D.2). La comparaison entre le tableau F-6 et le tableau G-10 indique également que les estimations de la composition du revenu imposable des hauts revenus issues des enquêtes "Revenus fiscaux" ne sauraient être utilisées à l'état brut.

L'INSEE a consacré des publications importantes à toutes les enquêtes "Revenus fiscaux". Mais l'objet de ces publications est l'étude de l'inégalité du revenu disponible des ménages, si bien que ces publications ne contiennent aucun tableau statistique concernant la distribution du revenu imposable des foyers que nous aurions pu utiliser. Les seules chiffres issus des enquêtes "Revenus fiscaux" et publiés par l'INSEE que nous puissions comparer aux estimations des tableaux G-1 à G-10 concernent le revenu fiscal total, qui coïncident toujours très bien avec les chiffres que nous avons estimés à partir des fichiers informatiques. ${ }^{144}$ Quelques tableaux statistiques issus des enquêtes "Revenus fiscaux" de 1970 et 1975 ont également été publiés dans le rapport sur le Conseil des Impôts a consacré à l'impôt sur le revenu en $1979,{ }^{145}$ et les estimations du nombre total de foyers et de revenu imposable total issus de ces tableaux coïncident parfaitement avec les estimations des tableaux F-1 et F-3. Les références des principales publications consacrées par I'INSEE aux enquêtes “ Revenus Fiscaux" de 1970, 1975, 1979, 1984 et 1990 sont les suivantes:

G. Banderier et P. Ghigliazza, "Les revenus des ménages en 1970 ”, Les collections de l'INSEE n¹47 (série M, n40), décembre 1974.

\footnotetext{
${ }^{143}$ De même que pour les échantillons légers, ces taux de sondage ont été calculés à partir de la centilisation en zfisf.

${ }^{144}$ La seule incohérence au niveau aggrégé concerne le nombre total de foyers fiscaux estimé par l'enquête de 1984 : I'INSEE annonce 24092880 foyers (cf. INSEE M139, p.20), alors que le tableau G-7 en annonce 24585220. Mais cette incohérence vient simplement du fait que l'INSEE a regroupé dans un même foyer les couples remplissant 3 déclarations durant l'année de leur mariage (cf. INSEE M139, pp.19-20), alors que nous avons dans toute cette étude suivi la convention habituelle des Etats 1921 (et des échantillons légers), consistant à traiter toutes les déclarations déposées comme des foyers fiscaux distincts. Ce "regroupement des déclarations multiples " n'avait pas été effectué dans le fichier SAS de l'enquête "RF" de 1984 possédé initialement au BEF, et nous avons donc utilisé ce fichier pour l'année 1984 (et non le fichier "RF" 1984 qui nous a été transmis par l'INSEE avec les fichiers des enquêtes de 1970, 1975 et 1990, qui effectue ce regroupement et qui conduit bien à un total de seulement 24092880 foyers). Ce problème ne semble pas se poser pour les autres années (le fichier 1979 possédé initialement au BEF coïncide parfaitement avec le fichier transmis par l'INSEE). ${ }^{145}$ Cf. S\&EF, série bleue, $n^{\circ} 361-362$ (1979), p.29 (tableau 9), p.42 (tableau 23) et p.43 (tableau 24).
} 
G. Canceill, A. Laferrere et P. Mercier, "Les revenus fiscaux des ménages en 1979 (et principaux résultats de 1975) ", Les collections de l'INSEE n570 (série M, n¹27), décembre 1987.

G. Canceill, "Les revenus fiscaux des ménages en 1984", Les collections de l'INSEE n605 (série $M, n^{\circ} 139$ ), mai 1989.

N. Chapagne, "Les revenus fiscaux des ménages en 1990 ", INSEE-Résultats n453 (série "Emploi-Revenus" $n^{\circ} 103$ ), avril 1996. (cf. également Synthèses $n^{\circ} 1$, "Revenus et patrimoine des ménages, édition 95 ", juin 1995). 


\section{Annexe H : Les tableaux statistiques des dossiers “ Modèle lourd ”, 1979-1993}

Jusqu'en 1993, le Bureau des Statistiques de la DGI (bureau IIC, anciennement bureau IIA4) avait l'habitude d'utiliser chaque année les échantillons lourds de déclarations de revenus pour produire un grand nombre de tableaux statistiques et les rassembler dans d'épais dossiers à usage principalement internes au Ministère. Les échantillons lourds utilisés étaient toujours les échantillons constitués au $31 / 03 / n+2$, dits " semi-définitifs " (les échantillons définitifs, i.e. au 31/12/n+2, ne sont jamais utilisés, car ils sont disponibles trop tard pour être encore d'actualité). Le dernier dossier "modèle lourd" porte sur les revenus de 1993 (situation au 31/03/1995). Depuis cette date, cette forme de diffusion a été abandonnée, vraisemblablement du fait de son coût. En rassemblant les dossiers " modèle lourd " conservés au BEF pour les années récentes et les dossiers plus anciens conservés par le SESDO, le SLF (bureau C 1) et la DGI (bureau IIC), nous avons pu constituer une série annuelle complète pour les années 1979-1993. II est probable que de tels dossiers n'existaient pas avant les revenus de l'année 1979, année qui doit correspondre à quelques années près avec les premiers échantillons de déclarations constitués par la DGI (cf. Annexe A.1). Par contre, des tableaux comparables aux tableaux des dossiers "modèles lourds" ont évidemment continué d'être produits pour les besoins internes de la DGI ou du SLF pour les revenus des années 19941996, même s'ils ne sont plus diffusés de façon systématique sous la forme de dossiers. De façon générale, il est probable que de multiples autres tableaux statistiques ont été produits dans le passé à partir des échantillons lourds. ${ }^{146}$ En l'absence de toute procédure d'archivage systématique, il semble cependant illusoire de chercher à reconstituer une série continue

Comparé aux tableaux statistiques des Etats 1921, qui existent sur une période beaucoup plus longue et qui portent sur l'ensemble des foyers fiscaux, le seul avantage des tableaux statistiques des dossiers “modèles lourds" est qu'ils contiennent pour certains d'entre eux des informations plus détaillées. Les dossiers "Modèle lourd" relatifs aux revenus des années 1983 à 1993 incluent notamment les "tableaux 10 ", qui indiquent le nombre de contribuables et le montant du revenu imposable par tranche de revenu imposable (tous nombres de parts de quotient familial confondus). De même que tous les autres tableaux des dossiers "Modèle lourd ", ce tableau est dressé séparément pour les foyers imposables et les foyers non-imposables. Les tranches de revenu imposable utilisées dans ces tableaux sont les mêmes (en francs courants) pour toute la période 1983-1993. Comparées à celles utilisées dans les Etats 1921, leur avantage est qu'elles incluent une tranche supérieure concernant les contribuables dont le revenu imposable est supérieur à 1 million de francs, ce qui permet d'effectuer des estimations plus fines de la part des très hauts revenus. Dans le cadre de cette étude, nous avons utilisé les tableaux 10 des dossiers "Modèle lourd" uniquement pour tester la fiabilité des estimations effectuées à partir des Etats 1921 (cf. tableaux E-5 à E-7, annexe E.3).

\footnotetext{
${ }^{146}$ Cf. par exemple le tableau publié dans Droit Fiscal (1986, n³2-38, pp.1493-1494), cité par David (1987, p.120), indiquant la répartition des contribuables par tranche d'imposition pour les barêmes de 1981, 1982, 1983 et 1984, et issu d' "informations statistiques données par l'Administration en réponse à des questions posées par des parlementaires ". Les tableaux issus des échantillons lourds sont d'ailleurs parfois diffusés dans la presse (cf. par exemple "Le Monde " (supplément "Fiscalité ") du 11/3/1998, qui publie un tableau indiquant pour les revenus de 1995 le nombre de contribuables et le revenu imposable total en fonction des mêmes tranches de revenu imposable qui étaient utilisées dans les tableaux 10 des dossiers "Modèle lourd " (tranche supérieure pour les revenus imposables supérieurs à 1 million de francs, etc...).
} 
Les dossiers "Modèle lourd " relatifs aux revenus des années 1983 à 1993 incluent également les "tableaux 20 ", qui indiquent le nombre et le montant des différents revenus catégoriels nets par tranche de revenu imposable. Les tranches utilisées dans les tableaux 20 sont les mêmes que celles utilisées dans les tableaux 10. Les tableaux 20 n'ont pas été utilisées dans le cadre de cette étude, mais ils pourraient être mobilisés pour affiner les estimations de la composition du revenu imposable des hauts revenus sur la période 1983-1993.

Enfin, les dossiers "Modèle lourd" relatifs aux revenus des années 1985 à 1993 incluent les "tableaux 30 ", qui indiquent le nombre de contribuables, le montant du revenu imposable par part de quotient familial et le montant des droits simples, par tranche de revenu imposable par part et par nombre de parts de quotient familial. ${ }^{147}$ Les tranches de revenu imposable par part utilisées dans ces tableaux sont les tranches du barème d'imposition de l'année considérée : par exemple, pour 1993, on dispose du nombre et du revenu des contribuables ayant 1 part de QF et dont le revenu imposable est supérieur à 273900 francs (tranche à 56,8\%), de ceux dont le revenu imposable est compris entre 222100 et 273900 francs (tranche à $65 \%$ ). Les tableaux 30 n'ont pas été utilisés dans le cadre de cette étude, mais ils pourraient être mobilisés pour affiner les estimations de la distribution du revenu imposable en fonction du nombre de parts de quotient familial sur la période 1985-1993. ${ }^{148}$

Les dossiers "Modèle lourd" relatifs aux revenus des années 1979 à 1982 ne contiennent aucun de ces tableaux, ce qui s'explique sans doute par le fait que ces dossiers étaient surtout utilisés pour obtenir certains types d'informations que les Etats 1921 ne couvraient pas (par exemple, les dossiers 1979-1982 incluent des tableaux sur les foyers inscrits dans des centres de gestion agréés, sur les foyers déclarant des déficits, etc..). ${ }^{149}$

\footnotetext{
${ }^{147}$ Des revenus de 1985 à ceux de 1990, les tableaux 30 contiennent des sous-tableaux séparés pour tous les nombres de parts de 1 à 6,5 (puis les foyers ayant 7 parts ou plus sont regroupés dans un même sous-tableau). Des revenus de 1991 aux revenus de 1993, des sous-tableaux séparés n'existent que pour les nombres de parts compris entre 1 et 4 (les foyers ayant 4,5 parts ou plus sont regroupés dans un même sous-tableau).

${ }^{148}$ Les tableaux 30 sont également complétés par les tableaux 31, qui décomposent les tableaux 30 en fonction de la nature du revenu dominant des contribuables (le "revenu dominant" doit être le revenu catégoriel le plus important et représenter au moins $1 / 3$ du revenu total), qui sont cependant difficiles à utiliser de façon fiable.

${ }^{149}$ Les seuls tableaux concernant la distribution du revenu imposable dans les dossiers 1979-1982 sont les tableaux 11, qui indiquent le nombre de contribuables par tranche de revenu imposable, mais qui omettent d'indiquer le montant du revenu imposable, si bien que ces tableaux ne permettent pas d'effectuer des approximations par une loi de Pareto (à moins d'utiliser la technique d'estimation de Feenberg-Poterba, qui est cependant moins fiable ; cf. annexe D.1). De plus, si les tableaux 11 des dossiers 81-82 utilisent les mêmes tranches de revenu imposable que les tableaux 10, les tableaux 11 des années 1979-1980 s'arrêtent à une tranche supérieure concernant les contribuables dont le revenu imposable est supérieur à 400000 francs (comparés aux Etats 1921, ils ne contiennent donc aucune information supplémentaire).
} 


\section{Annexe I : Les seuils de plafonnement du quotient familial, 1981-1997}

Le tableau I-1 indique le montant des réductions d'impôt maximales autorisées par le système de plafonnement des effets du quotient familial applicables aux revenus des années 1981 à 1997, ainsi que les seuils de revenu imposable correspondant pour les foyers fiscaux ayant 1,5, 2B, 2,5 ou 3 parts de quotient familial.

Insérer Tableau I-1 : Les seuils de plafonnement du quotient familial, 1981-1997

Les seuils de revenu imposable indiqués sur le tableau l-1 peuvent aisément être calculés à partir des barèmes " bruts" décrits dans le tableau 2-1 (section 2.1). Par exemple, le seuil de revenu imposable de 119440 francs indiqué pour les foyers ayant 1,5 parts de quotient familial en 1981 correspond au revenu imposable tel que la différence entre l'impôt dû avec 1,5 parts et l'impôt dû avec 1 part (en appliquant le barème de 1981) est exactement égale à 7500 francs (à un dizaine de francs près). Ces seuils de plafonnement sont également fournis chaque année dans les "Brochure Pratique" publiées par la DGI et dans les "Guide Pratique du Contribuable " publiés par le SNUI. ${ }^{150}$ Ces seuils de revenu imposable permettent de calculer les barèmes applicables aux foyers ayant 1,5, 2B, 2,5 ou 3 parts de quotient familial après prise en compte du plafonnement des effets du quotient familial.

Insérer Tableau I-2: Les barèmes de l'impôt sur le revenu de 1970 à 1997 applicables aux foyers ayant 1,5 parts de quotient familial (après plafonnement des effets du quotient familial)

Insérer Tableau I-3: Les barèmes de l'impôt sur le revenu de 1970 à 1997 applicables aux foyers ayant 2B parts de quotient familial (après plafonnement des effets du quotient familial)

Insérer Tableau I-4: Les barèmes de l'impôt sur le revenu de 1970 à 1997 applicables aux foyers ayant $2 \mathrm{~A}$ parts de quotient familial

Insérer Tableau I-5: Les barèmes de l'impôt sur le revenu de 1970 à 1997 applicables aux foyers ayant 2,5 parts de quotient familial (après plafonnement des effets du quotient familial)

Insérer Tableau I-6: Les barèmes de l'impôt sur le revenu de 1970 à 1997 applicables aux foyers ayant 3 parts de quotient familial (après plafonnement des effets du quotient familial)

Par exemple, en 1981, le seuil de 119440 francs applicables aux foyers ayant 1,5 parts signifie qu'en l'absence de plafonnement, le revenu imposable par part aurait été de $119440 / 1,5=79627$ francs, ce qui correspond à la tranche d'imposition à $40 \%$ (applicable aux revenus imposables par part compris entre 49680 et 82790 francs ; cf. tableau 2-1, section 2.1); du fait du plafonnement le revenu imposable par part est de 119400, ce qui correspond à la tranche d'imposition à 50\% (cf. tableau 2-1) : la mise en place du système de plafonnement des effets du quotient familial fait

\footnotetext{
${ }^{150}$ Les seuils applicables aux foyers ayant 2,5 ou 3 parts indiqués sur le tableau I- 1 ont été calculés en supposant que ces foyers étaient des foyers de couples mariés (les seuils applicables aux foyers de célibataires ayant 2,5 ou 3 parts sont sensiblement inférieurs). Le seuil applicable aux foyers ayant 1,5 parts en 1997 prend en compte la nouvelle réduction d'impôt maximale (6100 francs) applicable à la demi-part accordée aux personnes seules ayant élevé au moins un enfant (cf. section 2.1.3).
} 
Tableau I-1: Les seuils de plafonnement du quotient familial de 1981 à 1997

\begin{tabular}{ccccccc} 
& réduction d'impôt maximale & \multicolumn{3}{c}{ seuil de revenu imposable correspondant } \\
1981 & $1 / 2$ part & 1 part (2B) & QF=1,5 & QF=2B & QF=2,5 & QF=3 \\
1982 & 7500 & & 119440 & 138590 & 228550 & 238870 \\
1983 & 9250 & & 134390 & 155990 & 257190 & 268780 \\
1984 & 9960 & & 146930 & 170600 & 281220 & 293860 \\
1985 & 10520 & & 158170 & 183650 & 302720 & 316320 \\
1986 & 10770 & 13770 & 167040 & 193960 & 319710 & 334070 \\
1987 & 11130 & 14230 & 171020 & 116210 & 327310 & 342030 \\
1988 & 11420 & 14600 & 180050 & 124670 & 345400 & 360110 \\
1989 & 11800 & 15090 & 184740 & 127900 & 354370 & 369460 \\
1990 & 12180 & 15580 & 190850 & 132170 & 366110 & 381700 \\
1991 & 12550 & 16050 & 196980 & 136430 & 377860 & 393950 \\
1992 & 12910 & 16500 & 202930 & 140540 & 389280 & 405850 \\
1993 & 15400 & 19060 & 208690 & 144480 & 400310 & 417370 \\
1994 & 15620 & 19330 & 204090 & 161430 & 340590 & 408160 \\
1995 & 15900 & 19680 & 206980 & 163700 & 345390 & 413960 \\
1996 & 16200 & 20050 & 210700 & 166660 & 351600 & 421390 \\
1997 & 16380 & 20270 & 239340 & 173150 & 472960 & 478680 \\
& & & 104140 & 175060 & 478210 & 484020
\end{tabular}

Lecture: Au titre de l'imposition des revenus de 1981 (déclarés en 1982), la réduction d'impôt maximale que peut procurer chaque 1/2 part de quotient familial supérieure à 1 (pour les célibataires) ou à 2 (pour les couples mariés), quel que soit son motif d'attribution, est plafonnée à 7500 francs. Ce plafond est atteint par les contribuables ayant 1,5 parts de quotient familial et un revenu imposable supérieur ou égal à 119440 francs, par les contribuables ayant 2B parts et un revenu imposable supérieur ou égal à 138590 francs, par les contribuables (couples mariés) ayant 2,5 parts de quotient familial et un revenu imposable supérieur ou égal à 228550 francs, et par les contribuables (couples mariés) ayant 3 parts de quotient familial et un revenu imposable supérieur ou égal à 238870 francs. A compter des revenus de 1986, le plafond pour la part entière accordée au premier enfant à charge des contribuables célibataires $(\mathrm{QF}=2 \mathrm{~B})$ est inférieur au plafond général. 
Tableau I-2: Les barêmes de l'impôt sur le revenu de 1970 à 1997 applicables aux foyers fiscaux ayant 1,5 parts de quotient familial (après plafonnement des effets du quotient familial)

$\begin{array}{rrrrrrrrrrrrrr}1970 & & 1971 & & 1972 & & 1973 & & 1974 & & 1975 & 1976 & 5 \\ 4350 & 10 & 4650 & 10 & 4950 & 10 & 7425 & 5 & 8250 & 5 & 9188 & 5 & 10088 & 5 \\ 7650 & 15 & 8100 & 15 & 8625 & 15 & 7800 & 10 & 8738 & 10 & 9638 & 10 & 10575 & 10 \\ 12750 & 20 & 13425 & 20 & 14250 & 20 & 9375 & 15 & 10500 & 15 & 11550 & 15 & 12675 & 15 \\ 18900 & 30 & 19875 & 30 & 21075 & 30 & 14850 & 20 & 16650 & 20 & 18338 & 20 & 20100 & 20 \\ 30075 & 40 & 31575 & 40 & 33000 & 40 & 22350 & 30 & 22575 & 25 & 24863 & 25 & 26363 & 25 \\ 60150 & 50 & 63150 & 50 & 65250 & 50 & 33000 & 40 & 28500 & 30 & 31350 & 30 & 33225 & 30 \\ 120300 & 60 & 126300 & 60 & 129750 & 60 & 69488 & 50 & 36675 & 35 & 37875 & 35 & 40163 & 35 \\ & & & & & & 138188 & 60 & 39713 & 40 & 43688 & 40 & 46313 & 40 \\ & & & & & & & & 68738 & 45 & 75600 & 45 & 80138 & 45 \\ & & & & & & & & 97350 & 50 & 107063 & 50 & 110288 & 50 \\ & & & & & & & & 126000 & 55 & 138600 & 55 & 142763 & 55 \\ 1977 & & 1978 & & 1979 & & 1980 & & 1981 & & 1982 & & 1983 & \\ 10875 & 5 & 11888 & 5 & 13088 & 5 & 14835 & 5 & 16845 & 5 & 18930 & 5 & 20655 & 5 \\ 11400 & 10 & 12450 & 10 & 13688 & 10 & 15510 & 10 & 17610 & 10 & 19785 & 10 & 21585 & 10 \\ 13650 & 15 & 14888 & 15 & 16238 & 15 & 18405 & 15 & 20895 & 15 & 23460 & 15 & 25605 & 15 \\ 21600 & 20 & 23550 & 20 & 25688 & 20 & 29115 & 20 & 33045 & 20 & 37110 & 20 & 40485 & 20 \\ 28350 & 25 & 30938 & 25 & 33413 & 25 & 37425 & 25 & 42480 & 25 & 47715 & 25 & 52050 & 25 \\ 35700 & 30 & 38888 & 30 & 42000 & 30 & 47040 & 30 & 53385 & 30 & 59955 & 30 & 65415 & 30 \\ 43163 & 35 & 47025 & 35 & 50813 & 35 & 56955 & 35 & 64590 & 35 & 72555 & 35 & 79140 & 35 \\ 49800 & 40 & 54263 & 40 & 58613 & 40 & 65655 & 40 & 74520 & 40 & 83685 & 40 & 91305 & 40 \\ 86138 & 45 & 93900 & 45 & 97688 & 45 & 109410 & 45 & 119440 & 50 & 134390 & 50 & 146930 & 50 \\ 118538 & 50 & 129188 & 50 & 134363 & 50 & 150480 & 50 & 134650 & 55 & 151250 & 55 & 165010 & 55 \\ 151350 & 55 & 158925 & 55 & 158925 & 55 & 177990 & 55 & 153200 & 60 & 172040 & 60 & 187700 & 60 \\ 178650 & 60 & 187575 & 60 & 187575 & 60 & 202500 & 60 & & & 195000 & 65 & 212750 & 65\end{array}$

$\begin{array}{rrrrrrrrrrrrrr}1984 & & 1985 & & 1986 & & 1987 & & 1988 & & 1989 & & 1990 & \\ 22230 & 5 & 23475 & 5 & 24045 & 5 & 24840 & 5 & 25500 & 5 & 26355 & 5 & 27210 & 5 \\ 23235 & 10 & 24540 & 10 & 25140 & 10 & 25980 & 9,6 & 26670 & 9,6 & 27555 & 9,6 & 28440 & 9,6 \\ 27555 & 15 & 29100 & 15 & 29805 & 15 & 30795 & 14,4 & 31605 & 14,4 & 32655 & 14,4 & 33705 & 14,4 \\ 43575 & 20 & 46020 & 20 & 47130 & 20 & 48690 & 19,2 & 49965 & 19,2 & 51615 & 19,2 & 53280 & 19,2 \\ 56010 & 25 & 59160 & 25 & 60585 & 25 & 62595 & 24 & 64230 & 24 & 66360 & 24 & 68490 & 24 \\ 70380 & 30 & 74325 & 30 & 76110 & 30 & 78615 & 28,8 & 80655 & 28,8 & 83310 & 28,8 & 85980 & 28,8 \\ 85155 & 35 & 89925 & 35 & 92085 & 35 & 95130 & 33,6 & 97605 & 33,6 & 100830 & 33,6 & 104055 & 33,6 \\ 98250 & 40 & 103755 & 40 & 106245 & 40 & 109755 & 38,4 & 112605 & 38,4 & 116325 & 38,4 & 120045 & 38,4 \\ 158170 & 50 & 167040 & 50 & 171020 & 50 & 180050 & 49 & 184740 & 49 & 190850 & 49 & 196980 & 49 \\ 177550 & 55 & 187490 & 55 & 191990 & 55 & 198330 & 53,9 & 203490 & 53,9 & 210210 & 53,9 & 216940 & 53,9 \\ 201970 & 60 & 213280 & 60 & 218400 & 58 & 225610 & 56,8 & 231480 & 56,8 & 239120 & 56,8 & 246770 & 56,8 \\ 228920 & 65 & 241740 & 65 & & & & & & & & & & \\ & & & & & & & & & & & & 1997 & \\ 1991 & & 1992 & & 1993 & & 1994 & & 1995 & & 1996 & & 1996 \\ 28035 & 5 & 28830 & 5 & 32850 & 12 & 33315 & 12 & 33915 & 12 & 38415 & 10,5 & 38835 & 10,5 \\ 29295 & 9,6 & 30120 & 9,6 & 71850 & 25 & 72855 & 25 & 74160 & 25 & 75570 & 24 & 76395 & 24 \\ 34725 & 14,4 & 35700 & 14,4 & 126450 & 35 & 128220 & 35 & 130530 & 35 & 133005 & 33 & 104140 & 33 \\ 54885 & 19,2 & 56430 & 19,2 & 204090 & 50 & 206980 & 50 & 210700 & 50 & 215370 & 43 & 145160 & 43 \\ 70545 & 24 & 72525 & 24 & 273900 & 56,8 & 277730 & 56,8 & 282730 & 56,8 & 239340 & 48 & 236190 & 48 \\ 88560 & 28,8 & 91035 & 28,8 & & & & & & & 288100 & 54 & 291270 & 54 \\ 107175 & 33,6 & 110175 & 33,6 & & & & & & & & & & \end{array}$

Lecture : Au titre de l'imposition des revenus de 1970 (déclarés en 1971), la fraction du revenu imposable des foyers ayant 1,5 parts de quotient familial comprise entre 0 franc et 4350 francs est soumise à un taux marginal de $0 \%$, la fraction comprise entre 4350 francs et 7650 francs est soumise à un taux marginal de $10 \%$, etc..., et la fraction supérieure à 120300 francs est soumise à un taux marginal de $60 \%$. 
Tableau 1-3: Les barêmes de l'impôt sur le revenu de 1970 à 1997 applicables aux foyers fiscaux ayant $2 \mathrm{~B}$ parts de quotient familial (après plafonnement des effets du quotient familial)

\begin{tabular}{|c|c|c|c|c|c|c|c|c|c|c|c|c|c|}
\hline 1970 & & 1971 & & 1972 & & 1973 & & 1974 & & 1975 & & 1976 & \\
\hline 5800 & 10 & 6200 & 10 & 6600 & 10 & 9900 & 5 & 11000 & 5 & 12250 & 5 & 13450 & 5 \\
\hline 10200 & 15 & 10800 & 15 & 11500 & 15 & 10400 & 10 & 11650 & 10 & 12850 & 10 & 14100 & 10 \\
\hline 17000 & 20 & 17900 & 20 & 19000 & 20 & 12500 & 15 & 14000 & 15 & 15400 & 15 & 16900 & 15 \\
\hline 25200 & 30 & 26500 & 30 & 28100 & 30 & 19800 & 20 & 22200 & 20 & 24450 & 20 & 26800 & 20 \\
\hline 40100 & 40 & 42100 & 40 & 44000 & 40 & 29800 & 30 & 30100 & 25 & 33150 & 25 & 35150 & 25 \\
\hline 80200 & 50 & 84200 & 50 & 87000 & 50 & 44000 & 40 & 38000 & 30 & 41800 & 30 & 44300 & 30 \\
\hline \multirow[t]{6}{*}{160400} & 60 & 168400 & 60 & 173000 & 60 & 92650 & 50 & 48900 & 35 & 50500 & 35 & 53550 & 35 \\
\hline & & & & & & 184250 & 60 & 52950 & 40 & 58250 & 40 & 61750 & 40 \\
\hline & & & & & & & & 91650 & 45 & 100800 & 45 & 106850 & 45 \\
\hline & & & & & & & & 129800 & 50 & 142750 & 50 & 147050 & 50 \\
\hline & & & & & & & & 168000 & 55 & 184800 & 55 & 190350 & 55 \\
\hline & & & & & & & & 206300 & 60 & 226900 & 60 & 226900 & 60 \\
\hline 1977 & & 1978 & & 1979 & & 1980 & & 1981 & & 1982 & & 1983 & \\
\hline 14500 & 5 & 15850 & 5 & 17450 & 5 & 19780 & 5 & 22460 & 5 & 25240 & 5 & 27540 & 5 \\
\hline 15200 & 10 & 16600 & 10 & 18250 & 10 & 20680 & 10 & 23480 & 10 & 26380 & 10 & 28780 & 10 \\
\hline 18200 & 15 & 19850 & 15 & 21650 & 15 & 24540 & 15 & 27860 & 15 & 31280 & 15 & 34140 & 15 \\
\hline 28800 & 20 & 31400 & 20 & 34250 & 20 & 38820 & 20 & 44060 & 20 & 49480 & 20 & 53980 & 20 \\
\hline 37800 & 25 & 41250 & 25 & 44550 & 25 & 49900 & 25 & 56640 & 25 & 63620 & 25 & 69400 & 25 \\
\hline 47600 & 30 & 51850 & 30 & 56000 & 30 & 62720 & 30 & 71180 & 30 & 79940 & 30 & 87220 & 30 \\
\hline 57550 & 35 & 62700 & 35 & 67750 & 35 & 75940 & 35 & 86120 & 35 & 96740 & 35 & 105520 & 35 \\
\hline 66400 & 40 & 72350 & 40 & 78150 & 40 & 87540 & 40 & 99360 & 40 & 111580 & 40 & 121740 & 40 \\
\hline 114850 & 45 & 125200 & 45 & 130250 & 45 & 145880 & 45 & 138590 & 55 & 155990 & 55 & 170600 & 55 \\
\hline 158050 & 50 & 172250 & 50 & 179150 & 50 & 200640 & 50 & 153200 & 60 & 172040 & 60 & 187700 & 60 \\
\hline 201800 & 55 & 211900 & 55 & 211900 & 55 & 237320 & 55 & & & 195000 & 65 & 212750 & 65 \\
\hline 238200 & 60 & 250100 & 60 & 250100 & 60 & 270000 & 60 & & & & & & \\
\hline 1984 & & 1985 & & 1986 & & 1987 & & 1988 & & 1989 & & 1990 & \\
\hline 29640 & 5 & 31300 & 5 & 32060 & 5 & 33120 & 5 & 34000 & 5 & 35140 & 5 & 36280 & 5 \\
\hline 30980 & 10 & 32720 & 10 & 33520 & 10 & 34640 & 9,6 & 35560 & 9,6 & 36740 & 9,6 & 37920 & 9,6 \\
\hline 36740 & 15 & 38800 & 15 & 39740 & 15 & 41060 & 14,4 & 42140 & 14,4 & 43540 & 14,4 & 44940 & 14,4 \\
\hline 58100 & 20 & 61360 & 20 & 62840 & 20 & 64920 & 19,2 & 66620 & 19,2 & 68820 & 19,2 & 71040 & 19,2 \\
\hline 74680 & 25 & 78880 & 25 & 80780 & 25 & 83460 & 24 & 85640 & 24 & 88480 & 24 & 91320 & 24 \\
\hline 93840 & 30 & 99100 & 30 & 101480 & 30 & 104820 & 28,8 & 107540 & 28,8 & 111080 & 28,8 & 114640 & 28,8 \\
\hline 113540 & 35 & 119900 & 35 & 116210 & 40 & 124670 & 43,2 & 127900 & 43,2 & 132170 & 43,2 & 136430 & 43,2 \\
\hline 131000 & 40 & 138340 & 40 & 118020 & 45 & 167670 & 49,0 & 172030 & 49 & 177710 & 49 & 183400 & 49 \\
\hline 183650 & 55 & 193960 & 55 & 162310 & 50 & 198330 & 53,9 & 203490 & 53,9 & 210210 & 53,9 & 216940 & 53,9 \\
\hline 201970 & 60 & 213280 & 60 & 191990 & 55 & 225610 & 56,8 & 231480 & 56,8 & 239120 & 56,8 & 246770 & 56,8 \\
\hline 228920 & 65 & 241740 & 65 & 218400 & 58 & & & & & & & & \\
\hline 1991 & & 1992 & & 1993 & & 1994 & & 1995 & & 1996 & & 1997 & \\
\hline 37380 & 5 & 38440 & 5 & 43800 & 12 & 44420 & 12 & 45220 & 12 & 51220 & 10,5 & 51780 & 10,5 \\
\hline 39060 & 9,6 & 40160 & 9,6 & 95800 & 25 & 97140 & 25 & 98880 & 25 & 100760 & 24 & 101860 & 24 \\
\hline 46300 & 14,4 & 47600 & 14,4 & 161430 & 45 & 163700 & 45 & 166660 & 45 & 173150 & 43 & 175060 & 43 \\
\hline 73180 & 19,2 & 75240 & 19,2 & 222100 & 50 & 225210 & 50 & 229260 & 50 & 233620 & 48 & 236190 & 48 \\
\hline 94060 & 24 & 96700 & 24 & 273900 & 56,8 & 277730 & 56,8 & 282730 & 56,8 & 288100 & 54 & 291270 & 54 \\
\hline 118080 & 28,8 & 121380 & 28,8 & & & & & & & & & & \\
\hline 140540 & 43,2 & 144480 & 43,2 & & & & & & & & & & \\
\hline 188900 & 49 & 194190 & 49 & & & & & & & & & & \\
\hline 223450 & 53,9 & 229710 & 53,9 & & & & & & & & & & \\
\hline 254170 & 56,8 & 261290 & 56,8 & & & & & & & & & & \\
\hline
\end{tabular}

Lecture : Au titre de l'imposition des revenus de 1970 (déclarés en 1971), la fraction du revenu imposable des foyers ayant 2B parts de quotient familial comprise entre 0 franc et 5800 francs est soumise à un taux marginal de $0 \%$, la fraction comprise entre 5800 francs et 10200 francs est soumise à un taux marginal de $10 \%$, etc..., et la fraction supérieure à 160400 francs est soumise à un taux marginal de $60 \%$. 
Tableau I-4: Les barêmes de l'impôt sur le revenu de 1970 à 1997 applicables aux foyers fiscaux ayant $2 A$ parts de quotient familial

$\begin{array}{rrrrr}1970 & & 1971 & & 1972 \\ 5800 & 10 & 6200 & 10 & 6600 \\ 10200 & 15 & 10800 & 15 & 11500 \\ 17000 & 20 & 17900 & 20 & 19000 \\ 25200 & 30 & 26500 & 30 & 28100 \\ 40100 & 40 & 42100 & 40 & 44000 \\ 80200 & 50 & 84200 & 50 & 87000 \\ 160400 & 60 & 168400 & 60 & 173000\end{array}$

$\begin{array}{rr}10 & 1973 \\ 15 & 9900 \\ 20 & 10400 \\ 30 & 19800 \\ 40 & 29800 \\ 50 & 44000 \\ 60 & 92650 \\ & 184250\end{array}$

$\begin{array}{rr}5 & 1974 \\ 10 & 11000 \\ 15 & 11650 \\ 20 & 222000 \\ 30 & 30100 \\ 40 & 38000 \\ 50 & 48900 \\ 60 & 52950 \\ & 91650 \\ 129800 \\ 168000 \\ 206300\end{array}$

$\begin{array}{rr}5 & 1975 \\ 10 & 12250 \\ 15 & 12850 \\ 20 & 24450 \\ 25 & 33150 \\ 30 & 41800 \\ 35 & 50500 \\ 40 & 58250 \\ 45 & 100800 \\ 50 & 142750 \\ 55 & 184800 \\ 60 & 226900\end{array}$

$\begin{array}{rrr} & 1976 & \\ 5 & 13450 & 5 \\ 10 & 14100 & 10 \\ 15 & 16900 & 15 \\ 20 & 26800 & 20 \\ 25 & 35150 & 25 \\ 30 & 44300 & 30 \\ 35 & 53550 & 35 \\ 40 & 61750 & 40 \\ 45 & 106850 & 45 \\ 50 & 147050 & 50 \\ 55 & 190350 & 55 \\ 60 & 226900 & 60\end{array}$

$\begin{array}{rrrrr}1977 & & 1978 & & 1979 \\ 14500 & 5 & 15850 & 5 & 17450 \\ 15200 & 10 & 16600 & 10 & 18250 \\ 18200 & 15 & 19850 & 15 & 21650 \\ 28800 & 20 & 31400 & 20 & 34250 \\ 37800 & 25 & 41250 & 25 & 44550 \\ 47600 & 30 & 51850 & 30 & 56000 \\ 57550 & 35 & 62700 & 35 & 67750 \\ 66400 & 40 & 72350 & 40 & 78150 \\ 114850 & 45 & 125200 & 45 & 130250 \\ 158050 & 50 & 172250 & 50 & 179150 \\ 201800 & 55 & 211900 & 55 & 211900 \\ 238200 & 60 & 250100 & 60 & 250100\end{array}$

$\begin{array}{rr} & 1980 \\ 5 & 19780 \\ 10 & 20680 \\ 15 & 24540 \\ 20 & 38820 \\ 25 & 49900 \\ 30 & 62720 \\ 35 & 75940 \\ 40 & 87540 \\ 45 & 145880 \\ 50 & 200640 \\ 55 & 237320 \\ 60 & 270000\end{array}$

$\begin{array}{rr}5 & 1981 \\ 10 & 22460 \\ 15 & 27480 \\ 20 & 44060 \\ 25 & 56640 \\ 30 & 71180 \\ 35 & 86120 \\ 40 & 99360 \\ 45 & 165580 \\ 50 & 227720 \\ 55 & 269360 \\ 60 & 306400\end{array}$

$\begin{array}{rr}5 & 1982 \\ 10 & 25240 \\ 15 & 26380 \\ 20 & 49480 \\ 25 & 63620 \\ 30 & 79940 \\ 35 & 96740 \\ 40 & 111580 \\ 45 & 185940 \\ 50 & 255720 \\ 55 & 302500 \\ 60 & 344080 \\ & 390000\end{array}$

$\begin{array}{rrr} & 1983 & \\ 5 & 27540 & 5 \\ 10 & 28780 & 10 \\ 15 & 34140 & 15 \\ 20 & 53980 & 20 \\ 25 & 69400 & 25 \\ 30 & 87220 & 30 \\ 35 & 105520 & 35 \\ 40 & 121740 & 40 \\ 45 & 202860 & 45 \\ 50 & 279000 & 50 \\ 55 & 330020 & 55 \\ 60 & 375400 & 60 \\ 65 & 425500 & 65\end{array}$

1984
29640
30980
36740
58100
74680
93840
113540
131000
218280
300200
355100
403940
457840

$\begin{array}{rrrr} & 1985 & & 1986 \\ 5 & 31300 & 5 & 32060 \\ 10 & 32720 & 10 & 33520 \\ 15 & 38800 & 15 & 39740 \\ 20 & 61360 & 20 & 62840 \\ 25 & 78880 & 25 & 80780 \\ 30 & 99100 & 30 & 101480 \\ 35 & 119900 & 35 & 122780 \\ 40 & 138340 & 40 & 141660 \\ 45 & 230500 & 45 & 236040 \\ 50 & 317020 & 50 & 324620 \\ 55 & 374980 & 55 & 383980 \\ 60 & 426560 & 60 & 436800 \\ 65 & 483480 & 65 & \end{array}$

$\begin{array}{rrrrrr} & 1987 & & 1988 & & 1989 \\ 5 & 33120 & 5 & 34000 & 5 & 35140\end{array}$

1990

$\begin{array}{rrrrrrrrr}15 & 41060 & 14,4 & 42140 & 14,4 & 43540 & 14,4 & 44940 & 14,4\end{array}$

$\begin{array}{lllllllll}20 & 64920 & 19,2 & 66620 & 19,2 & 68820 & 19,2 & 71040 & 19,2\end{array}$

$\begin{array}{lllllllll}25 & 83460 & 24 & 85640 & 24 & 88480 & 24 & 91320 & 24\end{array}$

$\begin{array}{lllllllll}30 & 104820 & 28,8 & 107540 & 28,8 & 111080 & 28,8 & 114640 & 28,8\end{array}$

$\begin{array}{lllllllll}35 & 126840 & 33,6 & 130140 & 33,6 & 134440 & 33,6 & 138740 & 33,6\end{array}$

$\begin{array}{lllllllll}40 & 146340 & 38,4 & 150140 & 38,4 & 155100 & 38,4 & 160060 & 38,4\end{array}$

$\begin{array}{lllllllll}45 & 243820 & 43,2 & 250160 & 43,2 & 258420 & 43,2 & 266680 & 43,2\end{array}$

$\begin{array}{lllllllll}50 & 335340 & 49 & 344060 & 49 & 355420 & 49 & 366800 & 49\end{array}$

$\begin{array}{lllllllll}55 & 396660 & 53,9 & 406980 & 53,9 & 420420 & 53,9 & 433880 & 53,9\end{array}$

$\begin{array}{lllllllll}58 & 451220 & 56,8 & 462960 & 56,8 & 478240 & 56,8 & 493540 & 56,8\end{array}$

\begin{tabular}{|c|c|c|c|c|c|c|c|c|c|c|c|c|c|}
\hline 1991 & & 1992 & & 1993 & & 1994 & & 1995 & & 1996 & & 1997 & \\
\hline 37380 & 5 & 38440 & 5 & 43800 & 12 & 44420 & 12 & 45220 & 12 & 51220 & 10,5 & 51780 & 10,5 \\
\hline 39060 & 9,6 & 40160 & 9,6 & 95800 & 25 & 97140 & 25 & 98880 & 25 & 100760 & 24 & 101860 & 24 \\
\hline 46300 & 14,4 & 47600 & 14,4 & 168600 & 35 & 170960 & 35 & 174040 & 35 & 177340 & 33 & 179300 & 33 \\
\hline 73180 & 19,2 & 75240 & 19,2 & 273000 & 45 & 276820 & 45 & 281800 & 45 & 287160 & 43 & 290320 & 43 \\
\hline 94060 & 24 & 96700 & 24 & 444200 & 50 & 450420 & 50 & 458520 & 50 & 467240 & 48 & 472380 & 48 \\
\hline 808 & 28,8 & 121380 & 28,8 & 547800 & 56,8 & 555460 & 56,8 & 565460 & 56,8 & 576200 & 54 & 582540 & 54 \\
\hline
\end{tabular}

$\begin{array}{llll}118080 & 28,8 & 121380 & 28,8 \\ 142900 & 33,6 & 146900 & 33,6\end{array}$

$164860 \quad 38,4 \quad 169480 \quad 38,4$

$274680 \quad 43,2 \quad 282380 \quad 43,2$

$\begin{array}{llll}377800 & 49 & 388380 & 49\end{array}$

$\begin{array}{llll}446900 & 53,9 & 459420 & 53,9\end{array}$

$508340 \quad 56,8 \quad 522580 \quad 56,8$

Lecture : Au titre de l'imposition des revenus de 1970 (déclarés en 1971), la fraction du revenu imposable des foyers ayant $2 \mathrm{~A}$ parts de quotient familial comprise entre 0 franc et 5800 francs est soumise à un taux marginal de $0 \%$, la fraction comprise entre 5800 francs et 10200 francs est soumise à un taux marginal de $10 \%$, etc..., et la fraction supérieure à 160400 francs est soumise à un taux marginal de $60 \%$. 
Tableau I-5: Les barêmes de l'impôt sur le revenu de 1970 à 1997 applicables aux foyers fiscaux ayant 2,5 parts de quotient familial (après plafonnement des effets du quotient familial)

$\begin{array}{rrrrr}1970 & & 1971 & & 1972 \\ 7250 & 10 & 7750 & 10 & 8250 \\ 12750 & 15 & 13500 & 15 & 14375 \\ 21250 & 20 & 22375 & 20 & 23750 \\ 31500 & 30 & 33125 & 30 & 35125 \\ 50125 & 40 & 52625 & 40 & 55000 \\ 100250 & 50 & 105250 & 50 & 108750 \\ 200500 & 60 & 210500 & 60 & 216250\end{array}$

$\begin{array}{rr} & 1973 \\ 10 & 12375 \\ 15 & 13000 \\ 20 & 15625 \\ 30 & 24750 \\ 40 & 37250 \\ 50 & 55000 \\ 60 & 115813\end{array}$

$\begin{array}{rr}5 & 1974 \\ 10 & 13750 \\ 15 & 14563 \\ 20 & 27500 \\ 30 & 3750 \\ 40 & 47500 \\ 50 & 61125 \\ 60 & 66188 \\ & 114563 \\ & 162250 \\ & 210000 \\ & 257875\end{array}$

$\begin{array}{rr} & 1975 \\ 5 & 15313 \\ 10 & 16063 \\ 15 & 19250 \\ 20 & 30563 \\ 25 & 41438 \\ 30 & 52250 \\ 35 & 63125 \\ 40 & 72813 \\ 45 & 126000 \\ 50 & 178438 \\ 55 & 231000 \\ 60 & 283625\end{array}$

$\begin{array}{rrr}5 & 1976 & \\ 10 & 16813 & 5 \\ 15 & 21125 & 10 \\ 20 & 33500 & 15 \\ 25 & 43938 & 25 \\ 30 & 55375 & 30 \\ 35 & 66938 & 35 \\ 40 & 77188 & 40 \\ 45 & 133563 & 45 \\ 50 & 183813 & 50 \\ 55 & 237938 & 55 \\ 60 & 283625 & 60\end{array}$

$\begin{array}{rrr}1977 & & 1978 \\ 18125 & 5 & 19813 \\ 19000 & 10 & 20750 \\ 22750 & 15 & 24813 \\ 36000 & 20 & 39250 \\ 47250 & 25 & 51563 \\ 59500 & 30 & 64813 \\ 71938 & 35 & 78375 \\ 83000 & 40 & 90438 \\ 143563 & 45 & 156500 \\ 197563 & 50 & 215313 \\ 252250 & 55 & 264875 \\ 297750 & 60 & 312625\end{array}$

$\begin{array}{rr}5 & 1979 \\ 10 & 21813 \\ 15 & 22813 \\ 20 & 42863 \\ 25 & 55688 \\ 30 & 70000 \\ 35 & 84688 \\ 40 & 97688 \\ 45 & 162813 \\ 50 & 223938 \\ 55 & 264875 \\ 60 & 312625\end{array}$

$\begin{array}{rr}5 & 1980 \\ 10 & 24725 \\ 15 & 25850 \\ 20 & 48675 \\ 25 & 62375 \\ 30 & 78400 \\ 35 & 94925 \\ 40 & 109425 \\ 45 & 182350 \\ 50 & 250800 \\ 55 & 296650 \\ 60 & 337500\end{array}$

$\begin{array}{rr}5 & 1981 \\ 10 & 28075 \\ 15 & 29350 \\ 20 & 54825 \\ 25 & 70800 \\ 30 & 88975 \\ 35 & 107650 \\ 40 & 124200 \\ 45 & 206975 \\ 50 & 228550 \\ 55 & 269360 \\ 60 & 306400\end{array}$

$\begin{array}{rr}5 & 1982 \\ 10 & 31550 \\ 15 & 32975 \\ 20 & 61850 \\ 25 & 79525 \\ 30 & 99925 \\ 35 & 120925 \\ 40 & 139475 \\ 45 & 232425 \\ 50 & 257190 \\ 55 & 302500 \\ 60 & 344080 \\ & 390000\end{array}$

$\begin{array}{rrr}5 & 1983 & \\ 10 & 34425 & 5 \\ 15 & 42675 & 10 \\ 20 & 67475 & 15 \\ 25 & 86750 & 25 \\ 30 & 109025 & 30 \\ 35 & 131900 & 35 \\ 40 & 152175 & 40 \\ 45 & 253575 & 45 \\ 50 & 281220 & 50 \\ 55 & 330020 & 55 \\ 60 & 375400 & 60 \\ 65 & 425500 & 65\end{array}$

$\begin{array}{rrrrrrrrrrrrrr}1984 & & 1985 & & 1986 & & 1987 & & 1988 & & 1989 & & 1990 & \\ 37050 & 5 & 39125 & 5 & 40075 & 5 & 41400 & 5 & 42500 & 5 & 43925 & 5 & 45350 & 5 \\ 38725 & 10 & 40900 & 10 & 41900 & 10 & 43300 & 9,6 & 44450 & 9,6 & 45925 & 9,6 & 47400 & 9,6 \\ 45925 & 15 & 48500 & 15 & 49675 & 15 & 51325 & 14,4 & 52675 & 14,4 & 54425 & 14,4 & 56175 & 14,4 \\ 72625 & 20 & 76700 & 20 & 78550 & 20 & 81150 & 19,2 & 83275 & 19,2 & 86025 & 19,2 & 88800 & 19,2 \\ 93350 & 25 & 98600 & 25 & 100975 & 25 & 104325 & 24 & 107050 & 24 & 110600 & 24 & 114150 & 24 \\ 117300 & 30 & 123875 & 30 & 126850 & 30 & 131025 & 28,8 & 134425 & 28,8 & 138850 & 28,8 & 143300 & 28,8 \\ 141925 & 35 & 149875 & 35 & 153475 & 35 & 158550 & 33,6 & 162675 & 33,6 & 168050 & 33,6 & 173425 & 33,6 \\ 163750 & 40 & 172925 & 40 & 177075 & 40 & 182925 & 38,4 & 187675 & 38,4 & 193875 & 38,4 & 200075 & 38,4 \\ 272850 & 45 & 288125 & 45 & 295050 & 45 & 304775 & 43,2 & 312700 & 43,2 & 323025 & 43,2 & 333350 & 43,2 \\ 302720 & 50 & 319710 & 50 & 327310 & 50 & 345400 & 49 & 354370 & 49 & 366110 & 49 & 377860 & 49 \\ 355100 & 55 & 374980 & 55 & 383980 & 55 & 396660 & 53,9 & 406980 & 53,9 & 420420 & 53,9 & 433880 & 53,9 \\ 403940 & 60 & 426560 & 60 & 436800 & 58 & 451220 & 56,8 & 462960 & 56,8 & 478240 & 56,8 & 493540 & 56,8 \\ 457840 & 65 & 483480 & 65 & & & & & & & & & \end{array}$

$\begin{array}{rrrrrrrrrrrrrr}1991 & & 1992 & & 1993 & & 1994 & & 1995 & & 1996 & & 1997 & \\ 46725 & 5 & 48050 & 5 & 54750 & 12 & 55525 & 12 & 56525 & 12 & 64025 & 10,5 & 64725 & 10,5 \\ 48825 & 9,6 & 50200 & 9,6 & 119750 & 25 & 121425 & 25 & 123600 & 25 & 125950 & 24 & 127325 & 24 \\ 57875 & 14,4 & 59500 & 14,4 & 210750 & 35 & 213700 & 35 & 217550 & 35 & 221675 & 33 & 224125 & 33 \\ 91475 & 19,2 & 94050 & 19,2 & 340590 & 45 & 345390 & 45 & 351600 & 45 & 358950 & 43 & 362900 & 43 \\ 117575 & 24 & 120875 & 24 & 444200 & 50 & 450420 & 50 & 458520 & 50 & 472960 & 48 & 478210 & 48 \\ 147600 & 28,8 & 151725 & 28,8 & 547800 & 56,8 & 555460 & 56,8 & 565460 & 56,8 & 576200 & 54 & 582540 & 54 \\ 178625 & 33,6 & 183625 & 33,6 & & & & & & & & & & \\ 206075 & 38,4 & 211850 & 38,4 & & & & & & & & & & \\ 343350 & 43,2 & 352975 & 43,2 & & & & & & & & & & \\ 389280 & 49 & 400310 & 49 & & & & & & & & & & \end{array}$

Lecture : Au titre de l'imposition des revenus de 1970 (déclarés en 1971), la fraction du revenu imposable des foyers ayant 2,5 parts de quotient familial comprise entre 0 franc et 7250 francs est soumise à un taux marginal de $0 \%$, la fraction comprise entre 7250 francs et 12750 francs est soumise à un taux marginal de $10 \%$, etc..., et la fraction supérieure à 200500 francs est soumise à un taux marginal de $60 \%$. 
Tableau I-6: Les barêmes de l'impôt sur le revenu de 1970 à 1997 applicables aux foyers fiscaux ayant 3 parts de quotient familial (après plafonnement des effets du quotient familial)

$\begin{array}{rrrrrrr}1970 & & 1971 & & 1972 & & 1973 \\ 8700 & 10 & 9300 & 10 & 9900 & 10 & 14850 \\ 15300 & 15 & 16200 & 15 & 17250 & 15 & 15600 \\ 25500 & 20 & 26850 & 20 & 28500 & 20 & 18750 \\ 37800 & 30 & 39750 & 30 & 42150 & 30 & 29700 \\ 60150 & 40 & 63150 & 40 & 66000 & 40 & 44700 \\ 120300 & 50 & 126300 & 50 & 130500 & 50 & 66000 \\ 240600 & 60 & 252600 & 60 & 259500 & 60 & 138975 \\ & & & & & & 276375\end{array}$

$\begin{array}{rr}5 & 1974 \\ 10 & 16500 \\ 15 & 17475 \\ 20 & 21000 \\ 30 & 43300 \\ 40 & 57150 \\ 50 & 73350 \\ 60 & 79425 \\ & 137475 \\ & 194700 \\ & 252000 \\ & 309450\end{array}$

$\begin{array}{rr} & 1975 \\ 5 & 18375 \\ 10 & 19275 \\ 15 & 23100 \\ 20 & 36675 \\ 25 & 49725 \\ 30 & 62700 \\ 35 & 75750 \\ 40 & 87375 \\ 45 & 151200 \\ 50 & 214125 \\ 55 & 277200 \\ 60 & 340350\end{array}$

$\begin{array}{rrr}5 & 1976 & \\ 10 & 20175 & 5 \\ 15 & 21150 & 10 \\ 20 & 40250 & 15 \\ 25 & 52725 & 20 \\ 30 & 66450 & 30 \\ 35 & 80325 & 35 \\ 40 & 92625 & 40 \\ 45 & 160275 & 45 \\ 50 & 220575 & 50 \\ 55 & 285525 & 55 \\ 60 & 340350 & 60\end{array}$

$\begin{array}{rrr}1977 & & 1978 \\ 21750 & 5 & 23775 \\ 22800 & 10 & 24900 \\ 27300 & 15 & 29775 \\ 43200 & 20 & 47100 \\ 56700 & 25 & 61875 \\ 71400 & 30 & 77775 \\ 86325 & 35 & 94050 \\ 99600 & 40 & 108525 \\ 172275 & 45 & 187800 \\ 237075 & 50 & 258375 \\ 302700 & 55 & 317850 \\ 357300 & 60 & 375150\end{array}$

$\begin{array}{rr} & 1979 \\ 5 & 26175 \\ 10 & 27375 \\ 15 & 32475 \\ 20 & 51375 \\ 25 & 66825 \\ 30 & 84000 \\ 35 & 101625 \\ 40 & 117225 \\ 45 & 195375 \\ 50 & 268725 \\ 55 & 317850 \\ 60 & 375150\end{array}$

$\begin{array}{rr}5 & 1980 \\ 10 & 29670 \\ 15 & 31020 \\ 20 & 56810 \\ 25 & 74850 \\ 30 & 94080 \\ 35 & 113910 \\ 40 & 131310 \\ 45 & 218820 \\ 50 & 300960 \\ 55 & 355980 \\ 60 & 405000\end{array}$

$\begin{array}{rr}5 & 1981 \\ 10 & 33690 \\ 15 & 41790 \\ 20 & 66090 \\ 25 & 84960 \\ 30 & 106770 \\ 35 & 129180 \\ 40 & 149040 \\ 45 & 238870 \\ 50 & 269360 \\ 55 & 306400 \\ 60 & \end{array}$

$\begin{array}{rr}5 & 1982 \\ 10 & 37860 \\ 15 & 46570 \\ 20 & 74220 \\ 25 & 95430 \\ 30 & 119910 \\ 35 & 145110 \\ 40 & 167370 \\ 50 & 268780 \\ 55 & 302500 \\ 60 & 344080 \\ & 390000\end{array}$

$\begin{array}{rrr}5 & 1983 & \\ 10 & 41310 & 5 \\ 15 & 53170 & 10 \\ 20 & 80970 & 15 \\ 25 & 104100 & 25 \\ 30 & 130830 & 30 \\ 35 & 158280 & 35 \\ 40 & 182610 & 40 \\ 50 & 293860 & 50 \\ 55 & 330020 & 55 \\ 60 & 375400 & 60 \\ 65 & 425500 & 65\end{array}$

$\begin{array}{rrrrr}1984 & & 1985 & & 1986 \\ 44460 & 5 & 46950 & 5 & 48090 \\ 46470 & 10 & 49080 & 10 & 50280 \\ 55110 & 15 & 58200 & 15 & 59610 \\ 87150 & 20 & 92040 & 20 & 94260 \\ 112020 & 25 & 118320 & 25 & 121170 \\ 140760 & 30 & 148650 & 30 & 152220 \\ 170310 & 35 & 179850 & 35 & 184170 \\ 196500 & 40 & 207510 & 40 & 212490 \\ 316320 & 50 & 334070 & 50 & 342030 \\ 355100 & 55 & 374980 & 55 & 383980 \\ 403940 & 60 & 426560 & 60 & 436800 \\ 457840 & 65 & 483480 & 65 & \end{array}$

$\begin{array}{rrrrrrrrr} & 1987 & & 1988 & & 1989 & & 1990 & \\ 5 & 49680 & 5 & 51000 & 5 & 52710 & 5 & 54420 & 5 \\ 10 & 51960 & 9,6 & 53340 & 9,6 & 55110 & 9,6 & 56880 & 9,6 \\ 15 & 61590 & 14,4 & 63210 & 14,4 & 65310 & 14,4 & 67410 & 14,4 \\ 20 & 97380 & 19,2 & 99930 & 19,2 & 103230 & 19,2 & 106560 & 19,2 \\ 25 & 125190 & 24 & 128460 & 24 & 132720 & 24 & 136980 & 24 \\ 30 & 157230 & 28,8 & 161310 & 28,8 & 166620 & 28,8 & 171960 & 28,8 \\ 35 & 190260 & 33,6 & 195210 & 33,6 & 201660 & 33,6 & 208110 & 33,6 \\ 40 & 219510 & 38,4 & 225210 & 38,4 & 232650 & 38,4 & 240090 & 38,4 \\ 50 & 360110 & 49 & 369460 & 49 & 381700 & 49 & 393950 & 49 \\ 55 & 396660 & 53,9 & 406980 & 53,9 & 420420 & 53,9 & 433880 & 53,9 \\ 58 & 451220 & 56,8 & 462960 & 56,8 & 478240 & 56,8 & 493540 & 56,8\end{array}$

$\begin{array}{rrrrrrrrrrrrrr}1991 & & 1992 & & 1993 & & 1994 & & 1995 & & 1996 & & 1997 & \\ 56070 & 5 & 57660 & 5 & 65700 & 12 & 66630 & 12 & 67830 & 12 & 76830 & 10,5 & 77670 & 10,5 \\ 58590 & 9,6 & 60240 & 9,6 & 143700 & 25 & 145710 & 25 & 148320 & 25 & 151140 & 24 & 152790 & 24 \\ 69450 & 14,4 & 71400 & 14,4 & 252900 & 35 & 256440 & 35 & 261060 & 35 & 266010 & 33 & 268950 & 33 \\ 109770 & 19,2 & 112860 & 19,2 & 408160 & 45 & 413960 & 45 & 421390 & 45 & 430740 & 43 & 435480 & 43 \\ 141090 & 24 & 145050 & 24 & 444200 & 50 & 450420 & 50 & 458520 & 50 & 478680 & 48 & 484020 & 48 \\ 177120 & 28,8 & 182070 & 28,8 & 547800 & 56,8 & 555460 & 56,8 & 555460 & 56,8 & 576200 & 54 & 582540 & 54 \\ 214350 & 33,6 & 220350 & 33,6 & & & & & & & & & & \\ 247290 & 38,4 & 254220 & 38,4 & & & & & & & & & \end{array}$

Lecture : Au titre de l'imposition des revenus de 1970 (déclarés en 1971), la fraction du revenu imposable des foyers ayant 3 parts de quotient familial comprise entre 0 franc et 8700 francs est soumise à un taux marginal de $0 \%$, la fraction comprise entre 8700 francs et 15300 francs est soumise à un taux marginal de $10 \%$, etc..., et la fraction supérieure à 240600 francs est soumise à un taux marginal de $60 \%$. 
donc passer de $40 \%$ à $50 \%$ le taux marginal effectif de ces contribuables. Les barèmes après plafonnement décrits sur les tableaux $\mathrm{I}-2$ à $\mathrm{I}-6$ sont utilisés dans la section 4 pour estimer les taux marginaux effectifs en fonction du nombre de parts de quotient familial et du niveau de revenu imposable. 


\section{Annexe $\mathrm{J}$ : Liste des références citées dans le texte}

Cette liste contient l'ensemble des articles et ouvrages citées dans cette étude, à l'exclusion des publications d'origine administrative citées par ailleurs (rapports du Conseil des Impôts, études sur l'IR publiées dans "Statistiques et Etudes Financières", publications INSEE, etc..), dont les références sont données dans les autres annexes ou dans le texte de l'étude.

Blundell, R. (1995), " The Impact of Taxation on Labor Force Participation and Labor Supply", in Taxation, Employment and Unemployment (chapter 3), OECD.

Bourguignon, F. et P.A. Chiappori (1997), "Fiscalité et redistribution. Plans pour une réforme ", Notes de la Fondation Saint-Simon $n^{\circ} 88$.

Bourguignon, F. et T. Magnac (1985), "Labor Supply and Taxation in France", Journal of Human Ressources 25, 358-389.

Bourguignon, F. et M. Martinez (1997), "Decomposition of the Changes in the Distribution of Primary Family Incomes: a Microsimulation Approach Applied to France, 1979-1994 ", mimeo, Delta.

David, C. (1987), L'impôt sur le revenu des ménages, Economica.

Diamond, P. (1997), "Optimal Income Taxation: an Example with a U-shaped Pattern of Optimal Marginal Tax Rates ", American Economic Review 35(2), 589-632.

Feenberg, D. et J. Poterba (1993), “ Income Inequality and the Incomes of Very HighIncome Taxpayers : Evidence from Tax Returns", Tax Policy and the Economy 7, 145-177.

Feldstein, M. (1995), "The Effect of Marginal Tax Rates on Taxable Income : A Panel Study of the 1986 Tax Reform Act ", Journal of Political Economy 103, 551-572.

Fleurbaey, M., S.M. Ould Biya, P. Ralle et H. Rousse (1987), "Quelques réflexions à propos d'une estimation de la courbe de Laffer en France", Revue d'Economie Politique 97(2), 226-230.

Fourçans, A . (1985), "Taux de prélèvements obligatoires, recettes de l'Etat et production: une étude empirique du cas français", Revue d'Economie Politique 95(1), 51-65.

Gordon, R. and J. Slemrod (1998), "Are "Real " Responses to Taxes Simply Income Shifting Between Corporate and Personal Tax Bases ?", in Does Atlas Shrug? The Economic Consequences of Taxing the Rich, édité par J. Slemrod, Cambridge University Press.

Goolsbee, A. (1997), "What Happens When You Tax the Rich? Evidence from Executive Compensation ", NBER Working Paper nº633.

Hammermesh, D. (1993), Labor Demand, Princeton University Press. 
Jäntti, M. (1994), "A more efficient estimate of the effect of macroeconomic activity on the distribution of income ", The Review of Economics and Statistics LXXXVI(2), 372-378.

Lacoude, P. (1995), "Etude empirique de l'effet Laffer en France au cours des années 1980 ", Revue Française d'Economie 105(3), 101-156.

Loquet, R., J. Rafaliarison et A. Trannoy (1993), "L'évolution du caractère égalitaire du prélèvement fiscalo-social sous la cinquième République : un complément", Economie et Prévision 110-111, 81-104.

Lindsay, L. (1987), “Individual Taxpayer Response to Tax Cuts : 1982-1984 (With Implications for the Revenue Maximizing Tax Rate)", Journal of Public Economics 33, 173-206.

Maillard, D. (1989), “L'appréciation de la progressivité d'un impôt : une méthode et des illustrations ", Economie et Prévision 90, 117-124.

Navratil, J. (1995), "The Economic Recovery Tax Act of 1981: Evidence on Individual Taxpayer Behavior from Panel Tax Return Data", PhD Dissertation chapter, Harvard.

Nizet, J.-Y. (1991), Fiscalité, économie et politique : l'impôt en France, 1945-1990, LGDJ.

Nolan, B. (1987a), Income distribution and the macroeconomy, Cambridge University Press.

Nolan, B. (1987b), "Cyclical fluctuations in factor shares and the size distribution of income ", The Review of Income and Wealth 33(2), 193-210.

Nolan, B. (1989), "Macroeconomic conditions and the size distribution of income", Journal of Post-Keynesian Economics XI(2), 197-221.

Piketty, T. (1997a), L'économie des inégalités, La Découverte, collection " Repères ".

Piketty, T. (1997b), "La redistribution fiscale face au chômage ", Revue Française d’Economie XII(1), 157-201.

Piketty, T. (1997c), "Les créations d'emploi en France et aux Etats-Unis : "services de proximité " contre "petits boulots " ?", Notes de la Fondation Saint-Simon n ${ }^{\circ} 93$ (cf. également Revue de la CFDT $n^{\circ} 4$, novembre 1997).

Piketty, T. (1998), “L'impact des incitations financières au travail sur les comportements individuels: une estimation pour le cas français", Economie et Prévision (à paraître).

Quandt, R. (1966), "Old and New Methods of Estimation and the Pareto Distribution ", Metrika 10, 55-82. 
Saez, E. (1998a), "Responses to Federal Income Tax Changes in the Inter-war Period ", PhD Dissertation chapter, MIT.

Saez, E. (1998b), "Using Elasticities to Derive Optimal Income Tax Rates ", PhD Dissertation chapter, MIT.

Slemrod, J. (1995), “ Income Creation or Income Shifting ? Behavioral Responses to the Tax Reform Act of 1986 ", American Economic Review 85(2), 175-180.

Slemrod, J. (1996), " High Income Families and the Tax Changes of the 1980s: The Anatomy of Behavioral Response ", in Empirical Foundations of Household Taxation, University of Chicago Press.

Théret, B. et D. Uri (1987), "Pression fiscale limite, prélèvements obligatoires et production marchande: à propos de récentes estimations économétriques d'une courbe de Laffer pour la France ", Economie Appliquée XL(1), 115-159.

Trannoy, A., R. Jehanne, M. Lebreton et P. Moyes (1991), "L'évolution du caractère égalitaire du couple impôt sur le revenu-cotisations sociales sous la cinquième République ", Economie et Prévision 98, 1-19. 


\section{Table des matières}

\section{Introduction (p.1)}

1.1. Objectif et méthodologie de cette étude (p.1)

1.1.1. Les " expériences naturelles" utilisées (p.2)

1.1.2. La nécessité d'utiliser des données annuelles sur longue période (p.4)

1.2. Sources et données utilisées (p.5)

1.3. Organisation de ce texte (p.9)

2. L'impôt sur le revenu de 1970 à 1996 : quelques données de cadrage (p.11)

2.1. Les règles de calcul de l'IR de 1970 à 1996 (p.11)

2.1.1. L'évolution du barème de l'impôt sur le revenu (p.11)

2.1.2. Décote, majorations et minorations d'impôt (p.13)

2.1.3. Modifications apportées au mécanisme du quotient familial (p.17)

2.2. Le nombre de foyers, le revenu moyen et les recettes de l'IR de 1970 à 1996 (p.20)

3. L'évolution de la part et de la composition des hauts revenus de 1970 à 1996 (p.28)

3.1. Hauts revenus, très hauts revenus et très très hauts revenus : premiers repères (p.28)

3.2. L'évolution de la part des hauts revenus de 1970 à 1996 (p.32)

3.3. L'évolution de la composition des hauts revenus de 1970 à 1996 (p.41)

4. Estimations de l'élasticité du revenu imposable (p.47)

4.1. Estimations effectuées à partir des foyers fiscaux ayant 1 part de QF (p.47)

4.1.1. La création de la tranche à $65 \%(1981-1982)$ (p.51)

4.1.2. La suppression de la tranche à $65 \%$ (1986-1987) (p.57)

4.2. Estimations effectuées à partir des foyers de célibataires $(\mathrm{QF}=1,1,5$ et $2 \mathrm{~B})$ (p.59)

4.2.1. La création de la tranche à $65 \%$ et la mise en place du plafonnement des effets du quotient familial (1981-1982) (p.59)

4.2.2. La suppression de la tranche à $65 \%$ et l'abaissement du seuil de plafonnement du QF pour les foyers ayant 2B parts de quotient familial (19861987) (p.64)

4.3. Estimations effectuées à partir des foyers de couples mariés ( $Q F=2 A, 2,5$ et 3 ) (p.67)

4.3.1. La création de la tranche à $65 \%$ et la mise en place du plafonnement des effets du quotient familial (1981-1982) (p.67)

4.3.2. La suppression de la tranche à $65 \%$ (1986-1987) (p.68) 
5. Conclusions et perspectives en termes de politique économique (p.75)

Table des matières (p.169)

Liste des tableaux et graphiques du texte (p.171)

Liste des annexes (p.173)

Liste des tableaux des annexes (p.174) 


\section{Liste des tableaux et graphiques du texte}

Tableau 2-1: Les barèmes de l'IR de 1970 à 1997 (en francs courants) (p.12) Tableau 2-2: La croissance du nombre de foyers fiscaux de 1970 à 1996 (p.21)

Tableau 2-3: La croissance du revenu imposable de 1970 à 1996 (p.23)

Tableau 2-4: Les recettes de l'impôt sur le revenu de 1970 à 1996 (p.25)

Tableau 3-1: La distribution du revenu fiscal déclaré et du revenu imposable en 1995 (p.29)

Tableau 3-2: La composition du revenu fiscal déclaré en 1995 (p.31)

Tableau 3-3: L'évolution de la part des hauts revenus dans le revenu imposable de 1970 à 1996 (ensemble des foyers fiscaux) (p.34)

Tableau 3-4: L'évolution de la part des hauts revenus dans le revenu imposable de 1970 à 1996 (ensemble des foyers fiscaux) (p.35)

Tableau 3-5 : Les taux de croissance annuels de P95-99/P90-95, P99-99,5/P90-95, etc.., de 1971 à 1996 (ensemble des foyers fiscaux) (p.38)

Tableau 3-6 : L'évolution de la composition du revenu imposable des hauts revenus de 1970 à 1996 (ensemble des foyers fiscaux) (pp.42-43)

Tableau 4-1: L'évolution de la part des hauts revenus dans le revenu imposable de 1970 à 1996 (foyers fiscaux ayant 1 part de quotient familial) (p.48)

Tableau 4-2: L'évolution de la part des hauts revenus dans le revenu imposable de 1970 à 1996 (foyers fiscaux ayant 1 part de quotient familial) (p.49)

Tableau 4-3 : Les taux de croissance annuels de P98-99/P95-98, P99-99,5/P95-98, etc.., de 1971 à 1996 (foyers fiscaux ayant 1 part de quotient familial) (p.50)

Graphique 4-1 : Les seuils des fractiles et des tranches supérieures de 1970 à 1996 (foyers fiscaux ayant 1 part de quotient familial) (p.52)

Tableau 4-4: Estimation de l'élasticité du revenu imposable à partir des foyers fiscaux ayant 1 part de quotient familial (p.53)

Graphique 4-2 : Les seuils des fractiles et des tranches supérieures de 1970 à 1996 (foyers fiscaux ayant 1,5 parts de quotient familial) (p.60)

Tableau 4-5: Estimation de l'élasticité du revenu imposable à partir des foyers fiscaux ayant 1,5 parts de quotient familial (p.62)

Tableau 4-6: Estimation de l'élasticité du revenu imposable à partir des foyers fiscaux ayant 2B parts de quotient familial (p.63)

Tableau 4-7 : Comparaison entre les foyers de célibataires, 1985-1995 (p.66)

Tableau 4-8: Estimation de l'élasticité du revenu imposable à partir des foyers fiscaux ayant $2 \mathrm{~A}$ parts de quotient familial (p.69)

Tableau 4-9: Estimation de l'élasticité du revenu imposable à partir des foyers fiscaux ayant 2,5 parts de quotient familial (p.70) 
Tableau 4-10: Estimation de l'élasticité du revenu imposable à partir des foyers fiscaux ayant 3 parts de quotient familial (p.71)

Tableau 4-11 : Comparaison entre les foyers de couples mariés, 1985-1995 (p.74) 


\section{Liste des annexes}

Annexe A : Les sources statistiques sur l'impôt sur le revenu en France (p.77)

A.1. Les échantillons de déclarations constitués par la DGI (p.77)

A.2. Les enquêtes "Revenus fiscaux" réalisées par l'INSEE (p.79)

A.3. Les statistiques des émissions de rôles (Etats 1921) (p.80)

Annexe B : Les tableaux statistiques des Etats 1921 (p.83)

B.1. Les tableaux statistiques utilisés dans cette étude (p.83)

B.2. Les autres tableaux statistiques des Etats 1921 (p.85)

B.3. Références des numéros de "Statistiques et Etudes Financières " consacrés à l'impôt sur le revenu (p.86)

Annexe C: Méthodologie de l'estimation d'une série continue portant sur nombre de foyers fiscaux et le revenu imposable moyen sur la période 1970-1996 (p.89)

C.1. Estimation d'une série continue du nombre total de foyers fiscaux, 1970-1996 (p.89)

C.2. Estimation d'une série continue de revenu imposable total, 1970-1996 (p.93)

C.3. Estimations de séries continues pour le nombre de foyers fiscaux et le revenu imposable moyen en fonction du nombre de parts de quotient familial, 1970-1996 (p.96)

Annexe D: Méthodologie des estimations de la distribution du revenu imposable effectuées à partir des Etats 1921 (p.105)

D.1. L'estimation de la distribution du revenu imposable pour l'ensemble des foyers fiscaux (tous nombres de parts de quotient familial confondus) (p.105)

D.2. L'estimation de la distribution du revenu imposable en fonction du nombre de part de quotient familial (p.110)

D.3. L'estimation de la composition du revenu imposable (p.111)

Annexe E: Fiabilité des estimations de la distribution du revenu imposable effectuées à partir des Etats 1921 (p.125)

E.1. Comparaison avec les estimations issues des échantillons légers de déclarations de revenus, 1988-1995 (p.125)

E.2. Comparaison avec les estimations issues des enquêtes "Revenus fiscaux", 1970-1990 (p.128)

E.3. Comparaison avec les estimations issues des dossiers "Modèle lourd ", 19831993 (p.129)

Annexe F : Calculs effectués à partir des "échantillons légers", 1988-1995 (p.137)

Annexe G: Calculs effectués à partir des enquêtes "Revenus fiscaux", 1970-1990 (p.146)

Annexe H: Les tableaux statistiques des dossiers "Modèle lourd", 1979-1993 (p.155)

Annexe I : Les seuils de plafonnement du quotient familial, 1981-1997 (p.157) 
Annexe J : Liste des références citées dans le texte (p.165)

\section{Liste des tableaux des annexes}

Tableau C-1: Estimation du nombre de foyers fiscaux imposables et nonimposables, 1970-1996 (p.91)

Tableau C-2 : Estimation du revenu imposable total, 1970-1996 (p.94)

Tableau C-3 : L'évolution du nombre de foyers fiscaux en fonction du nombre de parts de quotient familial dans les Etats 1921 de 1970 à 1996 (p.97)

Tableau C-4 : L'évolution du revenu imposable moyen en fonction du nombre de parts de quotient familial dans les Etats 1921 de 1970 à 1996 (p.98)

Tableau C-5: L'évolution du nombre de foyers et du revenu imposable moyen en fonction du nombre de parts de quotient familial dans les enquêtes "Revenus fiscaux " de 1970 à 1990 (p.99)

Tableau C-6: Estimations du nombre de foyers fiscaux et du revenu imposable moyen en fonction du nombre de parts de quotient familial, 1970-1996 (p.100)

Tableau C-7: Estimations du nombre de foyers fiscaux et du revenu imposable moyen en fonction du nombre de parts de quotient familial, 1970-1996 (p.101)

Tableau D-1: Les seuils de revenu imposable et les ratios $y^{*}(y) / y$ utilisés pour l'estimation de la distribution du revenu imposable de l'ensemble des foyers fiscaux (tous nombres de parts confondus) (p.107)

Tableau D-2 : Les seuils P90, P95, P99, P99,5, P99,9 et P99,99 de la distribution du revenu imposable de l'ensemble des foyers fiscaux (p.109)

Tableau D-3: Les seuils de revenu imposable et les ratios $y^{*}(y) / y$ utilisés pour l'estimation de la distribution du revenu imposable des foyers fiscaux ayant 1 part de quotient familial (p.112)

Tableau D-4 : Les seuils P90, P95, P99, P99,5, P99,9 et P99,99 de la distribution du revenu imposable des foyers fiscaux ayant 1 part de quotient familial (p.113)

Tableau D-5: Les seuils de revenu imposable et les ratios $y^{*}(y) / y$ utilisés pour l'estimation de la distribution du revenu imposable des foyers fiscaux ayant 1,5 parts de quotient familial (p.114)

Tableau D-6: Résultats de l'estimation de la distribution du revenu imposable des foyers ayant 1,5 parts de quotient familial (p.115)

Tableau D-7: Les seuils de revenu imposable et les ratios $y^{*}(y) / y$ utilisés pour l'estimation de la distribution du revenu imposable des foyers fiscaux ayant 2B parts de quotient familial (p.116)

Tableau D-8 : Résultats de l'estimation de la distribution du revenu imposable des foyers ayant $2 B$ parts de quotient familial (p.117)

Tableau D-9: Les seuils de revenu imposable et les ratios $y^{*}(y) / y$ utilisés pour l'estimation de la distribution du revenu imposable des foyers fiscaux ayant $2 A$ parts de quotient familial (p.118)

Tableau D-10: Résultats de l'estimation de la distribution du revenu imposable des foyers ayant $2 A$ parts de quotient familial (p.119)

Tableau D-11: Les seuils de revenu imposable et les ratios $y^{*}(y) / y$ utilisés pour l'estimation de la distribution du revenu imposable des foyers fiscaux ayant 2,5 parts de quotient familial (p.120)

Tableau D-12: Résultats de l'estimation de la distribution du revenu imposable des foyers ayant 2,5 parts de quotient familial (p.121) 
Tableau D-13: Les seuils de revenu imposable et les ratios $\mathrm{y}^{*}(\mathrm{y}) / \mathrm{y}$ utilisés pour l'estimation de la distribution du revenu imposable des foyers fiscaux ayant 3 parts de quotient familial (p.122)

Tableau D-14: Résultats de l'estimation de la distribution du revenu imposable des foyers ayant 3 parts de quotient familial (p.123)

Tableau E-1 : Comparaison entre les chiffres bruts issus des échantillons légers et les estimations effectuées à partir des Etats 1921 (p.126)

Tableau E-2 : Comparaison entre les chiffres bruts issus des échantillons légers et les estimations effectuées à partir des échantillons légers (p.130)

Tableau E-3: Comparaison entre les chiffres bruts issus des enquêtes "Revenus fiscaux " et les estimations effectuées à partir des Etats 1921 (p.131)

Tableau E-4: Comparaison entre les chiffres bruts issus des enquêtes "Revenus fiscaux " et les estimations effectuées à partir des enquêtes " RF " (p.132)

Tableau E-5: Les seuils de revenu imposable et les ratios $y^{*}(y) / y$ utilisés pour les estimations effectuées à partir des dossiers "Modèle lourd" (p.133)

Tableau E-6 : Comparaison entre les estimations effectuées à partir des dossiers "Modèle lourd " et les estimations effectuées à partir des Etats 1921 (p.134)

Tableau E-7 : Comparaison entre les chiffres bruts issus des échantillons légers et les estimations effectuées à partir des dossiers "Modèle lourd " (p.135)

Tableau F-1: La distribution du revenu fiscal déclaré et du revenu imposable en 1988 (p.138)

Tableau F-2: La composition du revenu fiscal déclaré en 1988 (p.138)

Tableau F-3: La distribution du revenu fiscal déclaré et du revenu imposable en 1989 (p.139)

Tableau F-4: La composition du revenu fiscal déclaré en 1989 (p.139)

Tableau F-5: La distribution du revenu fiscal déclaré et du revenu imposable en 1990 (p.140)

Tableau F-6: La composition du revenu fiscal déclaré en 1990 (p.140)

Tableau F-7: La distribution du revenu fiscal déclaré et du revenu imposable en 1991 (p.141)

Tableau F-8: La composition du revenu fiscal déclaré en 1991 (p.141)

Tableau F-9: La distribution du revenu fiscal déclaré et du revenu imposable en 1992 (p.142)

Tableau F-10: La composition du revenu fiscal déclaré en 1992 (p.142)

Tableau F-11: La distribution du revenu fiscal déclaré et du revenu imposable en 1993 (p.143)

Tableau F-12: La composition du revenu fiscal déclaré en 1993 (p.143)

Tableau F-13: La distribution du revenu fiscal déclaré et du revenu imposable en 1994 (p.144)

Tableau F-14 : La composition du revenu fiscal déclaré en 1994 (p.144)

Tableau G-1: La distribution du revenu fiscal déclaré et du revenu imposable en 1970 (p.148)

Tableau G-2: La composition du revenu fiscal déclaré en 1970 (p.148) 
Tableau G-3: La distribution du revenu fiscal déclaré et du revenu imposable en 1975 (p.149)

Tableau G-4: La composition du revenu fiscal déclaré en 1975 (p.149)

Tableau G-5: La distribution du revenu fiscal déclaré et du revenu imposable en 1979 (p.150)

Tableau G-6: La composition du revenu fiscal déclaré en 1979 (p.150)

Tableau G-7: La distribution du revenu fiscal déclaré et du revenu imposable en 1984 (p.151)

Tableau G-8: La composition du revenu fiscal déclaré en 1984 (p.151)

Tableau G-9: La distribution du revenu fiscal déclaré et du revenu imposable en 1990 (p.152)

Tableau G-10: La composition du revenu fiscal déclaré en 1990 (p.152)

Tableau I-1 : Les seuils de plafonnement du quotient familial de 1981 à 1997 (p.158) Tableau I-2: Les barèmes de I'IR de 1970 à 1997 applicables aux foyers fiscaux ayant 1,5 parts de quotient familial (après plafonnement du quotient familial) (p.159) Tableau I-3: Les barèmes de I'IR de 1970 à 1997 applicables aux foyers fiscaux ayant 2B parts de quotient familial (après plafonnement du quotient familial) (p.160) Tableau I-4: Les barèmes de I'IR de 1970 à 1997 applicables aux foyers fiscaux ayant $2 A$ parts de quotient familial (p.161)

Tableau l-5: Les barèmes de I'IR de 1970 à 1997 applicables aux foyers fiscaux ayant 2,5 parts de quotient familial (après plafonnement du quotient familial) (p.162) Tableau I-6: Les barèmes de I'IR de 1970 à 1997 applicables aux foyers fiscaux ayant 3 parts de quotient familial (après plafonnement du quotient familial) (p.163) 